\title{
Development and Application of
} Europe-Wide Outdoor Air Pollution Exposure Models for Epidemiological Studies $\forall$

L

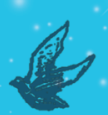
of Mortality Effects
$1 \times$
$E$
Jie Chen

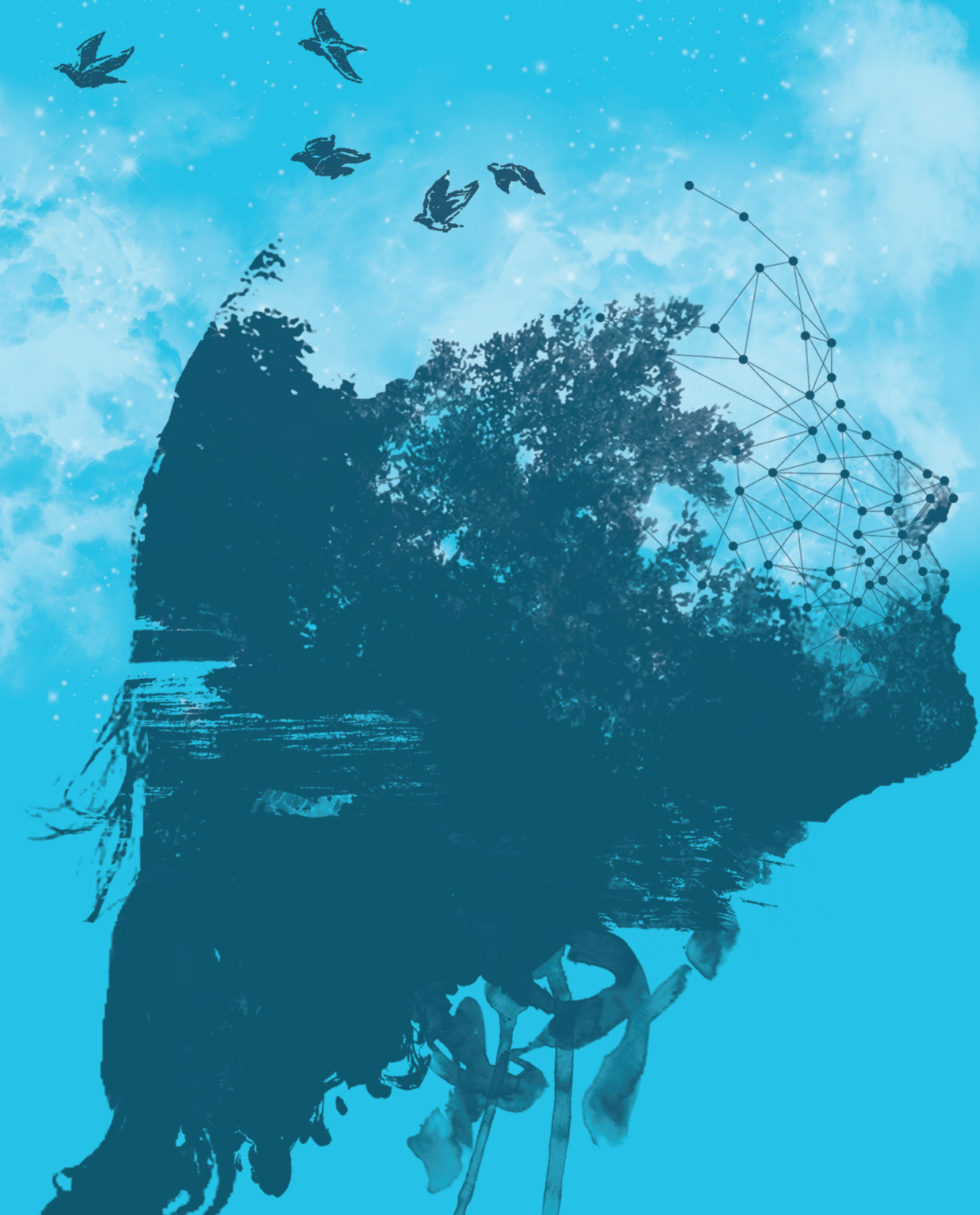





\section{Development and Application of Europe-Wide Outdoor Air Pollution Exposure Models for Epidemiological Studies of Mortality Effects}


Doctoral thesis, Utrecht University

(C) Jie Chen, 2021

All rights reserved. No part of this publication may be reproduced or transmitted in any form or by any means without written permission from the author. The copyrights of published manuscripts have been transferred to the respective journal publishers.

Cover art and layout by Xinye Cui and Zijun Xue

Content layout by Jie Chen

Print by Proefschrift-AIO

DOI: $10.33540 / 453$

ISBN: 978-94-93184-90-9 


\title{
Development and Application of Europe-Wide Outdoor Air Pollution Exposure Models for Epidemiological Studies of Mortality Effects
}

Ontwikkeling en toepassing van modellen voor het schatten van de blootstelling aan verontreiniging van de buitenlucht, ten behoeve van epidemiologisch onderzoek naar effecten op sterfte

(met een samenvatting in het Nederlands)

\section{Proefschrift}

\author{
ter verkrijging van de graad van doctor aan de \\ Universiteit Utrecht \\ op gezag van de \\ rector magnificus, prof.dr. H.R.B.M. Kummeling, \\ ingevolge het besluit van het college voor promoties \\ in het openbaar te verdedigen op \\ dinsdag 22 juni 2021 des middags te 4.15 uur
}

door

Jie Chen

geboren op 25 december 1990

te Quanzhou, China 


\section{Promotor:}

Prof. dr. B. Brunekreef

\section{Copromotoren:}

Dr. G. Hoek

Dr. M.M. Strak

This thesis was accomplished with financial support from the China Scholarship Council. 


\section{Table of Contents}

$\begin{array}{lll}\text { Chapter } 1 & \text { General Introduction } & 1\end{array}$

Chapter 2 Long-Term Exposure to PM and All-Cause and Cause- 11

Specific Mortality: A Systematic Review and Meta-Analysis

Chapter 3 Spatial $\mathrm{PM}_{2.5}, \mathrm{NO}_{2}, \mathrm{O}_{3}$ and $\mathrm{BC}$ Models for Western Europe Evaluation of Spatiotemporal Stability

Chapter 4 A Comparison of Linear Regression, Regularization, and Machine Learning Algorithms to Develop Europe-Wide Spatial Models of

Fine Particles and Nitrogen Dioxide

Chapter 5 Development of Europe-Wide Models for Particle

Elemental Composition Using Supervised Linear Regression and Random

Forest

Chapter 6 Long-Term Exposure to Fine Particle Elemental

Components and Natural and Cause-Specific Mortality - A Pooled Analysis

of Eight European Cohorts Within the ELAPSE Project

Chapter 7 General Discussion

Samenvatting

Affiliations of Coauthors 

Chapter

General Introduction 


\section{Air Pollution and Health Effects}

Exposure to air pollution has been associated with a variety of adverse health effects, ranging from subtle biochemical and physiological changes to severe illness and death ${ }^{1}$. The Global Burden of Disease Study (GBD 2015) identified air pollution as a leading contributor to the global disease burden². Mortality is one of the most often considered outcomes in epidemiological studies and risk and health impact analyses, because it is the most serious outcome and population mortality information is usually available from routinely collected data. Epidemiological studies from different regions have reported acute and chronic effects of air pollution exposure on mortality particularly from cardiovascular diseases and respiratory disease ${ }^{3-5}$. Acute effects of air pollution are usually investigated by time-series and case-crossover study designs linking short-term air pollution exposure to health outcomes ${ }^{6-8}$, while chronic effects are often investigated by cohort and case-control study designs investigating long-term air pollution exposure risks ${ }^{9-11}$. The epidemiological findings are supported by observations from controlled human exposure studies, animal toxicology and mechanistic in vitro studies, which suggest that systemic inflammation and oxidative stress are the possible underlying mechanisms of the associations between air pollution and morbidity and mortality ${ }^{12-15}$. This evidence has led to the adoption of ambient air quality standards to safeguard the public from the most common toxic air pollutants. In 2005, the World Health Organization (WHO) published air quality guidelines for worldwide use ${ }^{16}$. Local and national air quality standards were developed by organizations such as the European Union (EU) ${ }^{17}$ and the US Environmental Protection Agency (EPA) ${ }^{18}$ according to specific approaches to balancing risks to health, technological feasibility, economic considerations and other political and social factors. Over the last decades, levels of air pollution have declined significantly in North America and Europe with the introduction of cleaner fuels and the implementation of pollution control technologies ${ }^{19}$. Questions about associations at these lower pollution levels have been raised ${ }^{20}$. With the development of more accurate exposure assessment methods and the application of more sensitive statistical techniques, recent studies have suggested that the adverse health effects associated with air pollution persist at levels below the current air quality guidelines and standards ${ }^{5,21-23}$. This has raised questions about the appropriateness of the existing standards and guideline values. In 2016, WHO has started the update of Global Air Quality Guidelines for ambient air ${ }^{24}$.

Air pollution is a complex mixture of gases and particles from natural and anthropogenic sources. The current thesis focuses on ozone $\left(\mathrm{O}_{3}\right)$, nitrogen dioxide $\left(\mathrm{NO}_{2}\right)$, particulate matter $(\mathrm{PM})$ and black carbon (BC). $\mathrm{O}_{3}$ and most $\mathrm{NO}_{2}$ are secondary pollutants formed through a series of complex atmospheric chemical reactions. The principle sources of anthropogenic emissions of $\mathrm{NO}_{2}$ are the combustion processes in stationary sources (e.g., heating and electricity generation) and in mobile sources (e.g., motor vehicles). Most of $\mathrm{NO}_{2}$ is first emitted as nitric oxide (NO) and then converted to $\mathrm{NO}_{2}$ via reaction with atmospheric oxidants such as $\mathrm{O}_{3}$, where $\mathrm{O}_{3}$ is transformed into $\mathrm{O}_{2}$. As a result, the $\mathrm{O}_{3}$ concentrations are often low in busy urban centers, where strong emission sources of nitrogen oxides are present, and higher in suburban and adjacent rural areas ${ }^{1}$. The photostationary cycle is described in reactions (1) to (3) below. When high sunlight intensity and chemically reactive hydrocarbons are present, the photochemical equilibrium is disturbed. $\mathrm{NO}$ converted to $\mathrm{NO}_{2}$ without consuming $\mathrm{O}_{3}$, as presented in reaction (4), and thus high concentrations of $\mathrm{O}_{3}$ are produced.

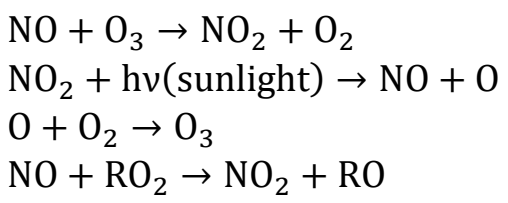

PM is a complex mixture of components with diverse chemical and physical characteristics. PM has been classified by aerodynamic diameter for current regulatory purpose ${ }^{16}$ : PM with an aerodynamic diameter $\leq 10 \mu \mathrm{m}\left(\mathrm{PM}_{10}\right)$ includes inhalable particles that are sufficiently small to penetrate to the thoracic region; $\mathrm{PM}$ with an aerodynamic diameter $\leq 2.5 \mu \mathrm{m}\left(\mathrm{PM}_{2.5}\right)$ is able to penetrate into and deposit 
in the smaller conducting airways and alveoli. The difference between $\mathrm{PM}_{10}$ and $\mathrm{PM}_{2.5}$ is often refer to as coarse particles $\left(\mathrm{PM}_{10-2.5}\right)$. The coarse particles are composed largely of crustal materials, sea salt, resuspended dust from roads and industrial activities, and biological material. The fine particles are largely formed in the atmosphere from gases. The main precursor gases are sulfur dioxide $\left(\mathrm{SO}_{2}\right), \mathrm{NO}_{2}$, ammonia and volatile organic compounds. For example, $\mathrm{SO}_{2}$ is oxidized to sulfuric acid, $\mathrm{NO}_{2}$ is oxidized to nitric acid, which in turn reacts with ammonia to form ammonium nitrate. The particles that are converted from gases in atmospheric reactions are called secondary particles. The fine fraction also includes primary particles generated from combustion processes, such as metals or organic compounds. Therefore, the fine fraction is typically composed of nitrate, sulfate, ammonium, organic compounds, trace metals and $\mathrm{BC}$. BC is the sooty black material emitted from burning fossil fuels and biomass, for example in diesel engines, coal-fired power plants and wood burning. $\mathrm{BC}$ in this thesis was measured as $\mathrm{PM}_{2.5}$ absorbance based on the blackness of $\mathrm{PM}_{2.5}$ filters ${ }^{25}$. $\mathrm{PM}_{2.5}$ absorbance is a proxy for elemental carbon (EC), which is predominantly in the fine particle fraction ${ }^{26} . \mathrm{PM}_{2.5}$ is considered to be particularly important among the pollutants because it can penetrate deeply in the lungs, it has long atmospheric lifetime and travels across large geographical areas, and it can easily infiltrate into homes, where people spend a large amount of their time.

$\mathrm{PM}_{2.5}$ has been associated with increases in mortality and morbidity more than any other regulated environmental pollutant ${ }^{2,15}$. Epidemiological studies around the world have generally reported associations between $\mathrm{PM}_{2.5}$ mass and mortality and morbidity, with substantial differences in the magnitude of effect estimates ${ }^{7,9,22,27-29}$. Part of these differences may be related to the fact that the composition of $\mathrm{PM}_{2.5}$ varies in time and space depending on sources of emission and atmospheric chemistry, which may in turn result in differences in toxicity and risk to health of $\mathrm{PM}_{2.5}$ mass ${ }^{30-33}$. This implies current regulations that focus on mass concentrations may not be using the most efficient metrics. Understanding which components of the PM mixture are of greater health concerns than others would help inform targeted policies to control for those sources that contribute most of the toxic components in the PM mixture. So far, studies have associated adverse health effects with a series of $\mathrm{PM}_{2.5}$ components including secondary inorganic aerosols (sulfate, nitrate), EC, metals and organic components ${ }^{34-39}$. Studies also used source apportionment techniques to identify the responsible sources of specific components such as coal combustion and traffic $\mathrm{c}^{40-42}$. However, the findings are inconsistent. The National Particle Component Toxicity (NPACT) studies funded by the Health Effect Institute (HEI) have performed a systematic evaluation on the relative toxicity of $\mathrm{PM}_{2.5}$ components and source categories by combining epidemiologic and toxicologic analyses ${ }^{43,44}$. The findings identified secondary sulfate and traffic sources were most consistently associated with health effects. However, other components and source categories could not be definitively excluded as having no adverse effects. Challenges in the analysis of component effects remain in the measurement errors of exposure assessment and the ability to disentangle effects of individual component or multiple components from the same source. Exposure assessment of PM component are more challenging than PM mass because of the scarcity of measurements and the high spatial heterogeneity for especially combustion-related components ${ }^{45}$. The ability to detect health effects of individual components or pollution sources is limited because components originate from the same source are usually highly correlated in concentrations and a specific component can be generated from several different sources ${ }^{41,42,45}$.

\section{Long-term air pollution exposure assessment}

Evaluating the mortality risk of long-term exposure to air pollution is usually addressed by cohort studies $^{4}$. Assessing individual long-term exposure to air pollution remains challenging in these studies. Early cohort studies compared mortality outcomes between cities using group level average exposures. Specifically, individual exposure of all subjects living in a city was characterized as the average concentration of air pollution measured at one or a few monitoring sites within this city ${ }^{9,10,28}$. However, numerous studies have documented that outdoor air pollution concentrations of especially $\mathrm{NO}_{2}$ and $\mathrm{BC}$ 
varied substantially at a small geographical scale ${ }^{25,46-48}$. Within-city spatial contrasts may be larger than between-city spatial contrasts ${ }^{25,48}$. The Women's Health Initiative study suggested larger cardiovascular mortality risks of $\mathrm{PM}_{2.5}$ related to within-city contrasts compared to between-city contrasts ${ }^{49}$.

Fixed-site monitoring networks are expensive and therefore not spatially dense enough to characterize within-city contrasts. Several exposure assessment approaches beyond direct assignment of monitoring data have been developed to better characterize intra-urban variations over the recent decades. These approaches include exposure indicator variables (e.g., traffic intensity in proximity to residence ${ }^{50}$ and distance to major roads ${ }^{51}$ ), interpolation techniques ${ }^{52,53}$ (e.g., kriging and inverse distance weighing), dispersion/chemical transport modeling ${ }^{54,55}$, satellite monitoring ${ }^{29,56,57}$ and land use regression (LUR) modeling ${ }^{22,58}$. One of the limitations of the exposure indicator variables is that they do not provide a quantitative pollution estimate. Although the interpolation techniques provide a spatially more resolved pattern than a simple average, the assumed smooth spatial variation of concentration may be too simple considering the complexity of local sources, street configurations and meteorological conditions. Dispersion/chemical transport models (DCTMs) are deterministic models based on physical principles and actual emission data, thus the models are subject to relatively low spatial resolution of meteorological data and require detailed input data including emission ${ }^{59}$. Satellite modeling combines remote sensing and chemical transport modeling for estimating vertical gradients ${ }^{60,61}$. Satellite observations are globally available but often coarse in spatial resolution, thus cannot represent finescale within-city variations. LUR is an empirical approach that models measured air pollution concentrations at (a limited number of) monitoring sites by (land use) variables collected via geographic information systems (GIS) ${ }^{62,63}$. LUR models are developed based upon locally measured air pollution data and may be difficult to transfer from one study area to another. LUR modeling has been widely used in air pollution exposure assessment in epidemiological studies because of the ability to capture small-scale variation in concentrations, the lower demand on input data compared to DCTMs and the typically good performance.

One recent development in air pollution epidemiology is the use of very large populations with sufficient statistical power to quantify effects of air pollution at low concentrations, where the air pollution contrasts are small. For this, national administrative cohorts or pooled data from multiple cohorts are often used ${ }^{22,64-67}$. Air pollution exposure estimates covering large geographical areas, such as a continent, at sufficiently fine spatial resolution are thus needed. LUR models have been extensively used to assess air pollution exposure at small spatial scales ${ }^{63,68,69}$. For application in large geographical areas, modeling the background concentrations is challenging for LUR models because they cannot explicitly model secondary formation of pollutants ${ }^{70}$. Recently so-called hybrid models have been developed incorporating satellite-derived data (SAT) and/or DCTM estimates as predictors with LUR to achieve a better prediction accuracy at large spatial scales ${ }^{71-74}$. Both SAT and DCTM are able to model the dispersion and chemical transformations and are relatively coarse in spatial resolution, thus represent area-averaged background concentrations. For LUR models based upon surface monitoring data, developing large-scale models with increasing number of monitoring sites helps to improve the robustness of models and the accuracy of subsequent health effect estimation ${ }^{75-77}$.

Another development in air pollution epidemiology is the necessity to evaluate health effects of particle metrics beyond the regulated $\mathrm{PM}_{2.5}$ and $\mathrm{PM}_{10}$, to identify the hazardous components within the complex PM mixture ${ }^{40,43}$. Exposure assessment for PM composition is more challenging than for routinely measured pollutants because of the limited monitoring data and the scarcity of input information such as emission factors. Studies conducted in the US based on routine monitoring measurements of PM composition mainly exploited between-city contrasts while within-city contrasts were not well captured because of the limited number of monitors within a city ${ }^{35,78,79}$. In the European Study of Cohorts for Air Pollution Effects (ESCAPE) based on purpose-designed monitoring campaigns, area-specific models were developed with 20 sites per area. In ESCAPE, model performance was good for traffic-related elements such as copper $(\mathrm{Cu})$ and iron $(\mathrm{Fe})$, but moderate to low for nickel $(\mathrm{Ni})$, vanadium $(\mathrm{V})$ and 
potassium $(\mathrm{K})$ because of the limited spatial variation within areas and the lack of key predictor variables representing specific sources ${ }^{80}$. In addition, exposure models for PM composition might not be specific thus limiting the ability to disentangle the independent effects of PM composition and PM mass in the subsequent epidemiological studies ${ }^{80,81}$. In recent years, dedicated short-term sampling and on-road mobile monitoring campaigns have been conducted to measure particle metrics such as UFP or $\mathrm{BC}$, which are characterized by high temporal and spatial variabilities ${ }^{82-85}$. Developments in satellite data and DCTMs make it possible to model the background concentrations of specific PM composition and to separate the components to some extent ${ }^{86}$.

LUR models are conventionally developed with linear regression algorithms ${ }^{87,88}$, with some possible modifications on the modeling process (e.g., predefined direction of predictor effect) ${ }^{68,89,90}$. Recent studies have attempted to use different modeling algorithms to address limitations of the linear regression algorithm and to achieve a better prediction accuracy. These approaches include the regularization algorithms ${ }^{91,92}$, which address collinearity between predictor variables, and machinelearning algorithms $\mathrm{s}^{72,93,94}$, which can model potential non-linearity and interactions within the data more flexible than linear regression. The more flexible algorithms such as machine-learning algorithms may be helpful in modeling PM composition exposure where complex relationships between pollutant concentrations and predictor variables might be present. One study suggested random forest, a machinelearning method, provided more accurate exposure assessment compared to linear regression in predicting $\mathrm{PM}_{2.5}$ elemental composition concentrations ${ }^{95}$. At the time the investigations described in the current thesis were conducted, few comparisons had been made regarding the prediction accuracy and interpretability of exposure models developed with different algorithms ${ }^{92,96,97}$. No studies had assessed whether application of these different models in air pollution epidemiology would lead to different health effect estimates.

\section{The ELAPSE project}

The Effects of Low-level Air Pollution: A Study in Europe (ELAPSE) is a Europe-wide collaboration that focuses on analyses of health effects of air pollutants at low concentrations, defined as less than current EU limit values, US EPA National Ambient Air Quality Standards (NAAQS) and/or WHO Air Quality Guideline values ${ }^{98}$. The ELAPSE study consists of a pooled cohort of more than 300,000 adults with detailed information on individual-level confounders and seven very large administrative cohorts of more than 28 million adults in total. In the pooled cohort, the data from existing cohorts in Sweden, Denmark, Germany, the Netherlands, Austria, France and Italy were pooled and analyzed centrally. Data from the administrative cohorts in Belgium, Denmark, England, the Netherlands, Norway and Switzerland and Rome, Italy were analyzed locally due to privacy regulations.

The analysis not only focuses on the routinely monitored pollutants $\mathrm{PM}_{2.5}, \mathrm{NO}_{2}$ and $\mathrm{O}_{3}$, but also exploits the dedicated monitoring data of $\mathrm{BC}$ and $\mathrm{PM}_{2.5}$ elemental composition available from the ESCAPE monitoring campaign. ELAPSE exposure assessment characterizes fine-scale intra-urban as well as between-urban air pollution contrasts using hybrid Europe-wide models combining land use regression, dispersion models and satellite observations. The Europe-wide models further allow the analyses to be conducted in the full national study areas within ELAPSE because of the geographical coverage.

ELAPSE was designed to analyze a wide variety of outcomes including 1) natural cause and causespecific mortality, 2) cancer incidence, 3) incidence of coronary and cerebrovascular events and 4) incidence of asthma and COPD. 


\section{Thesis aims and outline}

This thesis has the following specific aims:

1. To systematically evaluate the evidence of associations of long-term exposure to $\mathrm{PM}_{10}$ and $\mathrm{PM}_{2.5}$ with all-cause and cause-specific mortality with a focus on associations at low pollution levels

2. To develop Europe-wide LUR models for $\mathrm{PM}_{2.5}, \mathrm{NO}_{2}, \mathrm{O}_{3}, \mathrm{BC}$ and $\mathrm{PM}_{2.5}$ elemental composition

3. To compare LUR models developed with different algorithms in terms of prediction accuracy and interpretability

4. To investigate associations between long-term exposure to $\mathrm{PM}_{2.5}$ components and mortality, and to compare the health effects estimated using exposure models developed with different algorithms

In Chapter 2, we performed a systematic review on associations of long-term exposure to $\mathrm{PM}_{10}$ and $\mathrm{PM}_{2.5}$ with all-cause and cause-specific mortality with a focus on associations at low pollution levels to support the derivation of new air quality guidelines by the WHO. The remainder of the thesis was developed within the framework of the ELAPSE project to provide new empirical evidence at low pollution levels. In Chapter 3, we developed Europe-wide LUR models for $\mathrm{PM}_{2.5}, \mathrm{NO}_{2}, \mathrm{BC}$ and $\mathrm{O}_{3}$ using supervised linear regression algorithm, and evaluated the spatiotemporal stability of the models. In Chapter 4, we compared the Europe-wide $\mathrm{PM}_{2.5}$ and $\mathrm{NO}_{2}$ models developed with 16 different algorithms (linear regression, regularization and machine-learning algorithms), using the same measured air pollution data and potential predictor variables used in Chapter 3. We performed both cross-validation and external validation to strengthen the comparisons. In Chapter 5, we developed Europe-wide LUR models for $\mathrm{PM}_{2.5}$ elemental composition using supervised linear regression and random forest, a machine-learning algorithm. In Chapter 6, we assessed associations between $\mathrm{PM}_{2.5}$ elemental composition and mortality in the ELAPSE pooled cohort, using models developed in Chapter 5. We also compared the health effect estimates assessed using exposure models developed with supervised linear regression and random forest algorithms. In Chapter 7, the main findings of this thesis are summarized, followed by discussions on methodological issues in systematic reviews, spatial scale and statistical algorithms in developing exposure models, existing evidence on health effects of longterm PM component exposure, and the implications of this research. 


\section{References}

1. $\quad$ Brunekreef B, Holgate ST. Air pollution and health. The lancet 2002; 360(9341): 1233-42.

2. Cohen AJ, Brauer M, Burnett R, et al. Estimates and 25-year trends of the global burden of disease attributable to ambient air pollution: an analysis of data from the Global Burden of Diseases Study 2015. The Lancet 2017; 389(10082): 1907-18.

3. Liu C, Chen R, Sera F, et al. Ambient particulate air pollution and daily mortality in 652 cities. New England Journal of Medicine 2019; 381(8): 705-15.

4. Hoek G, Krishnan RM, Beelen R, et al. Long-term air pollution exposure and cardio- respiratory mortality: a review. Environmental health : a global access science source 2013; 12(1): 43.

5. Hales S, Blakely T, Woodward A. Air pollution and mortality in New Zealand: cohort study. Journal of epidemiology and community health 2012; 66(5): 468-73.

6. Katsouyanni K, Touloumi G, Samoli E, et al. Confounding and effect modification in the short-term effects of ambient particles on total mortality: results from 29 European cities within the APHEA2 project. Epidemiology 2001: 521-31. 7. Samet JM, Dominici F, Curriero FC, Coursac I, Zeger SL. Fine particulate air pollution and mortality in 20 US cities, 1987-1994. New England journal of medicine 2000; 343(24): 1742-9.

8. Franklin M, Zeka A, Schwartz J. Association between PM 2.5 and all-cause and specific-cause mortality in 27 US communities. Journal of exposure science \& environmental epidemiology 2007; 17(3): 279-87.

9. Dockery DW, Pope ICA, Xu X, et al. An association between air pollution and mortality in six U.S. cities. New England Journal of Medicine 1993; 329(24): 1753-9.

10. Pope CA, Thun MJ, Namboodiri MM, et al. Particulate air pollution as a predictor of mortality in a prospective study of U.S. Adults. American Journal of Respiratory and Critical Care Medicine 1995; 151(3 I): 669-74.

11. Rosenlund M, Bellander T, Nordquist T, Alfredsson L. Traffic-generated air pollution and myocardial infarction. Epidemiology 2009; 20(2): 265-71.

12. Sandstrom T. Respiratory effects of air pollutants: experimental studies in humans. European Respiratory Journal 1995; 8(6): 976-95.

13. Devlin RB, Frampton ML, Ghio AJ. In vitro studies: what is their role in toxicology? Experimental and Toxicologic Pathology 2005; 57: 183-8.

14. Sun Q, Wang A, Jin X, et al. Long-term air pollution exposure and acceleration of atherosclerosis and vascular inflammation in an animal model. Jama 2005; 294(23): 3003-10.

15. USEPA. Integrated Science Assessment (ISA) for Particulate Matter (Final Report, 2019). US Environmental Protection Agency, Washington, DC, EPA/600/R-19/188, 2019 (https://cfpubepagov/ncea/isa/recordisplaycfm?deid=347534, last access: 20200128) 2019.

16. WHO. Air quality guidelines: global update 2005: particulate matter, ozone, nitrogen dioxide, and sulfur dioxide: World Health Organization; 2006.

17. EU. https://ec.europa.eu/environment/air/quality/standards.htm.

18. USEPA. https://www.epa.gov/criteria-air-pollutants/naaqs-table.

19. Brauer M, Amann M, Burnett RT, et al. Exposure assessment for estimation of the global burden of disease attributable to outdoor air pollution. Environ Sci Technol 2012; 46(2): 652-60.

20. HEI. RFA 14-3 Assessing Health Effects of Long-term Exposure to Low Levels of Ambient Air Pollution. https://wwwhealtheffectsorg/research/funding/rfa/14-3-assessing-health-effects-long-term-exposure-low-levels-ambient-airpollution 2014.

21. Burnett R, Chen H, Szyszkowicz M, et al. Global estimates of mortality associated with long-term exposure to outdoor fine particulate matter. Proceedings of the National Academy of Sciences 2018; 115(38): 9592-7.

22. Beelen R, Raaschou-Nielsen O, Stafoggia M, et al. Effects of long-term exposure to air pollution on natural-cause mortality: an analysis of 22 European cohorts within the multicentre ESCAPE project. The Lancet 2014; 383(9919): 785-95. 23. Crouse DL, Peters PA, van Donkelaar A, et al. Risk of nonaccidental and cardiovascular mortality in relation to long-term exposure to low concentrations of fine particulate matter: a Canadian national-level cohort study. Environ Health Perspect 2012; 120(5): 708-14.

24. WHO. WHO expert consultation: available evidence for the future update of the WHO global air quality guidelines (AQGs). WHO: Geneva, Switzerland 2016.

25. Eeftens M, Tsai M-Y, Ampe C, et al. Spatial variation of PM2.5, PM10, PM2.5 absorbance and PMcoarse concentrations between and within 20 European study areas and the relationship with NO2 - Results of the ESCAPE project. Atmospheric Environment 2012; 62: 303-17.

26. Putaud J-P, Raes F, Van Dingenen R, et al. A European aerosol phenomenology-2: chemical characteristics of particulate matter at kerbside, urban, rural and background sites in Europe. Atmospheric environment 2004; 38(16): 2579-95.

27. Tseng E, Ho WC, Lin MH, Cheng TJ, Chen PC, Lin HH. Chronic exposure to particulate matter and risk of cardiovascular mortality: cohort study from Taiwan. BMC public health 2015; 15: 936.

28. Pope CA, Burnett RT, Thun MJ, et al. Lung cancer, cardiopulmonary mortality, and long-term exposure to fine particulate air pollution. Jama 2002; 287(9): 1132-41.

29. Wong CM, Lai HK, Tsang H, et al. Satellite-based estimates of long-term exposure to fine particles and association with mortality in elderly Hong Kong residents. Environmental health perspectives 2015; 123(11): 1167-72.

30. Laden F, Neas LM, Dockery DW, Schwartz J. Association of fine particulate matter from different sources with daily mortality in six US cities. Environmental health perspectives 2000; 108(10): 941-7.

31. Özkaynak H, Thurston GD. Associations between 1980 US mortality rates and alternative measures of airborne particle concentration. Risk analysis 1987; 7(4): 449-61. 
32. Sarnat JA, Marmur A, Klein M, et al. Fine particle sources and cardiorespiratory morbidity: an application of chemical mass balance and factor analytical source-apportionment methods. Environmental health perspectives 2008; 116(4): $459-66$.

33. Tsai FC, Apte MG, Daisey JM. An exploratory analysis of the relationship between mortality and the chemical composition of airborne particulate matter. Inhalation Toxicology 2000; 12(sup2): 121-35.

34. Ostro B, Hu J, Goldberg D, et al. Associations of mortality with long-term exposures to fine and ultrafine particles, species and sources: results from the California Teachers Study Cohort. Environ Health Perspect 2015; 123(6): 549-56.

35. Ostro B, Lipsett M, Reynolds P, et al. Long-term exposure to constituents of fine particulate air pollution and mortality: results from the California Teachers Study. Environ Health Perspect 2011; 118(3): 363-9.

36. Badaloni C, Cesaroni G, Cerza F, Davoli M, Brunekreef B, Forastiere F. Effects of long-term exposure to particulate matter and metal components on mortality in the Rome longitudinal study. Environment international 2017; 109: 146-54.

37. Beelen R, Hoek G, Raaschou-Nielsen O, et al. Natural-cause mortality and long-term exposure to particle components: an analysis of 19 European cohorts within the multi-center ESCAPE project. Environ Health Perspect 2015; 123(6): 525-33.

38. Raaschou-Nielsen O, Beelen R, Wang M, et al. Particulate matter air pollution components and risk for lung cancer. Environment international 2016; 87: 66-73.

39. Wang M, Beelen R, Stafoggia M, et al. Long-term exposure to elemental constituents of particulate matter and cardiovascular mortality in 19 European cohorts: results from the ESCAPE and TRANSPHORM projects. Environ Int 2014; 66: $97-106$

40. Thurston GD, Burnett RT, Turner MC, et al. Ischemic Heart Disease Mortality and Long-Term Exposure to SourceRelated Components of U.S. Fine Particle Air Pollution. Environ Health Perspect 2016; 124(6): 785-94.

41. Belis CA, Karagulian F, Larsen BR, Hopke PK. Critical review and meta-analysis of ambient particulate matter source apportionment using receptor models in Europe. Atmospheric Environment 2013; 69: 94-108.

42. Viana M, Kuhlbusch TAJ, Querol X, et al. Source apportionment of particulate matter in Europe: A review of methods and results. Journal of Aerosol Science 2008; 39(10): 827-49.

43. Lippmann M, Chen LC, Gordon T, Ito K, Thurston GD. National Particle Component Toxicity (NPACT) Initiative: integrated epidemiologic and toxicologic studies of the health effects of particulate matter components. Research Report (Health Effects Institute) 2013; (177): 5-13.

44. Vedal S, Campen M, McDonald J, et al. National Particle Component Toxicity (NPACT) initiative report on cardiovascular effects. Research Report (Health Effects Institute) 2013; (178): 5-8.

45. Tsai MY, Hoek G, Eeftens M, et al. Spatial variation of PM elemental composition between and within 20 European study areas--Results of the ESCAPE project. Environ Int 2015; 84: 181-92.

46. Lebret E, Briggs D, Van Reeuwijk H, et al. Small area variations in ambient NO2 concentrations in four European areas. Atmospheric Environment 2000; 34(2): 177-85.

47. Zhu Y, Hinds WC, Kim S, Shen S, Sioutas C. Study of ultrafine particles near a major highway with heavy-duty diesel traffic. Atmospheric environment 2002; 36(27): 4323-35.

48. Cyrys J, Eeftens M, Heinrich J, et al. Variation of NO2 and NOx concentrations between and within 36 European study areas: results from the ESCAPE study. Atmospheric Environment 2012; 62: 374-90.

49. Miller KA, Siscovick DS, Sheppard L, et al. Long-term exposure to air pollution and incidence of cardiovascular events in women. New England Journal of Medicine 2007; 356(5): 447-58.

50. Beelen R, Hoek G, van Den Brandt PA, et al. Long-term effects of traffic-related air pollution on mortality in a Dutch cohort (NLCS-AIR study). Environmental health perspectives 2008; 116(2): 196-202.

51. Hoffmann B, Moebus S, Stang A, et al. Residence close to high traffic and prevalence of coronary heart disease. European heart journal 2006; 27(22): 2696-702.

52. Lipsett MJ, Ostro BD, Reynolds P, et al. Long-term exposure to air pollution and cardiorespiratory disease in the California teachers study cohort. American journal of respiratory and critical care medicine 2011; 184(7): 828-35.

53. Chen LH, Knutsen SF, Shavlik D, et al. The association between fatal coronary heart disease and ambient particulate air pollution: are females at greater risk? Environmental Health Perspectives 2005; 113(12): 1723-9.

54. Carey IM, Atkinson RW, Kent AJ, Van Staa T, Cook DG, Anderson HR. Mortality associations with long-term exposure to outdoor air pollution in a national English cohort. American journal of respiratory and critical care medicine 2013; 187(11): 1226-33.

55. Huss A, Spoerri A, Egger M, Röösli M. Aircraft noise, air pollution, and mortality from myocardial infarction. Epidemiology 2010: 829-36.

56. Cakmak S, Hebbern C, Vanos J, Crouse DL, Burnett R. Ozone exposure and cardiovascular-related mortality in the Canadian Census Health and Environment Cohort (CANCHEC) by spatial synoptic classification zone. Environmental Pollution 2016; 214: 589-99.

57. Crouse DL, Peters PA, Hystad P, et al. Ambient PM2. 5, O3, and NO2 exposures and associations with mortality over 16 years of follow-up in the Canadian Census Health and Environment Cohort (CanCHEC). Environmental health perspectives 2015; 123(11): 1180-6.

58. Puett RC, Hart JE, Suh H, Mittleman M, Laden F. Particulate matter exposures, mortality, and cardiovascular disease in the health professionals follow-up study. Environmental health perspectives $2011 ; 119(8): 1130-5$.

59. Liu L-JS, Tsai M-Y, Keidel D, et al. Long-term exposure models for traffic related NO2 across geographically diverse areas over separate years. Atmospheric environment 2012; 46: 460-71.

60. Van Donkelaar A, Martin RV, Brauer M, et al. Global estimates of ambient fine particulate matter concentrations from satellite-based aerosol optical depth: development and application. Environmental health perspectives 2010; 118(6): 847 55. 
61. Van Donkelaar A, Martin RV, Spurr RJ, Burnett RT. High-resolution satellite-derived PM2. 5 from optimal estimation and geographically weighted regression over North America. Environmental science \& technology 2015; 49(17): 10482-91.

62. Hoek G. Methods for Assessing Long-Term Exposures to Outdoor Air Pollutants. Curr Environ Health Rep 2017; 4(4): 450-62.

63. Hoek G, Beelen R, De Hoogh K, et al. A review of land-use regression models to assess spatial variation of outdoor air pollution. Atmospheric environment 2008; 42(33): 7561-78.

64. Brauer M, Brook JR, Christidis T, et al. Mortality-Air Pollution Associations in Low-Exposure Environments (MAPLE): Phase 1. Research Reports: Health Effects Institute 2019; 2019.

65. Dominici F, Schwartz J, Di Q, Braun D, Choirat C, Zanobetti A. Assessing adverse health effects of long-term exposure to low levels of ambient air pollution: phase 1. Research Reports: Health Effects Institute 2019; 2019.

66. Pinault L, Tjepkema M, Crouse DL, et al. Risk estimates of mortality attributed to low concentrations of ambient fine particulate matter in the Canadian community health survey cohort. Environmental health : a global access science source 2016; 15: 18 .

67. Di Q, Wang Y, Zanobetti A, et al. Air Pollution and Mortality in the Medicare Population. The New England journal of medicine 2017 ; 376(26): 2513-22.

68. Eeftens M, Beelen R, de Hoogh K, et al. Development of Land Use Regression models for $\operatorname{PM}(2.5), \operatorname{PM}(2.5)$ absorbance, PM(10) and PM(coarse) in 20 European study areas; results of the ESCAPE project. Environ Sci Technol 2012; 46(20): 11195-205.

69. Crouse DL, Goldberg MS, Ross NA. A prediction-based approach to modelling temporal and spatial variability of traffic-related air pollution in Montreal, Canada. Atmospheric environment 2009; 43(32): 5075-84.

70. Kerckhoffs J, Wang M, Meliefste K, et al. A national fine spatial scale land-use regression model for ozone. Environmental research 2015; 140: 440-8.

71. de Hoogh K, Gulliver J, van Donkelaar A, et al. Development of West-European PM 2.5 and NO 2 land use regression models incorporating satellite-derived and chemical transport modelling data. Environmental research 2016; 151: $1-10$.

72. Di Q, Kloog I, Koutrakis P, Lyapustin A, Wang Y, Schwartz J. Assessing PM2. 5 exposures with high spatiotemporal resolution across the continental United States. Environmental science \& technology 2016; 50(9): 4712-21.

73. Di Q, Rowland S, Koutrakis P, Schwartz J. A hybrid model for spatially and temporally resolved ozone exposures in the continental United States. Journal of the Air \& Waste Management Association 2017; 67(1): 39-52.

74. Akita Y, Baldasano JM, Beelen R, et al. Large scale air pollution estimation method combining land use regression and chemical transport modeling in a geostatistical framework. Environmental science \& technology 2014; 48(8): 4452-9.

75. Basagana X, Aguilera I, Rivera M, et al. Measurement error in epidemiologic studies of air pollution based on landuse regression models. Am J Epidemiol 2013; 178(8): 1342-6.

76. Basagaña X, Rivera M, Aguilera I, et al. Effect of the number of measurement sites on land use regression models in estimating local air pollution. Atmospheric Environment 2012; 54: 634-42.

77. Wang M, Beelen R, Eeftens M, Meliefste K, Hoek G, Brunekreef B. Systematic evaluation of land use regression models for NO2. Environmental science \& technology 2012; 46(8): 4481-9.

78. Dominici F, Peng RD, Ebisu K, Zeger SL, Samet JM, Bell ML. Does the effect of PM10 on mortality depend on PM nickel and vanadium content? A reanalysis of the NMMAPS data. Environmental health perspectives 2007; 115(12): 1701-3.

79. Peng RD, Bell ML, Geyh AS, et al. Emergency admissions for cardiovascular and respiratory diseases and the chemical composition of fine particle air pollution. Environmental health perspectives 2009; 117(6): 957-63.

80. de Hoogh K, Wang M, Adam M, et al. Development of land use regression models for particle composition in twenty study areas in Europe. Environ Sci Technol 2013; 47(11): 5778-86.

81. Yang A, Wang M, Eeftens M, et al. Spatial variation and land use regression modeling of the oxidative potential of fine particles. Environmental health perspectives 2015; 123(11): 1187-92.

82. Abernethy RC, Allen RW, McKendry IG, Brauer M. A land use regression model for ultrafine particles in Vancouver, Canada. Environmental science \& technology 2013; 47(10): 5217-25.

83. Hankey S, Marshall JD. Land use regression models of on-road particulate air pollution (particle number, black carbon, PM2. 5, particle size) using mobile monitoring. Environmental science \& technology 2015; 49(15): 9194-202.

84. Larson T, Henderson SB, Brauer M. Mobile monitoring of particle light absorption coefficient in an urban area as a basis for land use regression. Environmental science \& technology 2009; 43(13): 4672-8.

85. Weichenthal S, Van Ryswyk K, Goldstein A, Bagg S, Shekkarizfard M, Hatzopoulou M. A land use regression model for ambient ultrafine particles in Montreal, Canada: A comparison of linear regression and a machine learning approach. Environmental research 2016; 146: 65-72.

86. van Donkelaar A, Martin RV, Li C, Burnett RT. Regional Estimates of Chemical Composition of Fine Particulate Matter Using a Combined Geoscience-Statistical Method with Information from Satellites, Models, and Monitors. Environ Sci Technol 2019; 53(5): 2595-611.

87. Briggs D. The role of GIS: coping with space (and time) in air pollution exposure assessment. Journal of Toxicology and Environmental Health, Part A 2005; 68(13-14): 1243-61.

88. Briggs DJ, Collins S, Elliott P, et al. Mapping urban air pollution using GIS: a regression-based approach. International Journal of Geographical Information Science 1997; 11(7): 699-718.

89. Brauer M, Hoek G, van Vliet $\mathrm{P}$, et al. Estimating long-term average particulate air pollution concentrations: application of traffic indicators and geographic information systems. Epidemiology 2003: 228-39. 


\section{Chapter 1}

90. Henderson SB, Beckerman B, Jerrett M, Brauer M. Application of land use regression to estimate long-term concentrations of traffic-related nitrogen oxides and fine particulate matter. Environmental science \& technology 2007; 41(7): $2422-8$.

91. Kim SY, Sheppard L, Bergen S, et al. Prediction of fine particulate matter chemical components with a spatiotemporal model for the Multi-Ethnic Study of Atherosclerosis cohort. J Expo Sci Environ Epidemiol 2016; 26(5): 520-8.

92. Van den Bossche J, De Baets B, Verwaeren J, Botteldooren D, Theunis J. Development and evaluation of land use regression models for black carbon based on bicycle and pedestrian measurements in the urban environment. Environmental Modelling \& Software 2018; 99: 58-69.

93. $\mathrm{Hu} \mathrm{X}$, Belle JH, Meng X, et al. Estimating PM2.5 Concentrations in the Conterminous United States Using the Random Forest Approach. Environ Sci Technol 2017.

94. Stafoggia M, Schwartz J, Badaloni C, et al. Estimation of daily PM10 concentrations in Italy (2006-2012) using finely resolved satellite data, land use variables and meteorology. Environ Int 2017; 99: 234-44.

95. Brokamp C, Jandarov R, Rao MB, LeMasters G, Ryan P. Exposure assessment models for elemental components of particulate matter in an urban environment: A comparison of regression and random forest approaches. Atmos Environ (1994) 2017; 151: 1-11.

96. Reid CE, Jerrett M, Petersen ML, et al. Spatiotemporal prediction of fine particulate matter during the 2008 northern California wildfires using machine learning. Environ Sci Technol 2015; 49(6): 3887-96.

97. Kerckhoffs J, Hoek G, Portengen L, Brunekreef B, Vermeulen RC. Performance of Prediction Algorithms for Modeling Outdoor Air Pollution Spatial Surfaces. Environmental science \& technology 2019.

98. ELAPSE. Effects of Low-Level Air Pollution: A Study in Europe. http://wwwelapseprojecteu/ 2016. 


\section{Chapter}

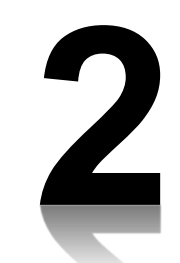

\section{Long-Term Exposure to PM and All- Cause and Cause-Specific Mortality: A Systematic Review and Meta-Analysis}

Jie Chen and Gerard Hoek

Environment International, 2020, 143: 105974 DOI: 10.1016/j.envint.2020.105974 


\begin{abstract}
As new scientific evidence on health effects of air pollution is generated, air quality guidelines need to be periodically updated. The objective of this review is to support the derivation of updated guidelines by the World Health Organization (WHO) by performing a systematic review of evidence of associations between long-term exposure to particulate matter with diameter under $2.5 \mu \mathrm{m}\left(\mathrm{PM}_{2.5}\right)$ and particulate matter with diameter under $10 \mu \mathrm{m}\left(\mathrm{PM}_{10}\right)$, in relation to all-cause and cause-specific mortality. As there is especially uncertainty about the relationship at the low and high end of the exposure range, the review needed to provide an indication of the shape of the concentration-response function (CRF).
\end{abstract}

We systematically searched MEDLINE and EMBASE from database inception to 9 October 2018. Articles were checked for eligibility by two reviewers. We included cohort and case-control studies on outdoor air pollution in human populations using individual level data. In addition to natural-cause mortality, we evaluated mortality from circulatory diseases (ischemic heart disease (IHD) and cerebrovascular disease (stroke) also specifically), respiratory diseases (Chronic Obstructive Pulmonary Disease (COPD) and acute lower respiratory infection (ALRI) also specifically) and lung cancer. A random-effect meta-analysis was performed when at least three studies were available for a specific exposure-outcome pair. Risk of bias was assessed for all included articles using a specifically developed tool coordinated by WHO. Additional analyses were performed to assess consistency across geographic region, explain heterogeneity and explore the shape of the CRF. An adapted GRADE (Grading of Recommendations Assessment, Development and Evaluation) assessment of the body of evidence was made using a specifically developed tool coordinated by WHO.

A large number $(\mathrm{N}=107)$ of predominantly cohort studies $(\mathrm{N}=104)$ were included after screening more than 3000 abstracts. Studies were conducted globally with the majority of studies from North America $(\mathrm{N}=62)$ and Europe $(\mathrm{N}=25)$. More studies used $\mathrm{PM}_{2.5}(\mathrm{~N}=71)$ as the exposure metric than $\mathrm{PM}_{10}(\mathrm{~N}=42)$. $\mathrm{PM}_{2.5}$ was significantly associated with all causes of death evaluated. The combined Risk Ratio (RR) for $\mathrm{PM}_{2.5}$ and natural-cause mortality was $1.08(95 \% \mathrm{CI} 1.06,1.09)$ per $10 \mu \mathrm{g} / \mathrm{m}^{3}$. Meta analyses of studies conducted at the low mean $\mathrm{PM}_{2.5}$ levels $\left(<25,20,15,12,10 \mu \mathrm{g} / \mathrm{m}^{3}\right)$ yielded RRs that were similar or higher compared to the overall RR, consistent with the finding of generally linear or supralinear CRFs in individual studies. Pooled RRs were almost identical for studies conducted in North America, Europe and Western Pacific region. $\mathrm{PM}_{10}$ was significantly associated with natural-cause and most but not all causes of death. Application of the risk of bias tool showed that few studies were at a high risk of bias in any domain. Application of the adapted GRADE tool resulted in an assessment of "high certainty of evidence" for $\mathrm{PM}_{2.5}$ with all assessed endpoints except for respiratory mortality (moderate). The evidence was rated as less certain for $\mathrm{PM}_{10}$ and cause-specific mortality ("moderate" for circulatory, IHD, COPD and "low" for stroke mortality.

Compared to the previous global WHO evaluation, the evidence base has increased substantially. However, studies conducted in low- and middle- income countries (LMICs) are still limited. There is clear evidence that both $\mathrm{PM}_{2.5}$ and $\mathrm{PM}_{10}$ were associated with increased mortality from all causes, cardiovascular disease, respiratory disease and lung cancer. Associations remained below the current WHO guideline exposure level of $10 \mu \mathrm{g} / \mathrm{m}^{3}$ for $\mathrm{PM}_{2.5}$.

Systematic review registration number (PROSPERO ID): CRD42018082577 


\section{Introduction}

Air pollution is a major environmental hazard to human health and a leading cause of mortality and morbidity worldwide (WHO, 2006; USEPA, 2019). Particulate matter (PM), which comprises multiple components and size fractions, is an important health relevant outdoor air pollutant regulated in many countries. Most earlier routine air quality monitoring systems measured particulate matter with diameter under $10 \mu \mathrm{m}\left(\mathrm{PM}_{10}\right)$, whereas more recent networks have added particulate matter with diameter under $2.5 \mu \mathrm{m}\left(\mathrm{PM}_{2.5}\right)$ measurements. $\mathrm{PM}_{10}$ includes both fine particles $\left(\mathrm{PM}_{2.5}\right)$ and coarse $\left(\mathrm{PM}_{10-2.5}\right)$ particles. $\mathrm{PM}_{2.5}$ originates primarily from combustion sources, while $\mathrm{PM}_{10-2.5}$ is composed largely of crustal material, sea salt and biological material (WHO, 2006). The proportion of particles in these two size ranges varies substantially depending on local geography, meteorology and specific PM sources such as construction work, unpaved roads or nearby deserts, all contributing to large amounts of coarse particles. Health effects of fine and coarse particles may differ because of different chemical composition and different penetration into the respiratory tract. In the 2019 Integrated Science Assessment (ISA) by the US Environmental Protection Agency (EPA), the association between $\mathrm{PM}_{2.5}$ and natural mortality was rated as "causal" while the association between $\mathrm{PM}_{10-2.5}$ and natural-cause mortality was rated as "suggestive" (USEPA, 2019). Causal or likely causal relationship between longterm exposure to fine PM and all-cause, cardiovascular, respiratory and lung cancer mortality have also been reported by the International Agency for Research on Cancer (IARC, 2013) and Health Canada (HC, 2013).The Global Burden of Disease (GBD) study estimated that ambient $\mathrm{PM}_{2.5}$ was the fifthranking mortality risk factor in 2015 , with 4.2 million deaths caused by exposure to $\mathrm{PM}_{2.5}$ (Cohen et al., 2017; WHO, 2018).

The World Health Organization (WHO) has published several volumes of Air Quality Guidelines (AQGs) to provide guidance to the public, especially to policy and other decision makers, on the health risks of air pollution. The latest version was published in 2006 with an annual average guideline exposure level of $10 \mu \mathrm{g} / \mathrm{m}^{3}$ for $\mathrm{PM}_{2.5}$ and $20 \mu \mathrm{g} / \mathrm{m}^{3}$ for $\mathrm{PM}_{10}$ (WHO, 2006). The guideline exposure levels represent the lower end of the range over which significant effects on survival were observed in the 2006 evaluation of the evidence. Guideline values are designed to advise national policy makers to what levels air pollution should be reduced to protect public health. The guideline was developed based on evaluation of a small number of cohort studies predominantly conducted in North America. Particularly, the American Cancer Society (ACS) study was important to derive the guideline. Concerns were raised regarding applying the guideline to other areas in the world where PM sources and population characteristics are different. A large number of new cohort studies has been published since 2006, including several large studies based on administrative databases (Hoek et al., 2013). A number of studies conducted in areas with PM levels below the current WHO guidelines (Cakmak et al., 2016; Dehbi et al., 2017; Gan et al., 2013; Pinault et al., 2016) suggested that health effects may occur at low pollution levels. This has increased the interest in the shape of the concentration-response function (CRF), including detection of a potential threshold in the CRF.

As new evidence is generated, the WHO air quality guidelines need to be periodically updated. The overall objective of the update of WHO Global AQGs is to develop public health recommendations for ambient air quality. To support the update of the guidelines, we performed a systematic review of evidence of associations between long-term exposure to PM and mortality. The most important goal of the systematic review was to provide quantitative information about the magnitude of risks, not to contribute to the debate about a potential causal relationship. The specific question formulated in terms of Population, Exposure, Comparator, Outcome, Study Design (PECOS) was: "In any population, including subgroups of susceptible individuals $(\mathrm{P})$, what is the increase in risk of all-cause and causespecific mortality (O) per 10 unit increase (C) in $\mu \mathrm{g} / \mathrm{m}^{3}$ of long-term exposure (in the order of months to years) to ambient concentrations of $\mathrm{PM}_{2.5}$ and $\mathrm{PM}_{10}(\mathrm{E})$, observed in cohort and case-control studies (S)? In these studies, is an increased risk observed at low levels, specifically below the current WHO guideline?" In particular, we used meta-analysis to quantitatively pool risk estimates across studies, and 
qualitatively summarized the concentration-response gradient evaluated in individual studies. The current review was based on a previous review which evaluated the epidemiological evidence for cardiovascular and respiratory mortality effects of long-term exposure to fine particulate matter (Hoek et al., 2013).

\section{Methods}

The study protocol was developed from a draft generic text provided by WHO to all systematic review teams. The protocol was registered at the International Prospective Register of Systematic Reviews (PROSPERO, registered ID: CRD42018082577).

\subsection{Eligibility criteria and search strategy}

We applied the following eligibility (inclusion and exclusion) criteria structured by PECOS items:

$\underline{\text { Population }}$

Studies reporting general human population exposed to $\mathrm{PM}_{2.5}$ and $\mathrm{PM}_{10}$ via inhalation through ambient air predominantly were included. Studies reporting on exposures of populations in the workplace exclusively were excluded. There were no restrictions on ages, geographical areas, occupations of population.

\section{Exposure}

Studies reporting long-term exposure (in the order of months to years) to ambient air $\mathrm{PM}_{2.5}$ and $\mathrm{PM}_{10}$ expressed in a concentration unit $\left(\mu \mathrm{g} / \mathrm{m}^{3}\right)$ were included. Studies that have translated other particle metrics such as total suspended particles (TSP) into $\mathrm{PM}_{10}$ or $\mathrm{PM}_{2.5}$ using local and time specific conversion factors were also included. Studies reporting exposure to $\mathrm{PM}_{2.5}$ and $\mathrm{PM}_{10}$ as a result of occupational exposure (measured in the workplace) or indoor exposure exclusively were excluded.

\section{Comparator}

In air pollution epidemiology, the association between a continuous exposure and the risk of death is evaluated. The risk of death is thus compared for subjects with relatively high and relatively low concentrations in each study. The comparator in each individual study was exposure to relatively low levels of $\mathrm{PM}_{2.5}$ and $\mathrm{PM}_{10}$ in the same population (cohort studies) or in a control population (case-control studies).

\section{Outcome}

Health outcomes were selected by the Guideline Development Group (GDG) based on evidence on causality according to the latest determination (causal or likely causal) from the US Environmental Protection Agency (EPA), the International Agency for Research on Cancer (IARC), Health Canada (HC) or other integrated science assessments available. Additional most severe health outcomes with suggestive causality were also included based on other considerations such as contribution to burden of disease (prevalence of disease, disability weight, etc), policy implications and expected increase in exposure to a pollutant in the future.

Health outcomes selected in relation to long-term exposure to $\mathrm{PM}_{2.5}$ and $\mathrm{PM}_{10}$ included (the $10^{\text {th }}$ revision of the International Classification of Diseases (ICD-10) codes, version 2016 in brackets): all-cause mortality (A00 - Z99) and cause-specific mortality including circulatory diseases (I00 - I99), ischemic heart diseases (IHD, I20 - I25), cerebrovascular diseases (stroke, I60 - I69), respiratory diseases (J00J99), chronic obstructive pulmonary diseases (COPD, J40 - J44, J47), acute lower respiratory infection (ALRI, J12 - J18, J20 - J22) and lung cancer mortality (C30 - C39). Natural-cause mortality or nonaccidental mortality (A00 - R99) is mortality from all-causes except external causes such as accidents, suicide and homicide. We considered natural-cause mortality equivalent to all-cause mortality as 
natural-cause mortality accounts for the majority of all-cause mortality and there is no clear evidence that air pollution is associated with accidental mortality. Equivalent definitions using ICD-9 or other versions of ICD-10 were included.

Study

Human epidemiological studies using prospective and retrospective cohort study designs, case-control and nested case-control study designs were included. Published journal articles in any language (abstract in English language) were included. If suitable articles were identified published in languages not known by the authors, further assistance was sought after.

Studies without individual level data (i.e. ecological studies with aggregated outcome, exposure and covariates data), studies where no original data were analyzed, methodological studies, non-human studies (in vivo, in vitro, other) and conference abstracts were excluded. Relevant reviews and systematic reviews were not included in the current systematic review but used to scan for references.

Ecological studies were excluded because they are not sufficiently informative for risks at the individual level. Studies analyzing exposure in categories were included in the review, but not in the meta-analysis, because of the uncertainty of transformations. Conference abstracts were excluded as they were expected to not contain sufficient information to perform data extraction and risk of bias assessment.

Studies matching the PECOS questions were searched systematically in the database MEDLINE using PubMed and the database EMBASE through EMBASE.com between database inception and 9 October 2018. Literature search strategies using free text and MeSH terms/ Emtree terms, considering exposure, health outcomes and study design, are presented in Appendix 1.

\subsection{Study selection and data collection}

We (GH and JC) independently screened references by titles and abstracts for potential relevance. We further assessed the full-text of the articles resulting from abstract screening independently for compliance with eligibility criteria in section 2.1. The specific reasons for excluding articles at this stage were recorded (Appendix 2). Any disagreement on inclusion was resolved by discussion.

We conducted data extraction in duplicate. When the data extraction did not agree, we went back to the original paper. We did not document the rate of agreement. We drafted a data extraction form in Excel and piloted the form with a few example studies by the two authors independently. We adapted the form based on these comparisons. The form was then reviewed by WHO with a few adaptations before being used in the current study (Appendix 3). The following characteristics of the included articles were extracted: citation details, study name, study design, study location; characteristics of the study population; follow up period(s); details on exposure; details on outcome assessment; details of confounders adjusted for; data to calculate the effect estimates and their confidence intervals; methods and results of assessment of the shape of the exposure response function; conflicts of interest. For a specific pollutant-outcome pair, most articles reported effect estimates from more than one singlepollutant models with increasing adjustment for potential confounders. When multiple estimates were reported, we extracted estimates from 1) the crude model (only adjusted for age and sex), 2) the most adjusted model, and 3) the authors favored model (usually shown in abstract). Additionally, we extracted estimates from two pollutant models with $\mathrm{NO}_{2}, \mathrm{O}_{3}$ or coarse particles as the second pollutant.

\subsection{Risk of bias (RoB) assessment}

A domain-based RoB assessment tool (WHO, 2020), developed by a group of experts convened by WHO, was used to assess all articles included in the meta-analyses. RoB assessments were conducted at outcome level; therefore, if a primary study reported on two relevant outcomes RoB was evaluated twice. There were six domains in the RoB assessment tool: confounding, selection bias, exposure assessment, outcome measurement, missing data, and selective reporting. Each domain contained several subdomains. Specifically, we examined the extent to which potential confounders were adjusted 
for, whether the methods for measuring and controlling for the potential confounders were valid; whether there was a selection of participants into the study that related to exposure or outcome; whether the methods used for exposure assessment were valid; whether the outcome measurement methods were valid; whether missing data were related to exposure or outcome; and whether all results were reported. In evaluating each article, we assigned a 'low', 'moderate' or 'high' RoB for each subdomain. To come to an overall assessment for a domain, the following approach was applied: if any of the subdomains had a rating of high risk of bias, the entire domain was rated as high risk of bias; if all the subdomains had a rating of low risk of bias, the entire domain was rated as low risk of bias; when at least one subdomain had a rating of moderate risk of bias and none of the other subdomains was at high risk of bias, the entire domain was rated as moderate risk of bias. No overall risk of bias was determined across domains for a single article, because we were uncomfortable with assigning equal weight to the different domains.

To judge the RoB per study, the tool contained specific guidelines, for example on what should be considered critical and potential confounders for the different outcomes (WHO2020). Critical confounders (age, sex, individual- or area-level socioeconomic status, body mass index/smoking) and additional potential confounders (year of enrolment, ethnicity, diet, physical activity, marital status) were identified prior to the evaluation. An article can only be classified as low risk of bias if all critical and additional potential confounders were adjusted for; if not all critical potential confounders were adjusted for, the article was classified as high RoB; otherwise, a moderate RoB was assigned. For other subdomains, criteria were also specified, though not always as straightforward as the list of confounders (WHO, 2020).

RoB assessment was conducted independently by one reviewer (JC) and checked for accuracy by a second reviewer $(\mathrm{GH})$. A 10\% selection of articles were assessed by a WHO methods expert (RM) for cross-checking. Reviewer assessments and rationales were recorded in an Excel file (Appendix 4).

\subsection{Meta-analysis}

In case three or more studies were identified for the same pollutant and health outcome, a meta-analysis was performed. Because of the expected differences in both populations and pollution, we a priori decided to pool estimates by a random-effect meta-analysis (DerSimonian-Laird estimator). We used Risk Ratios (RRs) as the effect measure of associations between health outcomes and per $10 \mu \mathrm{g} / \mathrm{m}^{3}$ increase in particulate air pollution. Hazard Ratios (HRs) were considered equivalent to RRs. If RR estimates were reported for increments other than per $10 \mu \mathrm{g} / \mathrm{m}^{3}$ (e.g. per IQR increase), we converted the estimates to RR per $10 \mu \mathrm{g} / \mathrm{m}^{3}$. We calculated slope (Beta) and standard error (SE) per $1 \mu \mathrm{g} / \mathrm{m}^{3}$, multiplied by 10 and then exponentiated.

We used the standard equations below.

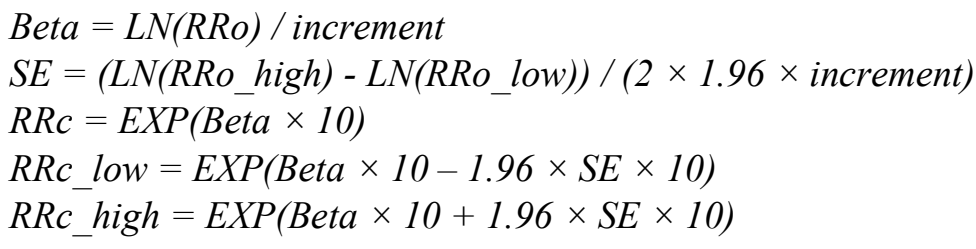

RRo is the effect estimate originally reported in the paper with its low (RRo_low) and high (RRo_high) end of the confidence interval (CI); RRc is the estimate we converted to.

In the main meta-analysis we did not include studies conducted in patient groups because the patient population is very different from the general population, and the main interest for WHO of the review is to develop a quantitative summary estimate that applies to the general population, although this was not explicitly stated. In sensitivity analysis, we tested the combined effect estimates after including patient populations. 
We only included in the meta-analysis the most recent published article when estimates for the same study population were reported in several articles, unless it has a smaller population or a different focus.

In meta-analysis, we used one estimate for a specific pollutant-health outcome from a single article. If an article reported two or more estimates for subgroups of the study population separately (e.g. male and female, age groups, regions) only, we combined the estimates by a fixed-effect meta-analysis. If an article reported more than one estimates from multiple single-pollutant models with increasing adjustment for potential confounders, we included the estimate from the authors favored model (usually shown in abstract). The authors favored model was always from adjusted models, but not necessarily the most adjusted model, for example in the case of testing for sensitivity to adding in variables that could both be a confounder and on the causal pathway from air pollution towards mortality, such as hypertension for cardiovascular mortality.

Statistical heterogeneity of effect estimates between studies were assessed using tau-squared, presented in the form of an $80 \%$ prediction interval around the mean effect in a random-effects meta-analysis (Borenstein et al., 2017). In addition, the Chi ${ }^{2}$ test (Cochran's Q) with a significance level $<0.1$ and the $\mathrm{I}^{2}$ value, where $\mathrm{I}^{2}$ values of $25 \%, 50 \%$ and $75 \%$ are taken as of low, moderate and high degree of heterogeneity, respectively (Woodward 2013).

The R program package 'metafor' was used to produce forest plots and to perform meta-analysis.

\subsection{Additional analyses}

In an attempt to explain heterogeneity, we further performed subgroup analysis for $\mathrm{PM}_{2.5}$ and naturalcause mortality in pre-specified subgroups: geographical location (WHO Regions (African Region, Region of Americas, South-East Asia Region, European Region, Eastern Mediterranean Region, and Western Pacific Region)); sex (men, women, men + women); age groups (average age $\leq 65$ years old or $>65$ years old); level of mean $\mathrm{PM}_{2.5}$ concentrations $\left(<10 \mu \mathrm{g} / \mathrm{m}^{3}, 10-25 \mu \mathrm{g} / \mathrm{m}^{3},>25 \mu \mathrm{g} / \mathrm{m}^{3}\right)$. A meta regression was conducted by including all subgroup factors as covariates.

For each meta-analysis, a funnel plot was made to detect any evidence of publication bias. Egger's test was also applied.

We assessed the shape of the CRF by meta-analysing studies with mean $\mathrm{PM}_{2.5}$ concentrations below certain cut-off values $\left(10,12,15,20,25 \mu \mathrm{g} / \mathrm{m}^{3}\right)$ for $\mathrm{PM}_{2.5}$ and natural-cause mortality. We further reviewed assessments within individual studies to assess the shape of the CRF, e.g. spline analyses, subset analyses, quartile/ quintile analyses or information from the discussion sections (Appendix 5).

For $\mathrm{PM}_{2.5}$ and natural-cause mortality, further sensitivity analyses were conducted: 1) Effect estimates from two pollutant models adjusting for coarse particles, $\mathrm{O}_{3}$ or $\mathrm{NO}_{2} ; 2$ ) Excluding studies at high risk of bias; 3) Excluding studies without individual level lifestyle confounders, specifically the large cohort studies based upon administrative databases; 4) Including studies in patient populations or infants.

\subsection{Evaluation of certainty of evidence}

A GRADE (Grading of Recommendations Assessment, Development and Evaluation) framework, adapted by a group of experts convened by WHO, was used to assess the overall certainty of evidence across studies for each exposure-outcome pair. A common guidance document for all assessors was prepared to assist the rating, which is added in Appendix 6a. Briefly, we started the rating process at moderate certainty evidence because of the risk of unmeasured confounding in observational studies. Then, we downgraded or upgraded the certainty of evidence based on five and four GRADE domains respectively. Reasons for downgrading included 1. Limitations in studies; 2. Indirectness; 3. Inconsistency; 4. Imprecision; 5. Publication bias. Reasons for upgrading include 1. Large magnitude of effect size; 2. All plausible confounding decreases observed RR; 3. Concentration-response gradient. The assessments for the GRADE domains were mostly based on results of the Risk of Bias assessment, 
heterogeneity, sensitivity and publication bias analyses, which were previously described in the methods section.

\section{Reasons for downgrade:}

Limitations in studies: the certainty of evidence was downgraded with one or two levels if serious or very serious risk of bias was present in studies that had a considerable weight in the meta-analysis. If high risk of bias studies differ in effect size from low/moderate risk of bias studies, consideration should be given to exclude high risk of bias studies from the meta-analysis.

Indirectness: the certainty of evidence was downgraded if the studies did not answer the PECOS question of the systematic review.

Inconsistency: the certainty of evidence was downgraded if severe heterogeneity was detected, for example, if there were studies in the body of evidence that show a harmful effect and also studies that show a preventive effect. Some heterogeneity is expected given differences in study location, type of population, level and composition of PM and methodological differences between studies. We assessed whether the $80 \%$ prediction interval of the meta-analytic risk estimate included unity and was more than twice the width of confidence interval. We further assessed whether heterogeneity could be explained by study-level factors and whether there was a sizable number of studies with HRs below 1 . The latter criterium was assessed because a mere difference in magnitude of positive effect estimates between studies is of less concern than a mix of positive and negative associations.

Imprecision: the certainty of evidence was downgraded if the number of person-years of follow-up was less than 940000 person-years.

Publication bias: the certainty of evidence was downgraded if publication bias was detected by visual inspection of the funnel plot in combination with the Egger's test. Careful consideration of heterogeneity as a cause for non-symmetric funnel plots and significant Egger tests was applied.

\section{Reasons for upgrade:}

Large effect size: the certainty of evidence had to be upgraded if the pooled effect size was large or very large. Calculation of a single E-value was proposed to evaluate how strong the relationship between an unmeasured confounder and both exposure and mortality needs to be to explain away the RRs we observed. We lacked the information to apply this procedure in our review. We had insufficient information to judge the strength of the relationships between exposure and confounders in the body of evidence. Furthermore, the relationship between a confounder and mortality is typically much stronger than between a confounder and air pollution exposure, so the use of a single E-value in our review is difficult to interpret. The certainty of evidence was therefore not upgraded based on this domain, consistent with RRs being typically low in well-executed air pollution epidemiological studies.

Confounding domain: the certainty of evidence was upgraded if all plausible confounding shifted the relative risk towards the null.

Concentration-response gradient domain: the certainty of evidence was upgraded if there was a concentration-response relationship between exposure and adverse mortality outcomes, either linearly or non-linearly."

\section{Results}

\subsection{Article Selection and Description}

After screening 3162 abstracts, we identified 216 records with potential relevance for the systematic review (Figure 1). We further excluded 54 records including 24 conference abstracts, 1 news report, 5 items with no full-text and 24 reviews. Reviews were not included in the study but used for references 
screening and thus not excluded with search terms. Titles of the reviews were recorded in Appendix 2.1. We considered the 5 items with no full-text very unlikely to be relevant for this systematic review and did not put more efforts obtaining the full texts (documented in Appendix 2.2). Of 162 articles which remained for full-text assessment based on the eligibility criteria, we excluded 57 records with rationales documented in Appendix 2.2. We categorized excluded studies by PECOS items in the flowchart (Figure 1). With 2 additional records identified from scanning references of the identified reviews, we included 107 articles for further data extraction (Appendix 2.3). The descriptive information of the included articles is shown in Table 1. More detailed information is available in the data extraction file (Appendix 3).

There is a large number of studies now that have evaluated mortality effects of long-term exposure to $\mathrm{PM}_{2.5}$ or $\mathrm{PM}_{10}$. The vast majority uses the cohort study design, with only three articles use a case-control study design (two in patient population and one in infants). Studies have been conducted in a wide range of countries, though the majority has been conducted in North America $(\mathrm{N}=62)$ and Europe $(\mathrm{N}=25)$. There is an increasing number of studies from Asia $(\mathrm{N}=19)$, but currently no studies from Africa, Central and South America. Widely different populations have been studied, including general population samples, elderly, specific occupational groups (nurses, agricultural workers). Studies further differ in their follow-up period, with the start of follow-up ranging from the 1970 to 2003 across studies. Several very large studies based upon administrative databases with more than a million subjects have been reported. Studies further differ in the detail of information available on potential confounders. Exposure assessment has evolved from assigning of city-average concentrations in the earlier studies to more individualized exposure assessment in later studies, using land use regression, dispersion modeling or interpolation.

\subsection{Meta analyses}

\section{Main analyses}

In addition to the forest plots showing articles included in the meta-analyses (Figure 2-5, Figure A7.1 - A7.11), we also presented all articles relevant for a specific exposure-outcome pair in a separate plot (Figure A7.12 - A7.26). The second set of plots (plot with all articles) were used to document our selection of a specific article in case of multiple articles from the same study population. Three studies that performed methodologically incorrect analyses were excluded from meta analyses as these results are biased (Kim et al., 2017; Zhang et al., 2014; Pun et al., 2017). Two of these studies assigned mean exposure over the follow-up on an individual basis, with subjects who did not die receiving the average of the full follow-up period (Kim et al., 2017; Zhang et al., 2014). Ostro et al., (2011) showed that assigning follow-up averages exposure to each individual while there was a downward trend in longterm ambient air pollution, resulted in severe overestimates of the air pollution risks. In Pun et al., (2017), HRs were biased upwards because of incorrect incorporation of exposure in analysis model: results affected by downward trend in exposure contrast in longer exposure window. The effect estimates extracted from these three studies were documented in the second set of plots (plot with all articles).

For $\mathrm{PM}_{2.5}$ and natural-cause mortality, the all-articles plot illustrates that several cohorts (e.g. ACS, CanCHEC) have been studied multiple times (Figure A7.12). The large majority of articles report a positive association between $\mathrm{PM}_{2.5}$ and natural-cause mortality. The overall summary estimate of the 25 studies is 1.08 (95\% CI 1.06, 1.09). No single study has a large weight (Figure 3). Similar patterns were observed for other pollutant-outcome combinations. For PM $\mathrm{PM}_{10}$ and ALRI mortality, no metaanalysis was performed as only two studies were identified (Figure A7.24).

The pooled effect estimates for all exposure-outcome pairs are shown in Table 2. $\mathrm{PM}_{2.5}$ was significantly associated with natural-cause mortality and all evaluated causes of death separately. HRs for all evaluated specific causes were moderately large than for natural-cause mortality. $\mathrm{PM}_{10}$ was significantly associated with natural-cause, ischemic heart disease, respiratory and lung cancer mortality. For $\mathrm{PM}_{10}$, effect estimates for the respiratory but not the cardiovascular outcomes were larger than for natural- 
cause mortality. For all outcomes, the number of studies included in meta-analysis for $\mathrm{PM}_{10}$ is less than that for $\mathrm{PM}_{2.5}$, thus might lead to less precise pooled effect estimates.

For most exposure-outcome pairs, there is a large degree of heterogeneity across studies as evidenced by the high $\mathrm{I}^{2}$ and the larger $80 \%$ prediction interval compared to the $95 \%$ random effects interval. High heterogeneity is to be expected, given differences in study location, population characteristics, level and composition of PM and methodological differences between the studies (Table 1).

(1) Records identified through MEDLINE $(\mathrm{n}=1855)$ and EMBASE $(\mathrm{n}=1962)$

(2) Records after duplicates removed $(n=3162)$

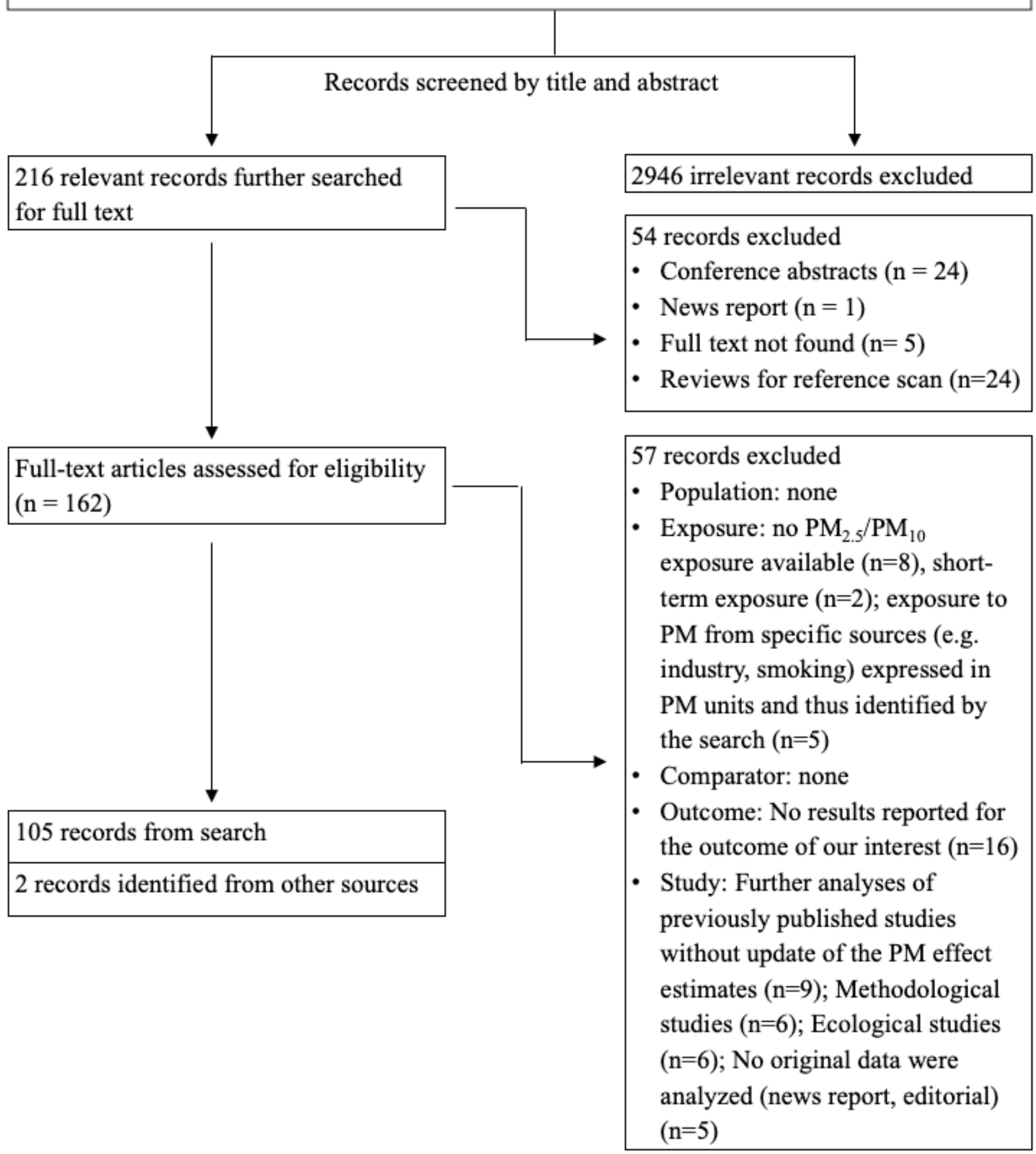

Figure 1. Flowchart of assessment of eligible studies 


\begin{tabular}{|c|c|c|c|c|c|c|c|c|c|c|c|c|c|c|c|c|c|c|}
\hline 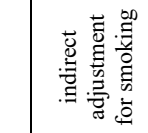 & $=$ & $>1$ & $=$ & $=$ & $=$ & $=$ & $=$ & $=$ & $=$ & $\lambda$ & $=$ & $=$ & $=$ & $=$ & $=$ & $=$ & $=$ & $=$ \\
\hline 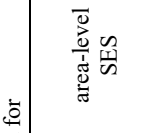 & $=$ & $>1$ & $\lambda$ & $\lambda$ & $\lambda$ & $\lambda$ & $\lambda$ & $\lambda$ & $>1$ & $=$ & $=$ & $\lambda$ & $\Rightarrow$ & $=$ & $\lambda$ & $=$ & $=$ & $\lambda$ \\
\hline 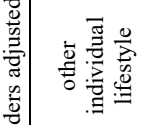 & $\lambda$ & $=$ & $=$ & $=$ & $\lambda$ & $\lambda$ & $\lambda$ & $\lambda$ & $=$ & $=$ & $=$ & $\lambda$ & $=$ & $\lambda$ & $\lambda$ & $\lambda$ & $\lambda$ & $=$ \\
\hline 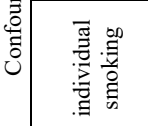 & $\lambda$ & $=1$ & $\lambda$ & $\lambda$ & $\lambda$ & $\lambda$ & $\lambda$ & $\lambda$ & $>1$ & $=$ & $=$ & $\lambda$ & $=$ & $\lambda$ & $\lambda$ & $\lambda$ & $\lambda$ & $=$ \\
\hline 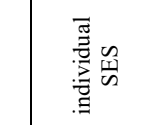 & $\lambda$ & $>1$ & $=$ & $=$ & $>$ & $\lambda$ & $\lambda$ & $=$ & $=$ & $\lambda$ & $\lambda$ & $=$ & $>$ & $\lambda$ & $\lambda$ & $\lambda$ & $\lambda$ & $\lambda$ \\
\hline 恖 & $\lambda$ & $>$ & $\lambda$ & $\lambda$ & $>$ & $>$ & $\lambda$ & $\lambda$ & $>$ & $\lambda$ & $\lambda$ & $>$ & $>$ & $\lambda$ & $\lambda$ & $\lambda$ & $\lambda$ & $\lambda$ \\
\hline 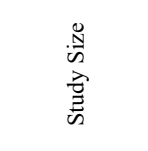 & 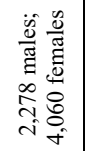 & 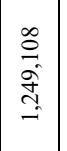 & 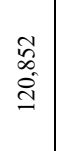 & 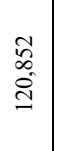 & 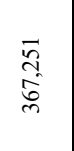 & $\begin{array}{l}\infty \\
\infty \\
0 \\
0 \\
0 \\
n\end{array}$ & $\begin{array}{l}\text { त्र } \\
\text { î. } \\
\text { in }\end{array}$ & $\begin{array}{l}\infty \\
\stackrel{\infty}{\div+} \\
\stackrel{+}{=}\end{array}$ & $\begin{array}{l}\hat{\tilde{A}} \\
\stackrel{\Xi}{\simeq}\end{array}$ & 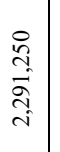 & 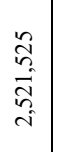 & 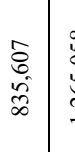 & 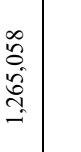 & ले & $\begin{array}{l}\stackrel{n}{\infty} \\
\infty \\
\infty \\
\infty\end{array}$ & $\begin{array}{l}\text { t. } \\
\text { o. } \\
\text { 今े }\end{array}$ & 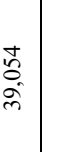 & 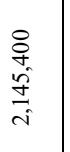 \\
\hline 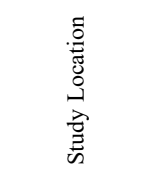 & 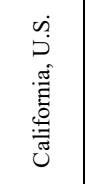 & 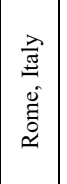 & 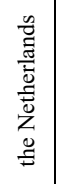 & 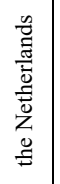 & $\begin{array}{l}\text { o. } \\
\text { 音 } \\
\text { 严 }\end{array}$ & 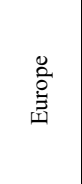 & $\begin{array}{l}\text { 莺 } \\
\text { 焉 }\end{array}$ & $\dot{S}$ & 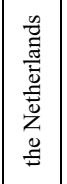 & 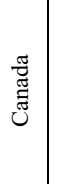 & $\begin{array}{l}\text { 焉 } \\
\text { 志 }\end{array}$ & 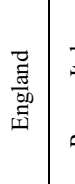 & 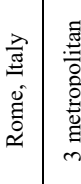 & 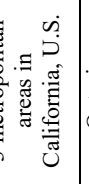 & : & 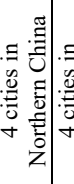 & 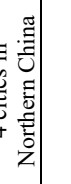 & 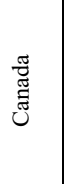 \\
\hline 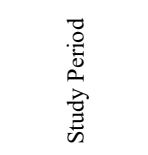 & 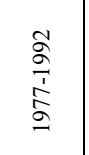 & 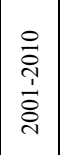 & 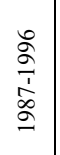 & 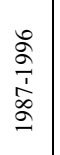 & $\begin{array}{l}\infty \\
\stackrel{1}{1} \\
\dot{1} \\
\Delta \\
\Delta \\
0\end{array}$ & 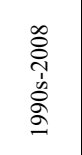 & 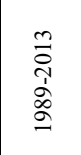 & 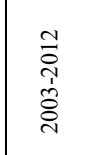 & 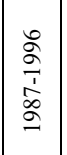 & $\begin{array}{l}\overline{\bar{a}} \\
\frac{1}{\Delta} \\
\frac{a}{2}\end{array}$ & $\begin{array}{l}8 \\
\stackrel{9}{9} \\
\frac{1}{2} \\
\frac{1}{2}\end{array}$ & 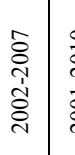 & | & $\begin{array}{l}\infty \\
\stackrel{\infty}{2} \\
\frac{1}{5} \\
\vdots \\
\frac{1}{2}\end{array}$ & $\begin{array}{l}\overline{\bar{a}} \\
\stackrel{1}{\Delta} \\
\text { aे }\end{array}$ & 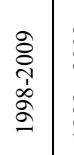 & 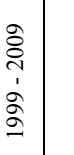 & 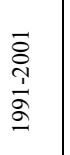 \\
\hline 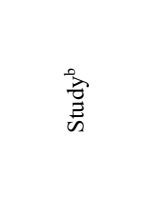 & 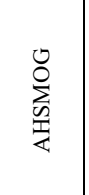 & 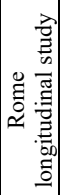 & $\begin{array}{l}v \\
\dot{z} \\
\dot{z}\end{array}$ & 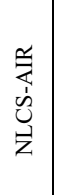 & 战 & 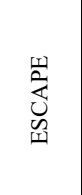 & $\begin{array}{l}\overline{\mathbb{E}} \\
\mathbb{J}\end{array}$ & 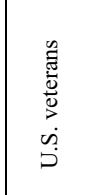 & $\begin{array}{l}\mathfrak{z} \\
\dot{d} \\
\dot{z} \\
\mathrm{z}\end{array}$ & 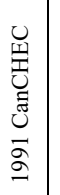 & 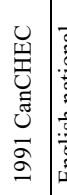 & 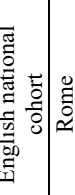 & 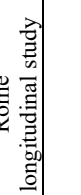 & 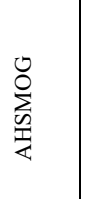 & 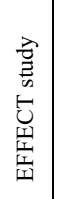 & 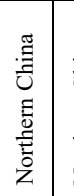 & 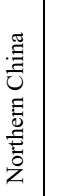 & 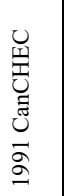 \\
\hline $\begin{array}{l}\text { है } \\
\text { : } \\
\text { 訁 }\end{array}$ & $\begin{array}{l}\underset{G}{0} \\
\ddot{\sim} \\
\ddot{4}\end{array}$ & \begin{tabular}{|c|}
0 \\
$\dddot{4}$
\end{tabular} & 0 & $\begin{array}{l}\underset{\cup}{u} \\
\ddot{\ddot{u}} \\
\ddot{4}\end{array}$ & $\varangle$ & 0 & $\begin{array}{l}\stackrel{\ddot{u}}{\ddot{4}} \\
\ddot{4}\end{array}$ & $\varangle$ & \begin{tabular}{|c|}
0 \\
$\ddot{\sim}$ \\
$\ddot{\ddot{x}}$ \\
$\ddot{\leftarrow}$
\end{tabular} & $\begin{array}{l}\stackrel{u}{u} \\
\ddot{\varkappa} \\
\ddot{4}\end{array}$ & $\stackrel{u}{\ddot{x}}$ & 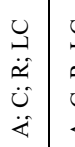 & 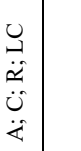 & 0 & $\stackrel{u}{\ddot{4}}$ & \begin{tabular}{l}
0 \\
\hdashline \\
\hdashline
\end{tabular} & $\simeq$ & 芹 \\
\hline 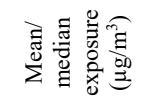 & 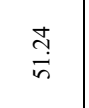 & $=0$ & & $\begin{array}{l}m \\
\dot{d} \\
\dot{d}\end{array}$ & & & $\because=$ & $\stackrel{\infty}{=}$ & $\mid \begin{array}{c}m \\
\stackrel{\infty}{\infty}\end{array}$ & & $\dot{\infty}$ & $=\stackrel{i}{i}$ & 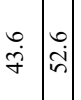 & ते & $\hat{\varrho}$ & 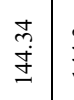 & $\stackrel{?}{g}$ & $\hat{\infty}$ \\
\hline 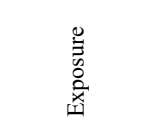 & 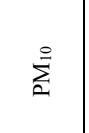 & $\sum_{\substack{n \\
n}}^{2}$ & $\sum_{n=1}^{n}$ & 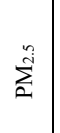 & $\sum_{\sum_{i=1}^{\infty}}^{\sum_{n=1}^{n}}$ & $\sum_{\substack{n \\
\text { ind }}}^{\infty}$ & $\sum_{\substack{a \\
n}}^{\infty}$ & $\sum_{n=1}^{n}$ & $\sum_{\substack{n \\
n}}^{n}$ & $\sum_{n=1}^{n}$ & $\sum_{a}^{n}$ & $=$ & $\sum_{i=0}^{n}$ & 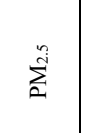 & $\sum_{a}^{n}$ & $\sum_{\beth}^{\circ}$ & $\sum_{i}^{\circ}$ & $\sum_{n=1}^{n}$ \\
\hline 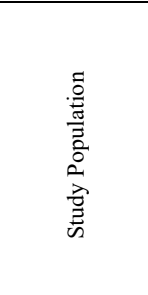 & 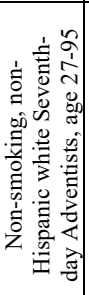 & 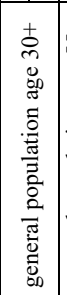 & 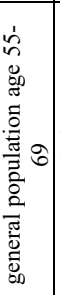 & 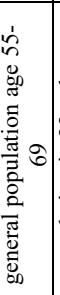 & 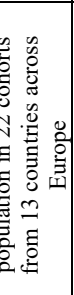 & 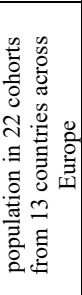 & 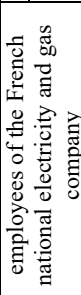 & 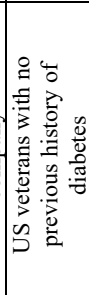 & 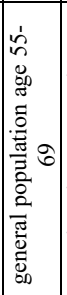 & 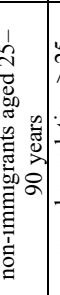 & 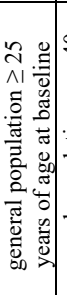 & 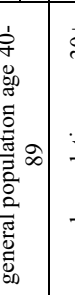 & 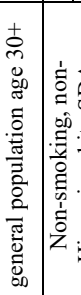 & 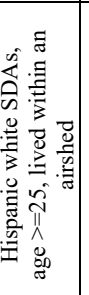 & 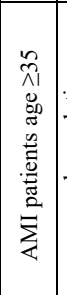 & 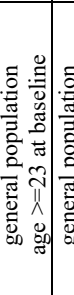 & 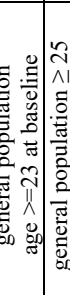 & 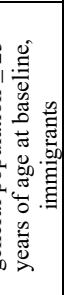 \\
\hline 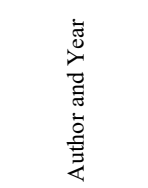 & 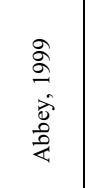 & 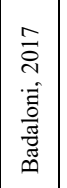 & 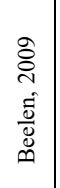 & 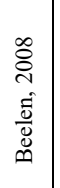 & 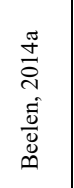 & 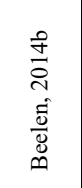 & 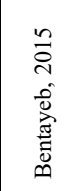 & 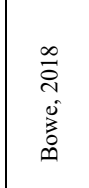 & 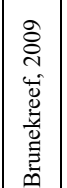 & 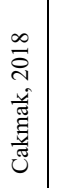 & 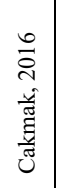 & 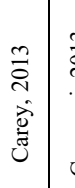 & 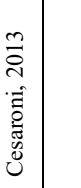 & 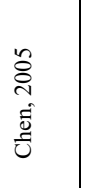 & 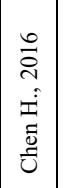 & 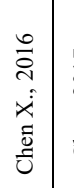 & 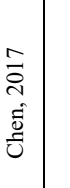 & 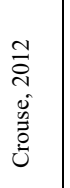 \\
\hline A & - & a & $m$ & + & in & 6 & - & $\infty$ & $a$ & 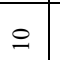 & $=$ & $\simeq \quad$ & $m$ & \pm & $\underline{n}$ & $\stackrel{\circ}{\circ}$ & $=$ & $\stackrel{\infty}{\prime}$ \\
\hline
\end{tabular}




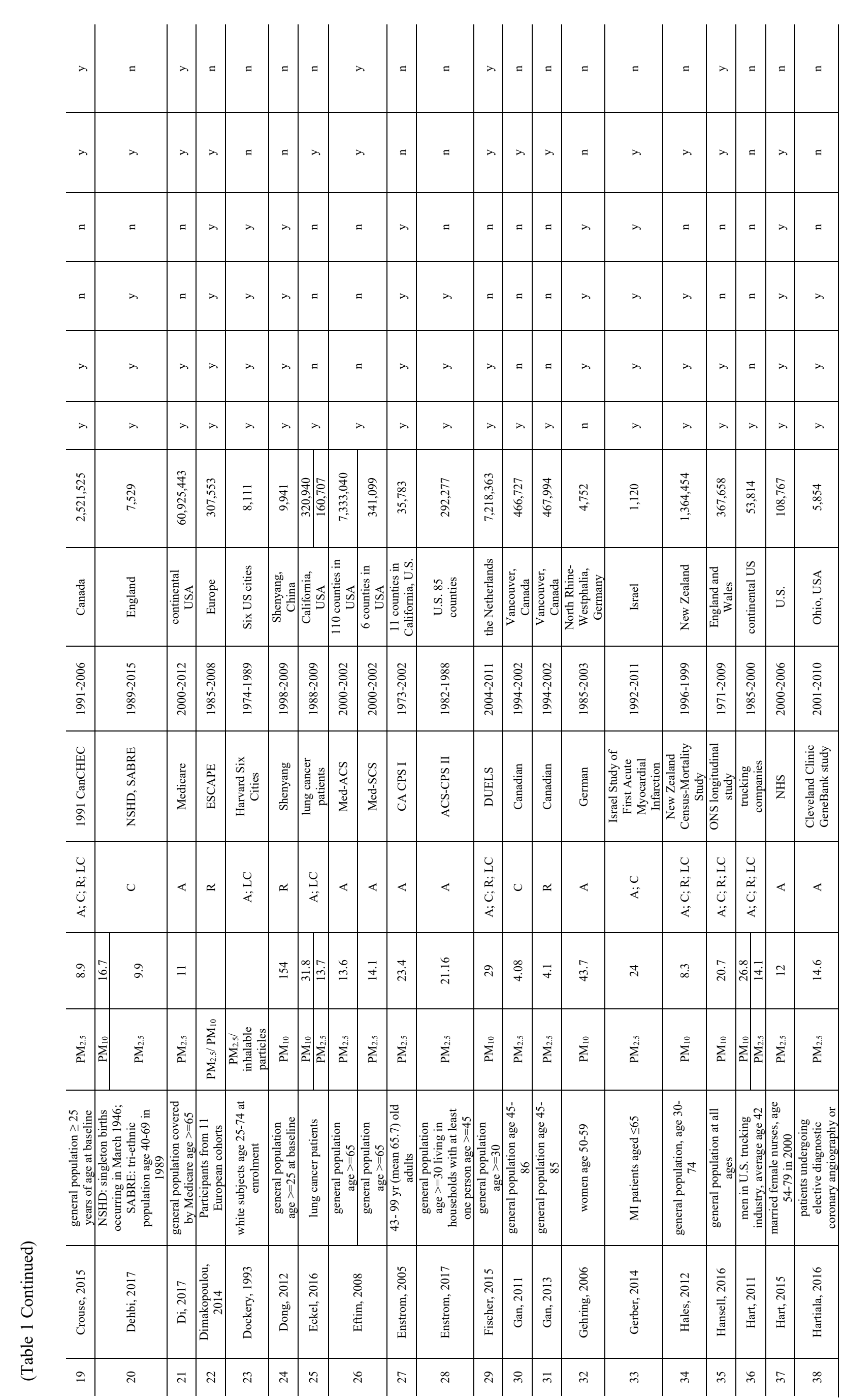




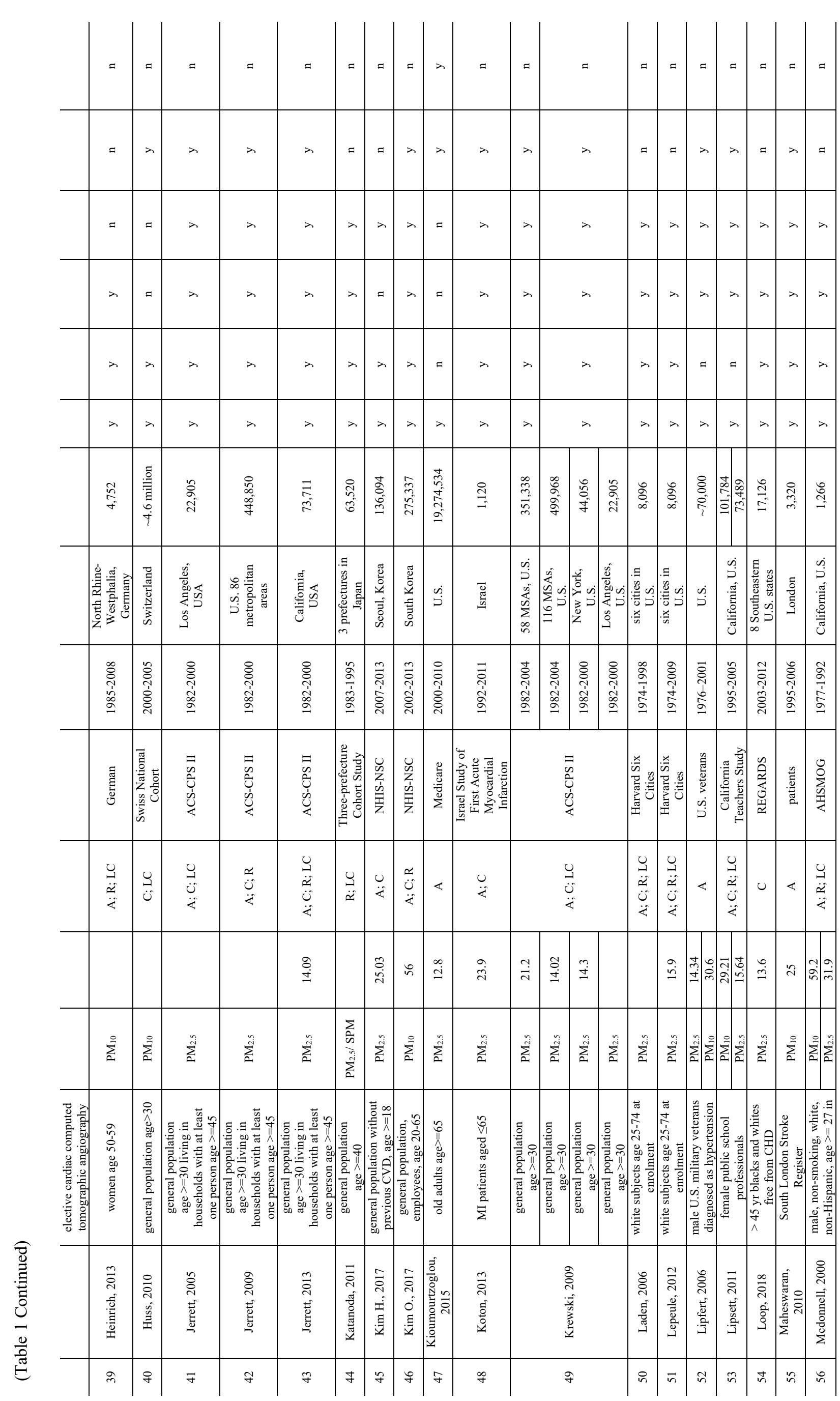




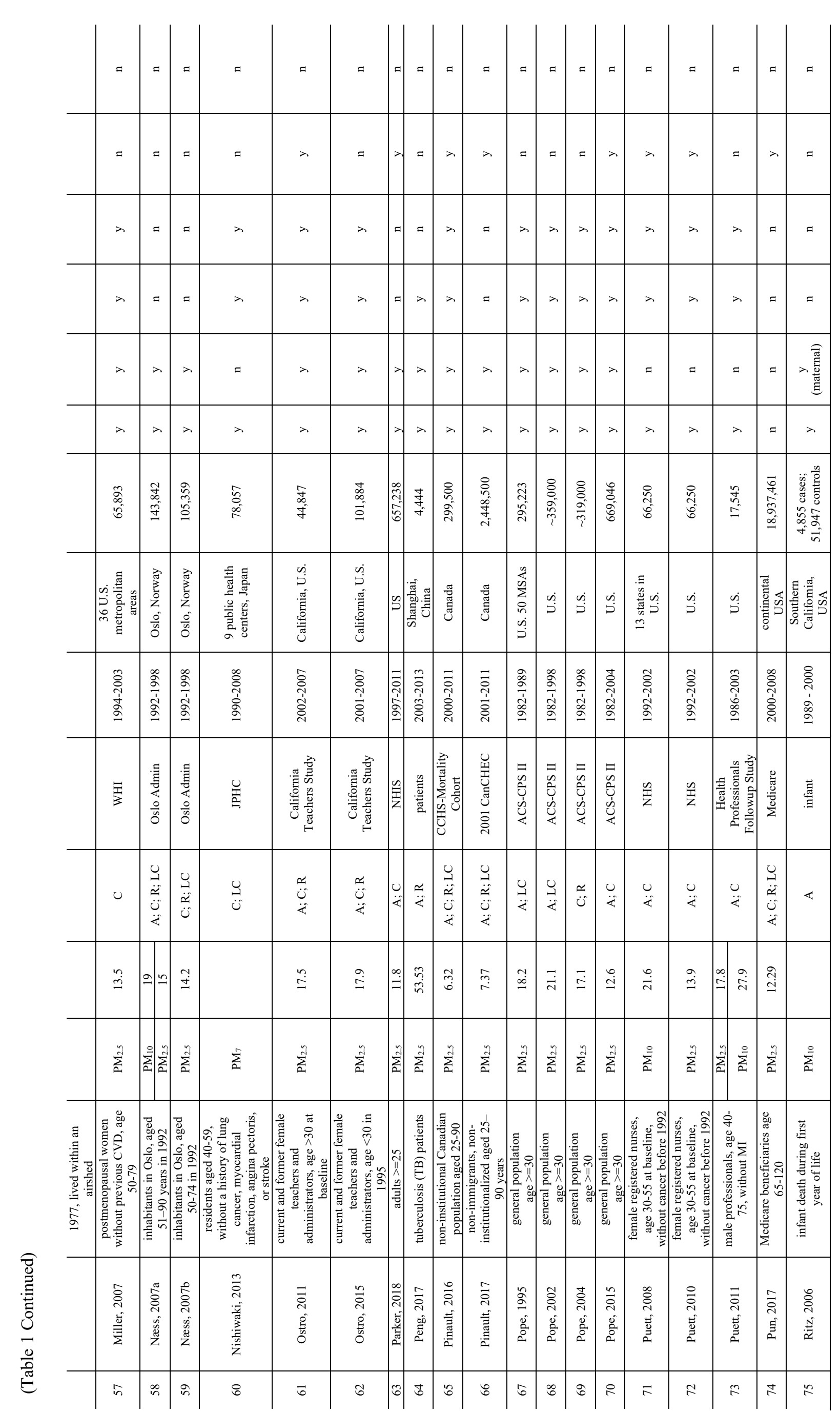




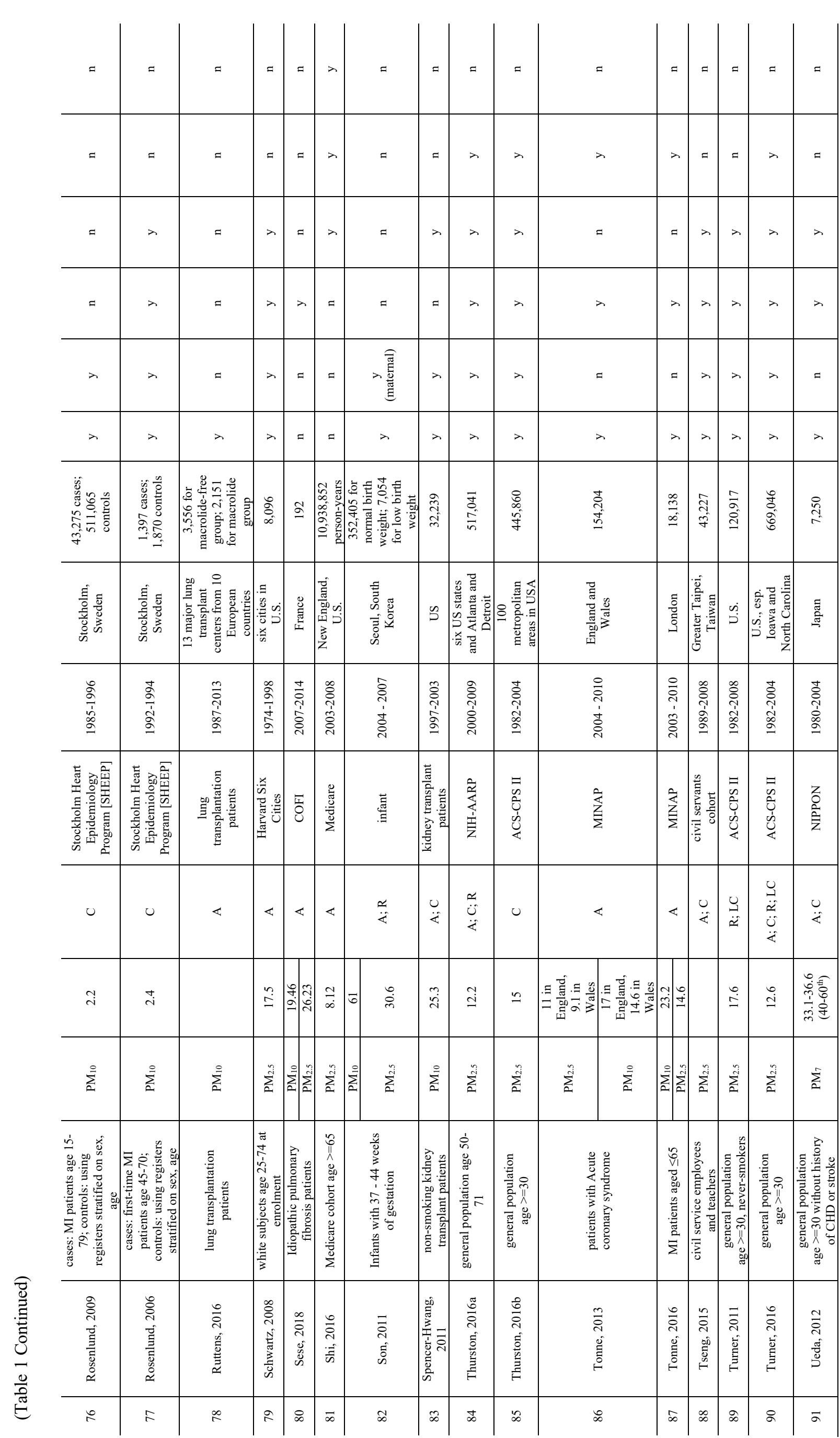




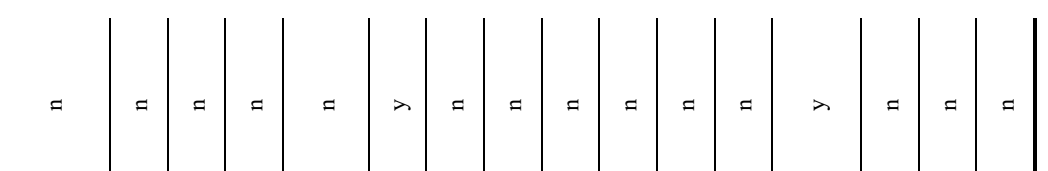

\begin{tabular}{|c|c|c|c|c|c|c|c|c|c|c|c|c|c|c|c|}
\hline$\lambda$ & $\lambda$ & $\lambda$ & $=$ & $\lambda$ & $\Rightarrow$ & $=$ & $\lambda$ & $\lambda$ & $\lambda$ & $=$ & $=$ & $\Rightarrow$ & $\lambda$ & $\lambda$ & $\lambda$ \\
\hline$\lambda$ & $\lambda$ & $\lambda$ & $=$ & $\lambda$ & $\Rightarrow$ & $=$ & $\lambda$ & $\lambda$ & $\lambda$ & $=$ & $=$ & $=$ & $\lambda$ & $\lambda$ & $\lambda$ \\
\hline
\end{tabular}

离

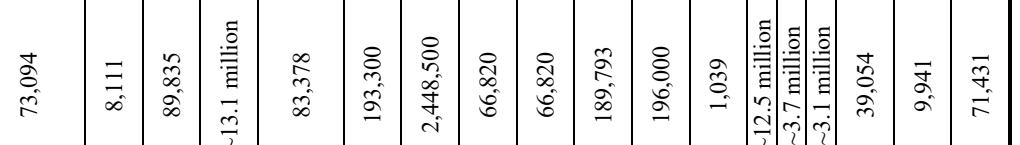

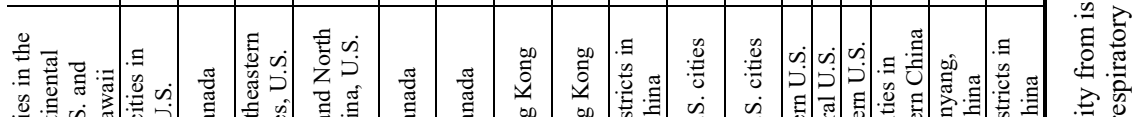

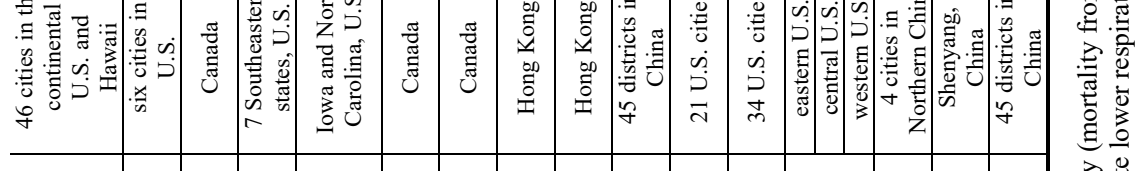

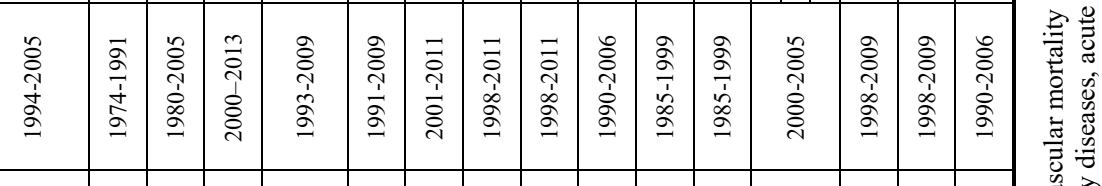

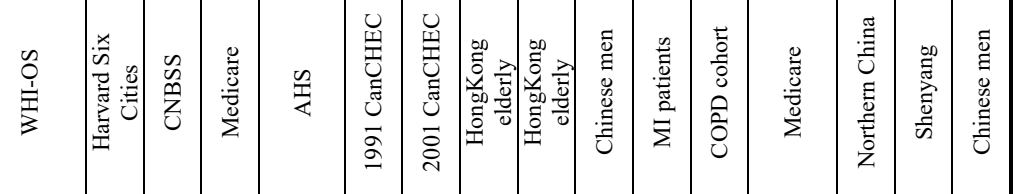

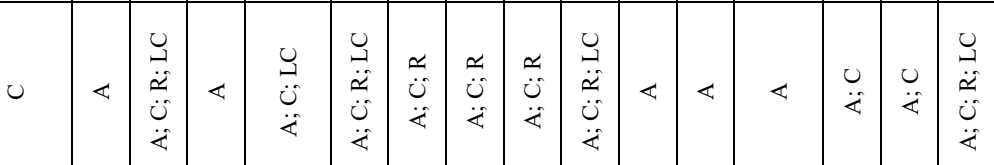

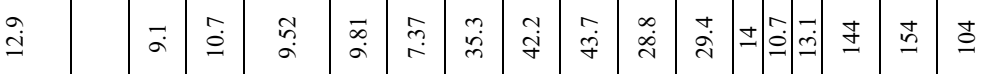

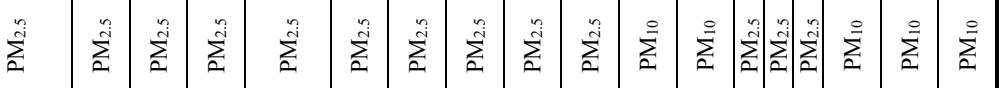

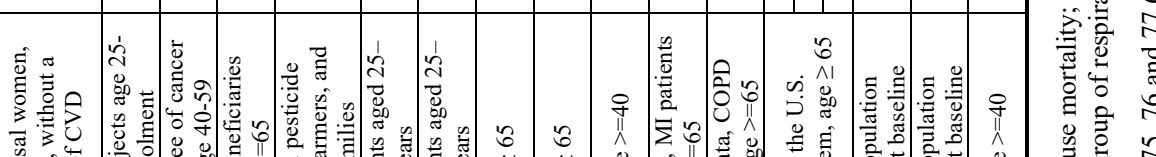

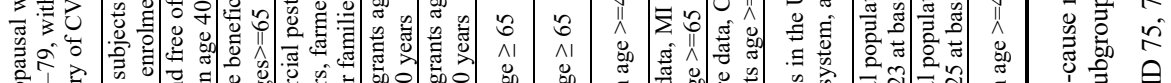

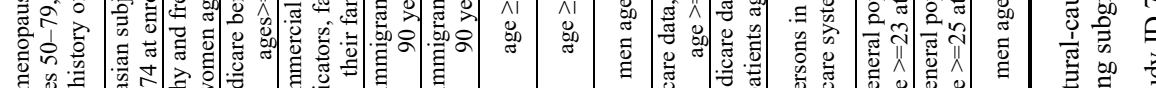

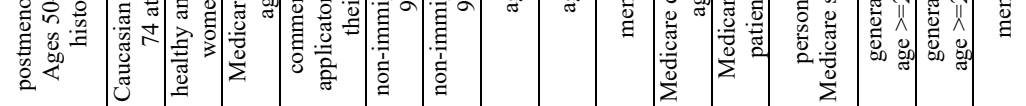

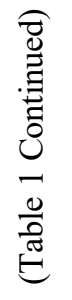

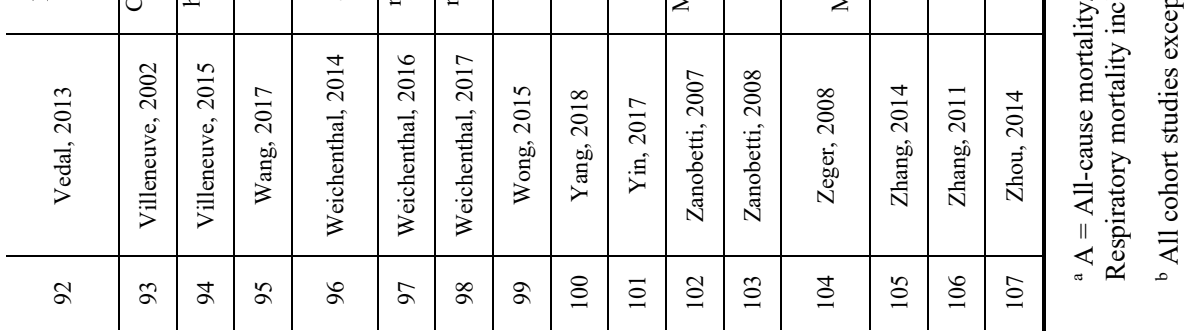




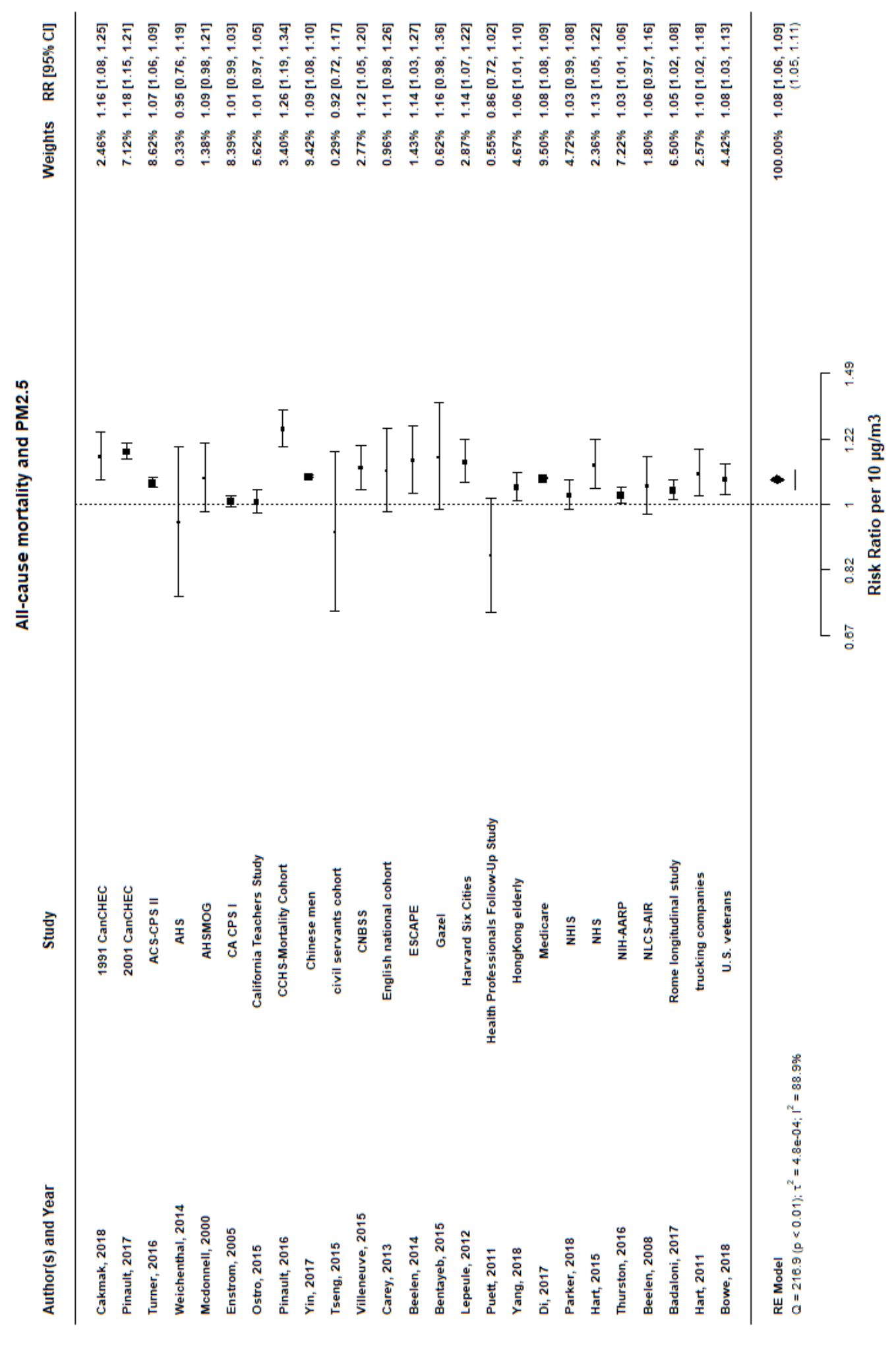

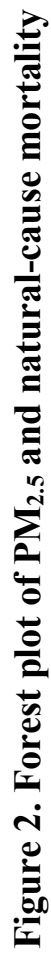




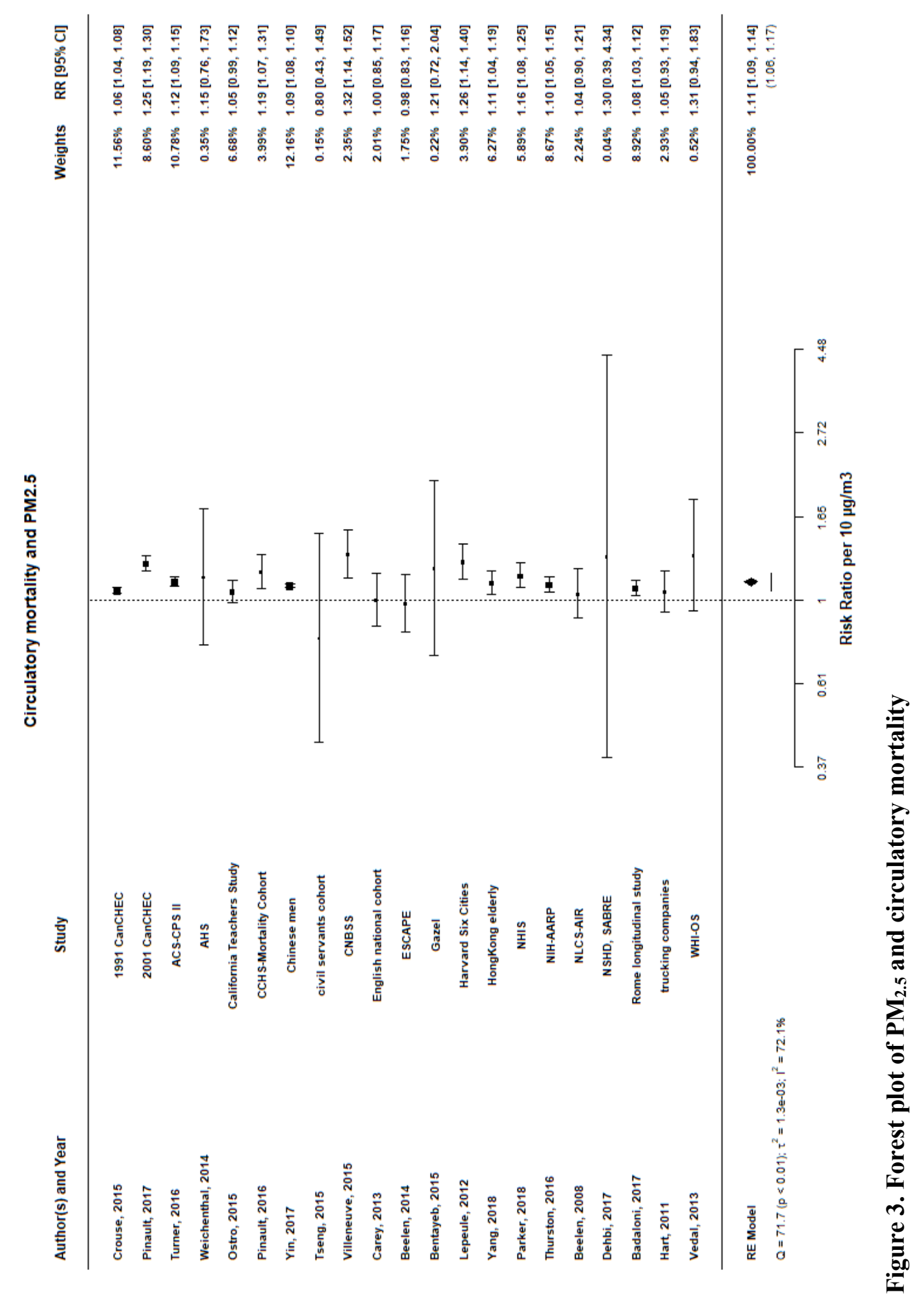




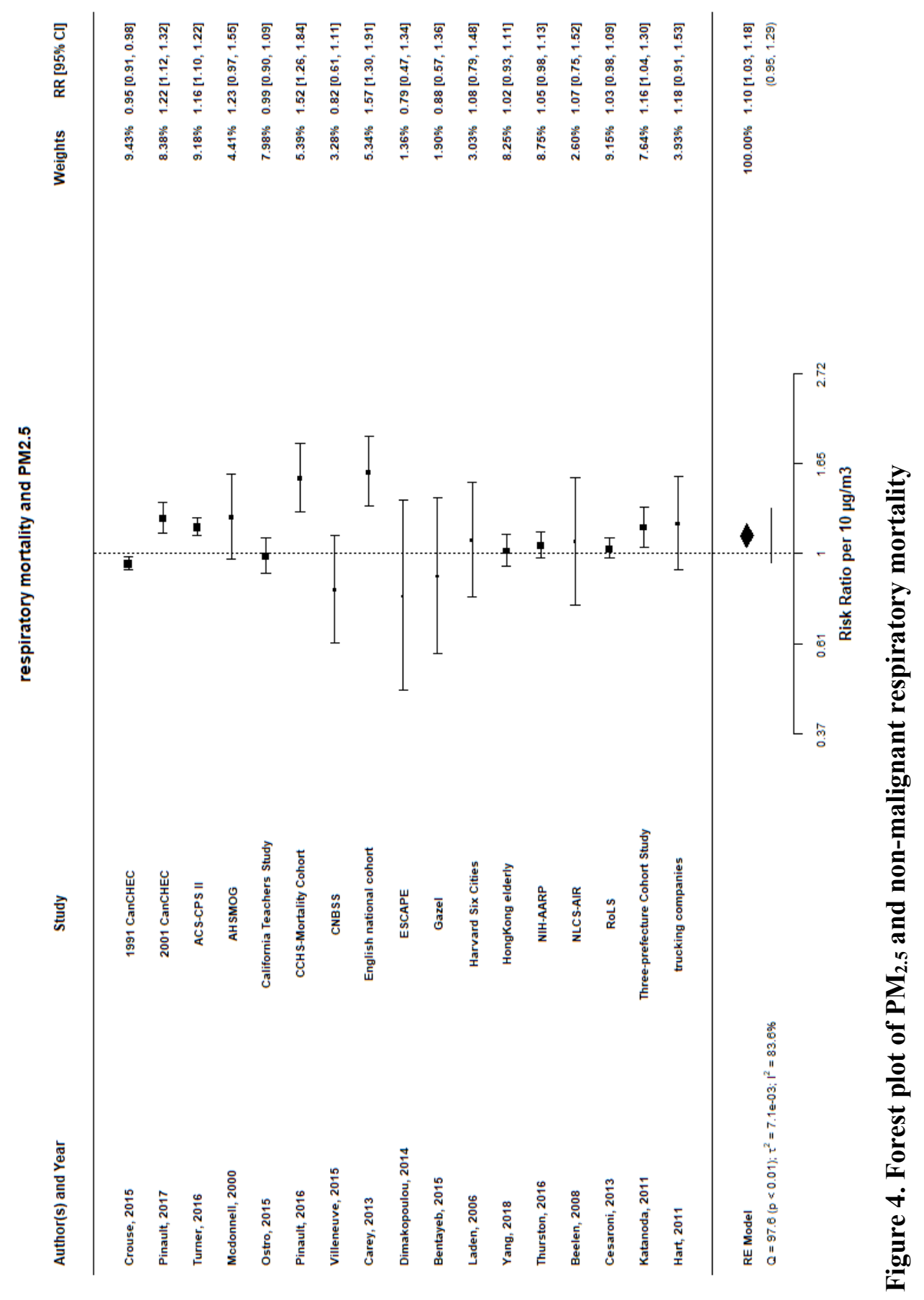


Chapter 2

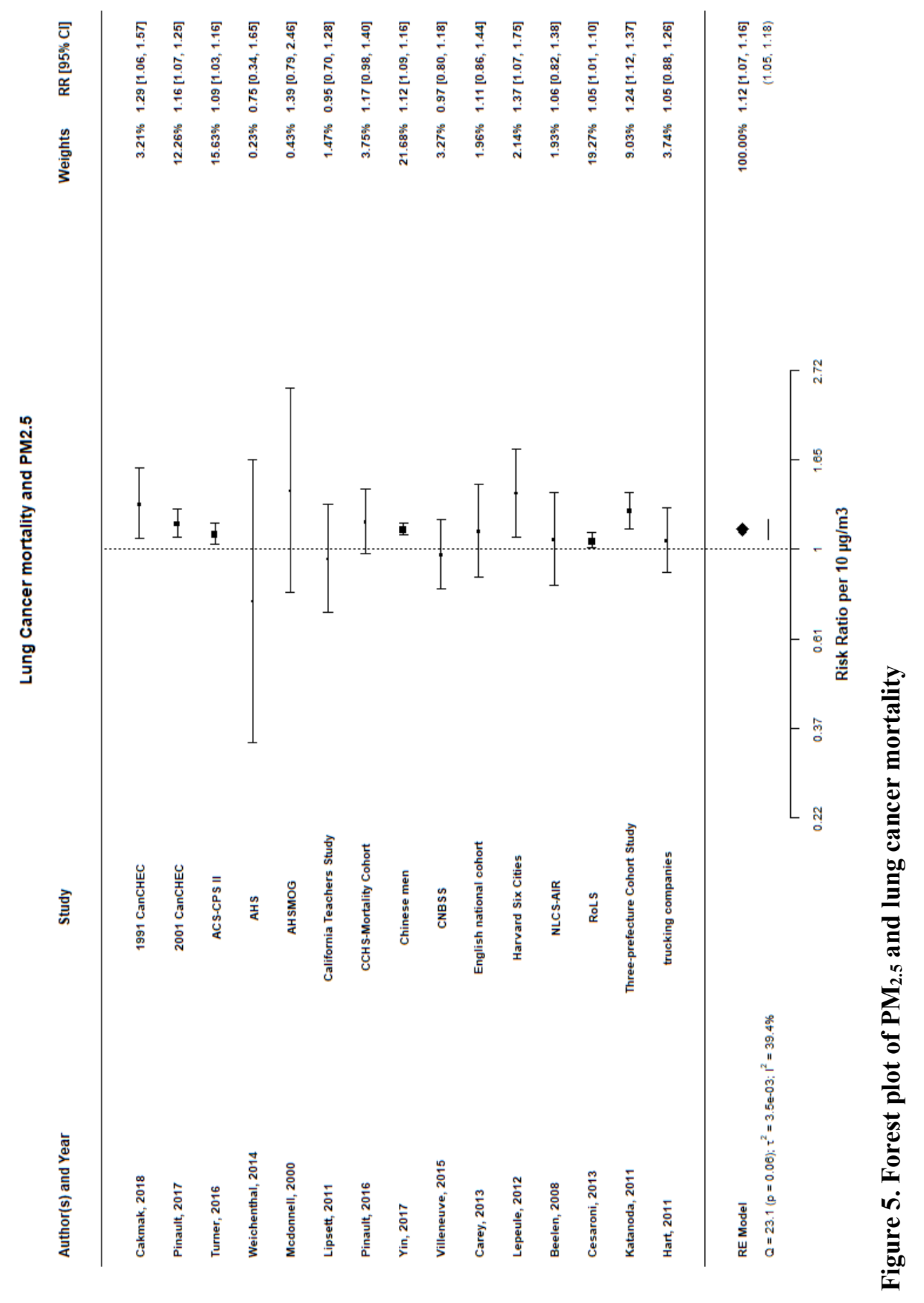




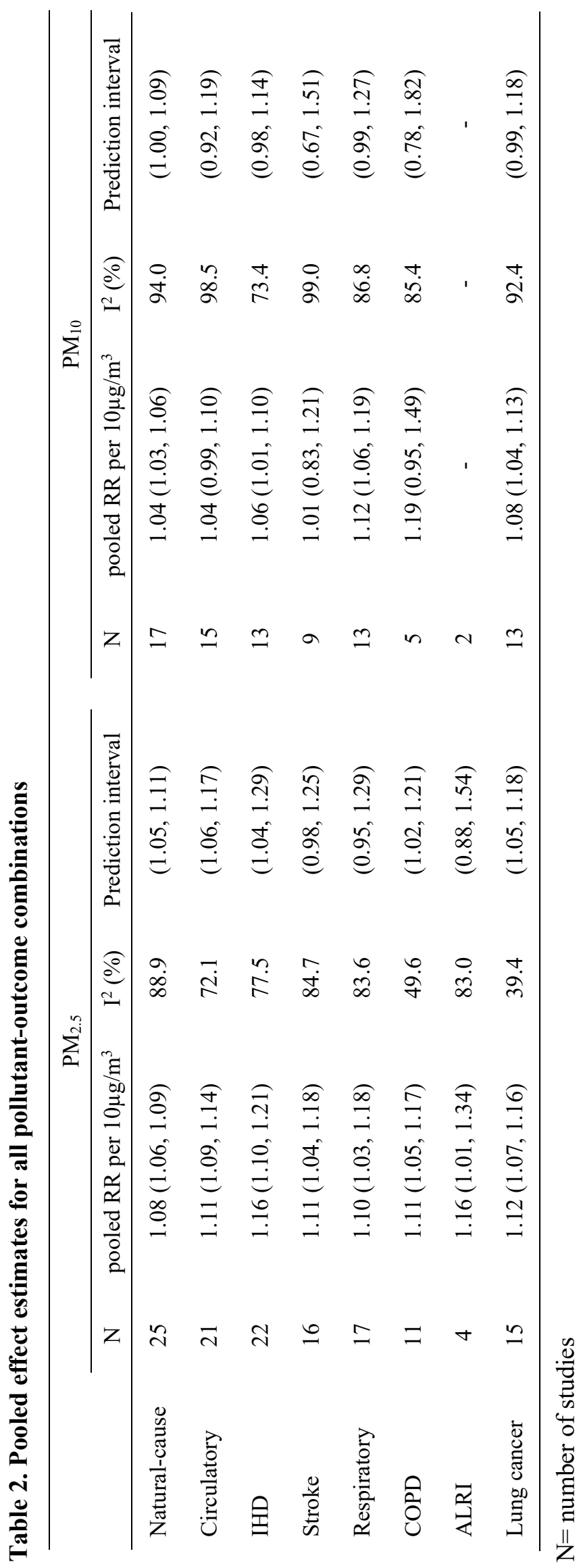




\section{Analyses of the shape of concentration-response function (CRF)}

Four studies assessed natural-cause mortality effects for participants exposed to $\mathrm{PM}_{2.5}$ concentrations below certain exposure levels (Figure A7.27). The positive associations remained below $10 \mu \mathrm{g} / \mathrm{m}^{3}$ in Medicare $\left(1.09\right.$ (95\% CI 1.01, 1.19)) and below $5 \mu \mathrm{g} / \mathrm{m}^{3}$ in CanCHEC $(1.27$ (95\%CI 1.09,1.49)). More assessments of the shape of the CRF by individual studies are documented in Appendix 5. These studies typically used non-parametric splines. The majority of studies which analyzed the CRF had no evidence of a threshold and showed linear or supra-linear functions.

We further combined effect estimates for studies with mean $\mathrm{PM}_{2.5}$ concentrations below certain cut-off exposure levels (Figure A7.28 - A7.32). The combined effect estimate is 1.17 (95\% CI 1.12, 1.23) for the five studies with a mean concentration below $10 \mu \mathrm{g} / \mathrm{m}^{3}$. The limitation of this approach is that subjects exposed to pollution concentration higher than the cut-off exposure levels in the cohorts were also included.

\section{Subgroup analyses}

Virtually the same effect estimates were found for European Region, Region of the Americas and Western Pacific Region (Figure 6). Heterogeneity especially remained within the large group of North American studies. Virtually no difference in effect estimates was found between studies in men, women or combined (Figure A7.34). Studies performed in predominantly elderly showed somewhat smaller RRs but the confidence intervals overlapped (Figure A7.35). RRs tended to be larger in the studies with a mean $\mathrm{PM}_{2.5}$ concentration below $10 \mu \mathrm{g} / \mathrm{m}^{3}$ (Figure A7.36). No single factor can explain the source of high heterogeneity between studies. Meta-regression did not explain the source of high heterogeneity between studies (residual heterogeneity $\left(\mathrm{I}^{2}\right)=86.53 \%$ ), probably because little effect-modifier information is available on study-level factors.

\section{Additional analyses}

Two-pollutant models adjusting for $\mathrm{NO}_{2}$ were specified by five studies and documented overall much lower RRs for $\mathrm{PM}_{2.5}$ compared to the single pollutant estimates in studies that specified two pollutant models (Figure A7.37 and A7.38: $1.07(95 \%$ CI 1.05, 1.08) in single pollutant models versus 1.02 (95\% CI 1.00, 1.04) in two-pollutant models). Two pollutant models can be difficult to interpret when the correlation between pollutants is high or exposure for pollutants is assessed with different methods or at a different spatial resolution. RRs remained stable after adjusting for coarse particles or $\mathrm{O}_{3}: 1.14(95 \%$ CI 1.05, 1.24) based upon three studies and 1.08 (95\% CI 1.04, 1.11) based upon seven studies respectively.

The combined effect estimate remained the same $(1.08$ (95\% CI 1.06, 1.09)) after we excluded one study that was at high risk of bias in any domain for $\mathrm{PM}_{2.5}$ and natural-cause mortality combination. The combined estimate slightly increased with a slightly wider confidence interval $(1.09$ (95\% CI 1.06, 1.11)) after we further excluded studies that score moderate on an item that all other studies score low (Figure A7.39). The combined effect estimate remained stable as these two studies had little weight on the metaanalysis.

Exclusion of the large administrative cohorts, which have limited information on individual lifestyle factors resulted in an identical effect for $\mathrm{PM}_{2.5}$ and natural-cause mortality, but with a slightly wider confidence interval: 1.08 (95\% CI 1.05, 1.10) (Figure A7.40).

Inclusion of studies conducted in the very diverse patient populations resulted in a modest increase in effect estimates and a further increase in heterogeneity for $\mathrm{PM}_{2.5}$ and natural-cause mortality (Figure A7.41: 1.11 (95\% CI 1.07, 1.14)). With the exception of a study in lung cancer patients, these typically smaller studies had less precise effect estimates than the general population studies. Including one small study reported effect estimate for $\mathrm{PM}_{2.5}$ and natural-cause mortality in infants with low precision did not change the overall effect estimate (Figure A7.42). 


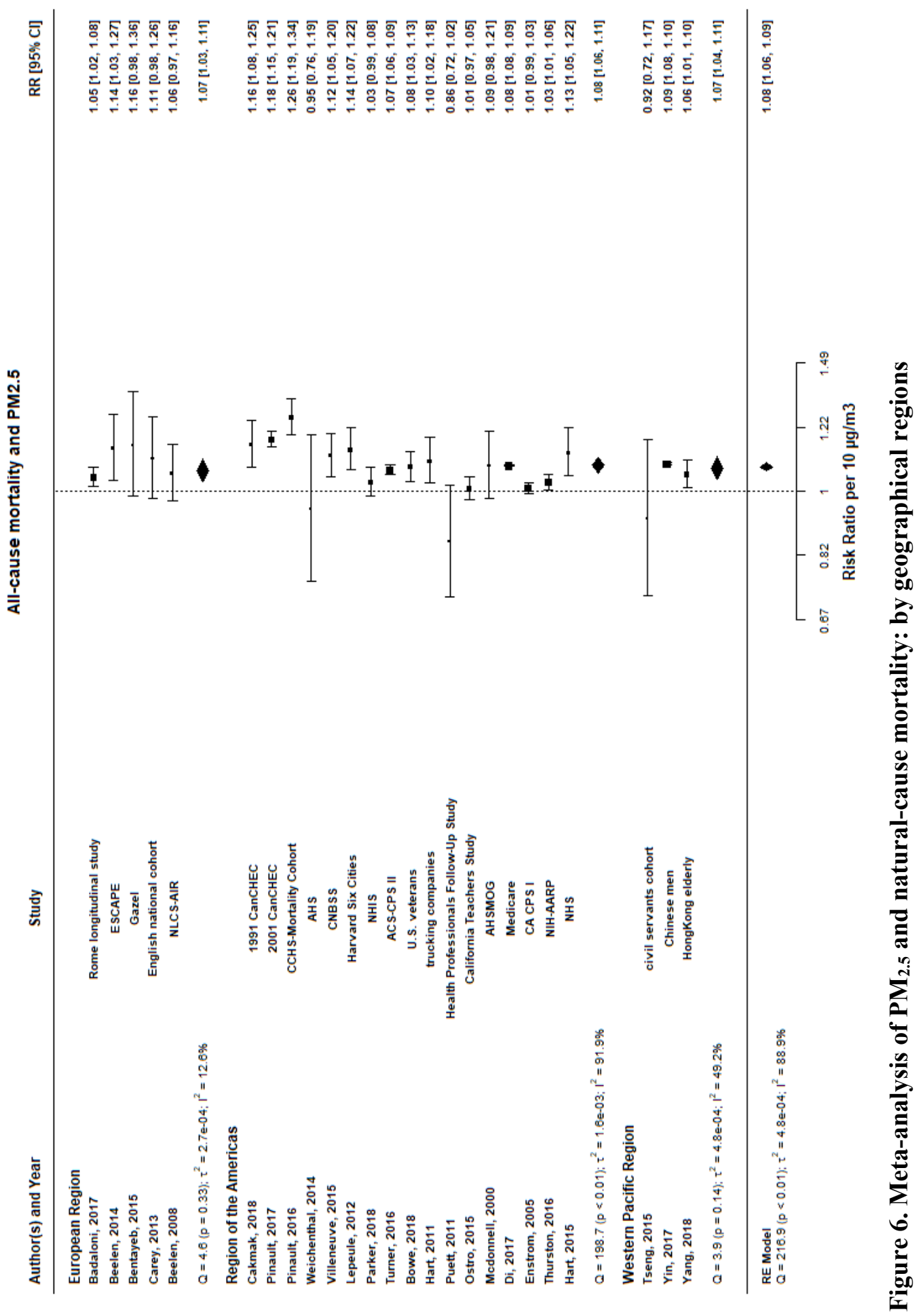




\subsection{Risk of Bias (RoB) assessment}

Figure 7 shows a summary of RoB assessments for all studies included in the meta-analyses. The individual RoB assessments are presented in Appendix 4b. Most but not all of the cohort studies had similar evaluations because of the similar study design they used - following the classical cohort studies conducted in the USA, specifically the Six city study (Dockery et al., 1993) and the American Cancer Society study (Pope et al., 2002).

Confounding Most of the studies were rated as 'moderate risk' in this domain. The most critical subdomain was "Were all confounders considered adjusted for in the analysis?". Individual-level smoking and BMI were usually not available in large administrative cohorts, and were indirectly adjusted for in some studies using an ancillary dataset (Badaloni et al., 2017; Crouse et al., 2015; Di et al., 2017; Fischer et al., 2015). Some studies were rated as 'moderate risk' because one or two of the confounders in the list of other/ additional potential confounders were not adjusted for. Studies that adjusted for a large number of individual- and area-level risk factors, such as the ACS study typically did not adjust for at least one of this list and we therefore rated the study as moderate risk of bias (Turner et al., 2016). This characterization is questionable however and some studies such as the ACS study would qualify as low risk of bias. Since the ACS study has adjusted for BMI and a large number of SES variables, it is highly likely that this will also adjust for effects of lack of physical activity. Similar examples apply to a large number of cohort studies, e.g. (Beelen et al., 2014; Carey et al., 2013; Thurston et al., 2016; Turner et al., 2016): 'low risk' might be more reasonable given the large number of covariates adjusted for.

Selection bias We found few studies where selection was related to exposure. Therefore, most of the studies were rated as 'low risk'.

Exposure assessment The exposure assessment methods varied across the studies. We considered most of the exposure assessment methods appropriate when they had documented validity such as good agreement between model predictions and measurements. Change in spatial exposure contrasts is not a potential risk for studies assigning time-varying exposures. In cohort studies where exposures were assigned to the participants for the same period, several reported the stability of spatial contrast. For studies that did not report stability of the contrasts, we made an assessment based on previous studies in the same study area and time period. We generally assessed spatial exposure contrasts did not change much in well-developed areas in North America and Europe. In strongly developing areas such as Asia, spatial exposure contrasts might have changed in the past decade(s). If the change of spatial exposure contrasts was not accounted for, we rated as 'moderate' or 'high' risk of bias.

Outcome measurement Most of the studies used a mortality registry to link health outcomes of the participants. Some earlier studies used interviews to confirm the vital status of participants. Deaths were classified according to the International Classification of Diseases (ICD) in most of the studies using the underlying cause of death. The same outcome measurement methods were applied for all subjects within a particular study, irrespective of the level of exposure, therefore all studies were rated as 'low risk'. We note that misclassification of cause of death may have contributed to additional noise in the data.

Missing data Studies measuring outcome by linkage to mortality registries were unlikely to have frequent missing outcome data. Most of the studies excluded subjects if they had missing exposure data. No studies used imputation of exposure. Percent of missing data of exposure was typically low. Therefore, most but not all of the studies were rated as 'low risk'.

Selective reporting Studies typically reported all risk estimates for the outcomes and pollutants identified in the Methods section therefore were rated as 'low risk'. One study (McDonnell et al., 2000) selectively reported effects for males only because effects for females were weak or inverse, thus was rated as "high risk". 


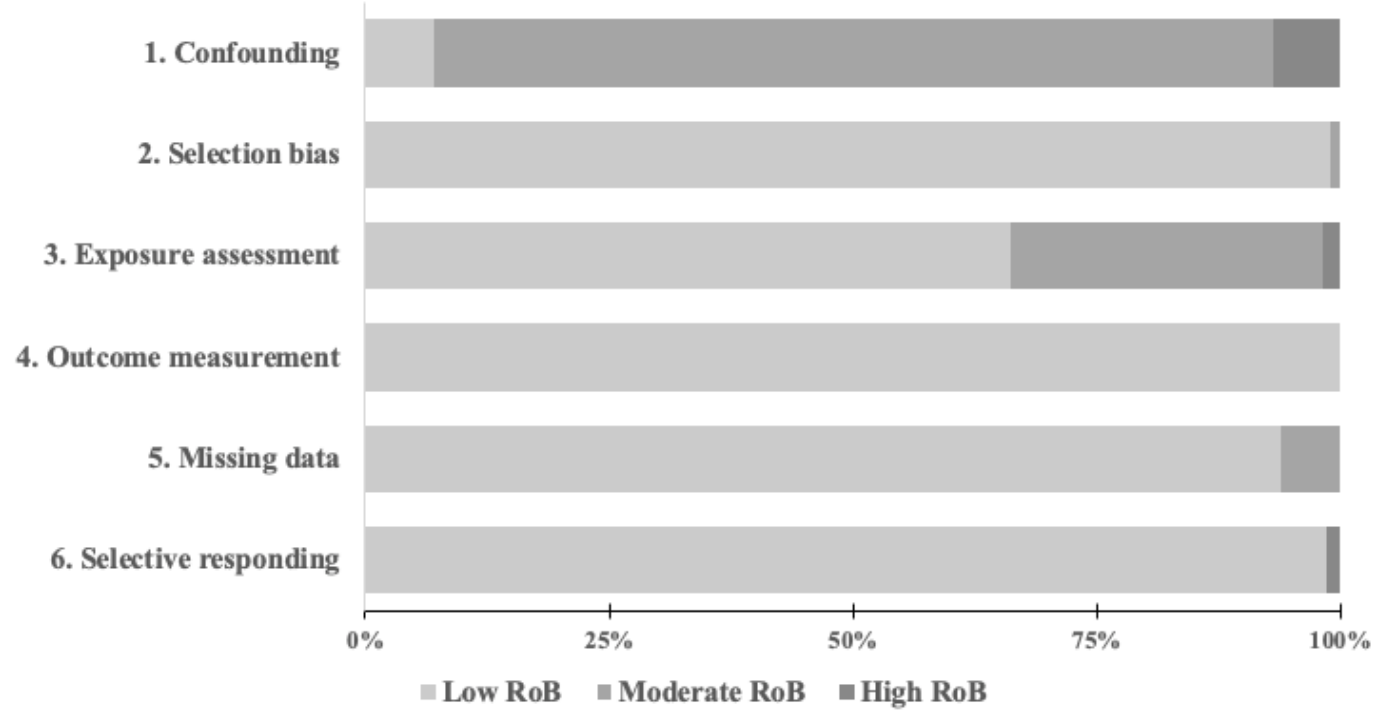

Figure 7. Summary of all RoB assessments (total number of assessments across all exposureoutcome pairs $=216$ )

\subsection{Assessment of the certainty of evidence}

Table 3 lists the application of the adapted GRADE tool to the body of evidence for $\mathrm{PM}_{2.5}$ and $\mathrm{PM}_{10}$ related to natural-cause and circulatory mortality. The complete evaluations are documented in Appendix $6 \mathrm{~b}$. The starting point was at moderate certainty of evidence reflecting the risk of unmeasured confounding in observational studies. We concluded an upgrade of the evidence with one level for all assessed endpoints associated with $\mathrm{PM}_{2.5}$ except respiratory mortality, and for natural-cause, respiratory and lung cancer mortality associated with $\mathrm{PM}_{10}$, resulting in a 'high certainty of evidence'. A downgrade with one level was concluded for $\mathrm{PM}_{10}$ and stroke mortality, resulting in a 'low certainty of evidence'. A 'moderate certainty of evidence' was assessed for the remainder of the exposure-endpoint combinations. Most of the rationales for the assessments in the various GRADE domains are documented in the previous sections. Briefly:

A1 Limitations in studies (risk of bias) No downgrading was applied as few studies were identified as 'high risk of bias' and exclusion of these studies had little impact on the overall effect estimate (section 3.2). This decision is supported by the stability of risk estimates excluding the large administrative cohorts with no direct information on individual lifestyle (section 3.2).

A2 Indirectness No downgrading was applied as all studies answered the PECOS (Population, Exposure, Comparator, Outcome and Study) question directly as shown in Table 1.

A3 Inconsistency $\mathrm{We}$ downgraded the certainty of the evidence for $\mathrm{PM}_{10}$ and circulatory mortality, $\mathrm{PM}_{10}$ and stroke mortality, and $\mathrm{PM}_{2.5}$ and respiratory mortality because the $80 \%$ prediction interval included unity; the width of the prediction interval was more than twice the width of confidence interval and there was a sizable number of studies with HRs below 1 . Heterogeneity was partly explained by the level of pollution and differences in population: higher HRs for studies with lower mean $\mathrm{PM}_{2.5}$ concentrations, somewhat lower HRs in elderly and higher HRs in patient populations.

A4 Imprecision No downgrading was applied as the included cohort studies followed a large population for several years, resulting in large number of person-years (Table 1). Several single studies included much more than 940000 person-years. All exposure outcome pairs (except PM 10 and ALRI, 2 studies) fulfilled the criterium by a wide margin. The small width of the confidence interval around the summary HR further supports that no downgrade is needed. 


\section{A5 Publication bias}

No downgrading was applied as no evidence of publication bias was found in funnel plots and Egger's tests. Upon visual inspection, no funnel plots showed that small studies with RRs below 1 were missing, indicating no evidence for publication bias (Figure A7.33). Egger's test has good performance for continuous outcomes but is less sensitive for the binary outcomes reported in the current review, especially for the outcomes with a small number of studies. The funnel plot is not sensitive with small number of studies for some outcome pollution pairs either. Cohort studies require a large effort often of different institutes and hence investigators generally try to get studies published irrespective of the results of the study. This qualitative interpretation cannot be investigated directly, because no registries of planned studies exist. We further do not imply that publication bias is absent in easier to conduct air pollution epidemiology studies. Most Funnel plots show a pattern with studies outside the area defined by uncertainty. This mostly reflects the large degree of heterogeneity across studies (Lau et al., 2006; Sterne et al., 2011). Egger's test is sometimes significant (e.g. natural-cause mortality and $\mathrm{PM}_{10}$, IHD and $\mathrm{PM}_{10}$ ), more reflecting heterogeneity than publication bias.

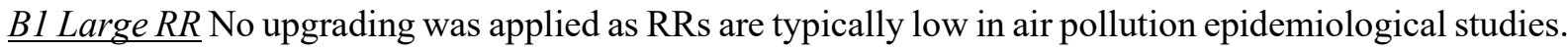
We did not apply the procedure based on a single E-value (section 2.6).

$\underline{B 2}$ All confounding decreases observed $R R$ No upgrading as confounding may decrease or increase observed RRs depending on the direction of the association between exposure and confounder in individual studies.

B3 Concentration-response gradient Upgrading was applied for most combinations. We considered upgrading for a specific combination when there was at least one study reported evidence of a concentration-response gradient (Appendix 5). 


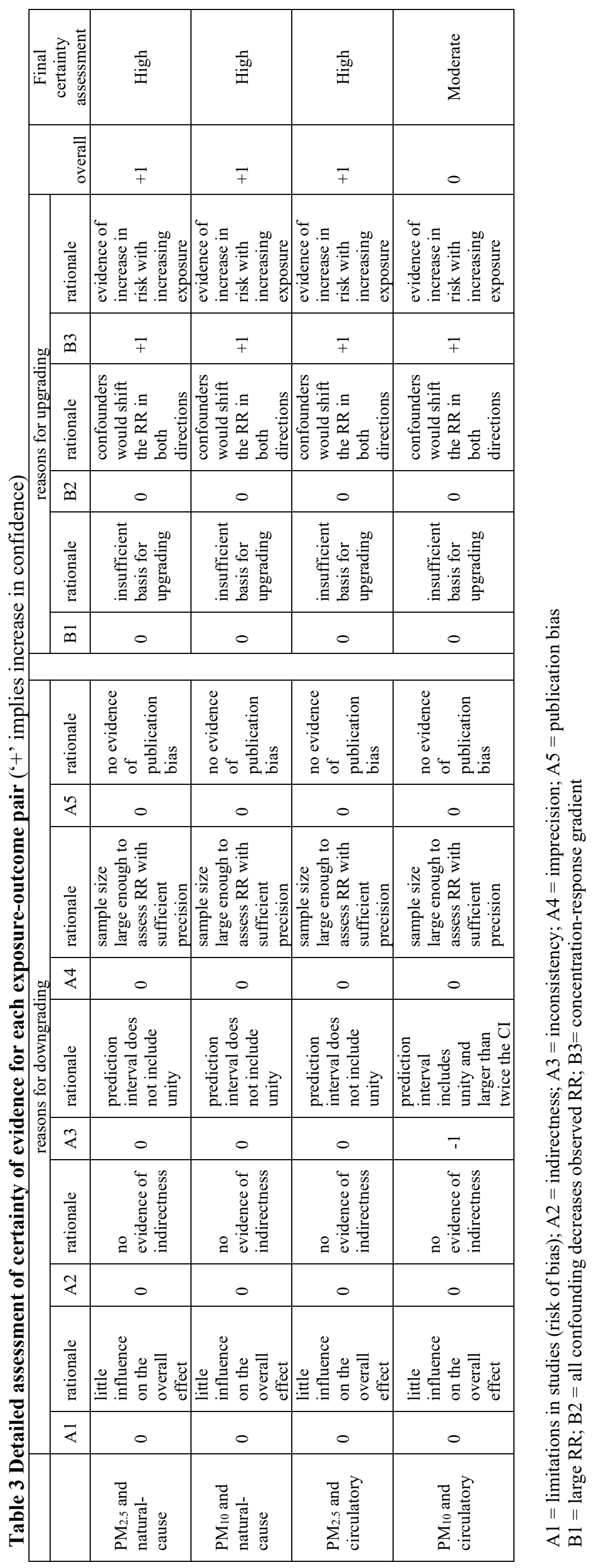




\section{Discussion}

\subsection{Summary of evidence}

\subsubsection{Quantitative effect estimates in meta-analysis}

In meta analyses, $\mathrm{PM}_{2.5}$ was associated with significantly increased risks of all causes of mortality evaluated. $\mathrm{PM}_{10}$ was associated with significantly increased risks of natural-cause and most but not all cause-specific mortality. The evidence base has increased substantially compared to the previous global WHO evaluation published in 2006 (WHO, 2006).

For natural-cause mortality, the combined effect estimate across 25 studies was 1.08 (95\%CI:1.06, 1.09) per $10 \mu \mathrm{g} / \mathrm{m}^{3}$ increase in $\mathrm{PM}_{2.5}$, which is slightly higher than the combined estimate of $1.06(95 \%$ CI:1.04, 1.08) across 11 studies reported in a 2013 review used extensively by the European Environment Agency for European health impact assessment (Hoek et al., 2013). The previous estimate was based on studies predominantly conducted in North America with two studies from Europe (Beelen et al., 2008; Cesaroni et al., 2013). The evidence was strengthened by including new evidence generated in Asia (Tseng et al., 2015; Yang et al., 2018; Yin et al., 2017), North America (Bowe et al., 2018; Pinault et al., 2017), Europe (Beelen et al., 2014b; Carey et al., 2013), and longer follow-up (Cakmak et al., 2018; Di et al., 2017). For $\mathrm{PM}_{10}$, the combined estimate increased from 1.035 (95\%CI:1.004, 1.066) reported in the Hoek et al. (2013) review to 1.04 (95\% CI:1.03, 1.06) in the current review. The previous review was based on only 6 cohort studies while the updated combined estimate was based on 17 cohort studies.

The combined effect estimate was larger for cardiovascular (particularly ischemic heart disease) than for natural-cause mortality associated with exposure to $\mathrm{PM}_{2.5}$. This pattern is consistent with findings in the previous reviews (Chen et al., 2008; Hoek et al., 2013; Liu et al., 2018). One potential source of heterogeneity for effects on IHD is misclassification of the underlying cause of death as Heart Failure rather than IHD. Both WHO and GBD consider heart failure as a "junk category" and re-assign most to IHD (WHO, 2006; Cohen et al., 2017). For stroke mortality, a significant increased risk was found to be associated with $\mathrm{PM}_{2.5}$ but not with $\mathrm{PM}_{10}$. A previous review derived the same conclusion but in addition, reported a significant increased risk for stroke incidence associated with both $\mathrm{PM}_{2.5}$ and $\mathrm{PM}_{10}$ (Scheers et al., 2015).

In the Hoek et al. (2013) review no significant association was found for $\mathrm{PM}_{2.5}$ and non-malignant respiratory mortality across six studies (Hoek et al., 2013). An increased risk of non-malignant respiratory mortality associated with both $\mathrm{PM}_{2.5}$ and $\mathrm{PM}_{10}$ in this review was found by including more recent findings. This increases the coherence with the time-series studies which have consistently shown short-term associations between PM and respiratory mortality (Brunekreef and Holgate 2002; WHO, 2006; Pope and Dockery 2006). Studies investigating long-term exposure and acute lower respiratory infection (ALRI) are still scarce.

Association between lung cancer and PM has been widely investigated as lung cancer is one of the most common cancers and has a poor prognosis. In this review we found significantly increased risk in lung cancer mortality associated with both $\mathrm{PM}_{2.5}$ and $\mathrm{PM}_{10}$. This pattern is consistent with several previous reviews (Chen et al., 2008; Cui et al., 2015; Yang et al., 2016). The effect estimates were slightly higher than that reported in (Cui et al., 2015): $1.09(95 \% \mathrm{CI} 1.06,1.11)$ for $10 \mu \mathrm{g} / \mathrm{m}^{3}$ increase in $\mathrm{PM}_{2.5}$ and 1.05 $(95 \%$ CI $1.03,1.07)$ for $\mathrm{PM}_{10}$. In the review resulting from the IARC evaluation of carcinogenicity of outdoor air pollution, the estimates were 1.09 (95\% CI 1.04, 1.14) and 1.08 (95\% CI 1.00, 1.17) associated with $10 \mu \mathrm{g} / \mathrm{m}^{3}$ increase in $\mathrm{PM}_{2.5}$ and $\mathrm{PM}_{10}$ respectively (Hamra et al., 2014). IARC has designated outdoor air pollution and particulate air pollution specifically as a Group 1 human carcinogen (IARC 2013). Studies investigating lung cancer incidence were not included in the current review. 
In general, associations with $\mathrm{PM}_{2.5}$ were more consistent than with $\mathrm{PM}_{10}$, particularly for cardiovascular outcomes. $\mathrm{PM}_{10}$ is made up of fine $\left(\mathrm{PM}_{2.5}\right)$ and coarse particles. The less consistent association for $\mathrm{PM}_{10}$ may reflect the smaller number of studies compared to $\mathrm{PM}_{2.5}$ and the lower risk of long-term exposure to coarse particles (Adar et al., 2014; Hoek et al., 2013). We note that we cannot compare the presented effect estimates for $\mathrm{PM}_{2.5}$ and $\mathrm{PM}_{10}$ as the applied increment of $10 \mu \mathrm{g} / \mathrm{m}^{3}$ represents a larger contrast for $\mathrm{PM}_{2.5}$ than for $\mathrm{PM}_{10}$.

\subsubsection{Heterogeneity of effect estimates}

In all meta analyses we observed a moderate to high degree of heterogeneity. This is to be expected given the wide diversity of studies conducted. Heterogeneity is likely due to a combination of differences in methodology, concentration and composition of PM, population, geographical location and time period. We primarily interpret this diversity of populations and methods as support for an association, as it decreases the likelihood that residual confounding explains the associations observed between PM and mortality. Documenting heterogeneity is important for health impact assessment of $\mathrm{PM}$ in different countries across the globe.

The exposure assessment methods varied across the studies, from assigned exposure to the nearest monitoring station, to land use regression or dispersion models. Exposures were assigned on very different spatial scales, ranging from residential address to US county. In the risk of bias assessment, we considered all exposure assessment methods appropriate if they had documented validity. However, differences of exposure assessment methodology may affect effect size estimation (Vodonos et al., 2018). For example, in studies characterizing exposure by an area-level value (Nishiwaki et al., 2013; Zhang et al., 2011), only a small number of exposure values were assigned to the population, resulting in difficulties to interpret associations.

The body of evidence includes several very large cohorts of several million subjects based upon administrative registry data, which is new compared to the REVIHAAP assessment (WHO, 2013). These large studies have strong statistical power but often lack individual lifestyle information. Importantly, we found identical RR with wider CI after excluding these studies without individual lifestyle factors. The wider CI is to be expected as the number of subjects in the meta-analysis decreased substantially and lowered the statistical power. Confounding may affect RR estimates for $\mathrm{PM}_{2.5}$ and $\mathrm{PM}_{10}$ in both directions. While risk factors such as smoking, high BMI or low SES affect mortality in the same (adverse) direction, the correlations between these factors and air pollution exposure actually vary across studies. In the Rome cohort study and the Canadian studies (Badaloni et al., 2017; Cakmak et al., 2018), lifestyle tends to be more favorable among the higher exposed subjects living in metropolitan areas, potentially leading to underestimation of air pollution RRs in case of insufficient confounder control. In other studies such as the ESCAPE study (Beelen et al., 2014a), adjustment for confounding reduced air pollution effect estimates. For the entire body of evidence it is therefore not likely that important confounding has occurred.

The body of evidence was based on studies conducted globally, though the majority of studies was from Europe and North America. Differences in geographical location lead to differences in population, concentration and composition of PM. Previous studies have suggested some population groups are more susceptible (Di et al., 2017) and some components are more harmful than others (Vedal 2013). An important observation of our study is that the combined effect estimates were similar across the three WHO regions (Region of the Americas, European Region and Western Pacific Region) where studies have been conducted. This comparison has become possible because of the increase of studies in different regions and addressed concerns about the applicability of results from in the past primarily North-American studies to assess health risks in Europe and other regions.

\subsubsection{Concentration-Response Function (CRF) at low pollution levels}

As the levels of ambient air pollution have declined significantly over the last few decades in North America, Europe, and in other developed regions, it is important to examine whether associations with 
adverse health effects continue to be observed at low levels. Two recently published studies indicated that health effects occur well below $12 \mu \mathrm{g} / \mathrm{m}^{3}$ (the US-EPA NAAQS exposure level) (Brauer et al., 2019; Dominici et al., 2019). In our review, a meta-analysis of studies investigating natural-cause mortality conducted at mean annual average $\mathrm{PM}_{2.5}$ levels below $25 \mu \mathrm{g} / \mathrm{m}^{3}$ (the EU limit exposure level) yielded a significantly positive RR, very similar to the overall RR estimate from all studies. An analysis of studies conducted at mean annual average $\mathrm{PM}_{2.5}$ levels below $10 \mu \mathrm{g} / \mathrm{m}^{3}$ (the current WHO guideline exposure level) yielded an even higher RR, coherent with an increasing number of studies showing linear or supra-linear concentration response relationships (Cesaroni et al., 2013; Crouse et al., 2015; Di et al., 2017; Hart et al., 2015; Pinault et al., 2016; Pinault et al., 2017; Pope et al., 2002). Monotonically rising concentration response relationships were also reported in individual studies for mortality from other diseases related to long-term exposure to $\mathrm{PM}_{2.5}$, including CVD, IHD, stroke, Respiratory disease, COPD and lung cancer (Cesaroni et al., 2013; H Chen et al., 2016; Crouse et al., 2012; Lepeule et al., 2012; Pinault et al., 2017; Thurston et al., 2016a; Weichenthal et al., 2014). Nonlinear CRFs were sometimes reported with usually wide CIs at both the higher and lower ends of the concentration distribution (Crouse et al., 2012; Gan et al., 2013; Villeneuve et al., 2015). Burnett et al., (2018) has recently reported on an analysis of a large number of cohorts included in the current review and showed a near-linear ensemble curve for natural-cause mortality and $\mathrm{PM}_{2.5}$. The study involved analysis using a standardized code allowing non-linear functions applied by local analysts and subsequent combination of the curves. The study also showed near-linear ensemble CRFs for lower respiratory infection, stroke, COPD, lung cancer and IHD associated with long-term exposure to $\mathrm{PM}_{2.5}$. While most studies suggested there is little evidence of a threshold for $\mathrm{PM}_{2.5}$ and mortality from all causes and specific causes (Di et al., 2017; Lepeule et al., 2012; Pinault et al., 2016; Schwartz et al., 2008), a threshold of $11 \mu \mathrm{g} / \mathrm{m}^{3}$ for $\mathrm{PM}_{2.5}$ and nonaccidental mortality was reported in (Villeneuve et al., 2015). Studies that evaluated the shape of the CRF for $\mathrm{PM}_{10}$ are more limited (Fischer et al., 2015).

\subsubsection{Certainty of evidence}

We applied an adapted GRADE method to assess certainty in the epidemiological body of evidence. In general, $\mathrm{PM}_{2.5}$ was more consistently associated with mortality than $\mathrm{PM}_{10}$, particularly for cardiovascular outcomes. $\mathrm{PM}_{10}$ is made up of fine $\left(\mathrm{PM}_{2.5}\right)$ and coarse particles. The less consistent association for $\mathrm{PM}_{10}$ may reflect the smaller number of studies compared to $\mathrm{PM}_{2.5}$ and the lower risk of long-term exposure to coarse particles (Adar et al., 2014; Hoek et al., 2013).

The assignment of high certainty of evidence to most long-term $\mathrm{PM}_{2.5}$ exposure and mortality associations agrees well with recent assessments made by the USEPA using a different methodology (USEPA, 2019). In the 2019 Integrated Science Assessment (ISA), the association between $P_{2.5}$ and natural mortality was rated as "causal" based on assessment of different scientific disciplines beyond the epidemiological air pollution mortality studies. For long-term $\mathrm{PM}_{10}$ exposure and mortality associations, the assessments are generally stronger than the ISA assessments for coarse particles. The 2019 PM ISA evaluated evidence from studies of $\mathrm{PM}_{10-2.5}$ and natural-cause mortality as "suggestive". The assessment for coarse $\mathrm{PM}$ is not directly comparable to $\mathrm{PM}_{10}$, as $\mathrm{PM}_{10}$ is the sum of $\mathrm{PM}_{2.5}$ and coarse PM. We also rated the evidence for specific causes of death lower for $\mathrm{PM}_{10}$ than for $\mathrm{PM}_{2.5}$. The high certainty assessment for lung cancer mortality agrees well with the assessment in 2013 by IARC, which designated outdoor air pollution and particulate air pollution specifically as a Group 1 human carcinogen (IARC, 2013).

The biological plausibility of associations between mortality and PM have been identified previously (USEPA, 2019). Inhalation of PM may result in injury, oxidative stress, and inflammation in the respiratory tract and lead to systemic inflammation and oxidative stress. Persistent or intermittent exposure to PM over months to years may lead to cumulative or chronic effects including mortality from respiratory, cardiovascular disease, lung cancer or possibly other diseases.

Application of the adapted GRADE approach was challenging in some domains. Limitations in studies were assessed based on results of the risk of bias assessments. Risk of Bias application is more complex 
than following a simple checklist and requires careful interpretations to make a proper judgment. The criteria for the confounding domain were clear, but probably a more refined list of confounders would have distinguished studies better. The missing data and exposure contrast subdomains required careful examination as data were not always reported in the epidemiological paper, but occasionally in a companion paper. Change in exposure contrast during follow-up also required additional information to be inspected. Consideration of heterogeneity was complex, as distinguishing differences in magnitude of effect size and differences in direction of effect across studies was needed. Publication bias was difficult to assess as the tools are somewhat problematic for non-continuous outcomes, for a small number of studies and settings with heterogeneity. The imprecision criterium was based upon comparison with a set number of person-years determined with a power calculation. Probably, the evaluation of the meta-analytical confidence interval would have been more direct. We did not apply the upgrade procedure for large RR, based upon the suggested evaluation of an E-value. None of these challenges likely has materially affected the overall certainty of evidence assessment.

\subsection{New studies published after last search}

A number of studies has been published after the date of our final search (October 2018), which is more than a year ago. Table A7.1 lists the new studies including RR estimates. Some of the new evidence is from an update of the included cohorts with longer follow-up or with more advanced methodology, including the National Health Interview Survey cohort (Pope et al., 2018; Pope et al., 2019) and the National Institutes of Health-AARP Diet and Health Study (Hayes et al., 2019). These new studies are unlikely to change our estimates as the same populations were already included. Some new studies were conducted in the included cohorts with a different focus (Lim et al., 2019; Sun et al., 2019), which would not fulfil the criteria to replace the estimates used in the meta analyses.

Two recently published reports funded by the Health Effect Institute (HEI) suggested health effects of air pollution exist at low levels, even below the current annual U.S. national ambient air quality standard for $\mathrm{PM}_{2.5}$ of $12 \mu \mathrm{g} / \mathrm{m}^{3}$ (Brauer et al., 2019; Dominici et al., 2019). This is coherent with findings from a number of studies included in the current review. These two reports were conducted in the Medicare cohort and four Canadian cohorts (three CanCHEC and CCHS). Estimates in the Medicare report were identical as previously reported in (Di et al., 2017), which was included in our review. Estimates in the Canadian report were published separately in Christidis et al., (2019) and Pappin et al., (2019). Three of the four Canadian cohorts were included in our review with a shorter follow-up, with exception of the 1996 CanCHEC.

The Dutch Environmental Longitudinal Study (DUELS) and the Korean National Health Insurance Service-based National Sample Cohort were included in the current review with effect estimates reported only for $\mathrm{PM}_{10}$ (Fischer et al., 2015; Kim et al., 2017). In the recent articles derived from these cohorts, health effect estimates associated with $\mathrm{PM}_{2.5}$ were reported (Fischer et al., 2020; Kim et al., 2019). However, the Korean cohort only reported $\mathrm{PM}_{2.5}$ estimates in a two-pollutant model adjusted for $\mathrm{O}_{3}$. Evidence was also generated from new cohorts including the Chinese Longitudinal Healthy Longevity Survey (CLHLS) (Li et al., 2018), the Danish Diet, Cancer and Health cohort (Hvidtfeldt et al., 2019) and the '45 and up study' cohort (Hanigan et al., 2019). Most of these studies have reported positive associations between $\mathrm{PM}_{2.5}$ and mortality. Importantly, sensitivity analysis including this new evidence did not change our combined estimates. The summary $\mathrm{RR}$ for $\mathrm{PM}_{2.5}$ and natural mortality was $1.08(95 \%$ CI 1.07, 1.10) after including 5 new studies (Figure A7.43). The body of evidence is already based on a large number of well conducted studies without a large weight from a single study, therefore including new evidence is unlikely to change the combined effect estimate materially.

\subsection{Strengths and limitations}

A strength of our study is the efforts made throughout the design and the conduct of the systematic review to ensure its validity, including the incorporation of risk of bias assessment. Another strength is the large number of studies included in our systematic review, with the diversity of populations. This 
decreases the likelihood that residual confounding explains the associations observed between PM and mortality.

This systematic review has a number of limitations. First, we acknowledge that our search strategy had limitations. The use of "NOT" operators in our search strategy and inclusion of study design might lead to missing relevant evidence. However, we also scanned references of identified reviews to identify papers that were potentially missed by our search strategy. Compared to a very recent review article (Pope et al., 2019), our systematic search only missed two studies published in the search period - one from a cohort we already included in our review (Wong et al., 2016), one from a patient group (Goss et al., 2004). We therefore think that the risk of missing key papers was small. Second, we found that relatively few studies were performed in low- and middle-income countries (LMICs) which typically experience higher air pollution levels than observed in the countries where the majority of cohort studies have been performed (Burns et al., 2019). Therefore, uncertainty about the shape of the CRF remains especially for the high end of the concentration distribution. To support health impact assessment in LMICs and global burden of disease assessment, new studies in LMICs are needed. Third, the review focused on $\mathrm{PM}_{2.5}$ and $\mathrm{PM}_{10}$ without assessing particle composition. As most included studies have been conducted in areas with combustion as the main source of (primary and secondary) particles, it is not clear whether the risk estimates can be applied in settings where other sources are dominant such as desert dust (Kotsyfakis et al., 2019; Naidja et al., 2018). More research investigating which components/ sources are most responsible for health effects is needed. Fourth, in a meta-analysis of published studies, we had limited possibilities to assess the shape of the CRF.

\subsection{Implications}

Results of this and other systematic reviews commissioned by WHO are currently being used in developing new air quality guidelines by WHO. Our results suggest that $\mathrm{PM}_{2.5}$ is associated with increased risk for mortality, even below the current WHO guideline exposure level of $10 \mu \mathrm{g} / \mathrm{m}^{3}$. If a threshold is present, it is at very low levels. These results suggest an update of the current guideline needs to be considered by WHO. An update of the $\mathrm{PM}_{10}$ guideline needs to be considered as well.

The large heterogeneity of effect estimates across studies suggests that health impact assessment in specific locations may have fairly large uncertainty. The full body of evidence should be used with caution in regions where no or few studies have been conducted such as the African Region, South-East Asia Region, and Eastern Mediterranean Region. Particularly in areas where dust contributes significantly to overall $\mathrm{PM}_{2.5}$ levels, our combined RRs may not apply. Also, as the summary RR estimates were derived assuming a linear relationship, application of this relationship in e.g. burden of disease assessments may be problematic when evaluating settings with very high concentrations such as in polluted regions of Asia (Pant et al., 2016).

\section{Conclusions}

The evidence base has increased substantially compared to the previous global WHO evaluation, however studies conducted in low- and middle-income countries (LMICs) are still scarce. There is clear evidence that both $\mathrm{PM}_{2.5}$ and $\mathrm{PM}_{10}$ are associated with increased mortality from all causes, cardiovascular disease, respiratory disease and lung cancer. The combined HRs for natural-cause mortality are 1.08 (95\% CI:1.06, 1.09) per $10 \mu \mathrm{g} / \mathrm{m}^{3}$ increase in $\mathrm{PM}_{2.5}$, and 1.04 (95\%CI:1.03, 1.06) per $10 \mu \mathrm{g} / \mathrm{m}^{3}$ increase in $\mathrm{PM}_{10}$. The associations with $\mathrm{PM}_{2.5}$ remained below the current WHO annual average guideline exposure level of $10 \mu \mathrm{g} / \mathrm{m}^{3}$. 


\section{References}

Abbey DE, Nishino N, McDonnell WF, et al., 1999. Long-term inhalable particles and other air pollutants related to mortality in nonsmokers. American Journal of Respiratory and Critical Care Medicine 159:373-382.

Adar SD, Filigrana PA, Clements N, et al., 2014. Ambient coarse particulate matter and human health: A systematic review and meta-analysis. Current environmental health reports 1:258-274.

Badaloni C, Cesaroni G, Cerza F, et al., 2017. Effects of long-term exposure to particulate matter and metal components on mortality in the rome longitudinal study. Environment international 109:146-154.

Beelen R, Hoek G, van den Brandt PA, et al., 2008. Long-term effects of traffic-related air pollution on mortality in a dutch cohort (nlcs-air study). Environmental health perspectives 116:196-202.

Beelen R, Hoek G, Houthuijs D, et al., 2009. The joint association of air pollution and noise from road traffic with cardiovascular mortality in a cohort study. Occupational and environmental medicine 66:243-250.

Beelen R, Raaschou-Nielsen O, Stafoggia M, et al., 2014a. Effects of long-term exposure to air pollution on natural-cause mortality: An analysis of 22 european cohorts within the multicentre escape project. Lancet (London, England) 383:785-795. Beelen R, Stafoggia M, Raaschou-Nielsen O, et al., 2014b. Long-term exposure to air pollution and cardiovascular mortality: An analysis of 22 european cohorts. Epidemiology 25:368-378.

Bentayeb M, Wagner V, Stempfelet M, et al., 2015. Association between long-term exposure to air pollution and mortality in france: A 25-year follow-up study. Environment International 85:5-14.

Borenstein M, Higgins J, Hedges LV, et al., 2017. Basics of meta-analysis: I2 is not an absolute measure of heterogeneity. Research synthesis methods 8:5-18.

Bowe B, Xie Y, Li T, et al., 2018. The 2016 global and national burden of diabetes mellitus attributable to pm $2 \cdot 5$ air pollution. The Lancet Planetary Health 2:e301-e312.

Brauer M, Brook JR, Christidis T, et al., 2019. Mortality-air pollution associations in low-exposure environments (maple): Phase 1. Research report (Health Effects Institute):1-87.

Brunekreef B, Holgate ST. 2002. Air pollution and health. The lancet 360:1233-1242.

Brunekreef B, Beelen R, Hoek G, et al., 2009. Effects of long-term exposure to traffic-related air pollution on respiratory and cardiovascular mortality in the netherlands: The nlcs-air study. Research report (Health Effects Institute):5-71; discussion 7389.

Burnett R, Chen H, Szyszkowicz M, et al., 2018. Global estimates of mortality associated with long-term exposure to outdoor fine particulate matter. Proceedings of the National Academy of Sciences 115:9592-9597.

Burns J, Boogaard H, Polus S, et al., 2019. Interventions to reduce ambient particulate matter air pollution and their effect on health. Cochrane Database Syst Rev 5:CD010919-CD010919.

Cakmak S, Hebbern C, Vanos J, et al., 2016. Ozone exposure and cardiovascular-related mortality in the canadian census health and environment cohort (canchec) by spatial synoptic classification zone. Environmental pollution (Barking, Essex : 1987) 214:589-599.

Cakmak S, Hebbern C, Pinault L, et al., 2018. Associations between long-term pm2.5 and ozone exposure and mortality in the canadian census health and environment cohort (canchec), by spatial synoptic classification zone. Environment international 111:200-211.

Carey IM, Atkinson RW, Kent AJ, et al., 2013. Mortality associations with long-term exposure to outdoor air pollution in a national english cohort. American journal of respiratory and critical care medicine 187:1226-1233.

Cesaroni G, Badaloni C, Gariazzo C, et al., 2013. Long-term exposure to urban air pollution and mortality in a cohort of more than a million adults in rome. Environmental health perspectives 121:324-331.

Chen H, Goldberg MS, Villeneuve PJ. 2008. A systematic review of the relation between long-term exposure to ambient air pollution and chronic diseases. Reviews on environmental health 23:243-297.

Chen H, Burnett RT, Copes R, et al., 2016. Ambient fine particulate matter and mortality among survivors of myocardial infarction: Population-based cohort study. Environmental health perspectives 124:1421-1428.

Chen LH, Knutsen SF, Shavlik D, et al., 2005. The association between fatal coronary heart disease and ambient particulate air pollution: Are females at greater risk? Environmental health perspectives 113:1723-1729.

Chen X, Zhang LW, Huang JJ, et al., 2016. Long-term exposure to urban air pollution and lung cancer mortality: A 12-year cohort study in northern china. The Science of the total environment 571:855-861.

Chen X, Wang X, Huang JJ, et al., 2017. Nonmalignant respiratory mortality and long-term exposure to pm10 and so2: A 12year cohort study in northern china. Environmental pollution (Barking, Essex : 1987) 231:761-767.

Christidis T, Erickson AC, Pappin AJ, et al., 2019. Low concentrations of fine particle air pollution and mortality in the canadian community health survey cohort. Environmental health : a global access science source 18:84-84.

Cohen AJ, Brauer M, Burnett R, et al., 2017. Estimates and 25-year trends of the global burden of disease attributable to ambient air pollution: An analysis of data from the global burden of diseases study 2015. The Lancet 389:1907-1918.

Crouse DL, Peters PA, van Donkelaar A, et al., 2012. Risk of nonaccidental and cardiovascular mortality in relation to longterm exposure to low concentrations of fine particulate matter: A canadian national-level cohort study. Environmental health perspectives 120:708-714.

Crouse DL, Peters PA, Hystad P, et al., 2015. Ambient pm2.5, o3, and no2 exposures and associations with mortality over 16 years of follow-up in the canadian census health and environment cohort (canchec). Environmental Health Perspectives 123:1180-1186.

Cui P, Huang Y, Han J, et al., 2015. Ambient particulate matter and lung cancer incidence and mortality: A meta-analysis of prospective studies. European journal of public health 25:324-329.

Dehbi HM, Blangiardo M, Gulliver J, et al., 2017. Air pollution and cardiovascular mortality with over 25years follow-up: A combined analysis of two british cohorts. Environment international 99:275-281. 
Di Q, Wang Y, Zanobetti A, et al., 2017. Air pollution and mortality in the medicare population. The New England journal of medicine 376:2513-2522.

Dimakopoulou K, Samoli E, Beelen R, et al., 2014. Air pollution and nonmalignant respiratory mortality in 16 cohorts within the escape project. American journal of respiratory and critical care medicine 189:684-696.

Dockery DW, Pope ICA, Xu X, et al., 1993. An association between air pollution and mortality in six u.S. Cities. New England Journal of Medicine 329:1753-1759.

Dominici F, Schwartz J, Di Q, et al., 2019. Assessing adverse health effects of long-term exposure to low levels of ambient air pollution: Phase 1. Research report (Health Effects Institute):1-51.

Dong GH, Zhang P, Sun B, et al., 2012. Long-term exposure to ambient air pollution and respiratory disease mortality in shenyang, china: A 12-year population-based retrospective cohort study. Respiration; international review of thoracic diseases 84:360-368.

Eckel SP, Cockburn M, Shu YH, et al., 2016. Air pollution affects lung cancer survival. Thorax 71:891-898.

Eftim SE, Samet JM, Janes H, et al., 2008. Fine particulate matter and mortality: A comparison of the six cities and american cancer society cohorts with a medicare cohort. Epidemiology 19:209-216.

Enstrom JE. 2005. Fine particulate air pollution and total mortality among elderly californians, 1973-2002. Inhalation toxicology 17:803-816.

Enstrom JE. 2017. Fine particulate matter and total mortality in cancer prevention study cohort reanalysis. Dose-response : a publication of International Hormesis Society 15:1559325817693345.

Fischer PH, Marra M, Ameling CB, et al., 2015. Air pollution and mortality in seven million adults: The dutch environmental longitudinal study (duels). Environmental Health Perspectives 123:697-704.

Fischer PH, Marra M, Ameling CB, et al., 2020. Particulate air pollution from different sources and mortality in 7.5 million adults - the dutch environmental longitudinal study (duels). The Science of the total environment 705:135778-135778.

Gan WQ, Koehoorn M, Davies HW, et al., 2011. Long-term exposure to traffic-related air pollution and the risk of coronary heart disease hospitalization and mortality. Environmental health perspectives 119:501-507.

Gan WQ, FitzGerald JM, Carlsten C, et al., 2013. Associations of ambient air pollution with chronic obstructive pulmonary disease hospitalization and mortality. American journal of respiratory and critical care medicine 187:721-727.

Gehring U, Heinrich J, Kramer U, et al., 2006. Long-term exposure to ambient air pollution and cardiopulmonary mortality in women. Epidemiology 17:545-551.

Gerber Y, Myers V, Broday DM, Steinberg DM, Yuval, Koton S, et al., 2014. Frailty status modifies the association between air pollution and post-myocardial infarction mortality: A 20-year follow-up study. Journal of the American College of Cardiology 63:1698-1699.

Goss CH, Newsom SA, Schildcrout JS, et al., 2004. Effect of ambient air pollution on pulmonary exacerbations and lung function in cystic fibrosis. American journal of respiratory and critical care medicine 169:816-821.

Hales S, Blakely T, Woodward A. 2012. Air pollution and mortality in new zealand: Cohort study. Journal of epidemiology and community health $66: 468-473$.

Hamra GB, Guha N, Cohen A, et al., 2014. Outdoor particulate matter exposure and lung cancer: A systematic review and meta-analysis. Environmental health perspectives.

Hanigan IC, Rolfe MI, Knibbs LD, et al., 2019. All-cause mortality and long-term exposure to low level air pollution in the ' 45 and up study' cohort, sydney, australia, 2006-2015. Environment international 126:762-770.

Hansell A, Ghosh RE, Blangiardo M, et al., 2016. Historic air pollution exposure and long-term mortality risks in england and wales: Prospective longitudinal cohort study. Thorax 71:330-338.

Hart JE, Garshick E, Dockery DW, et al., 2011. Long-term ambient multipollutant exposures and mortality. American journal of respiratory and critical care medicine 183:73-78.

Hart JE, Liao X, Hong B, et al., 2015. The association of long-term exposure to pm2.5 on all-cause mortality in the nurses' health study and the impact of measurement-error correction. Environmental health : a global access science source 14:38.

Hartiala J, Breton CV, Tang WH, et al., 2016. Ambient air pollution is associated with the severity of coronary atherosclerosis and incident myocardial infarction in patients undergoing elective cardiac evaluation. Journal of the American Heart Association 5.

Hayes RB, Lim C, Zhang Y, et al., 2019. Pm2.5 air pollution and cause-specific cardiovascular disease mortality. Int J Epidemiol:dyz114.

Heinrich J, Thiering E, Rzehak P, et al., 2013. Long-term exposure to no2 and pm10 and all-cause and cause-specific mortality in a prospective cohort of women. Occupational and Environmental Medicine 70:179-186.

Hoek G, Krishnan RM, Beelen R, et al., 2013. Long-term air pollution exposure and cardio- respiratory mortality: A review. Environmental health : a global access science source 12:43.

Huss A, Spoerri A, Egger M, et al., 2010. Aircraft noise, air pollution, and mortality from myocardial infarction. Epidemiology 21:829-836.

Hvidtfeldt UA, Sørensen M, Geels C, et al., 2019. Long-term residential exposure to pm2. 5, pm10, black carbon, no2, and ozone and mortality in a danish cohort. Environment international 123:265-272.

IARC (International Agency for Research on Cancer), 2013. Air pollution and cancer. IARC Scientific Publication No. 161. (http://publications.iarc.fr/Book-And-Report-Series/Iarc-Scientific-Publications/Air-Pollution-And-Cancer-2013, last access: 20200203)

Jerrett M, Burnett RT, Ma R, et al., 2005. Spatial analysis of air pollution and mortality in los angeles. Epidemiology 16:727736.

Jerrett M, Burnett RT, Arden Pope IC, et al., 2009. Long-term ozone exposure and mortality. New England Journal of Medicine 360:1085-1095.

Jerrett M, Burnett RT, Beckerman BS, et al., 2013. Spatial analysis of air pollution and mortality in california. American Journal of Respiratory and Critical Care Medicine 188:593-599. 
Katanoda K, Sobue T, Satoh H, et al., 2011. An association between long-term exposure to ambient air pollution and mortality from lung cancer and respiratory diseases in japan. Journal of epidemiology 21:132-143.

Kim H, Kim J, Kim S, Kang SH, Kim HJ, Kim H, et al., 2017. Cardiovascular effects of long-term exposure to air pollution: A population-based study with 900845 person-years of follow-up. Journal of the American Heart Association 6.

Kim I-S, Yang P-S, Lee J, et al., 2019. Long-term fine particulate matter exposure and cardiovascular mortality in the general population: A nationwide cohort study. J Cardiol:S0914-5087(0919)30344-30342.

Kim OJ, Kim SY, Kim H. 2017. Association between long-term exposure to particulate matter air pollution and mortality in a south korean national cohort: Comparison across different exposure assessment approaches. International journal of environmental research and public health 14.

Kioumourtzoglou MA, Austin E, Koutrakis P, et al., 2015. Pm2.5 and survival among older adults: Effect modification by particulate composition. Epidemiology 26:321-327.

Koton S, Molshatzki N, Yuval, et al., 2013. Cumulative exposure to particulate matter air pollution and long-term postmyocardial infarction outcomes. Preventive medicine 57:339-344.

Kotsyfakis M, Zarogiannis SG, Patelarou E. 2019. The health impact of saharan dust exposure. Int J Occup Med Environ Health 32:749-760.

Krewski D, Jerrett M, Burnett RT, et al., 2009. Extended follow-up and spatial analysis of the american cancer society study linking particulate air pollution and mortality. Research report (Health Effects Institute):5-114; discussion 115-136.

Laden F, Schwartz J, Speizer FE, et al., 2006. Reduction in fine particulate air pollution and mortality: Extended follow-up of the harvard six cities study. American journal of respiratory and critical care medicine 173:667-672.

Lau J, Ioannidis JP, Terrin N, et al., 2006. The case of the misleading funnel plot. Bmj 333:597-600.

Lepeule J, Laden F, Dockery D, et al., 2012. Chronic exposure to fine particles and mortality: An extended follow-up of the harvard six cities study from 1974 to 2009. Environmental health perspectives 120:965-970.

Li T, Zhang Y, Wang J, et al., 2018. All-cause mortality risk associated with long-term exposure to ambient pm2. 5 in china: A cohort study. The Lancet Public Health 3:e470-e477.

Lim CC, Hayes RB, Ahn J, et al., 2019. Long-term exposure to ozone and cause-specific mortality risk in the united states. American journal of respiratory and critical care medicine 200:1022-1031.

Lipfert F, Baty J, Miller J, et al., 2006. Pm2.5 constituents and related air quality variables as predictors of survival in a cohort of u.S. Military veterans. Inhalation Toxicology 18:645-657.

Lipsett MJ, Ostro BD, Reynolds P, et al., 2011. Long-term exposure to air pollution and cardiorespiratory disease in the california teachers study cohort. American journal of respiratory and critical care medicine 184:828-835.

Liu Z, Wang F, Li W, et al., 2018. Does utilizing who's interim targets further reduce the risk - meta-analysis on ambient particulate matter pollution and mortality of cardiovascular diseases? Environmental pollution (Barking, Essex : 1987) 242:1299-1307.

Loop MS, McClure LA, Levitan EB, et al., 2018. Fine particulate matter and incident coronary heart disease in the regards cohort. American heart journal 197:94-102.

Maheswaran R, Pearson T, Smeeton NC, et al., 2010. Impact of outdoor air pollution on survival after stroke: Populationbased cohort study. Stroke 41:869-877.

McDonnell WF, Nishino-Ishikawa N, Petersen FF, et al., 2000. Relationships of mortality with the fine and coarse fractions of long-term ambient pm10 concentrations in nonsmokers. Journal of exposure analysis and environmental epidemiology $10: 427-436$.

McGuire S. 2016. World cancer report 2014. Geneva, switzerland: World health organization, international agency for research on cancer, who press, 2015.Oxford University Press.

Miller KA, Siscovick DS, Sheppard L, et al., 2007. Long-term exposure to air pollution and incidence of cardiovascular events in women. The New England journal of medicine 356:447-458.

Morgan RL, Thayer KA, Santesso N, et al., 2019. A risk of bias instrument for non-randomized studies of exposures: A users' guide to its application in the context of grade. Environment international 122:168-184.

Naidja L, Ali-Khodja H, Khardi S. 2018. Sources and levels of particulate matter in north african and sub-saharan cities: A literature review. Environ Sci Pollut Res Int 25:12303-12328.

Næss Ø, Nafstad P, Aamodt G, Claussen B, Rosland P. 2007a. Relation between concentration of air pollution and causespecific mortality: Four-year exposures to nitrogen dioxide and particulate matter pollutants in 470 neighborhoods in oslo, norway. American Journal of Epidemiology 165:435-443.

Næss Ø, Piro FN, Nafstad P, Smith GD, Leyland AH. 2007b. Air pollution, social deprivation, and mortality: A multilevel cohort study. Epidemiology 18:686-694.

Nishiwaki Y, Michikawa T, Takebayashi T, et al., 2013. Long-term exposure to particulate matter in relation to mortality and incidence of cardiovascular disease: The jphc study. Journal of atherosclerosis and thrombosis 20:296-309.

Ostro B, Lipsett M, Reynolds P, et al., 2011. Long-term exposure to constituents of fine particulate air pollution and mortality: Results from the california teachers study. Environmental health perspectives 118:363-369.

Ostro B, Hu J, Goldberg D, et al., 2015. Associations of mortality with long-term exposures to fine and ultrafine particles, species and sources: Results from the california teachers study cohort. Environmental health perspectives 123:549-556.

Pant P, Guttikunda SK, Peltier RE. 2016. Exposure to particulate matter in india: A synthesis of findings and future directions. Environmental research 147:480-496.

Pappin AJ, Christidis T, Pinault LL, et al., 2019. Examining the shape of the association between low levels of fine particulate matter and mortality across three cycles of the canadian census health and environment cohort. Environmental health perspectives 127:107008-107008.

Parker JD, Kravets N, Vaidyanathan A. 2018. Particulate matter air pollution exposure and heart disease mortality risks by race and ethnicity in the united states: 1997 to 2009 national health interview survey with mortality follow-up through 2011. Circulation 137:1688-1697. 
Peng Z, Liu C, Xu B, et al., 2017. Long-term exposure to ambient air pollution and mortality in a chinese tuberculosis cohort. The Science of the total environment 580:1483-1488.

Pinault L, Tjepkema M, Crouse DL, et al., 2016. Risk estimates of mortality attributed to low concentrations of ambient fine particulate matter in the canadian community health survey cohort. Environmental health : a global access science source 15:18.

Pinault LL, Weichenthal S, Crouse DL, et al., 2017. Associations between fine particulate matter and mortality in the 2001 canadian census health and environment cohort. Environmental Research 159:406-415.

Pope CA, Ezzati M, Cannon JB, et al., 2018. Mortality risk and pm 2.5 air pollution in the USA: An analysis of a national prospective cohort. Air Quality, Atmosphere \& Health 11:245-252.

Pope CA, 3rd, Burnett RT, Thurston GD, et al., 2004. Cardiovascular mortality and long-term exposure to particulate air pollution: Epidemiological evidence of general pathophysiological pathways of disease. Circulation 109:71-77.

Pope CA, 3rd, Turner MC, Burnett RT, et al., 2015. Relationships between fine particulate air pollution, cardiometabolic disorders, and cardiovascular mortality. Circulation research 116:108-115.

Pope CA, 3rd, Coleman N, Pond ZA, et al., 2019. Fine particulate air pollution and human mortality: 25+ years of cohort studies. Environmental research:108924.

Pope CA, 3rd, Lefler JS, Ezzati M, et al., 2019. Mortality risk and fine particulate air pollution in a large, representative cohort of u.S. Adults. Environmental health perspectives 127:77007-77007.

Pope ICA, Thun MJ, Namboodiri MM, et al., 1995. Particulate air pollution as a predictor of mortality in a prospective study of u.S. Adults. American Journal of Respiratory and Critical Care Medicine 151:669-674.

Pope ICA, Burnett RT, Thun MJ, et al., 2002. Lung cancer, cardiopulmonary mortality, and long-term exposure to fine particulate air pollution. Journal of the American Medical Association 287:1132-1141.

Pope III CA, Dockery DW. 2006. Health effects of fine particulate air pollution: Lines that connect. Journal of the air \& waste management association 56:709-742.

Puett RC, Schwartz J, Hart JE, et al., 2008. Chronic particulate exposure, mortality, and coronary heart disease in the nurses' health study. American journal of epidemiology 168:1161-1168.

Puett RC, Hart JE, Yanosky JD, et al., 2010. Chronic fine and coarse particulate exposure, mortality, and coronary heart disease in the nurses' health study. Environmental health perspectives 117:1697-1701.

Puett RC, Hart JE, Suh H, et al., 2011. Particulate matter exposures, mortality, and cardiovascular disease in the health professionals follow-up study. Environmental health perspectives 119:1130-1135.

Pun VC, Kazemiparkouhi F, Manjourides J, Suh HH. 2017. Long-term pm2.5 exposure and respiratory, cancer, and cardiovascular mortality in older us adults. American journal of epidemiology 186:961-969.

Ritz B, Wilhelm M, Zhao Y. 2006. Air pollution and infant death in southern california, 1989-2000. Pediatrics 118:493-502. Rosenlund M, Berglind N, Pershagen G, et al., 2006. Long-term exposure to urban air pollution and myocardial infarction. Epidemiology 17:383-390.

Rosenlund M, Bellander T, Nordquist T, et al., 2009. Traffic-generated air pollution and myocardial infarction. Epidemiology 20:265-271.

Ruttens D, Verleden SE, Bijnens EM, et al., 2016. An association of particulate air pollution and traffic exposure with mortality after lung transplantation in europe. European Respiratory Journal 49.

Scheers H, Jacobs L, Casas L, et al., 2015. Long-term exposure to particulate matter air pollution is a risk factor for stroke: Meta-analytical evidence. Stroke 46:3058-3066.

Schwartz J, Coull B, Laden F, et al., 2008. The effect of dose and timing of dose on the association between airborne particles and survival. Environmental health perspectives 116:64-69.

Sese L, Nunes H, Cottin V, et al., 2018. Role of atmospheric pollution on the natural history of idiopathic pulmonary fibrosis. Thorax 73:145-150.

Shi L, Zanobetti A, Kloog I, et al., 2016. Low-concentration pm2.5 and mortality: Estimating acute and chronic effects in a population-based study. Environmental health perspectives 124:46-52.

Son JY, Bell ML, Lee JT. 2011. Survival analysis of long-term exposure to different sizes of airborne particulate matter and risk of infant mortality using a birth cohort in seoul, korea. Environmental health perspectives 119:725-730.

Spencer-Hwang R, Knutsen SF, Soret S, et al., 2011. Ambient air pollutants and risk of fatal coronary heart disease among kidney transplant recipients. American journal of kidney diseases : the official journal of the National Kidney Foundation 58:608-616.

Sterne JA, Sutton AJ, Ioannidis JP, et al., 2011. Recommendations for examining and interpreting funnel plot asymmetry in meta-analyses of randomised controlled trials. Bmj 343:d4002.

Straif K, Cohen A, Samet S. 2017. Air pollution and c? Ncer.International Agency for Research on Cancer (IARC).

Sun S, Cao W, Qiu H, et al., 2019. Benefits of physical activity not affected by air pollution: A prospective cohort study. Int J Epidemiol:dyz184.

Thurston GD, Ahn J, Cromar KR, et al., 2016a. Ambient particulate matter air pollution exposure and mortality in the nihaarp diet and health cohort. Environmental health perspectives 124:484-490.

Thurston GD, Burnett RT, Turner MC, et al., 2016b. Ischemic heart disease mortality and long-term exposure to source-related components of u.S. Fine particle air pollution. Environmental Health Perspectives 124:785-794.

Tonne C, Wilkinson P. 2013. Long-term exposure to air pollution is associated with survival following acute coronary syndrome. European heart journal 34:1306-1311.

Tonne C, Halonen JI, Beevers SD, et al., 2016. Long-term traffic air and noise pollution in relation to mortality and hospital readmission among myocardial infarction survivors. International journal of hygiene and environmental health 219:72-78.

Tseng E, Ho WC, Lin MH, et al., 2015. Chronic exposure to particulate matter and risk of cardiovascular mortality: Cohort study from taiwan. BMC public health 15:936. 
Turner MC, Krewski D, Pope ICA, et al., 2011. Long-term ambient fine particulate matter air pollution and lung cancer in a large cohort of never-smokers. American Journal of Respiratory and Critical Care Medicine 184:1374-1381.

Turner MC, Jerrett M, Pope CA, 3rd, et al., 2016. Long-term ozone exposure and mortality in a large prospective study. American journal of respiratory and critical care medicine 193:1134-1142.

Ueda K, Nagasawa SY, Nitta H, et al., 2012. Exposure to particulate matter and long-term risk of cardiovascular mortality in japan: Nippon data80. Journal of Atherosclerosis and Thrombosis 19:246-254.

U.S. EPA. Integrated Science Assessment (ISA) for Particulate Matter (Final Report, 2019). U.S. Environmental Protection Agency, Washington, DC, EPA/600/R-19/188, 2019. (https://cfpub.epa.gov/ncea/isa/recordisplay.cfm?deid=347534, last access: 20200128 )

Vedal S. 2013. National particle component toxicity (npact) initiative report on cardiovascular effects. Research report (Health Effects Institute):5-8.

Villeneuve PJ, Goldberg MS, Krewski D, et al., 2002. Fine particulate air pollution and all-cause mortality within the harvard six-cities study: Variations in risk by period of exposure. Annals of epidemiology 12:568-576.

Villeneuve PJ, Weichenthal SA, Crouse D, et al., 2015. Long-term exposure to fine particulate matter air pollution and mortality among canadian women. Epidemiology 26:536-545.

Vodonos A, Awad YA, Schwartz J. 2018. The concentration-response between long-term pm2.5 exposure and mortality; a meta-regression approach. Environmental research 166:677-689.

Wang Y, Shi L, Lee M, et al., 2017. Long-term exposure to pm2.5 and mortality among older adults in the southeastern us. Epidemiology 28:207-214.

Weichenthal S, Villeneuve PJ, Burnett RT, et al., 2014. Long-term exposure to fine particulate matter: Association with nonaccidental and cardiovascular mortality in the agricultural health study cohort. Environmental health perspectives 122:609615.

Weichenthal S, Crouse DL, Pinault L, et al., 2016. Oxidative burden of fine particulate air pollution and risk of cause-specific mortality in the canadian census health and environment cohort (canchec). Environmental research 146:92-99.

Weichenthal S, Pinault LL, Burnett RT. 2017. Impact of oxidant gases on the relationship between outdoor fine particulate air pollution and nonaccidental, cardiovascular, and respiratory mortality. Scientific reports 7:16401

Wong CM, Lai HK, Tsang H, et al., 2015. Satellite-based estimates of long-term exposure to fine particles and association with mortality in elderly hong kong residents. Environmental Health Perspectives 123:1167-1172.

Wong CM, Tsang H, Lai HK, et al., 2016. Cancer mortality risks from long-term exposure to ambient fine particle. Cancer Epidemiol Biomarkers Prev 25:839-845.

Woodward M. 2013. Epidemiology: Study design and data analysis:CRC press.

World Health Organization. 2006. Air quality guidelines: Global update 2005: Particulate matter, ozone, nitrogen dioxide, and sulfur dioxide:World Health Organization.

WHO for Europe. 2013. Review of evidence on health aspects of air pollution-revihaap project.

World Health Organization. 2018. Burden of disease from ambient air pollution for 2016. https://www.who.int/airpollution/data/AAP BoD results May2018 final.pdf?ua=1\#: :text=Summary\%20of\%20results,eac h\%20about\%201.3\%20million\%20deaths (last access: 20200619).

World Health Organization. 2020. Risk of bias assessment instrument for systematic reviews informing WHO global air quality guidelines. http://www.euro.who.int/en/health-topics/environment-and-health/air-quality/publications/2020/risk-ofbias-assessment-instrument-for-systematic-reviews-informing-who-global-air-quality-guidelines-2020 (last access: 20200224) Yang WS, Zhao H, Wang X, et al., 2016. An evidence-based assessment for the association between long-term exposure to outdoor air pollution and the risk of lung cancer. European journal of cancer prevention : the official journal of the European Cancer Prevention Organisation (ECP) 25:163-172.

Yang Y, Tang R, Qiu H, et al., 2018. Long term exposure to air pollution and mortality in an elderly cohort in hong kong. Environment international 117:99-106.

Yin P, Brauer M, Cohen A, et al., 2017. Long-term fine particulate matter exposure and nonaccidental and cause-specific mortality in a large national cohort of chinese men. Environmental health perspectives 125:117002.

Zanobetti A, Schwartz J. 2007. Particulate air pollution, progression, and survival after myocardial infarction. Environmental health perspectives 115:769-775.

Zanobetti A, Bind MAC, Schwartz J. 2008. Particulate air pollution and survival in a copd cohort. Environmental Health: A Global Access Science Source 7.

Zeger SL, Dominici F, McDermott A, et al., 2008. Mortality in the medicare population and chronic exposure to fine particulate air pollution in urban centers (2000-2005). Environmental health perspectives 116:1614-1619.

Zhang LW, Chen X, Xue XD, Sun M, Han B, Li CP, et al., 2014. Long-term exposure to high particulate matter pollution and cardiovascular mortality: A 12-year cohort study in four cities in northern china. Environment international 62:41-47.

Zhang P, Dong G, Sun B, et al., 2011. Long-term exposure to ambient air pollution and mortality due to cardiovascular disease and cerebrovascular disease in shenyang, china. PloS one 6:e20827.

Zhou M, Liu Y, Wang L, et al., 2014. Particulate air pollution and mortality in a cohort of chinese men. Environmental pollution (Barking, Essex : 1987) 186:1-6. 


\section{Supplementary Information}

(For space reasons, not all appendices are presented in this thesis. For more information, please refer to the online appendices: https://doi.org/10.1016/j.envint.2020.105974)

\section{Appendix $1 \quad$ Search Strategy}

\section{A1.1 Search strategy for MEDLINE}

\begin{tabular}{|c|c|c|}
\hline \multicolumn{2}{|c|}{ Search timeline: $1966-09.10 .2018$} & \multirow{2}{*}{$\begin{array}{r}\text { Abstracts } \\
1,855\end{array}$} \\
\hline$\# 20$ & 19 NOT "time series"[Title] & \\
\hline \#19 & 18 NOT "Air Pollution, Indoor"[Mesh] NOT "Occupational Exposure"[Mesh] & 1,873 \\
\hline \#18 & 17 NOT "Treatment Outcome"[MeSH] & 1,915 \\
\hline$\# 17$ & 16 NOT "animals"[Mesh:NoExp] & 2,157 \\
\hline \#16 & 15 NOT "Clinical Trial"[pt] & 2,235 \\
\hline \#15 & 5 AND 9 AND 14 & 2,362 \\
\hline \#14 & OR/ 10-13 & $2,880,275$ \\
\hline \#13 & case-control[Title/Abstract] & 111,292 \\
\hline$\# 12$ & $\begin{array}{l}\text { cohort[Title/Abstract] OR follow up[Title/Abstract] OR } \\
\text { Longitudinal[Title/Abstract] OR Prospective[Title/Abstract] OR } \\
\text { Retrospective[Title/Abstract] }\end{array}$ & $1,939,404$ \\
\hline$\# 11$ & Case-Control Studies[Mesh] & 945,436 \\
\hline$\# 10$ & Cohort Studies[Mesh] & $1,785,591$ \\
\hline$\# 9$ & $\mathrm{OR} / 5-8$ & $1,650,591$ \\
\hline$\# 8$ & mortality[Title/Abstract] OR death[Title/Abstract] & $1,182,902$ \\
\hline$\# 7$ & Death[Mesh] & 139,544 \\
\hline \#6 & mortality [Subheading] & 523,650 \\
\hline$\# 5$ & Mortality[Mesh] & 348,241 \\
\hline$\# 4$ & $\mathrm{OR} / 1-3$ & 293,389 \\
\hline$\# 3$ & $\begin{array}{l}\text { particulate matter[Title/Abstract] OR particulate air pollution[Title/Abstract] } \\
\text { OR PM[Title/Abstract] OR PM10[Title/Abstract] OR } \\
\text { PM2.5[Title/Abstract]OR particles[Title/Abstract] }\end{array}$ & 252,616 \\
\hline$\# 2$ & Particulate Matter[nm] & 13,598 \\
\hline$\# 1$ & Particulate Matter[Mesh] & 55,047 \\
\hline
\end{tabular}




\section{A1.2 Search strategy for EMBASE}

Search timeline: $1974-09.10 .2018$

Abstracts

\begin{tabular}{|c|c|c|}
\hline \#16 & 15 NOT 'time series':ti & 1,962 \\
\hline \#15 & 14 NOT ('occupational exposure'/exp/mj OR 'indoor air pollution'/exp/mj) & 1,971 \\
\hline$\# 14$ & $\begin{array}{l}13 \text { NOT ('case report'/de OR 'clinical trial'/de OR 'medical record review'/de OR } \\
\text { 'nonhuman'/de) }\end{array}$ & 2,027 \\
\hline \#13 & '12 AND 'human'/de & 2,275 \\
\hline$\# 12$ & 4 AND 7 AND 11 & 2,509 \\
\hline \#11 & $\mathrm{OR} / 8-10$ & $3,088,391$ \\
\hline$\# 10$ & $\begin{array}{l}\text { cohort:ab,ti OR 'follow up':ab,ti OR longitudinal:ab,ti OR prospective:ab,ti OR } \\
\text { retrospective: } \mathrm{ab}, \mathrm{ti}\end{array}$ & $2,921,977$ \\
\hline \#9 & 'case control study'/exp & 147,341 \\
\hline$\# 8$ & 'cohort analysis'/exp & 401,441 \\
\hline \#7 & $\mathrm{OR} / 4-6$ & $2,207,348$ \\
\hline$\# 6$ & 'death':ab,ti OR 'mortality':ab,ti & $1,641,979$ \\
\hline$\# 5$ & 'death'/exp & 669,233 \\
\hline$\# 4$ & 'mortality'/exp & 958,142 \\
\hline \#3 & 1 OR 2 & 302,300 \\
\hline$\# 2$ & $\begin{array}{l}\text { 'particulate matter':ab,ti OR 'particulate air pollution':ab,ti OR pm:ab,ti OR } \\
\text { pm10:ab,ti OR pm2.5:ab,ti OR particles:ab,ti }\end{array}$ & 292,768 \\
\hline$\# 1$ & "particulate matter'/exp & 36,016 \\
\hline
\end{tabular}




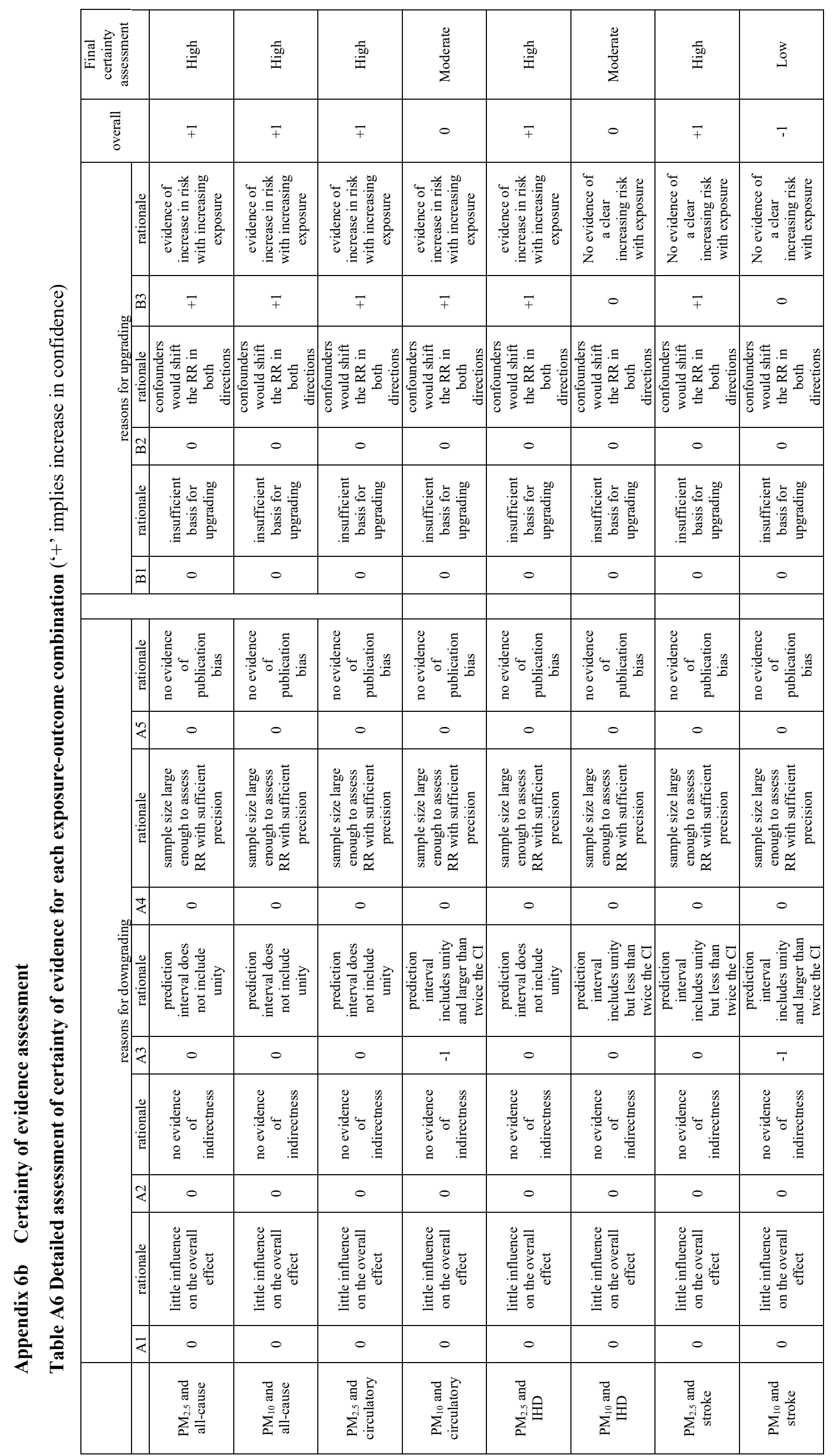




\begin{tabular}{|c|c|c|c|c|c|c|}
\hline 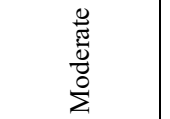 & 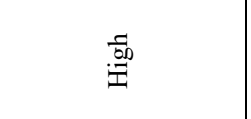 & 点 & $\begin{array}{l}\frac{0}{\pi} \\
\frac{5}{5} \\
\frac{5}{2}\end{array}$ & 离 & 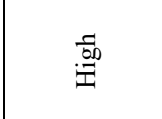 & $\stackrel{\cdot 50}{.00}$ \\
\hline 0 & $\mp$ & $\mp$ & 0 & $\mp$ & $\mp$ & $\mp$ \\
\hline 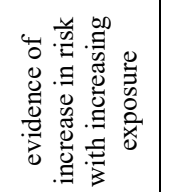 & 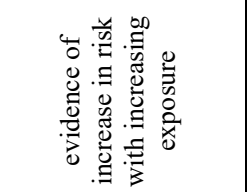 & 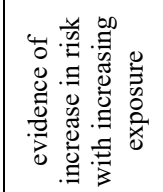 & 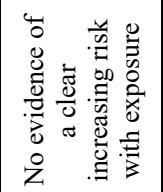 & 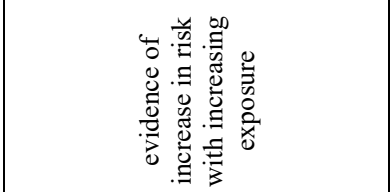 & 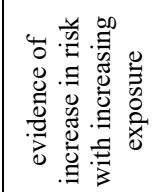 & 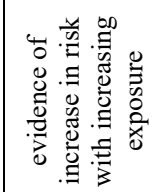 \\
\hline$\mp$ & $\mp$ & $\mp$ & 0 & $\mp$ & $\mp$ & $\mp$ \\
\hline 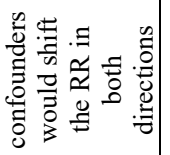 & 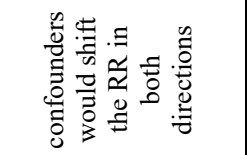 & 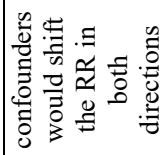 & 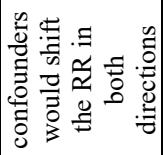 & 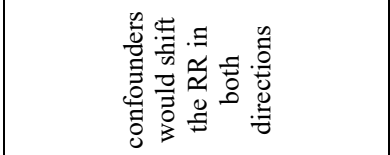 & 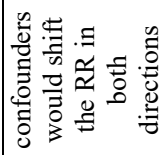 & 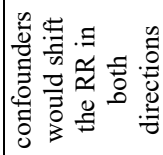 \\
\hline 0 & 0 & 0 & 0 & 0 & 0 & 0 \\
\hline 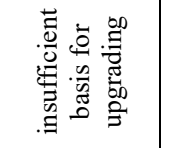 & 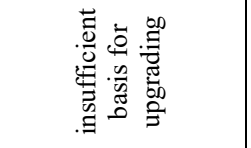 & 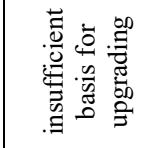 & 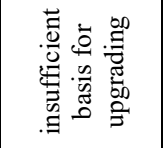 & 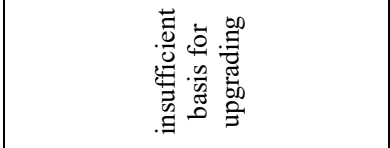 & 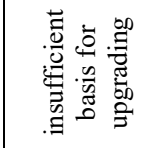 & 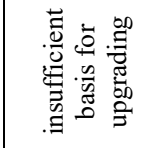 \\
\hline 0 & 0 & 0 & 0 & 0 & 0 & 0 \\
\hline 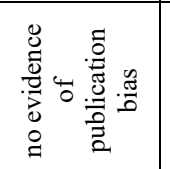 & 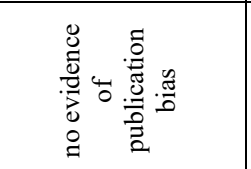 & 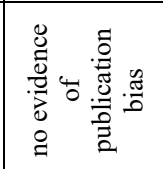 & 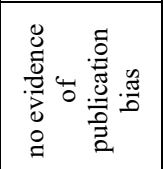 & 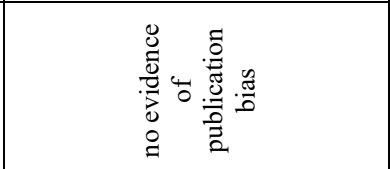 & 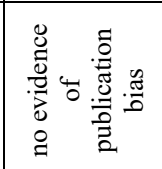 & 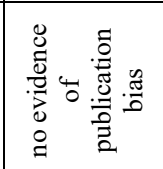 \\
\hline 0 & 0 & 0 & 0 & 0 & 0 & 0 \\
\hline 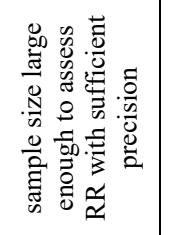 & 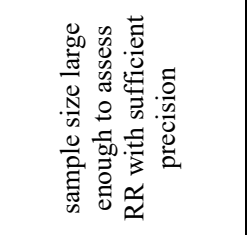 & 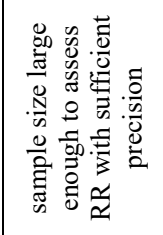 & 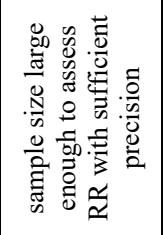 & 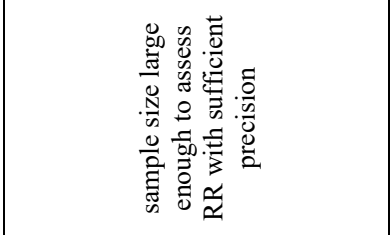 & 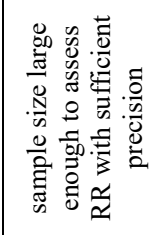 & 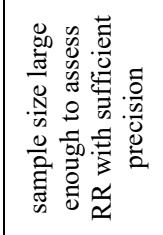 \\
\hline 0 & 0 & 0 & 0 & 0 & 0 & 0 \\
\hline 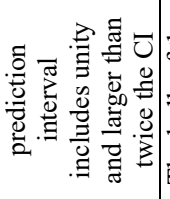 & 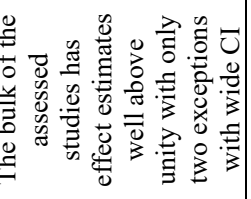 & 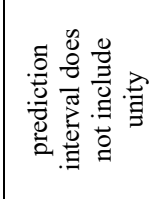 & 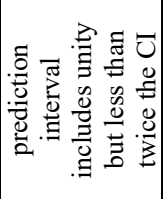 & 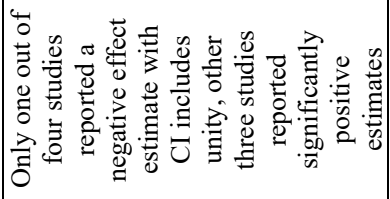 & 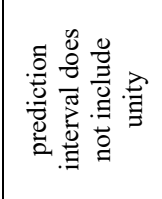 & 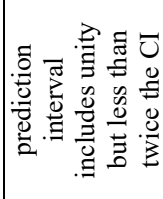 \\
\hline 7 & 0 & 0 & 0 & 0 & 0 & 0 \\
\hline 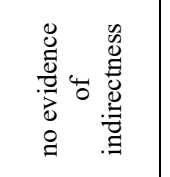 & 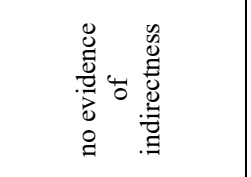 & 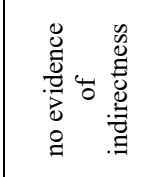 & 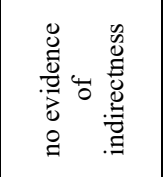 & 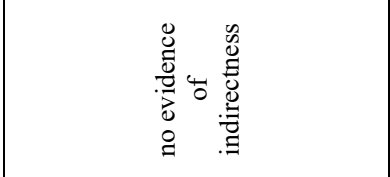 & 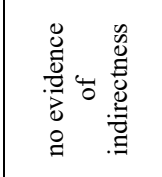 & 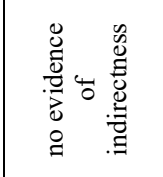 \\
\hline 0 & 0 & 0 & 0 & 0 & 0 & 0 \\
\hline 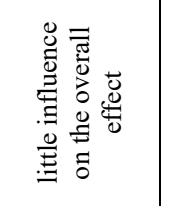 & 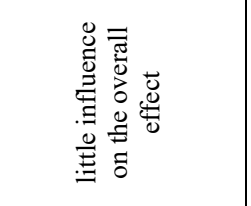 & 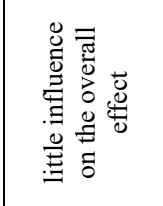 & 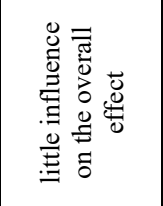 & 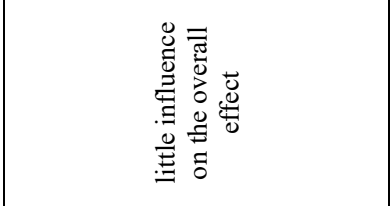 & 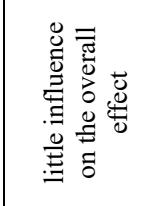 & 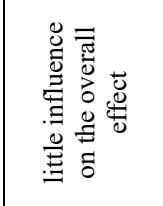 \\
\hline 0 & 0 & 0 & 0 & 0 & 0 & 0 \\
\hline 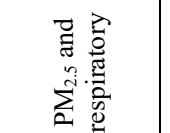 & 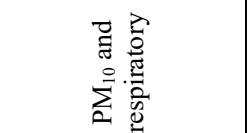 & 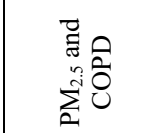 & $\begin{array}{l}\text { 룽 } \\
\sum_{0}^{0}\end{array}$ & 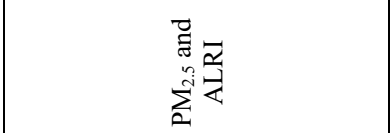 & 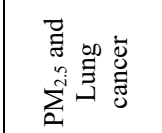 & 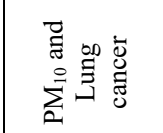 \\
\hline
\end{tabular}




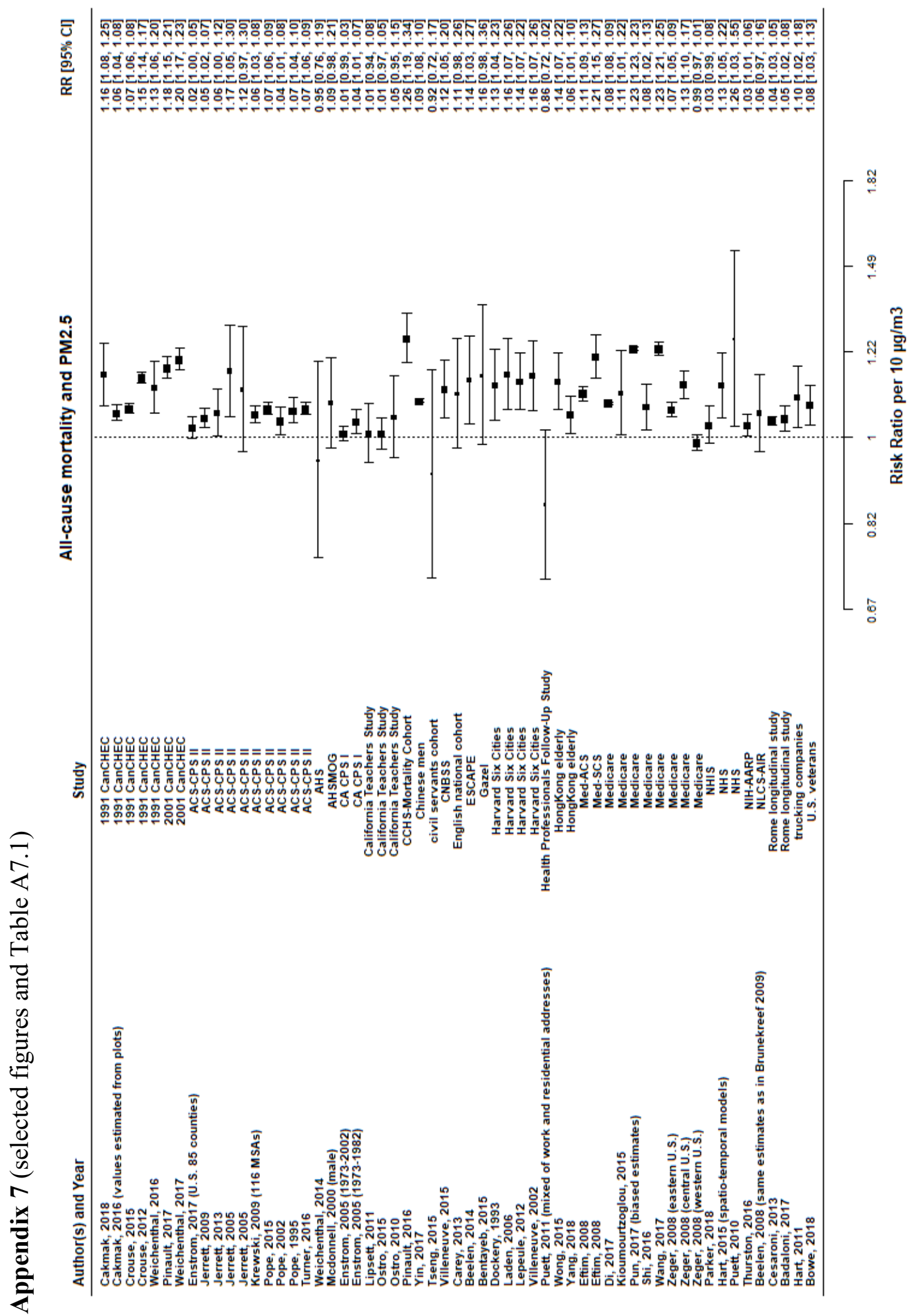

ב⿱艹 


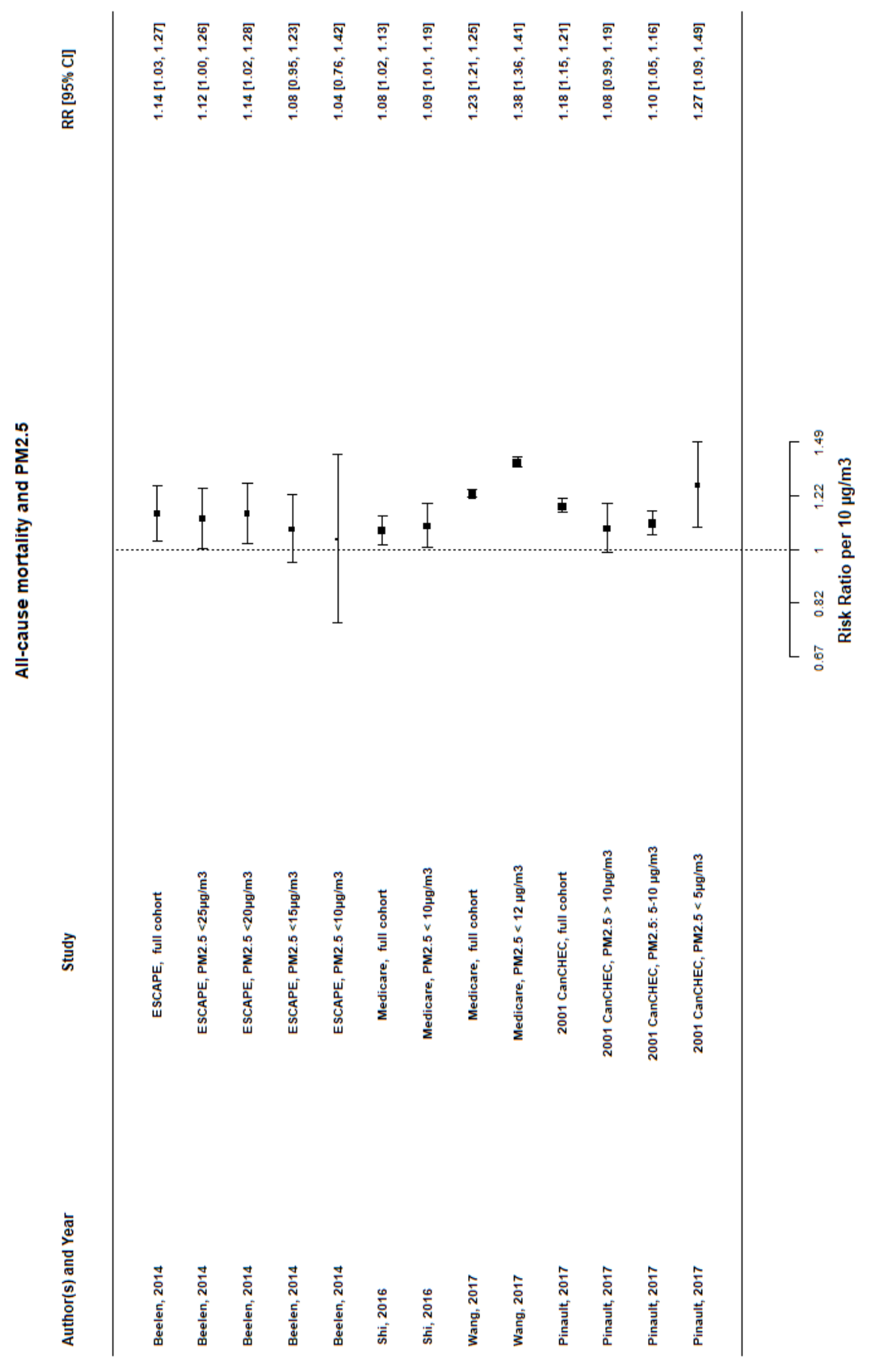

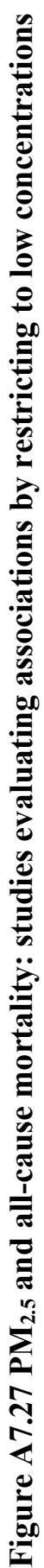




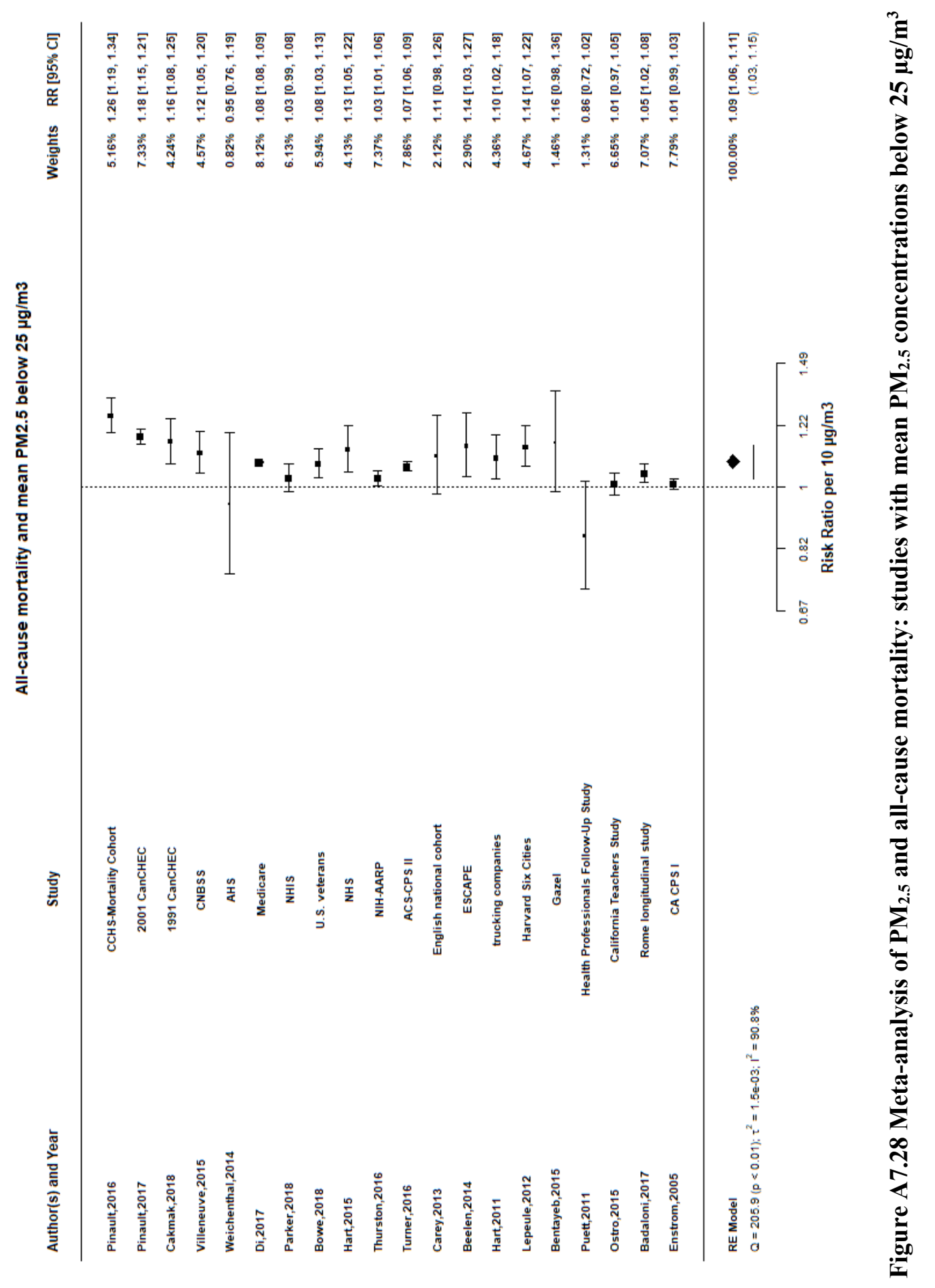




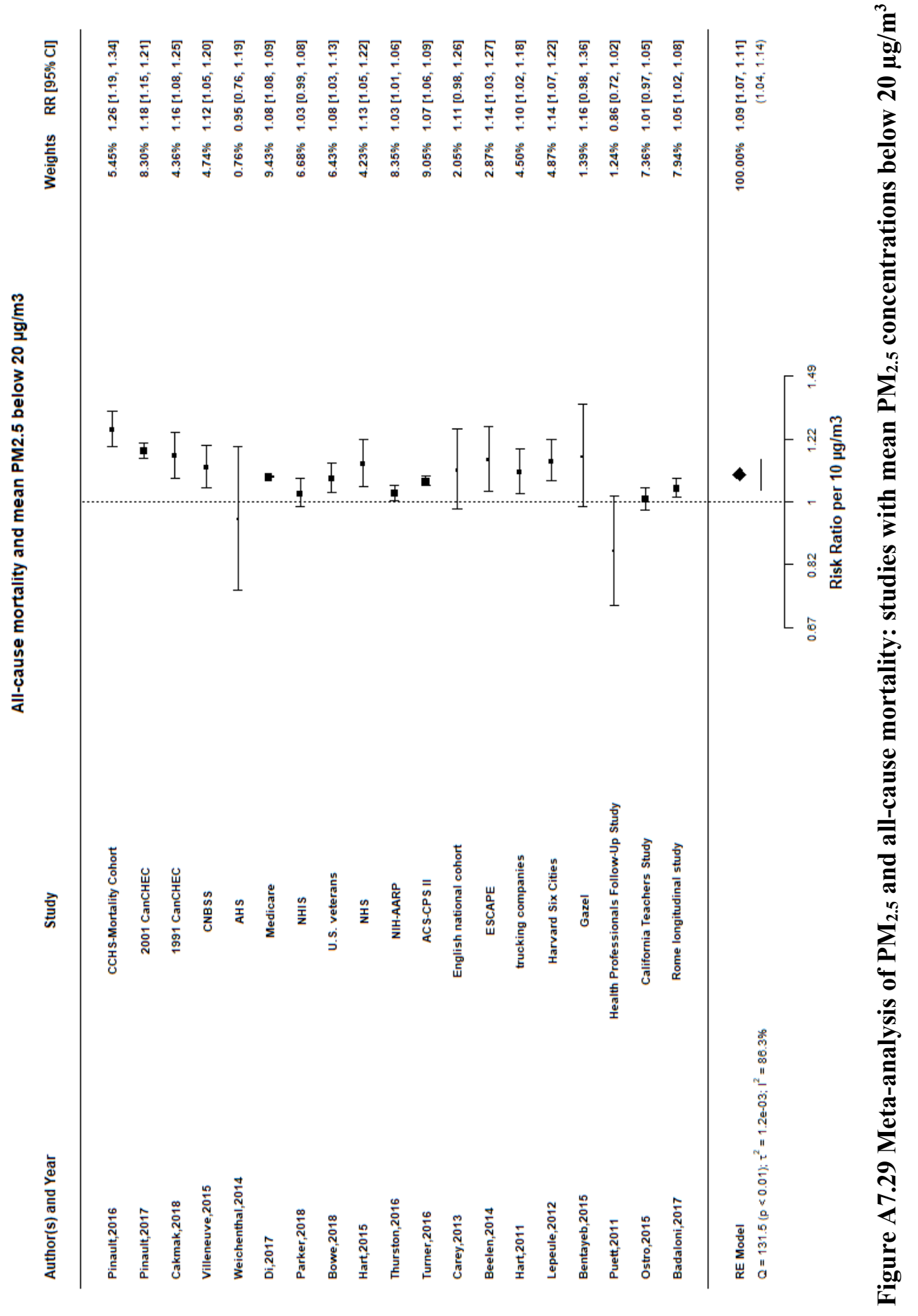




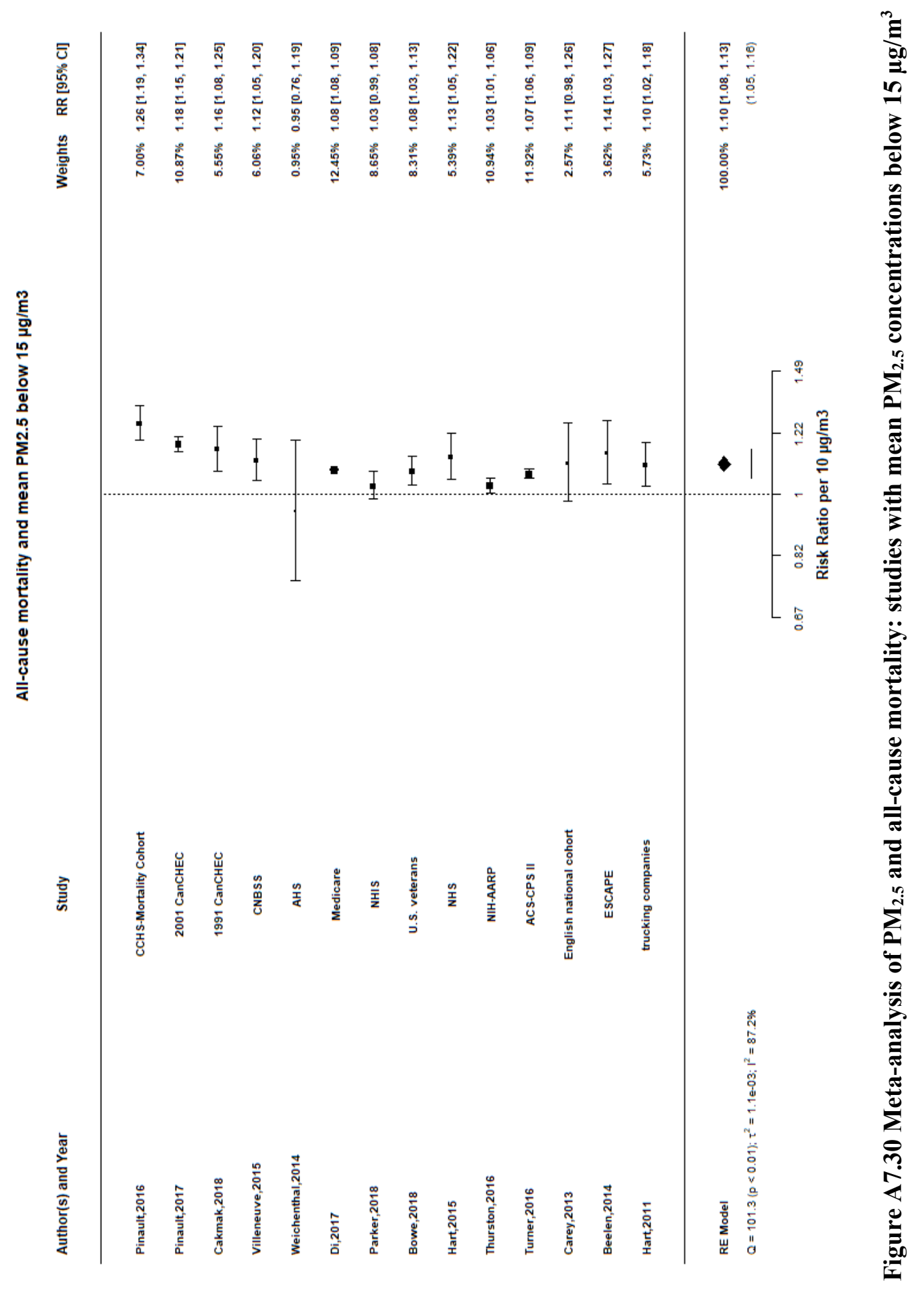




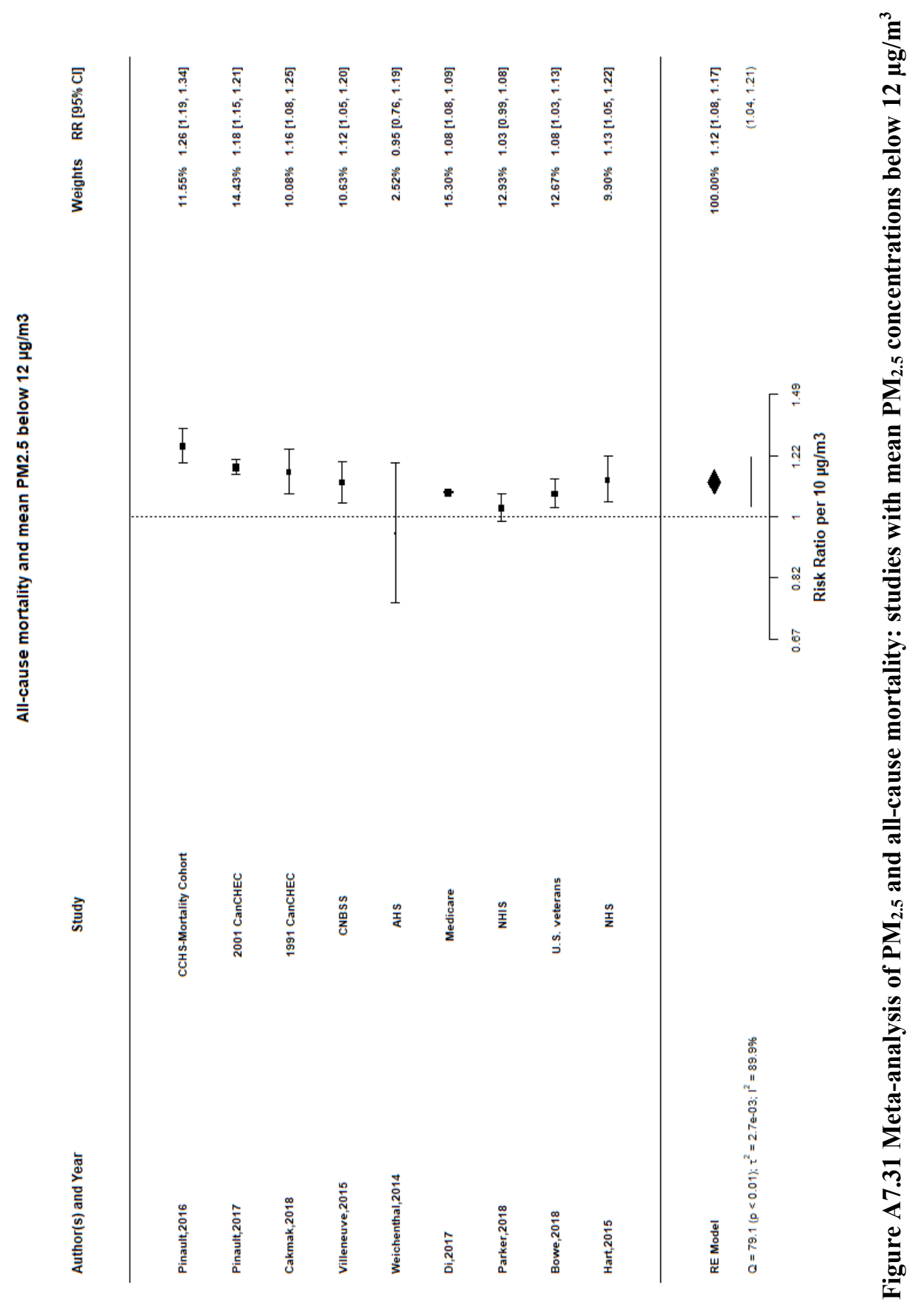


Chapter 2

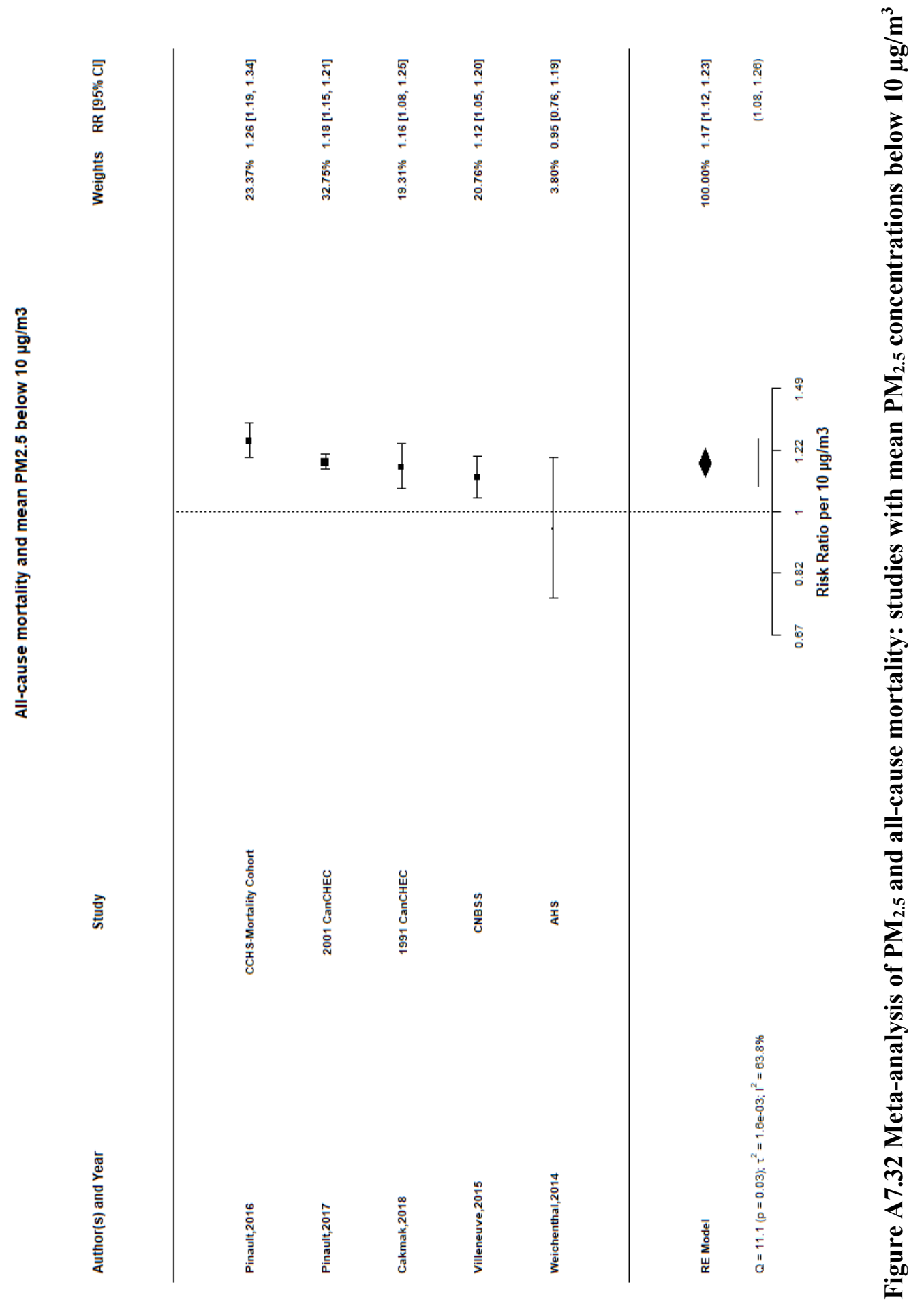




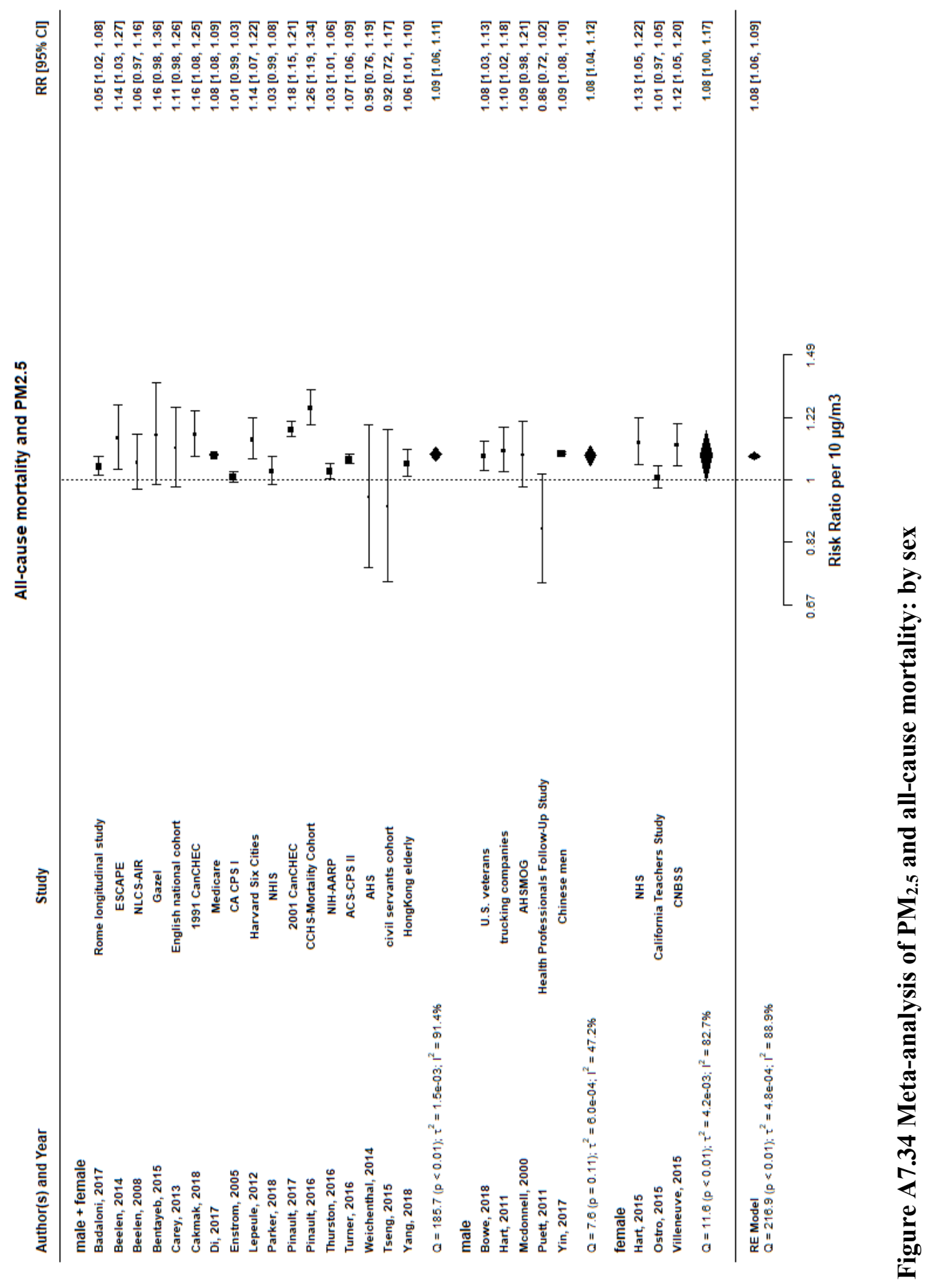




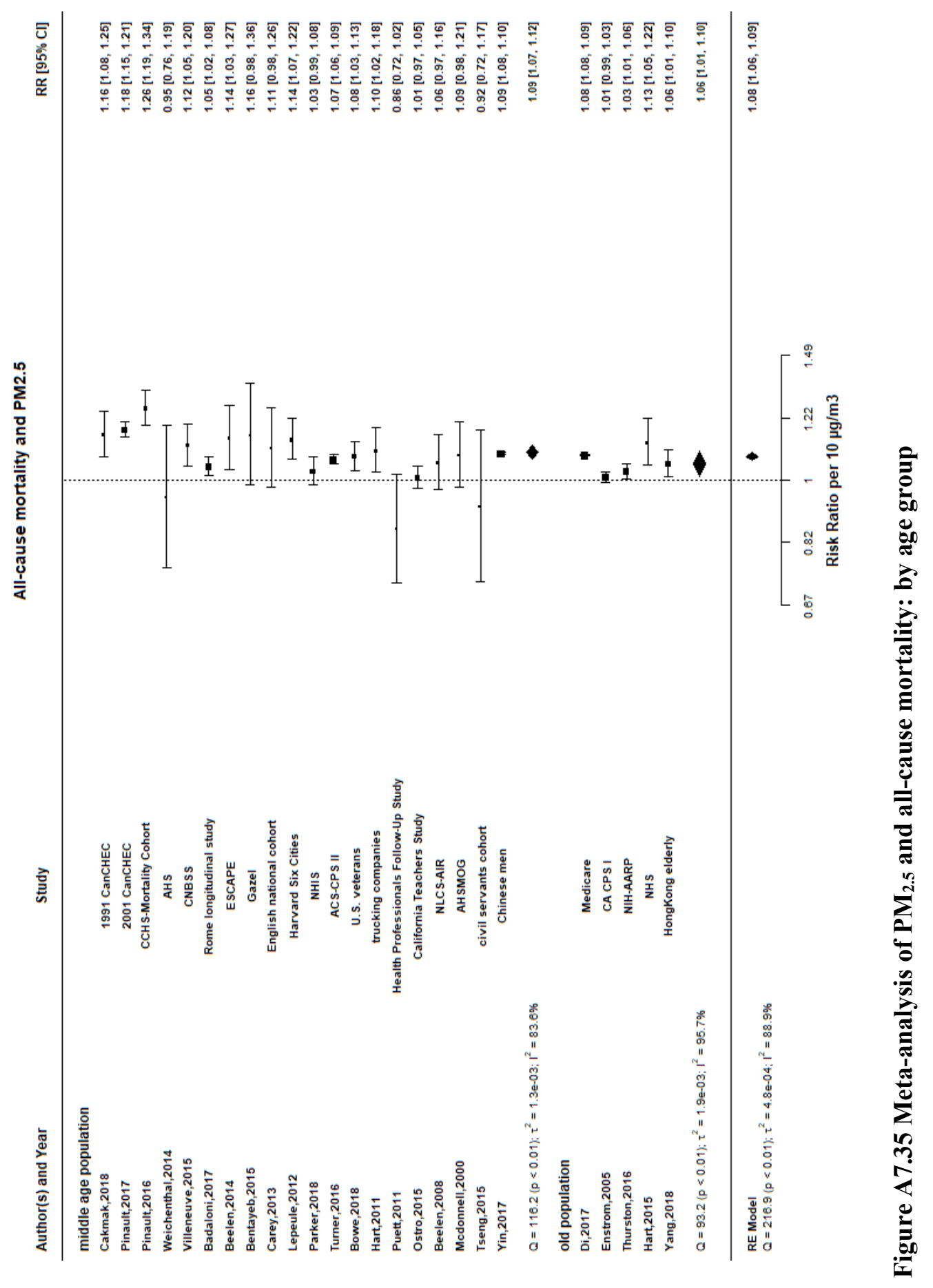




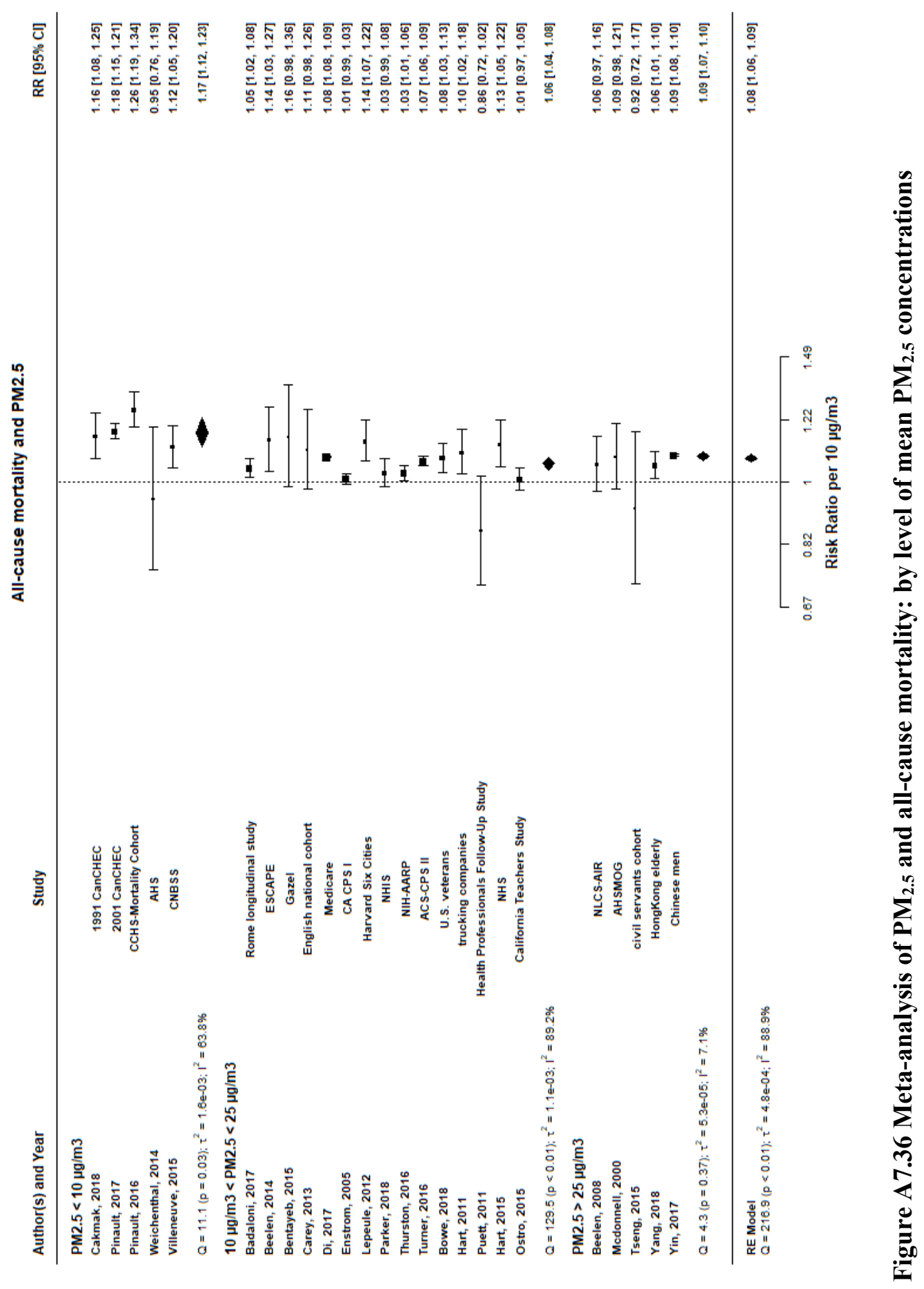


Chapter 2

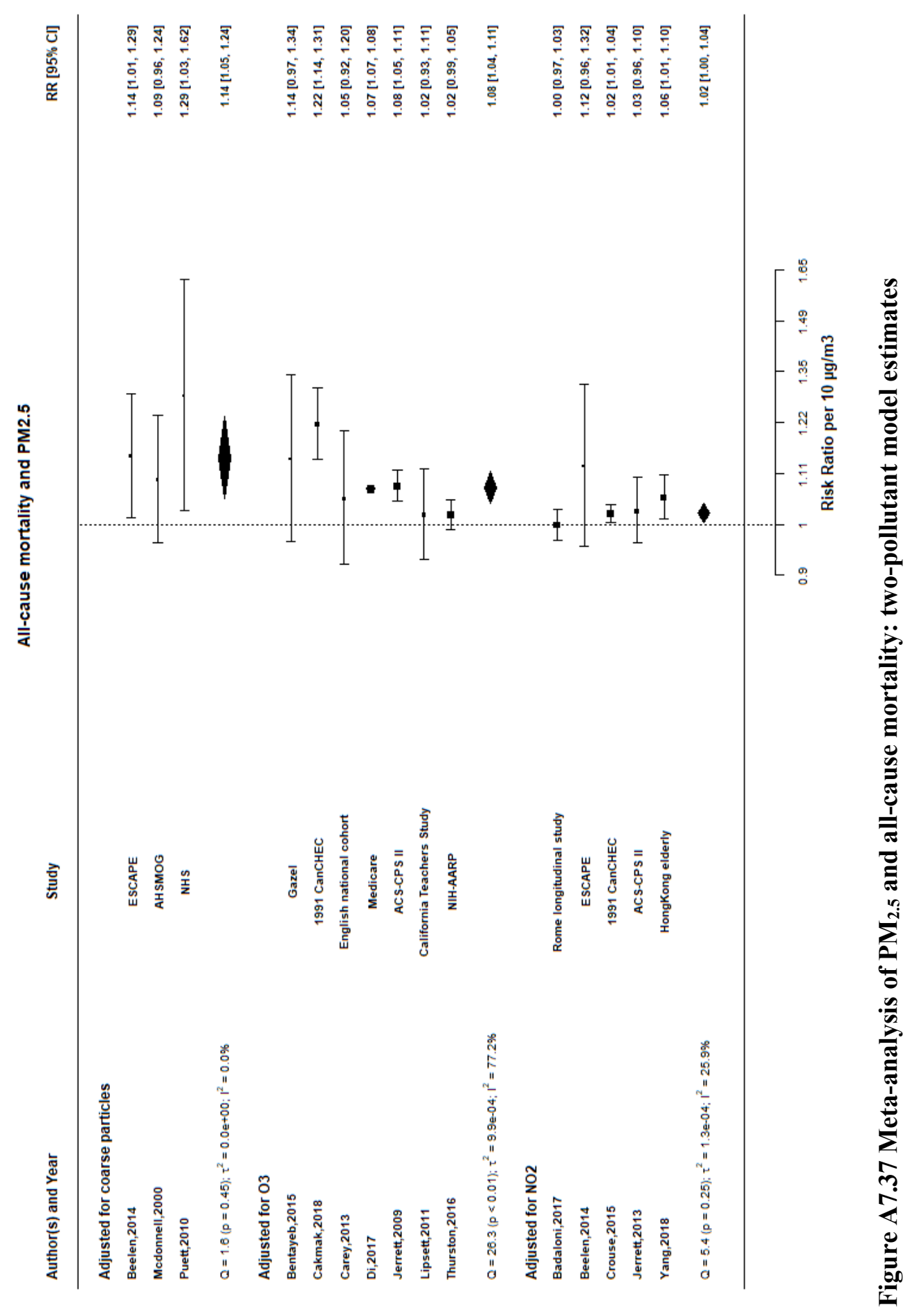




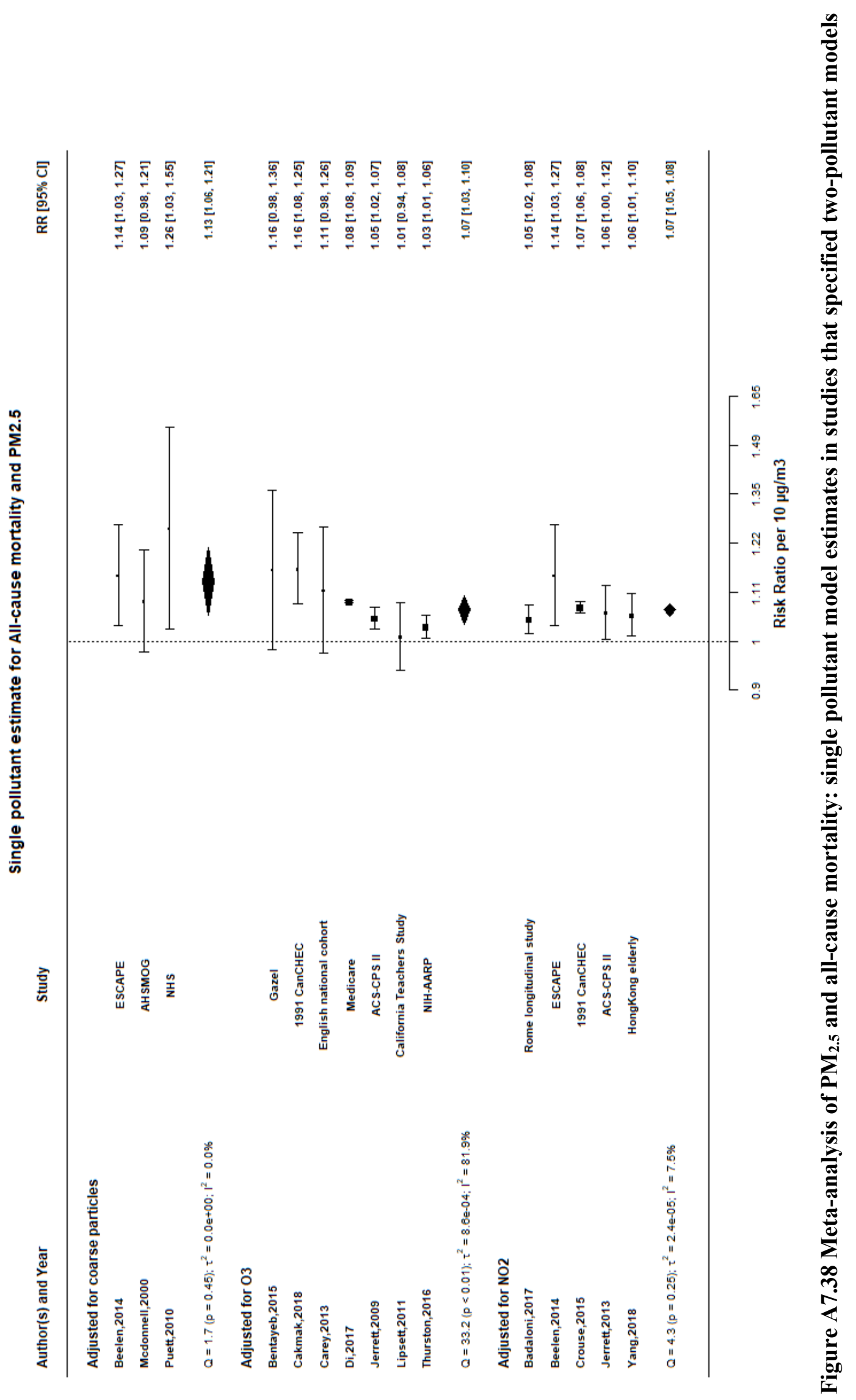




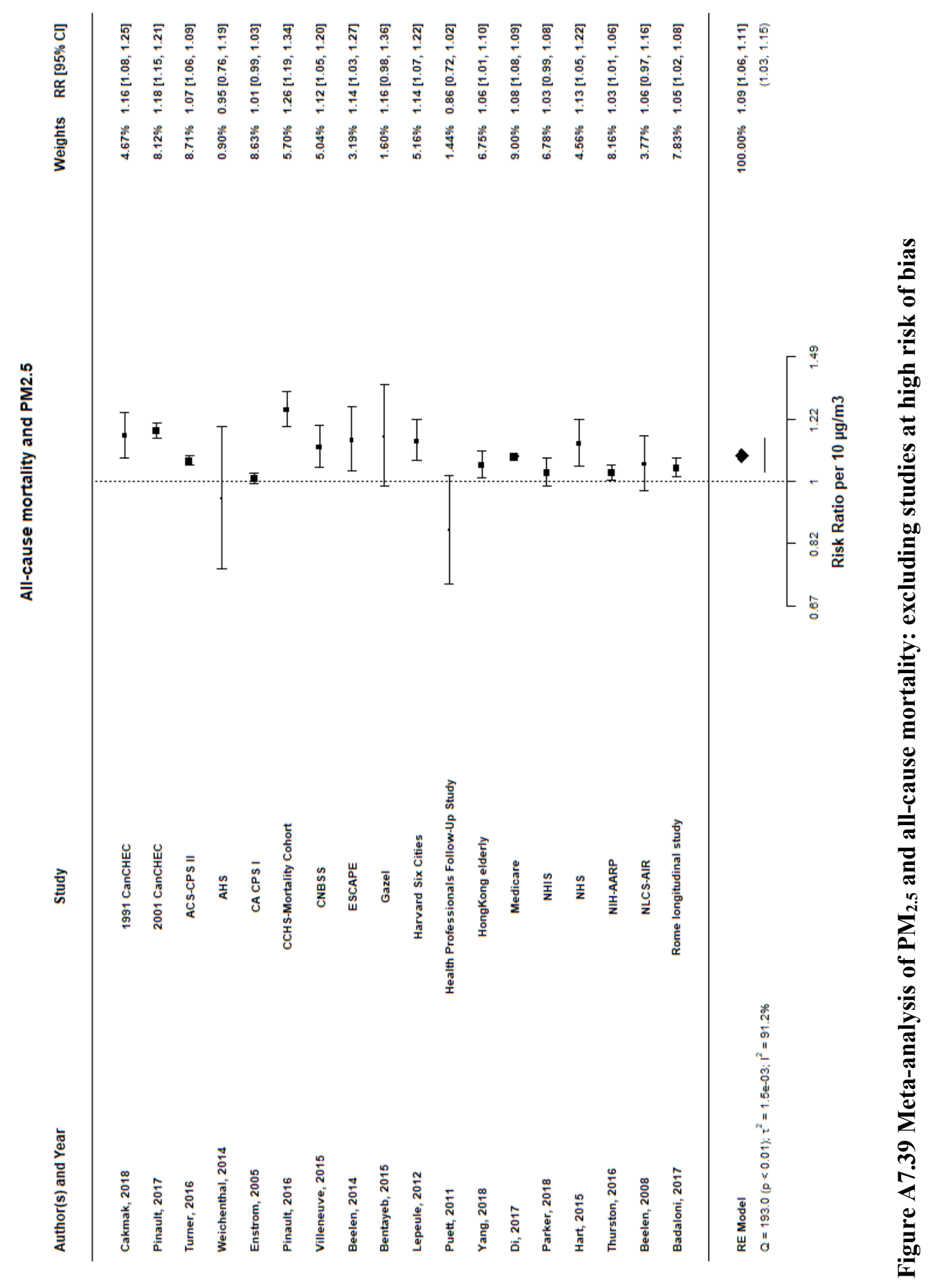




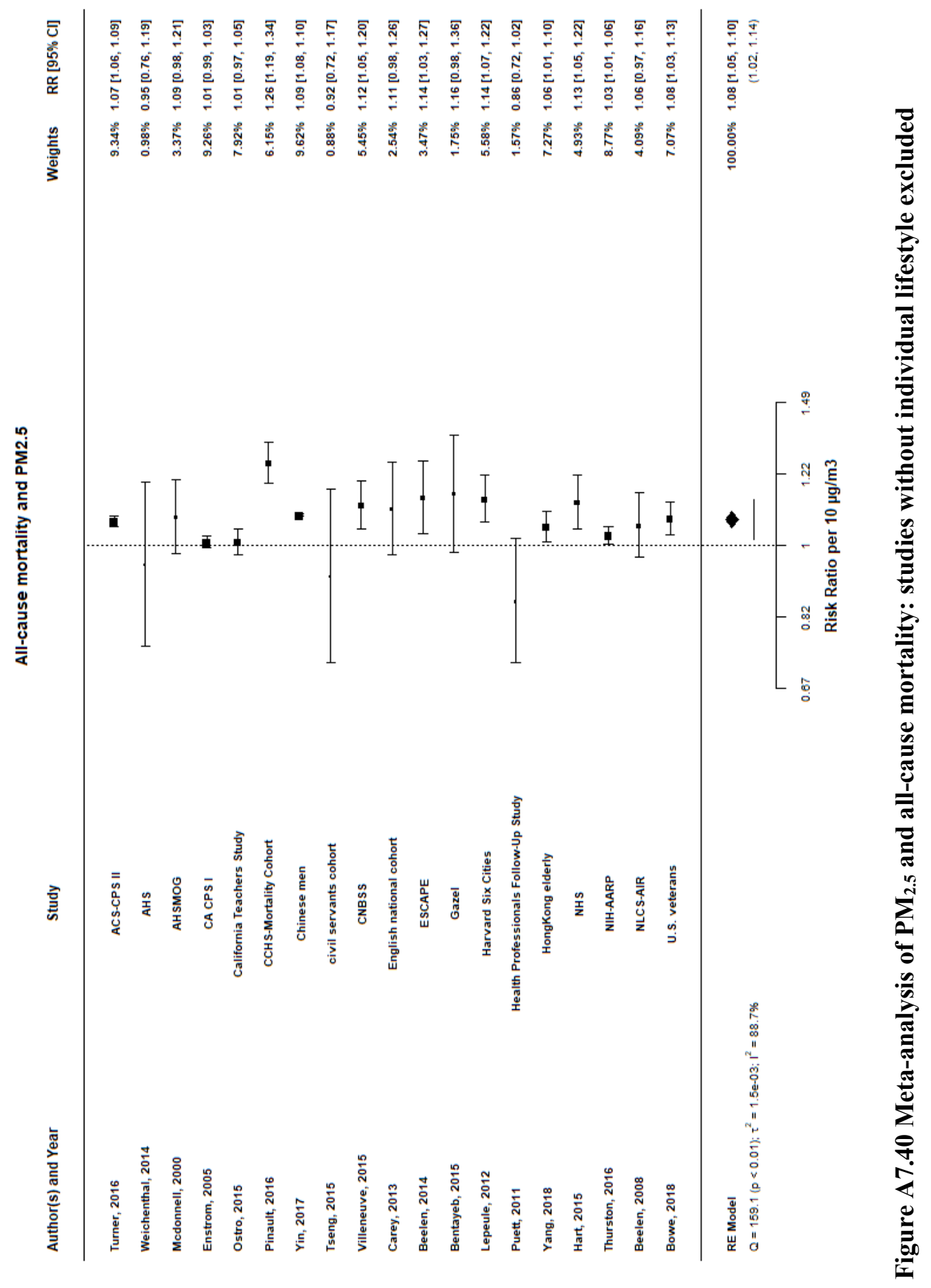




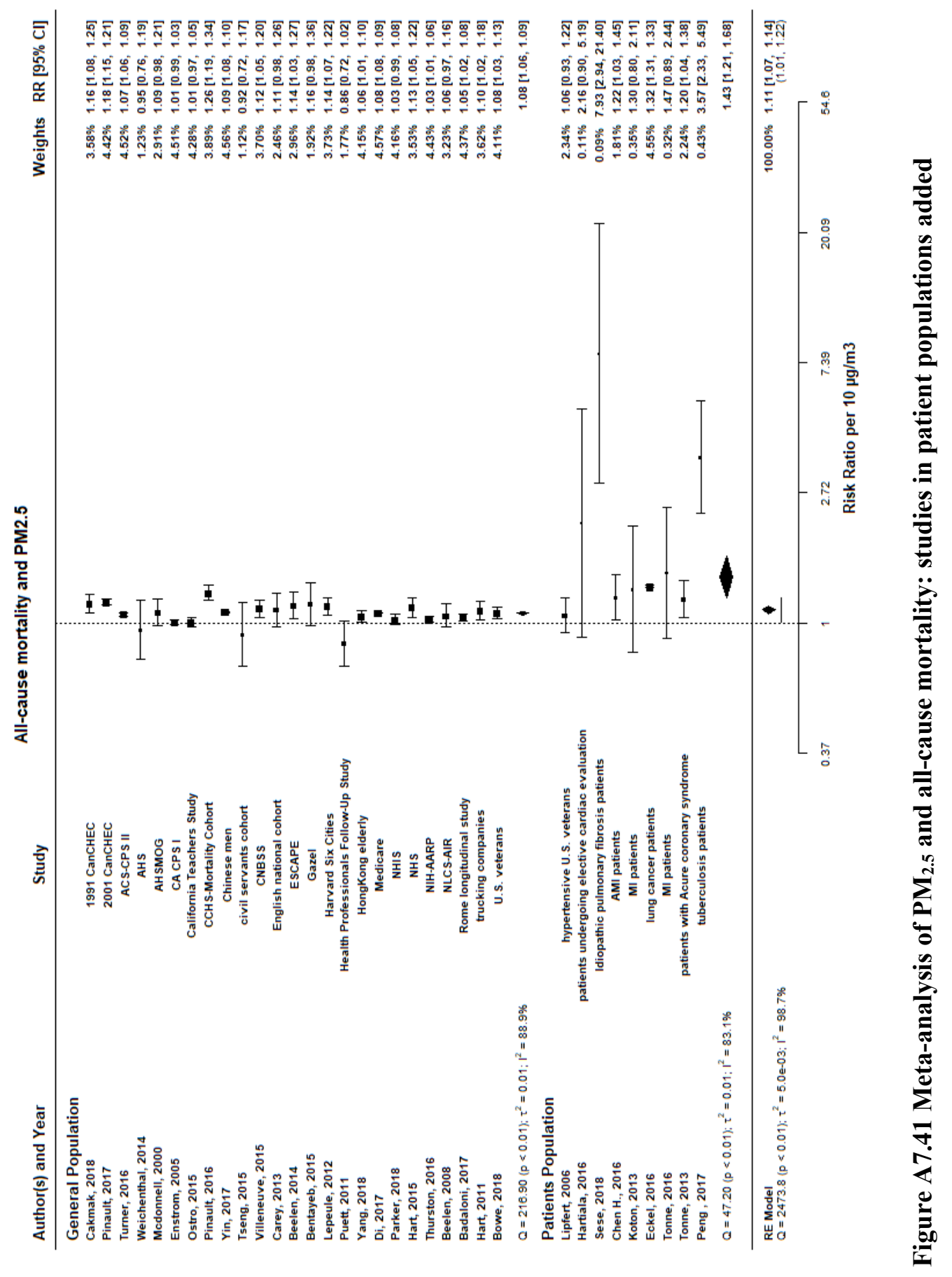




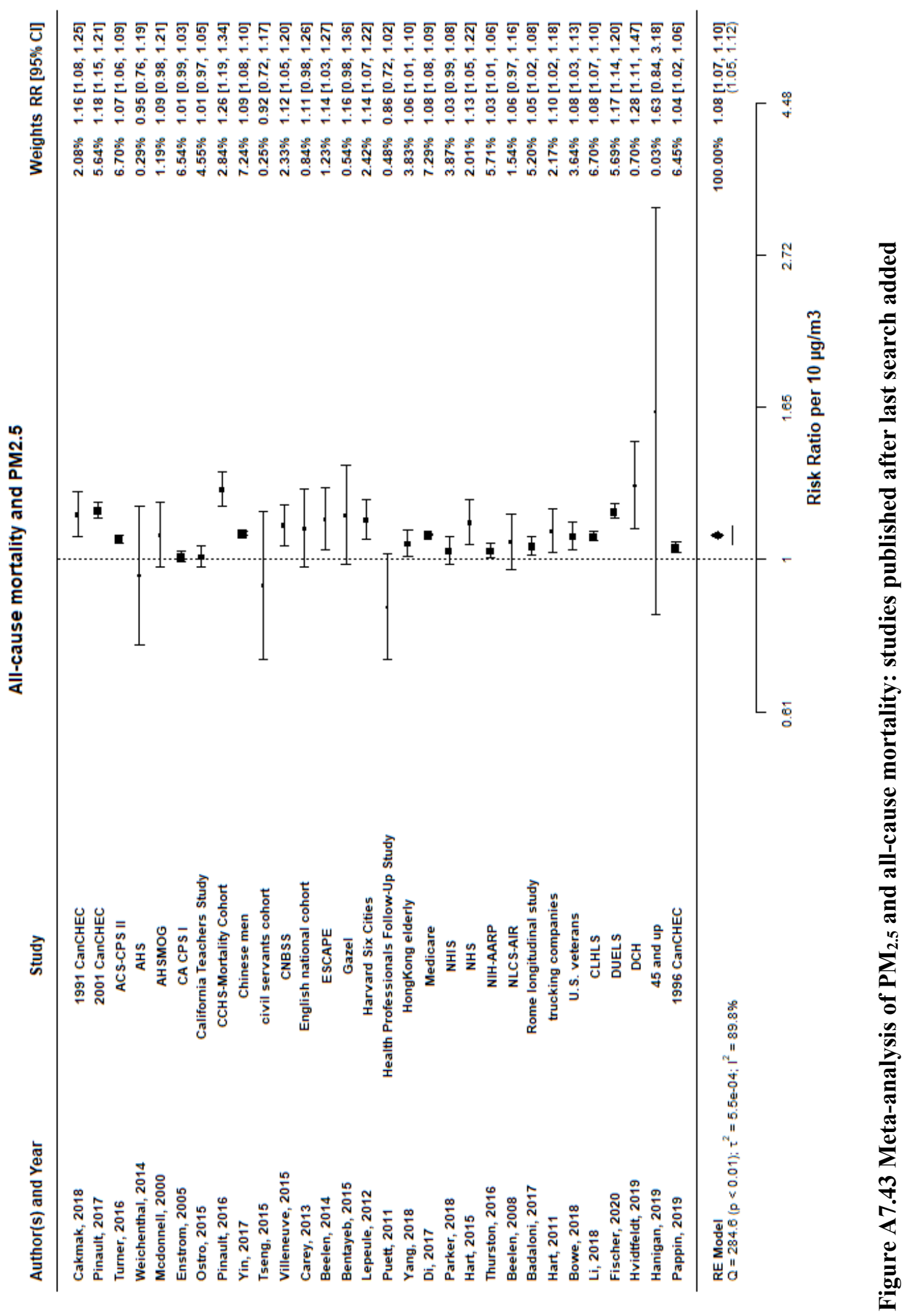




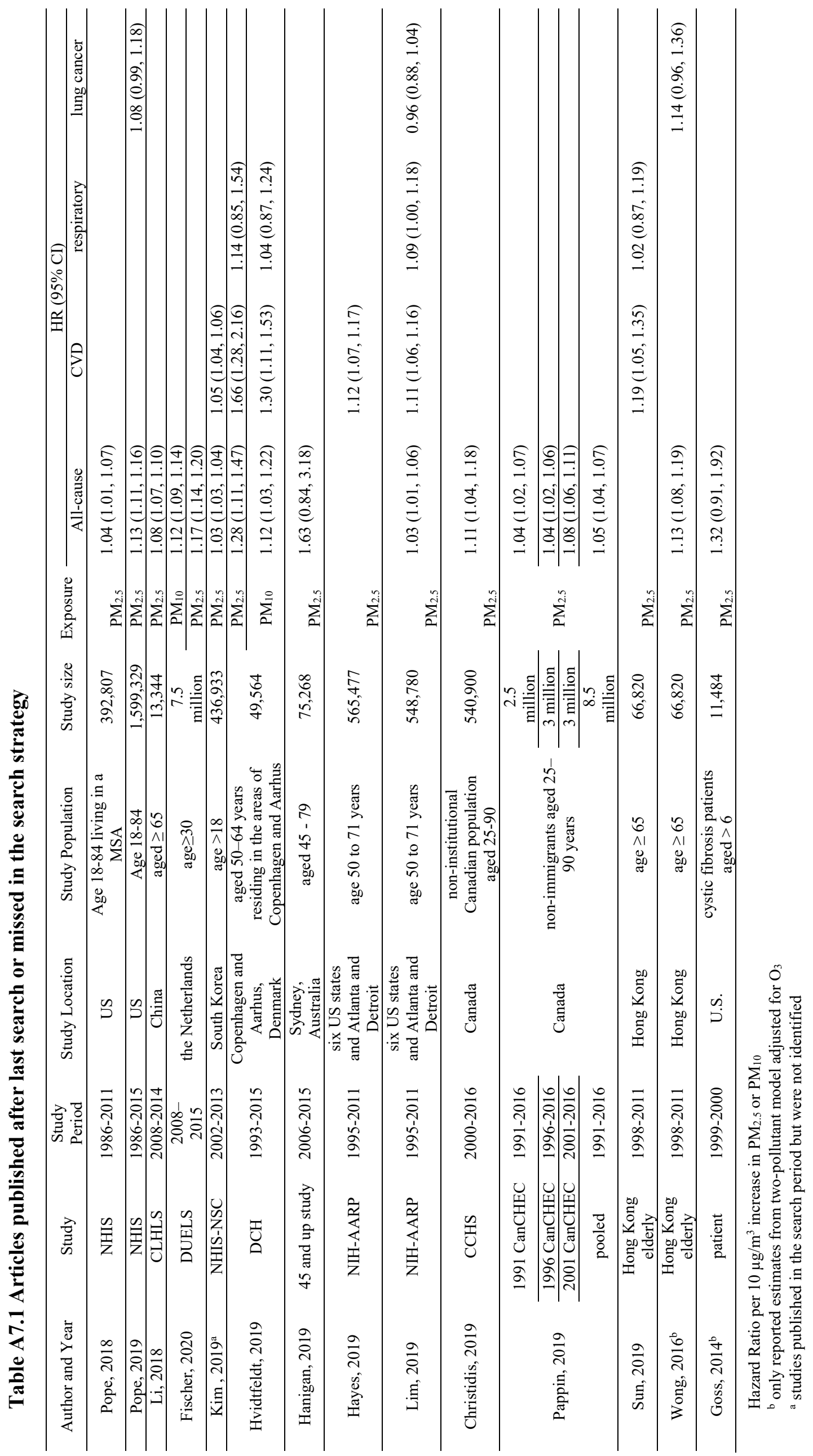




\section{Chapter}

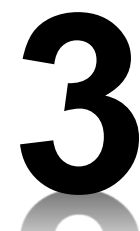

\section{Spatial $\mathrm{PM}_{2.5}, \mathrm{NO}_{2}, \mathrm{O}_{3}$ and $\mathrm{BC}$ Models for Western Europe - Evaluation of Spatiotemporal Stability}

Kees de Hoogh, Jie Chen, John Gulliver, Barbara Hoffmann, Ole Hertel, Matthias Ketzel, Mariska Bauwelinck, Aaron van Donkelaar, Ulla A. Hvidtfeldt, Klea Katsouyanni, Jochem Klompmaker, Randall V. Martin, Evangelia Samoli, Per E. Schwartz, Massimo Stafoggia, Tom Bellander, Maciej Strak, Kathrin Wolf, Danielle Vienneau, Bert Brunekreef and Gerard Hoek

Environment international, 2018, 120: 81-92

DOI: 10.1016/j.envint.2018.07.036 


\begin{abstract}
Background

In order to investigate associations between air pollution and adverse health effects consistent fine spatial air pollution surfaces are needed across large areas to provide cohorts with comparable exposures. The aim of this paper is to develop and evaluate fine spatial scale land use regression models for four major health relevant air pollutants $\left(\mathrm{PM}_{2.5}, \mathrm{NO}_{2}, \mathrm{BC}, \mathrm{O}_{3}\right)$ across Europe.
\end{abstract}

Methods

We developed West-European land use regression models (LUR) for 2010 estimating annual mean $\mathrm{PM}_{2.5}, \mathrm{NO}_{2}, \mathrm{BC}$ and $\mathrm{O}_{3}$ concentrations (including cold and warm season estimates for $\mathrm{O}_{3}$ ). The models were based on AirBase routine monitoring data $\left(\mathrm{PM}_{2.5}, \mathrm{NO}_{2}\right.$ and $\left.\mathrm{O}_{3}\right)$ and ESCAPE monitoring data (BC), and incorporated satellite observations, dispersion model estimates, land use and traffic data. Kriging was performed on the residual spatial variation from the LUR models and added to the exposure estimates. One model was developed using all sites (100\%). Robustness of the models was evaluated by performing a five-fold hold-out validation and for $\mathrm{PM}_{2.5}$ and $\mathrm{NO}_{2}$ additionally with independent comparison at ESCAPE measurements. To evaluate the stability of each model's spatial structure over time, separate models were developed for different years $\left(\mathrm{NO}_{2}\right.$ and $\mathrm{O}_{3}: 2000$ and 2005; $\mathrm{PM}_{2.5}$ : 2013).

Results

The $\mathrm{PM}_{2.5}, \mathrm{BC}, \mathrm{NO}_{2}, \mathrm{O}_{3}$ annual, $\mathrm{O}_{3}$ warm season and $\mathrm{O}_{3}$ cold season models explained respectively $72 \%, 54 \%, 59 \%, 65 \%, 69 \%$ and $83 \%$ of spatial variation in the measured concentrations. Kriging proved an efficient technique to explain a part of residual spatial variation for the pollutants with a strong regional component explaining respectively $10 \%, 24 \%$ and $16 \%$ of the $\mathrm{R}^{2}$ in the $\mathrm{PM}_{2.5}, \mathrm{O}_{3}$ warm and $\mathrm{O}_{3}$ cold models. Explained variance at fully independent sites vs the internal hold-out validation was slightly lower for $\mathrm{PM}_{2.5}(65 \%$ vs $66 \%)$ and lower for $\mathrm{NO}_{2}(49 \%$ vs $57 \%)$. Predictions from the 2010 model correlated highly with models developed in other years at the overall European scale.

\title{
Conclusions
}

We developed robust $\mathrm{PM}_{2.5}, \mathrm{NO}_{2}, \mathrm{O}_{3}$ and $\mathrm{BC}$ hybrid LUR models. At the West-European scale models were robust in time, becoming less robust at smaller spatial scales. Models were applied to $100 \times 100 \mathrm{~m}$ surfaces across Western Europe to allow for exposure assignment for 35 million participants from 18 European cohorts participating in the ELAPSE study.

\author{
Abbreviations \\ CTM Chemical Transport Models \\ SAT Satellite-derived predictions \\ FULL Models developed using 100\% of the monitoring sites \\ HOV Hold-Out-Validation models developed on $80 \%$ of the number of sites
}




\section{Introduction}

Ambient air pollution remains one of the main causes of morbidity and mortality in the world (Cohen et al., 2017). WHO's global assessment of ambient air pollution exposure estimated that one in nine deaths annually are caused by ambient air pollution (WHO, 2016). More recently, there is evidence showing that associations between mortality and morbidity and long-term exposure to outdoor air pollution might have no threshold, and extend to concentrations below current air quality limit values of the US EPA and EU (Beelen et al., 2015). Recent studies conducted in North-America have shown that long-term exposure to $\mathrm{PM}_{2.5}$ is associated with mortality also at low exposures (i.e. below the current WHO guideline of $10 \mu \mathrm{g} / \mathrm{m}^{3}$ ) (Crouse et al., 2015; Di et al., 2017; Pinault et al., 2017). Particularly in North-America and Europe, tougher air quality policies have led to a reduction in emissions and a gradual decline in ambient air pollution concentrations (EEA, 2017). Little, however, is known about the shape of the exposure-response curve at low concentrations, and thus the impact of low level concentrations on large populations remains uncertain.

The ELAPSE (Effects of Low-Level Air Pollution: A Study in Europe) study aims to fill this gap by investigating the relationship between long term air pollution and morbidity and mortality at low $\mathrm{PM}_{2.5}$ (Particulate Matter <2.5 $\mu \mathrm{g}$ ), nitrogen dioxide $\left(\mathrm{NO}_{2}\right)$, black carbon $(\mathrm{BC})$ and ozone $\left(\mathrm{O}_{3}\right)$ exposures. Low levels are defined as air pollutant concentrations below EU and/or US air quality limit values and/or WHO guidelines. ELAPSE includes 11 cohorts with in-depth individual data on lifestyle and 7 large administrative/national cohorts across Europe (http://www.elapseproject.eu/). Cohorts were selected to represent a contrast in air pollution exposures between and within study areas. The 11 detailed individual-level cohorts will be analyzed as a pooled cohort, whereas the administrative cohorts will be analyzed separately. Taken together, the evidence should allow collective consideration and evaluation. This study therefore needs consistent models that can provide valid exposures at two different spatial extents in Western Europe: combining all study regions of the detailed individual-level cohorts for the pooled analysis; and the national extents for the administrative/national cohorts. The previously developed ESCAPE LUR models (Beelen et al., 2013; Eeftens et al., 2012a) do not meet the requirements for the ELAPSE project because they do not cover the full national study areas. Secondly, methodological work by Basagana and Wang has shown that more stable models can be developed based on a larger number of model training sites than the 20 sites that the ESCAPE PM models were based upon (Basagaña et al., 2012; Wang et al., 2013). Finally, ESCAPE did not evaluate ozone.

Cohorts in the ELAPSE study have different recruitment and follow-up periods going back as early as the 1990's. Epidemiological studies have used the back-extrapolation method to estimate exposures back in time (Beelen et al., 2014; Chen et al., 2017). The method uses a well validated air pollution surface as the base and assumes that the spatial structure of this surface remains stable over time. Monitoring data from routine monitoring sites are then used to re-scale the surface back or forward in time (Cesaroni et al., 2012; Chen et al., 2010). Few studies have been able to document the stability of spatial surfaces, mostly focusing on $\mathrm{NO}_{2}$ and at the city level (Cesaroni et al., 2012; Eeftens et al., 2011; Wang et al., 2013) or national scale (Gulliver et al., 2013). We thus evaluated the stability of these surfaces over time by comparing modelled estimates with historic monitoring data and by developing models for other years.

The aims of the paper are to:

1. develop and evaluate performance of fine spatial scale hybrid land use regression models for four major health relevant pollutants $\mathrm{PM}_{2.5}, \mathrm{NO}_{2}, \mathrm{BC}, \mathrm{O}_{3}$ across Western Europe;

2. investigate the temporal stability of the spatial contrast at the West-European and national scale.

This paper follows our recently published West-European fine scale air pollution exposure models for $\mathrm{PM}_{2.5}$ and $\mathrm{NO}_{2}$ (de Hoogh et al., 2016). Models were based on both 2010 ESCAPE and the European 
Environment Agency (EEA) AirBase routine monitoring data, and documented the contribution of satellite data and chemical transport models (CTM) to LUR models. An important finding was that models performed well when validated with data from the other measurement network (i.e. ESCAPE model validated with AirBase sites and vice versa). In the current paper we substantially extended this work, firstly by adding $\mathrm{BC}$ and $\mathrm{O}_{3}$ which are both health relevant pollutants. We also improved the testing of the robustness of models by evaluating structure and predictions using five-fold hold-outvalidation (HOV), following a study on land use regression models for ultrafine particles (van Nunen et al., 2017). We further assessed improving the LUR models using kriging and added new predictor variables with improved granularity, including $1 \mathrm{x} 1 \mathrm{~km}$ satellite-derived $\mathrm{PM}_{2.5}$ to the previously used $10 \times 10 \mathrm{~km}$ satellite data. Finally we added an assessment of the temporal stability of the models.

\section{Materials and methods}

\subsection{Air pollution monitoring data}

$\mathrm{PM}_{2.5}, \mathrm{NO}_{2}$ and $\mathrm{O}_{3}$ daily concentration data for 2010 were derived from the AirBase $\mathrm{v} 8$ dataset (EEA, $2015)$. Only sites with $\geq 75 \%$ completeness of the total hours $\left(\mathrm{NO}_{2}\right.$ and $\left.\mathrm{O}_{3}\right)$ or days $\left(\mathrm{PM}_{2.5}\right)$ were accepted, and an annual average was calculated for $\mathrm{PM}_{2.5}$ and $\mathrm{NO}_{2}$. For $\mathrm{O}_{3}$, we calculated the maximum running 8-hour mean for each day and then averaged to obtain an annual, warm season (April through September) and cold season (January through March and October through December) average maximum running 8-hour mean. For BC, which is not available through AirBase, we used the ESCAPE annual mean $\mathrm{BC}$ concentrations (measured as $\mathrm{PM}_{2.5}$ absorbance based on reflectance measurement of the filters) reflecting the time period 2009-2010. Previous studies (Cyrys et al., 2012; Eeftens et al., 2012b) using AirBase data documented no difference in average BC concentrations between 2000 and 2010, therefore we treated all BC measurements as 2010 annual mean concentrations. A detailed description of the ESCAPE measurement campaign can be found elsewhere (Eeftens et al., 2012b). Table S1 describes the number of sites and summary statistics of the air pollution measurement data. The locations of the monitoring sites used for the 2010 models are shown in Figure S1. For temporal stability analysis we additionally included $\mathrm{NO}_{2}$ and $\mathrm{O}_{3}$ daily concentration data for 2000 and 2005 from AirBase v8 and daily PM$_{2.5}$ concentration data for 2013 from Air Quality e-Reporting (www.eea.europa.eu/data-and-maps/data/aqereporting-8). There were insufficient $\mathrm{PM}_{2.5}$ sites across Western Europe before 2010.

\subsection{Predictor variables}

\subsubsection{Satellite derived air pollution data}

In addition to the satellite-derived (SAT) $\mathrm{PM}_{2.5}$ product (v3.01) used in the previous paper (de Hoogh et al., 2016), we tested two additional different SAT $\mathrm{PM}_{2.5}$ products, which have become available only recently, as potential predictors. These were obtained from the global dataset reported in Van Donkelaar et al. (2015). Aerosol Optical Depth (AOD) retrievals from the NASA MODIS (Moderate Resolution Imaging Spectroradiometer), MISR (Multi-angle Imaging Spectroradiometer) and SeaWiFS instruments were related to near-surface concentrations using aerosol vertical profiles and scattering properties simulated by the GEOS-Chem CTM, to produce an annual average $\mathrm{PM}_{2.5}$ dataset at a $0.1^{\circ} \times 0.1^{\circ}(\sim 10 \mathrm{~km})$ resolution for 2010 . In the previous paper we used a dataset inferred from 2009-2011 (optimized for 2010), here we additionally tested the inferred data from 2010 data only. We further included the current, purely geophysical, global $\mathrm{PM}_{2.5}$ dataset (V4.GL.02.NoGWR), which includes some information at the finer resolution of $0.01^{\circ} \times 0.01^{\circ}(\sim 1 \mathrm{~km})$ published by van Donkelaar et al. (2016). The pre-Geographically Weighted Regression dataset used here includes AOD from multiple satellite products (MISR, MODIS Dark Target, MODIS and SeaWiFS Deep Blue, and MODIS MAIAC) together with simulation-based sources, with information content below $\sim 10 \mathrm{~km}$ provided by the MAIAC AOD retrieval. $\mathrm{PM}_{2.5}$ satellite data was offered as a predictor to the $\mathrm{PM}_{2.5}$ 
models. No $\mathrm{BC}$ satellite data were available and because $\mathrm{BC}$ is a major component of $\mathrm{PM}_{2.5}, \mathrm{PM}_{2.5}$ satellite data were also offered to the $\mathrm{BC}$ models.

$\mathrm{NO}_{2}$ SAT estimates for 2010 were derived from the tropospheric $\mathrm{NO}_{2}$ columns measured with the OMI (Ozone Monitoring Instrument) on board the Aura satellite. Like $\mathrm{PM}_{2.5}$, the satellite columnintegrated retrievals were related to ground-level concentrations using the global GEOS-Chem model, producing an annual gridded $\mathrm{NO}_{2}$ surface at a $10 \mathrm{~km}$ resolution (Bechle et al., 2013, 2015; Novotny et al., 2011). $\mathrm{NO}_{2}$ satellite predictors were offered to the $\mathrm{NO}_{2}$ models. No $\mathrm{O}_{3}$ satellite data were available but, because $\mathrm{NO}_{2}$ is related to $\mathrm{O}_{3}$ formation and scavenging, $\mathrm{NO}_{2}$ satellite data was also offered to the $\mathrm{O}_{3}$ models.

\subsubsection{Chemical transport model (CTM) data}

Pollutant estimates for 2010 from two long range CTM's were obtained as potential predictor variables for the models. Annual $\mathrm{PM}_{2.5}, \mathrm{NO}_{2}$ and $\mathrm{O}_{3}$ estimates were derived from the MACC-II ENSEMBLE model at a $0.1^{\circ}$ x $0.1^{\circ}(\sim 10 \mathrm{~km})$ resolution (Inness et al., 2013). The ENSEMBLE model provides a value at each pixel which is defined as the median value of seven individual CTMs: CHIMERE, EMEP, EURAD, LOTOS-EUROS, MATCH, MOCAGE and SILAM. Annual MACC-II ENSEMBLE averages for $\mathrm{PM}_{2.5}, \mathrm{NO}_{2}$ and $\mathrm{O}_{3}$ were offered to the respective LUR models. We additionally acquired a second CTM dataset from the Danish Eulerian Hemispheric Model (DEHM_v31102016) for $\mathrm{PM}_{2.5}, \mathrm{NO}_{2}, \mathrm{O}_{3}$ and $\mathrm{BC}$ at a monthly $50 \times 50 \mathrm{~km}$ resolution (Brandt et al., 2012). Annual DEHM averages were calculated for all pollutants and offered to the respective LUR models, while warm and cold averages of $\mathrm{O}_{3}$ were offered to the warm and cold season models.

\subsubsection{Other predictor variables}

The GIS predictor variables used in this study are described in more detail elsewhere (de Hoogh et al., 2016; Vienneau et al., 2013). In brief, road data, classified as 'all' and 'major' roads, were extracted from the 1:10,000 EuroStreets digital road network (version 3.1 based on TeleAtlas MultiNet TM, year 2008). Land cover data were extracted from European Corine Land Cover 2006 data (ETC-LC, 2013) except for Greece for which Corine Land Cover 2000 was used (ETC-LC, 2009). The $100 \mathrm{~m}$ resolution Corine datasets, with an initial 44 land classes, were grouped into six main land cover groups. Elevation was extracted from the SRTM Digital Elevation Database version 4.1 which has a resolution of one arc second (approximately $90 \mathrm{~m}$ ) and a vertical error $<16 \mathrm{~m}$ (CGIAR-CSI, 2013). We additionally obtained $1 \times 1 \mathrm{~km}$ population data for 2011 from Eurostat (EC, 2011).

Both road and land cover databases were intersected with a 100x100 m base polygon and the sum of road length (for 'all' and 'major' roads) and sum of land cover area (for the six grouped land classes) were calculated. The $100 \times 100 \mathrm{~m}$ polygons were converted to grids and a focalsum procedure was applied to calculate these predictor variables for different distances, i.e. "buffers". All potential predictor variables are listed in Table S2, and GIS analysis was conducted in ESRI ArcGIS 10.5.

\subsection{Model development and evaluation}

A two-stage statistical procedure was applied to explain the spatial variation in the measurement data. Firstly, separate standard LUR models were developed based on all measurements for each pollutant. LUR models were developed according to the ESCAPE protocol; i.e. supervised stepwise linear regression as used in our previous paper (de Hoogh et al., 2016). Predictor variables were only allowed to enter the model if they adhered to the predefined direction of effect (see Table S2). For example, road density, an indicator of traffic, has a positive $(+)$ effect in the prediction of $\mathrm{NO}_{2}, \mathrm{PM}_{2.5}$ and $\mathrm{BC}$ and therefore will increase concentrations. Other variables like altitude and natural land will have a negative (-) effect on the same pollutants, and will therefore decrease concentrations. We allowed significant predictor variables to enter the model when they added to the adjusted $\mathrm{R}^{2}$ of the previous model step. Secondly, using the urban and rural background sites only, we explored the remaining broad scale variation in the residuals. Ordinary kriging was applied to the residuals using 
the GSTAT R package (LUR + kriging). If kriging was not successful (i.e. we could not fit a kriging function through the residuals) we offered longitude and/or latitude to the LUR model as additional predictors.

For each pollutant, six LUR models for 2010 were developed. The main model was developed using all sites (FULL). To test the robustness and stability of this model we additionally developed five hold out validation (HOV) models (HOV1, HOV2,..., HOV5), each built on $80 \%$ of the monitoring sites with the remaining $20 \%$ used for validation. Sites were selected into five groups ( $20 \%$ of sites) at random, stratified by site type and country.

HOV was performed after the LUR modeling and after the kriging (when applicable) using the criteria $\mathrm{R}^{2}$ and root mean square error (RMSE). The main model (FULL, developed on all available sites) was evaluated against the 5 HOV samples.

For $\mathrm{PM}_{2.5}$ and $\mathrm{NO}_{2}$ we were able to perform an additional independent comparison with the ESCAPE monitoring datasets. Comparisons were performed at different scales: 1) overall (all ESCAPE sites); 2) overall ELAPSE (ESCAPE sites falling in ELAPSE study areas); and 3) matched to individual ELAPSE study areas (both detailed individual-level and administrative cohorts). Since the BC model was developed using the ESCAPE measurements, no independent comparison was possible.

\subsection{Stability of spatial structure}

In back extrapolation we assume that the spatial structure remains the same going back in time. To investigate the stability of the spatial structure of the models, and to test this assumption, we developed models for $\mathrm{NO}_{2}$ and $\mathrm{O}_{3}$ (2000 and 2005) using the same methods described in section 2.3. For $\mathrm{PM}_{2.5}$ it was not possible to develop models for 2000 and 2005 due to the lack of monitoring data (12 and 165 in 2000 and 2005 respectively), instead we developed a model for 2013 (number of included monitoring sites $=732$ ). The FULL models were mapped at a 100x100 m resolution across the study area and for the different years we visually inspected the spatial patterns.

As we did not have access to cohort geocodes, we created a random point file of 150,000 points across the full rectangular extent of the study area. After intersecting with the study area boundary, approximately 44,000 points remained which was considered a sufficient number to evaluate the stability. These points were intersected with all the raster surfaces: 2010 for $\mathrm{PM}_{2.5}, \mathrm{NO}_{2}$ and $\mathrm{O}_{3}$ (annual, cold season and warm season); 2013 for $\mathrm{PM}_{2.5}$; and 2005 and 2000 for $\mathrm{NO}_{2}$ and $\mathrm{O}_{3}$. Comparisons of model predictions were made for the West-European countries combined and at the national scale reporting $\mathrm{R}^{2}$, RMSE and fractional bias (FB). In addition we calculated population weighted annual means (see equation 1) (Briggs et al., 2007)for $\mathrm{PM}_{2.5}, \mathrm{NO}_{2}$ and $\mathrm{O}_{3}$, using the $1 \mathrm{x} 1 \mathrm{~km}$ GEOSTAT population database (EC, 2011).

Pop-w-exposure $=\Sigma($ pop $\mathrm{x}$ conc $) / \Sigma$ pop (equation 1)

We additionally evaluated the correlation of annual average measurements (plus summer and winter average for $\mathrm{O}_{3}$ ) for those AirBase stations with measurements going sufficiently back in time.

2.5 Population exposure

For 2010, we calculated the total population of West-European countries (based on the GEOSTAT 2011 population grid dataset (EC, 2011)) residing in $\mathrm{PM}_{2.5}$ and $\mathrm{NO}_{2}$ concentration classes.

\section{Results}

\subsection{Air pollution models 2010}

The performance statistics (squared Pearson correlation $\left(\mathrm{R}^{2}\right)$ and RMSE) and model structure of the FULL hybrid models for all pollutants are presented in Table 1 including the LUR component and, 
where applicable, the combined LUR + kriging component. The variograms of the kriging models for $\mathrm{PM}_{2.5}, \mathrm{O}_{3}$ in the warm and cold season are shown in Figure S2. A detailed model description, including constants, coefficients, incremental $\mathrm{R}^{2}$ and RMSE can be found in Table 2 for $\mathrm{PM}_{2.5}$ and the Supplementary material for the other pollutants (Table S3) and years (Table S4). Figure 1 shows the mapped surfaces at a 100x100 m resolution of the FULL models for all pollutants.

\subsection{1 $\mathrm{PM}_{2.5}$ models}

The $\mathrm{PM}_{2.5}$ LUR model developed on all available monitoring sites (FULL) explained $62 \%$ of spatial variation of the measured $\mathrm{PM}_{2.5}$ concentrations (Table 1). Apart from satellite and CTM estimates, the LUR model included altitude, all roads, natural areas, ports and residential area. The satellite variable was the strongest predictor in all models explaining approximately $48 \%$ of the spatial variation in measured $\mathrm{PM}_{2.5}$ concentrations. Comparing the predicted increase in $\mathrm{PM}_{2.5}$ across a change from the $1^{\text {st }}$ to the $99^{\text {th }}$ percentile of each predictor, satellite and $\mathrm{CTM} \mathrm{PM}_{2.5}$ were associated with the largest contrast in $\mathrm{PM}_{2.5}$. The model included large scale predictors (CTM, SAT at 10x10 km) and smallscale road, natural and residential land $(50-200 \mathrm{~m})$ predictors. Kriging increased the explained variation to $72 \%$.

The difference between the calibration and HOV R ${ }^{2}$ of the FULL $\mathrm{PM}_{2.5}$ model was small $(72 \% \mathrm{vs}$ $66 \%$ ) confirming that overfitting was unlikely to be a big problem in the model development (Table 2). Similar predictor variables as in the FULL model were retained in the validation models, with only ports and urban green not always present in each model. Consistently, predictions of the six models (FULL and $5 \mathrm{HOV}$ ) at the 44,000 randomly selected sites were very highly correlated documenting the robustness of the model (Figure S3).

The mapped FULL PM $\mathrm{PM}_{2.5}$ model (see Figure 1) showed predicted levels of $\mathrm{PM}_{2.5}>20 \mu \mathrm{g} / \mathrm{m}^{3}$ in major cities and the Po area (the Po river basin running from the Western Alps to the Adriatic Sea) in Italy. Large parts of Northern Europe had low $\left(<10 \mu \mathrm{g} / \mathrm{m}^{3}\right)$ predicted $\mathrm{PM}_{2.5}$ concentrations.

We tested the three different $\mathrm{PM}_{2.5}$ satellite products in preliminary $\mathrm{PM}_{2.5}$ model development and found that the $0.1^{\circ} \times 0.1^{\circ}$ inferred 2009-2011 product v3.01 produced the best results (see the Supplementary material section 1 and Table S5 for a more detailed description).

\subsection{2 $\mathrm{NO}_{2}$ models}

The FULL $\mathrm{NO}_{2}$ model explained $59 \%$ of the spatial variation (Table 1 and Table S3). In all models the CTM variable was the strongest predictor explaining approximately $29 \%$ of variation in $\mathrm{NO}_{2}$ concentrations, followed by the small $(100-300 \mathrm{~m})$ and larger scale $(2000 \mathrm{~m})$ road variables. All roads, major roads, natural and residential predictor variables consistently appeared in every model. Predictions of the six models (FULL and $5 \mathrm{HOV}$ ) models at the 44,000 randomly selected sites were very highly correlated (Figure S3). None of the variogram models adequately fit the residuals at the $\mathrm{NO}_{2}$ background monitoring sites, nor did including longitude and/or latitude help explain the residuals ( $\mathrm{p}$-value of coefficient not significant). The mapped $\mathrm{NO}_{2}$ estimates (Figure 1) showed more variation compared to $\mathrm{PM}_{2.5}$. Major roads and cities clearly stood out with predicted concentrations generally $>30 \mu \mathrm{g} / \mathrm{m}^{3}$. Away from sources in rural areas, $\mathrm{NO}_{2}$ levels dropped below $15 \mu \mathrm{g} / \mathrm{m}^{3}$.

\subsection{3 $\mathrm{O}_{3}$ models}

Around half of the spatial variation in the annual $\mathrm{O}_{3}$ measurements was explained by the CTM $\left(\mathrm{MACC}-\mathrm{O}_{3}\right)$ variable. Other variables consistently entering all 6 annual models were roads, residential land cover and altitude (Table S3). Ports entered the FULL model and 4 of the 5 HOV models. The CTM was associated with much larger contrast in $\mathrm{O}_{3}$ than the other predictors. Predictions of the 6 models (FULL and $5 \mathrm{HOV}$ ) models at the 44,000 randomly selected sites were very highly correlated (Figure S3). No reliable kriging function could be fit through the residuals of $\mathrm{O}_{3}$ background monitoring sites. However, latitude and longitude variables were fit to the models. The FULL model had a $\mathrm{R}^{2}$ of $65 \%$ (HOV models ranging from 63 to $68 \%$ ). 


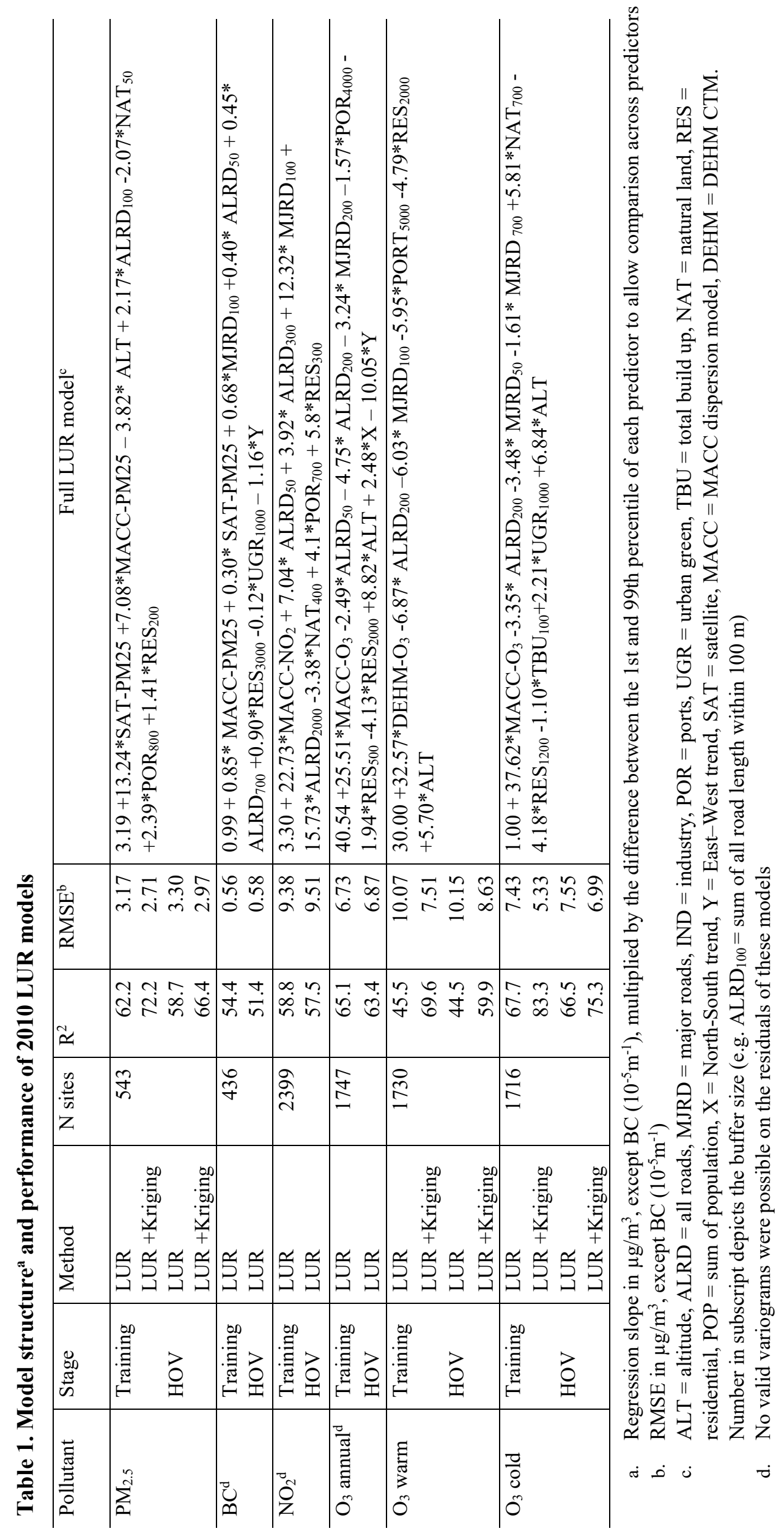


Table 2. Structure and performance of LUR models ${ }^{\text {a }}$ for $P M_{2.5}$ for full dataset and five hold-out validation datasets for 2010

\begin{tabular}{|c|c|c|c|c|c|c|c|}
\hline Theme & Variable $^{\mathrm{b}}$ & FULL $^{\mathrm{c}}$ & HOV1 & HOV2 & HOV3 & HOV4 & HOV5 \\
\hline & (Constant) & 3.19 & 3.46 & 3.53 & 3.14 & 3.49 & 3.32 \\
\hline Satellite & SAT-PM25 & 13.24 & 12.98 & 12.39 & 13.19 & 12.68 & 13.55 \\
\hline CTM & MACC-PM25 & 7.08 & 7.32 & 7.45 & 7.17 & 7.09 & 6.93 \\
\hline Altitude & ALT & -3.82 & -3.82 & -4.10 & -3.93 & -3.54 & -3.73 \\
\hline \multirow[t]{3}{*}{ Roads } & $\mathrm{ALRD}_{100}$ & 2.17 & 2.89 & & 2.23 & 2.00 & \\
\hline & $\mathrm{MJRD}_{50}$ & & & & & & 1.98 \\
\hline & $\mathrm{MJRD}_{100}$ & & & 2.26 & & & \\
\hline \multirow[t]{2}{*}{ Urban green } & $\mathrm{UGR}_{700}$ & & -1.08 & & & & \\
\hline & $\mathrm{UGR}_{800}$ & & & & & -0.98 & \\
\hline \multirow[t]{4}{*}{ Nature } & $\mathrm{NAT}_{50}$ & -2.07 & -2.24 & & & -2.72 & -2.26 \\
\hline & $\mathrm{NAT}_{100}$ & & & -2.31 & -2.12 & & \\
\hline & $\mathrm{NAT}_{300}$ & & & & & & \\
\hline & $\mathrm{NAT}_{400}$ & & & & & & \\
\hline \multirow{4}{*}{$\begin{array}{l}\text { Ports } \\
\text { Residential }\end{array}$} & $\mathrm{POR}_{800}$ & 2.39 & 3.19 & & 2.95 & 2.46 & 2.35 \\
\hline & $\mathrm{RES}_{50}$ & & 0.89 & & & & \\
\hline & $\mathrm{RES}_{200}$ & 1.41 & & 1.72 & 1.44 & 1.48 & \\
\hline & $\mathrm{RES}_{300}$ & & & & & & 1.39 \\
\hline Training & $\mathrm{R}^{2}$ & 62.2 & 62.0 & 63.1 & 61.1 & 60.8 & 66.0 \\
\hline (LUR) & RMSE & 3.17 & 3.26 & 3.10 & 3.30 & 3.22 & 2.95 \\
\hline $\mathrm{HOV}$ & $\mathrm{R}^{2}$ & 58.7 & 62.2 & 53.9 & 67.4 & 68.1 & 50.3 \\
\hline (LUR) & RMSE & 3.30 & 2.93 & 3.67 & 2.68 & 3.01 & 3.94 \\
\hline Training & $\mathrm{R}^{2}$ & 72.2 & 71.4 & 70.5 & 76.8 & 76.0 & 63.3 \\
\hline (LUR + Kriging) & RMSE & 2.71 & 2.55 & 2.94 & 2.26 & 2.61 & 3.38 \\
\hline $\mathrm{HOV}$ & $\mathrm{R}^{2}$ & 66.4 & 67.7 & 66.0 & 72.3 & 74.0 & 57.9 \\
\hline (LUR + Kriging) & RMSE & 2.97 & 2.71 & 3.15 & 2.47 & 2.72 & 3.61 \\
\hline
\end{tabular}

a. Regression slope $\mu \mathrm{g} / \mathrm{m}^{3}$ were multiplied by the difference between the 1 st and 99 th percentile of each predictor to allow comparison across predictors

b. $\quad \mathrm{ALT}=$ altitude, $\mathrm{ALRD}=$ all roads, $\mathrm{MJRD}=$ major roads, $\mathrm{IND}=$ industry, $\mathrm{POR}=$ ports, $\mathrm{UGR}=$ urban green, $\mathrm{TBU}=$ total build up, NAT $=$ natural land, $\mathrm{RES}=$ residential, $\mathrm{POP}=$ sum of population, $\mathrm{X}=$ North-South trend, Y = East-West trend, SAT = satellite, MACC = MACC dispersion model, DEHM = DEHM CTM. Number in subscript depicts the buffer size (e.g. ALRD ${ }_{100}=$ sum of all road length within $100 \mathrm{~m}$ )

c. FULL refers to all sites; HOV1 is first holdout validation dataset ( $80 \%$ stratified random sample) 


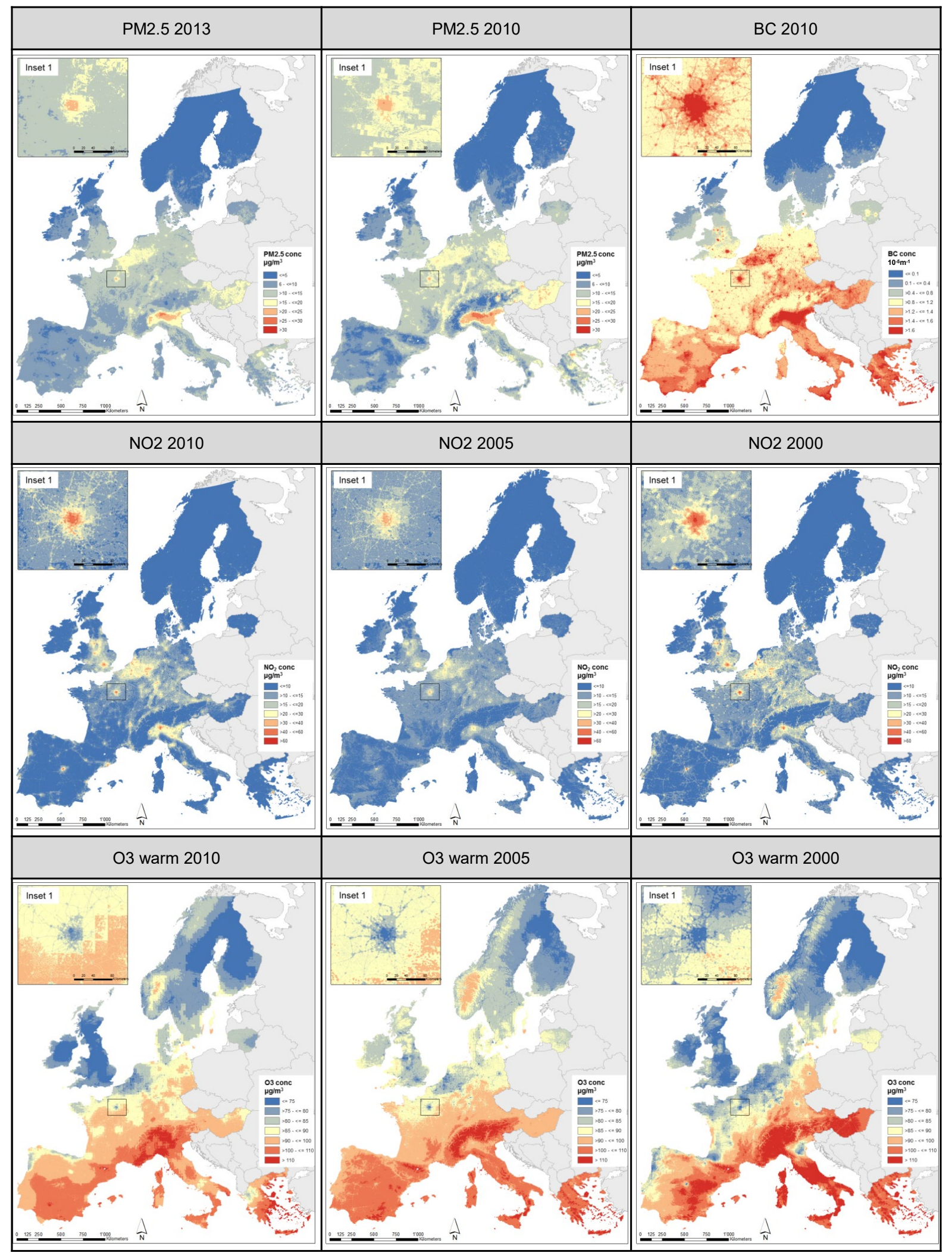

Figure 1. Mapping of hybrid West European LUR models for $\mathrm{PM}_{2.5}, \mathrm{BC}, \mathrm{NO}_{2}$ and $\mathrm{O}_{3}$ warm season at $100 \times 100 \mathrm{~m}\left(\mu \mathrm{g} / \mathrm{m}^{3}, \mathrm{BC} 10^{-5} \mathrm{~m}^{-1}\right)$. 
Like the annual $\mathrm{O}_{3}$ model, the cold season $\mathrm{O}_{3}$ model was dominated by the MACC predictor variable, explaining nearly $60 \%$ of the spatial variation in measured $\mathrm{O}_{3}$ concentrations. Roads, residential land and altitude variable entered in all 6 cold season models. Kriging explained, on average, an additional $16 \%$ of the spatial variation, bringing the final performance of the $\mathrm{FULL} \mathrm{O}_{3}$ cold model to $83 \%(80 \%$ to $85 \%$ for the 5 validation models).

The $\mathrm{O}_{3}$ warm season models also contained a CTM variable, but unlike the annual and cold season $\mathrm{O}_{3}$ models where the annual MACC CTM variable entered, here the warm season DEHM CTM variable was the stronger predictor. Other variables entering in all models were roads, ports, residential land and altitude. The performance of LUR models was moderate ( $\mathrm{R}^{2}$ ranging from 44 to $48 \%$ ) but with additionally fitted kriging functions, we increased the explained variation to $70 \%$ for the FULL model (67\% to $73 \%$ for the 5 validation models).

Maps of the FULL $\mathrm{O}_{3}$ models (Figure 1 and S4) showed similar general patterns for annual and cold season, with the highest predicted $\mathrm{O}_{3}$ concentrations in Southern Europe and lower concentrations in more central areas (England, the Netherlands, Germany and northern Italy). Areas of high altitude also tended to have higher predicted $\mathrm{O}_{3}$ levels compared areas of lower altitudes. Predicted $\mathrm{O}_{3}$ concentrations for the warm season showed a somewhat different spatial pattern with a much clearer negative North-South gradient than the cold season model.

\subsubsection{BC models}

For the FULL BC LUR model we achieved an explained variation of 54\% (FULL model) and between 52 and $57 \%$ for the 5 HOV models (Table 1, Table S3). For all 6 models, the CTM MACC$\mathrm{PM}_{2.5}$ contributed 24 to $30 \%$ of the explained spatial variation. Roads, $\mathrm{PM}_{2.5}$ SAT estimates, urban green land, residential land and natural land were also included consistently in FULL and HOV models. Predictions of the 6 (FULL and 5 HOV) models at the 44,000 randomly selected sites were very highly correlated (Figure S3). The BC model included large contributions from large-scale predictors (CTM PM 2.5 , Y-coordinate and residential density) and small-scale predictors (roads and residential density).

Due to the clustered nature of the $\mathrm{BC}$ monitoring data it was not possible to perform kriging. Latitude was best able to explain the residuals.

When mapped across Western Europe (Figure 1), BC predicted concentrations showed a distinct North - South division, with low $\left(<=0.810^{-5} \mathrm{~m}^{-1}\right) \mathrm{BC}$ concentrations in Scandinavia and the north of the UK, and higher $>0.810^{-5} \mathrm{~m}^{-1}$ in the rest of Western Europe. Mediterranean Europe had the highest concentration $>1.210^{-5} \mathrm{~m}^{-1}$. Traffic sources were also clearly identifiable in the inset with major roads visible around Paris.

\subsection{Comparison at ESCAPE sites}

We performed an independent external comparison for $\mathrm{PM}_{2.5}$ and $\mathrm{NO}_{2}$ FULL models using measured concentration data from the ESCAPE study. Table 3 shows the correlations at different scales including the mean and standard deviation of measured concentrations at the ESCAPE measurement sites.

The $\mathrm{PM}_{2.5}$ FULL model explained $65 \%$ of variance overall $(\mathrm{n}=416)$ with a small fractional bias $(\mathrm{FB}=$ $-2 \%$ ). The explained variance is almost identical to the HOV $\mathrm{R}^{2}$ of $66 \%$ (Table 1). Restricting the analysis to the overall area with ELAPSE cohorts $(n=255)$ led to a slight decrease in the explained variance $(59 \%)$ and a small overestimation $(\mathrm{FB}=-10 \%)$. The comparison at each ELAPSE study areas separately (detailed individual-level and administrative cohorts) revealed a large range in the explained variation, $8 \%$ for EPIC Oxford and English administrative cohort to $66 \%$ for HNR, also with the FB varying from -2 to $-30 \%$. We note that the number of sites is relatively small for the individual area comparisons. 
$\mathrm{NO}_{2}$ FULL models also showed reasonable associations for overall $(49 \%)$ and overall ELAPSE (46\%). The explained variance was modestly lower than the HOV R ${ }^{2}$ of $57 \%$ (Table 1). FB indicated a small overestimation of $13 \%$ for the ELAPSE overall area. At the ELAPSE detailed individual-level cohorts the correlations for $\mathrm{NO}_{2}$ were generally better than for $\mathrm{PM}_{2.5}$ : all were $>47 \%$ except for HUBRO (7\%) and EPIC VARESE (34\%). FB showed overestimation for all areas, except for ELAPSE areas in Italy.

Table 3. Comparison of $\mathrm{PM}_{2.5}$ and $\mathrm{NO}_{2}$ ELAPSE models at ESCAPE monitoring sites

\begin{tabular}{|c|c|c|c|c|c|c|c|}
\hline & \multirow{2}{*}{$\begin{array}{l}\text { Pollutant } \\
\text { Name ESCAPE area }\end{array}$} & \multicolumn{5}{|c|}{$\mathrm{PM}_{2.5}$} & \multirow[b]{2}{*}{$\mathrm{N}^{\mathrm{b}}$} \\
\hline & & $\mathrm{R}^{2}$ & RMSE & $\mathrm{FB}^{\mathrm{a}}$ & $\begin{array}{l}\text { Measu } \\
\text { Mean }\end{array}$ & $\begin{array}{l}\text { its } \\
\text { SD }\end{array}$ & \\
\hline Overall & & 64.8 & 3.41 & -0.02 & 15.86 & 5.73 & 416 \\
\hline Overall ELAPSE & & 58.7 & 2.85 & -0.10 & 14.16 & 4.43 & 255 \\
\hline \multicolumn{8}{|l|}{ ELAPSE cohorts } \\
\hline HUBRO & Oslo, NO & 18.4 & 2.04 & -0.30 & 8.59 & 2.20 & 19 \\
\hline CEANS & Stockholm County, SE & 39.0 & 1.32 & -0.04 & 8.29 & 1.64 & 19 \\
\hline $\mathrm{DCH}$ & Copenhagen, DK & 40.1 & 1.26 & -0.18 & 11.12 & 1.58 & 20 \\
\hline EPIC-NL & NL & 12.6 & 1.71 & -0.02 & 17.35 & 1.80 & 34 \\
\hline EPIC OXFORD & London- Oxford, Manchester, UK & 7.6 & 2.23 & -0.26 & 10.55 & 2.29 & 39 \\
\hline HNR & Ruhr Area, GER & 65.5 & 0.97 & -0.06 & 18.52 & 1.61 & 20 \\
\hline KORA & Munich-Augsburg, GER & 31.5 & 1.44 & -0.16 & 14.34 & 1.70 & 20 \\
\hline VHM\&PP & Vorarlberg, AU & 22.4 & 1.74 & -0.19 & 13.34 & 1.92 & 20 \\
\hline E3N & Paris, FR & 38.7 & 3.30 & -0.24 & 16.02 & 4.10 & 20 \\
\hline EPIC VARESE & n.a. & - & - & - & - & - & - \\
\hline DNC & n.a. & - & - & - & - & - & - \\
\hline \multicolumn{8}{|c|}{ Administrative ELAPSE cohorts } \\
\hline Dutch & NL & 12.6 & 1.71 & -0.02 & 17.35 & 1.80 & 34 \\
\hline English & London- Oxford, Manchester, UK & 7.6 & 2.23 & -0.26 & 10.55 & 2.29 & 39 \\
\hline Rome & Rome, IT & 43.0 & 2.51 & 0.16 & 19.77 & 3.24 & 20 \\
\hline Danish & n.a. & - & - & - & - & - & - \\
\hline \multicolumn{8}{|l|}{ Norwegian } \\
\hline Swiss $^{c}$ & Lugano. $\mathrm{CH}$ & - & - & - & - & - & \\
\hline \multirow[t]{4}{*}{ Belgian $^{c}$} & Antwerp, BE & - & - & - & - & - & \\
\hline & Pollutant & $\mathrm{NO}_{2}$ & & & & & \\
\hline & & & & & Measu & & \\
\hline & ESCAPE area & $\mathrm{R}^{2}$ & RSME & FB & Mean & SD & $\mathrm{N}$ \\
\hline Overall & & 49.4 & 11.47 & -0.08 & 29.32 & 16.12 & 1396 \\
\hline Overall ELAPSE & & 45.8 & 10.28 & -0.13 & 29.74 & 13.95 & 780 \\
\hline \multicolumn{8}{|l|}{ ELAPSE cohorts } \\
\hline HUBRO & Oslo, NO & 7.0 & 12.74 & -0.19 & 24.29 & 13.05 & 39 \\
\hline CEANS & Stockholm County, SE & 55.0 & 5.03 & -0.50 & 15.49 & 7.44 & 39 \\
\hline $\mathrm{DCH}$ & Copenhagen, DK & 59.0 & 5.99 & -0.54 & 17.82 & 9.21 & 41 \\
\hline EPIC-NL & NL & 75.9 & 5.10 & -0.26 & 28.76 & 10.32 & 68 \\
\hline EPIC OXFORD & London -Oxford, Manchester, Bradford, UK & 53.9 & 8.64 & -0.17 & 29.82 & 12.67 & 119 \\
\hline HNR & Ruhr Area, GER & 54.0 & 6.74 & -0.20 & 33.16 & 9.76 & 40 \\
\hline KORA & Munich-Augsburg, GER & 64.0 & 5.79 & -0.13 & 26.82 & 9.58 & 40 \\
\hline VHM\&PP & Vorarlberg, AU & 47.0 & 5.29 & -0.10 & 22.59 & 7.17 & 40 \\
\hline $\mathrm{E} 3 \mathrm{~N}$ & Paris, Grenoble, Lyon, Marseille, FR & 52.6 & 12.37 & -0.01 & 34.42 & 17.90 & 160 \\
\hline EPIC VARESE & Varese, IT & 34.0 & 13.78 & 0.10 & 36.53 & 16.54 & 20 \\
\hline $\mathrm{DNC}$ & n.a. & - & - & - & - & - & - \\
\hline \multicolumn{8}{|c|}{ Administrative ELAPSE cohorts } \\
\hline Dutch & NL & 75.9 & 5.10 & -0.26 & 28.76 & 10.32 & 68 \\
\hline English & London-Oxford, Manchester, Bradford, UK & 53.9 & 8.64 & -0.17 & 29.82 & 12.67 & 119 \\
\hline Rome & Rome, IT & 51.0 & 9.72 & 0.23 & 42.64 & 13.71 & 40 \\
\hline Danish & n.a. & - & - & - & - & - & - \\
\hline Norwegian & n.a. & - & - & - & - & - & - \\
\hline Swiss & Basel, Geneva, Lugano. CH & 13.7 & 7.55 & -0.16 & 30.03 & 8.09 & 121 \\
\hline Belgian $^{c}$ & Antwerp, BE & - & - & - & - & - & \\
\hline
\end{tabular}

\footnotetext{
a. $\quad \mathrm{FB}=$ Fractional Bias calculated as $2 *$ (mean observations - mean predictions $) /($ mean observations + mean predictions)

b. $\quad \mathrm{N}=$ number of ESCAPE monitoring sites (the same for black carbon and $\mathrm{PM}_{2.5}$ )

c. Covers only a small part of the area, with insufficient number of sites
} 


\title{
3.3 Air pollution models for different time periods and stability analysis
}

\author{
3.3.1 Models for 2000, $2005\left(\mathrm{NO}_{2}\right.$ and $\left.\mathrm{O}_{3}\right)$ and $2013\left(\mathrm{PM}_{2.5}\right)$
}

The performance statistics of the $\mathrm{PM}_{2.5}, \mathrm{NO}_{2}$ and $\mathrm{O}_{3}$ models for different years are presented in Table S4. The $2013 \mathrm{PM}_{2.5}$ LUR models explained $64 \%$ of spatial variation in the $\mathrm{PM}_{2.5}$ measurements. The LUR models had some similarities with the 2010 models, with MACC, SAT, roads and natural land entering all models. Neither reliable kriging models nor longitude/latitude variables improved the models.

No $\mathrm{NO}_{2}$ MACC CTM estimates were available for the years 2000 and 2005, so only DEHM $\mathrm{NO}_{2}$ for 2000 and 2005 estimates were offered to the $\mathrm{NO}_{2}$ model development. Otherwise the $\mathrm{NO}_{2}$ models showed a similar structure with the $2010 \mathrm{NO}_{2}$ LUR models (CTM, roads, natural land, residential land and ports in all models), but performed slightly less well $\left(\mathrm{R}^{2} \mathrm{NO}_{2} 2000=56 \% ; \mathrm{R}^{2} \mathrm{NO}_{2} 2005=52 \%\right)$.

$\mathrm{O}_{3}$ models for 2000 and 2005 were able to respectively explain $60 \%$ and $49 \%$ (annual), 82 and $42 \%$ (warm season), 52 and $70 \%$ (cold season) of the variation in measured concentrations. The 2000 and 2005 annual and warm $\mathrm{O}_{3}$ models contained DEHM CTM variables whereas no DEHM variable entered the cold season models. Kriging models explained an additional $\sim 25 \%$ of spatial variation in the 2000 warm season and the 2005 cold season models. Latitude and longitude variables were entered to the other models.

Figure 1 shows the maps of $\mathrm{PM}_{2.5}(2013,2010), \mathrm{NO}_{2}$ and $\mathrm{O}_{3}$ warm season $(2010,2005,2000)$. Similar patterns over multiple years were observed with, for example, high predicted $\mathrm{PM}_{2.5}$ concentrations for both 2010 and 2013 in the Po valley in North Italy and low PM $_{2.5}$ concentrations in Scandinavia. Spatial patterns in the $\mathrm{NO}_{2}$ and $\mathrm{O}_{3}$ concentrations maps for the 3 years also appeared broadly similar.

\subsubsection{Comparison of model predictions for Western Europe across years}

Table 4 (and Figure S5) shows the results of the stability tests at country level. Agreement in spatial variation was generally high at the overall EU country and combined ELAPSE country level $(>76 \%)$ for all comparisons, except for the $\mathrm{O}_{3}$ cold season surface (44\% when 2000 model compared to 2010). At the national level, focusing on ELAPSE countries only, we observed some heterogeneity in the associations. Both 2000 and $2005 \mathrm{NO}_{2}$ surfaces showed a high agreement with the $2010 \mathrm{NO}_{2}$ surface (all ELAPSE countries $>80 \%$ ). The agreement between $\mathrm{PM}_{2.5}$ surfaces developed for 2010 and 2013 showed more variability, with four ELAPSE countries $>80 \%$ (UK, Sweden, Belgium and Italy), the Netherlands $70 \%$ and the rest between 48 and $60 \%$. There was a high variability between the associations of the different $\mathrm{O}_{3}$ surfaces. The agreement between $\mathrm{O}_{3}$ annual surfaces of 2000 and 2005 with 2010 was reasonable, all ELAPSE countries had $>60 \%$ explained spatial variability, with the exception of Sweden (2000) with 45\%. Except for the $2005 \mathrm{O}_{3}$ cold (all ELAPSE countries $>60 \%$ ), the $\mathrm{O}_{3}$ cold and warm season surfaces were less stable over time with large ranges of explained spatial variability. Italy performed poorly with $1.6 \%, 11.9 \%$ and $16.6 \%$ for respectively 2000 warm season, 2005 warm season and 2000 cold season (combined with the largest RMSE's).

NUTS areas are standard administrative divisions of EU countries for statistical purposes. We performed the stability analysis using the same 44,000 random points at the NUTS1 area level (see Figure S6) to gain a better understanding of the stability at the sub-national level. Similar to the national level, there was a good agreement for all areas for $\mathrm{NO}_{2} 2000$ and 2005 when compared to the 2010 surface $\left(\mathrm{R}^{2}>0.60\right)$. For more details see the Supplementary material section 2 .

\subsubsection{Comparison of measurements}

We additionally evaluated the relationship between measured average concentrations for those AirBase stations with measurements going sufficiently back in time between 2010 to 2005 and 2000 (Table 5). In Western Europe the measured concentrations between the different years yielded high 


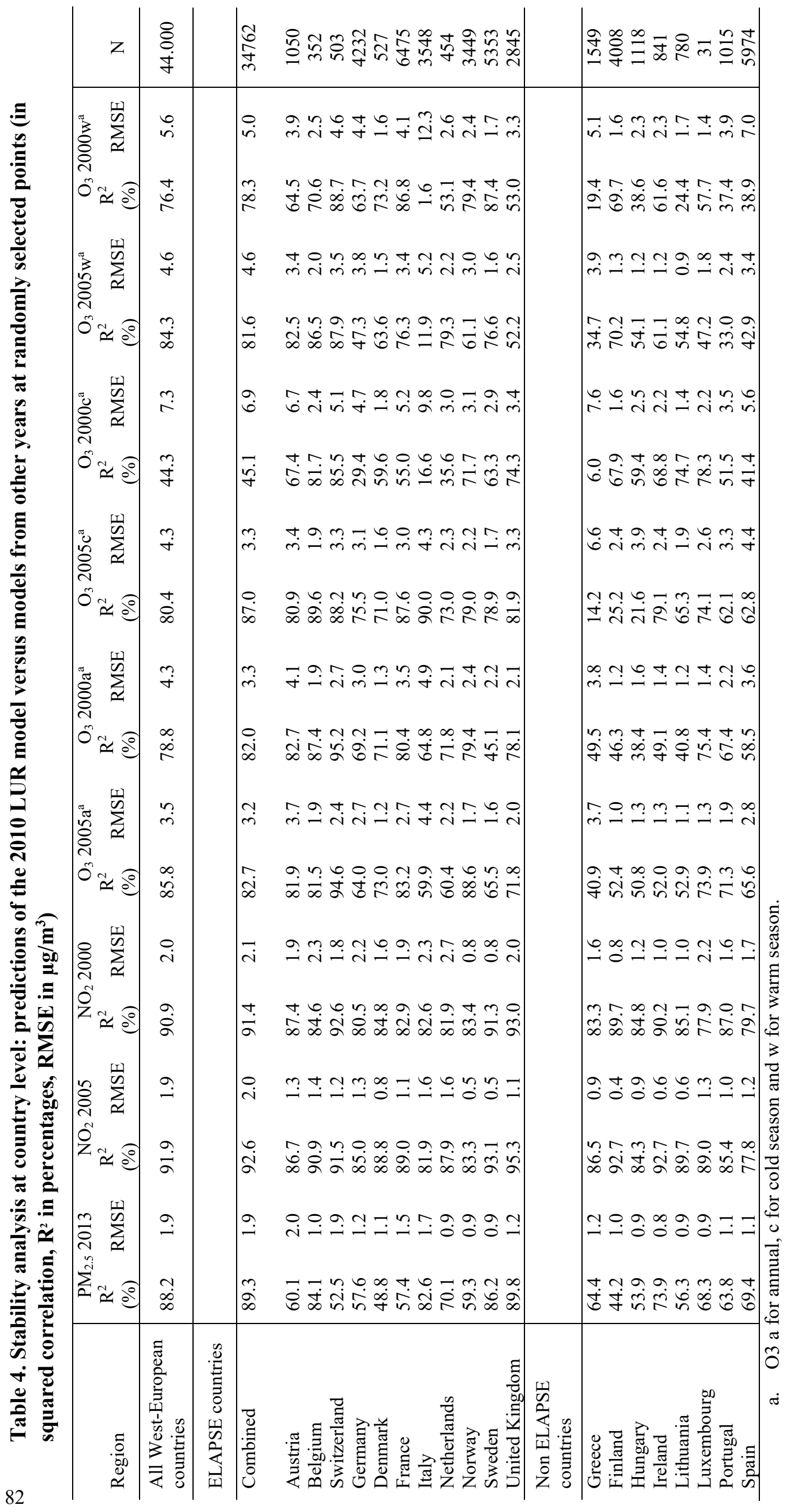




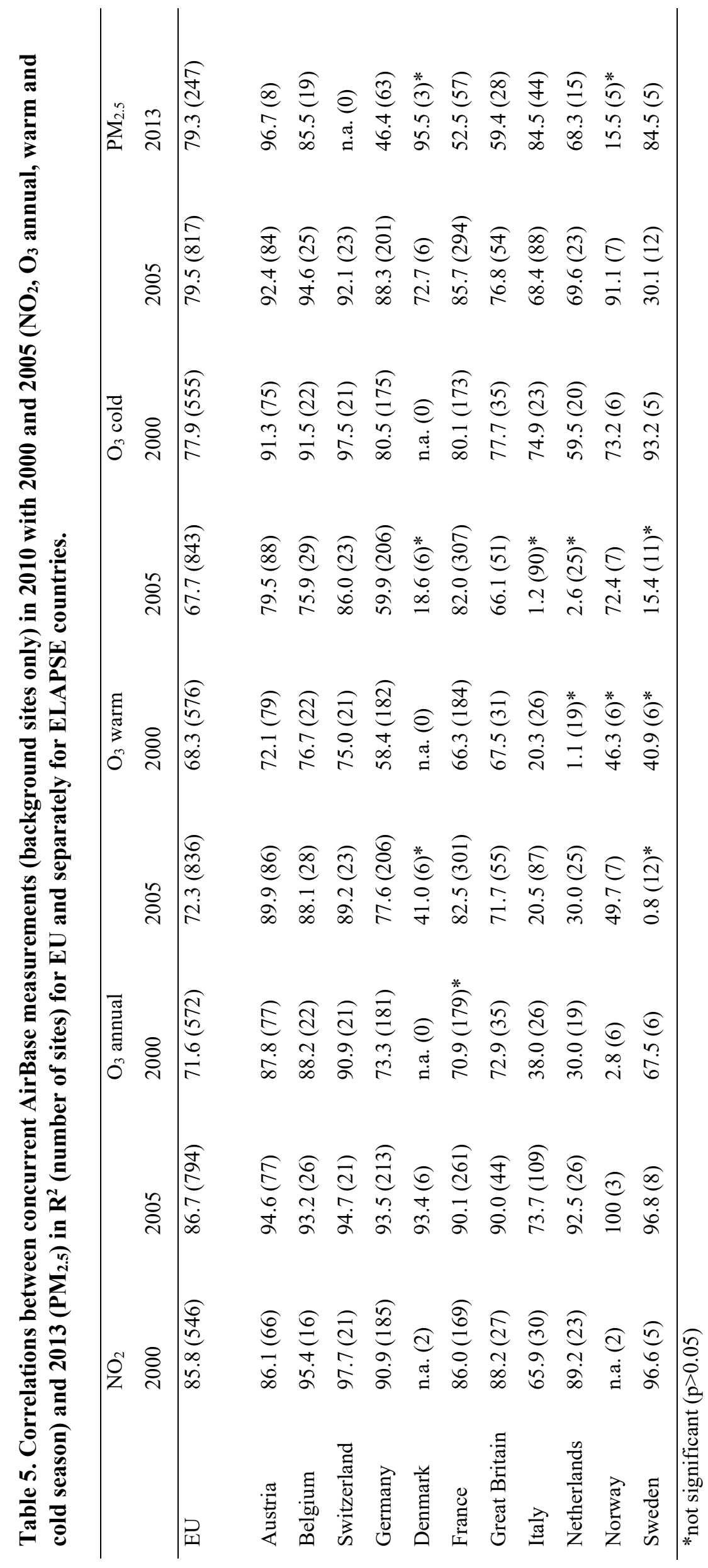


correlations. When focusing on ELAPSE participating countries, high correlations were also observed for the majority of the countries and years.

\subsection{Population exposure}

Based on our modelled concentrations (FULL models), a respective 8 million (2\%) and 371 million (89\%) people live in areas with estimated $\mathrm{PM}_{2.5}$ concentrations greater than the EU annual $\mathrm{PM}_{2.5}$ limit value of $25 \mu \mathrm{g} / \mathrm{m}^{3}$ and the WHO annual guideline of $10 \mu \mathrm{g} / \mathrm{m}^{3} .32$ million (8\%) of people live in areas with modelled $\mathrm{NO}_{2}$ concentration greater than the $\mathrm{EU}$ and $\mathrm{WHO}$ annual $\mathrm{NO}_{2}$ guideline of $40 \mu \mathrm{g} / \mathrm{m}^{3}$ (see Table S6). Table S7 shows that population weighted concentration levels across the whole of our study area do not drastically fluctuate over time and are generally low $\left(\mathrm{PM}_{2.5} \sim 11 \mu \mathrm{g} / \mathrm{m}^{3}\right.$ and $\mathrm{NO}_{2}<$ $\left.20 \mu \mathrm{g} / \mathrm{m}^{3}\right)$.

\section{Discussion}

We developed West-European LUR models at a 100x100 m spatial scale for four priority pollutants. The models including large scale satellite data and CTM and small-scale traffic and land use predictors explained between $54 \%(\mathrm{BC})$ and $83 \%\left(\mathrm{O}_{3}\right.$ cold season) of the measured variability in concentrations. The explained variance at fully independent sites was only slightly less than the internal hold-out validation: $65 \%$ vs $66 \%$ for $\mathrm{PM}_{2.5}$ and $49 \%$ vs $57 \%$ for $\mathrm{NO}_{2}$. Predictions from the 2010 model correlated highly with models developed for 2000 and 2005 (2013 for $\mathrm{PM}_{2.5}$ ) at the overall European scale, with squared correlations larger than 76\%, except for the $\mathrm{O}_{3}$ cold season of 2000 (44\%). The temporal correlation was more variable when evaluated at the country and especially at the NUTS1 level. Correlations between measured concentrations at the EU level between 2010 2005 and $2010-2000$ for $\mathrm{NO}_{2}$ and $\mathrm{O}_{3}\left(\mathrm{R}^{2}\right.$ between 68\% to 87\%) and for $\mathrm{PM}_{2.5} 2010-2013\left(\mathrm{R}^{2} 79 \%\right)$ were even higher than modeled concentrations. Based on our modelled surfaces, 371 million and 32 million people in Western Europe live in areas with air pollution levels exceeding the WHO annual guidelines for $\mathrm{PM}_{2.5}$ and $\mathrm{NO}_{2}$ respectively.

\subsection{Interpretation of 2010 models}

$\mathrm{PM}_{2.5}$ SAT and CTM available at a $10 \times 10 \mathrm{~km}$ scale were the strongest predictors in the $\mathrm{PM}_{2.5}$ models, consistent with $\mathrm{PM}_{2.5}$ being a largely regionally varying pollutant. Eeftens et al. (2012a) reported that $81 \%$ of the variability in the ESCAPE annual average $\mathrm{PM}_{2.5}$ concentrations was due to between study area contrast. The modest contrast related to the small-scale road variable is consistent with the overall mean ratio of 1.14 comparing traffic and background sites within ESCAPE (Eeftens et al., 2012a). Roads, ports and residential areas represent the contribution of local sources, with altitude, and nature/urban green representing pollution sinks. Applying kriging to the residuals of the LUR model explained an extra $10 \%$ of the variation, suggesting that the SAT and CTM predictors did not fully capture the large scale variation of $\mathrm{PM}_{2.5}$ across Europe. Alternatively, the number of sites was insufficient to train the model. Kriging was not feasible for the 2013 model, possibly due to the larger number of sites.

In the BC models, satellite and CTM PM 2.5 also contributed strongly, raising potential concerns when applying the $\mathrm{PM}_{2.5}$ and $\mathrm{BC}$ models in the epidemiological analysis as it might be difficult to tease apart their respective contribution to health effects. Compared to the $\mathrm{PM}_{2.5}$ models, small-scale road predictors contributed more to the BC prediction. The FULL model contained three road variables with a similar magnitude to the CTM and SAT predictors. This is consistent with the observation in ESCAPE that $52 \%$ of the variability was due to within-study area variability (Eeftens et al., 2012a). The overall ratio of $\mathrm{BC}$ concentrations measured at traffic/urban background sites was 1.38 (Eeftens et al., 2012a). The residuals of our initial model showed a clear north-south gradient, which was captured by a Y-coordinate in the model, documenting that the models did not predict the large scale contrast of BC across Europe sufficiently. MACC and satellites do not represent BC, whereas DEHM 
modelled $\mathrm{BC}$ at a larger scale $(50 \times 50 \mathrm{~km}$ scale). It is likely that limitations in emission data for $\mathrm{BC}$ may have impacted the performance of the models.

After the CTM predictor variable, small-scale road variables were the strongest predictors in the $\mathrm{NO}_{2}$ models. Motorized traffic is a dominant source of local $\mathrm{NO}_{2}$ concentrations, as illustrated by the overall ratio of 1.63 for concentrations measured at traffic vs. urban background ESCAPE monitoring sites (Cyrys et al., 2012). In ESCAPE, $60 \%$ of the variability of $\mathrm{NO}_{2}$ was due to within-study area variability (Cyrys et al., 2012). The $\mathrm{NO}_{2}$ models could not be further improved by kriging or geographical coordinates, suggesting that the CTM adequately captured the large scale variation across Europe. We previously suggested that CTM's were better developed for $\mathrm{NO}_{2}$ than for $\mathrm{PM}_{2.5}$ when discussing the contribution of CTM and SAT to $\mathrm{PM}_{2.5}$ and $\mathrm{NO}_{2}$ LUR models (de Hoogh et al., 2016).

In $\mathrm{O}_{3}$ models, CTM (the ensemble MACC for the annual and cold period and DEHM for the warm season) were the dominant predictor variables, consistent with $\mathrm{O}_{3}$ being a regional pollutant. The model further predicted higher concentrations at higher altitude, in accordance with a previous European LUR model (Beelen et al., 2009). Predicted lower concentrations near roads was consistent with scavenging of $\mathrm{O}_{3}$ by $\mathrm{NO}_{2}$. In both the warm and cold season, kriging substantially improved the models, likely illustrating limitations in the CTM. Kriging did not contribute to the annual model, possibly because the annual average combined the two different spatial patterns of the cold and warm seasons.

Few studies have combined LUR and kriging in air pollution models. Young et al. (2016) evaluated the additional value of satellite data and/or kriging on $\mathrm{NO}_{2}$ LUR models across the USA for 1990 2012. Models with both satellite data and kriging performed best, increasing the average crossvalidation $\mathrm{R}^{2}$ from 0.72 (just applying LUR) to 0.85 . Satellite or kriging alone yielded respective average $\mathrm{R}^{2}$ 's of 0.81 and 0.84 . Although we found improvement of model performance with kriging for the $\mathrm{PM}_{2.5}$ and $\mathrm{O}_{3}$ models, we did not see the same result in our $\mathrm{NO}_{2}$ models. This might be due to the difference in scale of the two studies. Young et al. (2016) estimated $\mathrm{NO}_{2}$ concentrations at a $25 \mathrm{x}$ $25 \mathrm{~km}$ resolution, thereby not explaining intra-urban variation but rather focusing on more regional background. This study operates at a much smaller resolution $(100 \times 100 \mathrm{~m})$ and, at least for $\mathrm{NO}_{2}$, the residual concentrations after LUR were too variable, even at background sites, for reliable kriging functions. In a previous study distinguishing global, regional and urban scales, universal kriging improved PM10, $\mathrm{O}_{3}$ and $\mathrm{NO}_{2}$ European models compared to regression models (Beelen et al., 2009). In that study, the analysis was based on $1 * 1 \mathrm{~km}$ estimates.

Relatively few studies have tested the robustness by developing HOV models and assessing the structure of the models. Johnson et al. (2010) evaluated $\mathrm{PM}_{2.5}, \mathrm{NO}_{\mathrm{x}}$ and benzene LUR models in New Haven, CT, USA by including hold-out validation using varying sizes of training/testing groups. van Nunen et al. (2017) performed a 10-fold cross validation when developing UFP LUR models in six study European areas. We observed that the model predictions from our FULL model correlated very highly with the $5 \mathrm{HOV}$ models at the 44,000 independent sites, suggesting that the developed models were robust. The correlations in our study were higher than that observed for the UFP models based on short-term monitoring at 160 sites in some of the cities (van Nunen et al., 2017).

\subsection{Comparison with other European models}

Previously we published the development of hybrid $\mathrm{PM}_{2.5}$ and $\mathrm{NO}_{2}$ LUR models for the same study area, showing that satellite-derived (SAT) estimates and CTM estimates contribute considerably to the explained variance in $\mathrm{PM}_{2.5}$ and $\mathrm{NO}_{2}$ measurements (de Hoogh et al., 2016). The models presented in this paper confirm our previous findings. Moreover, by additionally including kriging to explain residuals at background monitoring sites, we improved the $\mathrm{PM}_{2.5}$ hybrid models from 62 to $72 \%\left(\mathrm{R}^{2}\right)$. This improvement was also observed when tested using the independent ESCAPE monitoring dataset, showing an improvement from 53 to $65 \%\left(\mathrm{R}^{2}\right)$. For $\mathrm{NO}_{2}$ models, where the inclusion of longitude 
explained some of the residuals, the $\mathrm{R}^{2}$ remained the same (both 58\%); but the improved $\mathrm{NO}_{2}$ model described here yielded a higher independent validation $\left(\mathrm{R}^{2}\right)$ of $49 \%$ compared to $43 \%$ in de Hoogh et al. (2016). Additionally we evaluated the performance of SAT and CTM derived estimates by comparing monitored AIRBASE data and satellite derived $\mathrm{PM}_{2.5}\left(\mathrm{R}^{2}=0.48\right)$ and $\mathrm{NO}_{2}\left(\mathrm{R}^{2}=0.13\right)$ and CTM PM $2.5\left(\mathrm{R}^{2}=0.41\right)$ and $\mathrm{NO}_{2}\left(\mathrm{R}^{2}=0.29\right)$. SAT and CTM (MACC) surfaces explain less of the measured spatial variation than when these datasets are used within a hybrid LUR framework as presented in this paper.

Vienneau et al. (2013) also developed European $\mathrm{NO}_{2}$ and $\mathrm{PM}_{10}$ LUR models, for 2005-2007, showing that the inclusion of satellite data substantially improved model performance. The $\mathrm{NO}_{2}$ model explained a comparable fraction of the variation (46-56\%) to our models. The CTM predictor outperformed the satellite data in our $\mathrm{NO}_{2}$ model, a predictor variable not available in the study by Vienneau et al. (2013).

To date few studies have attempted to model pollutants other than $\mathrm{NO}_{2}$ and PM. European $\mathrm{O}_{3} \mathrm{LUR}$ models have been previously developed by Beelen et al. (2009) for the year 2001 at the global $\left(\mathrm{R}^{2}=\right.$ $0.53)$, rural $\left(\mathrm{R}^{2}=0.63\right)$ and urban $\left(\mathrm{R}^{2}=0.06\right)$ scale. Our annual $\mathrm{O}_{3}$ model performance for 2000 yielded a higher $\mathrm{R}^{2}(0.63)$ possibly due to the inclusion of DEHM estimates in our model. In addition we further developed seasonal $\mathrm{O}_{3}$ models.

\subsection{Application of 2010 models in epidemiological studies}

The models developed and described here will be used for the exposure assessment in ELAPSE for 7 administrative cohorts and a pooled cohort comprising of 11 local cohorts across 11 countries in Europe (Norway, Sweden, Denmark, United Kingdom, the Netherlands, Belgium, Germany, France, Switzerland, Austria and Italy). For the pooled cohort, the (moderately) high explained variance in hold-out validation and external validation over the full area suggests that exposure assessment is robust. For individual cohorts, comparison with ESCAPE data in the respective study areas showed more variable results, especially for $\mathrm{PM}_{2.5}$. This implies that our West European model should be applied with caution in a small area (part of a country) unless local validation is possible. The difference between $\mathrm{NO}_{2}$ and $\mathrm{PM}_{2.5}$ could be due to the relatively small number of sites for $\mathrm{PM}_{2.5}$ and the smaller contrast in $\mathrm{PM}_{2.5}$ within cohorts compared to $\mathrm{NO}_{2}$.

For the administrative cohorts, direct comparisons of the Dutch, Rome and to some extent national English and Swiss $\left(\mathrm{NO}_{2}\right.$ only) study areas with the ESCAPE data are possible due to overlaps between the ESCAPE and ELAPSE study areas/regions. The West European ELAPSE models explained variation well, except for $\mathrm{PM}_{2.5}$ in the Netherlands (possibly due to small variation) and $\mathrm{NO}_{2}$ in Switzerland. The findings for Switzerland do not directly apply to the Swiss cohort, as the evaluation was limited to three cities whereas the Swiss cohort includes the entire population including those in rural and Alpine areas. We have no ready explanation for these findings, and can only speculate that a more locally generated model may better capture area-specific small-scale concentration differences than a pan-European model, which tends to smooth intra-urban differences over several very different study areas.

\subsection{Spatial stability of models and measurements over time}

This is one of the few studies which has tested the stability of spatial structure of air pollution exposure models at a continental scale, by developing models for different time points and comparing the respective estimates. Most studies evaluated LUR models at a national or sub-national scale by linear regression using historical monitoring data, allowing the constant and coefficient to change (Cesaroni et al., 2012; Chen et al., 2010; Eeftens et al., 2011; Gulliver et al., 2013; Gulliver and de Hoogh, 2015; Levy et al., 2015). Gulliver et al. (2016), however, produced separate $\mathrm{NO}_{2}$ LUR models for 1991 and 2009 for the UK and found that the year-specific 1991 model yielded similar exposures as the back-extrapolated 2009 model. Wang et al. (2013) developed $\mathrm{NO}_{2}$ LUR models for 2003 and 2010 for Vancouver, Canada, and when applied to measurements of the other year were able to 
explain 52 to $61 \%$ (2003 model to 2010 measurements) and 44 to $49 \%$ (2010 model to 2003 measurements) of the spatial variation. These studies suggest that the spatial structure of the different models were similar, at least at a national or city level. It is difficult to compare the findings of the analyses carried out in this study with the studies conducted at the sub-continental scale. In this study we specifically assessed the stability of the spatial structure by comparing the concentration surfaces of the different models based on a set of $\sim 44,000$ random points spread across the study area. At the EU scale (all countries combined and ELAPSE countries combined) there was a high squared correlation $(>76 \%)$ between the other year models $\left(\mathrm{PM}_{2.5} 2013, \mathrm{NO}_{2}\right.$ and $\left.\mathrm{O}_{3} 2000,2005\right)$ and the corresponding 2010 models, with the only exception the $\mathrm{O}_{3} 2000$ cold season model ( $\left.45 \%\right)$. Other countries that performed poorly for $\mathrm{O}_{3} 2000$ cold were Germany and the Netherlands. The poorer temporal correlation for $\mathrm{O}_{3}$ may be due to the smaller spatial contrast when evaluating at a smaller spatial scale. Another explanation may be that there are different CTM predictions used in the LUR models for 2010 (MACC-O ${ }_{3}$ for annual and cold $\mathrm{O}_{3}$ ) compared to 2000 and 2005 for which only the DEHM model was available.

Correlations between annual average measured concentrations at sites that were in operation for an extended time period were even higher. The higher correlation for measurements was probably due to the only moderately high explained variance of the models and difference in availability of predictor variables across years. A difficulty in the interpretation of monitoring data is the limited number of sites with continuous data, especially for $\mathrm{PM}_{2.5}$.

The temporal stability of the estimated spatial surface for most of the pollutants has positive consequences for further application in long-term epidemiological studies especially those including cohorts which started one or two decades ago and which will have had several follow-ups since then. The 2010 surfaces produced here can be used with some confidence as the base for backextrapolation.

For several areas we now have study-area specific ESCAPE models and Europe wide ELAPSE models. The ESCAPE models are based upon a smaller number of training sites but may be more specific for the area. The spatial extent of ESCAPE PM models has limited the analysis of some ESCAPE cohorts (e.g. only Paris in the national French E3N cohort and Copenhagen in the Danish DCH cohort). The ELAPSE model can be applied to larger areas e.g. entire France, Denmark. In general, Europe wide models may be better when large areas are studied. In international multi-center studies, the use of a single harmonized model is important to standardize exposure assessment.

\section{Conclusions}

We were able to develop robust $\mathrm{PM}_{2.5}, \mathrm{NO}_{2}, \mathrm{BC}$ and $\mathrm{O}_{3}$ LUR models. At the West-European scale models were robust in time, becoming less robust at smaller spatial extents. In terms of model performance we improved on previously published European $\mathrm{NO}_{2}$ and $\mathrm{PM}_{2.5}$ models and developed new models for $\mathrm{BC}$ and $\mathrm{O}_{3}$ explaining large fractions of the variance. We showed, by five-fold holdout validation plus an independent comparison, that the models were spatially robust at the WestEuropean and, to a lesser degree, at the national scale. At the West-European scale, $\mathrm{PM}_{2.5}, \mathrm{NO}_{2}$ and $\mathrm{O}_{3}$ models were robust in time. For BC models we were not able to perform a stability analysis. At smaller spatial scales, models were less robust in time, especially for $\mathrm{O}_{3}$. The models presented here will be used to assign exposures in the ELAPSE study and will be made available for other studies in Europe. 


\section{References}

Basagaña X, Rivera M, Aguilera I, et al. 2012. Effect of the number of measurement sites on land use regression models in estimating local air pollution. Atmospheric Environment 54.

Bechle MJ, Millet DB, Marshall JD. 2013. Remote sensing of exposure to no2: Satellite versus ground-based measurement in a large urban area. Atmospheric Environment 69:345-353.

Bechle MJ, Millet DB, Marshall JD. 2015. National spatiotemporal exposure surface for no2: Monthly scaling of a satellitederived land-use regression, 2000-2010. Environmental science \& technology 49:12297-12305.

Beelen R, Hoek G, Pebesma E, et al. 2009. Mapping of background air pollution at a fine spatial scale across the european union. Science of the Total Environment 407:1852-1867.

Beelen R, Hoek G, Vienneau D, et al. 2013. Development of no2 and nox land use regression models for estimating air pollution exposure in 36 study areas in europe - the escape project. ATMOSPHERIC ENVIRONMENT 72:10-23.

Beelen R, Raaschou-Nielsen O, Stafoggia M, et al. 2014. Effects of long-term exposure to air pollution on natural-cause mortality: An analysis of 22 european cohorts within the multicentre escape project. The Lancet 383:785-795.

Beelen R, Hoek G, Raaschou-Nielsen O, et al. 2015. Natural-cause mortality and long-term exposure to particle components: An analysis of 19 european cohorts within the multi-center escape project. Environmental Health Perspectives 123:525-533.

Brandt J, Silver JD, Frohn LM, et al. 2012. An integrated model study for europe and north america using the danish eulerian hemispheric model with focus on intercontinental transport of air pollution. Atmospheric Environment 53:156-176.

Briggs DJ, Gulliver J, Fecht D, et al. 2007. Dasymetric modelling of small-area population distribution using land cover and light emissions data. Remote Sensing of Environment 108:451-466.

Cesaroni G, Porta D, Badaloni C, et al. 2012. Nitrogen dioxide levels estimated from land use regression models several years apart and association with mortality in a large cohort study. Environmental Health 11:48.

CGIAR-CSI. 2013. Srtm 90m digital elevation data.

Chen H, Goldberg MS, Crouse DL, et al. 2010. Back-extrapolation of estimates of exposure from current land-use regression models. Atmospheric Environment 44:4346-4354.

Chen H, Kwong JC, Copes R, et al. 2017. Exposure to ambient air pollution and the incidence of dementia: A populationbased cohort study. Environmental International 108:271-277.

Cohen AJ, Brauer M, Burnett R, et al. 2017. Estimates and 25-year trends of the global burden of disease attributable to ambient air pollution: An analysis of data from the global burden of diseases study 2015. The Lancet 389:1907-1918.

Crouse DL, Peters PA, Hystad P, et al. 2015. Ambient pm2.5, o3, and no2 exposures and associations with mortality over 16 years of follow-up in the canadian census health and environment cohort (canchec). Environmental Health Perspectives 123:1180-1186.

Cyrys J, Eeftens M, Heinrich J, et al. 2012. Variation of no2 and nox concentrations between and within 36 european study areas: Results from the escape study. Atmospheric Environment 62.

de Hoogh K, Gulliver J, Donkelaar Av, et al. 2016. Development of west-european pm2.5 and no2 land use regression models incorporating satellite-derived and chemical transport modelling data. Environmental Research 151:1-10.

Di Q, Wang Y, Zanobetti A, et al. 2017. Air pollution and mortality in the medicare population. New England Journal of Medicine 376:2513-2522.

EC. 2011. Geostat 2011 grid dataset.European Commission (Eurostat, Joint Research Centre and DG Regional Policy REGIO-GIS).

EEA. 2015. Airbase - the european air quality database, version 8. Available: http://www.eea.europa.eu/data-andmaps/data/airbase-the-european-air-quality-database-8 [accessed 13 January 2015].

EEA. 2017. Air quality in europe - 2017 report.

Eeftens M, Beelen R, Fischer P, et al. 2011. Stability of measured and modelled spatial contrasts in no2 over time. Occupational and Environmental Medicine.

Eeftens M, Beelen R, de Hoogh K, et al. 2012a. Development of land use regression models for pm2.5, pm2.5 absorbance, pm10 and pmcoarse in 20 european study areas; results of the escape project. Environmental Science \& Technology 46:1119511205.

Eeftens M, Tsai MY, Ampe C, et al. 2012b. Spatial variation of pm2.5, pm10, pm2.5 absorbance and pmcoarse concentrations between and within 20 european study areas and the relationship with no2 - results of the escape project. Atmospheric Environment 62:303-317.

ETC-LC. 2009. Corine land cover (clc2000), raster database (version 12/2009).

ETC-LC. 2013. Corine land cover (clc2006), raster database (version 12/2013).

Gulliver J, de Hoogh K, Hansell A, et al. 2013. Development and back-extrapolation of no2 land use regression models for historic exposure assessment in great britain. Environmental Science \& Technology 47:7804-7811.

Gulliver J, de Hoogh K. 2015. Environmental exposure assessment: Modelling air pollution concentrations. In: Oxford textbook of global public health Part 6 (Detels R, Gulliford M, Abdool Karim Q, et al., eds). Oxford:Oxford University Press. Gulliver J, de Hoogh K, Hoek G, et al. 2016. Back-extrapolated and year-specific no2 land use regression models for great britain - do they yield different exposure assessment? Environment International 92-93:202-209.

Inness A, Baier F, Benedetti A, et al. 2013. The macc reanalysis: An 8 yr data set of atmospheric composition. Atmos Chem Phys 13:4073-4109.

Johnson M, Isakov V, Touma JS, et al. 2010. Evaluation of land-use regression models used to predict air quality concentrations in an urban area. Atmospheric Environment 44.

Levy I, Levin N, Yuval, et al. 2015. Back-extrapolating a land use regression model for estimating past exposures to trafficrelated air pollution. Environmental Science \& Technology 49:3603-3610.

Novotny EV, Bechle MJ, Millet DB, et al. 2011. National satellite-based land-use regression: No2 in the united states. Environmental science \& technology 45:4407-4414. 
Novotny EV, Bechle MJ, Millet DB, et al. 2011. National satellite-based land-use regression: No2 in the united states. Environmental science \& technology 45:4407-4414.

Pinault L, van Donkelaar A, Martin RV. 2017. Exposure to fine particulate matter air pollution in canada. Health Reports 28:9-16.

Van Donkelaar A, Martin RV, Brauer M, et al. 2015. Use of satellite observations for long-term exposure assessment of global concentrations of fine particulate matter. Environmental health perspectives 123:135.

van Donkelaar A, Martin RV, Brauer M, et al. 2016. Global estimates of fine particulate matter using a combined geophysical-statistical method with information from satellites, models, and monitors. Environmental Science \& Technology 50:3762-3772.

van Nunen E, Vermeulen R, Tsai M-Y, et al. 2017. Land use regression models for ultrafine particles in six european areas. Environmental Science \& Technology 51:3336-3345.

Vienneau D, de Hoogh K, Bechle MJ, et al. 2013. Western european land use regression incorporating satellite- and groundbased measurements of no2 and pm10. Environmental Science \& Technology 47:13555-13564.

Wang M, Beelen R, Basagana X, et al. 2013. Evaluation of land use regression models for no2 and particulate matter in 20 european study areas: The escape project. Environmental Science \& Technology 47:4357-4364.

Wang R, Henderson SB, Sbihi H, et al. 2013. Temporal stability of land use regression models for traffic-related air pollution. Atmospheric Environment 64:312-319.

WHO. 2016. Ambient air pollution: A global assessment of exposure and burden of disease.

Young MT, Bechle MJ, Sampson PD, et al. 2016. Satellite-based no2 and model validation in a national prediction model based on universal kriging and land-use regression. Environmental Science \& Technology 50:3686-3694. 


\section{Supplementary material}

\section{Analysis of different $\mathbf{P M}_{2.5}$ satellite products}

We offered three different $\mathrm{PM}_{2.5}$ satellite products to the $\mathrm{PM}_{2.5}$ model development; (1) $10 \mathrm{~km}$ product inferred 2009-2011; (2) 10km product for 2010; (3) $1 \mathrm{~km}$ product for 2010. In preliminary models, the first data set led to better $\mathrm{PM}_{2.5}$ models compared to the other 2 datasets. We further investigated the raw squared correlation coefficients $\left(\mathrm{R}^{2}\right)$ of the 3 data products (annual mean) with the annual mean $\mathrm{PM}_{2.5}$ measurements from AirBase for the year 2010 (see Table S5 for more details). The difference in explained variance seems to be in the time period of the 3 products. The products 2 and 3 focusing on the year 2010 yielded similar correlations, irrespective of the 10 or $1 \mathrm{~km}$ spatial resolution, explaining around $40 \%$ of variation. Product 1, which was inferred for 2009 to 2011 and optimized for 2010 , explained $46 \%$ of variation. For the final $\mathrm{PM}_{2.5}$ model we therefore decided to only offer the first $\mathrm{PM}_{2.5}$ product.

\section{Stability analysis at regional (NUTS1) level}

NUTS areas are standard administrative divisions of EU countries for statistical purposes. The NUTS1 level is the first level. We also performed the stability analysis using the same 44,000 random points at the NUTS1 area level (see Figure S6). Like at the country level, there is a good agreement for all areas for $\mathrm{NO}_{2} 2000$ and 2005 when compared to the 2010 surface $\left(\mathrm{R}^{2}>0.60\right)$. For the other pollutants there is more heterogeneity in the correlation coefficients across areas. When comparing the $\mathrm{PM}_{2.5}$ surfaces (2010 vs. 2013), the majority of the NUTS1 areas have a correlation coefficient > 0.40 , with only a handful of areas dropping between 0.20 and 0.40 . The comparison of the $\mathrm{O}_{3}$ surfaces (2000, 2005 vs. 2013) shows a clear difference between annual and cold season versus the warm season. Both the 2000 and 2005 comparisons for warm season show a number of areas in the south of Europe with correlations of less than 0.20 . This pattern is not observed in the annual and cold season comparisons. 
Table S1. Descriptive statistics of $\mathrm{PM}_{2.5}, \mathrm{NO}_{2}, \mathrm{BC}, \mathrm{O}_{3}$ concentrations for 2010 used in the modeling procedure.

\begin{tabular}{|c|c|c|c|c|c|c|c|c|c|}
\hline \multirow[t]{2}{*}{ Air pollutant } & \multirow[t]{2}{*}{ Type } & \multirow[t]{2}{*}{$\mathrm{N}^{\mathrm{a}}$} & \multirow{2}{*}{$\begin{array}{c}\text { Mean } \\
(\mu \mathrm{g} / \\
\left.\mathrm{m}^{3}\right)\end{array}$} & \multirow[t]{2}{*}{$\begin{array}{l}\text { Median } \\
\left(\mu \mathrm{g} / \mathrm{m}^{3}\right)\end{array}$} & \multirow{2}{*}{$\begin{array}{c}\text { Std. } \\
\text { Deviation } \\
\left(\mu \mathrm{g} / \mathrm{m}^{3}\right)\end{array}$} & \multicolumn{4}{|c|}{$\begin{array}{c}\text { Percentiles } \\
\left(\mu \mathrm{g} / \mathrm{m}^{3}\right)\end{array}$} \\
\hline & & & & & & 5 & 25 & 75 & 95 \\
\hline \multirow[t]{4}{*}{$\mathrm{PM}_{2.5}$} & Traffic & 149 & 16.28 & 16.63 & 4.93 & 8.33 & 12.75 & 19.67 & 23.76 \\
\hline & Background & 341 & 15.75 & 15.77 & 5.16 & 7.25 & 12.65 & 18.78 & 23.92 \\
\hline & Industrial & 53 & 15.12 & 15.27 & 5.45 & 7.51 & 10.36 & 19.18 & 25.73 \\
\hline & All & 543 & 15.84 & 15.88 & 5.13 & 7.65 & 12.42 & 19.25 & 23.94 \\
\hline \multirow{4}{*}{$\mathrm{NO}_{2}$} & Traffic & 740 & 40.23 & 38.69 & 14.62 & 19.90 & 30.39 & 47.75 & 66.06 \\
\hline & Background & 1287 & 21.47 & 21.01 & 9.82 & 5.67 & 14.81 & 27.94 & 37.27 \\
\hline & Industrial & 372 & 19.43 & 18.22 & 10.13 & 4.47 & 11.52 & 26.72 & 38.06 \\
\hline & All & 2399 & 26.94 & 25.12 & 14.59 & 6.73 & 16.34 & 34.70 & 53.69 \\
\hline \multirow[t]{4}{*}{$\mathrm{BC}^{\mathrm{b}}$} & Traffic & 207 & 2.28 & 2.16 & 0.90 & 0.98 & 1.65 & 2.83 & 3.99 \\
\hline & Background & 229 & 1.51 & 1.47 & 0.54 & 0.74 & 1.08 & 1.85 & 2.44 \\
\hline & Industrial & 0 & n.a. & n.a. & n.a. & n.a. & n.a. & n.a. & n.a. \\
\hline & All & 436 & 1.88 & 1.78 & 0.83 & 0.84 & 1.26 & 2.33 & 3.50 \\
\hline \multirow[t]{4}{*}{$\mathrm{O}_{3}$ annual $^{\mathrm{c}}$} & Traffic & 228 & 65.01 & 63.86 & 12.26 & 45.80 & 57.10 & 73.44 & 88.72 \\
\hline & Background & 1323 & 72.10 & 70.92 & 10.66 & 56.25 & 65.71 & 77.76 & 91.86 \\
\hline & Industrial & 194 & 75.77 & 76.59 & 11.76 & 57.42 & 67.12 & 84.31 & 94.49 \\
\hline & $\mathrm{All}^{\mathrm{c}}$ & 1747 & 71.59 & 70.61 & 11.35 & 53.75 & 64.54 & 78.31 & 91.85 \\
\hline \multirow{4}{*}{$\mathrm{O}_{3}$ warm $^{\mathrm{c}}$} & Traffic & 225 & 81.62 & 81.34 & 15.03 & 57.65 & 70.69 & 92.51 & 106.04 \\
\hline & Background & 1311 & 89.99 & 89.30 & 12.99 & 69.09 & 82.22 & 98.45 & 111.84 \\
\hline & Industrial & 192 & 90.70 & 92.20 & 13.45 & 67.13 & 81.61 & 100.18 & 110.61 \\
\hline & All $^{\mathrm{d}}$ & 1730 & 88.98 & 88.95 & 13.62 & 66.39 & 80.97 & 97.96 & 111.08 \\
\hline \multirow[t]{4}{*}{$\mathrm{O}_{3}$ cold $^{\mathrm{c}}$} & Traffic & 223 & 48.31 & 47.13 & 12.29 & 29.96 & 39.44 & 57.52 & 72.03 \\
\hline & Background & 1304 & 54.05 & 53.18 & 12.69 & 35.34 & 44.92 & 62.17 & 77.63 \\
\hline & Industrial & 188 & 60.16 & 62.26 & 13.55 & 37.90 & 49.77 & 69.77 & 82.50 \\
\hline & $\mathrm{All}^{\mathrm{e}}$ & 1716 & 53.98 & 53.04 & 13.05 & 34.11 & 44.34 & 62.72 & 77.42 \\
\hline
\end{tabular}

\footnotetext{
a. Number of sites

b. BC monitoring data from ESCAPE (2009-2010), no industrial type monitoring sites were used. Measured as absorbance of $\mathrm{PM}_{2.5}\left(10^{-5} \mathrm{~m}^{-1}\right)$

c. $\mathrm{O}_{3}$ concentrations were calculated as the average of the daily maximum running 8-hour mean; warm season is from 1 April to 30 September; cold season is from 1 October to 31 March falling in the same year.

d. These include 2 sites with site type 'Unknown'

e. These include 1 site with site type 'Unknown'
} 
Table S2. GIS predictor variables

\begin{tabular}{|c|c|c|c|c|c|}
\hline Data set & Predictor variable & Name variable & Year & $\begin{array}{l}\text { Buffer Size } \\
\text { (radius in m) or } \\
\text { point estimate }\end{array}$ & $\begin{array}{l}\text { Pre-specified } \\
\text { direction of } \\
\text { effect for } \\
\mathrm{PM}_{2.5}, \mathrm{NO}_{2} \text {, } \\
\mathrm{BC}\left(\mathrm{O}_{3}\right)\end{array}$ \\
\hline $\begin{array}{l}\mathrm{PM}_{2.5}\left(\mu \mathrm{g} / \mathrm{m}^{3}\right) \\
\text { derived from } \\
\text { MODIS on board } \\
\text { the Terra satellite: } \\
\sim 10 \mathrm{~km}\end{array}$ & $\begin{array}{l}\text { Surface } \mathrm{PM}_{2.5} \\
\text { concentration derived } \\
\text { from satellite }\end{array}$ & SAT-PM25 & 2010,2013 & Point & + \\
\hline $\begin{array}{l}\mathrm{PM}_{2.5}\left(\mu \mathrm{g} / \mathrm{m}^{3}\right) \\
\text { derived from } \\
\text { MODIS on board } \\
\text { the Terra satellite: } \\
\sim 1 \mathrm{~km}\end{array}$ & $\begin{array}{l}\text { Surface } \mathrm{PM}_{2.5} \\
\text { concentration derived } \\
\text { from satellite }\end{array}$ & SAT-PM25-1k & 2010,2013 & Point & + \\
\hline $\begin{array}{l}\mathrm{NO}_{2}\left(\mu \mathrm{g} / \mathrm{m}^{3}\right) \\
\text { derived from OMI } \\
\text { on board the Aura } \\
\text { satellite: } \sim 10 \mathrm{~km}\end{array}$ & $\begin{array}{l}\text { Surface } \mathrm{NO}_{2} \\
\text { concentration derived } \\
\text { from satellite }\end{array}$ & $\mathrm{SAT}-\mathrm{NO}_{2}$ & 2010 & Point & $+(-)$ \\
\hline $\begin{array}{l}\mathrm{PM}_{2.5}, \mathrm{NO}_{2}, \mathrm{O}_{3} \\
\left(\mu \mathrm{g} / \mathrm{m}^{3}\right) \text { estimated } \\
\text { by MACC-II } \\
\text { Ensemble model: } \\
\sim 10 \mathrm{~km}\end{array}$ & $\begin{array}{l}\text { Surface } \mathrm{PM}_{2.5}, \mathrm{NO}_{2} \text { and } \\
\mathrm{O}_{3} \text { concentration from } \\
\text { dispersion model }\end{array}$ & $\begin{array}{l}\text { MACC-PM25 } \\
\text { MACC-NO } \\
\text { MACC-O }\end{array}$ & 2010,2013 & Point & $+(-)$ \\
\hline $\begin{array}{l}\mathrm{PM}_{2.5}, \mathrm{NO}_{2}, \mathrm{O}_{3} \\
\text { and BC }\left(\mu \mathrm{g} / \mathrm{m}^{3}\right) \\
\text { estimated by } \\
\text { DEHM: } \sim 50 \mathrm{~km}\end{array}$ & $\begin{array}{l}\text { Surface } \mathrm{PM}_{2.5}, \mathrm{NO}_{2}, \\
\mathrm{O}_{3} \text { and } \mathrm{BC} \\
\text { concentration from } \\
\text { dispersion model }\end{array}$ & $\begin{array}{l}\text { DEHM-PM } \\
\text { DEHM-NO }_{2} \\
\text { DEHM-O }_{3} \\
\text { DEHM-BC }\end{array}$ & $\begin{array}{l}2000,2005 \\
2010\end{array}$ & Point & $+(-)$ \\
\hline $\begin{array}{l}\text { EuroStreets roads } \\
\text { (length in } \mathrm{m} \text { ) }\end{array}$ & $\begin{array}{l}\text { Major roads } \\
\text { All roads }\end{array}$ & $\begin{array}{l}\text { MJRD } \\
\text { ALRD }\end{array}$ & 2010 & $\begin{array}{l}50 ; 100 ; 200 ; \\
300 ; 400 ; 500 ; \\
700 ; 1000 ; 2000 ; \\
5000 ; 10000\end{array}$ & $+(-)$ \\
\hline $\begin{array}{l}\text { Corine land cover: } \\
100 \mathrm{~m}\end{array}$ & $\begin{array}{l}\text { Industry/commercial } \\
\text { Ports } \\
\text { Urban green } \\
\text { Total built up } \\
\text { Natural land } \\
\text { Residential }^{\mathrm{b}}\end{array}$ & $\begin{array}{l}\text { IND } \\
\text { POR } \\
\text { UGR } \\
\text { TBU } \\
\text { NAT } \\
\text { RES }\end{array}$ & 2006 & $\begin{array}{l}50 ; 100 ; 200 ; \\
300 ; 400 ; 500 ; \\
600 ; 700 ; 800 ; \\
1000 ; 1200 ; \\
1500 ; 1800 ; \\
2000 ; 2500 ; \\
3000 ; 3500 ; \\
4000 ; 5000 ; \\
6000 ; 7000 ; \\
8000 ; 10000\end{array}$ & $\begin{array}{l}+(-) \\
+(-) \\
-(+) \\
+(-) \\
-(+) \\
+(-)\end{array}$ \\
\hline $\begin{array}{l}\text { GEOSTAT } 2011 \\
\text { population grid } \\
\text { dataset: } \sim 1 \mathrm{~km} \\
\end{array}$ & Sum of population & POP & 2011 & Point & $+(-)$ \\
\hline $\begin{array}{l}\text { Altitude SRTM } \\
\text { DTM: } \sim 90 \mathrm{~m}\end{array}$ & $\begin{array}{l}\text { Altitude - } \\
\text { transformed }^{\mathrm{c}}\end{array}$ & ALT & & Point & $-(+)$ \\
\hline Trend & $\begin{array}{l}\text { North-South and East- } \\
\text { West trend }\end{array}$ & $X, Y^{d}$ & & Point & n.a. \\
\hline
\end{tabular}

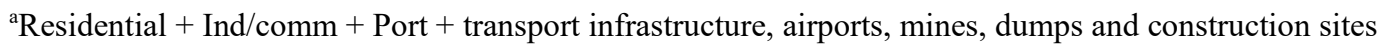

${ }^{\mathrm{b}}$ continuous urban fabric ( high density housing) + discontinuous urban fabric (low density housing)

${ }^{\mathrm{c}}$ Transformed altitude is calculated as $\sqrt{ }($ nalt $/ \max ($ nalt $))$, where nalt $=$ altitude $-\min ($ altitude $)$.

${ }^{\mathrm{d}}$ Coordinates were truncated $: \mathrm{X}=\mathrm{x}-\mathrm{xmin} /(\mathrm{xmax}-\mathrm{xmin}) ; \mathrm{Y}=\mathrm{y}-\mathrm{ymin} /(\mathrm{ymax}-\mathrm{ymin})$ 
Table S3. Structure and performance of $\mathrm{LUR}$ models for $\mathrm{BC}, \mathrm{NO}_{2}$ and $\mathrm{O}_{3}$ for full dataset and five hold-out validation datasets

\section{BC models ${ }^{a}$}

\begin{tabular}{|c|c|c|c|c|c|c|c|}
\hline Theme & Variables $^{\mathrm{b}}$ & FULL & HOV1 & HOV2 & HOV3 & HOV4 & HOV5 \\
\hline & Constant & 0.99 & 0.85 & 0.99 & 0.94 & 0.99 & 1.10 \\
\hline CTM & MACC-PM25 & 0.85 & 0.95 & 0.80 & 0.92 & 0.95 & 0.65 \\
\hline Satellite & SAT-PM25 & 0.30 & 0.34 & 0.39 & 0.32 & 0.22 & 0.34 \\
\hline Major roads & $\mathrm{MJRD}_{50}$ & & 0.64 & & & & \\
\hline & $\mathrm{MJRD}_{100}$ & 0.68 & & 0.68 & 0.67 & 0.65 & 0.44 \\
\hline All roads & $\mathrm{ALRD}_{50}$ & 0.40 & & & 0.39 & 0.40 & 0.52 \\
\hline & $\mathrm{ALRD}_{1000}$ & & & & 0.34 & & \\
\hline & $\mathrm{ALRD}_{700}$ & 0.45 & 0.67 & 0.40 & & 0.43 & 0.57 \\
\hline Residential & $\mathrm{RES}_{2500}$ & & & 0.93 & 0.86 & & \\
\hline & $\mathrm{RES}_{3000}$ & 0.90 & 0.86 & & & 0.86 & 0.94 \\
\hline Urban green & $\mathrm{UGR}_{1000}$ & -0.12 & -0.18 & -0.14 & -0.11 & -0.19 & -0.08 \\
\hline Ycoord & $\mathrm{Y}$ & -1.16 & -1.09 & -1.16 & -1.16 & -1.16 & -1.30 \\
\hline Training & $\mathrm{R}^{2}$ & 54.4 & 52.2 & 52.3 & 53.9 & 57.6 & 55.3 \\
\hline (LUR) & RMSE & 0.56 & 0.59 & 0.56 & 0.58 & 0.54 & 0.572 \\
\hline $\mathrm{HOV}$ & $\mathrm{R}^{2}$ & 51.4 & 56.7 & 56.9 & 53.8 & 43.2 & 49.3 \\
\hline (LUR) & RMSE & 0.58 & 0.52 & 0.62 & 0.52 & 0.67 & 0.54 \\
\hline
\end{tabular}

\section{$\mathrm{NO}_{2}$ models ${ }^{\mathrm{a}}$}

\begin{tabular}{|c|c|c|c|c|c|c|c|}
\hline Theme & Variable $^{\mathrm{b}}$ & FULL & HOV1 & HOV2 & HOV3 & HOV4 & HOV5 \\
\hline \multirow{4}{*}{$\begin{array}{l}\text { CTM } \\
\text { All Roads }\end{array}$} & Constant & 3.30 & 3.30 & 3.70 & 4.70 & 3.30 & \multirow{2}{*}{$\begin{array}{r}3.20 \\
22.73\end{array}$} \\
\hline & $\mathrm{MACC}-\mathrm{NO}_{2}$ & 22.73 & 22.73 & 23.30 & 22.73 & \multirow{2}{*}{$\begin{array}{r}22.16 \\
9.38\end{array}$} & \\
\hline & $\mathrm{ALRD}_{50}$ & 7.04 & \multirow{4}{*}{9.68} & \multirow[t]{2}{*}{8.60} & 6.65 & & \multirow{4}{*}{10.29} \\
\hline & $\mathrm{ALRD}_{100}$ & \multirow{3}{*}{3.92} & & & \multirow{5}{*}{7.50} & \multirow{5}{*}{12.27} & \\
\hline \multirow{6}{*}{ Major roads } & $\mathrm{ALRD}_{300}$ & & & & & & \\
\hline & $\operatorname{ALRD}_{1000}$ & & & & & & \\
\hline & $\mathrm{ALRD}_{2000}$ & 15.73 & 16.71 & 16.71 & & & 15.73 \\
\hline & $\mathrm{MJRD}_{50}$ & & 5.70 & & & & \\
\hline & $\mathrm{MJRD}_{100}$ & 12.32 & & 12.32 & 10.67 & 12.32 & 12.32 \\
\hline & $\mathrm{MJRD}_{200}$ & & 6.95 & & & & \\
\hline Natural & $\mathrm{NAT}_{400}$ & -3.38 & & & -3.97 & -3.09 & -3.14 \\
\hline & $\mathrm{NAT}_{500}$ & & -3.24 & -3.89 & & & \\
\hline Ports & $\mathrm{POR}_{200}$ & & & 1.82 & & & 1.80 \\
\hline & $\mathrm{POR}_{700}$ & 4.10 & 4.51 & & & 2.87 & \\
\hline & $\mathrm{POR}_{1800}$ & & & & 4.14 & & \\
\hline Residential & $\mathrm{RES}_{200}$ & & & & 2.73 & & \\
\hline & $\mathrm{RES}_{300}$ & 5.80 & & 6.38 & & & \\
\hline & $\mathrm{RES}_{400}$ & & 6.86 & & & & 6.37 \\
\hline & $\begin{array}{l}\mathrm{RES}_{2500} \\
\mathrm{RFS}\end{array}$ & & & & 14.10 & 700 & \\
\hline Total build up & $\mathrm{TBU}_{300}$ & & & & & $\begin{array}{l}7.09 \\
6.09\end{array}$ & \\
\hline & $\mathrm{UGR}_{100}$ & & & & -3.20 & & \\
\hline Urban green & $\mathrm{UGR}_{200}$ & & & -2.40 & & & \\
\hline Training & $\mathrm{R}^{2}$ & 58.8 & 59.1 & 58.3 & 58.4 & 59.0 & 59.6 \\
\hline (LUR) & RMSE & 9.38 & 9.38 & 9.39 & 9.38 & 9.36 & 9.36 \\
\hline $\mathrm{HOV}$ & $\mathrm{R}^{2}$ & 57.5 & 57.8 & 59.9 & 60.2 & 54.8 & 54.7 \\
\hline (LUR) & RMSE & 9.51 & 9.36 & 9.44 & 9.44 & 9.81 & 9.51 \\
\hline
\end{tabular}

${ }^{\text {a }}$ Regression slope in $10^{-5} \mathrm{~m}^{-1}(\mathrm{BC})$ or $\mu \mathrm{g} / \mathrm{m}^{3}\left(\mathrm{NO}_{2}\right)$ were multiplied by the difference between the $1 \mathrm{st}$ and 99 th percentile of each predictor to allow comparison across predictors

${ }^{\mathrm{b}} \mathrm{ALT}=$ altitude, $\mathrm{ALRD}=$ all roads, $\mathrm{MJRD}=$ major roads, $\mathrm{IND}=$ industry, $\mathrm{POR}=$ ports, $\mathrm{UGR}=$ urban green, $\mathrm{TBU}=$ total build up, NAT = natural land, RES = residential, $\mathrm{POP}=$ sum of population, $\mathrm{X}=$ North-South trend, $\mathrm{Y}=$ EastWest trend, SAT $=$ satellite, MACC $=$ MACC dispersion model, DEHM = DEHM CTM. Number in subscript depicts the buffer size (e.g. $A_{L R D} D_{100}=$ sum of all road length within $100 \mathrm{~m}$ ) 


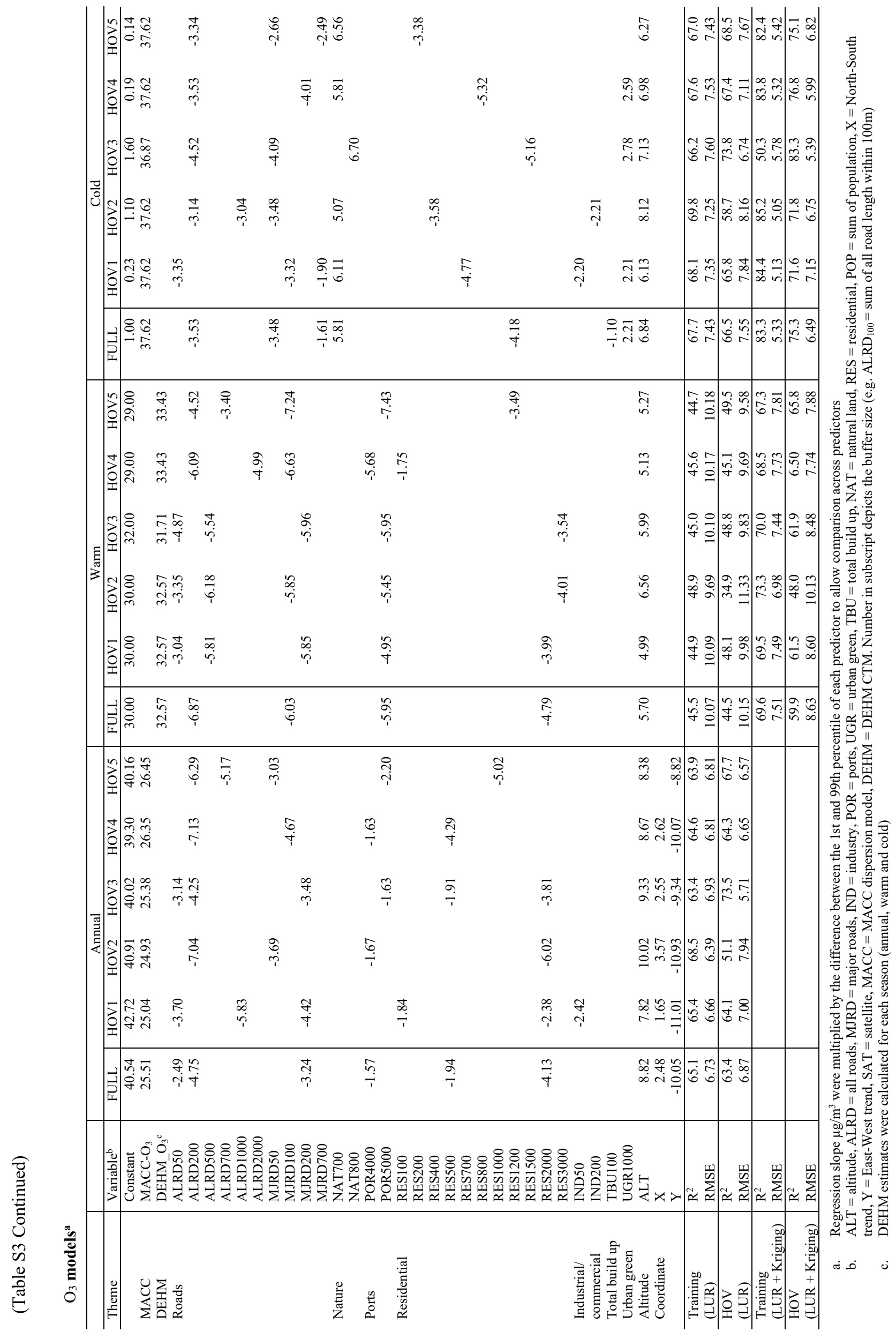


Table S4. Details of 2000, $2005\left(\mathrm{NO}_{2}\right.$ and $\left.\mathrm{O}_{3}\right)$ and $2013\left(\mathrm{PM}_{2.5}\right)$ FULL models ${ }^{\mathrm{a}}$

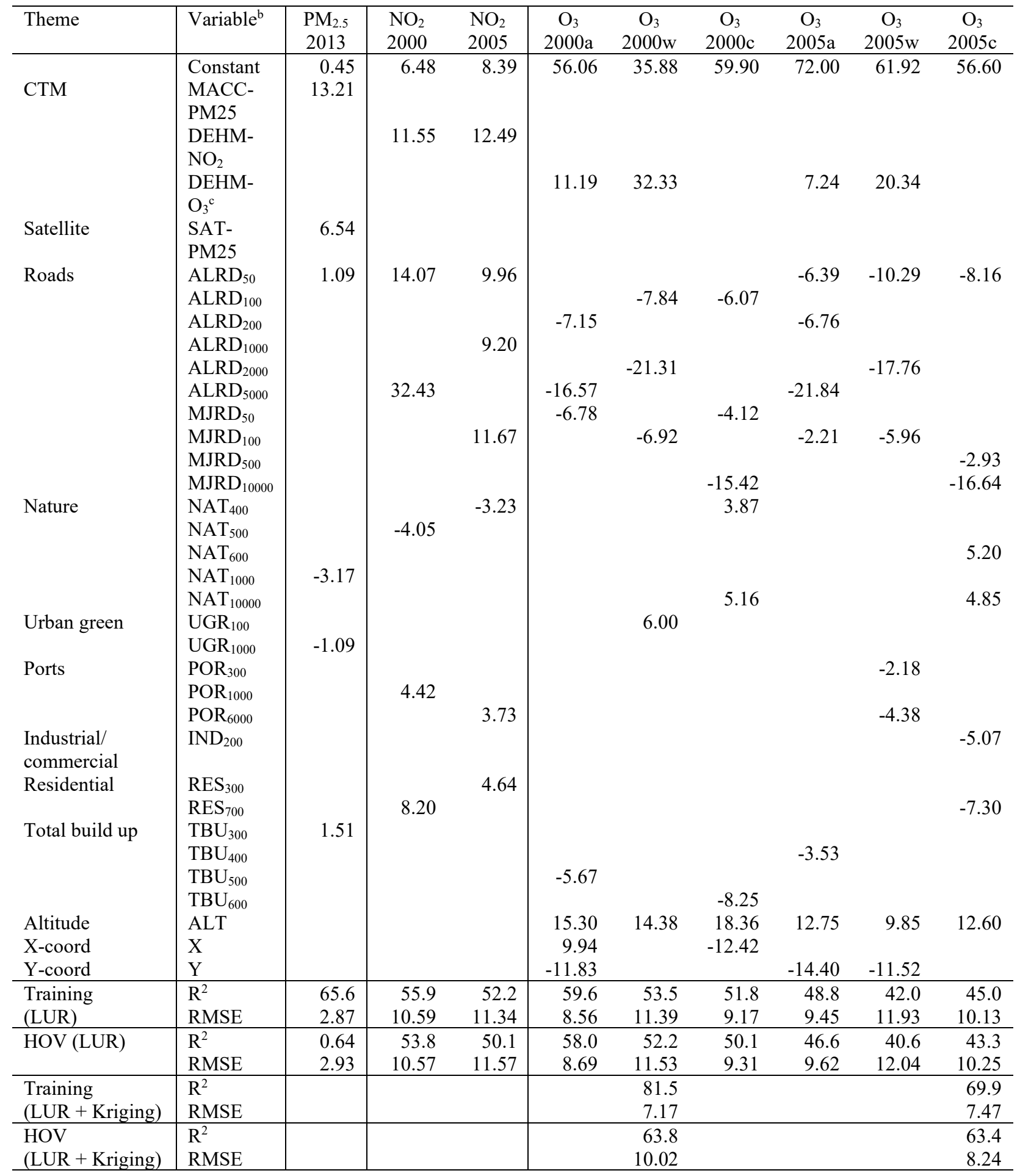

a. Regression slope $\mu \mathrm{g} / \mathrm{m}^{3}$ were multiplied by the difference between the 1 st and 99 th percentile of each predictor to allow comparison across predictors

b. $\quad \mathrm{ALT}=$ altitude, $\mathrm{ALRD}=$ all roads, $\mathrm{MJRD}=$ major roads, $\mathrm{IND}=$ industry, $\mathrm{POR}=$ ports, $\mathrm{UGR}=$ urban green, $\mathrm{TBU}=$ total build up, NAT $=$ natural land, RES $=$ residential, POP $=$ sum of population, $\mathrm{X}=$ North-South trend, $\mathrm{Y}=$ East-West trend, SAT $=$ satellite, MACC $=$ MACC dispersion model, DEHM $=$ DEHM CTM. Number in subscript depicts the buffer size (e.g. $\mathrm{ALRD}_{100}=$ sum of all road length within $100 \mathrm{~m}$ )

c. $\mathrm{DEHM} \mathrm{O}_{3}$ estimates were calculated for each season (annual, warm and cold) 
Table S5. Comparison of predictions of different satellite derived $\mathbf{P M}_{2.5}$ (SAT) products with routine $\mathbf{P M}_{2.5}$ concentrations.

\begin{tabular}{|c|c|c|c|c|c|c|c|}
\hline \multirow[t]{2}{*}{ AirBase Site type } & \multicolumn{2}{|c|}{$\begin{array}{c}\text { Inferred 2009-2011, } \\
10 \times 10 \mathrm{~km}, \mathrm{v} 3.01\end{array}$} & \multicolumn{2}{|c|}{$\begin{array}{c}\text { Inferred } 2010, \\
10 \times 10 \mathrm{~km}, \\
\text { v3.01 }\end{array}$} & \multicolumn{2}{|c|}{$\begin{array}{c}\text { Inferred } 2010, \\
1 \times 1 \mathrm{~km}, \\
\text { v4.GL.02.NoGWR }\end{array}$} & \multirow[b]{2}{*}{$\mathrm{N}$} \\
\hline & $\mathrm{R}^{2}$ & RMSE & $\mathrm{R}^{2}$ & RMSE & $\mathrm{R}^{2}$ & RMSE & \\
\hline All sites & 0.46 & 3.77 & 0.39 & 4.01 & 0.40 & 3.98 & 545 \\
\hline No industrial sites & 0.47 & 3.71 & 0.38 & 4.02 & 0.38 & 4.00 & 492 \\
\hline Traffic only & 0.46 & 3.62 & 0.42 & 3.75 & 0.42 & 3.76 & 149 \\
\hline Background only ${ }^{a}$ & 0.49 & 3.68 & 0.37 & 4.11 & 0.38 & 4.07 & 342 \\
\hline
\end{tabular}

a. Monitoring site located away from major pollutant sources

Table S6. Population (2010) by classes of modelled air pollution estimates $\left(\mathrm{PM}_{2.5}\right.$ and $\mathrm{NO}_{2}$ FULL models)

\begin{tabular}{|c|c|c|c|c|c|}
\hline Pollutant & $\begin{array}{l}\text { Concentration } \\
\text { class }\left(\mu \mathrm{g} / \mathrm{m}^{3}\right)\end{array}$ & $\begin{array}{l}\text { Area }(1,000,000 \\
\text { m2) }\end{array}$ & $\begin{array}{l}\text { Percentage of } \\
\text { total }(\%)\end{array}$ & $\begin{array}{l}\text { Population }^{\mathrm{a}} \\
\text { (millions) }\end{array}$ & $\begin{array}{l}\text { Percentage of } \\
\text { total }(\%)\end{array}$ \\
\hline \multirow[t]{7}{*}{$\mathrm{PM}_{2.5}$} & $<5$ & 1769.41 & 11.32 & 4.51 & 1.08 \\
\hline & $5-<10$ & 3592.20 & 22.98 & 42.31 & 10.12 \\
\hline & $10-<15$ & 7016.49 & 44.88 & 173.87 & 41.57 \\
\hline & $15-<20$ & 2762.81 & 17.67 & 155.38 & 37.15 \\
\hline & $20-<25$ & 404.38 & 2.59 & 33.68 & 8.05 \\
\hline & $25-<30$ & 87.30 & 0.56 & 7.96 & 1.90 \\
\hline & $>30$ & 2.17 & 0.01 & 0.53 & 0.13 \\
\hline Total & & 15634.76 & & 418.24 & \\
\hline \multirow[t]{7}{*}{$\mathrm{NO}_{2}$} & $<10$ & 7335.38 & 46.93 & 32.72 & 7.82 \\
\hline & $10-<15$ & 4156.22 & 26.59 & 60.36 & 14.43 \\
\hline & $15-<20$ & 2351.02 & 15.04 & 77.17 & 18.45 \\
\hline & $20-<30$ & 1500.96 & 9.60 & 139.49 & 33.35 \\
\hline & $30-<40$ & 240.35 & 1.54 & 76.54 & 18.30 \\
\hline & $40-<60$ & 45.95 & 0.29 & 30.92 & 7.39 \\
\hline & $>60$ & 1.54 & 0.01 & 1.07 & 0.26 \\
\hline Total & & 15631.42 & & 418.26 & \\
\hline
\end{tabular}

a. Population was derived from 2011 census data

Table S7. Population weighted annual concentration $\left(\mu \mathrm{g} / \mathrm{m}^{3}\right)$ averaged over Western Europe

\begin{tabular}{cccccc}
\hline & \multicolumn{3}{c}{ Pollutant $\left(\mu \mathrm{g} / \mathrm{m}^{3}\right)$} \\
Year & $\mathrm{PM}_{2.5}$ & $\mathrm{NO}_{2}$ & $\mathrm{O}_{3}$ annual & $\mathrm{O}_{3}$ cold & $\mathrm{O}_{3}$ warm \\
\hline 2000 & & 20.09 & 55.37 & 39.94 & 70.02 \\
2005 & 15.41 & 57.97 & 43.48 & 71.86 \\
2010 & 11.17 & 18.85 & 57.11 & 42.70 & 70.51 \\
2013 & 10.58 & & & & \\
\hline
\end{tabular}




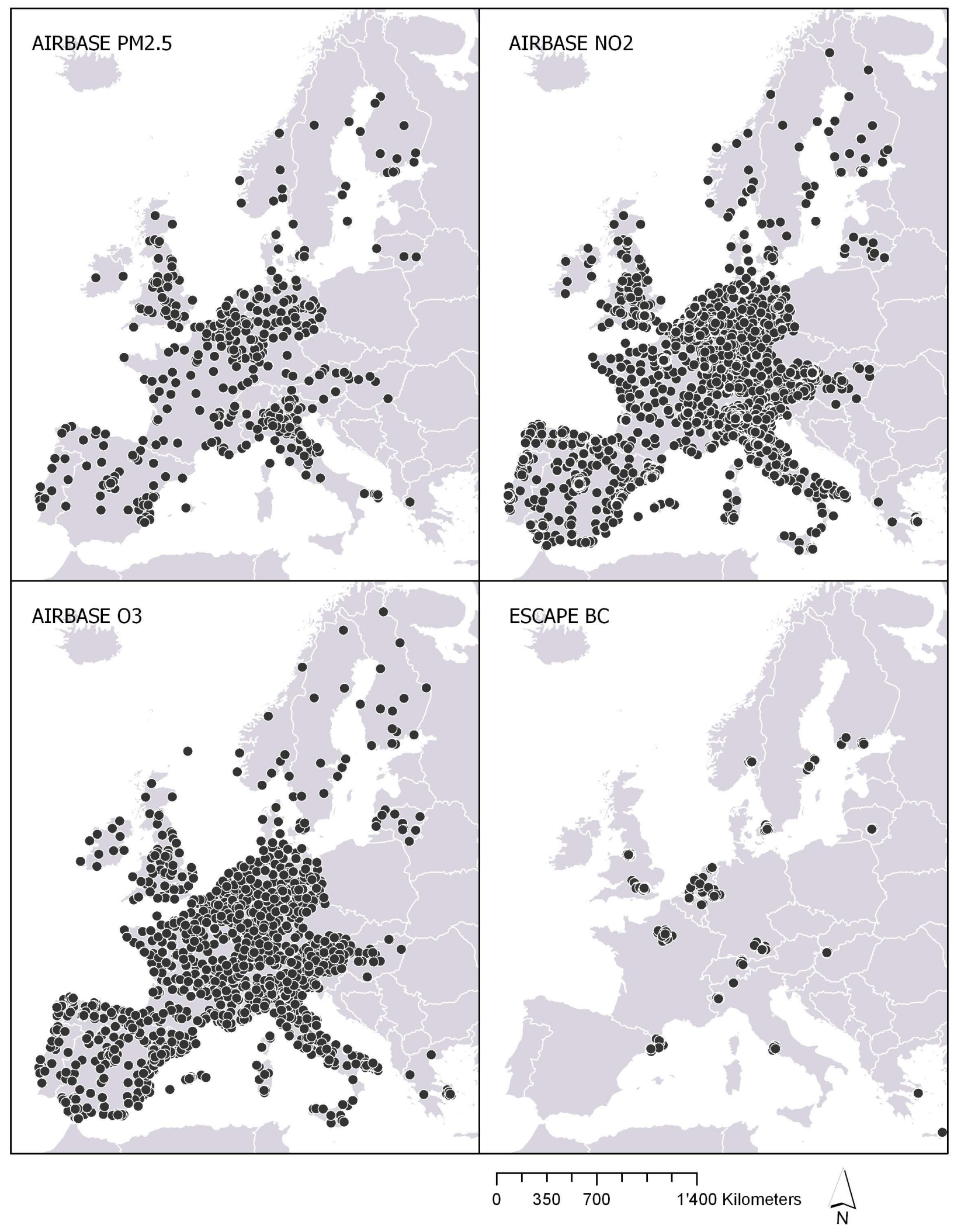

Figure S1. Location of $\mathrm{PM}_{2.5}, \mathrm{NO}_{2}, \mathrm{O}_{3}$ and $\mathrm{BC}$ monitoring sites for the year 2010 


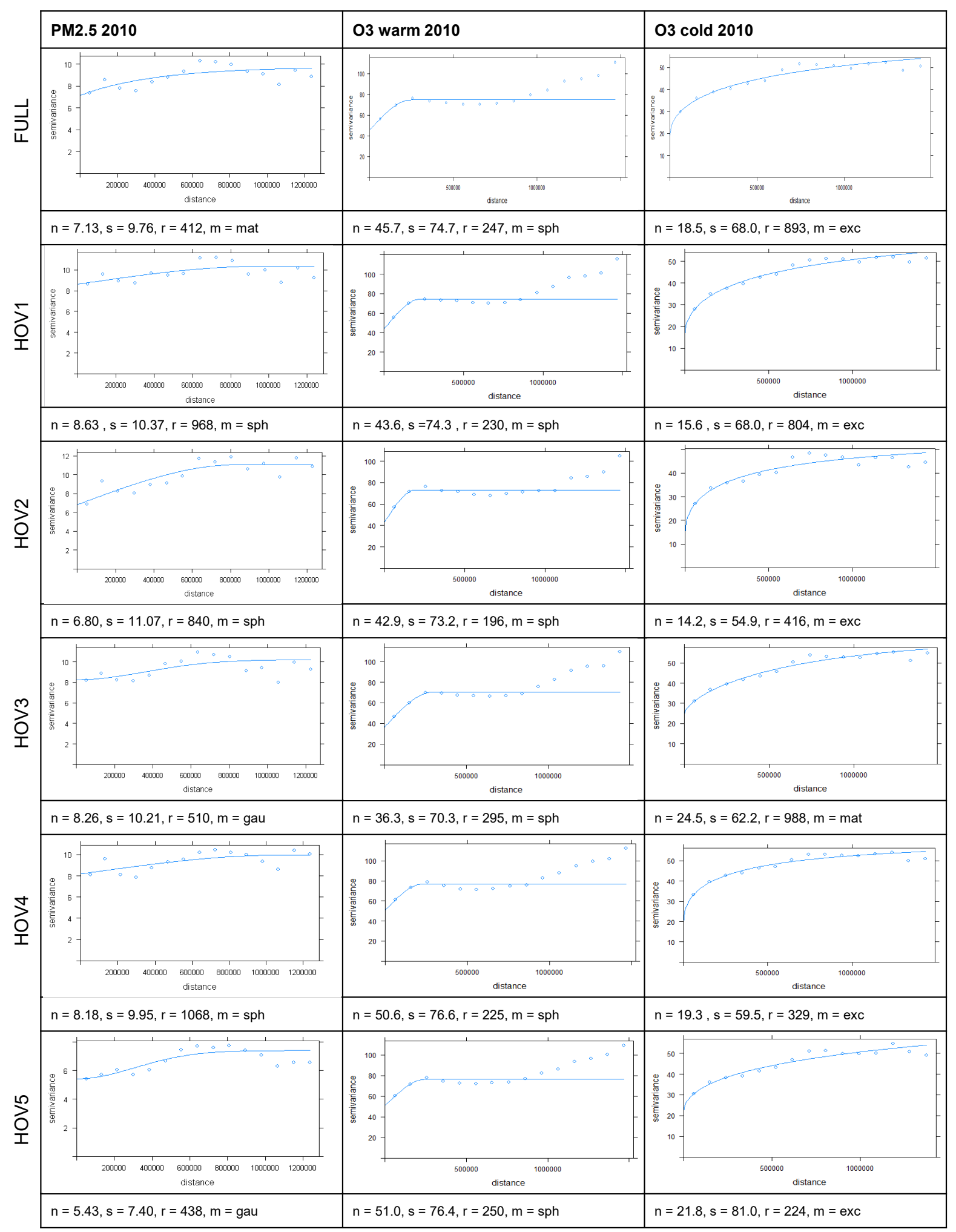

Figure S2. Variograms for $\mathbf{P M}_{2.5}, \mathbf{O}_{3}$ warm and $\mathbf{O}_{3}$ cold models for 2010. $\mathrm{n}=\operatorname{nugget}\left(\mu \mathrm{g} / \mathrm{m}^{3}\right), \mathrm{s}=$ sill $\left(\mu \mathrm{g} / \mathrm{m}^{3}\right), \mathrm{r}=$ range $(\mathrm{km}), \mathrm{m}$ (model); mat matern; $\mathrm{sph}=$ spherical; gau = gaussian, exc $=$ exclass (exponential class/stable) 


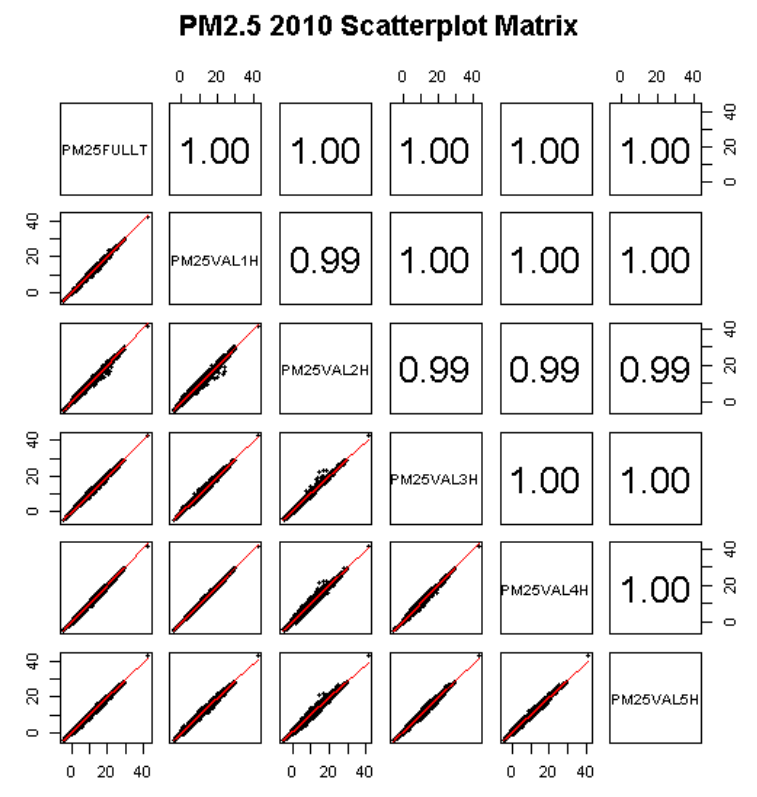

NO2 2010 Scatterplot Matrix

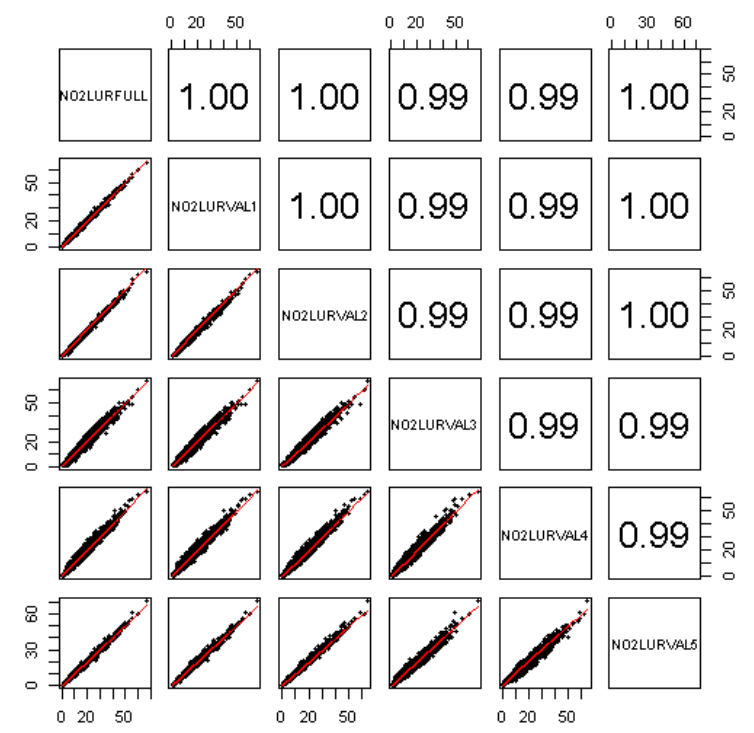

BC 2010 Scatterplot Matrix

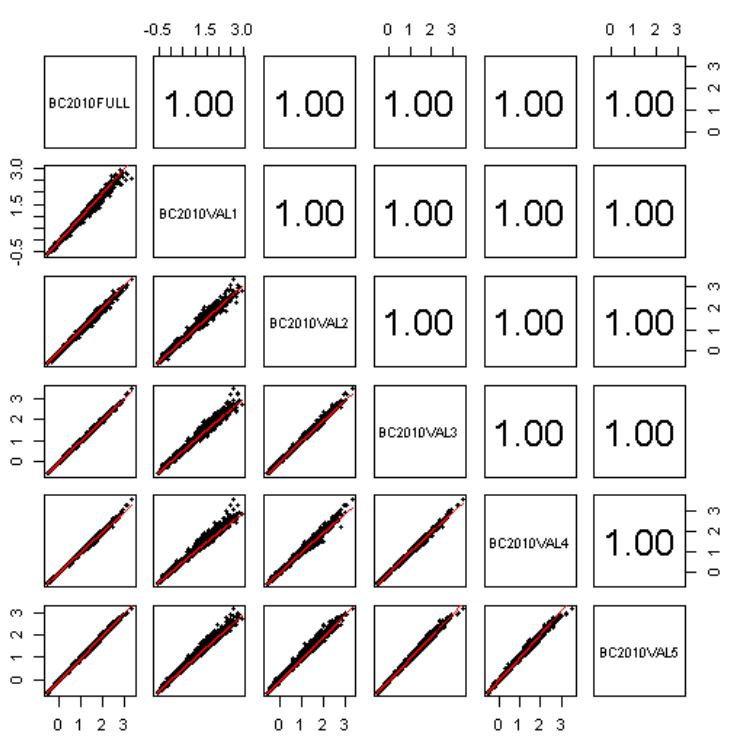

O3 warm 2010 Scatterplot Matrix

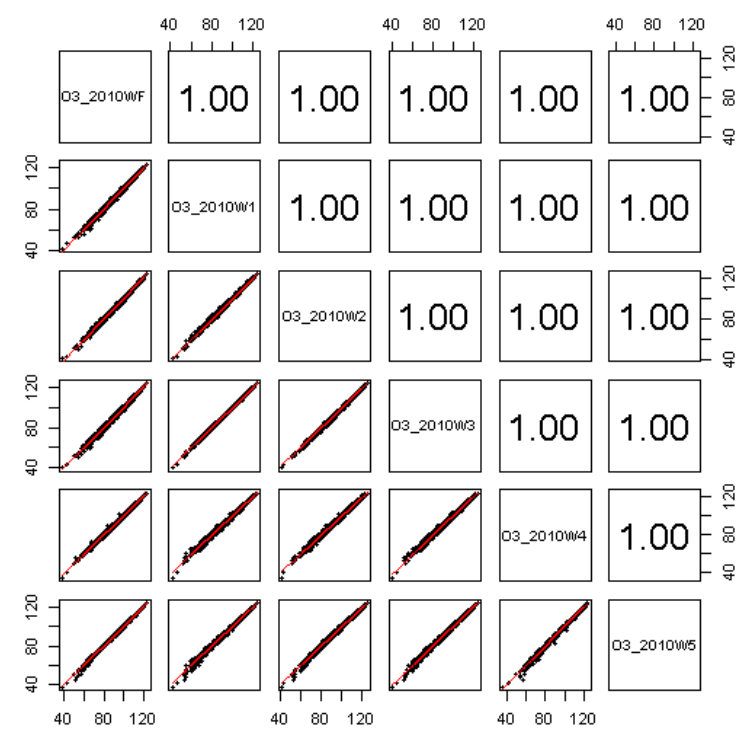

Figure S3. Scatterplot matrix of predicted concentrations of FULL and HOV models for all pollutants at $\sim \mathbf{4 4 , 0 0 0}$ random points across Western Europe 


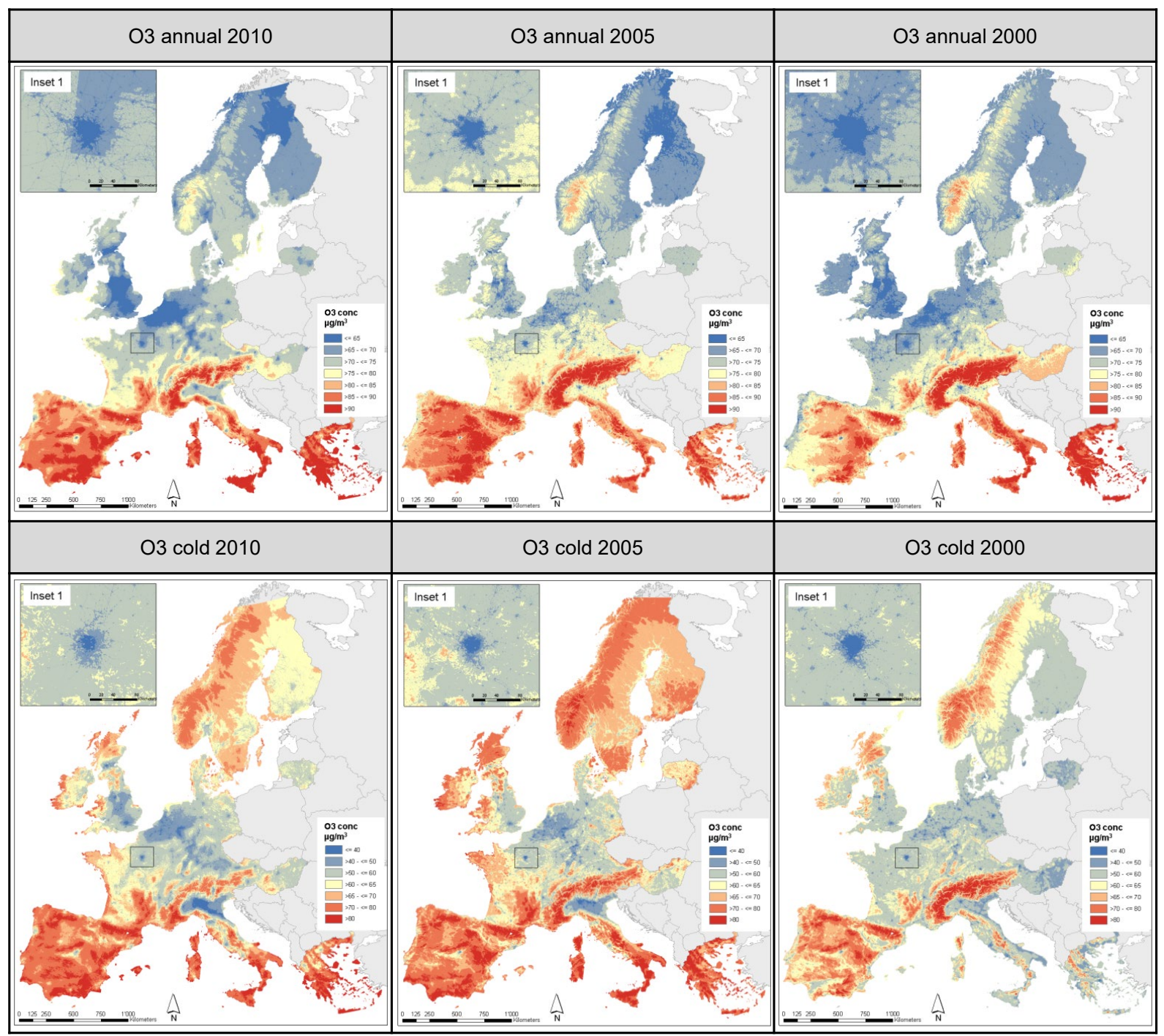

Figure S4. Mapping of FULL hybrid LUR models for $\mathrm{O}_{3}$ annual and warm season $(2010,2005$, 2000). 


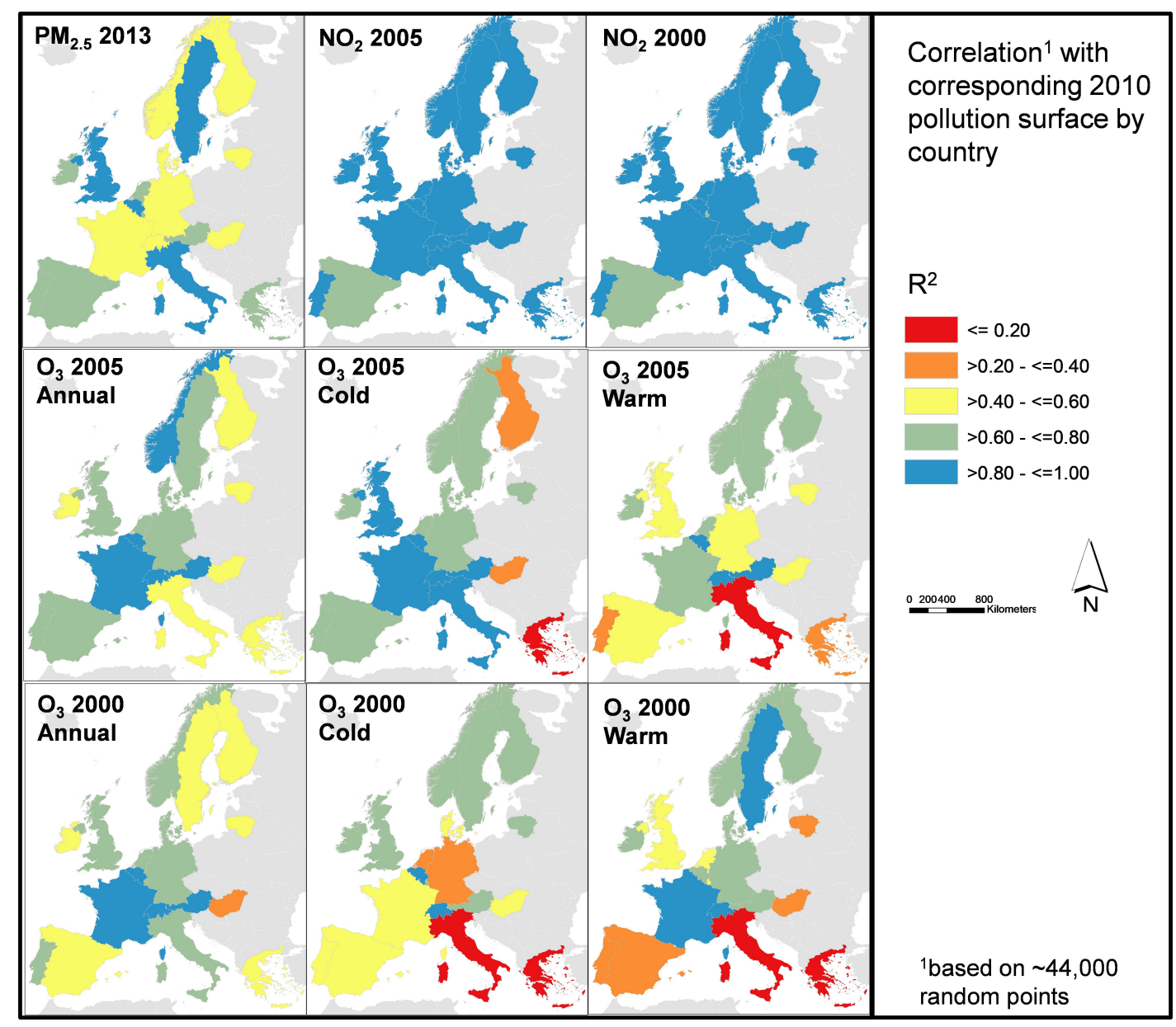

Figure S5. Stability analysis at country level 


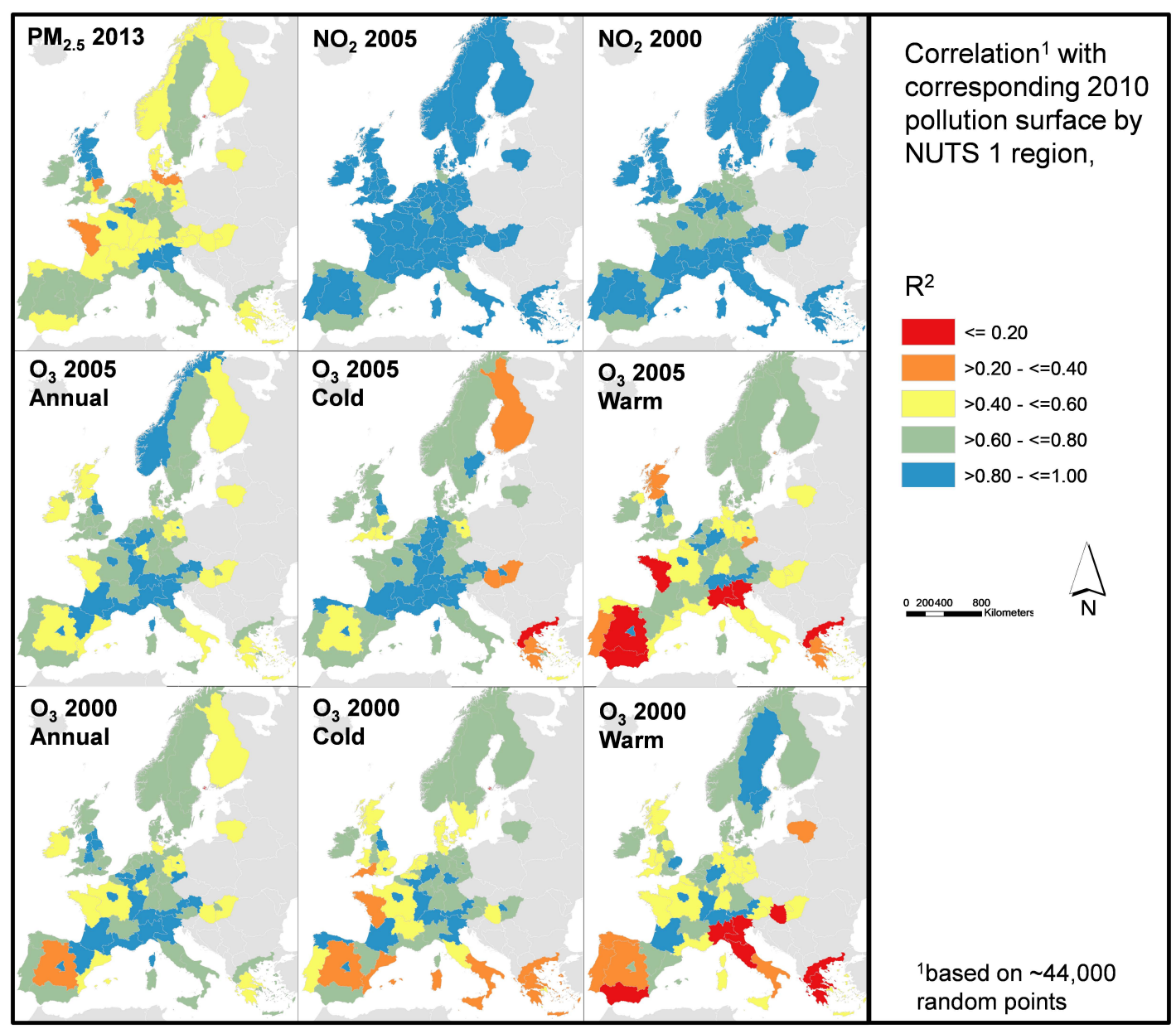

Figure S6. Stability analysis at NUTS 1 level (on average $\sim 600$ random points per NUTS1 area) 


\section{Chapter}

\section{4}

\section{A Comparison of Linear Regression, Regularization, and Machine Learning Algorithms to Develop Europe-Wide Spatial Models of Fine Particles and Nitrogen Dioxide}

Jie Chen, Kees de Hoogh, John Gulliver, Barbara Hoffmann, Ole Hertel, Matthias Ketzel, Mariska Bauwelinck, Aaron van Donkelaar, Ulla A. Hvidtfeldt, Klea Katsouyanni, Nicole AH Janssen, Randall V. Martin, Evangelia Samoli, Per E. Schwartz, Massimo Stafoggia, Tom Bellander, Maciej Strak, Kathrin Wolf, Danielle Vienneau, Roel Vermeulen, Bert Brunekreef and Gerard Hoek

Environment International, 2019, 130: 104934

DOI: 10.1016/j.envint.2019.104934 


\begin{abstract}
Empirical spatial air pollution models have been applied extensively to assess exposure in epidemiological studies with increasingly sophisticated and complex statistical algorithms beyond ordinary linear regression. However, different algorithms have rarely been compared in terms of their predictive ability.
\end{abstract}

This study compared 16 algorithms to predict annual average fine particle $\left(\mathrm{PM}_{2.5}\right)$ and nitrogen dioxide $\left(\mathrm{NO}_{2}\right)$ concentrations across Europe. The evaluated algorithms included linear stepwise regression, regularization techniques and machine learning methods. Air pollution models were developed based on the 2010 routine monitoring data from the AIRBASE dataset maintained by the European Environmental Agency (543 sites for $\mathrm{PM}_{2.5}$ and 2399 sites for $\mathrm{NO}_{2}$ ), using satellite observations, dispersion model estimates and land use variables as predictors. We compared the models by performing five-fold cross-validation $(\mathrm{CV})$ and by external validation (EV) using annual average concentrations measured at $416\left(\mathrm{PM}_{2.5}\right)$ and 1396 sites $\left(\mathrm{NO}_{2}\right)$ from the ESCAPE study. We further assessed the correlations between predictions by each pair of algorithms at the ESCAPE sites.

For $\mathrm{PM}_{2.5}$, the models performed similarly across algorithms with a mean $\mathrm{CV} \mathrm{R}^{2}$ of 0.59 and a mean $\mathrm{EV} \mathrm{R}^{2}$ of 0.53 . Generalized boosted machine, random forest and bagging performed best $\left(\mathrm{CV} \mathrm{R}^{2} \sim 0.63\right.$; EV R ${ }^{2}$ 0.58-0.61), while backward stepwise linear regression, support vector regression and artificial neural network performed less well (CV R ${ }^{2} 0.48-0.57$; EV R ${ }^{2}$ 0.39-0.46). Most of the $\mathrm{PM}_{2.5}$ model predictions at ESCAPE sites were highly correlated $\left(\mathrm{R}^{2}>0.85\right.$, with the exception of predictions from the artificial neural network). For $\mathrm{NO}_{2}$, the models performed even more similarly across different algorithms, with $\mathrm{CV} \mathrm{R}^{2} \mathrm{~s}$ ranging from 0.57 to 0.62 , and $\mathrm{EV} \mathrm{R}^{2} \mathrm{~s}$ ranging from 0.49 to 0.51 . The predicted concentrations from all algorithms at ESCAPE sites were highly correlated $\left(\mathrm{R}^{2}>0.9\right)$. For both pollutants, biases were low for all models except the artificial neural network. Dispersion model estimates and satellite observations were two of the most important predictors for $\mathrm{PM}_{2.5}$ models whilst dispersion model estimates and traffic variables were most important for $\mathrm{NO}_{2}$ models in all algorithms that allow assessment of the importance of variables.

Different statistical algorithms performed similarly when modelling spatial variation in annual average air pollution concentrations using a large number of training sites. 


\section{Abbreviations}

\begin{tabular}{ll}
\hline ANN & Artificial Neural Network \\
BLR & Backward stepwise Linear Regression \\
CTM & Chemical Transport Models \\
CV & Cross Validation \\
DSA & Deletion/ Substitution/ Addition \\
EN25/50/75 & Elastic Net with $\alpha=0.25 / 0.50 / 0.75$ \\
EV & External Validation \\
FLR & Forward stepwise Linear Regression \\
GAM & Generalized Additive Model \\
GBM & Generalized Boosted Machine \\
KRLS & Kernel-based Regularized Least Squares \\
LASSO & Least Absolute Shrinkage and Selection Operator \\
LUR & Land Use Regression \\
NO 2 & Nitrogen dioxide \\
PM 2.5 & Particulate Matter with an aerodynamic diameter smaller than 2.5 micrometers \\
RF & Random Forest \\
RMSE & Root-mean-square error \\
SAT & Satellite-derived \\
SLR & Supervised Linear Regression \\
SVR & Support Vector Regression \\
WLR & Stepwise Linear Regression \\
\hline
\end{tabular}

\section{Introduction}

Research in developed countries is currently focusing on health effects of long-term exposure to ambient air pollution at low concentrations, where the concentration contrast is small (Beelen et al., 2014; Di et al., 2017; Pinault et al., 2017). In order to do so, accurately assessing exposure for study subjects is particularly important.

Land Use Regression (LUR) models are frequently used to assess air pollution exposure in epidemiological studies on long-term health effects of air pollution. These are empirical models derived by combining air pollution concentrations monitored at a limited number of locations and potential predictor variables collected in a geographic information systems (GIS) (Hoek, 2017). In a LUR model, a linear regression with an automatic variable selection algorithm has often been used to maximize the within-sample explained variation of measured air pollution concentrations (Crouse et al., 2009; Hoek et al., 2008). Some LUR models are fitted with a supervised linear regression algorithm to include only predictor variables following the plausible direction of effect, e.g. a positive traffic slope, in order to increase the physical interpretability and potentially transferability of the models (Brauer et al., 2003; Briggs et al., 1997; Henderson et al., 2007).

There are several concerns about the standard linear regression algorithm. One is that the algorithm may overfit the data when there are relatively few monitoring sites to train a model and a large number of potential predictor variables offered (Basagaña et al., 2012; Friedman et al., 2001; Wang et al., 2012). Second, the algorithm may fail to capture potentially complex relationships within the data, since it assumes the relationship between air pollution and a predictor is linear across the whole range of the predictor values, and the impacts of different predictors to be independent (no interaction) (Friedman et al., 2001; Tibshirani et al., 2013). Third, the algorithm may result in unstable and uninterpretable 
coefficient estimates when highly correlated predictors are included in one model (Crouse et al., 2009; Tibshirani et al., 2013).

A number of different algorithms beyond standard linear regression have increasingly been applied to fit LUR models in air pollution exposure assessment. The Deletion/ Substitution/ Addition (DSA) algorithm selects the subset of predictors that minimize the cross-validation mean squared errors (Basagaña et al., 2012; Beckerman et al., 2013). The Generalized Additive Model (GAM) algorithm estimates nonlinear relationships between air pollution and predictors (Y. Liu et al., 2009). The LASSO (Least Absolute Shrinkage and Selection Operator) algorithm addresses collinearity by shrinking the coefficients of correlated predictors toward zero (Kim et al., 2016). Machine learning algorithms can detect previously unknown relationships within the data by modelling nonlinearity and interactions. Applications of the machine learning algorithms include Artificial Neural Network (ANN) (Di et al., 2016b; Zou et al., 2015), Random Forests (RF) (Brokamp et al., 2018; Hu et al., 2017; Zhan et al., 2018), Support Vector Regression (SVR) (de Hoogh et al., 2018b; Stafoggia et al., 2017; Van den Bossche et al., 2018), Generalized Boosted Machine (GBM) (Reid et al., 2015; Zhan et al., 2017), and Kernel-based Regularized Least Squares (KRLS) (Weichenthal et al., 2016).

Few studies have compared the performance of different algorithms in building LUR models for exposure assessment. The generalized boosted machine algorithm outperformed 10 other algorithms in a study modelling spatiotemporal variation of daily $\mathrm{PM}_{2.5}$ concentrations during wildfires (Reid et al., 2015). The random forest algorithm outperformed the linear stepwise regression algorithm in a study modelling spatial variation of $\mathrm{PM}_{2.5}$ and its components (Brokamp et al., 2017). In other studies modelling spatial variation of air pollution concentrations, similar performance was found using the Deletion/ Substitution/ Addition algorithm and the linear regression algorithm (Basagaña et al., 2012), the Kernel-based Regularized Least Squares algorithm and the linear regression algorithm (Weichenthal et al., 2016), the linear regression algorithm, the LASSO algorithm and the Support Vector Regression algorithm (Van den Bossche et al., 2018). Only modest differences in performance across algorithms were found in a recent comprehensive algorithm comparison study based on mobile monitoring of ultrafine particles (Kerckhoffs et al., 2019).

Most of the previous air pollution exposure assessment studies evaluated model performance based on cross-validation, which assesses a model's predictive ability within the monitoring domain. For crossvalidation to be meaningful, the monitoring sites need to be representative of the locations to which the model is applied (e.g. residential addresses versus routine monitoring sites other than in residential areas or on-road mobile monitoring). A model that performs well in cross-validation does not necessarily transfer well to application in epidemiological studies (Hystad et al., 2011; Kerckhoffs et al., 2016). Therefore, it is valuable to evaluate models using pollution data collected from monitoring sites which represent the application locations.

We have recently developed spatial air pollution models across Europe, using a Supervised Linear Regression (SLR) algorithm (de Hoogh et al., 2018a). The aim of the current study was to compare 16 different algorithms, including the SLR, in their ability to predict spatial variation of $\mathrm{PM}_{2.5}$ and $\mathrm{NO}_{2}$ concentrations across Europe. To strengthen our comparisons, we used two ground-based monitoring datasets to perform both cross-validation (AIRBASE dataset; EEA) and external validation (ESCAPE (European Study of Cohorts for Air Pollution Effects) dataset) (Cyrys et al., 2012; Eeftens et al., 2012). The selected algorithms follow a recent evaluation of model development of mobile monitoring data by our group (Kerckhoffs et al., 2019).

\section{Methods}

Measured air pollution concentration data and GIS predictor variables were the same as in our recently published Europe-wide modelling study (de Hoogh et al., 2018a). 


\subsection{Air pollution monitoring data}

To build the spatial empirical models, we used annual mean concentrations for $\mathrm{PM}_{2.5}$ (available for 543 sites) and $\mathrm{NO}_{2}$ (available for 2399 sites) for 2010 from the AIRBASE v8 dataset (EEA, 2015) (Figure S1). AIRBASE is a database maintained by the European Environmental Agency (EEA) containing monitoring data reported by EU member states and associated countries. Air pollution data are from routine regulatory networks in individual countries, measured by a diversity of methods. The monitoring locations are chosen to check for compliance with the European Union air quality standards (http://ec.europa.eu/environment/air/quality/standards.htm) at background sites, near busy roads or in industrial zones. The annual mean concentrations were aggregated by EEA based on the primary observations uploaded by countries and successfully tested by automated quality control. The primary observations were reported with different frequency (hour, day, or week). Based on the frequency of reported air pollution values, an annual average was calculated only when valid measurements coverage $\geq 75 \%$ of a year. AIRBASE monitoring sites were randomly divided into five groups $(20 \%$ each), stratified by site type and region (de Hoogh et al., 2018a). Main models were built using all measurements (100\% sites) in the AIRBASE dataset. Each of the 5 hold-out validation models was developed based on $80 \%$ of the monitoring sites, with the remaining $20 \%$ used for validation.

Air pollution monitoring data from the ESCAPE study were used for external validation. Three 2weekly measurement campaigns were held at 416 monitoring sites for $\mathrm{PM}_{2.5}$ and 1396 sites for $\mathrm{NO}_{2}$, using Harvard Impactors and Ogawa badges respectively (Cyrys et al., 2012; Eeftens et al., 2012). The annual mean concentrations reflecting the period 2009-2010 were derived based on measurements in the three seasons with temporal adjustment. Measurement sites in ESCAPE were specifically selected to represent spatial variation of air pollution at home addresses of subjects in the included cohorts, thus the monitoring sites were clustered (Fig S1).

Summary statistics of the training and validation datasets are presented in Table S1.

\subsection{Predictor variables}

Potential predictor variables used in this study are described in more detail elsewhere (de Hoogh et al., 2016; de Hoogh et al., 2018a; Vienneau et al., 2013). The predictor variables are integrated into a 100 $\mathrm{m}$ gridded GIS database covering Western Europe. All potential predictor variables and summary statistics are shown in Table S2. We offered 150 potential predictor variables.

\subsubsection{Satellite-derived air pollution estimates and chemical transport model estimates}

Satellite-derived (SAT) estimates of $\mathrm{PM}_{2.5}$ were obtained from global datasets (V3.GL.01; Van Donkelaar et al., 2015). A gridded surface of the 2010 annual average $\mathrm{PM}_{2.5}$ was produced at a $0.1^{\circ} \mathrm{X}$ $0.1^{\circ}(\sim 10 \times 10 \mathrm{~km})$ resolution by relating aerosol optical depth (AOD) retrievals from the NASA MODIS (Moderate Resolution Imaging Spectroradiometer), MISR (Multi-angle Imaging Spectroradiometer) and SeaWiFS (Sea-viewing Wide Field-of-view Sensor) instruments to nearsurface concentrations using aerosol vertical profiles and scattering properties simulated by the GEOSChem chemical transport model. For $\mathrm{NO}_{2}$, SAT estimates for 2010 were derived from the tropospheric $\mathrm{NO}_{2}$ columns measured with the OMI (Ozone Monitoring Instrument) on board the Aura satellite. The satellite column-integrated retrievals were related to ground-level concentrations using the global GEOS-Chem model to produce a $10 \times 10 \mathrm{~km}$ resolution dataset (Bechle et al., 2013, 2015; Novotny et al., 2011).

Annual $\mathrm{PM}_{2.5}$ and $\mathrm{NO}_{2}$ chemical transport models (CTM) estimates for 2010 were derived from the MACC-II ENSEMBLE model at a $0.1^{\circ}$ x $0.1^{\circ}(\sim 10 \times 10 \mathrm{~km})$ resolution (Inness et al., 2013). In the ENSEMBLE model, the median value of seven individual regional CTMs (CHIMERE, EMEP, EURAD, LOTOS-EUROS, MATCH, MOCAGE and SILAM) was provided for each pixel. 


\subsubsection{Traffic, land use and altitude predictors}

Road data were extracted from the 1:10,000 EuroStreets digital road network (version 3.1 based on TeleAtlas MultiNet TM, year 2008), classified into 'all' and 'major' roads. These were then intersected with a $100 \mathrm{~m}$ base polygon and the sum of the road lengths was calculated within each grid cell.

The European Corine Land Cover 2006 dataset (ETC-LC, 2009) was used to extract land cover variables for all study areas except for Greece, which has missing data. We used the Corine Land Cover 2000 (ETC-LC, 2013) to extract data for Greece. Six main groups (residential, industry, ports, urban green space, total built up land and natural land) were derived from the initial 44 land classes. A moving window procedure was used to calculate both road and land cover data for selected radii, which ranged from $50 \mathrm{~m}$ to $10,000 \mathrm{~m}$ (Focalstatistics using sum with a circle).

Elevation was obtained from the SRTM Digital Elevation Database version 4.1 with a resolution of 3 arc second (approximately $90 \mathrm{~m}$ ) with vertical error of $<16 \mathrm{~m}$ (CGIAR-CSI).

\subsection{Model development}

We applied 16 statistical algorithms to build the models. These algorithms cover almost all algorithms applied in previous LUR models and have been assessed in a model comparison paper using mobile monitoring ultrafine particle concentration data (Kerckhoffs et al., 2019).

For each algorithm, 6 models ( 1 main model plus 5 hold-out validation models) were developed for both pollutants (see Section 2.1). We used grid search to optimize hyperparameters (whose values were set before the training process) for each model, based on the minimum mean cross-validated error. This approach helped to minimize the risk of overfitting and ensured that the models we derived had the best predictive power. Hyperparameters for each algorithm were specified in Table S3.

Linear stepwise regression algorithms assume that the relationships between the pollutants and the predictors are linear and additive (Tibshirani et al., 2013). We used 3 automatic variable selection methods to choose the best subset of predictors. Forward stepwise Linear Regression (FLR) started with a null model, then the predictor that generated the highest increase in the adjusted $\mathrm{R}^{2}$ was added to the model at each subsequent step. This process was repeated until the model adjusted $\mathrm{R}^{2}$ stopped maximizing. Backward stepwise Linear Regression (BLR) began with all variables in the model and deleted the variable with the highest $\mathrm{P}$-value one at a time. The procedure stopped when it generated a model that had only significant predictors (significance level of 0.1 ) with the maximum adjusted $\mathrm{R}^{2}$. Stepwise Linear Regression (WLR) allowed variables to be added or deleted as modelling progresses. The algorithm started off in a forward approach with a null model, and then removed variables if they became statistically insignificant (significance level of 0.1). We also used a Supervised Linear Regression (SLR) algorithm that was described previously in de Hoogh et al. (2018a). In this algorithm, a univariate linear regression model was run for each potential predictor to choose the model with the highest adjusted $\mathrm{R}^{2}$ as the starting point. Additional significant predictor variables were allowed to enter the model if they added to the adjusted $\mathrm{R}^{2}$ of the previous model step, and only if they adhered to the plausible direction of effect. Variables with variance inflation factor (VIF) larger than 3 were removed from the model to avoid multicollinearity.

Regularization or shrinkage algorithms are used to estimate reliable predictor coefficients when the predictors are highly correlated. By imposing different penalties, ridge regression keeps all predictors in the final model, while LASSO ensures sparsity of the results by shrinking some coefficients exactly to zero. Elastic Net is a hybrid of ridge regression and LASSO by adjusting the values of hyperparameter $\alpha$ (Friedman et al., 2009). Elastic net is the same as lasso when $\alpha=1$, it approaches ridge regression as $\alpha$ reduces towards 0. In this study, $\alpha=0.25$ (EN25), 0.5 (EN50) and 0.75 (EN75) were used to build separate elastic net models.

The Generalized Additive Model (GAM) algorithm (Wood and Wood, 2015) extends the standard linear regression by introducing non-linear functions for predictors while keeping the additive 
assumption. We used "gam" function in the "mgcv" R package, which performs automatic smoothing parameter estimation and allows adding an extra penalty to remove redundant variables from the model. A smoothing spline was fit for potential predictors with at least 5 unique values. Variables with less than 5 differing values, i.e. the land use variables in the smallest buffers (TBU50, NAT50, IND50, POR50, UGR50, RES50), were deleted because the function could not estimate the smoothing parameters for them. The roughness of the smoothing spline was selected via restricted maximum likelihood method (REML).

Machine learning algorithms are able to model nonlinearity as well as the potentially complex interactions among predictors. One type of machine learning algorithms is the ensemble learning machine based on decision trees. Bagging, also known as the bootstrap aggregation, repeatedly draws separate subsets from the full training dataset. The final predictions were calculated by averaging the results from all the decision trees built on bootstrapped training subsets. Random Forest (RF) (Breiman et al., 2011) adds an additional layer of randomness to bagging by forcing each split to consider only a randomly chosen subset of candidate predictors, instead of the full set. Instead of building independent trees using bootstrapped samples, Generalized Boosted Machine (GBM) (Ridgeway et al., 2013) grows trees sequentially: each tree is fit on the residuals of the given model. Other types of machine learning algorithms include the Support Vector Regression (SVR) algorithm (Friedman et al., 2001; Meyer et al., 2017), which uses kernel functions to enlarge the feature space and produces non-linear boundaries by constructing a linear boundary in a transformed high-dimensional feature space; the Kernel-based Regularized Least Squares (KRLS) (Ferwerda et al., 2017; Hazlett and Hainmueller 2017) algorithm, whose kernel function measures the similarity between covariates while the regularization imposes a preference for a smoother function; and the Artificial Neural Network (ANN)(Ripley et al., 2016) algorithm, which consist of interconnected "neurons" (represent predictors) in layers that can account for possible nonlinearities and interactions.

We additionally use two approaches to make ensemble predictions that leverage information from all models. In Ensemble 1 model, the median value of 16 model predictions was provided for each site (Inness et al., 2013). In Ensemble 2 model, a weighted average of the 16 model predictions was provided for each site. The weight $\left(w_{i}\right)$ of each model was calculated based on the inverse of average crossvalidation absolute bias at all AIRBASE sites. The ensemble prediction at each site $(\hat{y})$ was defined as:

$$
\hat{y}=\frac{\sum_{i=1}^{16} w_{i} y_{i}}{\sum_{i=1}^{16} w_{i}}
$$

where $y_{i}$ is the prediction of the individual models.

\subsection{Model evaluation and comparison}

We evaluated model performance by regression-based $R^{2}\left(R^{2}\right)$, mean square error based $R^{2}\left(M S E-R^{2}\right)$, and root-mean-square error (RMSE). $\mathrm{R}^{2}$ was derived from correlations between predicted and observed values. MSE- $\mathrm{R}^{2}$ can be seen as a rescaling of MSE. It measures fit about the 1:1 line rather than fit about the best fit line in regression-based $\mathrm{R}^{2}$. The formula was defined as:

$$
\text { MSE }-\mathrm{R}^{2}=1-\frac{M S E}{\left(\frac{1}{n} \sum_{i=1}^{n}\left(y_{i}-\bar{y}\right)^{2}\right)}
$$

where $\bar{y}$ is the average of the observed values. MES- $\mathrm{R}^{2}$ can yield negative values when the average of the observed values performs better than the predictions of the model (Wang et al., 2012). RMSE was computed as the square root of the mean of squared difference between predicted and observed values. Training $\mathrm{R}^{2}$ and RMSE were calculated by comparing the predictions and the observations at all AIRBASE sites. Cross-validated (CV) $\mathrm{R}^{2}, \mathrm{MSE}^{2} \mathrm{R}^{2}$, and RMSE were computed by comparing the assembled predictions at 5 held-out sets to the corresponding observations at AIRBASE sites (see section 2.1). External-validated (EV) $\mathrm{R}^{2}$, MSE-R ${ }^{2}$, and RMSE were computed by comparing the 
predictions, which were derived from the main models, and the observations at all ESCAPE sites. In external validation, bias (mean difference between predictions and measurements) was additionally calculated for each model to evaluate the transferability of models.

We also evaluated model performance in subsets of ESCAPE sites, including areas with low air pollution concentrations (annual average concentration below 10, 12, 15, 20 and $25 \mu \mathrm{g} / \mathrm{m}^{3}$ for $\mathrm{PM}_{2.5}$; annual average concentration below 20,30 and $40 \mu \mathrm{g} / \mathrm{m}^{3}$ for $\mathrm{NO}_{2}$ ), sites of different types (street, rural background and urban background), and different regions (north, west, central, and south). Externalvalidated $\mathrm{R}^{2}$, RMSE and bias were calculated for each evaluation.

The predicted concentrations can correlate poorly between models even if the models have similar explained variance $\left(\mathrm{R}^{2}\right)$. Therefore, for each pair of models, scatter plots of predicted concentrations at all ESCAPE sites were made to visually assess the correlations at these independent locations. Pearson correlation coefficients were also calculated. Scatter plots of the predictions versus observations at ESCAPE sites were also made for each of the models.

We compared the structure of models by looking at the number of predictors included in a model and the direction and magnitude of coefficients, where applicable. In linear regression models, regression slopes were multiplied by the difference between the $1^{\text {st }}$ and $99^{\text {th }}$ percentile of each predictor to allow comparison across predictors.

\subsection{Sensitivity analysis}

\subsection{1 $\mathrm{NO}_{2}$ models based on a reduced number of sites}

The main $\mathrm{NO}_{2}$ models were built on 2399 monitoring sites while the $\mathrm{PM}_{2.5}$ models were developed on 543 sites. To separate the impacts of the number of training sites and the differences in pollutant characteristics, we built additional $\mathrm{NO}_{2}$ models using a random subset of 543 measurements extracted from all AIRBASE $\mathrm{NO}_{2}$ monitoring sites (stratified by region and site type). The $\mathrm{NO}_{2}$ sample models were developed and evaluated by the methods described above.

\subsubsection{Models with a reduced number of potential predictors}

Three variable selection methods were applied to explore the effect of the number of potential predictors offered. For each set of potential predictors derived from the methods described below, we arbitrarily selected two linear regression-based algorithms (SLR and Elastic Net $(\alpha=0.75)$ ) and two machine learning algorithms (RF and $\mathrm{ANN}$ ) to fit the models. The training $\mathrm{R}^{2}, \mathrm{CV} \mathrm{R}^{2}$ and $\mathrm{EV} \mathrm{R}^{2}$ were calculated for each model.

Firstly, the predictor variables were ranked by their absolute correlation coefficients with pollutant concentrations, based on univariate correlation. In separate models, the first 80, 40 and 20 variables with the highest absolute correlation were used as potential predictor variables.

Secondly, the predictor variables were ranked by their variable importance, calculated as percentage increase in mean squared errors after a random permutation of the values of a variable, derived from the RF algorithm. In separate models, the first 80,40 and 20 variables were used as potential predictor variables.

Finally, we reduced the number of buffers for road length and land use variables. Only variables with radii of $50 \mathrm{~m}, 100 \mathrm{~m}, 300 \mathrm{~m}, 500 \mathrm{~m}, 1000 \mathrm{~m}, 2000 \mathrm{~m}, 5000 \mathrm{~m}, 10000 \mathrm{~m}$ were offered as potential predictors, resulting in a total of 64 predictors.

\subsection{3 $\mathrm{PM}_{2.5}$ models with kriging}

In the recently published Europe-wide modelling study (de Hoogh et al., 2018a), kriging proved an efficient technique to explain a part of residual spatial variation for the $\mathrm{PM}_{2.5}$ SLR model. To examine whether the residual variation explained by kriging had been captured by a more flexible algorithm, we performed kriging on the residuals from the selected $\mathrm{PM}_{2.5}$ models (BLR, SLR, LASSO, GBM and 
ANN). Ordinary kriging was applied to the residuals of background sites only, and added to the pollution estimates of the models. Models were evaluated by the metrics described in section 2.4. Scatter plots comparing the predictions at all ESCAPE sites were made, and Pearson correlation coefficients were calculated.

All statistical analyses were conducted in R v 3.4.1(Team, 2013).

\section{Results}

\subsection{PM 2.5 models}

All models had moderate to good performance when evaluated by cross-validation (CV), with $\mathrm{CV}^{2} \mathrm{~S}$ ranging from 0.48 to 0.63 , and CV RMSEs ranging from 3.1 to $3.9 \mu \mathrm{g} / \mathrm{m}^{3}$ (Table 1). CV MSE- $\mathrm{R}^{2} \mathrm{~s}$ were similar as $C V R^{2} s$. Higher $C V R^{2} s$ and lower CV RMSEs were found for machine learning models based on decision trees (the GBM, the bagging, and the RF). The lowest $C V \mathrm{R}^{2}$ and the highest $\mathrm{CV}$ RMSE were found for the ANN model. Among all linear regression-based models, the BLR model had the lowest $\mathrm{CV} \mathrm{R}^{2}$ and the highest CV RMSE, while it had the highest training $\mathrm{R}^{2}$ among these linear models.

Model performance measured by external validation (EV) showed good agreement with the results measured by $\mathrm{CV}$, though less of the variation $\left(\mathrm{R}^{2}\right)$ in the external data was explained (Table 1). MSE$\mathrm{R}^{2} \mathrm{~s}$ were on average $5 \%$ lower than the $\mathrm{R}^{2} \mathrm{~s}$. The decision tree-based ensemble models performed moderately better than others whilst the BLR, the SVR, and the ANN models performed moderately worse. Biases were lower than $1 \mu \mathrm{g} / \mathrm{m}^{3}$ for all models, except the ANN. The better performance of the decision tree-based models disappeared when restricting validation dataset to sites with low $\mathrm{PM}_{2.5}$ concentrations (Table S4). For all algorithms, validation $\mathrm{R}^{2}$ decreased and bias increased when restricting to lower pollution levels. Similar differences in model performance across algorithms were observed for street and urban background sites (Table S5). For all algorithms, bias was higher for background sites than for street sites. The pattern between algorithms was similarly when evaluated at regional scale (Table S6). We noted only small differences of both ensemble models performances compared to the best individual models.

Figure 1 shows the scatter plots of $\mathrm{PM}_{2.5}$ predictions at all ESCAPE sites by each pair of models. Most of the predicted concentrations were highly correlated, with correlation coefficients above 0.85 . Almost identical predictions were found for several models, such as the LASSO and three Elastic Net models, the FLR and the WLR models, as well as the Bagging and the RF models. Predictions by the ANN model and other models were less correlated. All models tended to overpredict at low concentrations and underpredict at high concentrations (Figure S2).

For linear regression-based models (except for the Ridge regression model), the number of predictors included in the main model, the direction and magnitude of regression slope are summarized in Figure 2 (details shown in Table S7). The SLR model included the lowest number of predictors (7) in the model while the BLR retained the highest number (48). All models included CTM and SAT estimates as well as all roads, natural areas, ports, residential areas, and altitude as predictors. CTM and SAT estimates were positively correlated with $\mathrm{PM}_{2.5}$ in all linear regression models, while altitude was always negatively correlated with $\mathrm{PM}_{2.5}$. All models except the SLR model included predictors with counterintuitive direction of slope, such as negative slopes for road length and ports. CTM and SAT estimates were consistently two of the most important predictors in all models, as shown by the large regression slopes, except in the BLR model. CTM and SAT estimates were also identified as the most important predictors in variable importance plots from the RF model and the GBM model, followed by altitude (Figure S4). A rapid drop in variable importance was observed after CTM and SAT estimates. 
Table 1. Performance of $\mathrm{PM}_{2.5}$ spatial models using different model building algorithms

\begin{tabular}{|c|c|c|c|c|c|c|c|c|c|}
\hline \multirow{2}{*}{ Algorithm $^{\mathrm{a}}$} & \multicolumn{2}{|c|}{$\begin{array}{l}\text { Training } \\
(\mathrm{N}=543)\end{array}$} & \multicolumn{3}{|c|}{$\begin{array}{l}\text { Cross Validation } \\
\qquad(\mathrm{N}=543)\end{array}$} & \multicolumn{4}{|c|}{$\begin{array}{l}\text { External Validation } \\
\qquad(\mathrm{N}=416)\end{array}$} \\
\hline & $\mathrm{R}^{2}$ & $\begin{array}{l}\mathrm{RMSE}^{\mathrm{b}} \\
\left(\mu \mathrm{g} / \mathrm{m}^{3}\right)\end{array}$ & $\mathrm{R}^{2}$ & MSE-R ${ }^{2}$ & $\begin{array}{l}\text { RMSE } \\
\left(\mu \mathrm{g} / \mathrm{m}^{3}\right)\end{array}$ & $\mathrm{R}^{2}$ & $\begin{array}{l}\mathrm{MSE}- \\
\mathrm{R}^{2}\end{array}$ & $\begin{array}{l}\text { RMSE } \\
\left(\mu \mathrm{g} / \mathrm{m}^{3}\right)\end{array}$ & $\begin{array}{c}\text { Bias } \\
\left(\mu \mathrm{g} / \mathrm{m}^{3}\right)\end{array}$ \\
\hline FLR & 0.657 & 3.0 & 0.600 & 0.598 & 3.3 & 0.517 & 0.481 & 4.1 & 0.7 \\
\hline BLR & 0.704 & 2.8 & 0.506 & 0.472 & 3.7 & 0.463 & 0.445 & 4.3 & 0.8 \\
\hline WLR & 0.657 & 3.0 & 0.600 & 0.598 & 3.3 & 0.517 & 0.481 & 4.1 & 0.7 \\
\hline SLR & 0.622 & 3.2 & 0.595 & 0.594 & 3.3 & 0.529 & 0.478 & 4.1 & 0.9 \\
\hline Ridge & 0.665 & 3.0 & 0.592 & 0.592 & 3.3 & 0.535 & 0.485 & 4.1 & 0.7 \\
\hline EN25 & 0.643 & 3.1 & 0.608 & 0.607 & 3.2 & 0.545 & 0.483 & 4.1 & 0.8 \\
\hline EN50 & 0.642 & 3.1 & 0.609 & 0.608 & 3.2 & 0.546 & 0.486 & 4.1 & 0.8 \\
\hline EN75 & 0.641 & 3.1 & 0.609 & 0.609 & 3.2 & 0.547 & 0.486 & 4.1 & 0.8 \\
\hline LASSO & 0.641 & 3.1 & 0.610 & 0.609 & 3.2 & 0.547 & 0.487 & 4.1 & 0.8 \\
\hline GAM & 0.652 & 3.0 & 0.608 & 0.608 & 3.2 & 0.557 & 0.498 & 4.1 & 0.9 \\
\hline Bagging & 0.954 & 1.2 & 0.627 & 0.626 & 3.1 & 0.575 & 0.531 & 3.9 & 0.4 \\
\hline $\mathrm{RF}$ & 0.955 & 1.2 & 0.626 & 0.624 & 3.1 & 0.583 & 0.530 & 3.9 & 0.4 \\
\hline GBM & 0.895 & 1.8 & 0.631 & 0.630 & 3.1 & 0.610 & 0.548 & 3.9 & 0.4 \\
\hline SVR & 0.799 & 2.3 & 0.569 & 0.568 & 3.4 & 0.457 & 0.432 & 4.3 & 0.3 \\
\hline KRLS & 0.726 & 2.7 & 0.590 & 0.586 & 3.3 & 0.525 & 0.466 & 4.2 & 0.6 \\
\hline ANN & 0.723 & 2.7 & 0.477 & 0.428 & 3.9 & 0.391 & 0.286 & 4.8 & 1.2 \\
\hline Ensemble1 & 0.698 & 2.8 & 0.618 & 0.617 & 3.2 & 0.553 & 0.495 & 4.1 & 0.7 \\
\hline Ensemble2 & 0.762 & 2.6 & 0.622 & 0.622 & 3.2 & 0.573 & 0.513 & 4.0 & 0.7 \\
\hline
\end{tabular}

${ }^{a}$ FLR $=$ Forward stepwise Linear Regression; BLR = Backward stepwise Linear Regression; WLM = Stepwise Linear Regression; SLR = Supervised Linear Regression; EN25 = Elastic Net with $\alpha=0.25$; EN50 = Elastic Net with $\alpha=0.50$; EN75 = Elastic Net with $\alpha=0.75 ;$ LASSO $=$ Least Absolute Shrinkage and Selection Operator; GAM $=$ Generalized Additive Model; RF $=$ Random Forest; GBM = Generalized Boosted Machine; SVR = Support Vector Regression; KRLS = Kernel-based Regularized Least Squares; ANN = Artificial Neural Network; Ensemble1 = Ensemble model based on median prediction; Ensemble2 $=$ Ensemble model based on weighted average

${ }^{\mathrm{b}} \mathrm{RMSE}=$ Root-mean-square error 


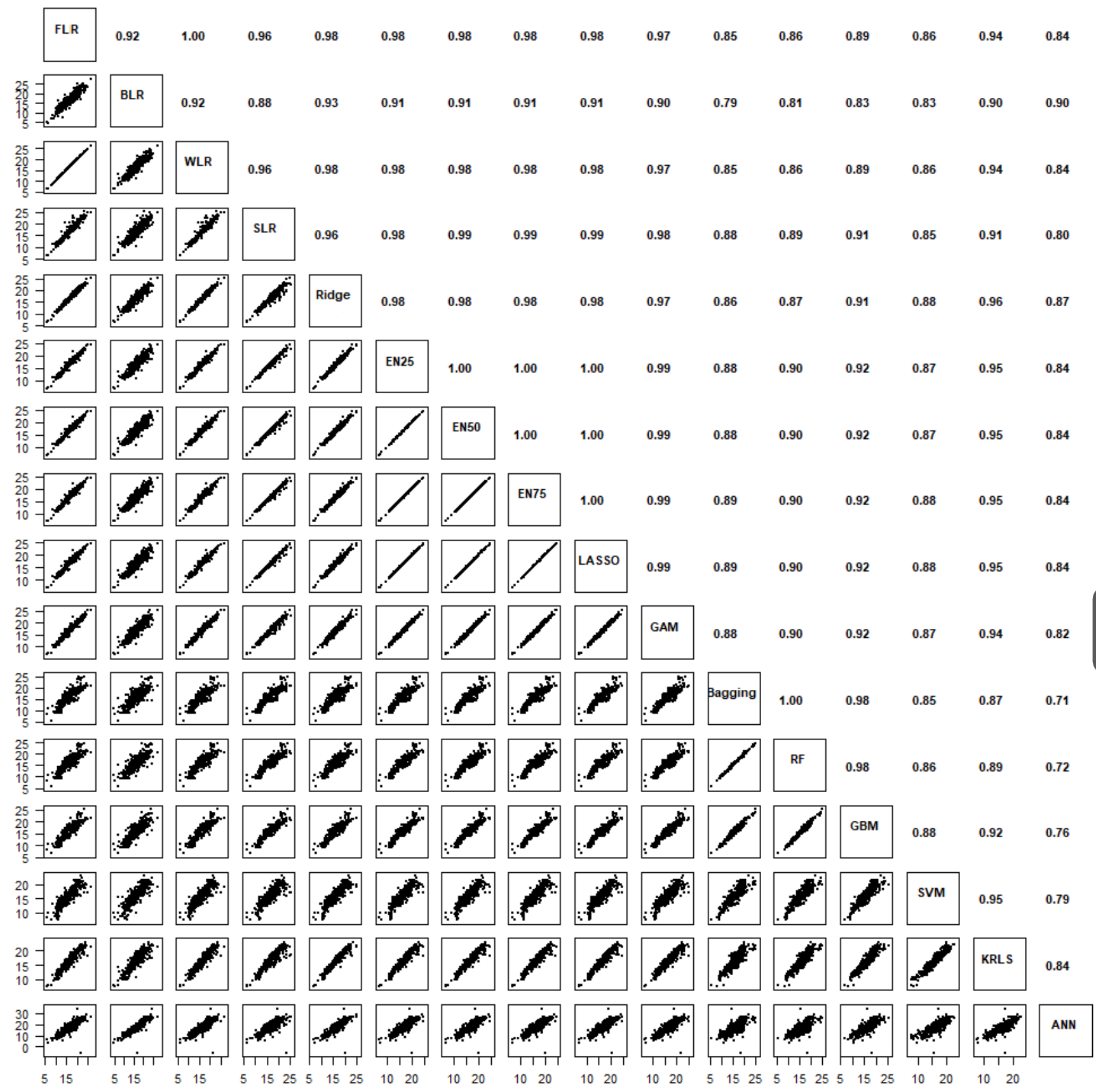

Figure 1. Correlations between $\mathrm{PM}_{2.5}$ predictions at ESCAPE sites. The upper triangle shows the correlation coefficients, the lower triangle shows the scatter plots

FLR $=$ Forward stepwise Linear Regression; BLR $=$ Backward stepwise Linear Regression; WLM $=$ Stepwise Linear Regression; SLR $=$ Supervised Linear Regression; EN25 = Elastic Net with $\alpha=0.25$; EN50 = Elastic Net with $\alpha=0.50$; EN75 = Elastic Net with $\alpha=0.75 ;$ LASSO $=$ Least Absolute Shrinkage and Selection Operator; GAM $=$ Generalized Additive Model; RF $=$ Random Forest; GBM $=$ Generalized Boosted Machine; SVR $=$ Support Vector Regression; KRLS = Kernel-based Regularized Least Squares; ANN = Artificial Neural Network 

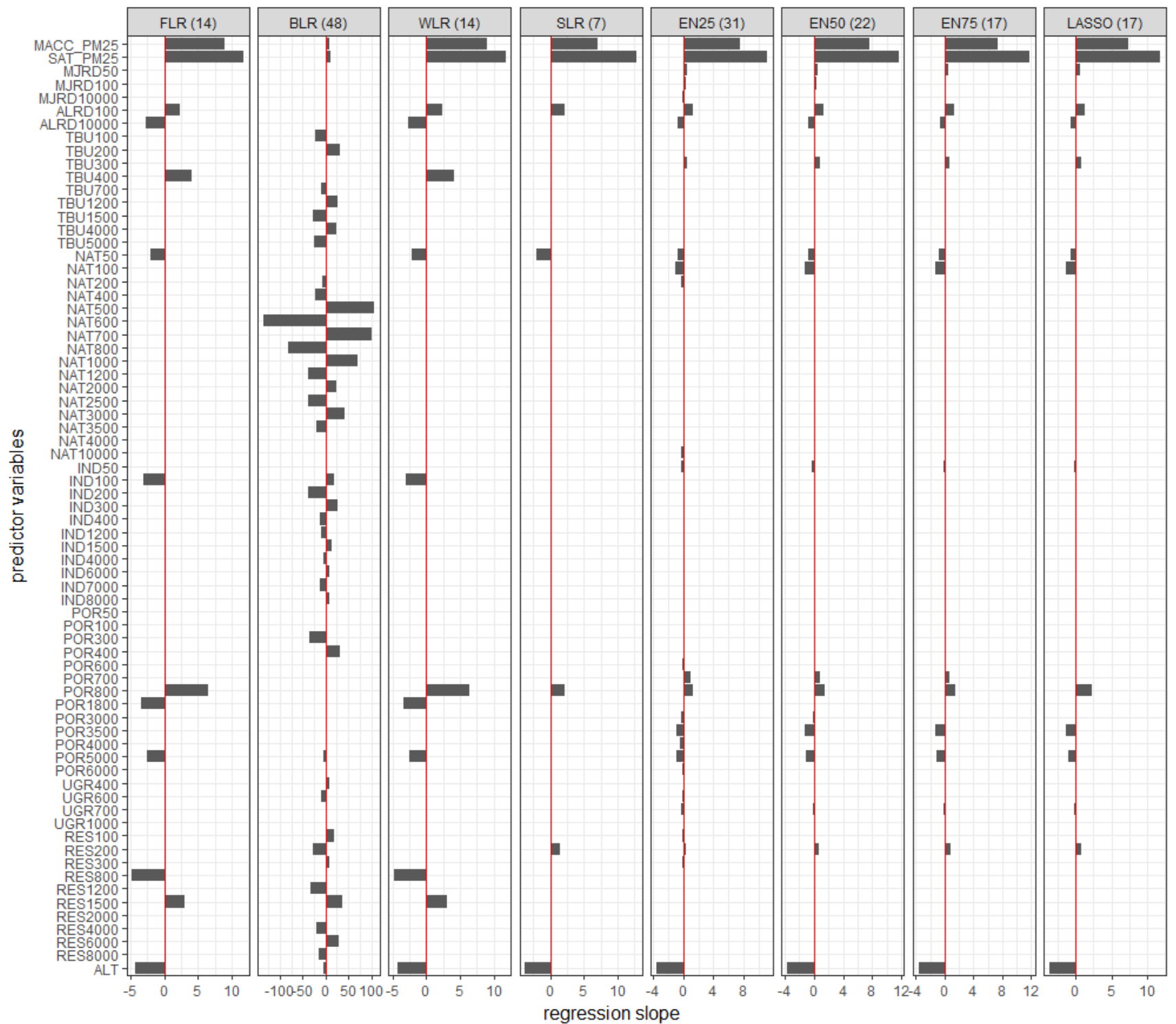

Figure 2. Predictors included in linear $\mathrm{PM}_{2.5}$ models. The figures in the blanket show the number of predictors included in each model

Regression slopes were multiplied by the difference between the 1th and 99th percentile of each predictor to allow comparison across predictors

MACC $=$ MACC dispersion model, $\mathrm{SAT}=$ Satellite-derived, MJRD $=$ Major Roads, ALRD $=$ All Roads, $\mathrm{TBU}=$ Total Build Up, NAT $=$ Natural Land, IND $=$ Industry, POR $=$ Ports, UGR $=$ Urban Green, RES $=$ Residential, ALT $=$ Altitude

FLR $=$ Forward stepwise Linear Regression; BLR $=$ Backward stepwise Linear Regression; WLM $=$ Stepwise Linear Regression; SLR $=$ Supervised Linear Regression; EN25 $=$ Elastic Net with $\alpha=0.25$; EN50 = Elastic Net with $\alpha=0.50$; EN75 $=$ Elastic Net with $\alpha=0.75 ;$ LASSO $=$ Least Absolute Shrinkage and Selection Operator 


\section{2 $\mathrm{NO}_{2}$ models}

Table 2 shows the performance of the different $\mathrm{NO}_{2}$ models. Though the non-linear models had higher training $\mathrm{R}^{2} \mathrm{~s}$ than the linear regression-based models, all models had similar performances when measured by cross-validation (CV R 20.57 to 0.62 , CV RMSE 9.0 to $9.6 \mu \mathrm{g} / \mathrm{m}^{3}$ ), and when measured by external validation (EV R ${ }^{2} 0.49$ to 0.51 , EV RMSE 11.6 to $14.6 \mu \mathrm{g} / \mathrm{m}^{3}$ ). Biases were low (1.2 to 3.3 $\left.\mu \mathrm{g} / \mathrm{m}^{3}\right)$ for all models except the ANN $\left(8.8 \mu \mathrm{g} / \mathrm{m}^{3}\right)$. Model performance also did not vary much across algorithms when restricting validation subsets to less polluted sites (Table S8) and specific type of sites (Table S9). For all algorithms, validation $\mathrm{R}^{2}$ decreased and bias increased when restricting to lower pollution levels (Table S8). For all algorithms, validation $\mathrm{R}^{2}$ was lower for street sites compared to background sites. A small negative bias was observed for street sites and a more substantial positive bias for background sites, again with small differences across algorithms. All algorithms performed similarly when evaluated at regional scale. Only small differences were found for both ensemble models performances compared to the best individual models.

The predictions at the ESCAPE sites by each pair of models were highly correlated, with Pearson correlation coefficients ranging from 0.91 to 1.00 (Figure 3). All models tended to overpredict at low concentrations and underpredict at high concentrations (Figure S3).

Even though the $\mathrm{NO}_{2}$ model predictions were similar, their structures were different (Figure 4 and Table S11). The SLR model included the lowest number of predictors (8), while the EN25 model included the highest number (55). CTM estimates were positively associated with $\mathrm{NO}_{2}$ in all models. All models included SAT estimates with a counterintuitive negative slope, except the SLR which did not include SAT at all. Counterintuitive slopes were also found for road length, ports, natural areas and residential areas in BLR model and regularization models. The variable importance plots derived from the RF and the GBM indicate that the CTM estimates and road variables were strong predictors (Figure S5).

\subsection{Sensitivity analysis}

\subsection{1 $\mathrm{NO}_{2}$ models based on a reduced number of sites}

The performances of $\mathrm{NO}_{2}$ models built on a subset of 543 sites (the number of $\mathrm{PM}_{2.5}$ sites) are summarized in Table S12. Compared to $\mathrm{NO}_{2}$ models built on all 2399 sites, the CV R ${ }^{2}$ s were virtually the same, while the EV R ${ }^{2} \mathrm{~s}$ were about 0.05 lower. More variation in the model performances across different algorithms was found compared to the original $\mathrm{NO}_{2}$ models. The $\mathrm{ANN}$ model performed the most poorly when evaluated by CV. The BLR and the ANN performed moderately worse than other models when evaluated by EV. Other models performed similarly when comparing CV and EV results.

\subsubsection{Models with a reduced number of potential predictors}

For $\mathrm{PM}_{2.5}$ models, the training $\mathrm{R}^{2} \mathrm{~s}, \mathrm{CV} \mathrm{R}^{2} \mathrm{~s}$ and $\mathrm{EV} \mathrm{R}^{2} \mathrm{~s}$ were relatively stable in relation to the number of potential predictors offered to fit the SLR, the EN75 and the RF algorithms (Figure S6). The ANN model had lower training $\mathrm{R}^{2}$ but higher $\mathrm{CV} \mathrm{R}^{2}$ and $E V \mathrm{R}^{2}$ when fitted with fewer potential predictors.

$\mathrm{NO}_{2}$ models fitted with different algorithms show consistent patterns in relation to the number of potential predictors offered (Figure S7). The plots on the left show the relatively poor performance of $\mathrm{NO}_{2}$ models built with only 20 predictors selected based on univariate correlation, where $\mathrm{NO}_{2} \mathrm{CTM}$ estimates ranked 30 and were not included in the first 20 predictors. The model training $R^{2} s, C^{2} R^{2} s$ and $E V R^{2} s$ all increased rapidly when the number of potential predictors offered increased from 20 to 40. The training $R^{2} s, C V R^{2} s$ and $E V R^{2} s$ further increased mildly when 80 potential predictors were offered. In contrast, the training $R^{2} s, C V R^{2} s$ and $E V R^{2} s$ were not affected by the number of potential predictors offered when variables were selected based on RF or a priori reduction of the number of buffers. 
Table 2. Performance of $\mathrm{NO}_{2}$ spatial models using different model building algorithms

\begin{tabular}{|c|c|c|c|c|c|c|c|c|c|}
\hline \multirow{2}{*}{ Algorithm $^{\mathrm{a}}$} & \multicolumn{2}{|c|}{$\begin{array}{c}\text { Training } \\
(\mathrm{N}=2399)\end{array}$} & \multicolumn{3}{|c|}{$\begin{array}{c}\text { Cross Validation } \\
(\mathrm{N}=2399)\end{array}$} & \multicolumn{4}{|c|}{$\begin{array}{c}\text { External Validation } \\
\qquad(\mathrm{N}=1396)\end{array}$} \\
\hline & $\mathrm{R}^{2}$ & $\begin{array}{l}\mathrm{RMSE}^{\mathrm{b}} \\
\left(\mu \mathrm{g} / \mathrm{m}^{3}\right)\end{array}$ & $\mathrm{R}^{2}$ & $\begin{array}{l}\text { MSE- } \\
\mathrm{R}^{2}\end{array}$ & $\begin{array}{l}\text { RMSE } \\
\left(\mu \mathrm{g} / \mathrm{m}^{3}\right)\end{array}$ & $\mathrm{R}^{2}$ & $\begin{array}{l}\text { MSE- } \\
\mathrm{R}^{2}\end{array}$ & $\begin{array}{l}\text { RMSE } \\
\left(\mu \mathrm{g} / \mathrm{m}^{3}\right)\end{array}$ & $\begin{array}{c}\text { Bias } \\
\left(\mu \mathrm{g} / \mathrm{m}^{3}\right)\end{array}$ \\
\hline FLR & 0.596 & 9.3 & 0.584 & 0.583 & 9.4 & 0.499 & 0.485 & 11.6 & 1.3 \\
\hline BLR & 0.614 & 9.1 & 0.573 & 0.571 & 9.5 & 0.496 & 0.481 & 11.6 & 1.8 \\
\hline WLR & 0.596 & 9.3 & 0.584 & 0.583 & 9.4 & 0.499 & 0.485 & 11.6 & 1.3 \\
\hline SLR & 0.588 & 9.4 & 0.575 & 0.575 & 9.5 & 0.495 & 0.468 & 11.8 & 2.5 \\
\hline Ridge & 0.606 & 9.2 & 0.586 & 0.586 & 9.4 & 0.500 & 0.471 & 11.7 & 2.5 \\
\hline EN25 & 0.605 & 9.2 & 0.588 & 0.588 & 9.4 & 0.504 & 0.483 & 11.6 & 2.0 \\
\hline EN50 & 0.606 & 9.2 & 0.588 & 0.588 & 9.4 & 0.505 & 0.485 & 11.6 & 1.9 \\
\hline EN75 & 0.606 & 9.2 & 0.588 & 0.588 & 9.4 & 0.505 & 0.485 & 11.6 & 1.9 \\
\hline LASSO & 0.606 & 9.2 & 0.588 & 0.588 & 9.4 & 0.505 & 0.485 & 11.6 & 1.9 \\
\hline GAM & 0.639 & 8.8 & 0.609 & 0.609 & 9.1 & 0.506 & 0.486 & 11.6 & 2.3 \\
\hline Bagging & 0.950 & 3.6 & 0.612 & 0.612 & 9.1 & 0.490 & 0.449 & 12.0 & 3.2 \\
\hline $\mathrm{RF}$ & 0.951 & 3.6 & 0.613 & 0.612 & 9.1 & 0.487 & 0.444 & 12.0 & 3.3 \\
\hline GBM & 0.807 & 6.5 & 0.621 & 0.621 & 9.0 & 0.499 & 0.471 & 11.7 & 2.7 \\
\hline SVR & 0.708 & 8.0 & 0.607 & 0.601 & 9.2 & 0.492 & 0.481 & 11.6 & 1.2 \\
\hline KRLS & 0.687 & 8.2 & 0.613 & 0.613 & 9.1 & 0.505 & 0.480 & 11.6 & 2.4 \\
\hline ANN & 0.623 & 9.0 & 0.570 & 0.568 & 9.6 & 0.488 & 0.181 & 14.6 & 8.8 \\
\hline Ensemble1 & 0.628 & 8.9 & 0.597 & 0.60 & 9.3 & 0.509 & 0.49 & 11.6 & 2.1 \\
\hline Ensemble2 & 0.706 & 8.0 & 0.611 & 0.61 & 9.1 & 0.518 & 0.49 & 11.5 & 2.5 \\
\hline
\end{tabular}

${ }^{a}$ FLR $=$ Forward stepwise Linear Regression; BLR $=$ Backward stepwise Linear Regression; WLM $=$ Stepwise Linear Regression; SLR = Supervised Linear Regression; EN25 = Elastic Net with $\alpha=0.25$; EN50 = Elastic Net with $\alpha=0.50$; EN75 = Elastic Net with $\alpha=0.75 ;$ LASSO $=$ Least Absolute Shrinkage and Selection Operator; GAM $=$ Generalized Additive Model; RF $=$ Random Forest; GBM = Generalized Boosted Machine; SVR $=$ Support Vector Regression; KRLS $=$ Kernel-based Regularized Least Squares; ANN = Artificial Neural Network; Ensemble1 $=$ Ensemble model based on median prediction; Ensemble2 $=$ Ensemble model based on weighted average

${ }^{\mathrm{b}} \mathrm{RMSE}=$ Root-mean-square error 


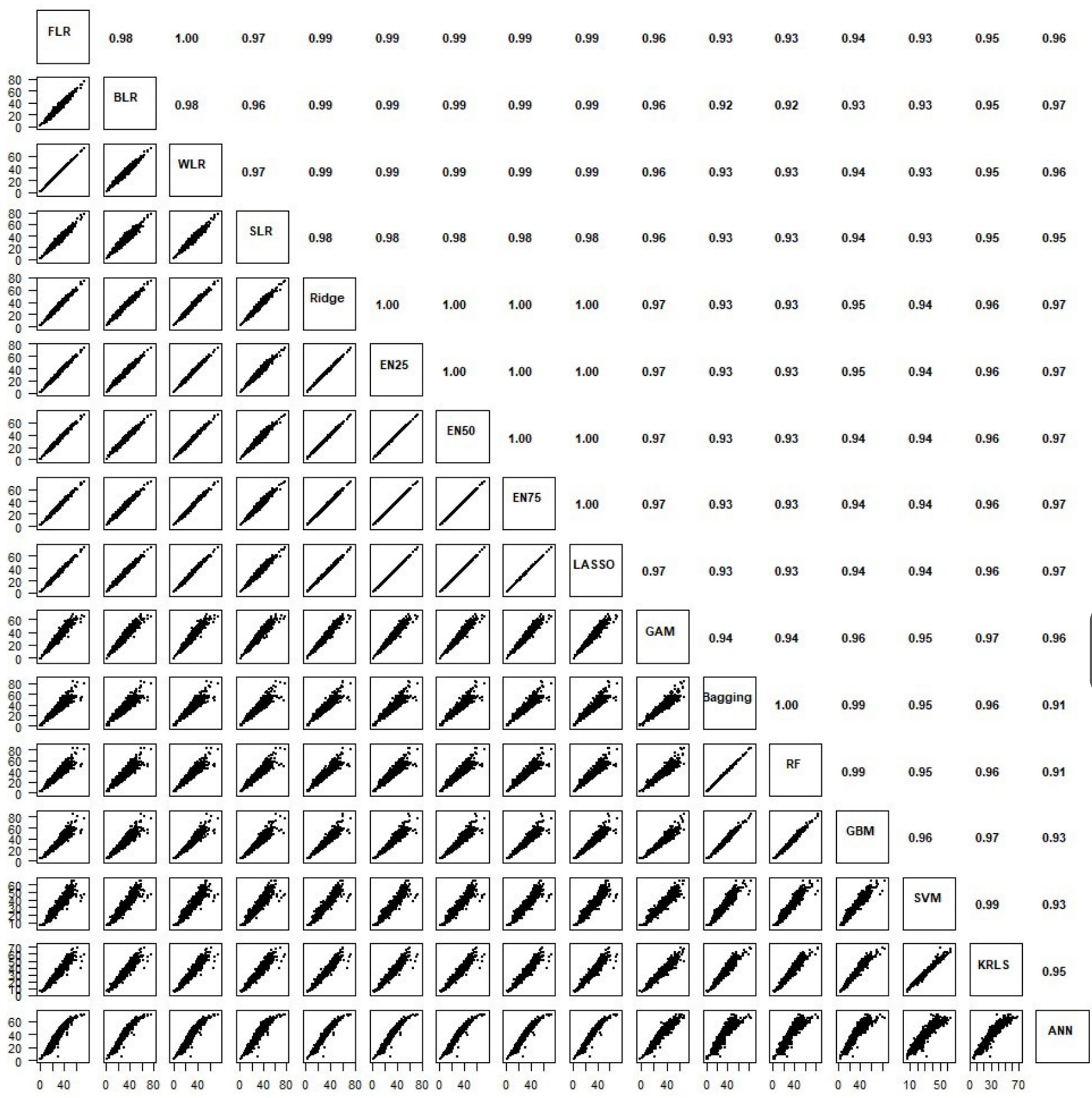

Figure 3. Correlations between $\mathrm{NO}_{2}$ predictions at ESCAPE sites. The upper triangle shows the correlation coefficients, the lower triangle shows the scatter plots

FLR $=$ Forward stepwise Linear Regression; BLR $=$ Backward stepwise Linear Regression; WLM $=$ Stepwise Linear Regression; SLR $=$ Supervised Linear Regression; EN25 = Elastic Net with $\alpha=0.25$; EN50 = Elastic Net with $\alpha=0.50$; EN75 = Elastic Net with $\alpha=0.75$; LASSO $=$ Least Absolute Shrinkage and Selection Operator; GAM = Generalized Additive Model; RF = Random Forest; GBM = Generalized Boosted Machine; SVR = Support Vector Regression; KRLS $=$ Kernel-based Regularized Least Squares; ANN = Artificial Neural Network 


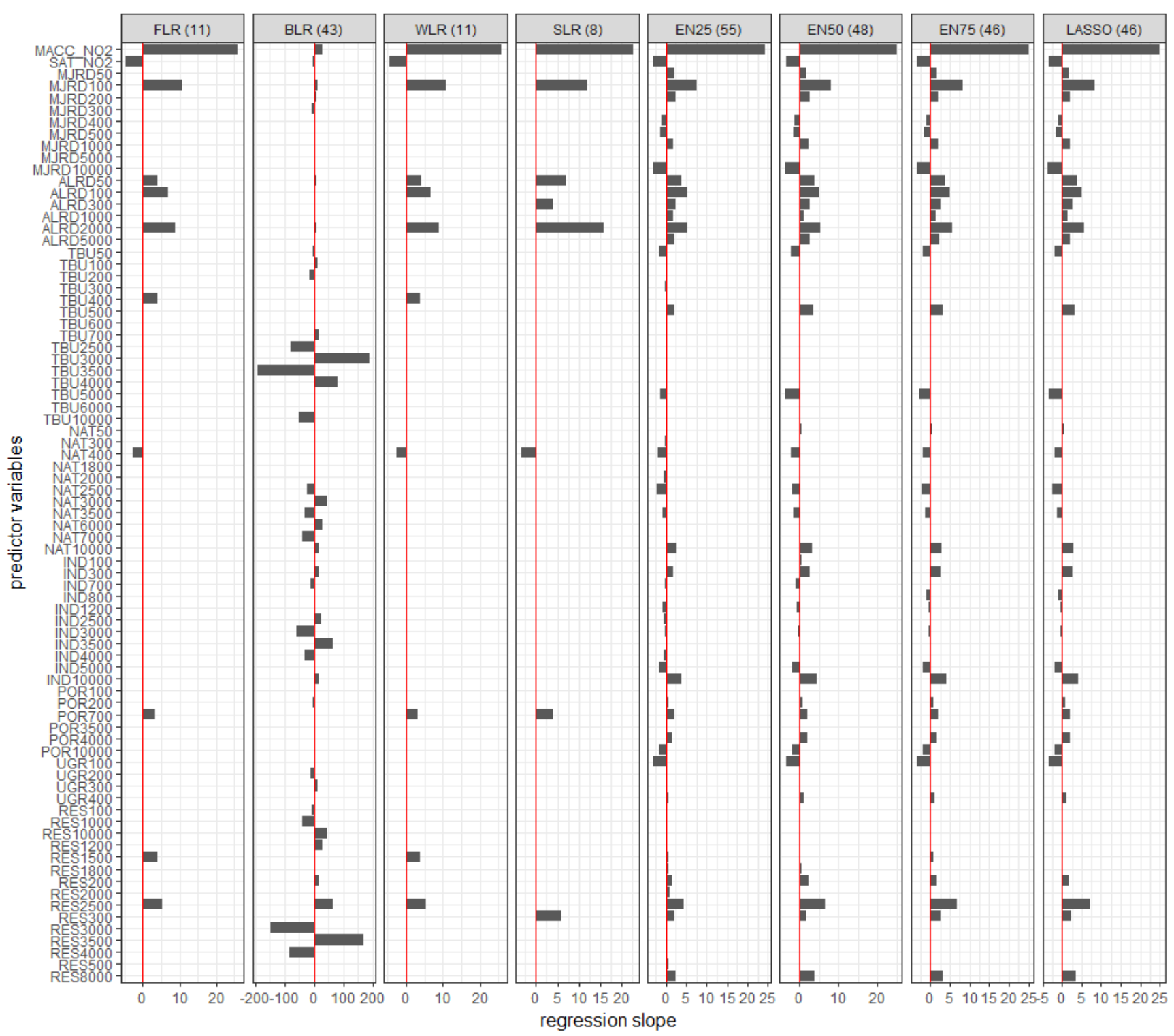

Figure 4. Predictors included in linear $\mathrm{NO}_{2}$ models. The figures in the blanket show the number of predictors included in each model

Regression slopes were multiplied by the difference between the 1th and 99th percentile of each predictor to allow comparison across predictors

MACC $=$ MACC dispersion model, SAT $=$ Satellite-derived, MJRD = Major Roads, ALRD = All Roads, TBU $=$ Total Build Up, NAT $=$ Natural Land, IND $=$ Industry, POR $=$ Ports, UGR $=$ Urban Green, RES $=$ Residential, ALT $=$ Altitude FLR = Forward stepwise Linear Regression; BLR = Backward stepwise Linear Regression; WLM = Stepwise Linear Regression; SLR = Supervised Linear Regression; EN25 = Elastic Net with $\alpha=0.25$; EN50 = Elastic Net with $\alpha=0.50$; EN75 = Elastic Net with $\alpha=0.75$; LASSO = Least Absolute Shrinkage and Selection Operator 


\subsection{3 $\mathrm{PM}_{2.5}$ models with kriging}

The kriging technique performed on residuals further increased the $\mathrm{CV} \mathrm{R} \mathrm{R}^{2} \mathrm{~s}$ and $\mathrm{EV} \mathrm{R}^{2} \mathrm{~s}$ of the linear models (BLR, SLR, LASSO) by $4.0 \%$ and $12.4 \%$ on average, while it increased less for the GBM and ANN models (the CV R $\mathrm{R}^{2}$ and EV $\mathrm{R}^{2}$ of the GBM model increased by $0.8 \%$ and $3.8 \%$ respectively, the $\mathrm{CV} \mathrm{R}^{2}$ and $\mathrm{EV} \mathrm{R}^{2}$ of the ANN model increased by $2.3 \%$ and $6.1 \%$ respectively) (Table S13). The correlation coefficients of model predictions at ESCAPE sites were both 0.90 for SLM + kriging and $\mathrm{GBM}+$ kriging, and LASSO + kriging and GBM + kriging (Figure S8).

\section{Discussion}

We compared 16 algorithms to develop Europe-wide models predicting annual average $\mathrm{PM}_{2.5}$ and $\mathrm{NO}_{2}$ concentrations in 2010. For both validation methods, $\mathrm{PM}_{2.5}$ models developed on 543 sites performed similarly across algorithms, though models developed with the generalized boosted machine, random forest and bagging performed slightly better than others in the full datasets. The $\mathrm{PM}_{2.5}$ predictions at all ESCAPE sites derived from different models were highly correlated, except for predictions from the artificial neural network. For both validation methods, $\mathrm{NO}_{2}$ models developed on 2399 sites performed even more similarly across different algorithms. The $\mathrm{NO}_{2}$ predictions at external sites were all highly correlated. For both pollutants, low biases were found when different models were applied on all ESCAPE sites, except for the ANN models.

\subsection{Predictive ability}

Our study found small differences in performance and in predictions at all external sites derived from different algorithms. The algorithms identified the same key predictor variables. The small differences between algorithms may be the result of the large number of training sites, the use of relatively stable annual average concentrations to develop models and the lack of complex relationships between predictors and annual average concentrations.

Previous algorithm comparison studies were based on either a smaller number of sites (Brokamp et al., 2017) or on mobile monitoring with much more variation in the measured concentration data (Kerckhoffs et al., 2019; Van den Bossche et al., 2018; Weichenthal et al., 2016). In our study, all algorithms may have the advantage of a lower risk of overfitting because of the combination of a large number of training sites and stable annual average concentrations. The $\mathrm{NO}_{2}$ models built on 2399 sites performed more similarly across algorithms than the $\mathrm{PM}_{2.5}$ models built on 543 sites, which could be due to the fact that the $\mathrm{NO}_{2}$ models were built on four times more training sites. This is supported by the sensitivity analysis where the performance of $\mathrm{NO}_{2}$ models built on 543 sites (the same number of training sites available for $\mathrm{PM}_{2.5}$ ) varied more across algorithms than the original $\mathrm{NO}_{2}$ models built on 2399 sites.

The machine learning algorithms did not perform better in our study. However, their ability to model complex relationships among the data is a clear benefit in studies modelling spatiotemporal variations of air pollution, where the variability in concentration is often larger and the relationships between pollution concentration and predictors are more complicated (e.g. nonlinear relationships between pollution and satellite data exist under different meteorological conditions and emission features (Liu et al., 2009; Schaap et al., 2009)). The RF algorithm was fitted to assess spatiotemporal patterns of air pollution in Japan (Araki et al., 2018), the United States (Hu et al., 2017) and China (Zhan et al., 2018). The neural network algorithm was trained to predict daily $\mathrm{PM}_{2.5}$ concentrations over the continental United States from 2000 to 2012 (Di et al., 2016a). The SVR algorithm was used in recent European studies to estimate daily $\mathrm{PM}_{2.5}$ concentrations across Switzerland (de Hoogh et al., 2018b) and daily $\mathrm{PM}_{10}$ concentrations in Italy (Stafoggia et al., 2017). The GBM algorithm outperformed 10 other algorithms, including linear-regression based algorithms, to model spatiotemporal variation of $\mathrm{PM}_{2.5}$ concentrations during a wildfire (Reid et al., 2015). In the Reid et al., algorithm comparison study, the 
larger variability in pollution concentrations and the more complicated relationships between predictors and pollution may explain why the more sophisticated algorithms, which are able to model nonlinearity and complex interactions, outperformed the simple algorithms. However, if there are no strong nonlinear relationships or complex interactions in the data, as we have good reasons to assume is the case in our data based on annual average spatial variation, the more sophisticated algorithms do not add to the simple linear regression-based algorithms. In a recent algorithm comparison study trained on mobile and short-term measurements (Kerckhoffs et al., 2019), differences in performance evaluated by external long-term exposure estimates were also small. The investigators used spatial average concentrations for 368 short-term sites and over 8000 road segments, resulting in much less stable estimates of site-specific averages probably due to the shorter sampling time.

We noted only small differences of both ensemble models performances compared to the best individual models. The fact that ensemble models did not improve upon the individual algorithms in our setting could be due to the similar performances and highly correlated predictions across algorithms. Ensemble models are attractive because the researcher does not have to make (arbitrary) choices of what model to choose for final exposure assignment. We used global weights for the different algorithms. If there is evidence for spatially different performance of the different algorithm, spatially varying weights could be used.

Comparing $\mathrm{PM}_{2.5}$ and $\mathrm{NO}_{2}$ models built on the same number of training sites, the ensemble learning algorithms (Bagging, RF and GBM) performed slightly better than other algorithms for $\mathrm{PM}_{2.5}$ but not for $\mathrm{NO}_{2}$. We speculate that this might be due to the different characteristics of $\mathrm{PM}_{2.5}$ and $\mathrm{NO}_{2}$. $\mathrm{PM}_{2.5}$ concentrations vary at large regional scales (Eeftens et al., 2012), whereas $\mathrm{NO}_{2}$ concentrations, strongly influenced by local traffic emissions, vary more widely at smaller scales (Cyrys et al., 2012). The ensemble learning algorithms modelled detailed fluctuations of the measurements - as indicated by the extremely high training $\mathrm{R}^{2} \mathrm{~s}$, which may not transfer to the validation dataset for $\mathrm{NO}_{2}$.

The results of the GAM models might not be comparable with other models because of the slightly different input variables. However, we would not expect much deviation from the current results as none of the deleted small buffer land use variables was identified as important predictors in other models. The ANN models as specified in the current study did not perform well among the algorithms. One possible reason is that the large number of predictors and relatively small number of observations in the training dataset required more careful training. Our sensitivity analysis also supported that with less potential predictors or more training data, the ANN algorithm tended to perform better. In this study, we used one hidden layer to build ANN models because, as suggested by Schalkoff (1997), one hidden layer is sufficient for avoiding overfitting in most applications. However, we cannot rule out the possibility of better performance by using more than one hidden layer.

Kriging is a technique which can be used to explain spatial variation within the data. In the sensitivity analysis, kriging on residual variation did not significantly improve the performance for $\mathrm{PM}_{2.5} \mathrm{GBM}$ and ANN models, indicating the machine learning algorithms have some ability to address spatial autocorrelation in air pollution concentrations. The CV-R ${ }^{2}$ of SLR, LASSO and GBM models became closer after adding kriging surfaces, indicating part of the residual variance of the SLR and LASSO models explained by kriging had been accounted for by a more flexible GBM algorithm. For $\mathrm{NO}_{2}$, kriging did not explain the residual spatial variation (de Hoogh et al., 2018a).

\subsection{Model structure and interpretation}

Although our main interest is in the predictive performance of the models, it is informative to interpret the structure of the models. The importance of specific determinants such as traffic in the model may be helpful to compare risks across epidemiological studies in different areas.

The machine learning algorithms are often considered "black boxes" (Zhang and Ding, 2017) since the models derived from these algorithms are difficult to interpret. Even though some algorithms provide variable importance measures, such as the RF and the GBM (Breiman et al., 2011; Ridgeway et al., 


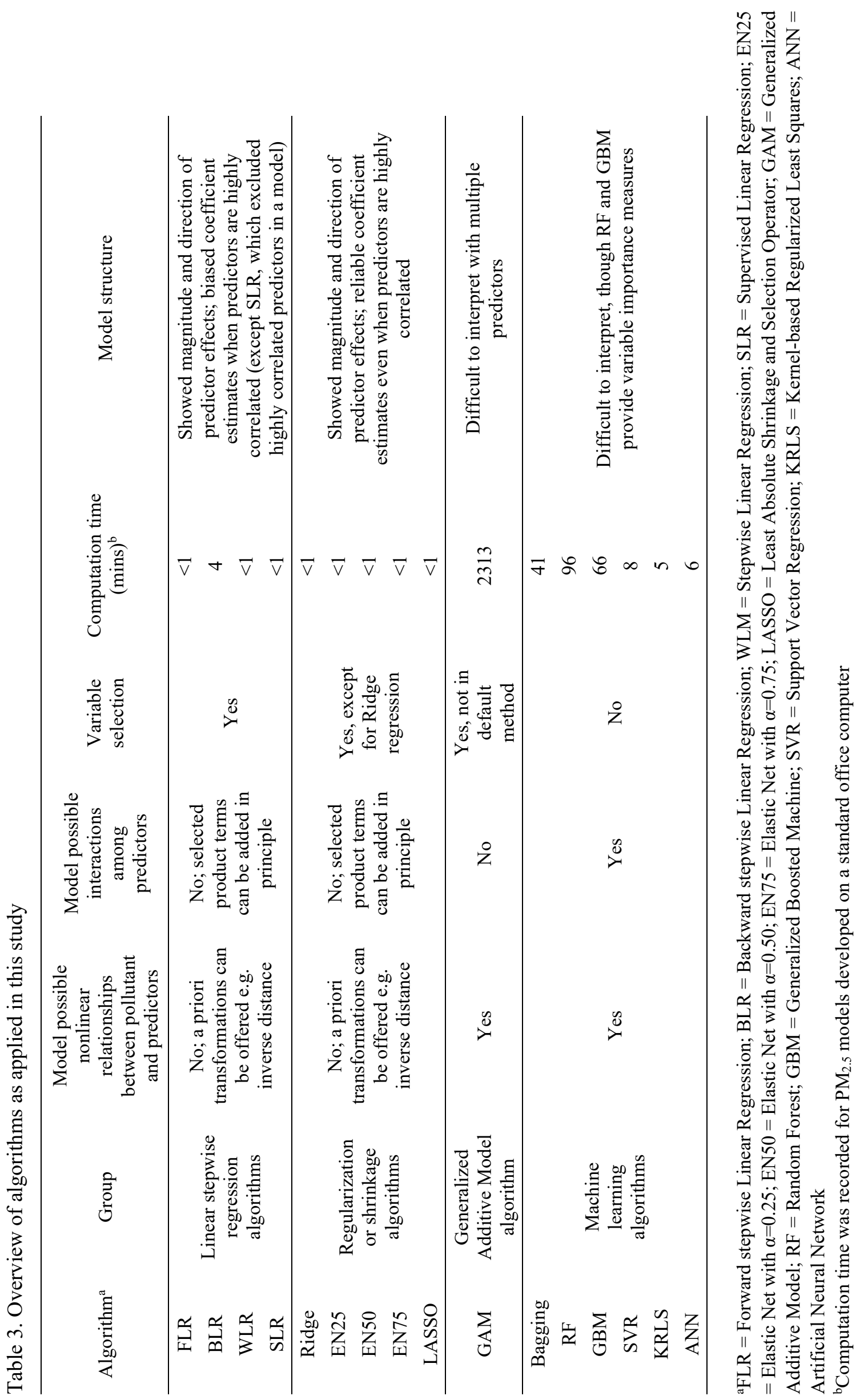


2013), the magnitude and direction of the predictor effects are unknown. Models built with linear stepwise regression and regularization algorithms are easier to interpret, both in terms of included predictors and the magnitude and direction of predictor effects. An overview of the applied algorithms is shown in Table 3.

For both pollutants, the SLR models by definition included only predictors following the plausible direction of effect, resulting in a substantially smaller number of predictors than other models. Models developed with all other algorithms included predictors with counterintuitive directions of effect, though in most cases not for the key predictor variables. For example in the $\mathrm{NO}_{2}$ models, satellite $\mathrm{NO}_{2}$ was included with a negative slope possibly to compensate for overprediction by the other large spatial scale predictor variable (CTM estimates). Restricting the inclusion of predictors did not affect model performance in our study and may be considered more a philosophical choice (Brauer et al., 2003; Vienneau et al., 2010). However, model prediction may deviate when the models are applied in another domain than the Europe-wide training domain such as a smaller area within Europe. This is supported by our subset validation where the $\mathrm{NO}_{2}$ SLR model outperformed automatic variable selection linear models at rural background sites.

In our study, correlated potential predictors were offered to build linear-regression based models. These predictors are usually the same land use feature/ road length in buffers with different radii. Offering highly correlated predictors would lead to incorrect selection of predictors in a model (Agier et al., 2016), and including highly correlated predictors in an ordinary least squares-based model would lead to biased coefficient estimates (Tibshirani et al., 2013). Clear evidence for this is found in the backward stepwise selection algorithm, which included very different predictor variables than all other algorithms, and had a lower explained variance in the validation dataset. The SLR algorithm deals with collinearity by deleting predictors with a variance inflation factor larger than 3, at the expense of including fewer predictor variables. The regularization algorithms impose a penalty to shrink the coefficients of the least informative predictors towards zero, which have been shown to be more efficient in identifying correct predictors than the ordinary least squares-based algorithms (Agier et al., 2016). Compared to the SLR algorithm, regularization algorithms included more buffers of the same variable, which is consistent with the notion of smooth changes of pollution with increasing buffer size: if a road length variable contributes to the pollution estimate, one would expect road length variables with other buffer sizes also to add to the pollution estimate. However, interpretation is hampered because the same land use feature/ road length with different radii entered the model with both plausible and implausible directions of effect.

\subsection{Strengths and limitations}

One strength of our study is that we compared multiple algorithms with very different assumptions. Most of the previous algorithm comparison studies only compared two or three algorithms (Basagaña et al., 2012; Brokamp et al., 2017; Van den Bossche et al., 2018; Weichenthal et al., 2016), therefore results between studies are difficult to compare. Studies have assessed more algorithms in different settings such as assessing spatiotemporal variation of $\mathrm{PM}_{2.5}$ during a wildfire (Reid et al., 2015), or assessing spatial variation of ultrafine particles using mobile monitoring data (Kerckhoffs et al., 2019). Our study gives new insight into the predictive ability of these algorithms because the number of training sites, variation in the monitoring data and complexity of relationships within the data can all affect relative performance of the algorithms.

Secondly, we used both cross-validation and external validation to strengthen our comparison. CV is commonly used to evaluate model performance in air pollution exposure assessment (Kim et al., 2016; Y. Liu et al., 2009). Because CV is restricted to the monitoring domain, a good performance evaluated by $\mathrm{CV}$ does not necessarily mean the model can accurately predict residential exposure, which is often used as surrogates for long-term exposure of subjects in epidemiological studies (Beelen et al., 2014; Di et al., 2017). Concerns about overestimating exposures at residential addresses have been raised for models based upon on-road mobile monitoring (Hoek, 2017; Kerckhoffs et al., 2016). In this situation, 
$\mathrm{CV}$ alone cannot reflect true transferability because the mobile monitoring sites are located on roads and the measurements usually have significant variations. Validation of such models using an external dataset reflecting residential exposure is important (Kerckhoffs et al., 2019). In our study, monitoring data from the regulatory AIRBASE network were used to develop air pollution models. With the aim to check for compliance with the European Union air quality standards, the monitors in the AIRBASE network have not primarily been located with the goal of characterizing residential exposure in mind. On the other hand, the location of ESCAPE sites was purposely selected to be representative for air pollution exposure at home addresses of study subjects. In this sense, results of our EV using pollution data from the ESCAPE study reflects the transferability of models in application. Besides the choice of monitoring locations, there are also a number of differences between the two datasets that could impact the evaluation. Firstly, ESCAPE monitoring sites do not cover the same geographical area as the AIRBASE monitoring sites. Secondly, the two datasets differed in measurement techniques. A comprehensive comparison showed limited systematic differences between the ESCAPE and AIRBASE methods for $\mathrm{NO}_{2}$ (Cyrys et al., 2012). $\mathrm{NO}_{2}$ was mainly measured by chemiluminescence in AIRBASE versus Ogawa badges in ESCAPE. Chemiluminescence is subject to interference from other reactive nitrogen species that can vary spatially (Suzuki et al., 2011), which could, at least partially, explain the higher bias at low concentration and background ESCAPE sites. For $\mathrm{PM}_{2.5}$ no methods comparison was made. Thirdly, the ESCAPE measurements are based on 2-week sampling in three seasons with temporal adjustment versus continuous measurements in AIRBASE. Lastly, ESCAPE measurements were conducted following the same standard measurement methods and strict quality control procedures, while the AIRBASE were measured by inconsistent methods and reported with different frequency across countries. Nevertheless, the agreement in results showed by CV and EV help us to strengthen the comparison.

Thirdly, we used grid search to optimize hyperparameters for some algorithms before fitting the models, based on the best performance in 5-fold cross-validation. This approach helped to minimize the risk of overfitting and avoid overly optimistic performance estimates (Van den Bossche et al., 2018). This adds to a recent algorithm comparison performed by Kerckhoffs et al., (2019) which used default parameters for algorithms. Though the grid search approach added to the computation load in model development for the GAM and machine learning algorithms, the computation time is generally short for all algorithms, and was not a decisive factor for choosing one algorithm over another in this moderately small dataset (Table 3).

Despite similarities across algorithms, the model performances remained moderate in our study for both pollutants. This is probably a result of missing explanatory variables. A previous European $\mathrm{PM}_{2.5}$ model developed using supervised linear regression algorithm obtained a CV R ${ }^{2}$ of 0.80 (Wang et al., 2014), which was driven by the inclusion of local traffic intensity and measured regional background concentrations. Such variables are often only available at local level, and were not available in the current study. The relevant influence of missing explanatory variables is also supported by our sensitivity analysis where performance of different $\mathrm{NO}_{2}$ models all reduced dramatically when the CTM estimates were not offered as a potential predictor. Additionally, the overall model performance we evaluated in the study could be dominated by regional variation and might not reflect within-city variations well. Decreased and varying $\mathrm{R}^{2} \mathrm{~s}$ were reported when the SLR model was validated at individual study areas (de Hoogh et al., 2018a). However, the overall model performance was our primary interest as the models developed in this study were aimed to estimate air pollution exposure for participants across Europe. Our sensitivity analyses showed moderate performances when models were validated at regional scale (Tables S6 and S10). Another limitation is that none of the algorithms explicitly include handling spatial autocorrelation in air pollution concentrations in model building. We addressed this with a 2-step kriging sensitivity analysis. Our results showed that kriging significantly improved model performance of linear regression algorithms, and suggest that the machine learning algorithms have some ability to handle autocorrelation within data. Our 2-step kriging approach likely underestimates the value of including spatial autocorrelation (Mercer et al., 2011). The predictive power 
of these models might be further improved by applying universal kriging, which can be seen as a LUR with addition of correlated residuals.

\subsection{Conclusion}

Different statistical algorithms performed similarly when modelling spatial variation of annual average air pollution concentrations using a large number of training sites.

The results of our study and the previous algorithm comparisons suggest that the relative performance of algorithms may differ with the study setting, therefore generic recommendations for one algorithm cannot be made. To take appropriate decisions for a particular study, future studies may opt for models developed using more than one algorithm. 


\section{References}

Agier L, Portengen L, Chadeau-Hyam M, et al., 2016. A systematic comparison of linear regression-based statistical methods to assess exposome-health associations. Environmental health perspectives 124:1848.

Araki S, Shima M, Yamamoto K. 2018. Spatiotemporal land use random forest model for estimating metropolitan no 2 exposure in japan. Science of The Total Environment 634:1269-1277.

Basagaña X, Rivera M, Aguilera I, et al., 2012. Effect of the number of measurement sites on land use regression models in estimating local air pollution. Atmospheric environment 54:634-642.

Bechle MJ, Millet DB, Marshall JD. 2013. Remote sensing of exposure to no 2: Satellite versus ground-based measurement in a large urban area. Atmospheric Environment 69:345-353.

Bechle MJ, Millet DB, Marshall JD. 2015. National spatiotemporal exposure surface for no2: Monthly scaling of a satellitederived land-use regression, 2000-2010. Environmental science \& technology 49:12297-12305.

Beckerman BS, Jerrett M, Martin RV, et al., 2013. Application of the deletion/substitution/addition algorithm to selecting land use regression models for interpolating air pollution measurements in california. Atmospheric Environment 77:172-177.

Beelen R, Raaschou-Nielsen O, Stafoggia M, et al., 2014. Effects of long-term exposure to air pollution on natural-cause mortality: An analysis of 22 european cohorts within the multicentre escape project. Lancet (London, England) 383:785-795. Brauer M, Hoek G, van Vliet P, et al., 2003. Estimating long-term average particulate air pollution concentrations: Application of traffic indicators and geographic information systems. Epidemiology (Cambridge, Mass):228-239.

Breiman L, Cutler A, Liaw A, et al., 2011. Package'randomforest'. software available at URL: http://stat-www berkeley edu/users/breiman/RandomForests.

Briggs DJ, Collins S, Elliott P, et al., 1997. Mapping urban air pollution using gis: A regression-based approach. International Journal of Geographical Information Science 11:699-718.

Brokamp C, Jandarov R, Rao MB, et al., 2017. Exposure assessment models for elemental components of particulate matter in an urban environment: A comparison of regression and random forest approaches. Atmos Environ (1994) 151:1-11.

Brokamp C, Jandarov R, Hossain M, et al., 2018. Predicting daily urban fine particulate matter concentrations using a random forest model. Environ Sci Technol 52:4173-4179.

CGIAR-CSI. Srtm 90m digital elevation data.

Crouse DL, Goldberg MS, Ross NA. 2009. A prediction-based approach to modelling temporal and spatial variability of traffic-related air pollution in montreal, canada. Atmospheric Environment 43:5075-5084.

Cyrys J, Eeftens M, Heinrich J, et al., 2012. Variation of no2 and nox concentrations between and within 36 european study areas: Results from the escape study. Atmospheric Environment 62:374-390.

de Hoogh K, Gulliver J, van Donkelaar A, et al., 2016. Development of west-european pm 2.5 and no 2 land use regression models incorporating satellite-derived and chemical transport modelling data. Environmental research 151:1-10.

de Hoogh K, Chen J, Gulliver J, et al., 2018a. Spatial pm2.5, no2, o3 and bc models for western europe - evaluation of spatiotemporal stability. Environment international 120:81-92.

de Hoogh K, Heritier H, Stafoggia M, et al., 2018b. Modelling daily pm2.5 concentrations at high spatio-temporal resolution across switzerland. Environ Pollut 233:1147-1154.

Di Q, Kloog I, Koutrakis P, et al., 2016a. Assessing pm2. 5 exposures with high spatiotemporal resolution across the continental united states. Environmental science \& technology 50:4712-4721.

Di Q, Koutrakis P, Schwartz J. 2016b. A hybrid prediction model for pm2. 5 mass and components using a chemical transport model and land use regression. Atmospheric Environment 131:390-399.

Di Q, Wang Y, Zanobetti A, et al., 2017. Air pollution and mortality in the medicare population. The New England journal of medicine 376:2513-2522.

EEA, 2015. Airbase - The European Air Quality Database, Version 8 (Available). http:// www.eea.europa.eu/data-andmaps/data/airbase-the-european-air-quality-database-8, Accessed date: 13 January 2015.

Eeftens M, Tsai M-Y, Ampe C, et al., 2012. Spatial variation of pm2.5, pm10, pm2.5 absorbance and pmcoarse concentrations between and within 20 european study areas and the relationship with no2 - results of the escape project. Atmospheric Environment 62:303-317.

ETC-LC. Corine land cover (clc2006), raster database (version 12/2013).

ETC-LC. Corine land cover (clc2000), raster database (version 12/2009).

Ferwerda J, Hainmueller J, Hazlett CJ. 2017. Kernel-based regularized least squares in r (krls) and stata (krls). Journal of Statistical Software 79.

Friedman J, Hastie T, Tibshirani R. 2001. The elements of statistical learning:Springer series in statistics New York.

Friedman J, Hastie T, Tibshirani R. 2009. Glmnet: Lasso and elastic-net regularized generalized linear models. R package version 1.

Hazlett JHSC, Hainmueller MJ. 2017. Package 'krls'.

Henderson SB, Beckerman B, Jerrett M, et al., 2007. Application of land use regression to estimate long-term concentrations of traffic-related nitrogen oxides and fine particulate matter. Environmental science \& technology 41:2422-2428.

Hoek G, Beelen R, de Hoogh K, et al., 2008. A review of land-use regression models to assess spatial variation of outdoor air pollution. Atmospheric Environment 42:7561-7578.

Hoek G. 2017. Methods for assessing long-term exposures to outdoor air pollutants. Current environmental health reports 4:450-462.

Hu X, Belle JH, Meng X, et al., 2017. Estimating pm2.5 concentrations in the conterminous united states using the random forest approach. Environ Sci Technol.

Hystad P, Setton E, Cervantes A, et al., 2011. Creating national air pollution models for population exposure assessment in canada. Environmental health perspectives 119:1123. 
Inness A, Baier F, Benedetti A, et al., 2013. The macc reanalysis: An 8 yr data set of atmospheric composition. Atmospheric chemistry and physics 13:4073-4109.

Kerckhoffs J, Hoek G, Messier KP, et al., 2016. Comparison of ultrafine particle and black carbon concentration predictions from a mobile and short-term stationary land-use regression model. Environmental science \& technology 50:12894-12902.

Kerckhoffs J, Hoek G, Portengen L, et al., 2019. Performance of prediction algorithms for modeling outdoor air pollution spatial surfaces. Environmental science \& technology.

Kim SY, Sheppard L, Bergen S, et al., 2016. Prediction of fine particulate matter chemical components with a spatio-temporal model for the multi-ethnic study of atherosclerosis cohort. J Expo Sci Environ Epidemiol 26:520-528.

Liu Y, Paciorek CJ, Koutrakis P. 2009. Estimating regional spatial and temporal variability of pm(2.5) concentrations using satellite data, meteorology, and land use information. Environmental health perspectives 117:886-892.

Liu Y, Paciorek CJ, Koutrakis P. 2009. Estimating regional spatial and temporal variability of pm2. 5 concentrations using satellite data, meteorology, and land use information. Environmental health perspectives 117:886.

Mercer LD, Szpiro AA, Sheppard L, et al., 2011. Comparing universal kriging and land-use regression for predicting concentrations of gaseous oxides of nitrogen (NOx) for the Multi-Ethnic Study of Atherosclerosis and Air Pollution (MESA Air). Atmospheric Environment 45 (26): 4412-4420.

Meyer D, Dimitriadou E, Hornik K, et al., 2017. Package 'e1071'

Novotny EV, Bechle MJ, Millet DB, et al., 2011. National satellite-based land-use regression: No2 in the united states. Environmental science \& technology 45:4407-4414.

Pinault LL, Weichenthal S, Crouse DL, et al., 2017. Associations between fine particulate matter and mortality in the 2001 canadian census health and environment cohort. Environmental Research 159:406-415.

Reid CE, Jerrett M, Petersen ML, et al., 2015. Spatiotemporal prediction of fine particulate matter during the 2008 northern california wildfires using machine learning. Environ Sci Technol 49:3887-3896.

Ridgeway G, Southworth MH, RUnit S. 2013. Package 'gbm'. Viitattu 10:40.

Ripley B, Venables W, Ripley MB. 2016. Package 'nnet'. R package version:7.3-12.

Schaap M, Apituley A, Timmermans R, et al., 2009. Exploring the relation between aerosol optical depth and pm 2.5 at cabauw, the netherlands. Atmospheric Chemistry and Physics 9:909-925.

Stafoggia M, Schwartz J, Badaloni C, et al., 2017. Estimation of daily pm10 concentrations in italy (2006-2012) using finely resolved satellite data, land use variables and meteorology. Environment international 99:234-244.

Suzuki H, Miyao Y, Nakayama T, et al., 2011. Comparison of laser-induced fluorescence and chemiluminescence measurements of no2 at an urban site. Atmospheric environment 45:6233-6240.

Team RC. 2013. R: A language and environment for statistical computing.

Tibshirani R, James G, Witten D, et al., 2013. An introduction to statistical learning-with applications in r.New York, NY: Springer.

Van den Bossche J, De Baets B, Verwaeren J, et al., 2018. Development and evaluation of land use regression models for black carbon based on bicycle and pedestrian measurements in the urban environment. Environmental Modelling \& Software 99:58-69.

Van Donkelaar A, Martin RV, Brauer M, et al., 2015. Use of satellite observations for long-term exposure assessment of global concentrations of fine particulate matter. Environmental health perspectives 123:135.

Vienneau D, de Hoogh K, Beelen R, et al., 2010. Comparison of land-use regression models between great britain and the netherlands. Atmospheric Environment 44:688-696.

Vienneau D, De Hoogh K, Bechle MJ, et al., 2013. Western european land use regression incorporating satellite-and groundbased measurements of no2 and pm10. Environmental science \& technology 47:13555-13564.

Wang M, Beelen R, Eeftens M, et al., 2012. Systematic evaluation of land use regression models for no2. Environmental science \& technology 46:4481-4489.

Wang M, Beelen R, Bellander T, et al., 2014. Performance of multi-city land use regression models for nitrogen dioxide and fine particles. Environmental health perspectives 122:843-849.

Weichenthal S, Ryswyk KV, Goldstein A, et al., 2016. A land use regression model for ambient ultrafine particles in montreal, canada: A comparison of linear regression and a machine learning approach. Environ Res 146:65-72.

Wood S, Wood MS. 2015. Package 'mgcv'. R package version:1-7.

Zhan Y, Luo Y, Deng X, et al., 2017. Spatiotemporal prediction of continuous daily pm 2.5 concentrations across china using a spatially explicit machine learning algorithm. Atmospheric Environment 155:129-139.

Zhan Y, Luo Y, Deng X, et al., 2018. Satellite-based estimates of daily no2 exposure in china using hybrid random forest and spatiotemporal kriging model. Environ Sci Technol 52:4180-4189.

Zhang J, Ding W. 2017. Prediction of air pollutants concentration based on an extreme learning machine: The case of hong kong. Int J Environ Res Public Health 14.

Zou B, Wang M, Wan N, et al., 2015. Spatial modeling of pm 2.5 concentrations with a multifactoral radial basis function neural network. Environmental Science and Pollution Research 22:10395-10404 


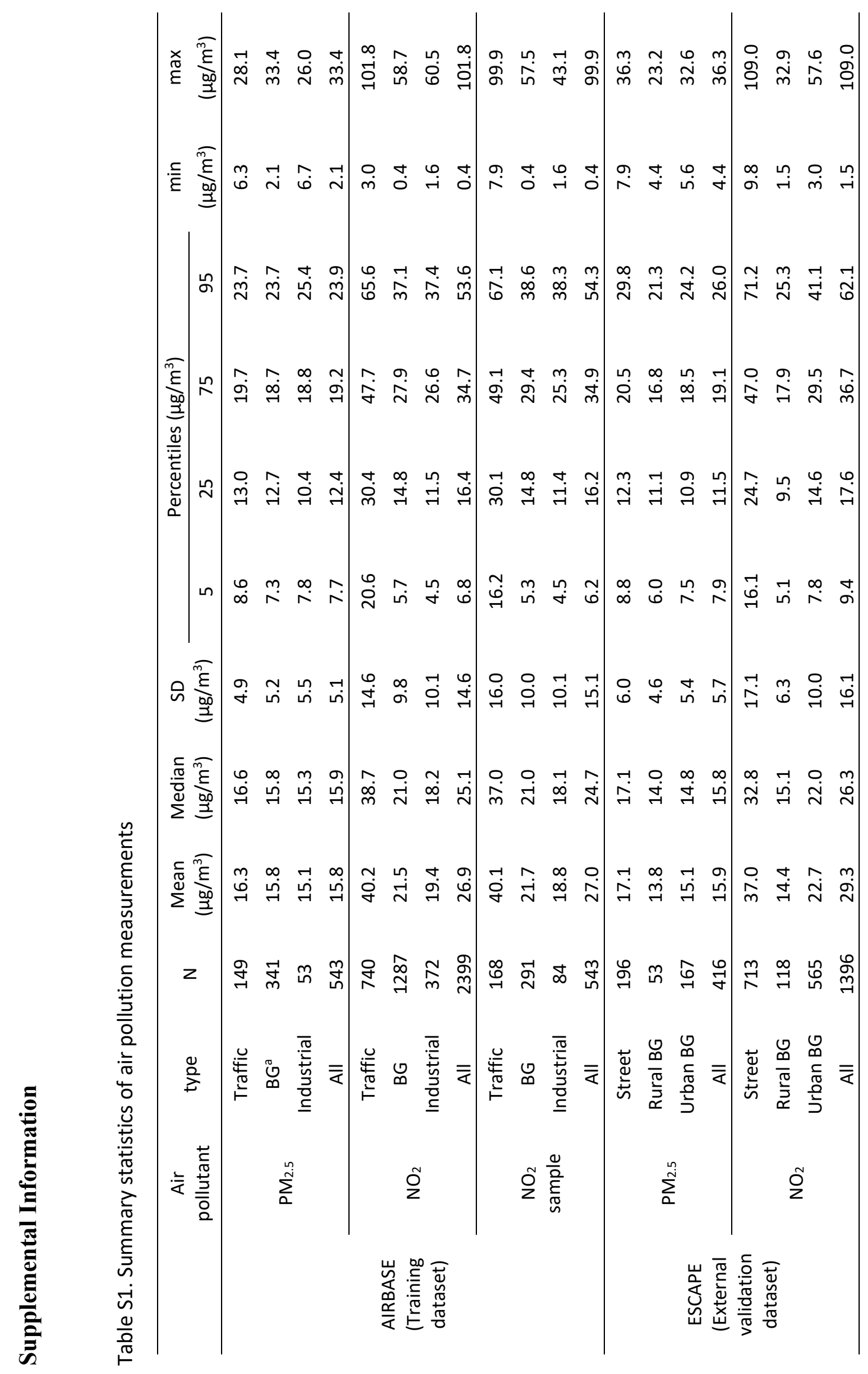




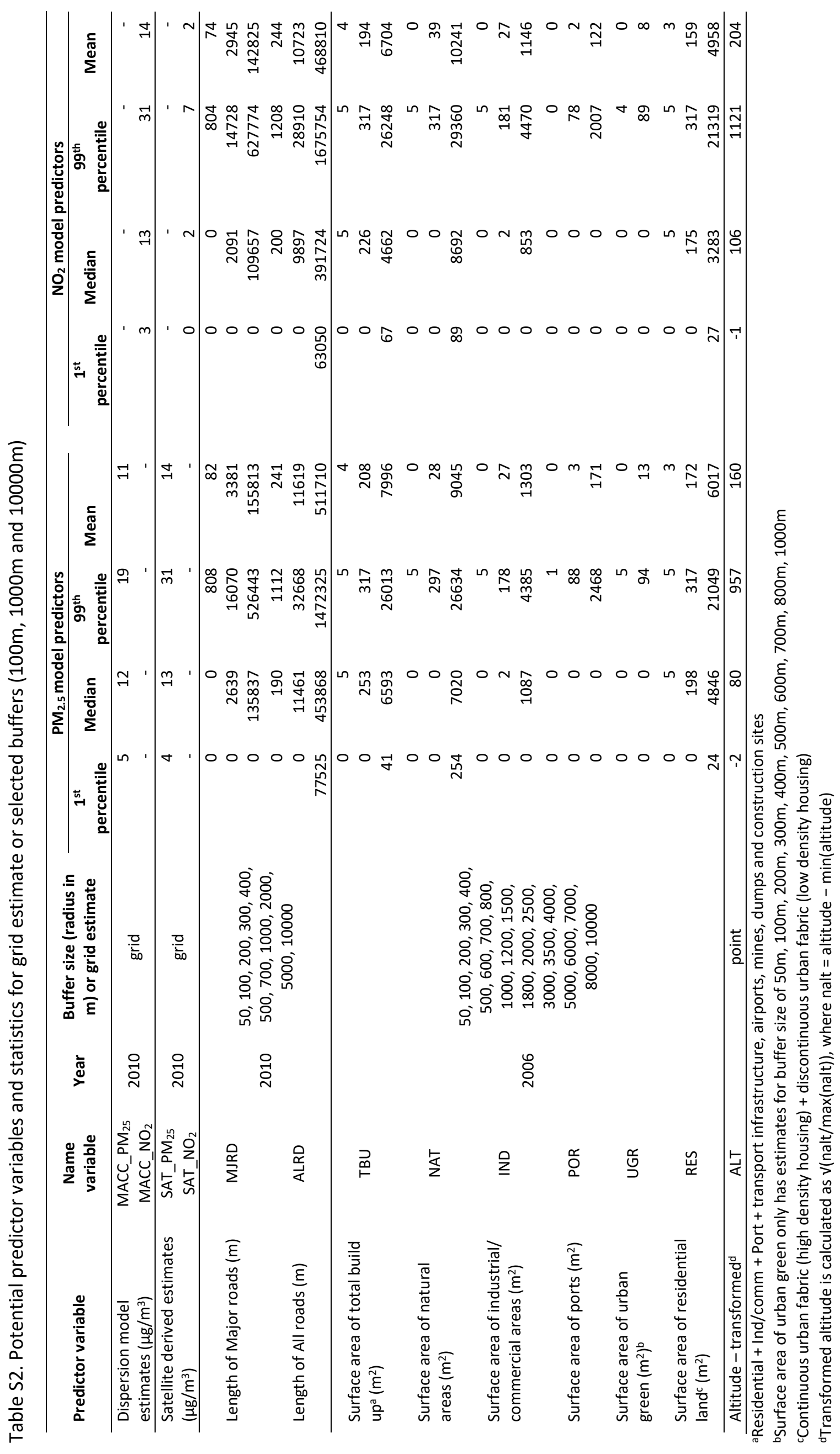



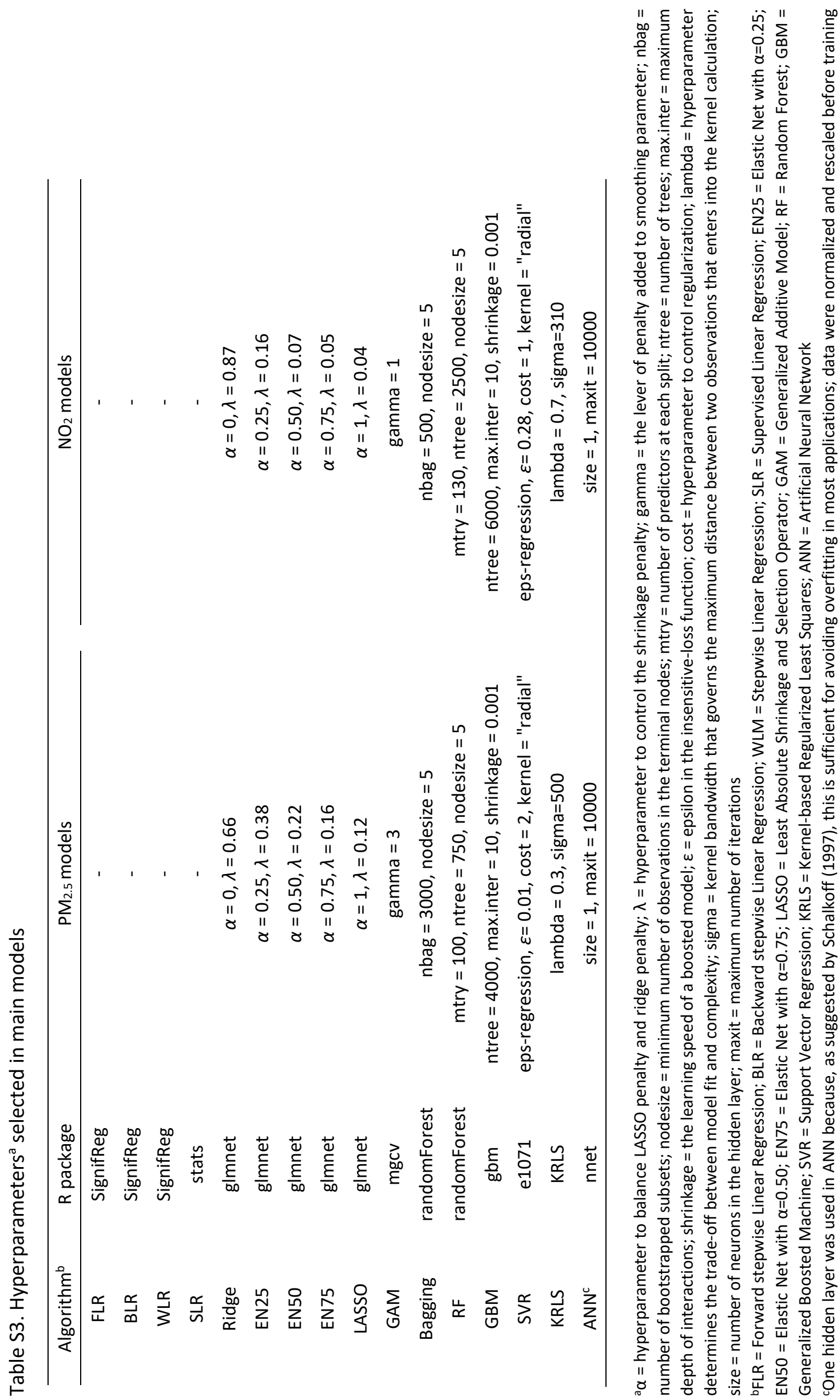

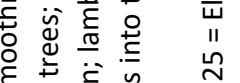

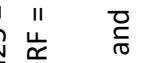

है

है

贾

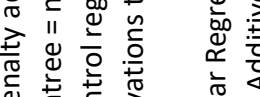

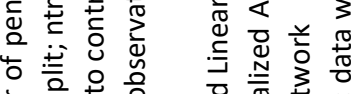

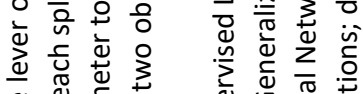

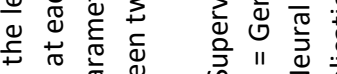

II

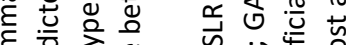

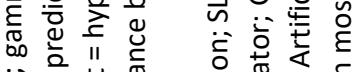

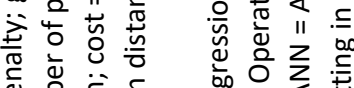

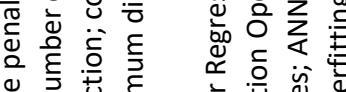

D.

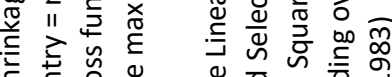

员 $\begin{aligned} & \underline{1} \\ & 0\end{aligned}$

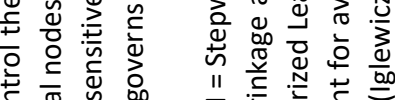

다에

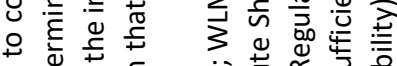

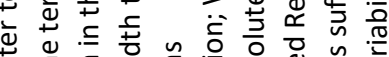

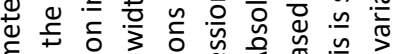

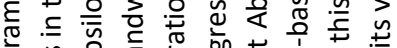

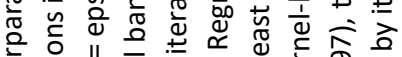

बे

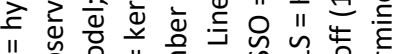

$<$ ○े ह

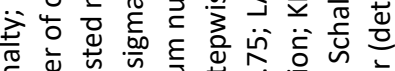

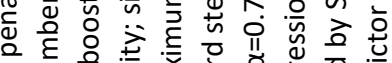

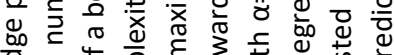

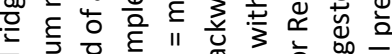

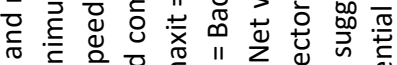

글

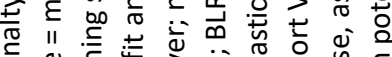

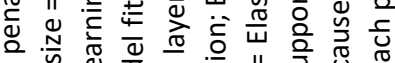

可

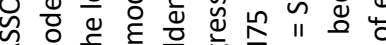

S

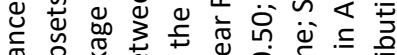

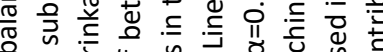

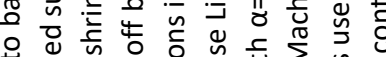

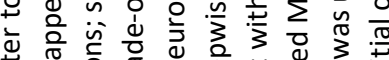

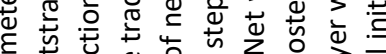

है

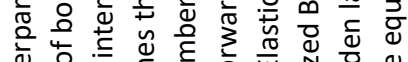

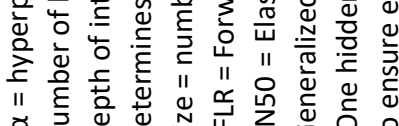




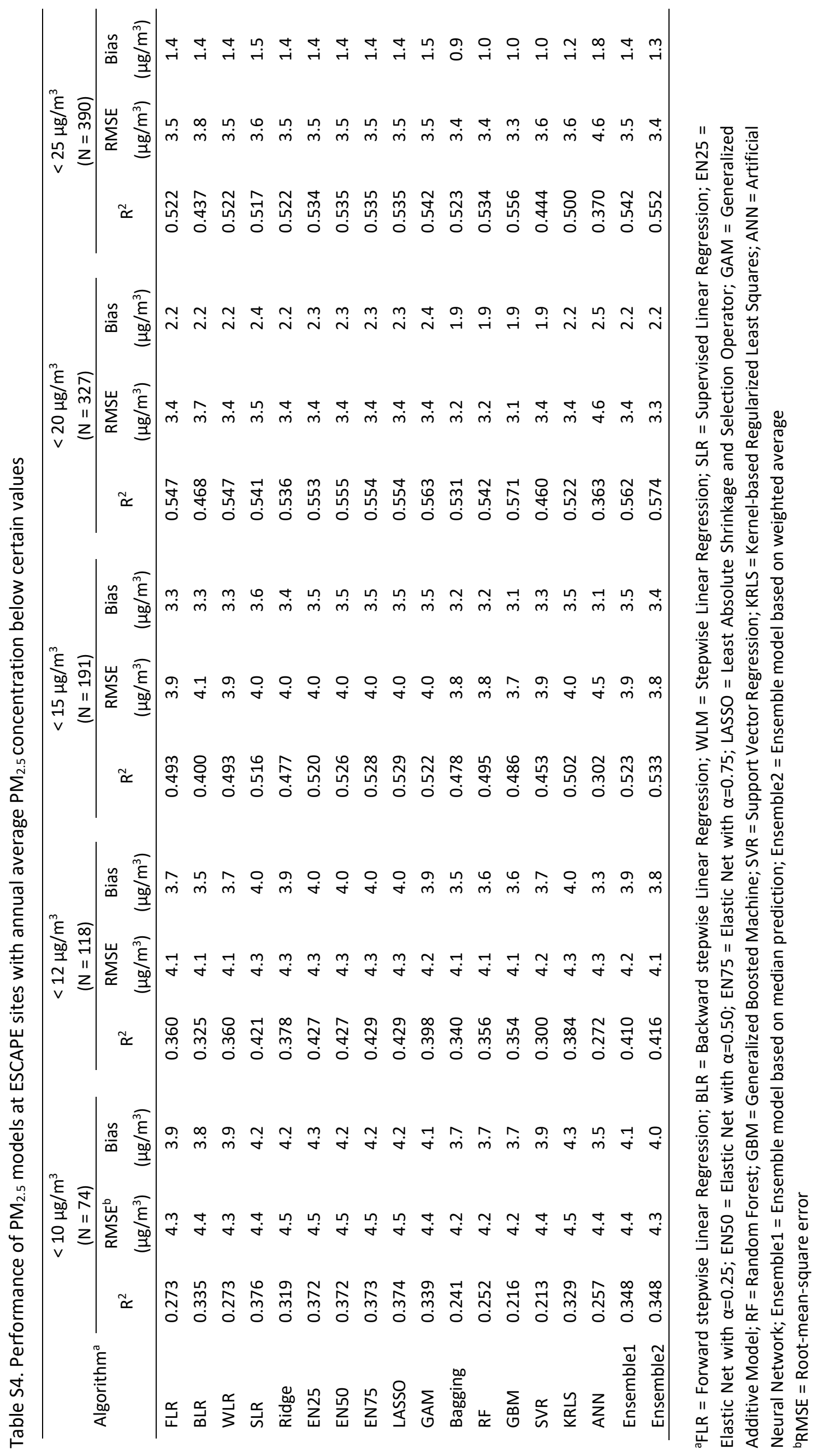


Table S5. Performance of $\mathrm{PM}_{2.5}$ models at different type of sites

\begin{tabular}{|c|c|c|c|c|c|c|c|c|c|}
\hline \multirow{2}{*}{ Algorithm ${ }^{a}$} & \multicolumn{3}{|c|}{$\begin{array}{c}\text { Street } \\
(\mathrm{N}=196)\end{array}$} & \multicolumn{3}{|c|}{$\begin{array}{l}\text { Rural Background } \\
\qquad(\mathrm{N}=53)\end{array}$} & \multicolumn{3}{|c|}{$\begin{array}{l}\text { Urban Background } \\
\qquad(\mathrm{N}=167)\end{array}$} \\
\hline & $R^{2}$ & $\begin{array}{l}\text { RMSE }^{\mathrm{b}} \\
\left(\mu \mathrm{g} / \mathrm{m}^{3}\right)\end{array}$ & $\begin{array}{c}\text { Bias } \\
\left(\mu \mathrm{g} / \mathrm{m}^{3}\right)\end{array}$ & $R^{2}$ & $\begin{array}{c}\text { RMSE } \\
\left(\mu \mathrm{g} / \mathrm{m}^{3}\right)\end{array}$ & $\begin{array}{c}\text { Bias } \\
\left(\mu \mathrm{g} / \mathrm{m}^{3}\right)\end{array}$ & $\mathrm{R}^{2}$ & $\begin{array}{c}\text { RMSE } \\
\left(\mu \mathrm{g} / \mathrm{m}^{3}\right)\end{array}$ & $\begin{array}{c}\text { Bias } \\
\left(\mu \mathrm{g} / \mathrm{m}^{3}\right)\end{array}$ \\
\hline FLR & 0.482 & 4.4 & 0.0 & 0.675 & 3.2 & 1.7 & 0.504 & 4.1 & 1.3 \\
\hline BLR & 0.469 & 4.4 & -0.1 & 0.508 & 4.0 & 2.4 & 0.449 & 4.2 & 1.2 \\
\hline WLR & 0.482 & 4.4 & 0.0 & 0.675 & 3.2 & 1.7 & 0.504 & 4.1 & 1.3 \\
\hline SLR & 0.495 & 4.4 & 0.1 & 0.669 & 3.2 & 1.7 & 0.520 & 4.1 & 1.6 \\
\hline Ridge & 0.504 & 4.4 & -0.1 & 0.662 & 3.3 & 1.9 & 0.532 & 4.0 & 1.3 \\
\hline EN25 & 0.519 & 4.3 & -0.1 & 0.665 & 3.3 & 1.8 & 0.538 & 4.1 & 1.4 \\
\hline EN50 & 0.519 & 4.3 & -0.1 & 0.669 & 3.3 & 1.8 & 0.541 & 4.1 & 1.5 \\
\hline EN75 & 0.520 & 4.3 & -0.1 & 0.670 & 3.3 & 1.8 & 0.543 & 4.1 & 1.5 \\
\hline LASSO & 0.520 & 4.3 & -0.1 & 0.671 & 3.2 & 1.8 & 0.544 & 4.1 & 1.5 \\
\hline GAM & 0.536 & 4.2 & 0.0 & 0.679 & 3.3 & 2.0 & 0.553 & 4.0 & 1.6 \\
\hline Bagging & 0.537 & 4.3 & -0.5 & 0.677 & 3.1 & 1.4 & 0.597 & 3.8 & 1.1 \\
\hline $\mathrm{RF}$ & 0.552 & 4.2 & -0.5 & 0.670 & 3.1 & 1.5 & 0.601 & 3.8 & 1.1 \\
\hline GBM & 0.586 & 4.1 & -0.5 & 0.711 & 3.0 & 1.5 & 0.616 & 3.7 & 1.1 \\
\hline SVR & 0.423 & 4.7 & -0.8 & 0.677 & 3.5 & 2.3 & 0.493 & 4.1 & 1.0 \\
\hline KRLS & 0.487 & 4.5 & -0.4 & 0.663 & 3.4 & 2.1 & 0.551 & 4.0 & 1.2 \\
\hline ANN & 0.411 & 4.7 & 0.3 & 0.542 & 4.8 & 3.2 & 0.363 & 5.0 & 1.7 \\
\hline Ensemble1 & 0.522 & 4.3 & -0.1 & 0.680 & 3.2 & 1.8 & 0.554 & 4.0 & 1.4 \\
\hline Ensemble2 & 0.544 & 4.3 & -0.2 & 0.688 & 3.2 & 1.9 & 0.579 & 3.9 & 1.3 \\
\hline
\end{tabular}

${ }^{\mathrm{a}} \mathrm{FLR}=$ Forward stepwise Linear Regression; BLR = Backward stepwise Linear Regression; WLM = Stepwise Linear Regression; SLR = Supervised Linear Regression; EN25 = Elastic Net with $\alpha=0.25$; EN50 = Elastic Net with $\alpha=0.50$; EN75 = Elastic Net with $\alpha=0.75$; LASSO = Least Absolute Shrinkage and Selection Operator; GAM = Generalized Additive Model; RF = Random Forest; GBM = Generalized Boosted Machine; SVR = Support Vector Regression; KRLS = Kernel-based Regularized Least Squares; ANN = Artificial Neural Network; Ensemble1 = Ensemble model based on median prediction; Ensemble2 = Ensemble model based on weighted average

${ }^{\mathrm{b}} \mathrm{RMSE}=$ Root-mean-square error 


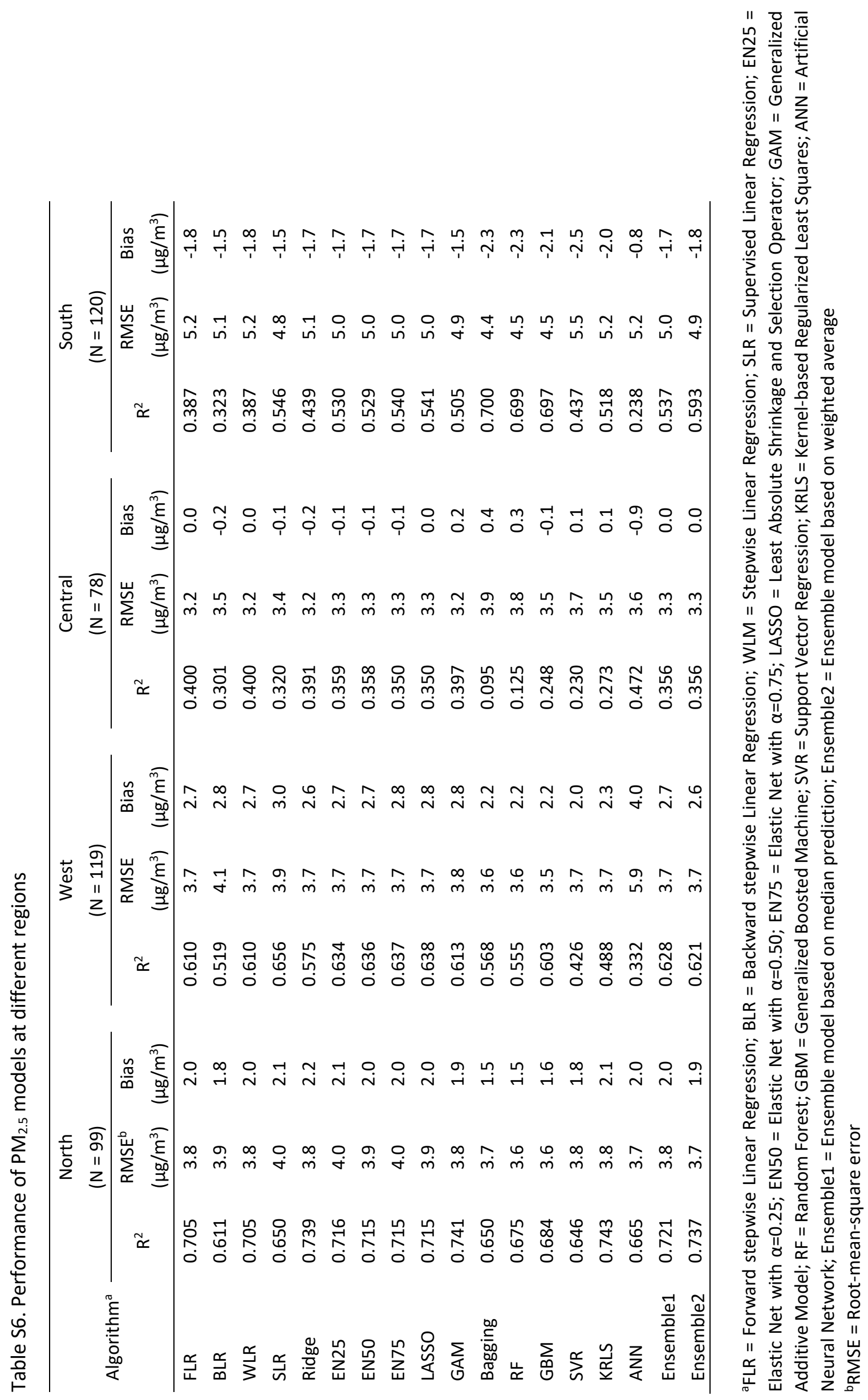


Table S7. Predictors ${ }^{\mathrm{a}}$ included and corresponding coefficients ${ }^{\mathrm{b}}$ of parametric $\mathrm{PM}_{2.5}$ models

\begin{tabular}{|c|c|}
\hline Algorithm ${ }^{c}$ & main model \\
\hline FLR & 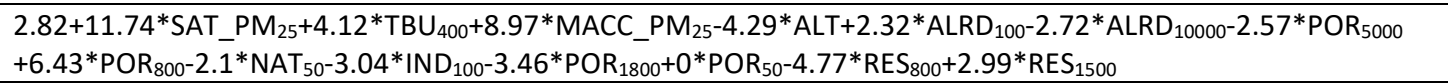 \\
\hline BLR & 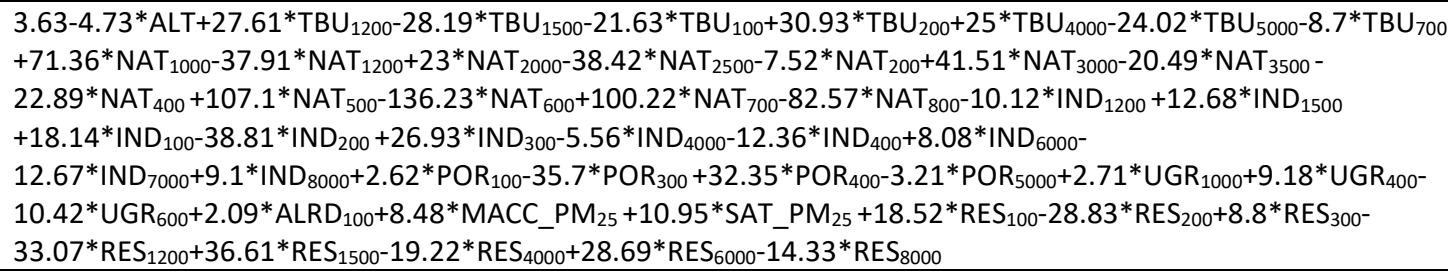 \\
\hline WLR & $\begin{array}{l}\text { 2.82+11.74*SAT_PM } 25+4.12 * \mathrm{TBU}_{400}+8.97 * \mathrm{MACC}_{2} \mathrm{PM}_{25}-4.29 * \mathrm{ALT}+2.32 * \mathrm{ALRD}_{100}-2.72 * \mathrm{ALRD}_{10000}-2.57 * \mathrm{POR}_{5000} \\
+6.43 * \mathrm{POR}_{800}-2.1 * \mathrm{NAT}_{50}-3.04 * \mathrm{IND}_{100}-3.46 * \mathrm{POR}_{1800}+0 * \mathrm{POR}_{50}-4.77 * \mathrm{RES}_{800}+2.99 * \mathrm{RES}_{1500}\end{array}$ \\
\hline SLR & 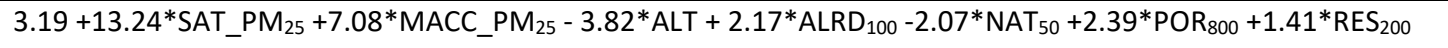 \\
\hline Ridge & 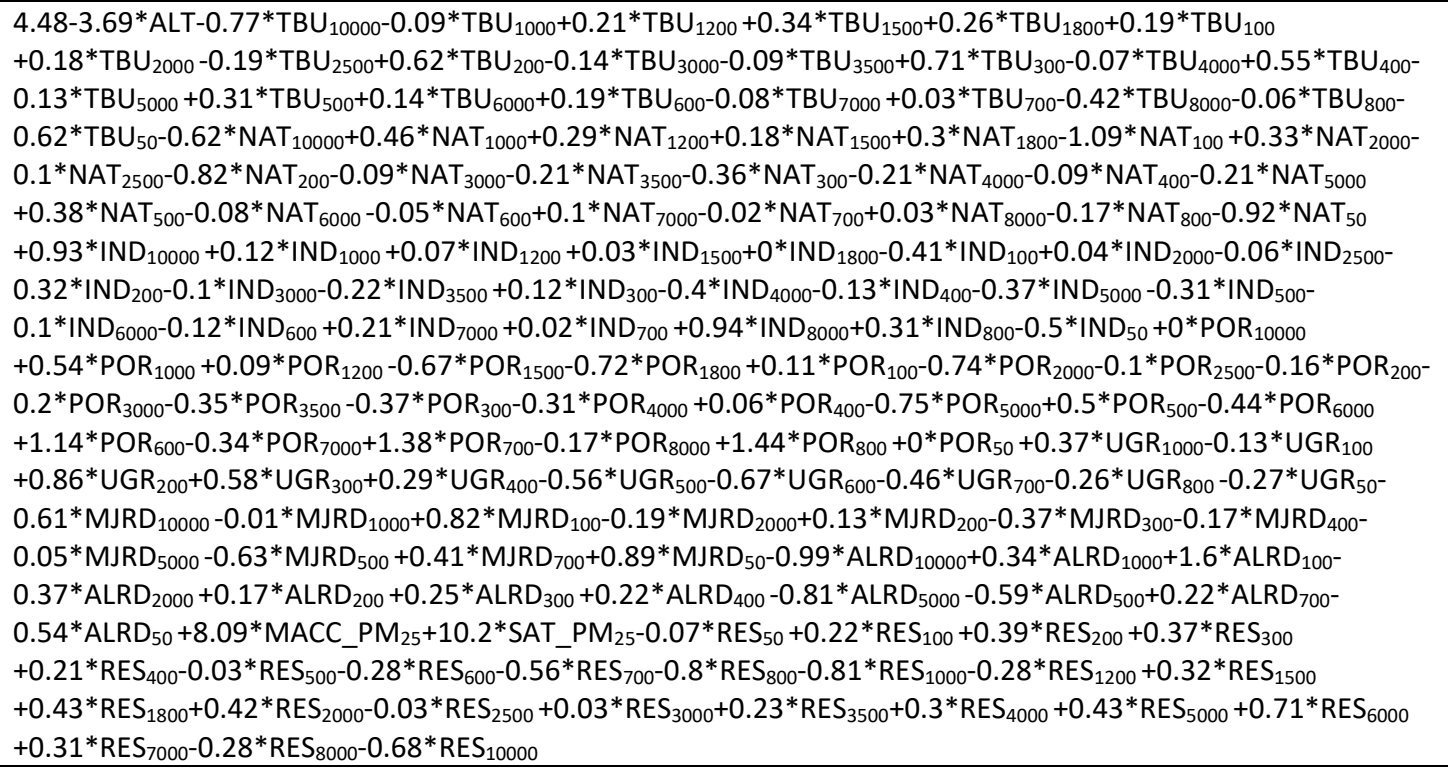 \\
\hline EN25 & 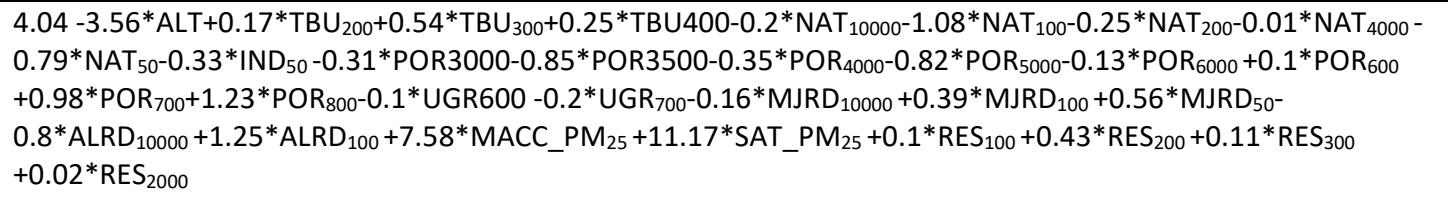 \\
\hline EN50 & 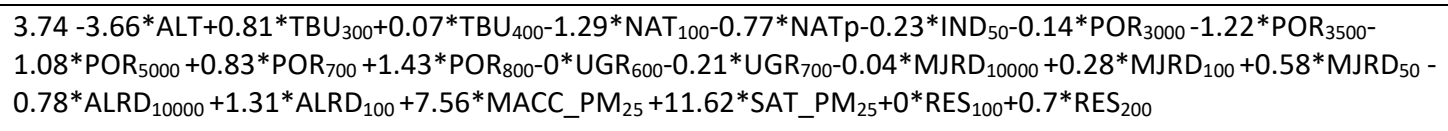 \\
\hline EN75 & $\begin{array}{l}\text { 3.73 -3.63*ALT+0.73*TBU }{ }_{300}-1.31 * \mathrm{NAT}_{100}-0.7 * \mathrm{NAT}_{50}-0.09 * \mathrm{IND}_{50}-1.26 * \mathrm{POR}_{3500}-1.04 * \mathrm{POR}_{5000}+0.69 * \mathrm{POR}_{700} \\
+1.51 * \mathrm{POR}_{800}-0.17 * \mathrm{UGR}_{700}+0.21 * \mathrm{MJRD}_{100}+0.59 * \mathrm{MJRD}_{50}-0.7 * \mathrm{ALRD}_{10000}+1.3 * \mathrm{ALRD}_{100}+7.44 * \mathrm{MACC}_{-} \mathrm{PM}_{25} \\
+11.78 * \mathrm{SAT} \mathrm{PM}_{25}+0.81 * \mathrm{RES}_{200}\end{array}$ \\
\hline LASSO & $\begin{array}{l}\text { 3.67-3.65*ALT+0.74*TBU }{ }_{300}-1.29 * \mathrm{NAT}_{100}-0.69 * \mathrm{NAT}_{50}-0.08 * \mathrm{IND}_{50}-1.33 * \mathrm{POR}_{3500}-1 * \mathrm{POR}_{5000}+0.04 * \mathrm{POR}_{700} \\
+2.2 * \mathrm{POR}_{800}-0.15 * \mathrm{UGR}_{700}+0.18 * \mathrm{MJRD}_{100}+0.59 * \mathrm{MJRD}_{50}-0.72 * \mathrm{ALRD}_{10000}+1.33 * \mathrm{ALRD}_{100}+7.45 * \mathrm{MACC}_{2} \mathrm{PM}_{25} \\
\text { +11.9*SAT_PM } \\
25+0.8 * \mathrm{RES}_{200}\end{array}$ \\
\hline
\end{tabular}

${ }^{\mathrm{a}} \mathrm{MACC}=\mathrm{MACC}$ dispersion model, $\mathrm{SAT}=$ satellite, $\mathrm{MJRD}=$ major roads, $\mathrm{ALRD}=$ all roads, $\mathrm{TBU}=$ total build up, NAT = natural land, IND = industry, POR = ports, UGR = urban green, RES = residential, ALT = altitude

${ }^{\mathrm{b}}$ Regression slope were multiplied by the difference between the $1^{\text {st }}$ and $99^{\text {th }}$ percentile of each predictor to allow comparison across predictors

${ }^{c}$ FLR = Forward stepwise Linear Regression; BLR = Backward stepwise Linear Regression; WLM = Stepwise Linear Regression; SLR = Supervised Linear Regression; EN25 = Elastic Net with $\alpha=0.25$; EN50 = Elastic Net with $\alpha=0.50 ;$ EN75 = Elastic Net with $\alpha=0.75$; LASSO = Least Absolute Shrinkage and Selection Operator 
Table S8. Performance of $\mathrm{NO}_{2}$ models at ESCAPE sites with annual average $\mathrm{NO}_{2}$ concentration below certain values

\begin{tabular}{|c|c|c|c|c|c|c|c|c|c|}
\hline \multirow{2}{*}{ Algorithm $^{a}$} & \multicolumn{3}{|c|}{$\begin{array}{c}<20 \mu \mathrm{g} / \mathrm{m}^{3} \\
(\mathrm{~N}=433)\end{array}$} & \multicolumn{3}{|c|}{$\begin{array}{c}<30 \mu \mathrm{g} / \mathrm{m}^{3} \\
(\mathrm{~N}=844)\end{array}$} & \multicolumn{3}{|c|}{$\begin{array}{l}<40 \mu \mathrm{g} / \mathrm{m}^{3} \\
(\mathrm{~N}=1116)\end{array}$} \\
\hline & $\mathrm{R}^{2}$ & $\begin{array}{l}\text { RMSE }^{\mathrm{b}} \\
\left(\mu \mathrm{g} / \mathrm{m}^{3}\right)\end{array}$ & $\begin{array}{c}\text { Bias } \\
\left(\mu \mathrm{g} / \mathrm{m}^{3}\right)\end{array}$ & $\mathrm{R}^{2}$ & $\begin{array}{c}\text { RMSE } \\
\left(\mu \mathrm{g} / \mathrm{m}^{3}\right)\end{array}$ & $\begin{array}{c}\text { Bias } \\
\left(\mu \mathrm{g} / \mathrm{m}^{3}\right)\end{array}$ & $\mathrm{R}^{2}$ & $\begin{array}{c}\text { RMSE } \\
\left(\mu \mathrm{g} / \mathrm{m}^{3}\right)\end{array}$ & $\begin{array}{c}\text { Bias } \\
\left(\mu \mathrm{g} / \mathrm{m}^{3}\right)\end{array}$ \\
\hline FLR & 0.339 & 9.6 & 7.9 & 0.463 & 8.8 & 6.6 & 0.488 & 8.4 & 5.1 \\
\hline BLR & 0.313 & 9.8 & 8.0 & 0.451 & 9.2 & 6.9 & 0.473 & 8.9 & 5.5 \\
\hline WLR & 0.339 & 9.6 & 7.9 & 0.463 & 8.8 & 6.6 & 0.488 & 8.4 & 5.1 \\
\hline SLR & 0.385 & 10.2 & 8.7 & 0.492 & 9.5 & 7.6 & 0.508 & 9.2 & 6.3 \\
\hline Ridge & 0.341 & 10.3 & 8.8 & 0.471 & 9.6 & 7.6 & 0.492 & 9.3 & 6.3 \\
\hline EN25 & 0.337 & 10.0 & 8.4 & 0.467 & 9.2 & 7.2 & 0.492 & 8.9 & 5.8 \\
\hline EN50 & 0.333 & 9.9 & 8.3 & 0.464 & 9.2 & 7.0 & 0.490 & 8.8 & 5.7 \\
\hline EN75 & 0.334 & 9.9 & 8.3 & 0.465 & 9.1 & 7.0 & 0.491 & 8.8 & 5.7 \\
\hline LASSO & 0.334 & 9.9 & 8.2 & 0.465 & 9.1 & 7.0 & 0.490 & 8.8 & 5.7 \\
\hline GAM & 0.367 & 9.3 & 7.4 & 0.491 & 9.3 & 6.8 & 0.507 & 9.2 & 5.8 \\
\hline Bagging & 0.320 & 10.9 & 9.3 & 0.448 & 10.2 & 8.1 & 0.481 & 9.8 & 6.9 \\
\hline RF & 0.323 & 11.0 & 9.5 & 0.447 & 10.3 & 8.3 & 0.480 & 9.9 & 7.0 \\
\hline GBM & 0.344 & 10.0 & 8.4 & 0.476 & 9.6 & 7.5 & 0.498 & 9.4 & 6.3 \\
\hline SVR & 0.311 & 9.3 & 7.6 & 0.455 & 8.7 & 6.4 & 0.483 & 8.5 & 5.0 \\
\hline KRLS & 0.326 & 9.9 & 8.4 & 0.470 & 9.5 & 7.3 & 0.495 & 9.2 & 6.1 \\
\hline ANN & 0.332 & 14.6 & 12.6 & 0.470 & 14.9 & 12.8 & 0.489 & 14.6 & 12.1 \\
\hline Ensemble1 & 0.352 & 9.9 & 8.4 & 0.478 & 9.2 & 7.2 & 0.500 & 8.9 & 5.9 \\
\hline Ensemble2 & 0.356 & 10.1 & 8.6 & 0.487 & 9.5 & 7.5 & 0.511 & 9.1 & 6.3 \\
\hline
\end{tabular}

${ }^{\mathrm{a}} \mathrm{FLR}=$ Forward stepwise Linear Regression; BLR = Backward stepwise Linear Regression; WLM = Stepwise Linear Regression; SLR = Supervised Linear Regression; EN25 = Elastic Net with $\alpha=0.25 ;$ EN50 = Elastic Net with $\alpha=0.50$; EN75 = Elastic Net with $\alpha=0.75$; LASSO = Least Absolute Shrinkage and Selection Operator; GAM = Generalized Additive Model; RF = Random Forest; GBM = Generalized Boosted Machine; SVR = Support Vector Regression; KRLS = Kernel-based Regularized Least Squares; ANN = Artificial Neural Network; Ensemble1 = Ensemble model based on median prediction; Ensemble2 = Ensemble model based on weighted average

${ }^{\mathrm{b}} \mathrm{RMSE}=$ Root-mean-square error 
Table S9. Performance of $\mathrm{NO}_{2}$ models at different type of sites

\begin{tabular}{|c|c|c|c|c|c|c|c|c|c|}
\hline \multirow{2}{*}{ Algorithm ${ }^{\mathrm{a}}$} & \multicolumn{3}{|c|}{$\begin{array}{c}\text { Street } \\
(\mathrm{N}=713)\end{array}$} & \multicolumn{3}{|c|}{$\begin{array}{l}\text { Rural Background } \\
\qquad(\mathrm{N}=118)\end{array}$} & \multicolumn{3}{|c|}{$\begin{array}{l}\text { Urban Background } \\
\qquad(\mathrm{N}=565)\end{array}$} \\
\hline & $R^{2}$ & $\begin{array}{l}\text { RMSE }^{\mathrm{b}} \\
\left(\mu \mathrm{g} / \mathrm{m}^{3}\right)\end{array}$ & $\begin{array}{c}\text { Bias } \\
\left(\mu \mathrm{g} / \mathrm{m}^{3}\right) \\
\end{array}$ & $R^{2}$ & $\begin{array}{c}\text { RMSE } \\
\left(\mu \mathrm{g} / \mathrm{m}^{3}\right)\end{array}$ & $\begin{array}{c}\text { Bias } \\
\left(\mu \mathrm{g} / \mathrm{m}^{3}\right)\end{array}$ & $\mathrm{R}^{2}$ & $\begin{array}{c}\text { RMSE } \\
\left(\mu \mathrm{g} / \mathrm{m}^{3}\right) \\
\end{array}$ & $\begin{array}{c}\text { Bias } \\
\left(\mu \mathrm{g} / \mathrm{m}^{3}\right) \\
\end{array}$ \\
\hline FLR & 0.392 & 13.7 & -3.2 & 0.486 & 7.6 & 5.3 & 0.584 & 9.0 & 6.1 \\
\hline BLR & 0.400 & 13.5 & -2.7 & 0.466 & 8.1 & 5.7 & 0.573 & 9.4 & 6.6 \\
\hline WLR & 0.392 & 13.7 & -3.2 & 0.486 & 7.6 & 5.3 & 0.584 & 9.0 & 6.1 \\
\hline SLR & 0.384 & 13.6 & -2.0 & 0.594 & 8.9 & 7.2 & 0.612 & 9.7 & 7.2 \\
\hline Ridge & 0.398 & 13.4 & -2.0 & 0.534 & 8.7 & 6.8 & 0.596 & 9.8 & 7.4 \\
\hline EN25 & 0.403 & 13.5 & -2.5 & 0.514 & 8.3 & 6.2 & 0.595 & 9.4 & 6.8 \\
\hline EN50 & 0.405 & 13.5 & -2.6 & 0.505 & 8.2 & 6.1 & 0.593 & 9.4 & 6.7 \\
\hline EN75 & 0.404 & 13.5 & -2.6 & 0.506 & 8.2 & 6.1 & 0.594 & 9.4 & 6.7 \\
\hline LASSO & 0.404 & 13.5 & -2.7 & 0.505 & 8.2 & 6.1 & 0.594 & 9.3 & 6.7 \\
\hline GAM & 0.400 & 13.4 & -1.8 & 0.541 & 8.4 & 5.9 & 0.604 & 9.5 & 6.7 \\
\hline Bagging & 0.353 & 13.8 & -0.6 & 0.535 & 8.9 & 7.0 & 0.570 & 9.9 & 7.3 \\
\hline $\mathrm{RF}$ & 0.346 & 13.8 & -0.5 & 0.537 & 9.0 & 7.2 & 0.570 & 9.9 & 7.4 \\
\hline GBM & 0.363 & 13.7 & -1.0 & 0.563 & 8.4 & 6.6 & 0.583 & 9.4 & 6.7 \\
\hline SVR & 0.365 & 13.9 & -3.0 & 0.536 & 8.6 & 6.9 & 0.581 & 8.5 & 5.4 \\
\hline KRLS & 0.381 & 13.5 & -1.7 & 0.568 & 8.4 & 6.7 & 0.576 & 9.4 & 6.6 \\
\hline ANN & 0.388 & 14.3 & 5.1 & 0.527 & 13.3 & 11.3 & 0.579 & 15.1 & 13.0 \\
\hline Ensemble1 & 0.401 & 13.4 & -2.3 & 0.530 & 8.3 & 6.4 & 0.603 & 9.3 & 6.8 \\
\hline Ensemble2 & 0.404 & 13.3 & -1.7 & 0.544 & 8.5 & 6.6 & 0.611 & 9.5 & 7.1 \\
\hline
\end{tabular}

${ }^{\mathrm{a}} \mathrm{FLR}=$ Forward stepwise Linear Regression; BLR = Backward stepwise Linear Regression; WLM = Stepwise Linear Regression; SLR = Supervised Linear Regression; EN25 = Elastic Net with $\alpha=0.25$; EN50 = Elastic Net with $\alpha=0.50$; EN75 = Elastic Net with $\alpha=0.75 ;$ LASSO = Least Absolute Shrinkage and Selection Operator; GAM = Generalized Additive Model; RF = Random Forest; GBM = Generalized Boosted Machine; SVR = Support Vector Regression; KRLS = Kernel-based Regularized Least Squares; ANN = Artificial Neural Network; Ensemble1 = Ensemble model based on median prediction; Ensemble2 = Ensemble model based on weighted average

${ }^{\mathrm{b}} \mathrm{RMSE}=$ Root-mean-square error 


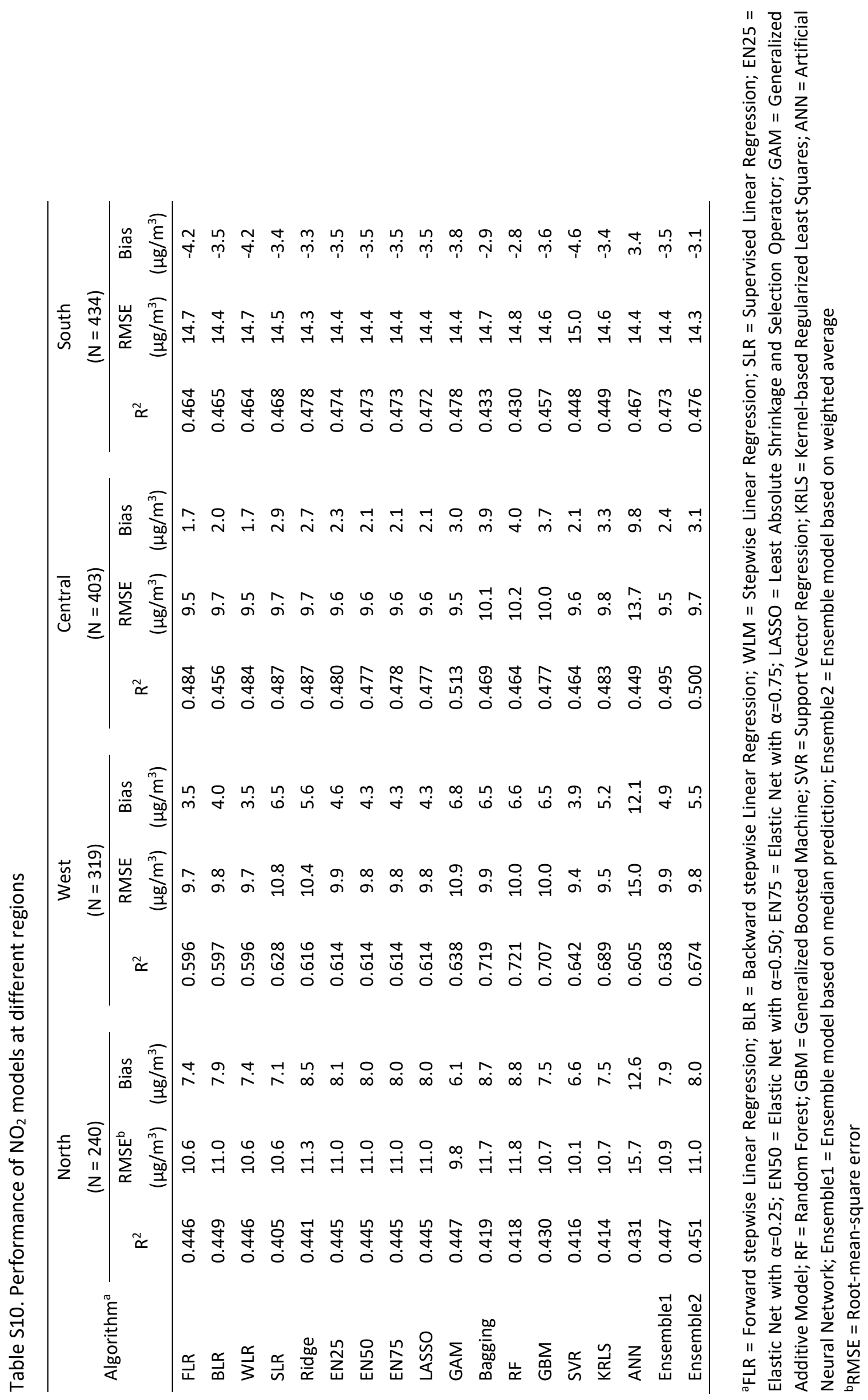


Algorithm Comparison for Exposure Modeling

Table S11. Predictors ${ }^{\mathrm{a}}$ included and corresponding coefficients ${ }^{\mathrm{b}}$ of parametric $\mathrm{NO}_{2}$ models

\begin{tabular}{|c|c|}
\hline Algorithmc & main model \\
\hline FLR & $\begin{array}{l}\text { 3.00+8.82*ALRD } 2000+25.98 * \mathrm{MACC}_{2} \mathrm{NO}_{2}+6.83 * \mathrm{ALRD}_{100}+3.91 * \mathrm{RES}_{1500}+10.74 * \mathrm{MJRD}_{100}+3.92 * \mathrm{TBU}_{400}+3.31 * \mathrm{POR}_{700^{-}} \\
\text {4.47*SAT_NO} 2+4.12 * \mathrm{ALRD}_{50}-2.71 * \mathrm{NAT}_{400}+5.26 * \mathrm{RES}_{2500}\end{array}$ \\
\hline BLR & 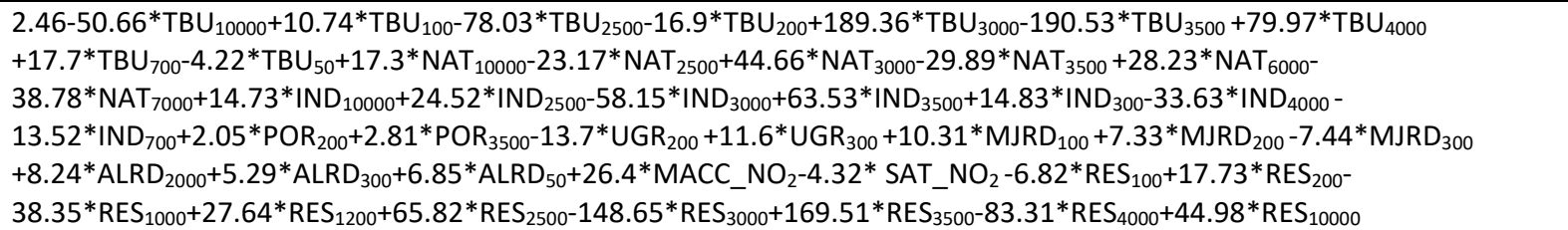 \\
\hline WLR & $\begin{array}{l}\text { 3.00+8.82*ALRD } 2000+25.98 * M A C C \_N_{2}+6.83 * \mathrm{ALRD}_{100}+3.91 * \mathrm{RES}_{1500}+10.74 * \mathrm{MJRD}_{100}+3.92 * \mathrm{TBU}_{400}+3.31 * \mathrm{POR}_{700^{-}} \\
\text {4.47*SAT_NO} 2+4.12 * \mathrm{ALRD}_{50}-2.71 * \mathrm{NAT}_{400}+5.26 * \mathrm{RES}_{2500}\end{array}$ \\
\hline SLR & $\begin{array}{l}3.30+22.73 * \mathrm{MACC} \mathrm{NO}_{2}+7.04 * \mathrm{ALRD}_{50}+3.92 * \mathrm{ALRD}_{300}+12.32 * \mathrm{MJRD}_{100}+15.73 * \mathrm{ALRD}_{2000}-3.38 * \mathrm{NAT}_{400}+4.1 * \mathrm{POR}_{700} \\
+5.8 * \mathrm{RES}_{300}\end{array}$ \\
\hline Ridge & 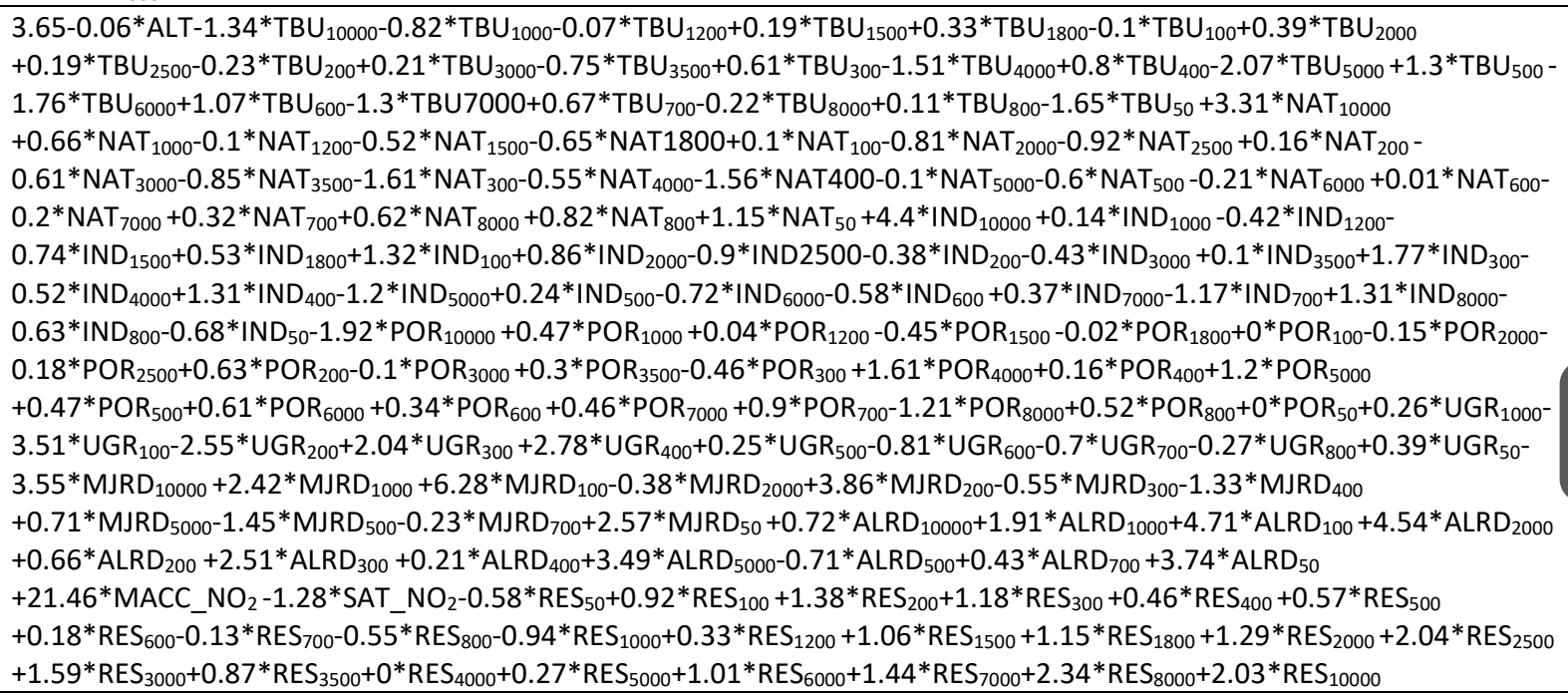 \\
\hline EN25 & 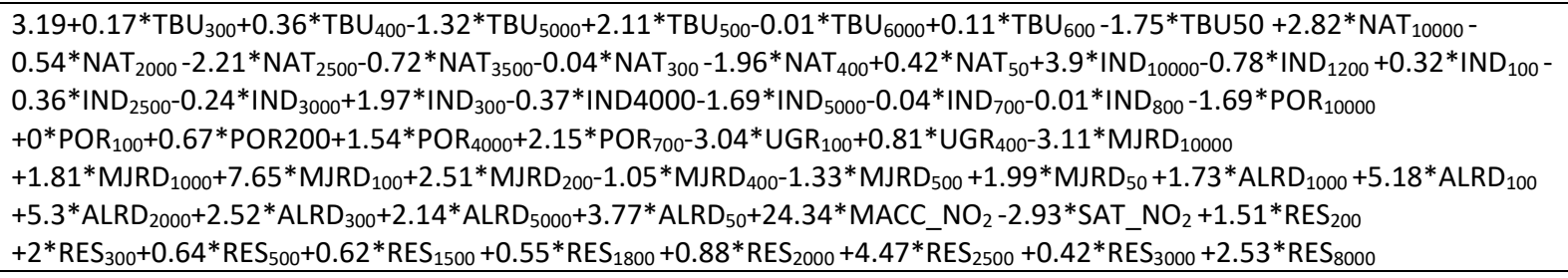 \\
\hline EN50 & 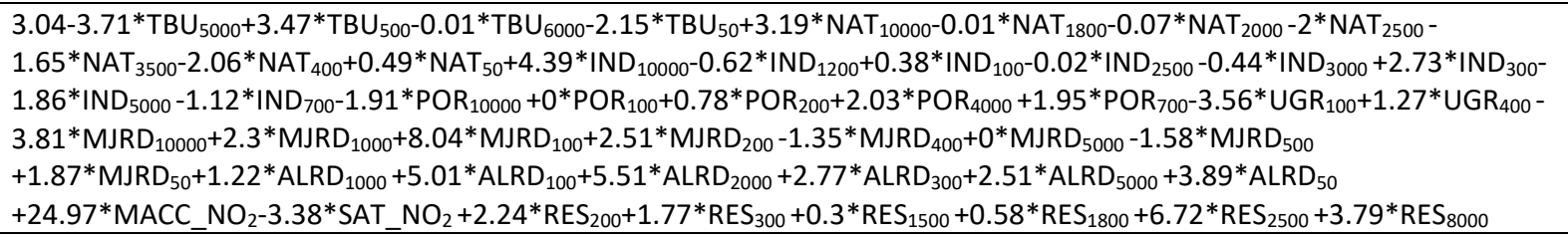 \\
\hline EN75 & 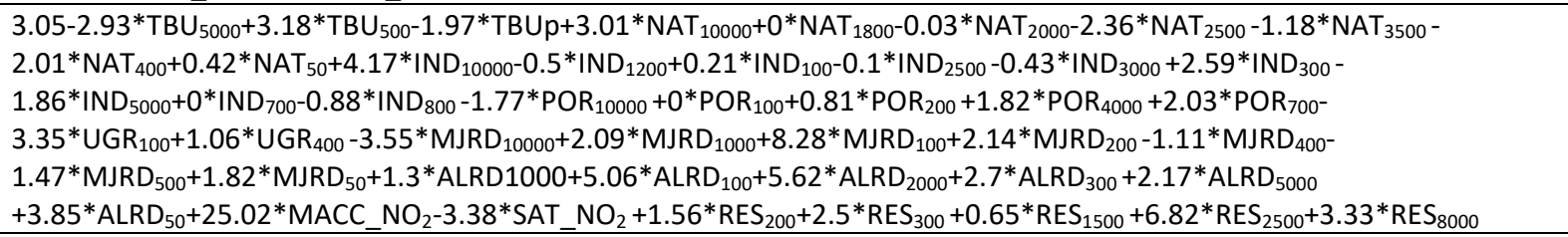 \\
\hline LASSO & 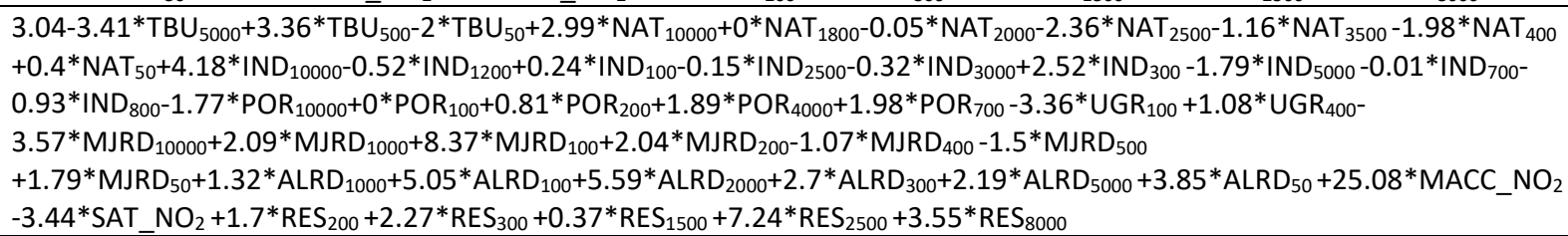 \\
\hline
\end{tabular}

${ }^{a} \mathrm{MACC}=\mathrm{MACC}$ dispersion model, SAT = satellite, MJRD = major roads, $\mathrm{ALRD}=$ all roads, $\mathrm{TBU}=$ total build up, NAT = natural land, IND = industry, $\mathrm{POR}=$ ports, $\mathrm{UGR}=$ urban green, $\mathrm{RES}=$ residential, $\mathrm{ALT}=$ altitude

${ }^{\mathrm{b}}$ Regression slope were multiplied by the difference between the $1^{\text {st }}$ and $99^{\text {th }}$ percentile of each predictor to allow comparison across predictors ${ }^{c} \mathrm{FLR}=$ Forward stepwise Linear Regression; BLR = Backward stepwise Linear Regression; WLM = Stepwise Linear Regression; SLR $=$ Supervised Linear Regression; EN25 = Elastic Net with $\alpha=0.25$; EN50 = Elastic Net with $\alpha=0.50$; EN75 = Elastic Net with $\alpha=0.75 ;$ LASSO = Least Absolute Shrinkage and Selection Operator 


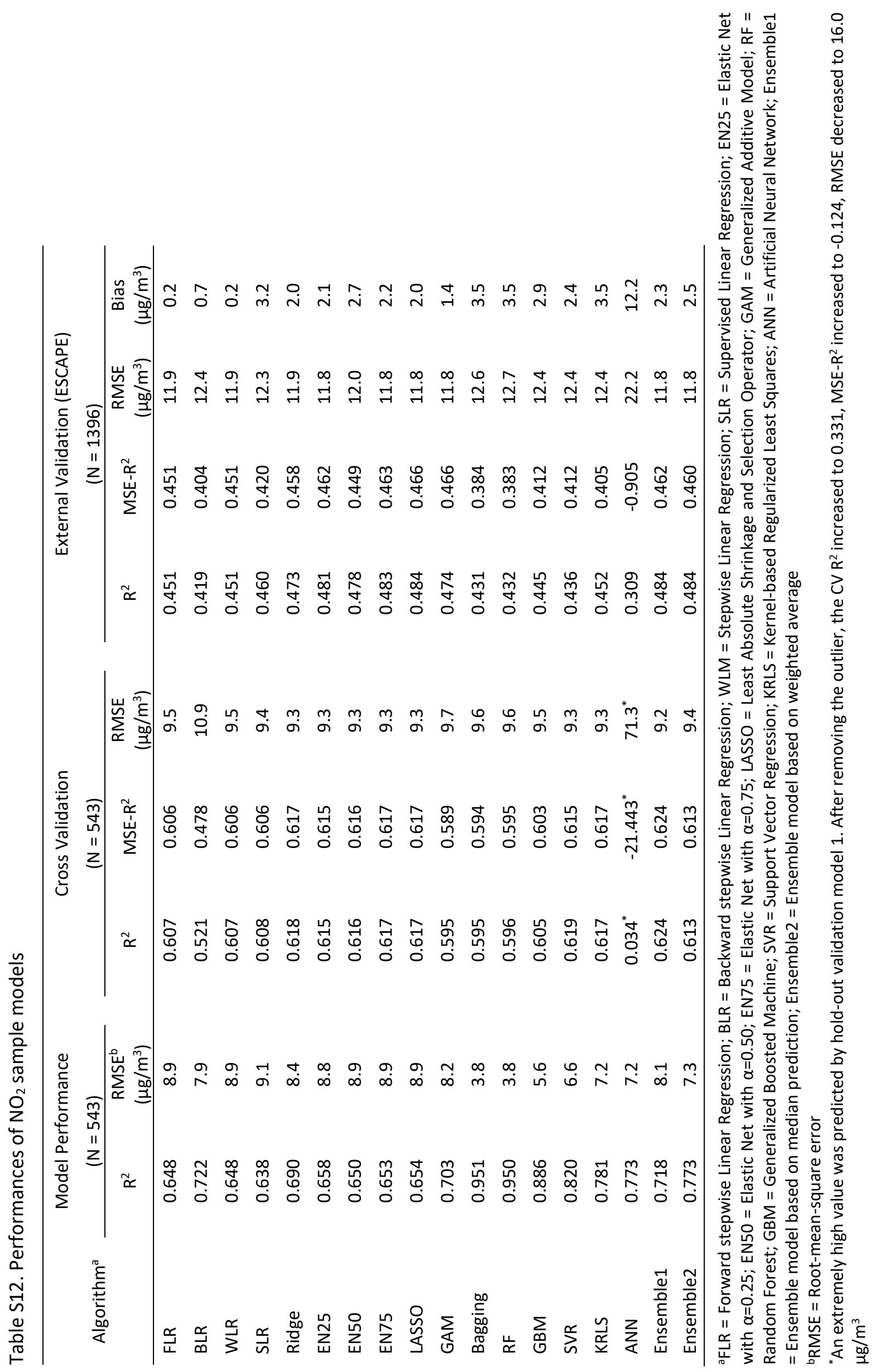




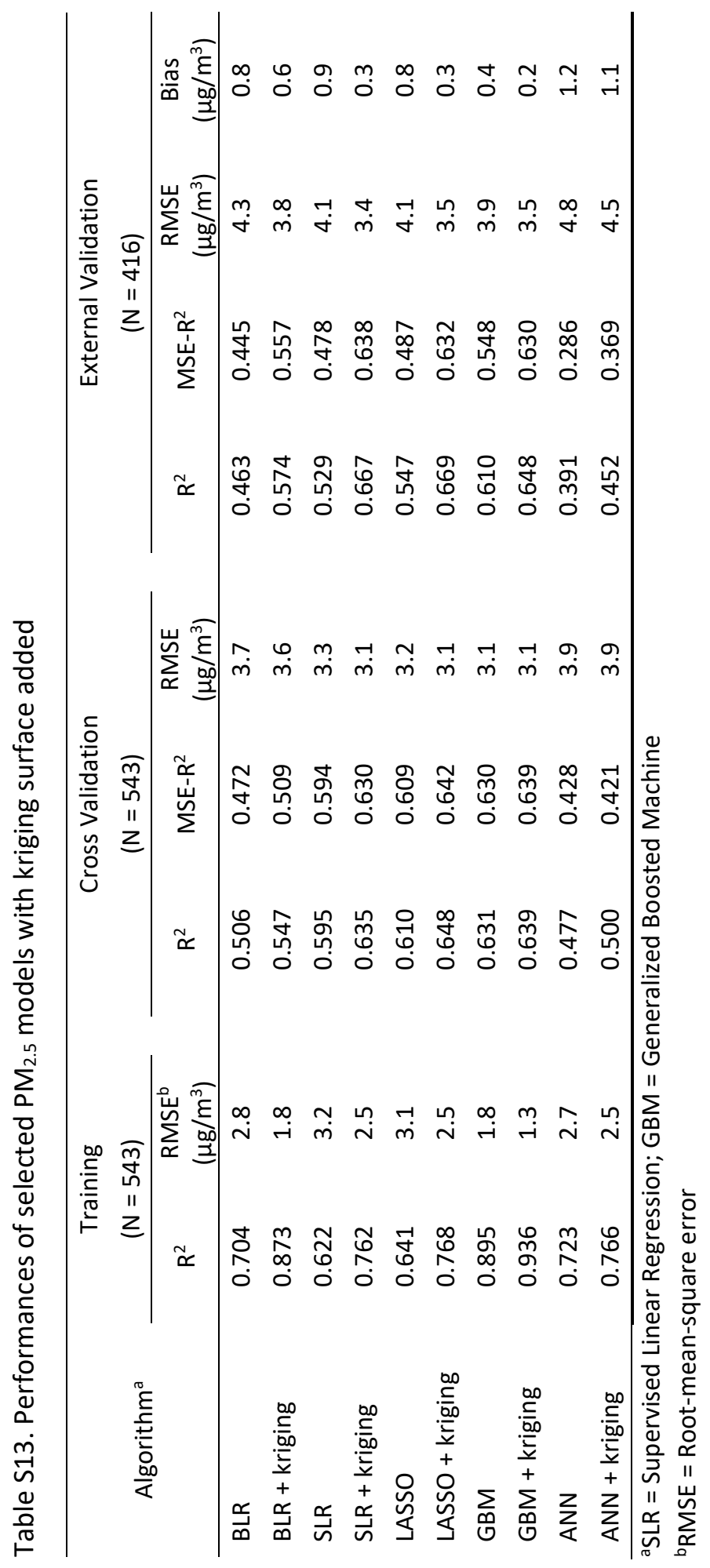




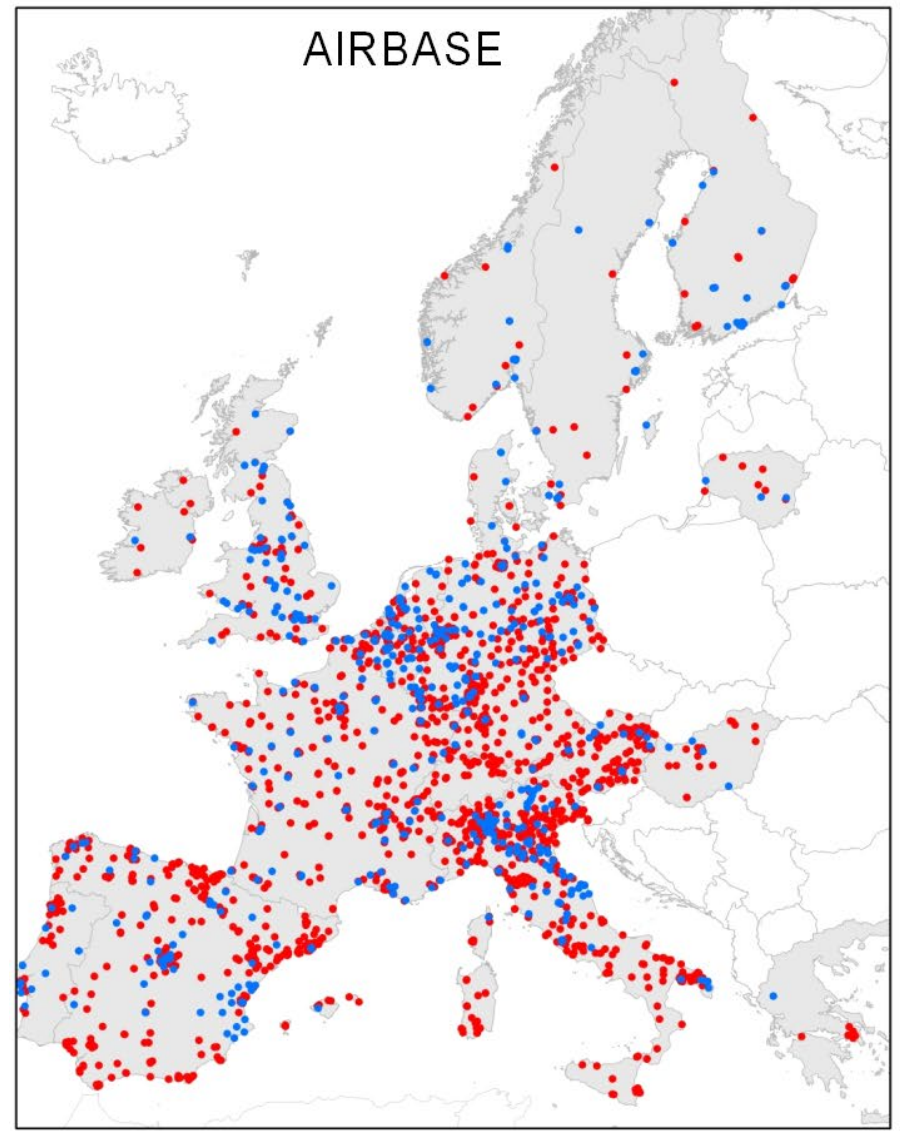

\begin{tabular}{llll}
0 & 200 & $400 \quad 800 \quad 1 ' 200$ & \\
\hline
\end{tabular}

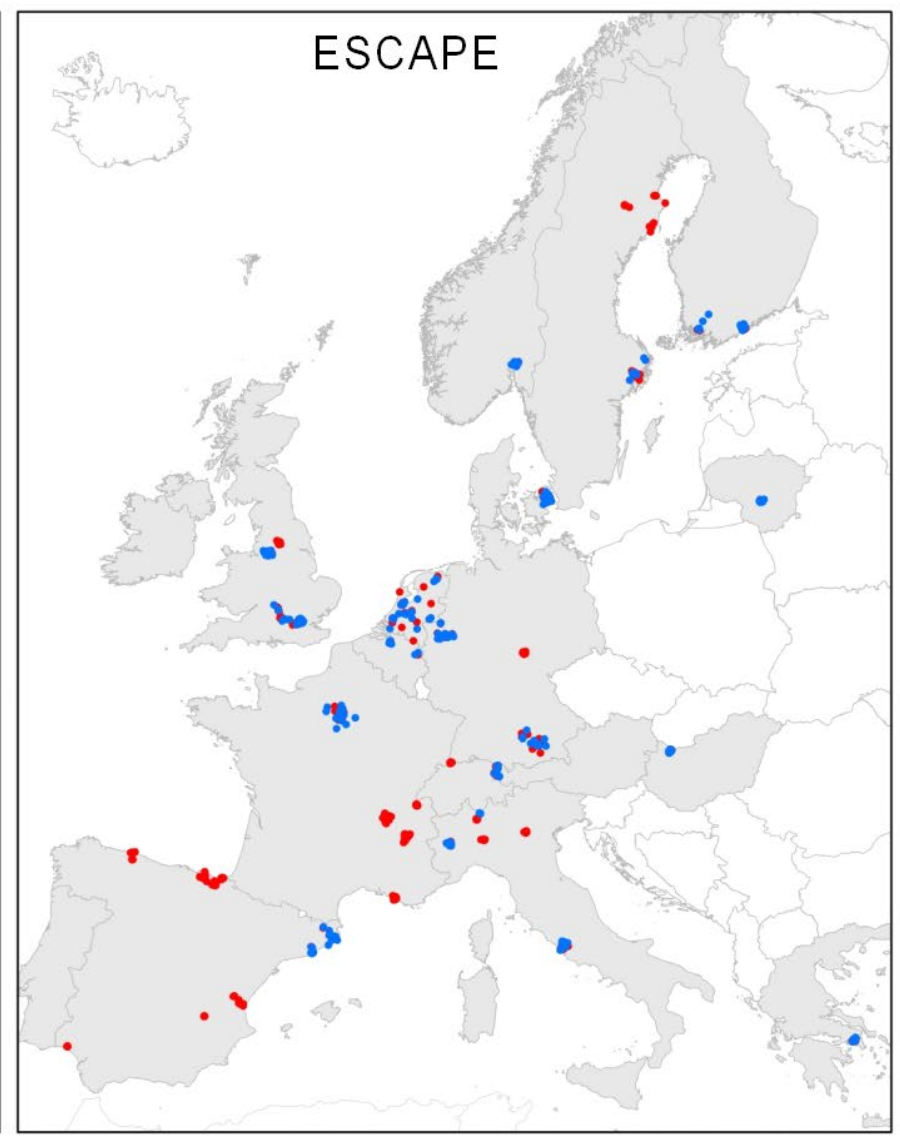

- $\mathrm{NO}_{2}$ monitoring sites

Figure S1. location of AirBase and ESCAPE monitoring sites (de Hoogh et al., 2016) 

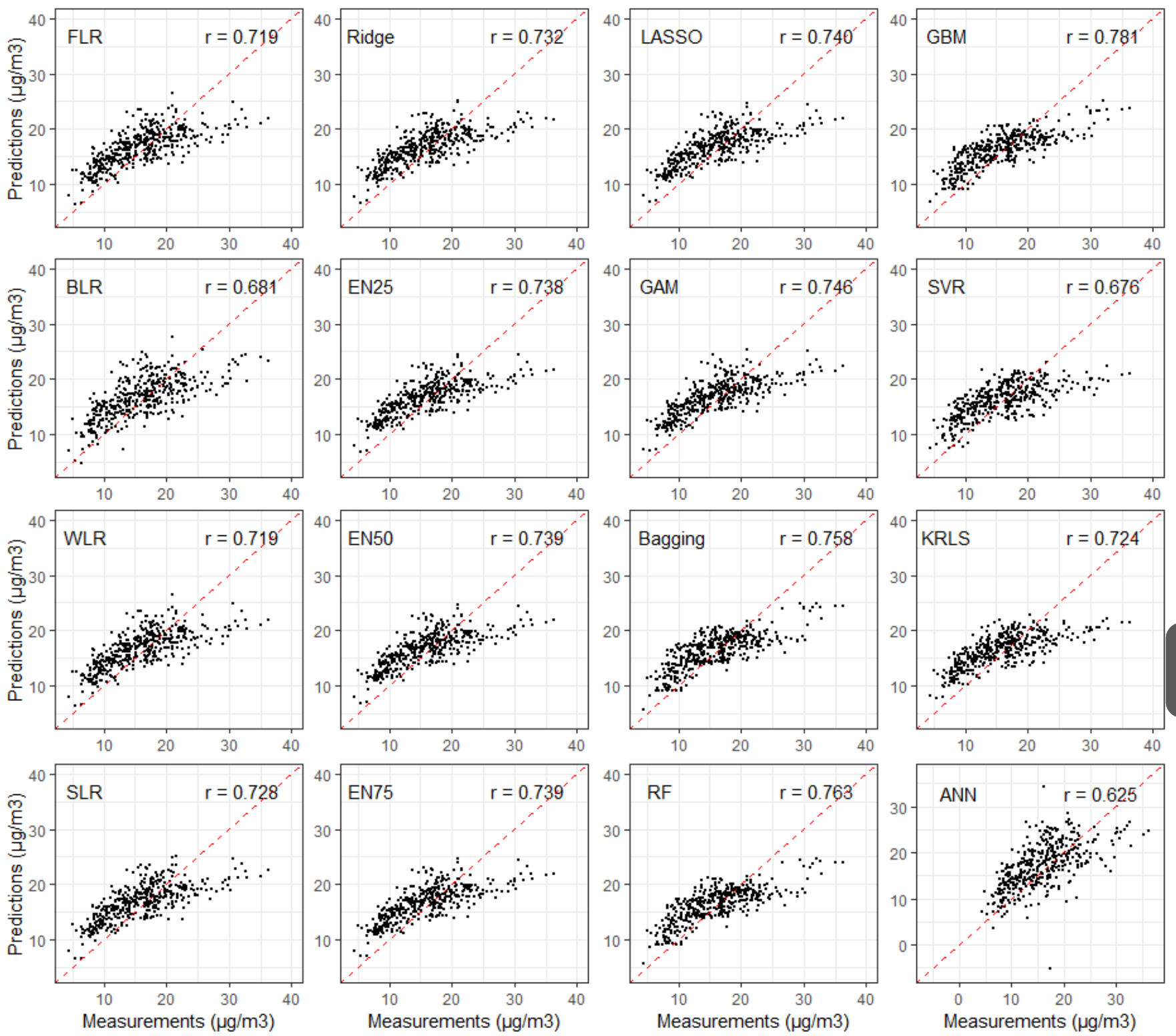

Figure S2. Correlations between $\mathrm{PM}_{2.5}$ predictions and measurements at ESCAPE sites $(r=$ Pearson correlation coefficient) 

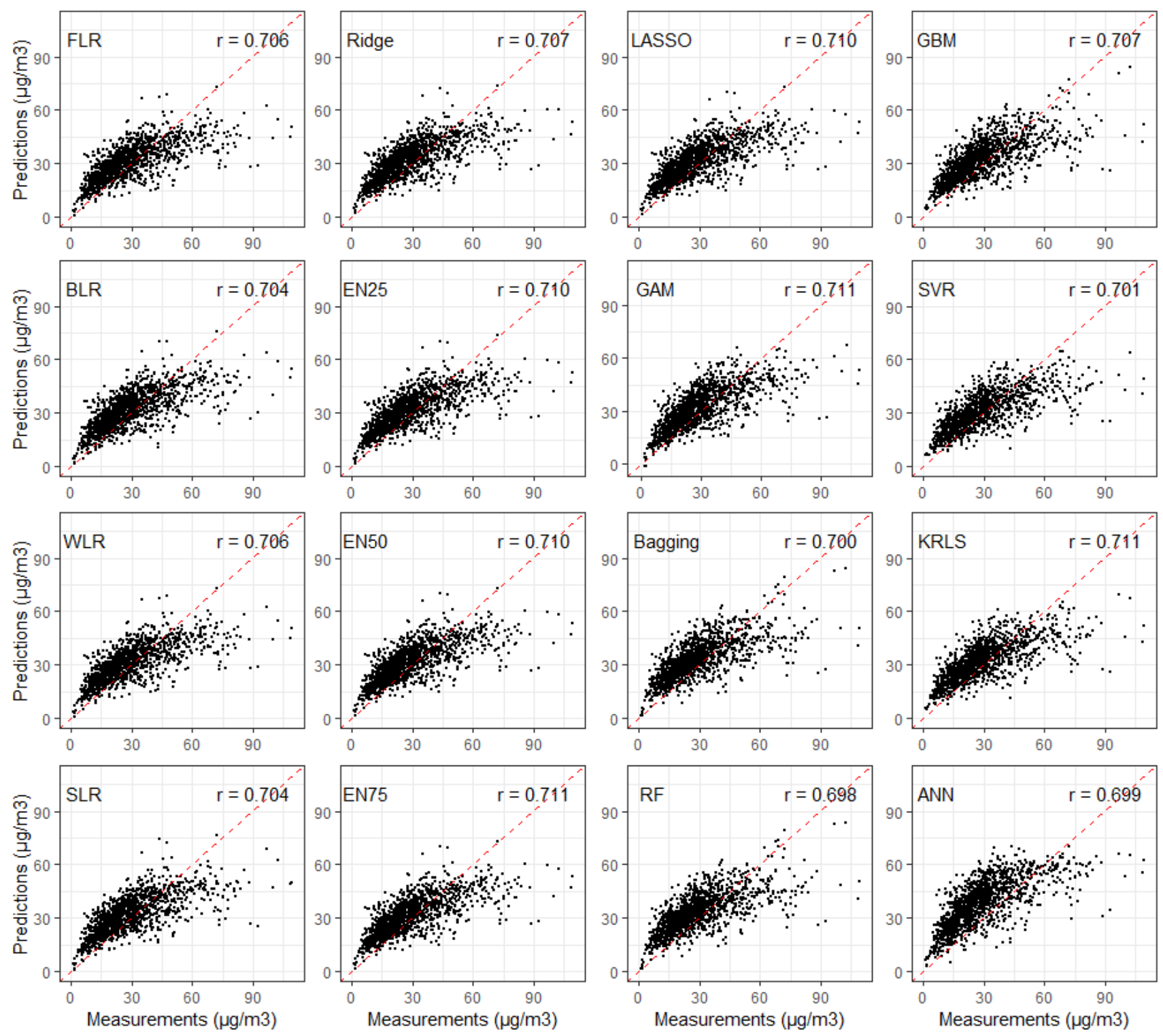

Figure S3. Correlations between $\mathrm{NO}_{2}$ predictions and measurements at ESCAPE sites ( $r=$ Pearson correlation coefficient) 


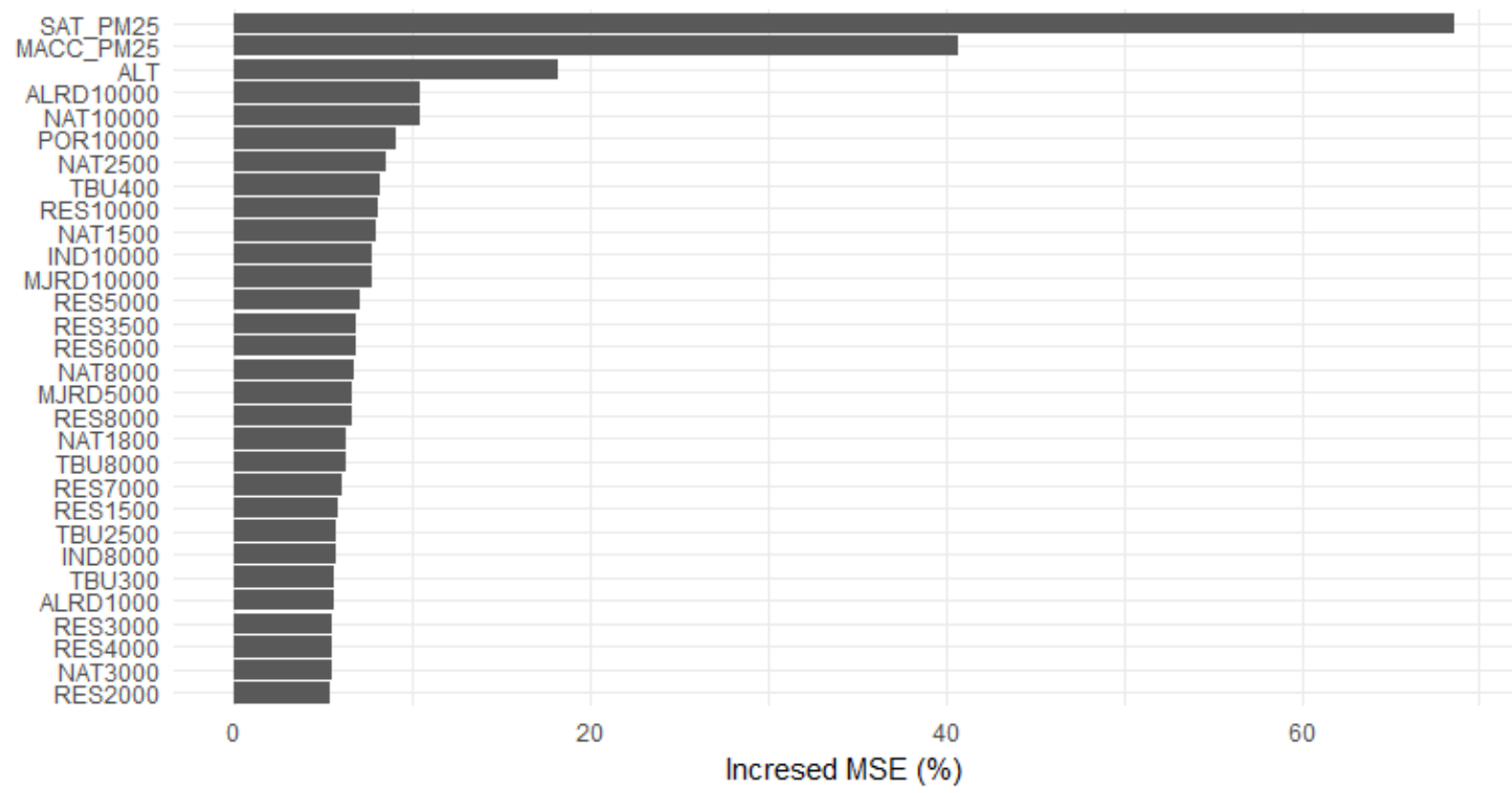

(A) Percentage increase in MSE (Mean Squared Errors) after a random permutation of the values of a variable (Random Forest)

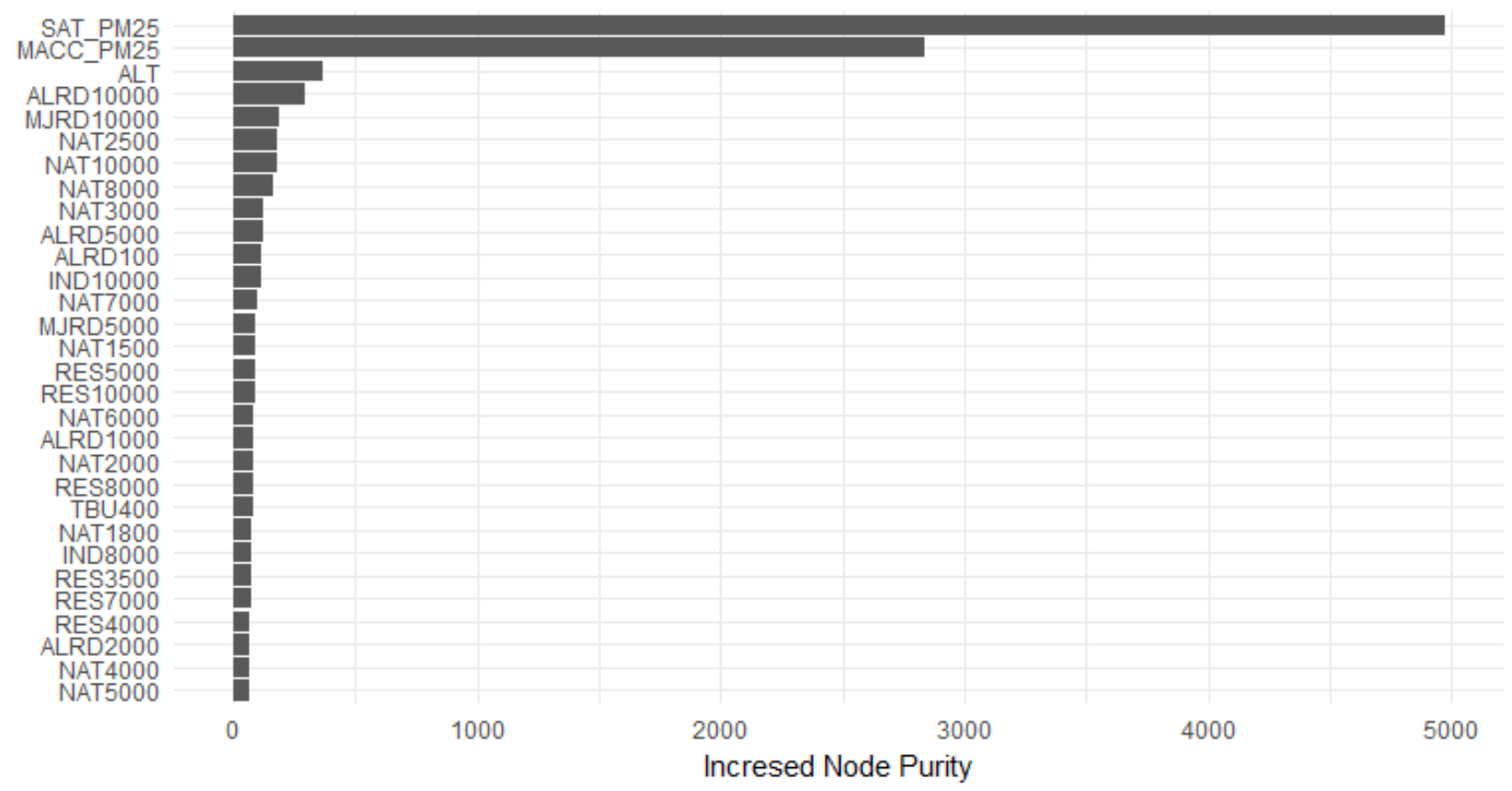

(B) Increase in MSE after a split on a variable (Random Forest) 

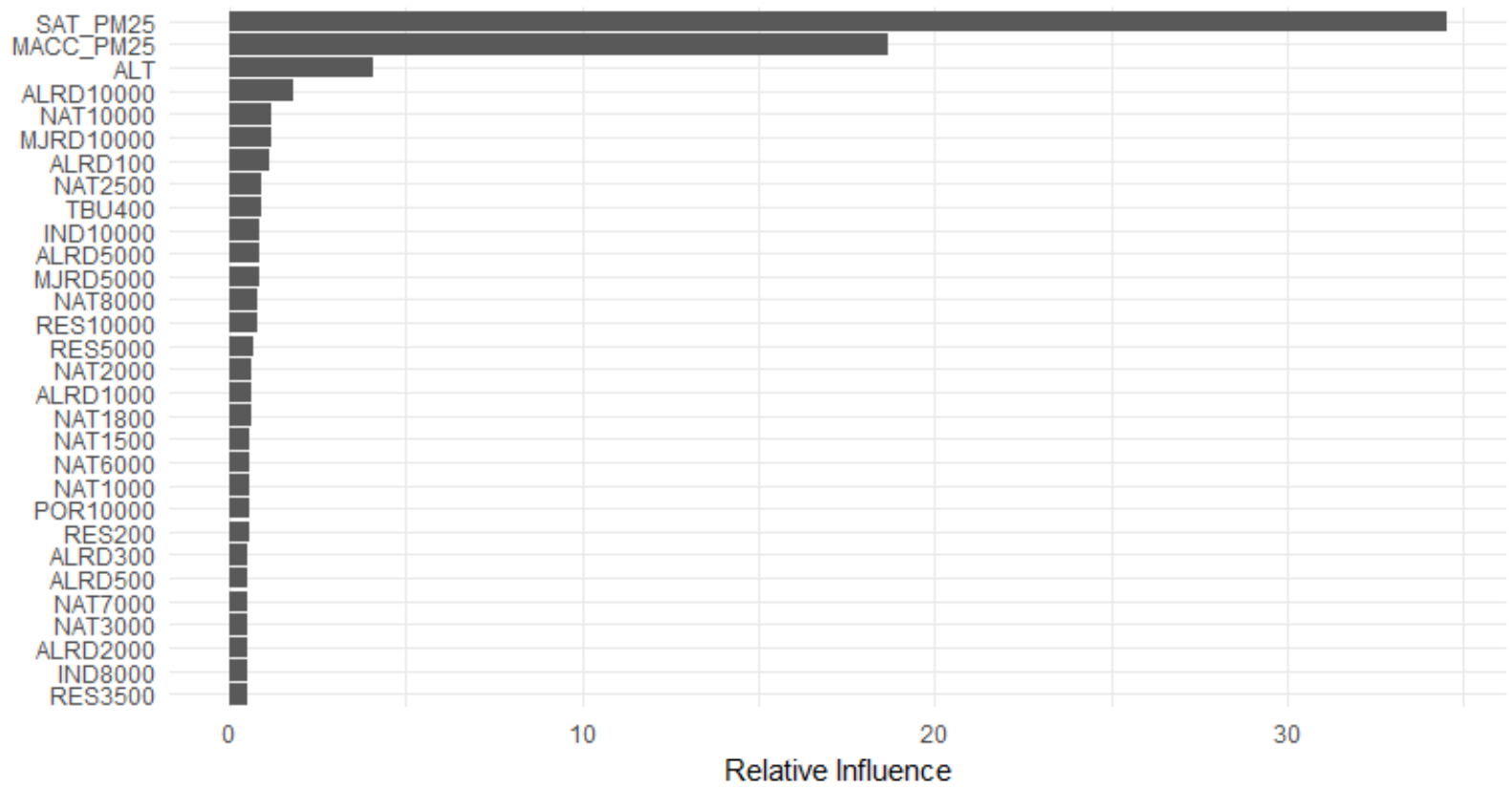

(C) Relative influence of predictors (Generalized Boosted Machine)

Figure S4. Variable importance plots of $\mathrm{PM}_{2.5}$ random forest and Generalized Boosted Machine models

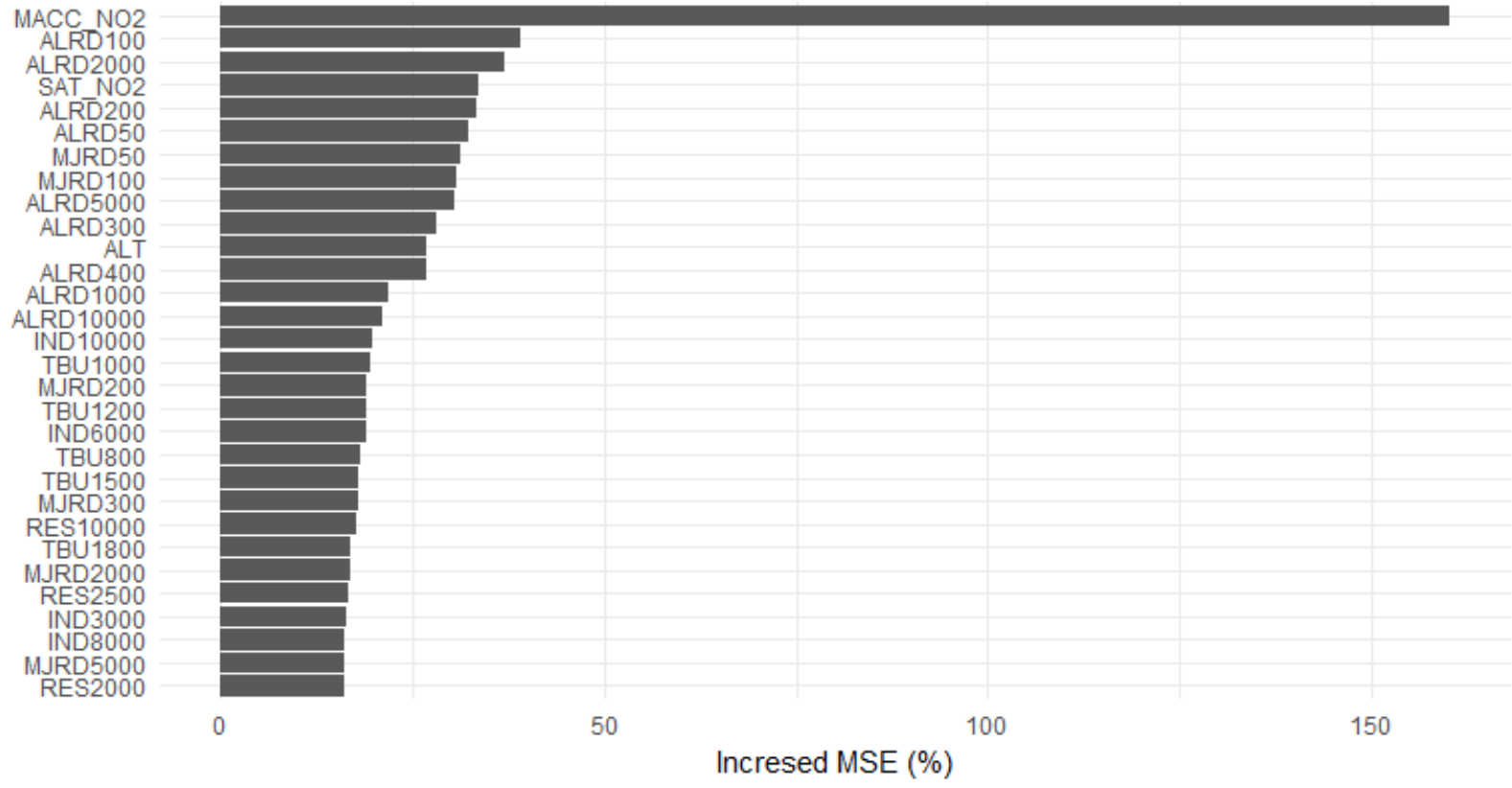

(A) Percentage increase in MSE after a random permutation of the values of a variable (random Forest) 


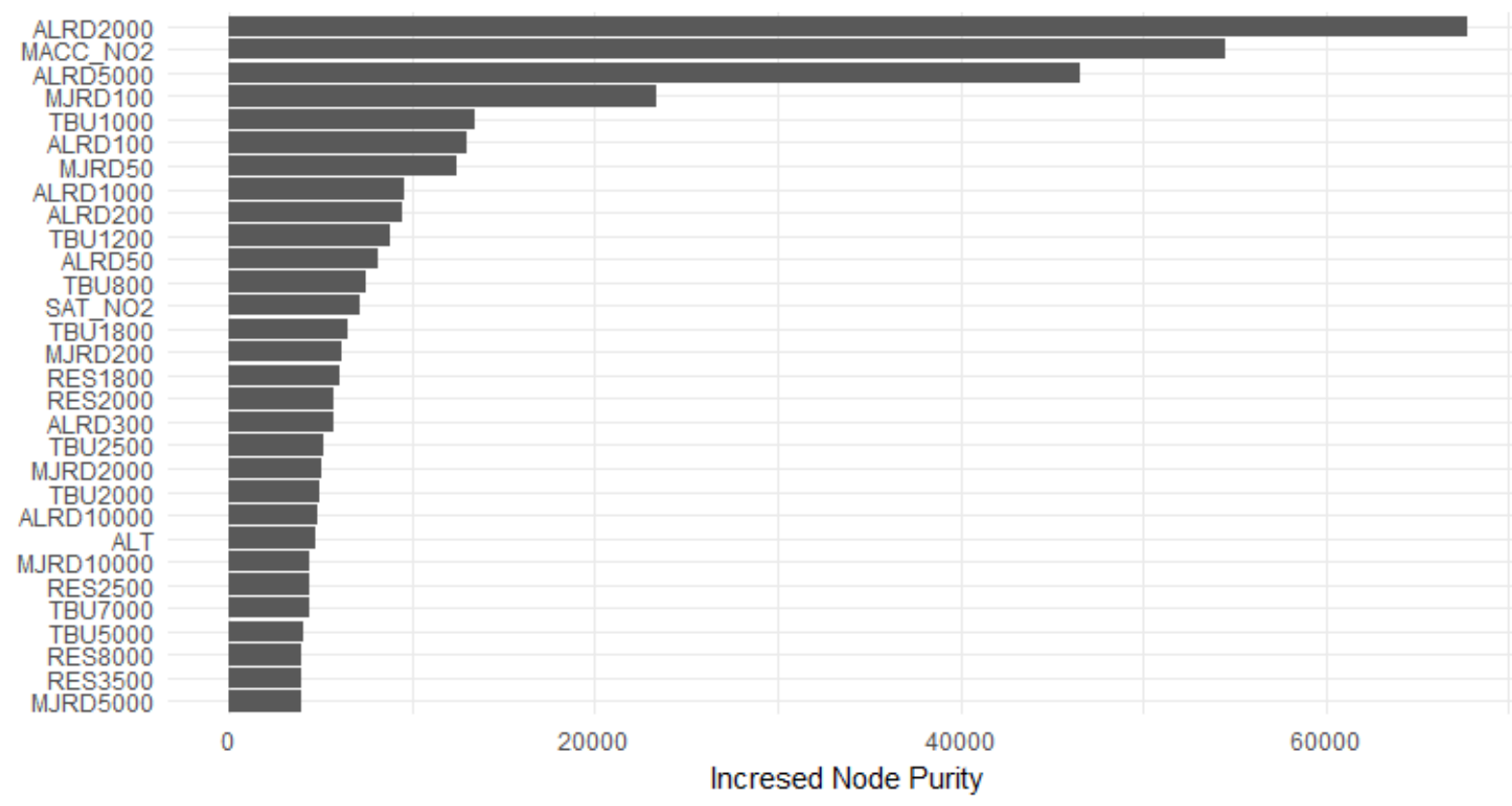

(B) Increase in MSE after a split on a variable (Random Forest)

MACC NO2
ALRD2000
ALRD5000
MJRD100
ALRD 50
MJRD50
ALRD100
ALRD200 200
ALRD1000
TBU1000
RES2500
ALRD 300
TBU 800
TBU1200
MJRD200
SAT N0O2
MJRD2000
ALRD 400
RES3000
ALRD500
RES3500
RES1800
ALRD700
RES2000
MJRD5000
RES8000
ALT
TBU1800
IND 3000
MJRD10000

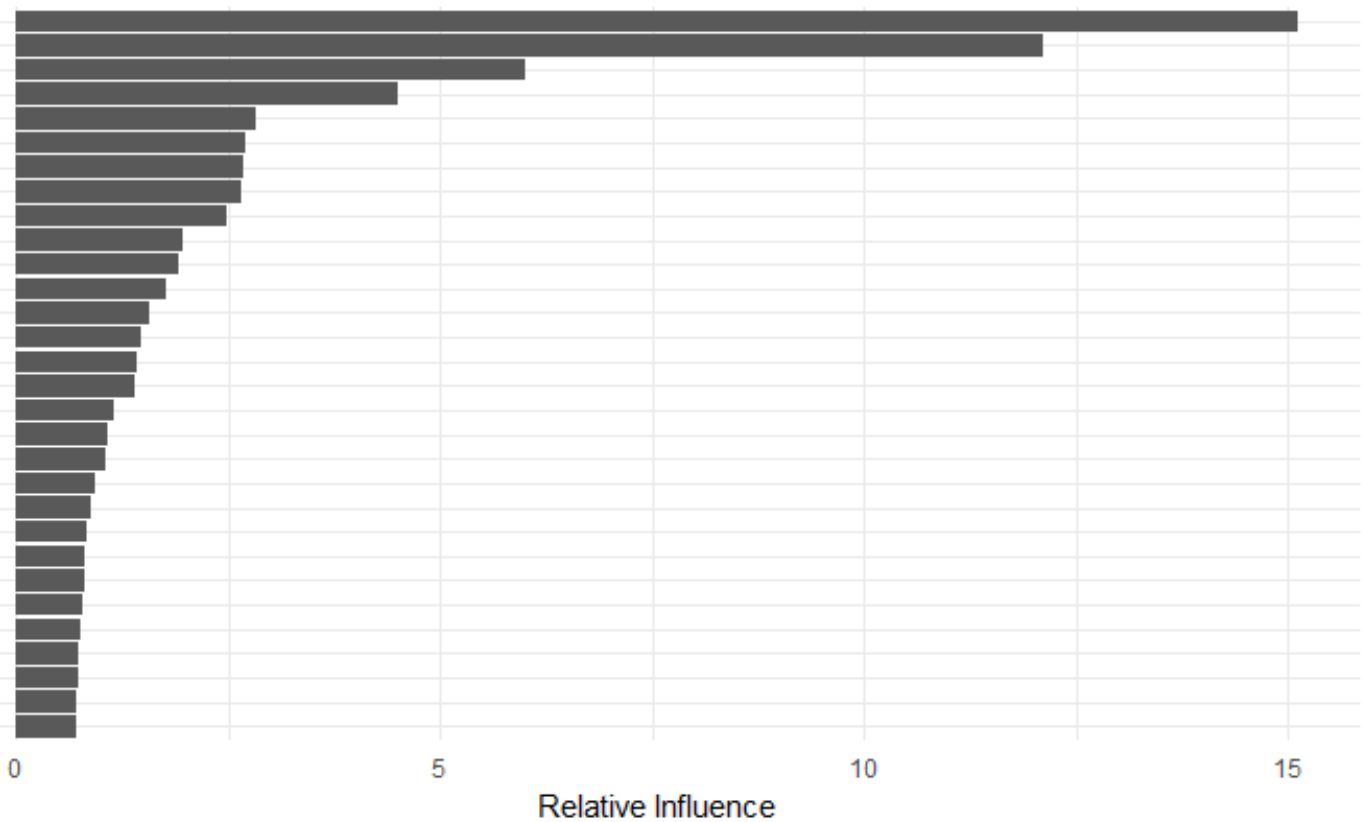

(C) Relative influence of predictors (Generalized Boosted Machine)

Figure S5. Variable importance plots of $\mathrm{NO}_{2}$ Random Forest and Generalized Boosted Machine models 

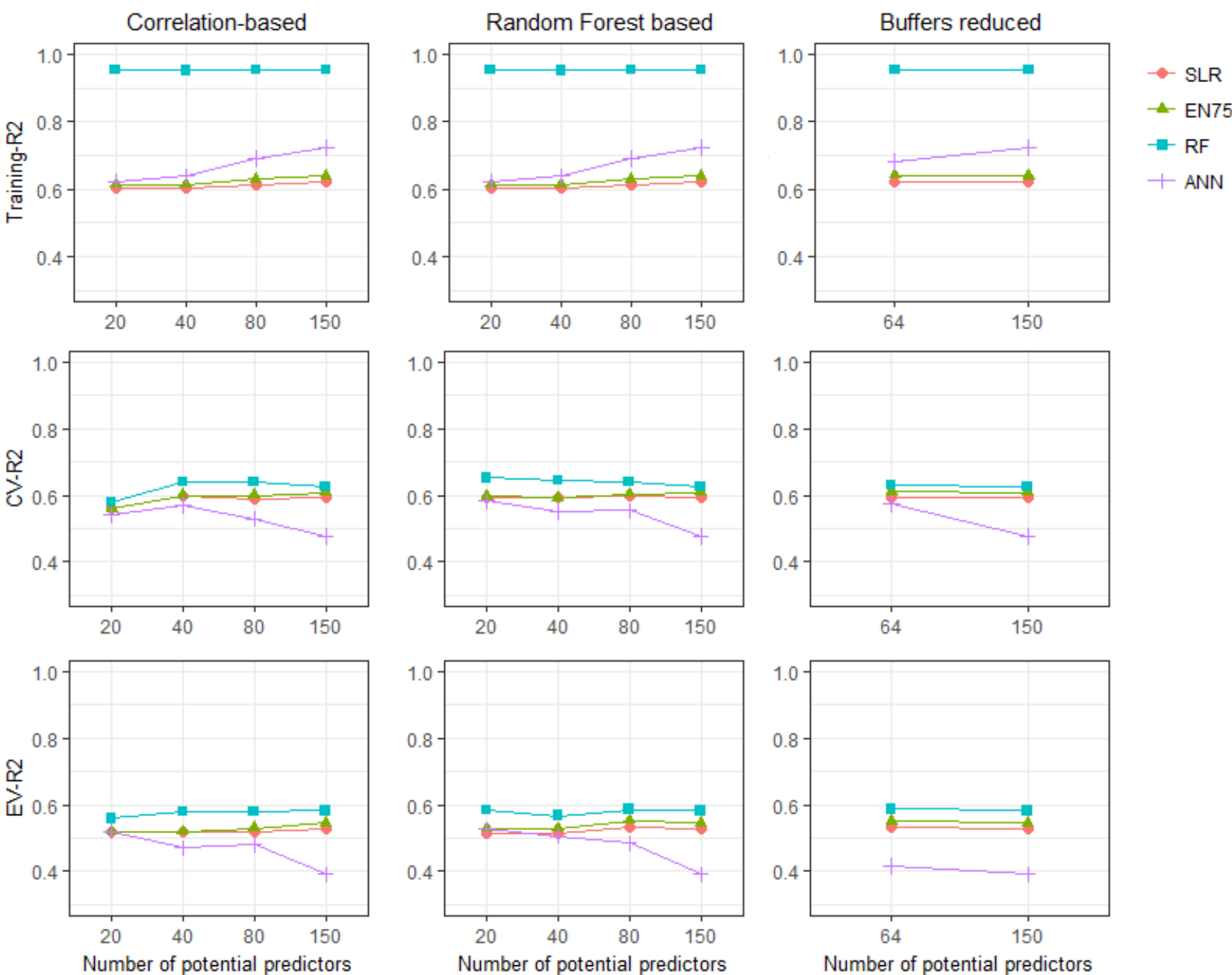

Figure S6. Model Training- $\mathrm{R}^{2}, \mathrm{CV}^{2}$ and $\mathrm{EV} \mathrm{R}^{2}$ of $\mathrm{PM}_{2.5}$ models with reduced number of potential predictors offered (the number of all potential predictors is 150 )

SLR = Supervised Linear Regression; EN75 = Elastic Net with $\alpha=0.75 ; \mathrm{RF}=$ Random Forest; ANN = Artificial Neural Network; CV = Cross Validation; EV = External Validation 

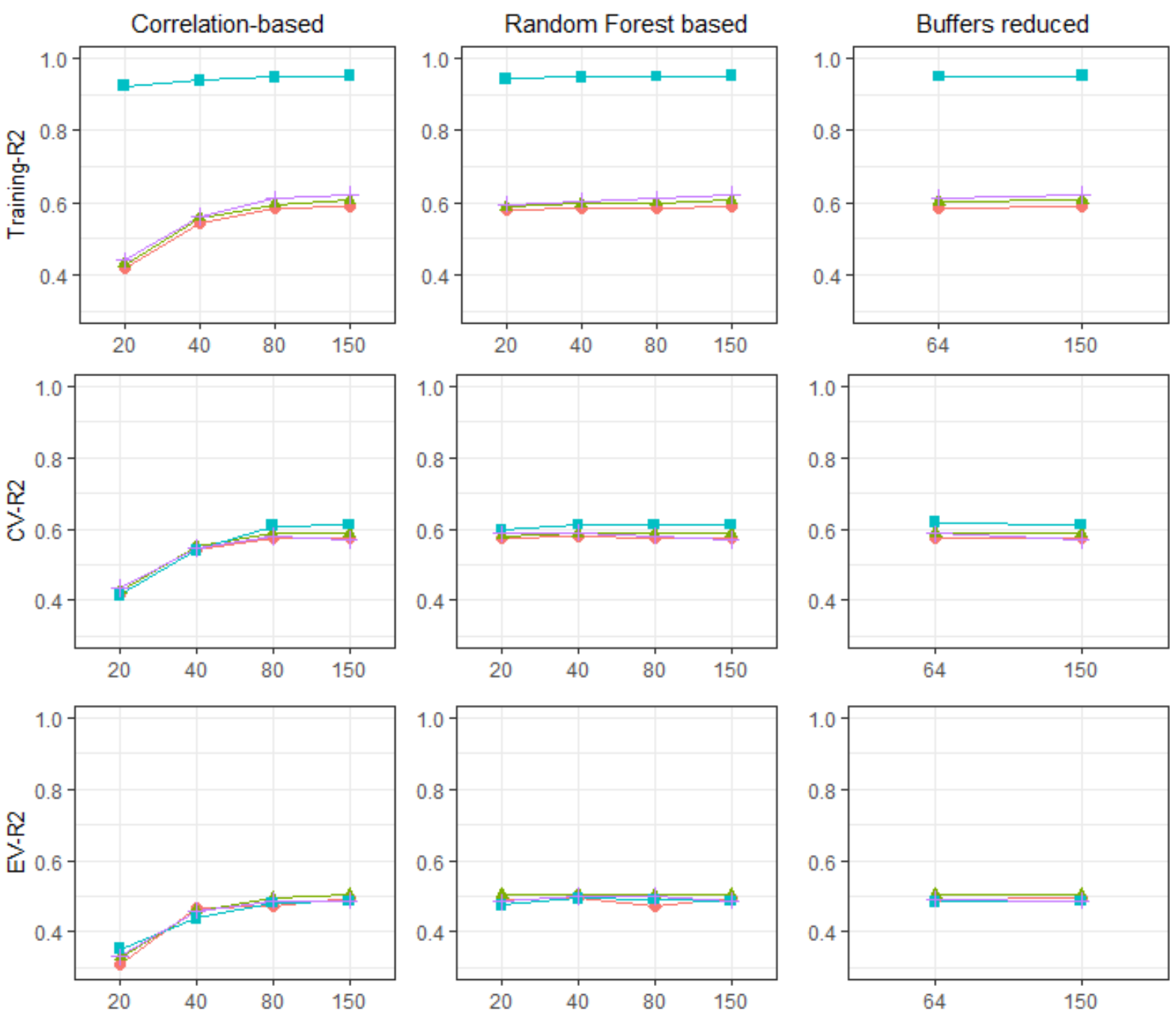

Number of potential predictors

Number of potential predictors

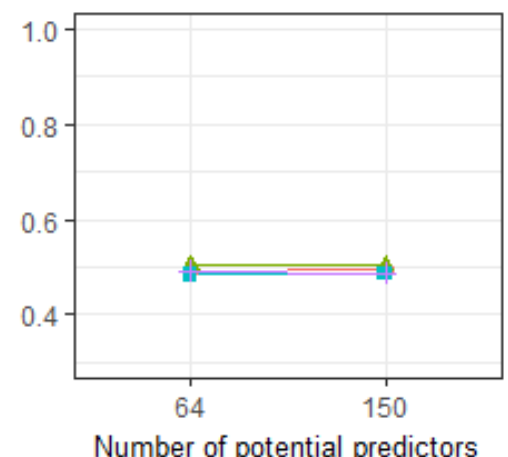

Figure S7. Model Training- $\mathrm{R}^{2}, \mathrm{CV} \mathrm{R}^{2}$ and $\mathrm{EV} \mathrm{R}^{2}$ of $\mathrm{NO}_{2}$ models with reduced number of potential predictors offered (the number of all potential predictors is 150)

SLR = Supervised Linear Regression; EN75 = Elastic Net with $\alpha=0.75 ;$ RF = Random Forest; ANN = Artificial Neural Network; CV = Cross Validation; EV = External Validation 

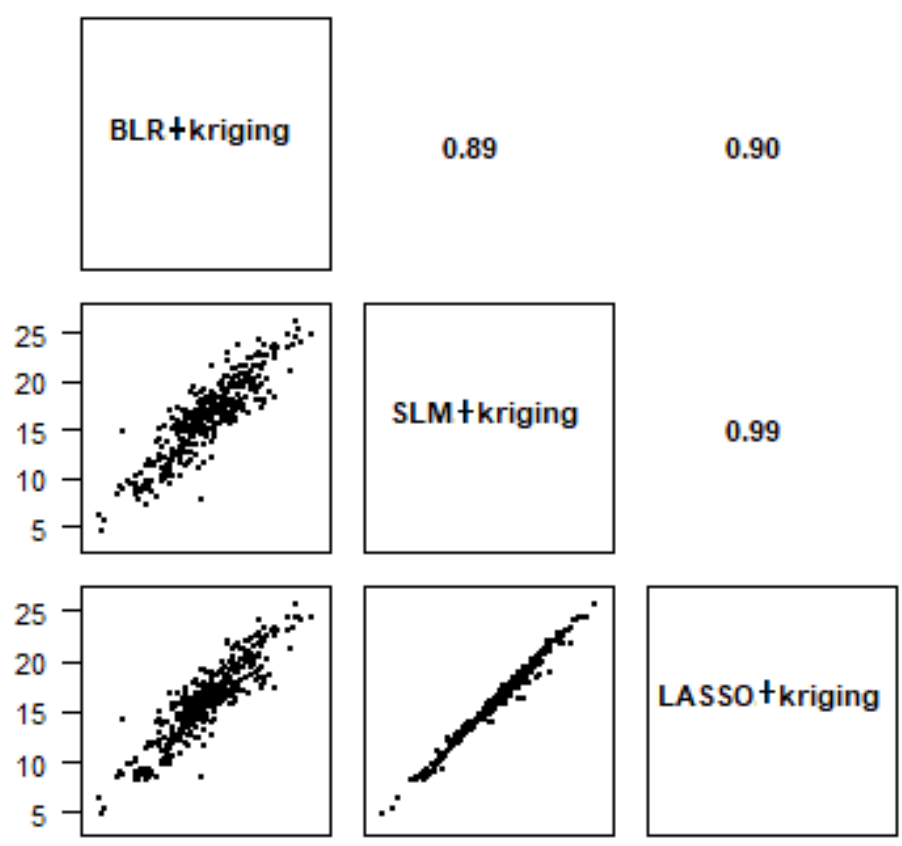

0.90

0.83
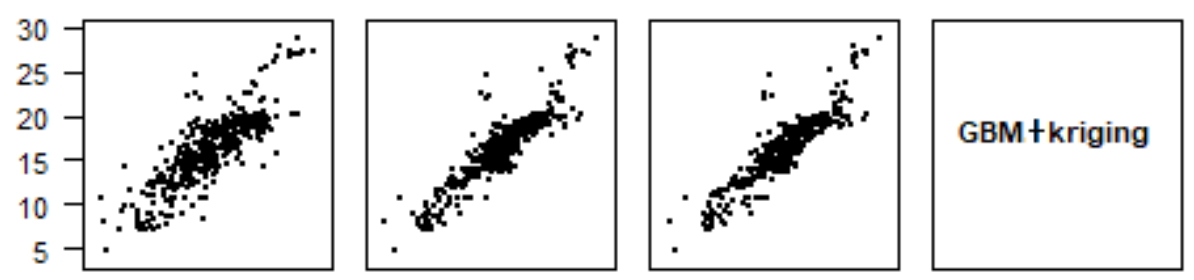

0.91
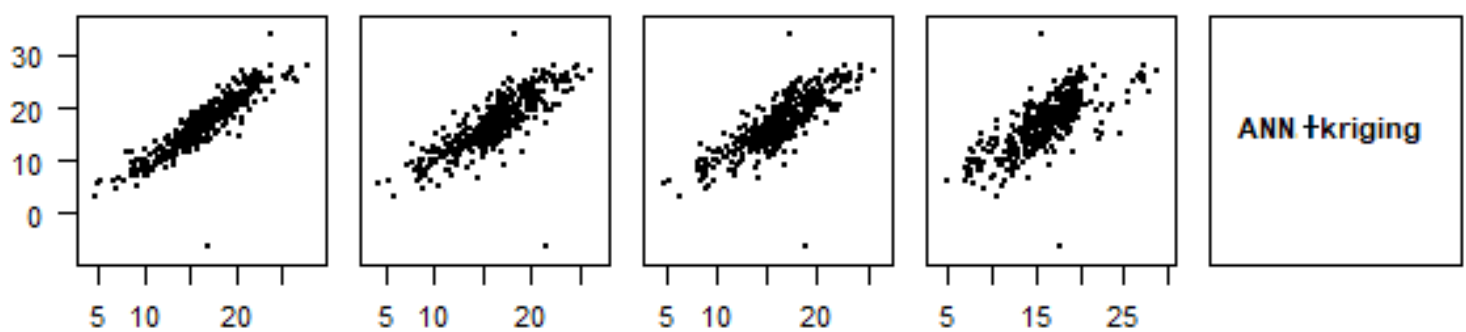

Figure S8. Correlation of $\mathrm{PM}_{2.5}$ estimations at ESCAPE sites predicted by selected models plus kriging

BLR = Backward stepwise Linear Regression; SLR = Supervised Linear Regression; LASSO = Least Absolute Shrinkage and Selection Operator; GBM = Generalized Boosted Machine; ANN = Artificial Neural Network

\section{References}

de Hoogh K, Gulliver J, van Donkelaar A, et al. 2016. Development of west-european pm 2.5 and no 2 land use regression models incorporating satellite-derived and chemical transport modelling data. Environmental research 151:1-10.

Iglewicz B. 1983. Robust scale estimators and confidence intervals for location:New York: Wiley.

Schalkoff RJ. 1997. Artificial neural networks:McGraw-Hill New York. 


\section{Chapter}

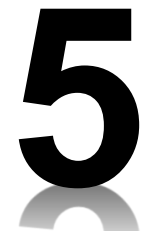

\section{Development of Europe-Wide Models for Particle Elemental Composition Using Supervised Linear Regression and Random Forest}

Jie Chen, Kees de Hoogh, John Gulliver, Barbara Hoffmann, Ole Hertel, Matthias Ketzel, Gudrun Weinmayr, Mariska Bauwelinck, Aaron van Donkelaar, Ulla A. Hvidtfeldt, Richard Atkinson, Nicole AH Janssen, Randall V. Martin, Evangelia Samoli, Zorana J. Andersen, Bente M. Oftedal, Massimo Stafoggia, Tom Bellander, Maciej Strak, Kathrin Wolf, Danielle Vienneau, Bert Brunekreef and Gerard Hoek

Environmental Science \& Technology, 2020, 54, 24, 15698-15709

DOI: 10.1021/acs.est.0c06595 


\begin{abstract}
We developed Europe-wide models of long-term exposure to 8 elements (copper, iron, potassium, nickel, sulfur, silicon, vanadium and zinc) in particulate matter with diameter $<2.5 \mu \mathrm{m}\left(\mathrm{PM}_{2.5}\right)$ using standardized measurements for one-year periods between October 2008 and April 2011 in 19 study areas across Europe, with supervised linear regression (SLR) and random forest (RF) algorithms. Potential predictor variables were obtained from satellites, chemical transport models, land-use, traffic and industrial point source databases to represent different sources. Overall model performance across Europe was moderate to good for all elements with hold-out-validation R-squared ranging from 0.41 to 0.90 . RF consistently outperformed SLR. Models explained within-area variation much less than the overall variation, with similar performance for RF and SLR. Maps proved a useful additional model evaluation tool. Models differed substantially between elements regarding major predictor variables, broadly reflecting known sources. Agreement between the two algorithm predictions was generally high at the overall European level and varied substantially at the national level. Applying the two models in epidemiological studies could lead to different associations with health. If both between- and withinarea exposure variability are exploited, RF may be preferred. If only within-area variability is used, both methods should be interpreted equally.
\end{abstract}

\title{
1. Introduction
}

Exposure to particulate matter (PM) is associated with adverse health outcomes ${ }^{1,2}$. PM is a complex mixture of components that differ spatially and temporally. Identifying which components are main contributors to adverse health effects is important for targeted policymaking. Multiple studies have attempted to associate health effects with PM components including metals, organic compounds, inorganic carbonaceous material and inorganic secondary aerosols $\mathrm{s}^{3-5}$. Findings, however, are inconsistent. Epidemiological studies have been limited due to the scarcity of air quality monitors that routinely measure PM composition. In Europe, a PM monitoring campaign was conducted in 20 ESCAPE (European Study of Cohorts for Air Pollution Effects) study areas following a common sampling protocol ${ }^{6}$. Most study areas consisted of a metropolitan area with some small towns around the main city. The PM samples were analyzed for elemental composition ${ }^{7}$. Based on the measurements from 20 sites in each study area, area-specific land use regression (LUR) models were developed to assess long-term exposure to elemental composition ${ }^{8}$. The models were applied to cohorts within the study areas to assess health effects related to particle composition ${ }^{9}$.

The geographical extent of the study-area specific ESCAPE models is limited and predictions from these models cannot reliably be used for other cohorts, such as large multi-center studies. The models were furthermore developed on 20 sites per area. Methodological studies suggested that more stable models can be developed based on larger number of sites in the model training dataset ${ }^{10,11}$. In addition, the ESCAPE study-area specific models had rather good performance for traffic-related elements such as copper $(\mathrm{Cu})$ and iron $(\mathrm{Fe})$, but had poor performance for elements such as sulfur $(\mathrm{S})$, nickel $(\mathrm{Ni})$ and vanadium $(\mathrm{V})$ for which spatial variation was limited within areas and key predictors were missing ${ }^{8}$. The lack of large-scale European models for particle elemental composition hampers large-area epidemiological studies. Our previous studies showed the possibility to develop European LUR models with good performance using a combined dataset from the ESCAPE study areas for PM with diameter $<2.5 \mu \mathrm{m}\left(\mathrm{PM}_{2.5}\right)$, Black Carbon $(\mathrm{BC})$ and nitrogen dioxide $\left(\mathrm{NO}_{2}\right)^{12,13}$.

The supervised Linear Regression (SLR) algorithm is often used in air pollution modeling ${ }^{14,15}$, and was used to develop ESCAPE models of elemental composition ${ }^{8}$ and European models of $\mathrm{PM}_{2.5}, \mathrm{NO}_{2}$ and $\mathrm{BC}^{12}$. The SLR algorithm shows good predictive ability and interpretability but has strong statistical assumptions such as linearity. SLR models can, however, take into account non-linear relationships by offering a priori transformed predictor variables (e.g. inverse distance to a source), include only predictor variables following plausible direction of effect (e.g. a positive traffic slope) and add interaction terms. A number of more flexible algorithms including machine-learning algorithms have 
increasingly been applied in air pollution exposure assessment ${ }^{16,17}$. Random Forest (RF) has been widely used in recent years ${ }^{18,19}$. RF is a classification tree analysis. It can model potentially complex relationships including non-linearity and interactions within data but gives little information regarding the prediction process ${ }^{20}$. A key feature of RF is the "bagging" procedure adopted in both observation and variable selection: this allows even marginally important predictors to contribute, even in presence of high multicollinearity. A previous study found RF outperformed linear regression in modeling spatial variation of particle elemental composition ${ }^{21}$.

LUR models for multiple particle components are more useful for epidemiological studies if they include more specific predictors. Developments in satellite and chemical transport modeling and availability of industrial point source data have made it possible to develop more specific models.

The aim of this study was to assess the performance of Europe-wide models for particle elemental composition, developed using SLR and RF algorithms. The models have been developed in the "Effects of Low-Level Air Pollution: A Study in Europe" (ELAPSE), a Europe-wide project investigating longterm health effects of low-level air pollution.

\section{Materials and Methods}

\subsection{Air pollution data}

The $\mathrm{PM}_{2.5}$ elemental composition concentration data originated from the ESCAPE monitoring campaigns conducted in 19 study areas across Europe (Figure S1). PM sampling and analysis methods have been described previously ${ }^{6,7}$. Briefly, measurements were made at 20 sites in each study area (40 in the large Catalunya and Netherlands/Belgium areas) for three 2-week periods in a 1-year period between October 2008 and April 2011. Monitoring sites were selected to represent pollution levels at regional background, urban background, and street locations using a common sampling protocol. $\mathrm{PM}_{2.5}$ samples were collected on Teflon filters using Harvard Impactors and analyzed for elemental composition using energy-dispersive X-ray fluorescence. Annual average concentrations were calculated based on three 14-day average measurements spread over the seasons (warm, cold and intermediate) with temporal adjustment from a reference background site in each study area. Our measurement campaign was restricted temporally, as previous sampling campaigns used to develop land use regression models ${ }^{22}$. While this design does not formally estimate absolute annual average concentrations as in regulatory monitoring, it has been shown to be useful to assess spatial contrast of long-term average concentrations because of specific design elements ${ }^{6,22,23}$. We performed temporal adjustment, using a continuous reference site located at a regional or urban background location (not directly influenced by local sources), where measurements were made for the full 12-month period. Three 14-day average samples were taken in different seasons at all locations, which are less sensitive to the very short-term variations caused by daily variation in weather. Five sites and the reference site were measured simultaneously representing all different site types (regional background, urban background and street) $)^{6,23}$.

Eight elements were a priori selected within ESCAPE to represent major pollution sources: $\mathrm{Cu}, \mathrm{Fe}$ and $\mathrm{Zn}$ representing non-tailpipe traffic emissions, $\mathrm{S}$ representing long-range transport, $\mathrm{Ni}$ and $\mathrm{V}$ representing mixed oil burning/industry, silicon ( $\mathrm{Si}$ ) representing crustal material, and potassium (K) representing biomass burning ${ }^{7,8}$.

\subsection{Potential predictor variables}

\subsubsection{Traffic, population, altitude and land use variables}

We used the same road density, population and elevation variables as in our previous exposure modeling paper for $\mathrm{PM}_{2.5}, \mathrm{NO}_{2}$, ozone $\left(\mathrm{O}_{3}\right)$ and $\mathrm{BC}$ across Europe ${ }^{12}$. In short, road data were extracted from the 1:10,000 EuroStreets digital road network (version 3.1 based on TeleAtlas MultiNet TM, year 2008), classified into 'all' and 'major' roads, and road density calculated in a 100x100 m grid. Population 
density in $1 \mathrm{x} 1 \mathrm{~km}$ grid for 2011 were obtained from Eurostat ${ }^{24}$. Elevation was obtained from the SRTM Digital Elevation Database ${ }^{25}$ version 4.1 with a resolution of 3 arc second (approximately $90 \mathrm{~m}$ ) with vertical error of $<16 \mathrm{~m}$. X and/or Y coordinates were offered to represent the East-West/ North-South gradient.

Land use variables were newly extracted from an updated European CORINE Land Cover surface in 100x100 m grid $^{26}$. The initial 44 land cover classes were grouped to six main classes: residential, industry, ports, urban green space, total built up land and natural land.

\subsubsection{Additional component/ source-specific variables}

Special attention was taken in obtaining specific potential predictor variables representing different sources of the eight selected elements. We hypothesized that this would allow us to develop better and more specific models, such that the independent associations of the different elements with health could be studied better. For each component, only plausible variables were offered for model development. The restrictions of offering specific potential predictors are specified in Table S1.

Satellite-model (SAT) estimates of 2010 annual average sulfate $\left(\mathrm{SO}_{4}{ }^{2-}\right)$, organic matter $(\mathrm{OM}), \mathrm{BC}$ and mineral dust (SOIL) in $\mathrm{PM}_{2.5}$ were extracted from a gridded surface $\left(0.01^{\circ} \times 0.01^{\circ}, \sim 1.11 \mathrm{~km}\right)$ over Europe. These estimates are an application of simulated relative composition to the total $\mathrm{PM}_{2.5}$ estimates produced by the methods described elsewhere ${ }^{27}$, and do not incorporate compositional ground-based measurements over Europe. In brief, $\mathrm{PM}_{2.5}$ mass estimates were produced by relating a combined aerosol optical depth (AOD) retrieval involving multiple satellite products and simulation to near-surface $\mathrm{PM}_{2.5}$ concentrations using the spatiotemporally varying geophysical relationship simulated by the GEOSChem Chemical Transport Model (CTM). Ground-based observations of total $\mathrm{PM}_{2.5}$ were then incorporated into these initial values using geographically weighted regression, and the resulting total mass estimates partitioned into chemical composition using their relative contributions according to the GEOS-Chem CTM simulations.

Chemical Transport Model estimates of BC AOD, Sulfate AOD, total column $\mathrm{SO}_{2}$, and sea-salt AOD were obtained from the European Centre for Medium-Range Weather Forecasts ${ }^{28}$. Daily estimates in 2010 were extracted from a gridded surface $\left(0.125^{\circ} \times 0.125^{\circ}, \sim 13.9 \mathrm{~km}\right)$ produced by the MACC-II ENSEMBLE model $^{29}$, and then aggregated to derive the annual average.

In addition to the unspecific industry land-use category, information on major industrial point sources was obtained including facility location, pollutant and emission amount from the European Pollutant Release and Transfer Register ${ }^{30}$. The industrial facility points were intersected with a $100 \mathrm{~m}$ base polygon, and then the number of facility sites and emissions were summed within each 100x100 m cell. Density of general industries and industries emitting specific aerosols (metal, $\mathrm{Cu}, \mathrm{Ni}, \mathrm{PM}_{10}, \mathrm{SO}_{\mathrm{x}}, \mathrm{Zn}$ ) were calculated accordingly. Sum of emissions were calculated for $\mathrm{PM}_{10}, \mathrm{Cu}, \mathrm{Ni}, \mathrm{SO}_{\mathrm{x}}$ and $\mathrm{Zn}$.

All predictor variables were integrated into a $100 \mathrm{~m}$ gridded GIS database covering Europe. For road density, land use and industrial information, a moving window procedure was used to calculate the sum of values for selected buffers (focal statistics using sum within a circle). The influence of industrial point sources was calculated by inverse distance weighting (1/d). The processing of variable surfaces was done in ArcMap 10.6.

\subsection{Model Development}

The number of monitoring sites available for particle composition ranged from 400 to 414 because of failed PM composition measurements ${ }^{8}$. We used both Supervised Linear Regression (SLR) and Random Forest (RF) algorithms to develop models.

The SLR approach has been described in detail before ${ }^{12}$. Briefly, a univariate linear regression model was applied for each potential predictor to find the predictor that explained the maximum variance in the measurements. At each subsequent step, the significant predictor variable $(\mathrm{P}<0.1)$ that generated 
the highest increase in model adjusted coefficient of determination (adjusted $\mathrm{R}^{2}$ ) was added. Predictors only entered the model if they adhered to the plausible direction of effect (Table S1). This process was repeated until the model adjusted $\mathrm{R}^{2}$ could not be increased anymore. Predictor variables with variance inflation factor (VIF) larger than 3 were removed from the model to avoid multicollinearity.

$\mathrm{RF}$ is an ensemble machine learning technique based on decision trees ${ }^{31}$. It builds independent trees in parallel, each based on a random sample drawn from the full set of measurements. At each node, a random subset of potential predictors is split. The final predictions are derived by averaging predictions from all decision trees. RF does not perform variable selection. It produces variable importance, calculated as percentage increase in mean squared errors after a random permutation of the values of a variable. We used the R package 'randomForest' to develop the RF models.

One-step and two-step modeling processes were used to offer geographical coordinates (X and $\mathrm{Y}$ ) to the models. Following our previous exposure modeling procedure ${ }^{12}$, for SLR we used a 2-step approach, in which we first developed a SLR model without offering X or Y, then added X and Y only if they increased the model adjusted $\mathrm{R}^{2}$. The rationale for the 2-step procedure is that we preferred spatial variation to be explained first by specific predictor variables and the residual variation to be further explained by the X, Y coordinates added in the second step. In RF we applied one-step modeling as our primary approach: $\mathrm{X}$ and $\mathrm{Y}$ were offered together with the other predictor variables. This allowed us to take advantage of the possibilities of RF algorithm to model the potential interactions between coordinates and other predictors. For comparison, we also developed one-step models for SLR and 2step models for RF: we first developed a RF model without offering X or Y, then developed a second RF model with $\mathrm{X}, \mathrm{Y}$ coordinates only, explaining variations in the residuals of the step1 RF model. The predictions of these two RF models were later added together. We further performed a sensitivity analysis offering a few non-linear transformations of the X- and Y-coordinates to the SLR model, including $\mathrm{X}^{2}, \mathrm{Y}^{2}, \sqrt{\mathrm{X}}, \sqrt{ } \mathrm{Y}$ and $\mathrm{XY}$, to allow more flexible functions of the coordinates than the linear function. We were not able to perform kriging due to the clustered nature of the monitoring data ${ }^{12}$.

\subsection{Model Evaluation and Comparison}

For each model, we calculated Model $\mathrm{r}^{2}$ (squared Pearson correlation) and RMSE (root-mean-square error) by comparing main model predictions to the measurements.

We performed five-fold hold-out validation (HOV). The full set of measurements were randomly divided into 5 groups ( $20 \%$ each), stratified by site type (Street, Rural and Urban Background) and region (North, West, Central and South). For each element-model combination, 5 additional HOV models were built, each based on $80 \%$ of the monitoring sites, with the remaining $20 \%$ for validation. HOV regression-based $\mathrm{r}^{2}$ and RMSE were computed by comparing the stacked predictions at the 5 holdout validation test sets to the corresponding measurements. We also calculated mean square error-based $\mathrm{R}^{2}\left(\mathrm{MSE}-\mathrm{R}^{2}\right)$, defined as:

$$
\mathrm{MSE}-\mathrm{R}^{2}=1-\frac{M S E}{\left(\frac{1}{n} \sum_{i=1}^{n}\left(y_{i}-\bar{y}\right)^{2}\right)}
$$

where $\bar{y}$ is the average of the measurements. MSE- $\mathrm{R}^{2}$ can be seen as a rescaling of MSE. It measures fit about the 1:1 line rather than fit about the best fit line in regression-based $r^{2}$. The HOV $r^{2}$ and RMSE are relevant for multi-city studies that exploit both within and between city variability of air pollution contrasts.

To test how the European models predict within-area variability, we calculated within-area $r^{2}$ and RMSE by comparing the stacked HOV predictions and measurements within each individual study area. Since the monitors are spatially clustered over Europe and nearby locations might have auto-correlations in their measurements, we additionally performed leave-one-area-out cross-validation (LOAOCV) ${ }^{32,33}$. We developed Europe-wide models by excluding all observations from one study area at a time and applied the models to the sites that were left out. Therefore, 19 additional models were developed for each 
pollutant-algorithm combination. Within-area $r^{2}$ and RMSE were computed by comparing the predictions and measurements in the area that was excluded from model development. We focus interpretation on the average of the within-area $r^{2} s$ and RMSEs because the performance statistics of the individual study areas may be affected strongly by random error since they were based on only 20 sites in each study area.

For each main model, predictor variables selected in SLR models and the 15 most important variables in RF models were compared.

Each main model was mapped at a 100x100 m resolution across the whole study area, allowing for visual comparison between maps. Additionally, we compared predictions from models at 41,936 random locations across Europe used previously ${ }^{12}$. Comparisons of model predictions were made for the entire study area and at the national scale reporting Pearson correlation coefficient (r) and RMSE. Truncations were performed to deal with unrealistic predictions of the SLR approach: predictions at the high end were truncated to the maximum final two-step modeled value, calculated by fitting the model with the maximum predictor values at monitoring sites for positive slopes (or the minimum predictor values for negative slopes); the negative predictions were set to zeros.

\section{Results and Discussion}

\subsection{Distribution of $\mathbf{P M}_{2.5}$ component measurements}

Boxplots of the annual mean concentration for $\mathrm{PM}_{2.5}$ components in the full dataset and in individual study areas are shown in Figure S2. For the majority of pollutants, pollution concentrations varied substantially within and between study areas. A positive north-south gradient was observed with higher pollution levels in southern study areas. A more detailed interpretation of the measured concentrations can be found elsewhere?

\subsection{Model performance}

Performance of models across Europe is shown in Table 1. Models for most components had moderate to good performance based upon HOV. Model performance was almost the same evaluating by regression-based $\mathrm{r}^{2}$ or MSE-based $\mathrm{R}^{2}$ (Table S2), consistent with the observation that the fitted regression slopes between observed vs. predicted values are close to the 1-1 line (Figure S3). Models with the highest $\mathrm{HOV} \mathrm{r}^{2} \mathrm{~s}$ were developed for $\mathrm{PM}_{2.5} \mathrm{~S}$, having large between-area concentration variability for which large-scale predictor variables from CTM were available to explain the contrast. Sulfate (represented by S) is a secondary pollutant formed by oxidation of sulfur dioxide for which the ratio of between- and within-area variability is larger than for the other elements ${ }^{7}$. RF models consistently outperformed SLR models for all elements. This agrees with a previous study, which found more accurate exposure assessed for elemental components by RF than SLR, based on 24 monitoring sites $^{21}$. The better performance of RF is different from two previous comparisons ${ }^{16,17}$, where similar performance of spatial models was observed for SLR and RF. One study compared Europe-wide models for $\mathrm{PM}_{2.5}$ and $\mathrm{NO}_{2}$ developed using similar predictor variables as in the current study ${ }^{16}$, the second study compared LUR models for ultrafine particles based upon mobile monitoring ${ }^{17}$. One possible explanation for the difference in findings is that there might be more complex relationships between predictors and elemental composition than with the mass of $\mathrm{PM}_{2.5}, \mathrm{NO}_{2}$ and UFP. RF can capture unknown non-linear relationships and interactions not predefined in SLR, without introducing overfitting of the data. Another important difference is that in the current study, the data were clustered within Europe whereas in the earlier study on $\mathrm{PM}_{2.5}$ and $\mathrm{NO}_{2}$, models were developed based upon routine monitoring, with a more even distribution of sites across Europe. We hypothesize that the RF model accounted for spatial trends across Europe better than the linear model.

For most components, HOV r ${ }^{2}$ s were similar for the one-step model and the final two-step model and higher than for the first step of the two-step model, documenting that spatial trends account for the 
residual variance not explained by the available predictors. Offering a priori transformed X- and Ycoordinates did not further improve performance for SLR models. The differences between model $\mathrm{r}^{2}$ and HOV $\mathrm{r}^{2}$ in SLR models were small, suggesting the models do not over-fit. The perfect performance on training set (Model $\mathrm{r}^{2}$ ) for RF models is "by design" and basically meaningless. This is because the $\mathrm{RF}$ algorithm generally does not prune the individual trees, relying instead on the ensemble of trees to control overfitting ${ }^{20}$.

While the models performed well to explain overall variability across Europe, models performed less well in explaining variation within individual study areas (Table 2). Results are similar by performing 5-fold HOV and leave-one-area-out cross-validation, suggesting the model performance is stable regardless of CV methods. The better overall performance is explained by a combination of larger variability of concentrations between areas than within areas and the better availability of predictor variables for describing between- compared to within-area variability. Specifically, the addition of largescale satellite and chemical transport models has contributed to assess the study-area background. The average within-area $\mathrm{r}^{2} \mathrm{~s}$ were moderate for $\mathrm{Cu}$ and $\mathrm{Fe}$ and relatively poor for other components. $\mathrm{Cu}$ and Fe represent mechanically generated traffic-related particles and thus their particle size distribution within $\mathrm{PM}_{2.5}$ is skewed towards coarse particles ${ }^{7,34}$. Therefore, $\mathrm{Cu}$ and $\mathrm{Fe}$ do not travel far and may show large within-area variation. The better within-area performance for $\mathrm{Cu}$ and $\mathrm{Fe}$ is thus possibly due to the combination of higher within-area variation of the concentrations in most areas and the availability of data on traffic networks within individual areas. Within-area $\mathrm{r}^{2} \mathrm{~s}$ were poor for components that have limited within-area variation such as S. S represents secondary inorganic aerosols (sulfates) produced by atmospheric chemistry of precursor gases (sulfur oxides) originating from combustion of sulfurcontaining fossil fuels (e.g., in power plants) ${ }^{35}$. Much of transported sulfate are in the sub-micron range and travel far, resulting in fairly uniform spatial variation in the scale of cities. Ni and V are often emitted from coal, oil or residual oil burning in buildings and ships. The emission height of buildings and ships are relatively low so that within-city variation can be observed. Despite the consistently better performance of RF models than SLR models in overall HOV, the average within-area ${ }^{2} \mathrm{~s}$ were similar across models for each element. This further supports our hypothesis that the RF model accounted for spatial trends across Europe better than the SLR model. Within-area $\mathrm{r}^{2} \mathrm{~s}$ varied substantially across study areas and were low in areas with small contrasts in measured concentrations shown by low RMSE (Figure S4).

In summary, the generally moderate within-area performance of the developed models is likely related to a combination of limited availability of predictor variables e.g. targeting especially non-exhaust traffic emissions, the clustered nature of the monitoring data and the lack of exposure contrasts within specific areas. Especially predictor variables at the local scale are insufficient.

\subsection{Model structure}

Predictor variables selected in SLR models and the 15 most important variables in RF models are shown in Figure 1. For each element, some consistency was found between SLR models and RF models in terms of the variable categories that were included. To some extent, however, different buffer sizes were included. Variables within each algorithm were very similar. X, Y coordinates usually contributed to the models when offered and were considered relatively important variables in the one-step RF.

The major predictors in the models differed substantially between the eight elements, broadly reflecting the different sources.

In $\mathrm{Cu}$ and $\mathrm{Fe}$ models, traffic-related predictor variables dominated the other source categories in SLR models while they were also considered relatively important in RF models. This is consistent with previous LUR models of $\mathrm{Cu}$ and $\mathrm{Fe}$ where a large proportion of the variability in the measured concentrations was explained by traffic related variables ${ }^{8,36,37}$. Some of the industrial point sources were picked up in the SLR models for $\mathrm{Cu}$ and $\mathrm{Fe}$, possibly reflecting emission released by metallurgic 
industries ${ }^{35}$. A previous study suggested that industrial sources were major predictors for $\mathrm{Cu}$ and $\mathrm{Fe}$ models in $\mathrm{PM}$ with diameter $<1 \mu \mathrm{m}\left(\mathrm{PM}_{1}\right)^{38}$.

In $\mathrm{Zn}$ models, predictors representing industrial $\mathrm{Zn}$ emission and combustion sources contributed a large proportion to the overall $\mathrm{r}^{2}$. This is consistent with LUR models in other studies ${ }^{36-38}$. In ESCAPE modeling, specific industrial predictors were not available ${ }^{8}$. The large contribution of industrial point sources to the $\mathrm{Zn}$ models is consistent with results of source apportionment analyses in MESA (MultiEthnic Study of Atherosclerosis) showing that $\mathrm{Zn}$-rich features were indicative of incinerators at nearby fixed locations ${ }^{34}$. Previous studies have used $\mathrm{Zn}$ as a tracer for metallurgic industries and nonmetallurgic industries for frit production ${ }^{35}$.

In $\mathrm{Ni}$ and $\mathrm{V}$ models, ports were important predictors, as a proxy for shipping emissions. Density of Niemitting industries and more general industrial density predictors were included in the SLR model for $\mathrm{Ni}$ and $\mathrm{V}$, consistent with the identification that $\mathrm{Ni}$ and $\mathrm{V}$ shared the same mixed industrial/fuel-oil combustion source ${ }^{39}$. Large-scale satellite-model dust showed a large contribution in the $\mathrm{Ni}$ and $\mathrm{V}$ models, which possibly accounts for the observed north-south trend in the absence of a specific largescale Ni and V CTM or satellite predictors. We offered satellite-model dust to all elements as windblown dust can be a source for all components.

In $\mathrm{S}$ models, variation in the measured concentrations was predominantly explained by large-scale satellite and chemical transport model estimates and predictors in large buffers. Sulfate from the CTM and satellite-model dust were virtually equally important in the models. Satellite-model sulfate did not enter the model possibly because sulfate from the CTM was in the model and they are highly correlated. Satellite-model dust likely accounts for the observed north-south trend in concentration. In area-specific ESCAPE models, less well performing models were developed for S mainly due to the small withinstudy area variability ${ }^{8}$. Predictors representing industrial point sources also contributed to the $\mathrm{S}$ models, indicative of transformation of emissions from combustion ${ }^{34}$.

In $\mathrm{K}$ models, satellite-model estimates for organic matter explained a large proportion of the variation, indicative of the main source of biomass burning for fine particle $\mathrm{K}^{35}$. Small-scale variables contributed little to $\mathrm{K}$ models, resulting in limited ability in explaining within-area variability. In our current models, we are still missing fine spatial scale biomass burning source terms because of the lack of reliable predictor variables.

Si models were dominated by satellite-model dust estimates and population density, reflecting its crustal dust source ${ }^{35}$. Road length and industry areas from CORINE land cover also contributed to the models. These variables contributed a large fraction also in models for $\mathrm{Si}$ in $\mathrm{PM}_{1}{ }^{38}$. In a previous study in New York, Si was strongly associated with an indicator for areas of industrial structures. This indicator includes a wide range of industrial, manufacturing and commercial activities thus it is difficult to identify the main source ${ }^{36}$.

\subsection{Maps and prediction at random locations}

The truncation frequency for prediction at random locations is shown in Table S3. A large number of negative SLR predictions were truncated to zero for some elements - e.g., 41.3\% of the 41,936 random locations across Europe for $\mathrm{Cu}$ in the final two-step SLR model predictions. Most of the negative values were located in the low population density areas of Northern Europe, covered mostly by natural land. When we applied the final two-step SLR models to a large Europe-wide pooled dataset of ESCAPE cohorts with 393,064 subjects (including a Swedish and Danish cohort), truncation frequencies were much smaller: $10.5 \%$ for $\mathrm{PM}_{2.5} \mathrm{Cu}, 0.5 \%$ for $\mathrm{PM}_{2.5} \mathrm{Fe}, 11.3 \%$ for $\mathrm{PM}_{2.5} \mathrm{Ni}, 14.2 \%$ for $\mathrm{PM}_{2.5} \mathrm{~V}, 2.7 \%$ for $\mathrm{PM}_{2.5} \mathrm{Zn}$. Therefore, we do not expect this to be a big issue when applying the SLR models to participants in epidemiological studies. No truncation was needed for RF models.

Although we a priori considered one-step RF models as our main RF models, we observed large concentration jumps along horizontal or vertical lines in several maps (Figure S5). This counterintuitive 
Table 1. Performance of $\mathbf{P M}_{2.5}$ composition models over Europe

\begin{tabular}{|c|c|c|c|c|c|c|c|c|c|c|}
\hline & $\begin{array}{l}\text { Inclusion of } X \text {, } \\
Y \text { coordinates }\end{array}$ & $\begin{array}{l}\text { Component } \\
\text { No. of sites }\end{array}$ & $\begin{array}{l}\mathrm{Cu} \\
414\end{array}$ & $\begin{array}{l}\mathrm{Fe} \\
413\end{array}$ & $\begin{array}{c}K \\
414\end{array}$ & $\begin{array}{l}\mathrm{Ni} \\
402\end{array}$ & $\begin{array}{c}S \\
404\end{array}$ & $\begin{array}{r}\text { Si } \\
400\end{array}$ & $\begin{array}{c}\mathrm{V} \\
402\end{array}$ & $\begin{array}{l}\mathrm{Zn} \\
413\end{array}$ \\
\hline \multicolumn{11}{|c|}{ Model building } \\
\hline \multirow{2}{*}{\multicolumn{2}{|c|}{ One-step }} & Model $\mathrm{r}^{2}$ & 0.56 & 0.55 & 0.61 & 0.62 & 0.79 & 0.52 & 0.70 & 0.48 \\
\hline & & Model RMSE ${ }^{a}$ & 3.3 & 65.5 & 64.6 & 0.9 & 146.5 & 59.7 & 1.7 & 11.8 \\
\hline \multirow{4}{*}{ SLR } & \multirow{2}{*}{$\begin{array}{l}\text { Two-step, } \\
\text { step1 }\end{array}$} & Model $\mathrm{r}^{2}$ & 0.52 & 0.53 & 0.52 & 0.56 & 0.80 & 0.48 & 0.66 & 0.47 \\
\hline & & Model RMSE & 3.4 & 67.4 & 71.1 & 1.0 & 142.2 & 61.9 & 1.8 & 11.9 \\
\hline & \multirow{2}{*}{$\begin{array}{l}\text { Two-step, } \\
\text { step2 }\end{array}$} & Model $r^{2}$ & 0.56 & 0.53 & 0.60 & 0.60 & 0.82 & 0.50 & 0.69 & 0.48 \\
\hline & & Model RMSE & 3.3 & 67.4 & 65.0 & 0.9 & 135.4 & 61.1 & 1.7 & 11.8 \\
\hline \multirow{6}{*}{$\mathbf{R F}^{\mathbf{b}}$} & \multirow{2}{*}{ One-step } & Model $r^{2}$ & 0.95 & 0.95 & 0.97 & 0.95 & 0.98 & 0.95 & 0.97 & 0.95 \\
\hline & & Model RMSE & 1.1 & 20.8 & 16.8 & 0.3 & 40.2 & 19.9 & 0.5 & 3.5 \\
\hline & \multirow{2}{*}{$\begin{array}{l}\text { Two-step, } \\
\text { step1 }\end{array}$} & Model $r^{2}$ & 0.95 & 0.95 & 0.97 & 0.95 & 0.98 & 0.94 & 0.97 & 0.96 \\
\hline & & Model RMSE & 1.1 & 20.9 & 17.4 & 0.3 & 41.8 & 20.3 & 0.5 & 3.4 \\
\hline & \multirow{2}{*}{$\begin{array}{l}\text { Two-step, } \\
\text { step2 }\end{array}$} & Model $r^{2}$ & 0.98 & 0.98 & 0.99 & 0.98 & 0.99 & 0.98 & 0.99 & 0.99 \\
\hline & & Model RMSE & 0.6 & 12.4 & 9.5 & 0.2 & 27.0 & 12.2 & 0.3 & 1.8 \\
\hline \multicolumn{11}{|c|}{$\underline{\text { Hold-Out Validation }}$} \\
\hline \multirow{6}{*}{ SLR } & \multirow{2}{*}{ One-step } & $\mathrm{HOV} \mathrm{r}^{2}$ & 0.47 & 0.48 & 0.58 & 0.57 & 0.76 & 0.50 & 0.63 & 0.41 \\
\hline & & HOV RMSE & 3.6 & 70.5 & 66.4 & 1.0 & 156.4 & 60.8 & 1.8 & 12.5 \\
\hline & \multirow{2}{*}{$\begin{array}{l}\text { Two-step, } \\
\text { step1 }\end{array}$} & $\mathrm{HOV} \mathrm{r}{ }^{2}$ & 0.44 & 0.46 & 0.50 & 0.51 & 0.76 & 0.46 & 0.60 & 0.42 \\
\hline & & HOV RMSE & 3.7 & 71.7 & 72.6 & 1.0 & 154.9 & 63.4 & 1.9 & 12.4 \\
\hline & \multirow{2}{*}{$\begin{array}{l}\text { Two-step, } \\
\text { step2 }\end{array}$} & $\mathrm{HOV} \mathrm{r}{ }^{2}$ & 0.48 & 0.48 & 0.59 & 0.56 & 0.79 & 0.46 & 0.63 & 0.41 \\
\hline & & HOV RMSE & 3.6 & 70.5 & 66.1 & 1.0 & 147.0 & 62.9 & 1.8 & 12.5 \\
\hline \multirow{6}{*}{ RF } & \multirow{2}{*}{ One-step } & $\mathrm{HOV} \mathrm{r}^{2}$ & 0.60 & 0.60 & 0.82 & 0.74 & 0.91 & 0.62 & 0.85 & 0.68 \\
\hline & & HOV RMSE & 3.2 & 61.7 & 44.1 & 0.7 & 97.0 & 52.9 & 1.2 & 9.3 \\
\hline & \multirow{2}{*}{$\begin{array}{l}\text { Two-step, } \\
\text { step1 }\end{array}$} & $\mathrm{HOV} \mathrm{r}{ }^{2}$ & 0.59 & 0.59 & 0.79 & 0.74 & 0.90 & 0.60 & 0.84 & 0.68 \\
\hline & & HOV RMSE & 3.2 & 62.4 & 47.4 & 0.7 & 102.1 & 54.2 & 1.2 & 9.2 \\
\hline & \multirow{2}{*}{$\begin{array}{l}\text { Two-step, } \\
\text { step2 }\end{array}$} & $\mathrm{HOV} \mathrm{r}^{2}$ & 0.59 & 0.61 & 0.80 & 0.76 & 0.90 & 0.62 & 0.86 & 0.71 \\
\hline & & HOV RMSE & 3.2 & 61.3 & 45.8 & 0.7 & 99.5 & 53.1 & 1.1 & 8.7 \\
\hline
\end{tabular}

SLR = Supervised Linear Regression; RF = Random Forest; $r^{2}=$ squared Pearson correlation; RMSE $=$ RootMean-Square Error; HOV = Five-fold Hold-Out Validation ${ }^{\mathrm{a}}$ unit of RMSE: $\mathrm{ng} / \mathrm{m}^{3}$

${ }^{b}$ Performance of Random Forest on training set cannot be interpreted 


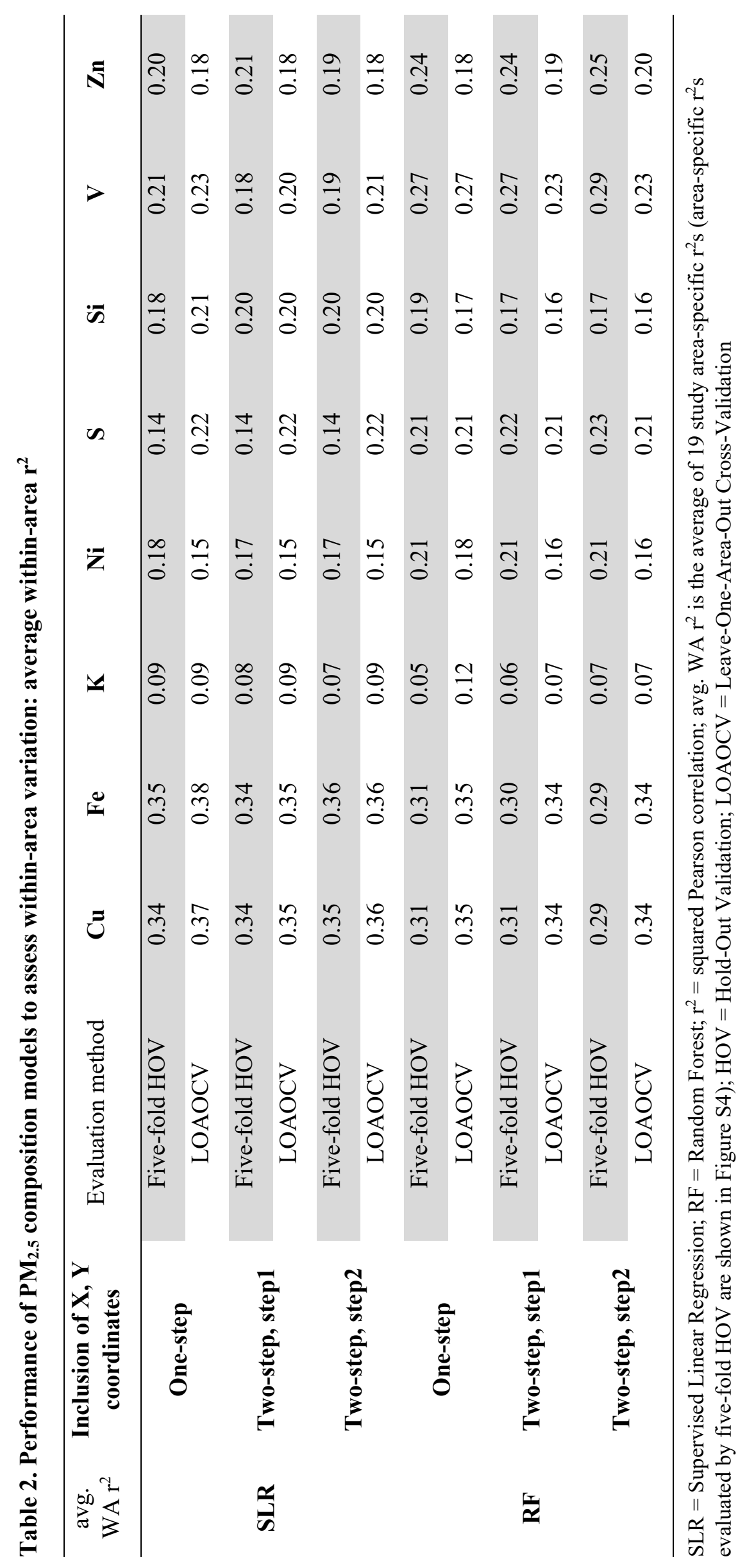



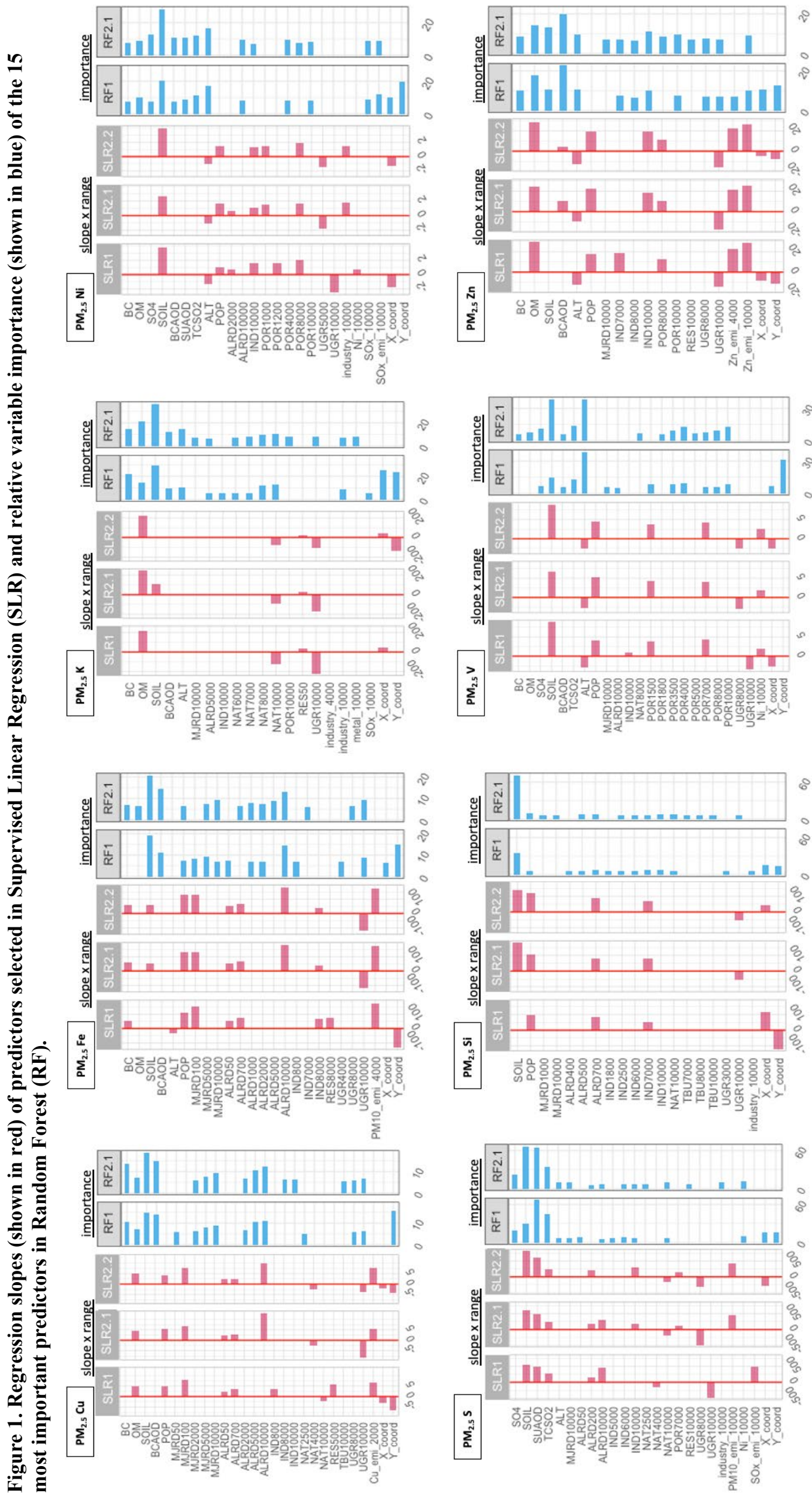

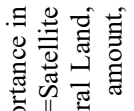
家品昰

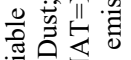
政

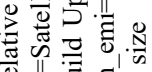

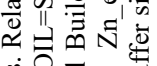
영 푱

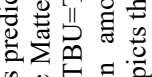
के

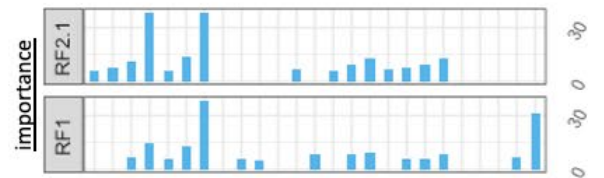

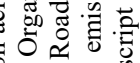

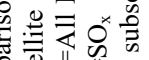

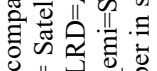

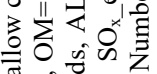

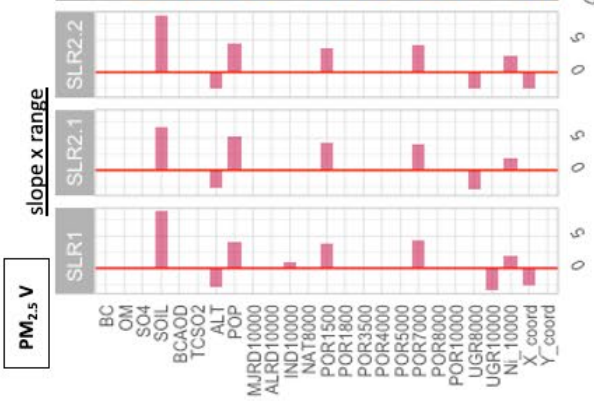

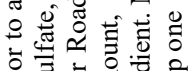

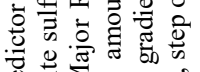

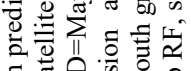

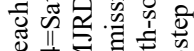
政专

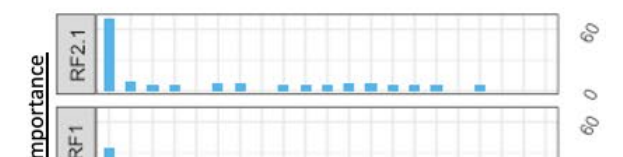

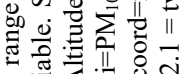

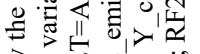

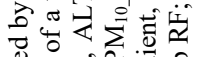
总甜㣢 实

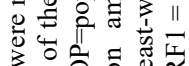
후의.
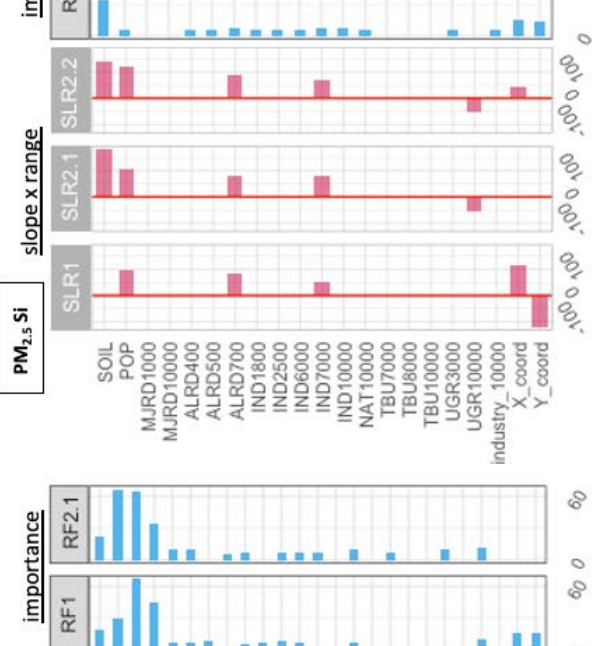

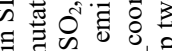
के

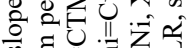

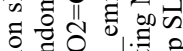
政记

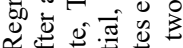

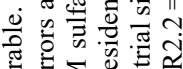

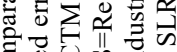

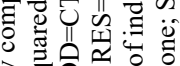
중

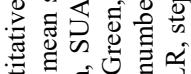

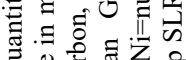
可 论

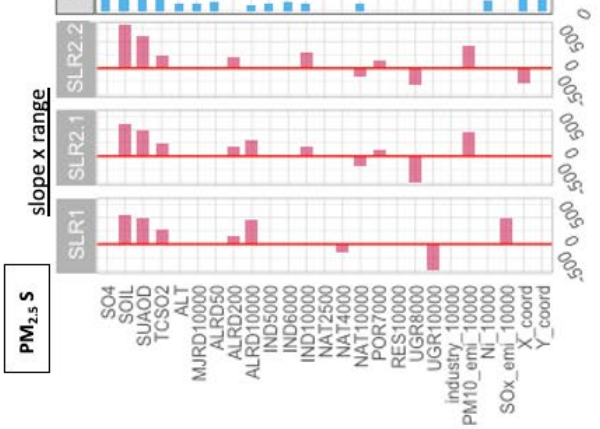

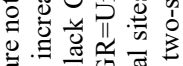

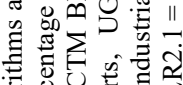

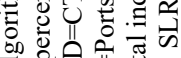
ज़ 政造的 ष्ष 造券要

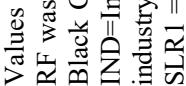


pattern possibly reflects the role of the $\mathrm{X}, \mathrm{Y}$ coordinates in RF modeling and relative importance attributed to these variables. Using $\mathrm{X}$ and $\mathrm{Y}$ in $\mathrm{RF}$ introduces strong boundary effects because, depending on the value where trees are split, large difference in predictions will be produced below and above that value. The concentration jumps were also observed in the final two-step RF model maps with $\mathrm{X}, \mathrm{Y}$ coordinates. The RF models without offering X, Y coordinates produced clearly different maps while the HOV $r^{2}$ s were marginally lower than for the RF models with coordinates (Table 1). We therefore prefer the first step in the two-step RF and the final two-step SLR (maps in Figure 2), and show maps deriving from the other procedures in the appendix (Figure S5). The maps showing strong boundary effects might require smoothing before application in epidemiological studies. Our results clearly indicate the value in evaluating plausibility of maps as an important last step in air pollution exposure assessment studies. Comparing models solely by HOV statistics is not sufficient. We did not observe sharp gradients in the SLR model maps.

There are clear agreements between maps produced by our main SLR and RF for some elements and differences for other elements (Figure 2). In both maps for $\mathrm{Cu}$, high levels of pollution are shown in big cities, and transport networks can be clearly seen in the inset map of the area around Paris. Maps for $\mathrm{PM}_{2.5} \mathrm{~S}$ are broadly similar with higher pollution levels in the south and east, while quite different patterns were observed for east Germany and Spain. Both maps for Zn show high concentrations close to industrial sites. The same industrial sites were picked up in the area around Paris shown in the inset. Comparing the predictions at a total of 41,936 random locations, agreement was high at all European countries-level and the ELAPSE countries combined-level for $\mathrm{PM}_{2.5} \mathrm{Cu}, \mathrm{PM}_{2.5} \mathrm{~K}, \mathrm{PM}_{2.5} \mathrm{~S}, \mathrm{PM}_{2.5} \mathrm{Zn}(\mathrm{r}>$ 0.7 ), and moderately high for other elements (Table 3 ). Correlation between all model predictions at the ELAPSE countries combined-level is presented in Figure S6. For most components, correlations were high for predictions derived from the same algorithm, and lower for predictions derived from different algorithms.

While correlations between predictions derived from SLR and RF were moderate to high at the European level, they are lower than the very high correlations ( $\mathrm{r}$ generally $>0.9$ ) reported previously for Europewide models of $\mathrm{PM}_{2.5}$ and $\mathrm{NO}_{2}{ }^{16}$. Agreement between predictions from the two algorithms at national level varied substantially across countries (Table 3 ). There was no consistently good agreement between predictions for a specific country. Poor agreement between predictions were observed for areacomponent combinations that had small contrasts in measured concentrations shown by low RMSE (e.g. most components in Norway and Sweden).

Computation time for mapping differed substantially for RF and SLR - around 40 hours for RF and less than 1 hour for SLR to map pollution concentrations across Europe on a standard office computer.

\subsection{Strengths and limitations}

With the development of Europe-wide models, we are able to assess long-term exposures to $\mathrm{PM}_{2.5}$ components in a large European project, which consists of several nation-wide cohorts and smaller cohorts in which participants were recruited in specific study areas. The use of a single harmonized model allows a standardized exposure assessment in international multi-center studies.

Our Europe-wide models had the advantage of a large training dataset with large contrasts in measured concentrations by combining measurements from individual ESCAPE study areas. In contrast, the previous ESCAPE area-specific models could not be developed for some composition-area combinations because of missing data (e.g. in Lugano), small within-area variability (e.g. S) and poor precision of the measurements in areas with low concentrations $(\mathrm{Ni} \text { and } \mathrm{V})^{8}$. The moderate to good performance of our models across Europe suggests that the models would perform well in multi-center studies that exploit both within and between area variability of air pollution contrasts.

Another strength of our study is that we made efforts in collecting specific large-scale predictors, from satellites and chemical transport models, representing different pollution sources such as soil, industrial sources and biomass burning, which could not be applied in prior area-specific models. The availability 


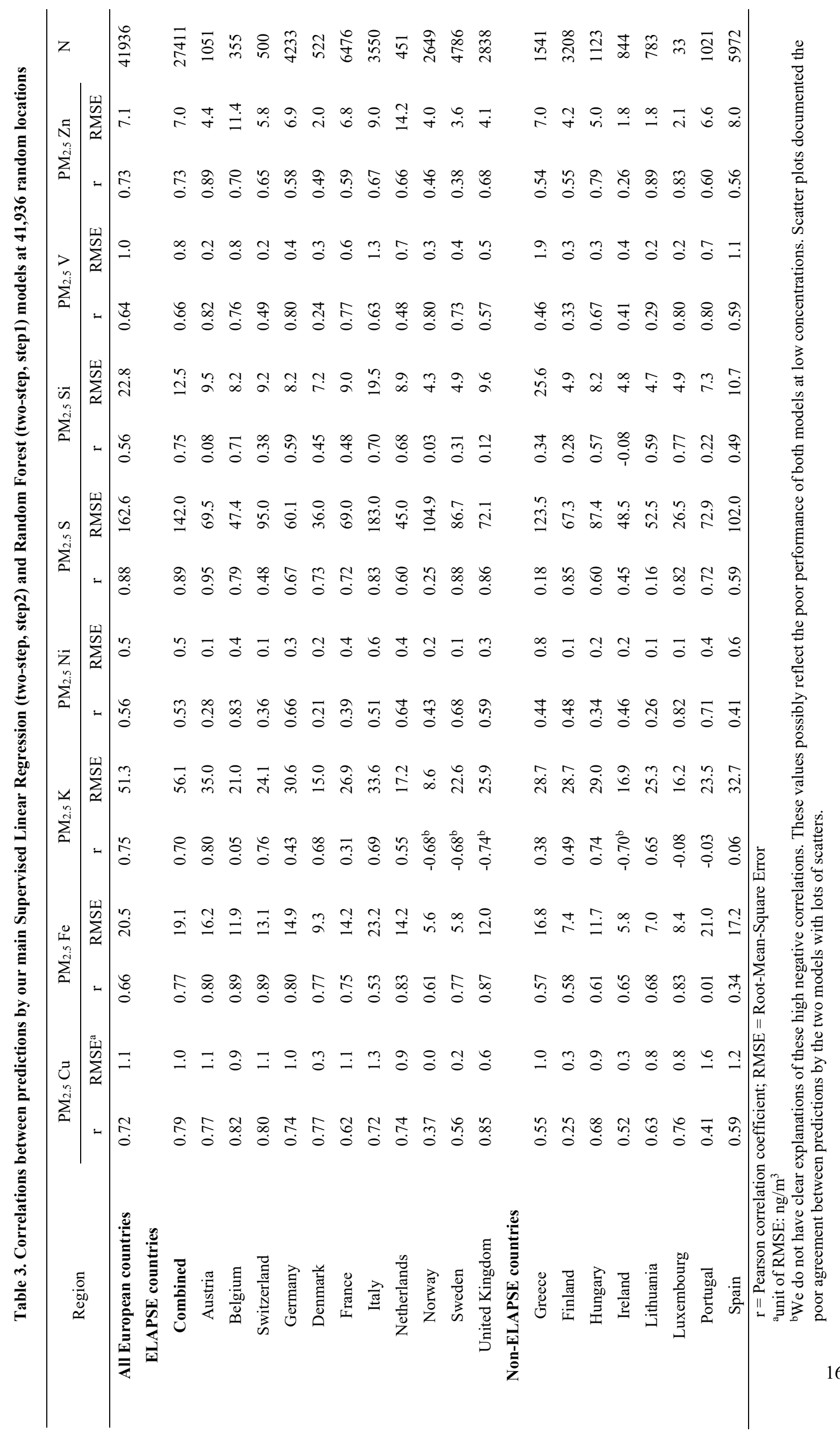


(1) $\mathrm{PM}_{2.5} \mathrm{Cu}$
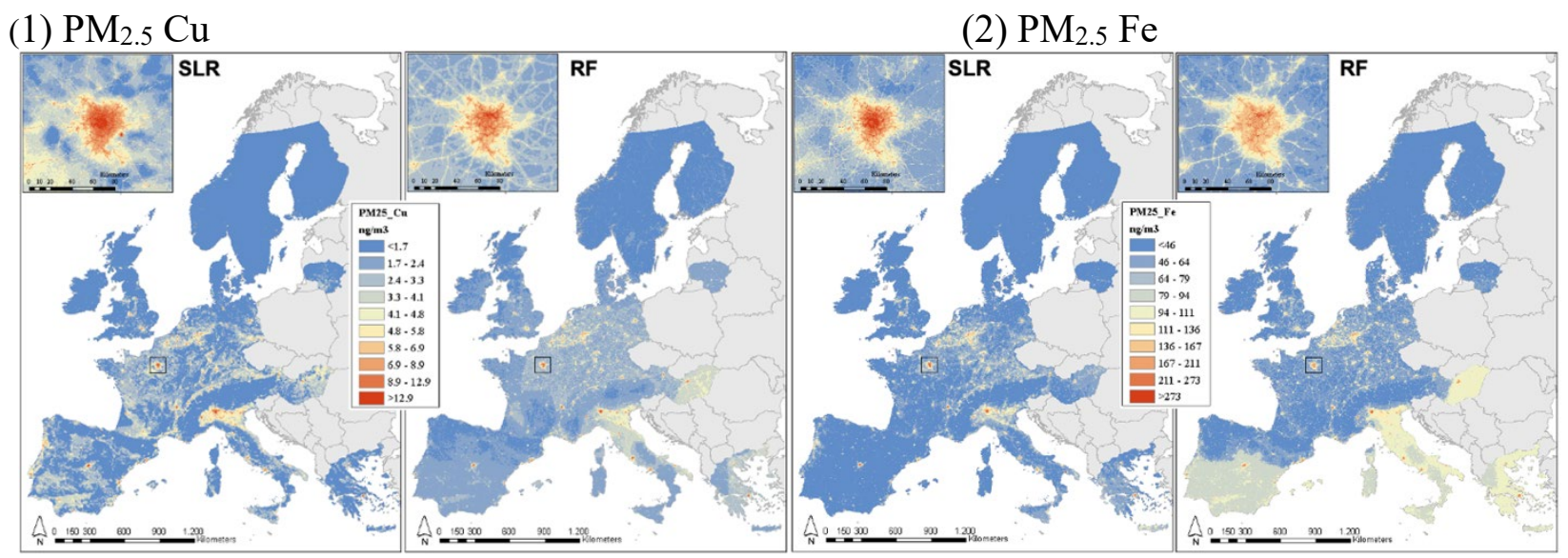

(3) $\mathrm{PM}_{2.5} \mathrm{~K}$
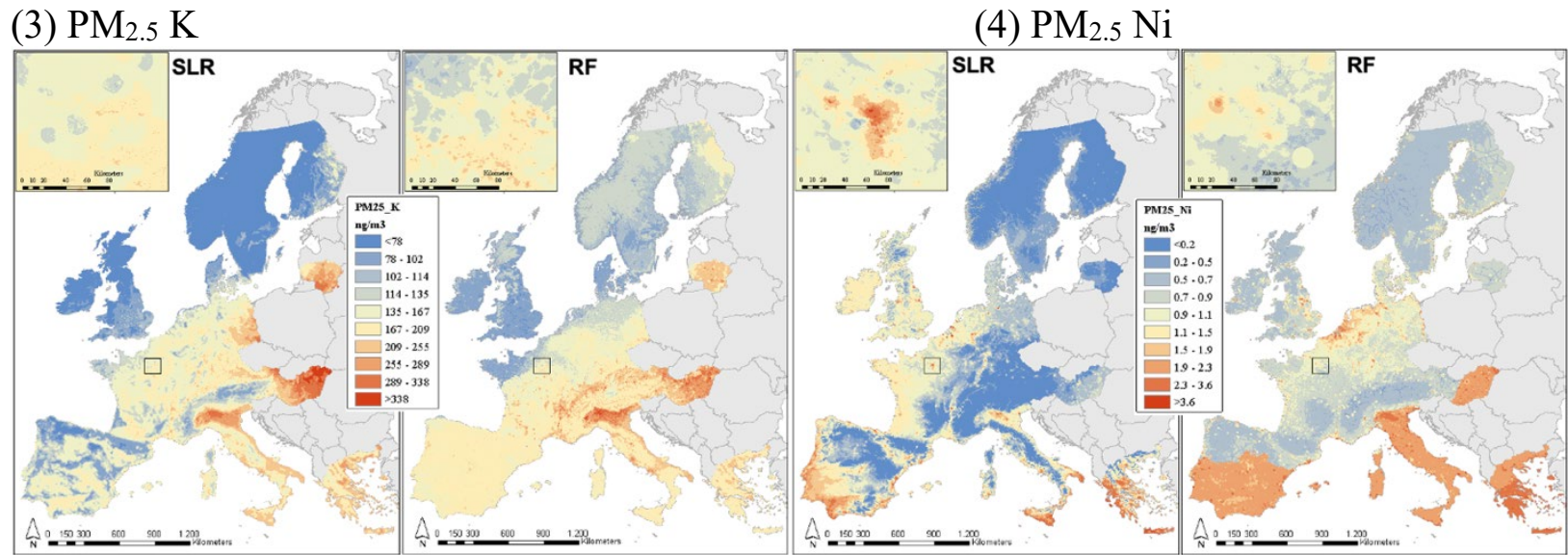

(5) $\mathrm{PM}_{2.5} \mathrm{~S}$
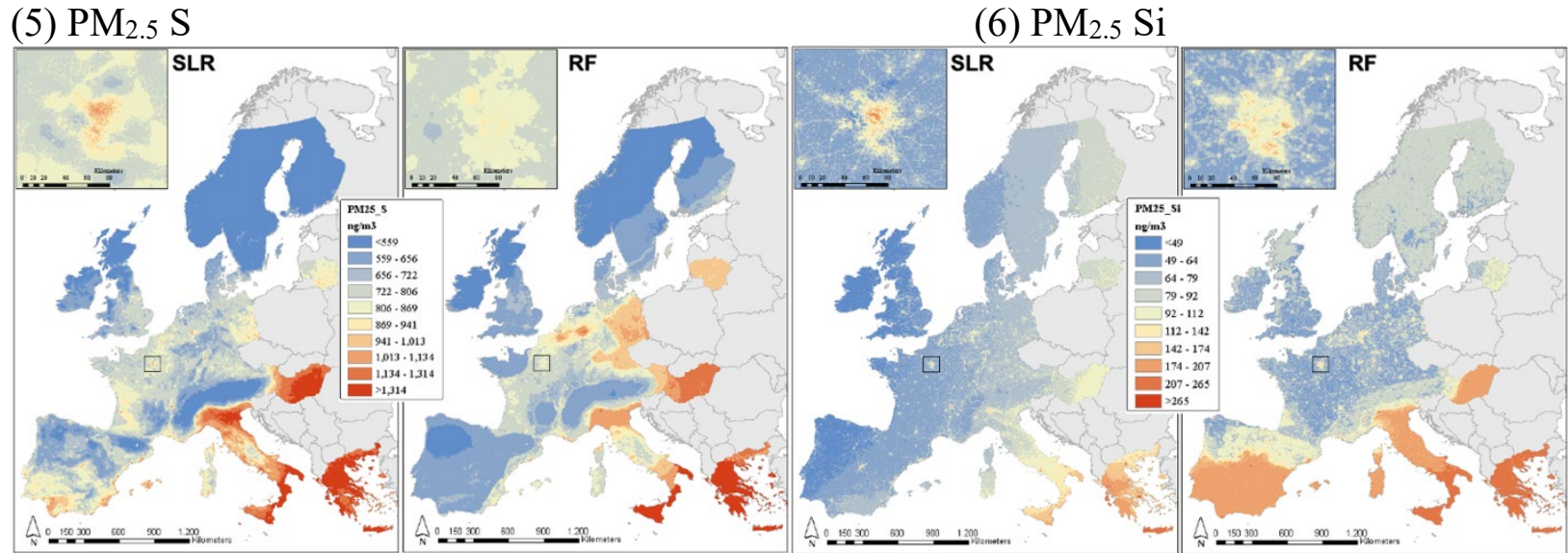

(7) $\mathrm{PM}_{2.5} \mathrm{~V}$

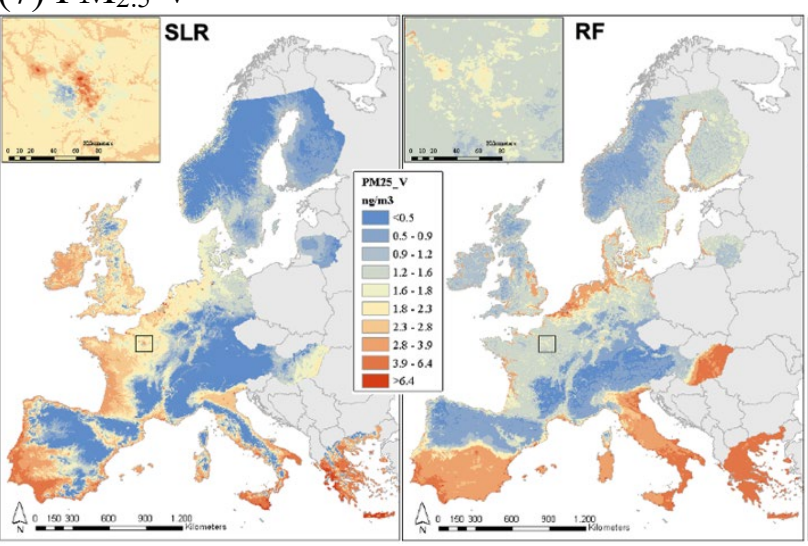

(8) $\mathrm{PM}_{2.5} \mathrm{Zn}$

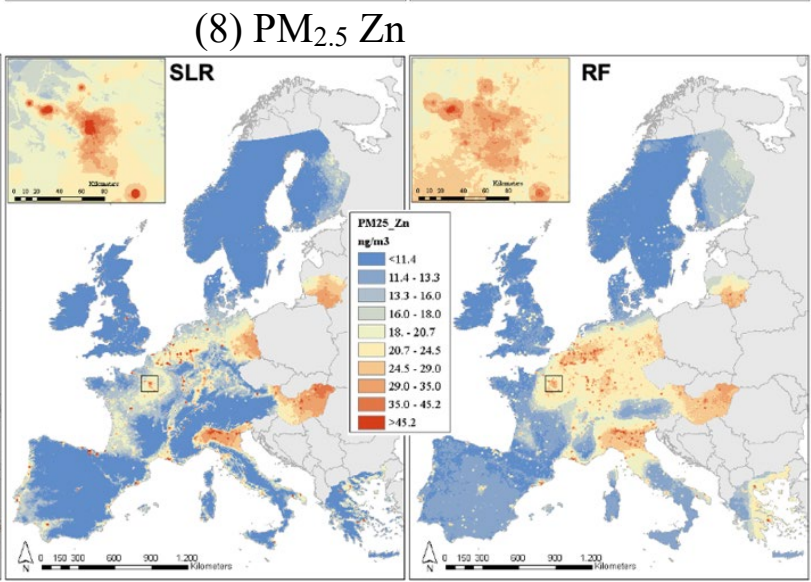

Figure 2. Maps of $\mathbf{P M}_{2.5}$ components developed by our main Supervised Linear Regression (two-step, step2) and Random Forest (two-step, step1) models 
of these predictors increased the specificity of our models, which is useful to study associated health effects of specific single components.

While inclusion of industrial point source data was an improvement over the simple land use categories available in CORINE land cover, a dispersion model for point sources would have been the method of choice. We did not have the possibility to use Europe-wide small-scale dispersion modeling, and we did not have information on chimney height and wind direction around chimneys. We therefore used inverse distance weighting to create variables from industrial point sources, which can lead to overestimation of pollution levels in areas very close to the industrial sites ${ }^{40}$. The misclassification is expected to be minimal given that it is unlikely that many people live very close to the large point source chimneys included in the European Pollutant Release and Transfer Register databases. The small truncation frequency $(<0.1 \%)$ above the maximum values in a total of around 42,000 random locations across Europe suggested the overestimation might not have a large impact.

The prediction ability of our Europe-wide model at small-scale, however, is limited, especially in areas without main sources present. The lack of specificity of the small-scale land use predictors might have contributed to the poor predictive ability for some elements. The poor within-area predictive ability suggests our Europe-wide models should be applied with caution in small-scale individual study areas, with the possible exception of the $\mathrm{Cu}$ and Fe models. Our model is more suited for multi-center studies.

Moderate to high overall correlations between our Europe-wide SLR and previous area-specific ESCAPE model predictions at monitoring sites were observed except for $\mathrm{K}$ and $\mathrm{Zn}$ (Table S4). The within-area correlations between SLR and ESCAPE varied considerably and the average correlations were high for $\mathrm{Cu}$ and $\mathrm{Fe}$. The results suggested applying the newly developed Europe-wide models in epidemiological studies could lead to different findings from the ESCAPE study. The leave-one-outcross-validation (LOOCV) $\mathrm{r}^{2} \mathrm{~s}$ of the area-specific ESCAPE models are not quantitatively comparable with the within-area 5-fold cross-validation $r^{2}$ s in this study as the LOOCV is based on a small number of sites and tends to overestimate predictive ability ${ }^{10,11}$.

Given the discrepancies in predictions derived from the two methods, applying the two sets of models in epidemiological studies could lead to different associations with health. SLR and RF model performances were similar for the within-area concentration variability, while RF model explained overall concentration variability (including between-area variability) better than SLR. In SLR, we did not add fixed or random intercepts for study area as such models could not be applied outside the specific study areas. In a previous study on $\mathrm{PM}_{2.5}$ and $\mathrm{NO}_{2}{ }^{33}$, we found that adding indicators for study area or the measured regional background in each study area, improved the overall explained variability. Therefore, when applied in epidemiological studies, it depends on the contrast exploited in the epidemiological study which method is the preferred method. If both between- and within-area variability are exploited, RF would be the method of choice based on the cross-validation statistics. If an epidemiological study only includes within-area exposure contrast, then both methods should be interpreted equally, without $a$ prior preference for one of the methods. Given the moderate performance of both models, it would be important to observe robustness of the findings in epidemiological studies. If health effects are found with only one model, this should be interpreted cautiously.

Because of the lack of external validation data, we cannot draw strong conclusions about the preferred method. We note that RF models might be more difficult to interpret in terms of how predictor variables act in the models, although the "importance" statistics provide useful information on the relative importance of individual predictors. The classification nature of RF led to visible boundary effects in some exposure maps, which might require smoothing before application in epidemiological studies. On the other hand, SLR might fail to capture some complex non-linear relationships and/or interactions between predictors and pollutant, or might induce overfitting if multiple non-linear and interaction terms were added to the model. Despite the discrepancies in predictions, we believe our models are stable and the results are robust, as different cross-validation methods and several sensitivity analyses showed moderate to good performance especially at the overall Europe-wide scale and similar results. 


\section{References}

1. Adar SD, Filigrana PA, Clements N, Peel JL. Ambient coarse particulate matter and human health: a systematic review and meta-analysis. Current environmental health reports 2014; 1(3): 258-74.

2. Vodonos A, Awad YA, Schwartz J. The concentration-response between long-term PM2.5 exposure and mortality; A meta-regression approach. Environ Res 2018; 166: 677-89.

3. Adams K, Greenbaum DS, Shaikh R, van Erp AM, Russell AG. Particulate matter components, sources, and health: Systematic approaches to testing effects. J Air Waste Manag Assoc 2015; 65(5): 544-58.

4. Badaloni C, Cesaroni G, Cerza F, Davoli M, Brunekreef B, Forastiere F. Effects of long-term exposure to particulate matter and metal components on mortality in the Rome longitudinal study. Environment international 2017; 109: 146-54.

5. Pennington AF, Strickland MJ, Gass K, et al. Source-Apportioned PM2. 5 and Cardiorespiratory Emergency Department Visits: Accounting for Source Contribution Uncertainty. Epidemiology 2019; 30(6): 789-98.

6. Eeftens M, Tsai M-Y, Ampe C, et al. Spatial variation of PM2.5, PM10, PM2.5 absorbance and PMcoarse concentrations between and within 20 European study areas and the relationship with NO2 - Results of the ESCAPE project. Atmospheric Environment 2012; 62: 303-17.

7. Tsai MY, Hoek G, Eeftens M, et al. Spatial variation of PM elemental composition between and within 20 European study areas--Results of the ESCAPE project. Environ Int 2015; 84: 181-92.

8. de Hoogh K, Wang M, Adam M, et al. Development of land use regression models for particle composition in twenty study areas in Europe. Environ Sci Technol 2013; 47(11): 5778-86.

9. Beelen R, Hoek G, Raaschou-Nielsen O, et al. Natural-cause mortality and long-term exposure to particle components: an analysis of 19 European cohorts within the multi-center ESCAPE project. Environ Health Perspect 2015; 123(6): 525-33.

10. Basagaña X, Rivera M, Aguilera I, et al. Effect of the number of measurement sites on land use regression models in estimating local air pollution. Atmospheric Environment 2012; 54: 634-42.

11. Wang M, Beelen R, Eeftens M, Meliefste K, Hoek G, Brunekreef B. Systematic evaluation of land use regression models for NO2. Environmental science \& technology 2012; 46(8): 4481-9.

12. De Hoogh K, Chen J, Gulliver J, et al. Spatial PM2. 5, NO2, O3 and BC models for Western Europe-Evaluation of spatiotemporal stability. Environment international 2018; 120: 81-92.

13. de Hoogh K, Gulliver J, van Donkelaar A, et al. Development of West-European PM 2.5 and NO 2 land use regression models incorporating satellite-derived and chemical transport modelling data. Environmental research 2016; 151: $1-10$.

14. Brauer M, Hoek G, van Vliet P, et al. Estimating long-term average particulate air pollution concentrations: application of traffic indicators and geographic information systems. Epidemiology 2003; 14: 228-39.

15. Henderson SB, Beckerman B, Jerrett M, Brauer M. Application of land use regression to estimate long-term concentrations of traffic-related nitrogen oxides and fine particulate matter. Environmental science \& technology 2007; 41(7): 2422-8.

16. Chen J, de Hoogh K, Gulliver J, et al. A comparison of linear regression, regularization, and machine learning algorithms to develop Europe-wide spatial models of fine particles and nitrogen dioxide. Environment international 2019; 130: 104934 .

17. Kerckhoffs J, Hoek G, Portengen Lt, Brunekreef B, Vermeulen RC. Performance of Prediction Algorithms for Modeling Outdoor Air Pollution Spatial Surfaces. Environmental science \& technology 2019; 53(3): 1413-21.

18. Meng X, Hand JL, Schichtel BA, Liu Y. Space-time trends of PM2.5 constituents in the conterminous United States estimated by a machine learning approach, 2005-2015. Environ Int 2018; 121(Pt 2): 1137-47.

19. Hu X, Belle JH, Meng X, et al. Estimating PM2. 5 concentrations in the conterminous United States using the random forest approach. Environmental science \& technology 2017; 51(12): 6936-44.

20. $\quad$ Breiman L. Random forests. Machine learning 2001; 45(1): 5-32.

21. Brokamp C, Jandarov R, Rao MB, LeMasters G, Ryan P. Exposure assessment models for elemental components of particulate matter in an urban environment: A comparison of regression and random forest approaches. Atmos Environ (1994) 2017; 151: 1-11.

22. Hoek G, Beelen R, De Hoogh K, et al. A review of land-use regression models to assess spatial variation of outdoor air pollution. Atmospheric environment 2008; 42(33): 7561-78.

23. Eeftens M, Beelen R, de Hoogh K, et al. Development of Land Use Regression models for $\operatorname{PM}(2.5), \operatorname{PM}(2.5)$ absorbance, PM(10) and PM(coarse) in 20 European study areas; results of the ESCAPE project. Environ Sci Technol 2012; 46(20): 11195-205.

24. GEOSTAT. (version 2.0.1): https://ec.europa.eu/eurostat/web/gisco/geodata/reference-data/population-distributiondemography/geostat (last access 20190719). 2011.

25. CGIAR-CSI. Srtm 90m digital elevation data. 2013.

26. CLC. https://land.copernicus.eu/pan-european/corine-land-cover/clc-2012 (last access 20190719). 2012.

27. van Donkelaar A, Martin RV, Li C, Burnett RT. Regional Estimates of Chemical Composition of Fine Particulate Matter Using a Combined Geoscience-Statistical Method with Information from Satellites, Models, and Monitors. Environ Sci Technol 2019; 53(5): 2595-611.

28. ECMWF. https://apps.ecmwf.int/datasets/data/macc-reanalysis/levtype $=$ sfc/ (last access 20190723).

29. Inness A, Baier F, Benedetti A, et al. The MACC reanalysis: an $8 \mathrm{yr}$ data set of atmospheric composition. Atmospheric Chemistry and Physics 2013; 13(8): 4073-109.

30. E-PRTR. https://www.eea.europa.eu/data-and-maps/data/member-states-reporting-art-7-under-the-europeanpollutant-release-and-transfer-register-e-prtr-regulation-18 (version 16, last access 20190719).

31. Liaw A, Wiener M. Classification and regression by randomForest. $R$ news $2002 ; 2$ (3): 18-22. 
32. Murray NL, Holmes HA, Liu Y, Chang HH. A Bayesian ensemble approach to combine PM2.5 estimates from statistical models using satellite imagery and numerical model simulation. Environ Res 2019; 178: 108601.

33. Wang M, Beelen R, Bellander T, et al. Performance of multi-city land use regression models for nitrogen dioxide and fine particles. Environ Health Perspect 2014; 122(8): 843-9.

34. Vedal S. National Particle Component Toxicity (NPACT) initiative report on cardiovascular effects. Research report (Health Effects Institute) 2013; (178): 5-8.

35. Belis CA, Karagulian F, Larsen BR, Hopke PK. Critical review and meta-analysis of ambient particulate matter source apportionment using receptor models in Europe. Atmospheric Environment 2013; 69: 94-108.

36. Ito K, Johnson S, Kheirbek I, et al. Intraurban Variation of Fine Particle Elemental Concentrations in New York City. Environ Sci Technol 2016; 50(14): 7517-26.

37. Tripathy S, Tunno BJ, Michanowicz DR, et al. Hybrid land use regression modeling for estimating spatio-temporal exposures to PM2.5, BC, and metal components across a metropolitan area of complex terrain and industrial sources. The Science of the total environment 2019; 673: 54-63.

38. Zhang JJY, Sun L, Barrett O, Bertazzon S, Underwood FE, Johnson M. Development of land-use regression models for metals associated with airborne particulate matter in a North American city. Atmospheric Environment 2015; 106: 165-77. 39. Viana M, Kuhlbusch TAJ, Querol X, et al. Source apportionment of particulate matter in Europe: A review of methods and results. Journal of Aerosol Science 2008; 39(10): 827-49.

40. Michanowicz DR, Shmool JLC, Tunno BJ, et al. A hybrid land use regression/AERMOD model for predicting intraurban variation in PM2.5. Atmospheric Environment 2016; 131: 307-15. 


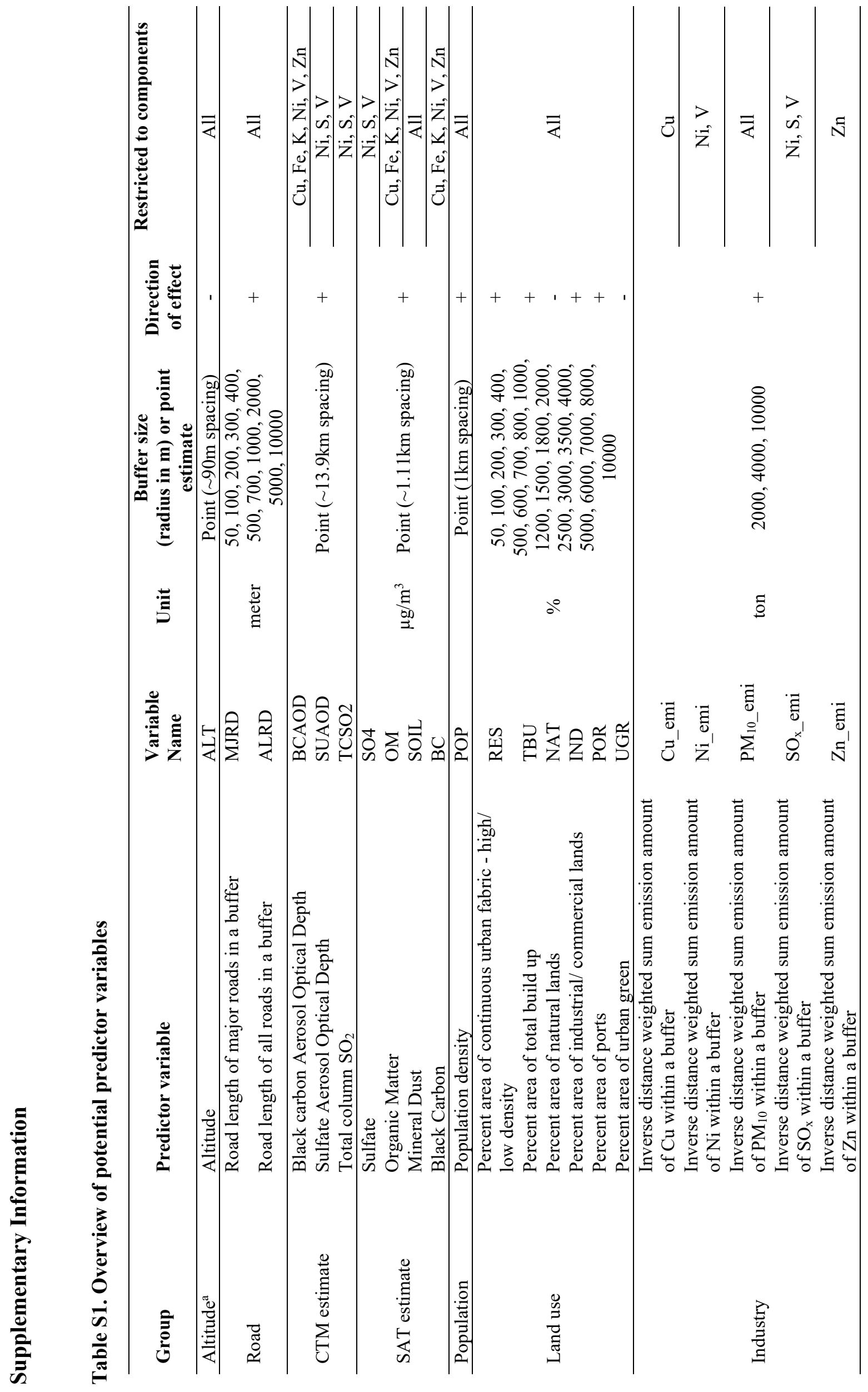




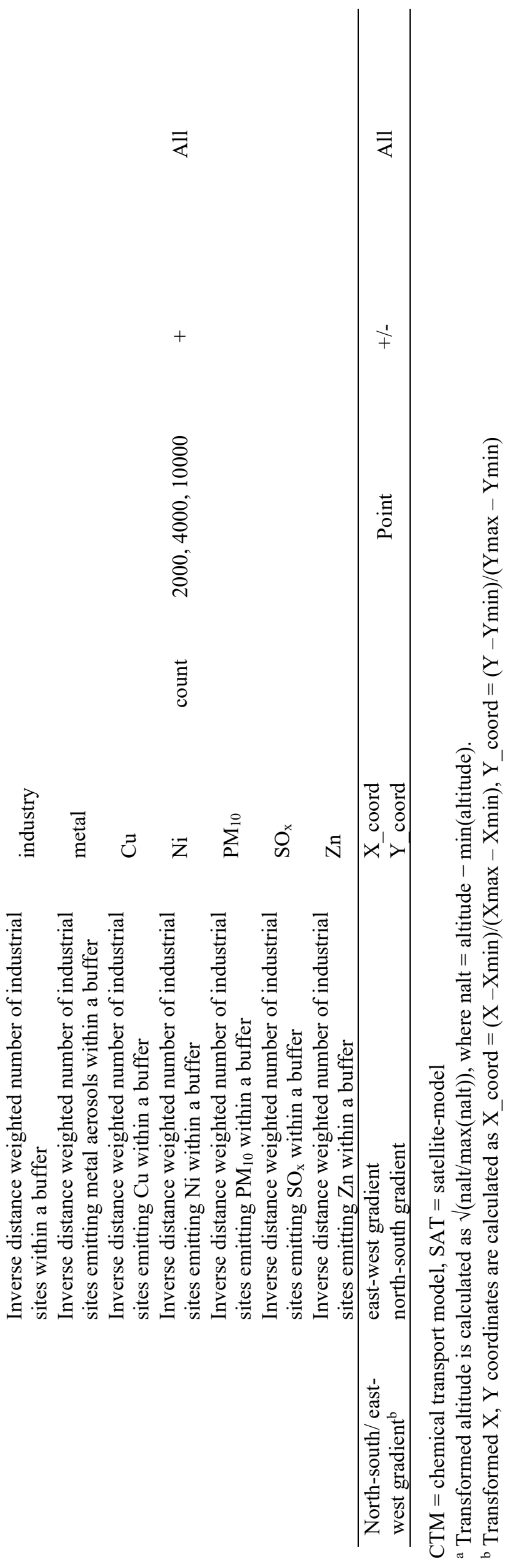




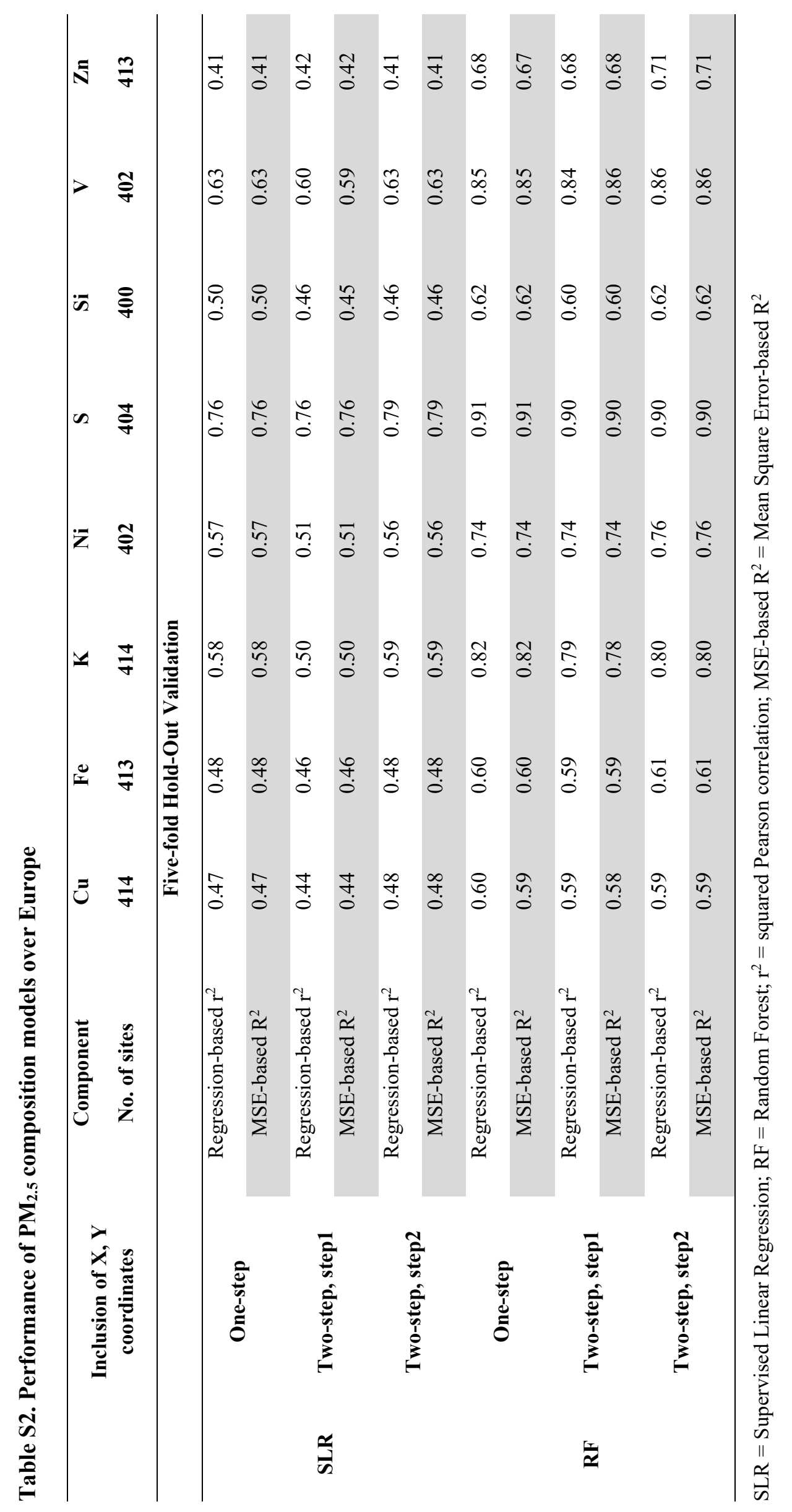


Table S3. Truncation frequency (\%) for model predictions at 41,936 random locations

\begin{tabular}{ccccccccccc}
\hline & $\begin{array}{c}\text { Inclusion of X, } \\
\text { Y coordinates }\end{array}$ & & $\mathrm{Cu}$ & $\mathrm{Fe}$ & $\mathrm{K}$ & $\mathrm{Ni}$ & $\mathrm{S}$ & $\mathrm{Si}$ & $\mathrm{V}$ & $\mathrm{Zn}$ \\
\hline SLR & \multirow{2}{*}{ one-step } & exceed maximum & 0 & 0 & 0 & 0 & 0 & 0 & 0 & 0.1 \\
& & negative & 38.9 & 36.3 & 12 & 26.2 & 0 & 3.9 & 23.7 & 18.1 \\
& Two-step, step1 & exceed maximum & 0 & 0 & 0 & 0 & 0 & 0 & 0 & 0.1 \\
& & negative & 53.6 & 10 & 0 & 26.7 & 0 & 0 & 24.3 & 19.4 \\
& \multirow{2}{*}{ Two-step, step2 } & exceed maximum & 0 & 0 & 0 & 0 & 0 & 0 & 0 & 0.1 \\
& & negative & 41.3 & 10 & 11.5 & 21.8 & 0 & 0 & 20.5 & 19.8 \\
RF & exceed maximum & 0 & 0 & 0 & 0 & 0 & 0 & 0 & 0 \\
& & negative & 0 & 0 & 0 & 0 & 0 & 0 & 0 & 0 \\
& Two-step, step1 & exceed maximum & 0 & 0 & 0 & 0 & 0 & 0 & 0 & 0 \\
& & negative & 0 & 0 & 0 & 0 & 0 & 0 & 0 & 0 \\
& Two-step, step2 & exceed maximum & 0 & 0 & 0 & 0 & 0 & 0 & 0 & 0 \\
& & negative & 0 & 0 & 0 & 0 & 0 & 0 & 0 & 0 \\
\hline
\end{tabular}

$\mathrm{SLR}=$ Supervised Linear Regression; $\mathrm{RF}=$ Random Forest

Table S4. Correlation of predictions at monitoring sites between Europe-wide models ${ }^{\mathrm{a}}$ and ESCAPE models

\begin{tabular}{cccc}
\hline & Overall $\mathrm{r}^{2}$ & Average of within-area $\mathrm{r}^{2}$ & Range of within-area $\mathrm{r}^{2}$ \\
\hline $\mathrm{Cu}$ & 0.63 & 0.50 & $(0.02,0.77)$ \\
$\mathrm{Fe}$ & 0.55 & 0.47 & $(0.07,0.75)$ \\
$\mathrm{K}$ & 0.26 & 0.10 & $(0.00,0.47)$ \\
$\mathrm{Ni}$ & 0.69 & 0.22 & $(0.00,0.78)$ \\
$\mathrm{S}$ & 0.84 & 0.22 & $(0.00,0.73)$ \\
$\mathrm{Si}$ & 0.49 & 0.27 & $(0.00,0.68)$ \\
$\mathrm{V}$ & 0.73 & 0.27 & $(0.00,0.79)$ \\
$\mathrm{Zn}$ & 0.13 & 0.22 & $(0.00,0.74)$ \\
\hline
\end{tabular}

SLR = two-step Supervised Linear Regression step2; ESCAPE = area-specific ESCAPE model predictions; $\mathrm{r}^{2}=$ squared Pearson correlation

a We only presented correlations between ESCAPE and SLR predictions, as correlations between ESCAPE and RF predictions cannot be interpreted because RF models have "by design" perfect predictions at training sites. 


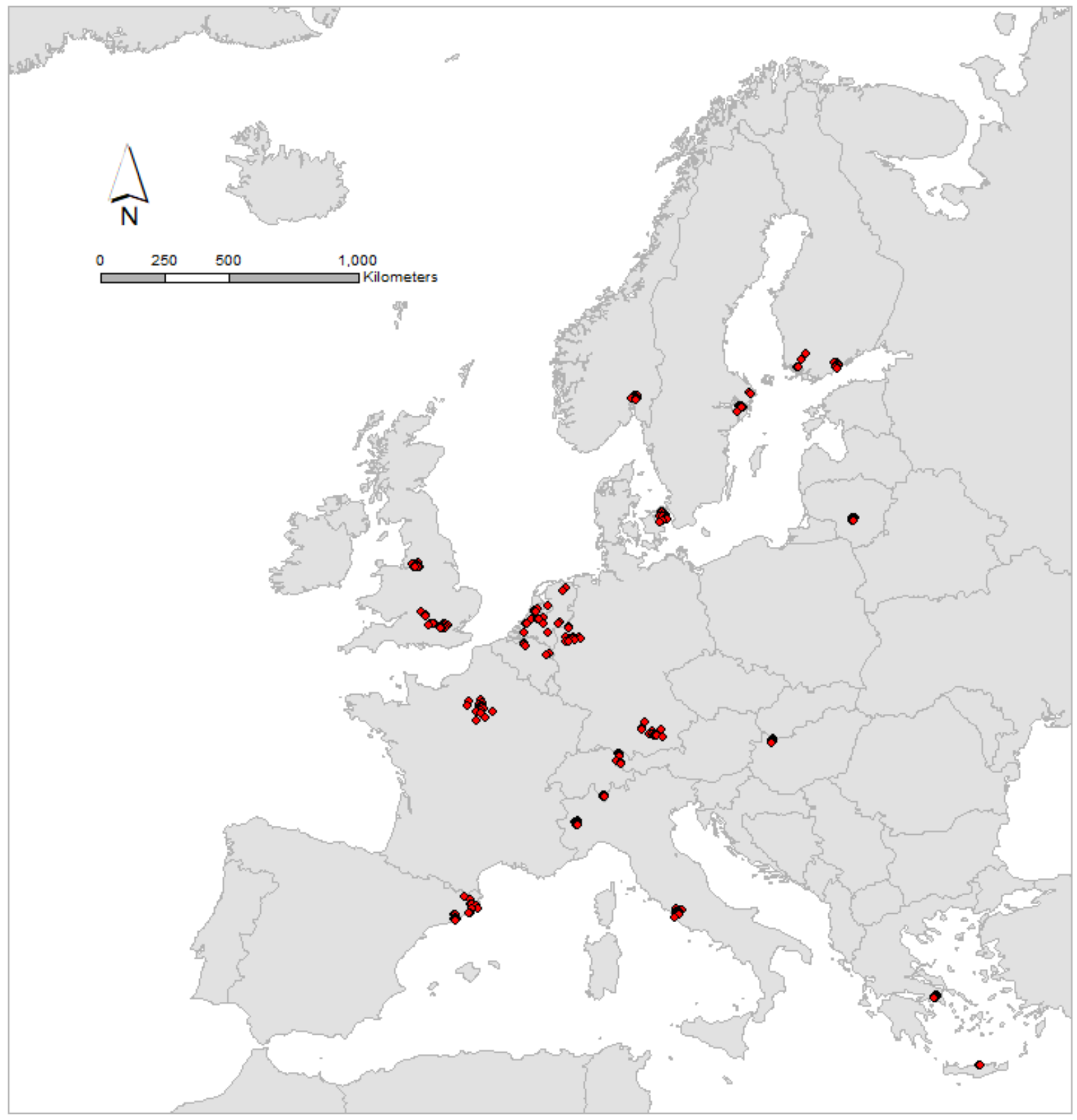

Figure S1. Distribution of 416 ESCAPE monitoring sites across 19 study areas. Each area has 20 sites (40 sites in the Netherlands/Belgium and Catalunya) 
PM25_Cu

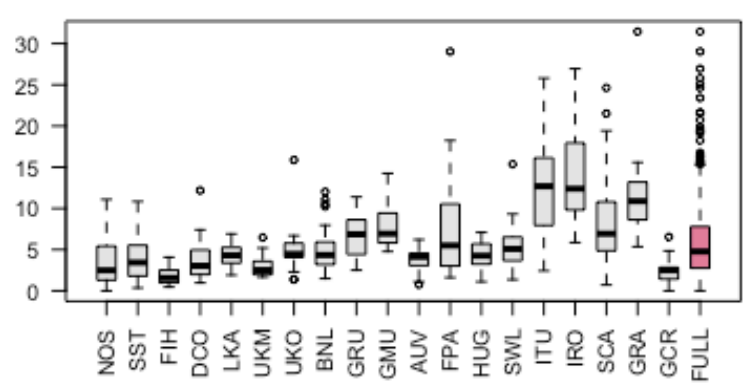

PM25 K

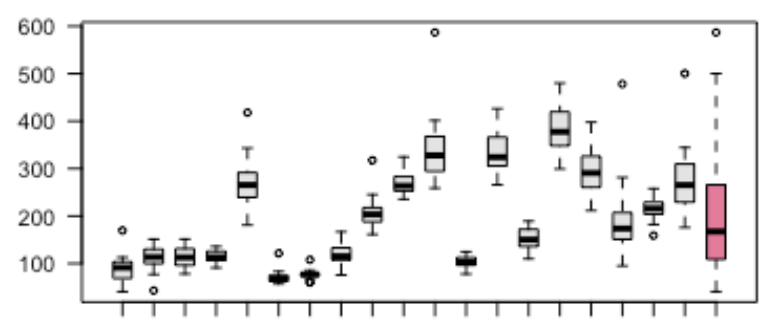

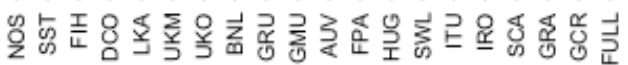

PM25_S

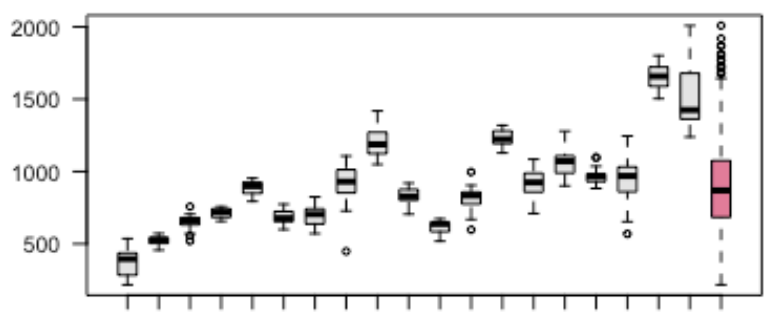

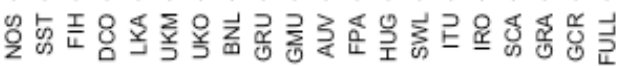

PM25_V

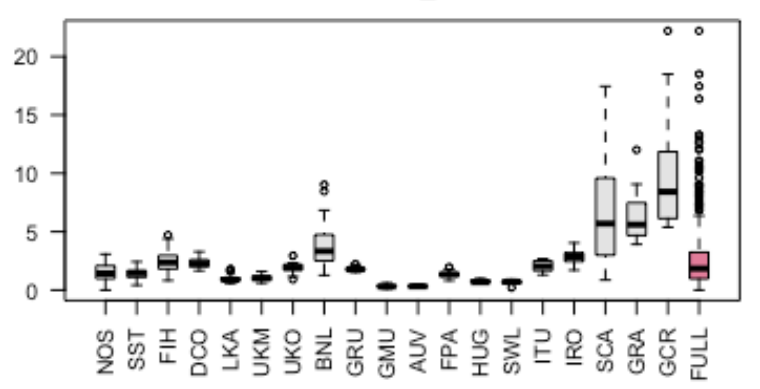

PM25_Fe

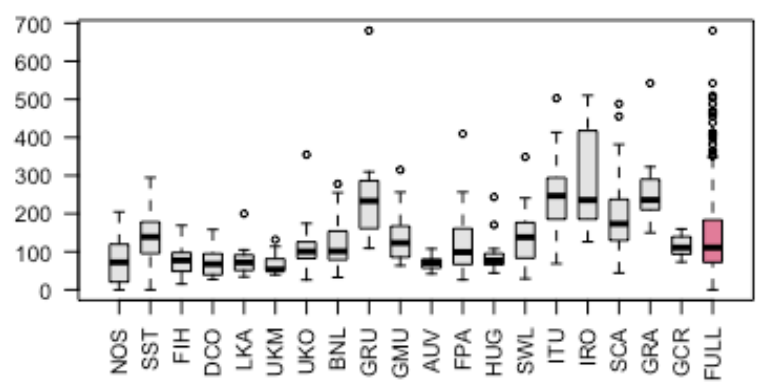

PM25_Ni

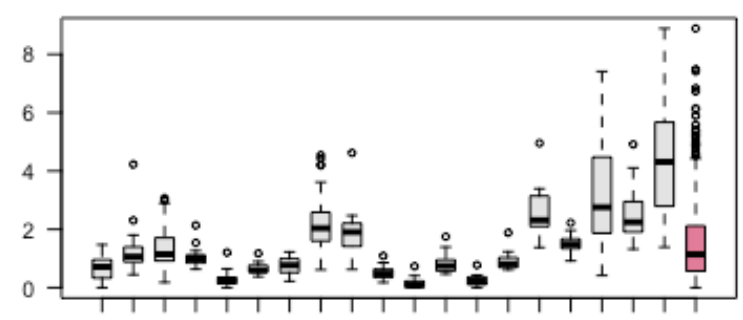

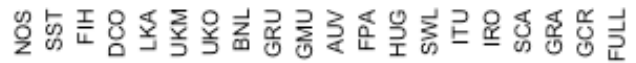

PM25_Si

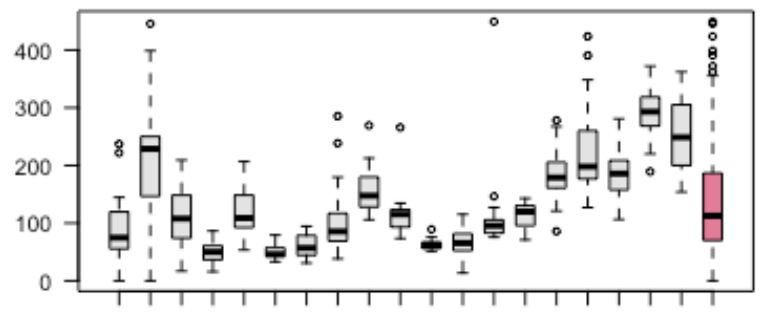

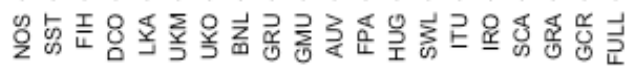

PM25_Zn

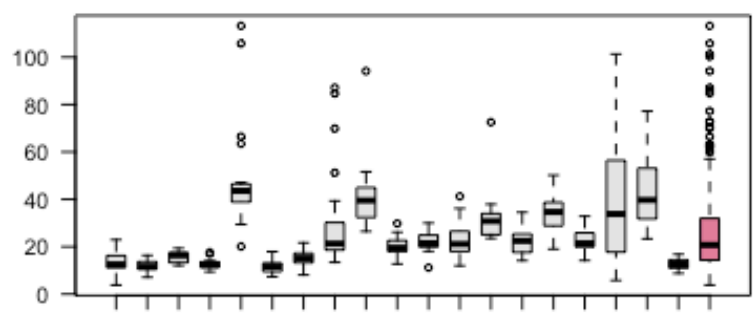

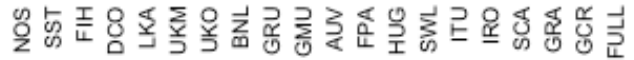

Figure S2. Boxplots of annual mean concentrations for $\mathbf{P M}_{2.5}$ composition $\left(\mathrm{ng} / \mathrm{m}^{3}\right)$ in individual study areas and in the full dataset (box shown in red). Individual study areas are shown from north to south.

NOS: Oslo (Norway); SST: Stockholm County (Sweden); FIH: Helsinki/Turku (Finland); DCO: Copenhagen (Denmark); LKA: Kaunas (Lithuania); UKM: Manchester (United Kingdom); UKO: London/Oxford (United Kingdom); BNL: Netherlands/Belgium; GRU: Ruhr Area (Germany); GMU: Munich/Augsburg (Germany); AUV: Vorarlberg (Austria); FPA: Paris (France); HUG: Gyor (Hungary); SWL: Lugano (Switzerland); ITU: Turin (Italy); IRO: Rome (Italy); SCA: Catalunya (Spain); GRA: Athens (Greece); GCR: Heraklion (Greece). 


\section{Figure S3. Scatter plots of the stacked predictions at 5 held-out sites versus measurements, obtained from 5-fold hold-out validation analyses}

SLR $=$ Supervised Linear Regression; RF $=$ Random Forest

AUV: Vorarlberg (Austria); BNL: Netherlands/Belgium; DCO: Copenhagen (Denmark); FIH: Helsinki/Turku (Finland); FPA: Paris (France); GCR: Heraklion (Greece); GMU: Munich/Augsburg (Germany); GRA: Athens (Greece); GRU: Ruhr Area (Germany); HUG: Gyor (Hungary); IRO: Rome (Italy); ITU: Turin (Italy); LKA: Kaunas (Lithuania); NOS: Oslo (Norway); SCA: Catalunya (Spain); SST: Stockholm County (Sweden); SWL: Lugano (Switzerland); UKM: Manchester (United Kingdom); UKO: London/Oxford (United Kingdom)

(1) $\mathrm{PM}_{2.5} \mathrm{Cu}$ (Left two-step SLR step2, Right two-step RF step 1)
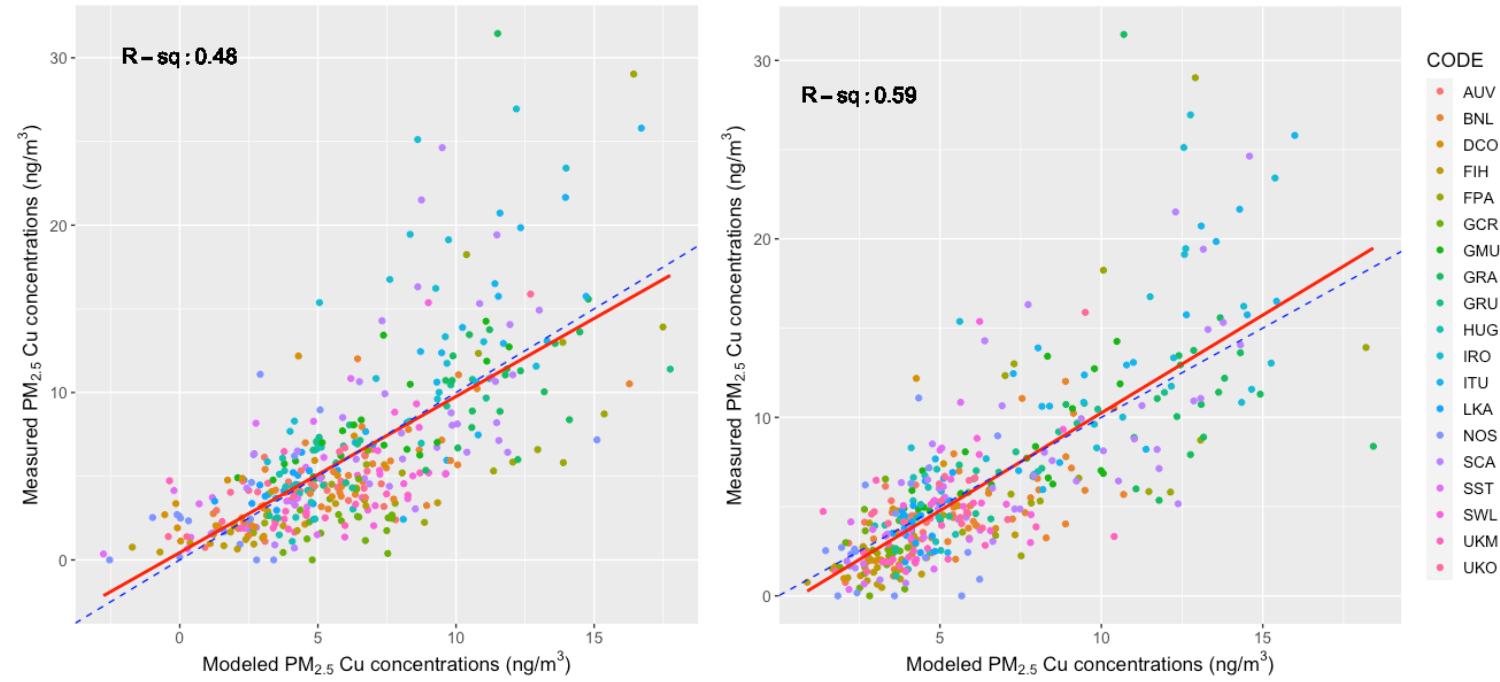

(2) $\mathrm{PM}_{2.5} \mathrm{Fe}$ (Left two-step SLR step2, Right two-step RF step 1)
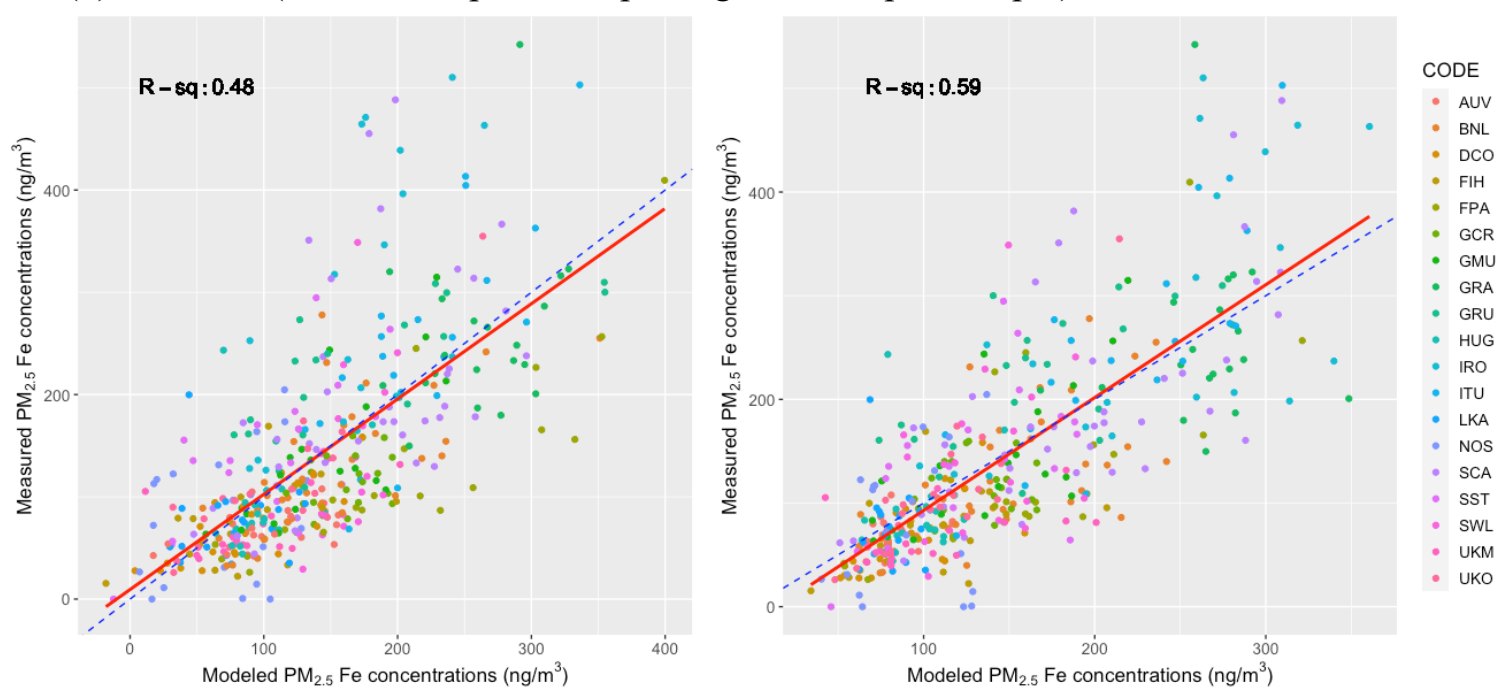
(3) $\mathrm{PM}_{2.5} \mathrm{~K}$ (Left two-step SLR step2, Right two-step RF step 1)
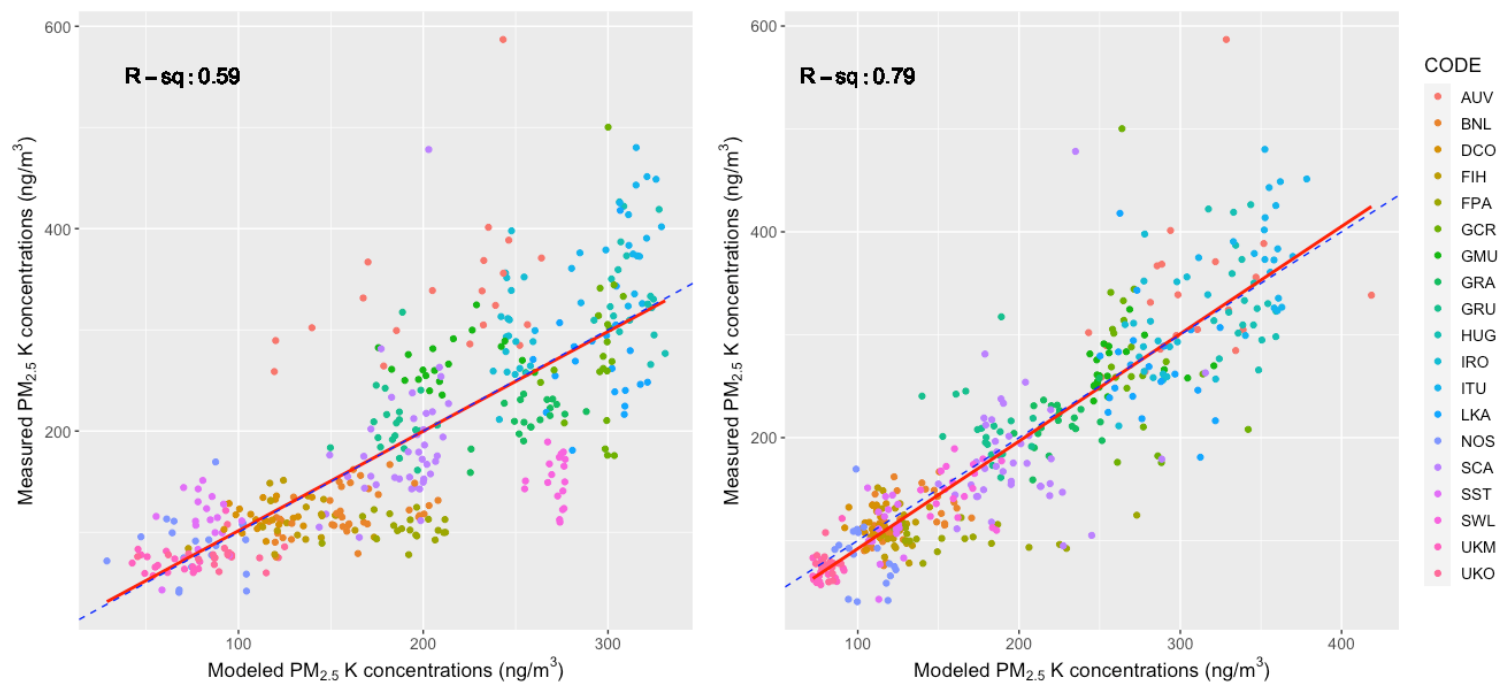

(4) $\mathrm{PM}_{2.5} \mathrm{Ni}$ (Left two-step SLR step2, Right two-step RF step 1)
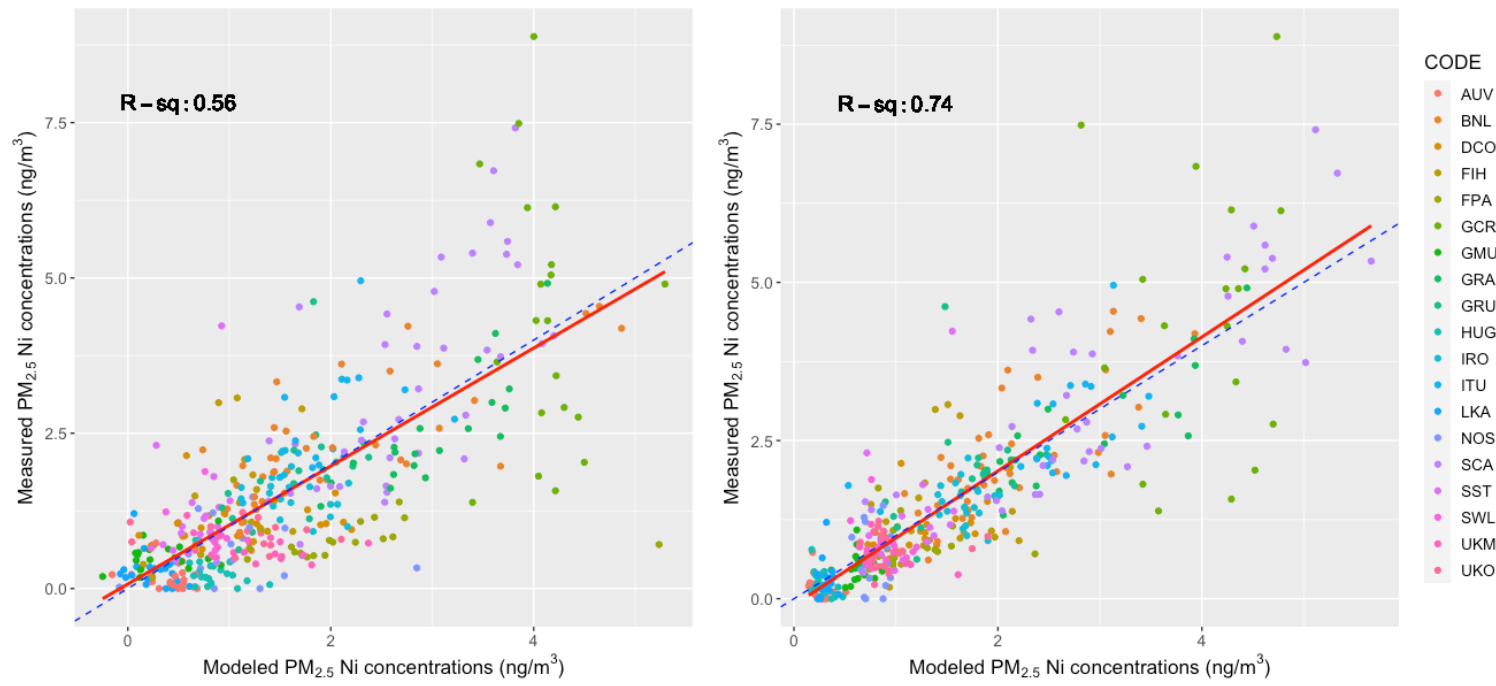

(5) $\mathrm{PM}_{2.5} \mathrm{~S}$ (Left two-step SLR step2, Right two-step RF step 1)
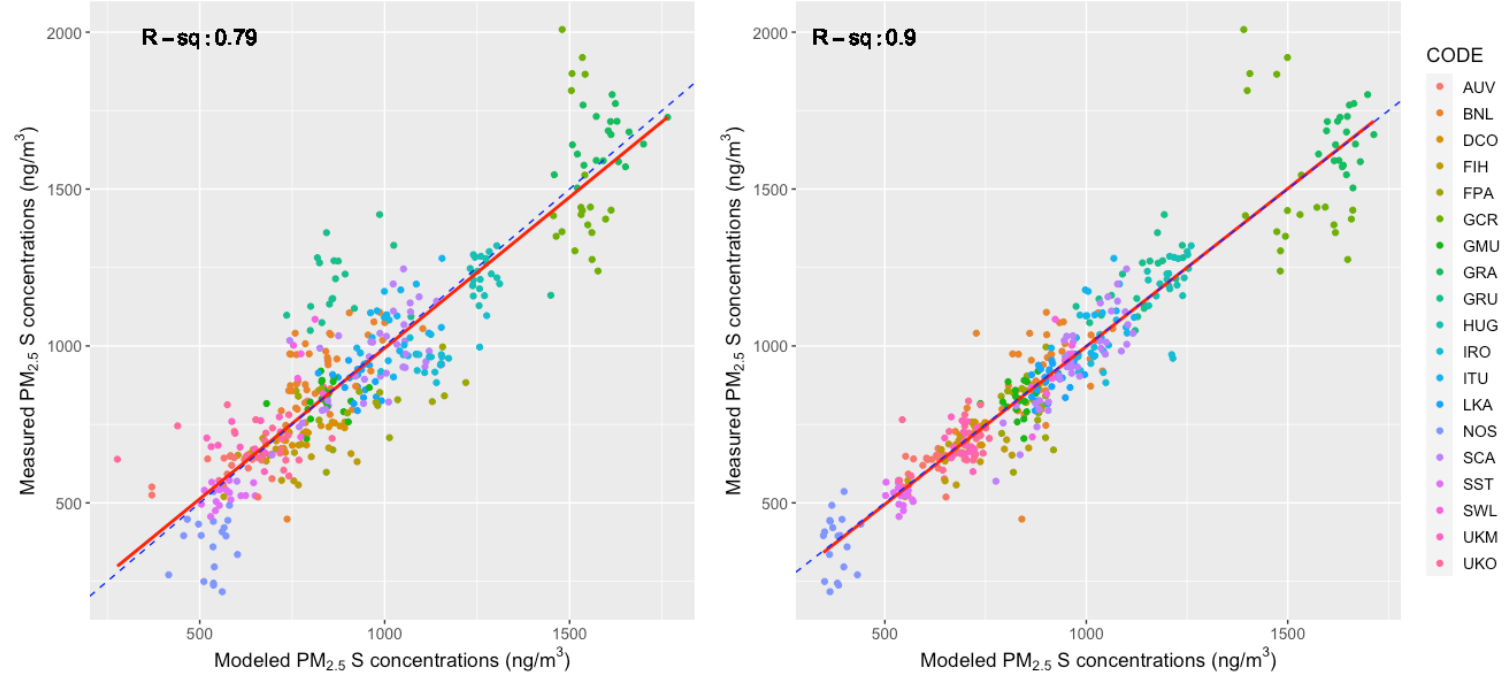
(6) $\mathrm{PM}_{2.5} \mathrm{Si}$ (Left two-step SLR step2, Right two-step RF step 1)
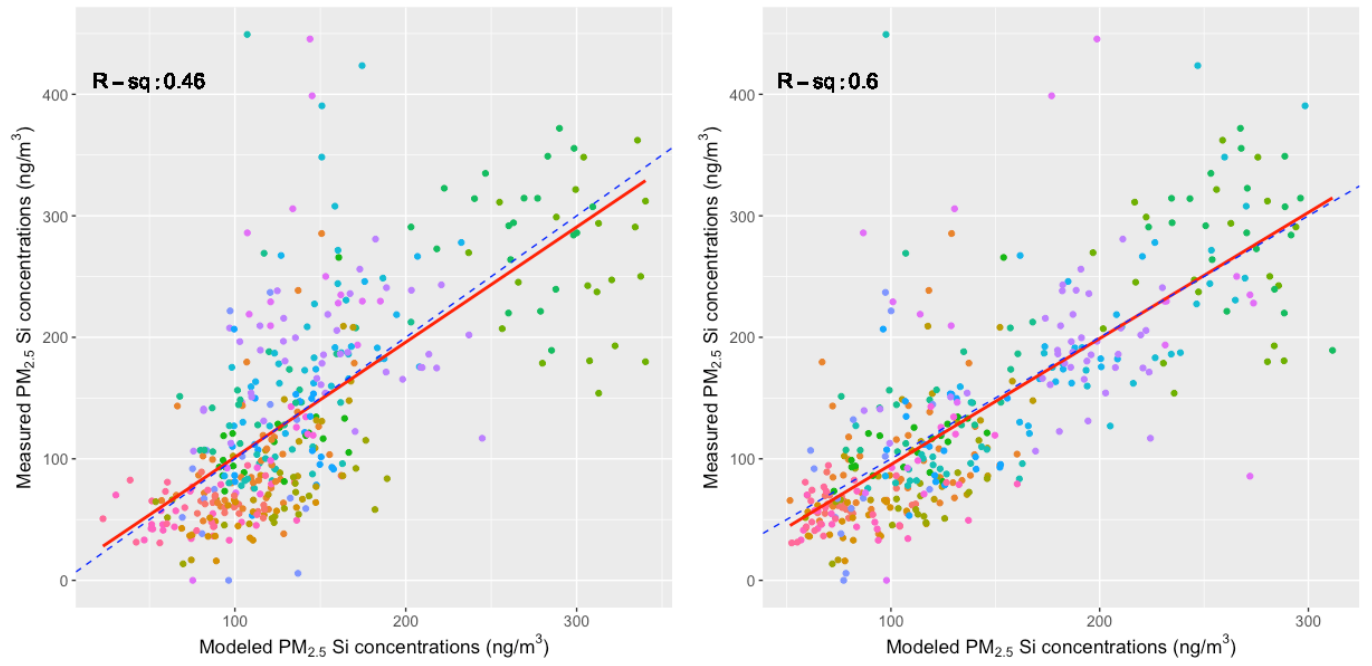

- GRA

- HUG

- IRO

ITU

LKA

- NOS

- SCA

SWL

- UKM

(7) $\mathrm{PM}_{2.5} \mathrm{~V}$ (Left two-step SLR step2, Right two-step RF step 1)
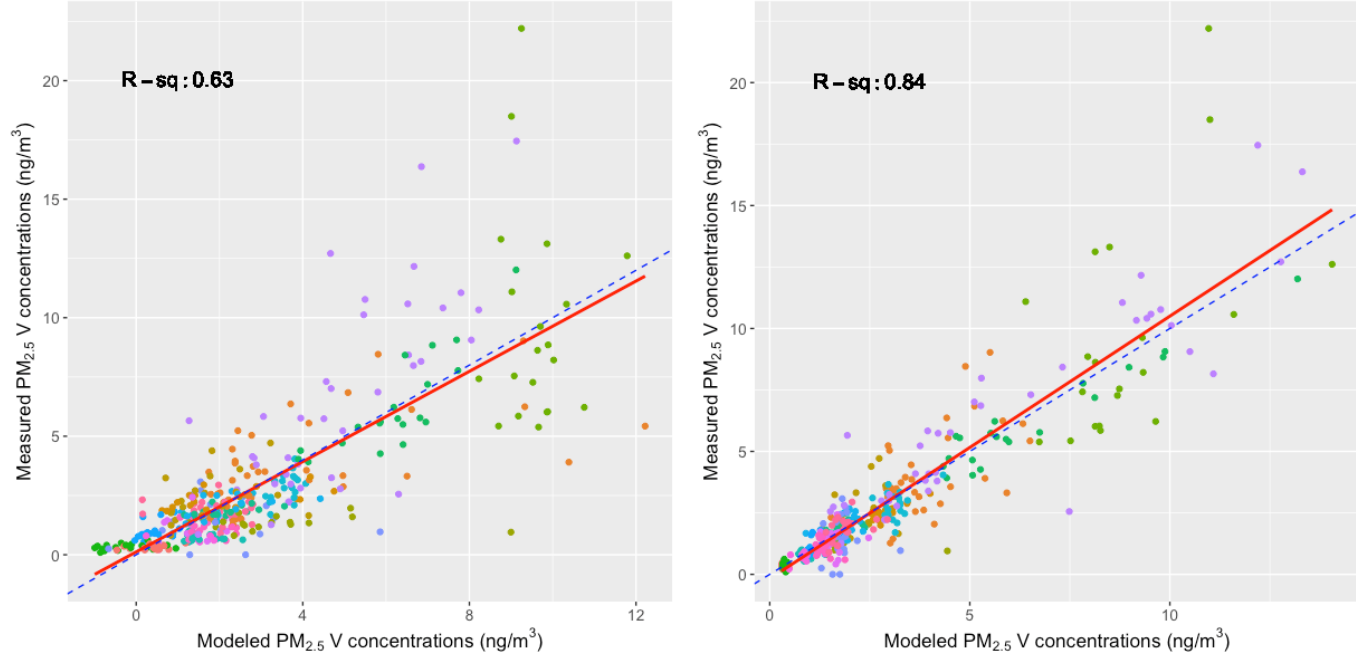

(8) $\mathrm{PM}_{2.5} \mathrm{Zn}$ (Left two-step SLR step2, Right two-step RF step 1)
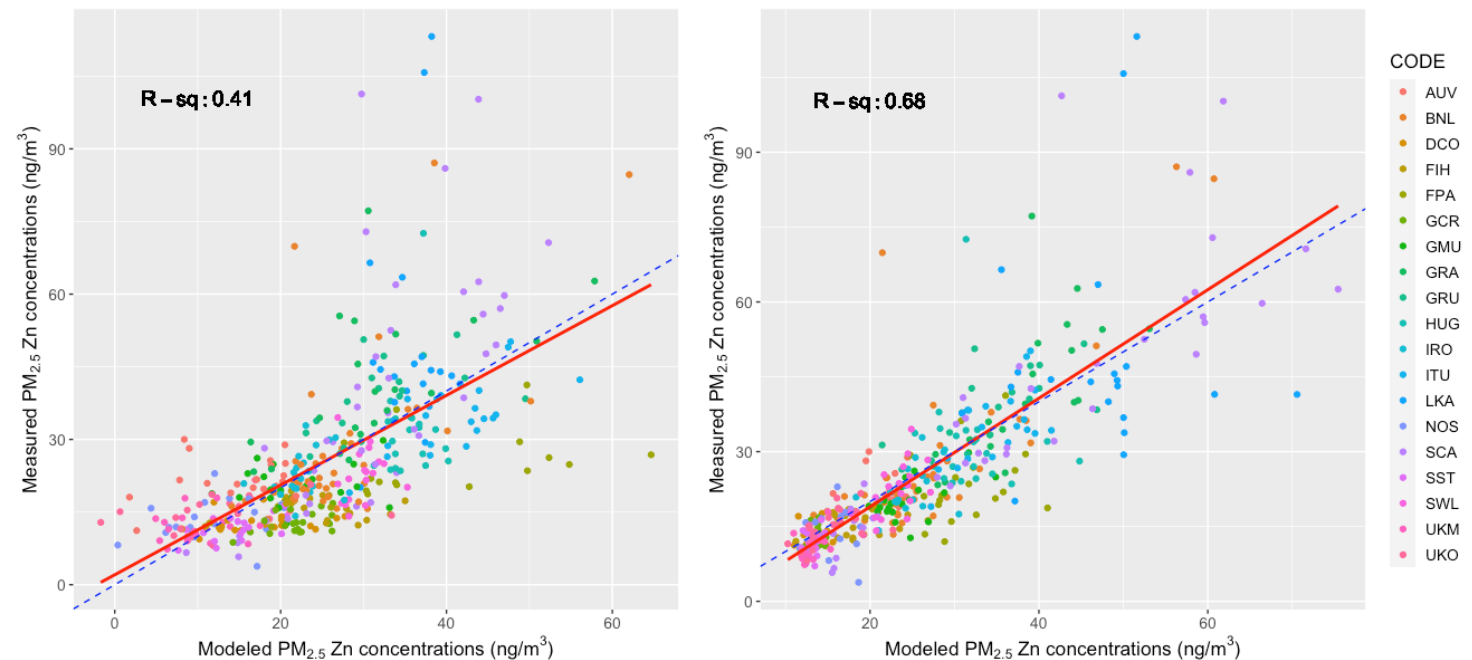
Figure S4. Within-area $\mathbf{r}^{2} \mathbf{s}$ (bars and scale on left) and Root-Mean-Square Errors (RMSEs) (plus signs and scale on right) of $\mathrm{PM}_{2.5}$ composition models evaluated by five-fold hold-out-validation

(a) One-step Supervised Linear Regression

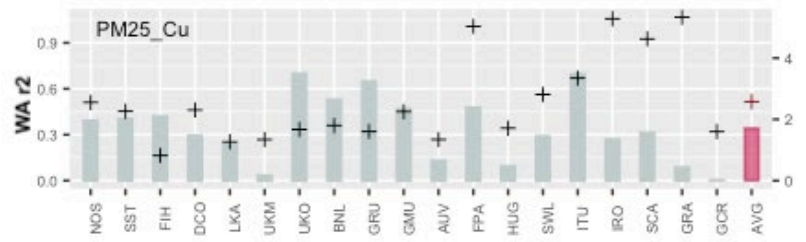

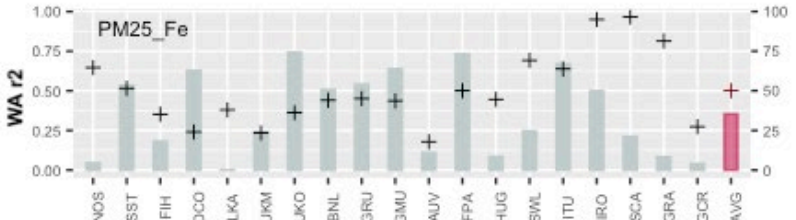



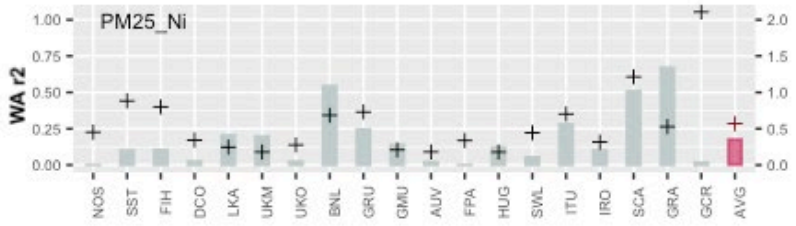

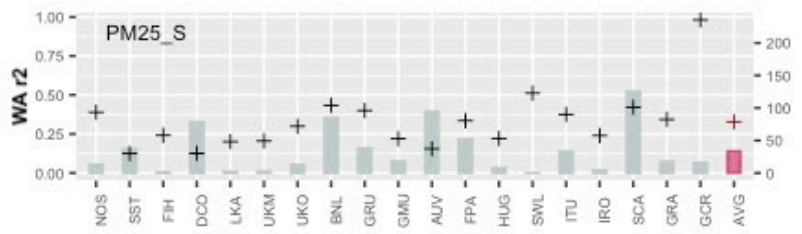

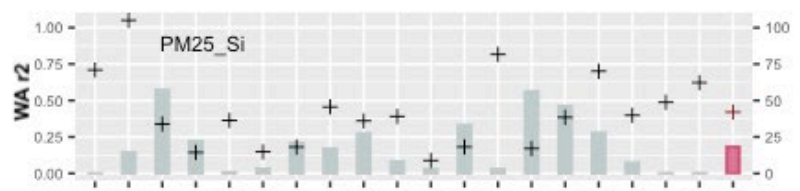

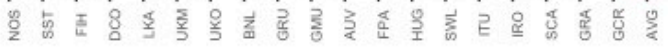
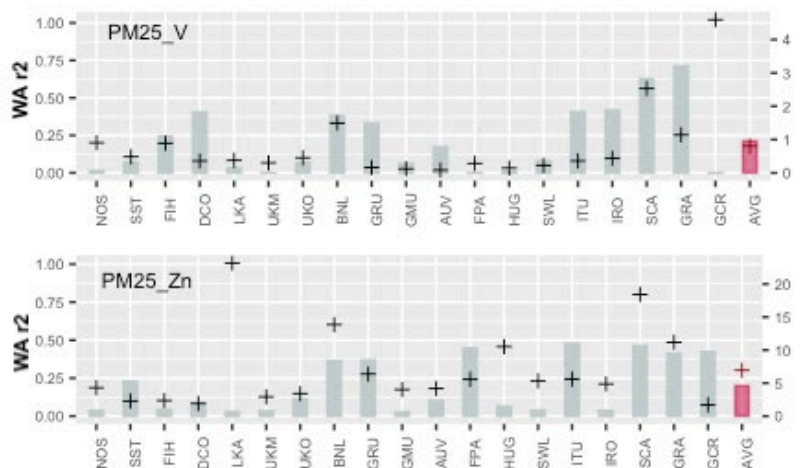

One-step Random Forest
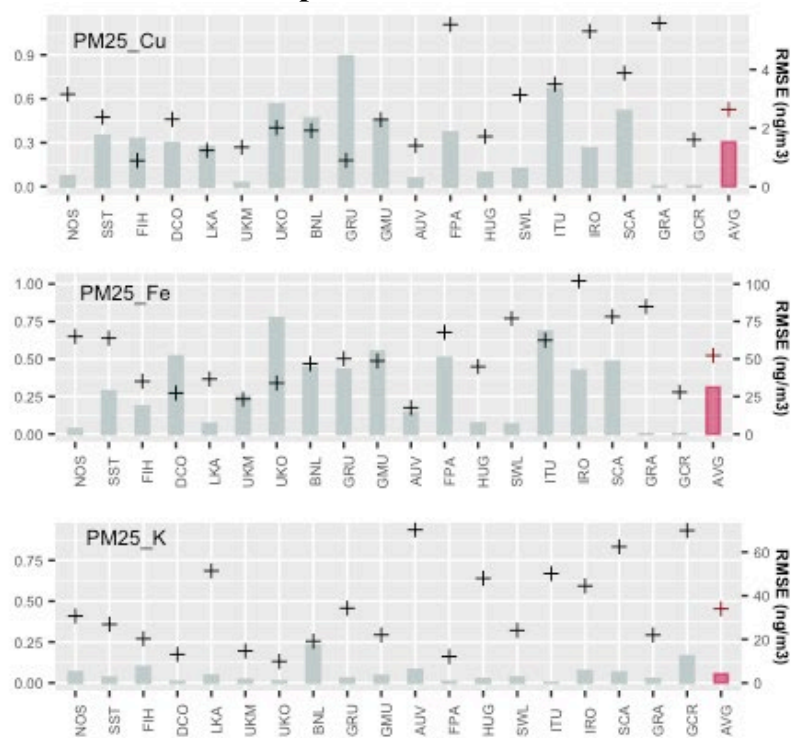

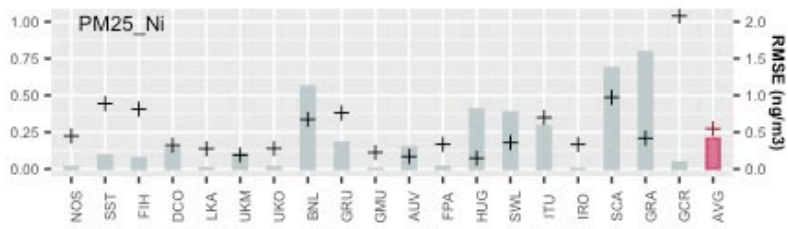
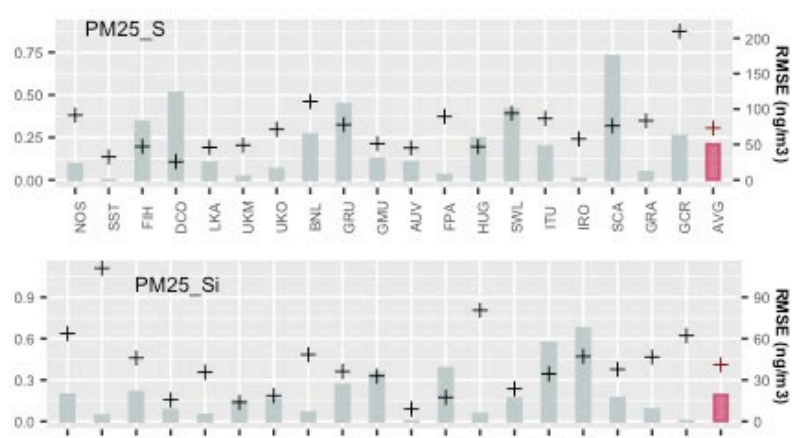

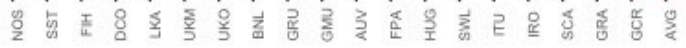
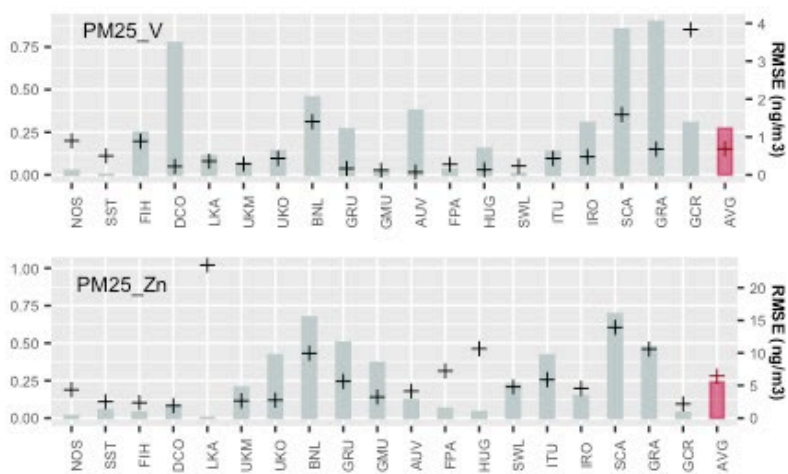
(b) Two-step Supervised Linear Regression, step1

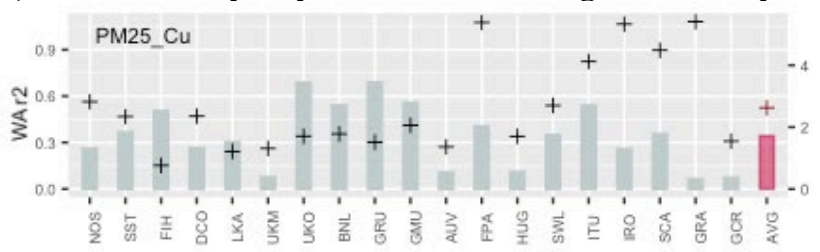
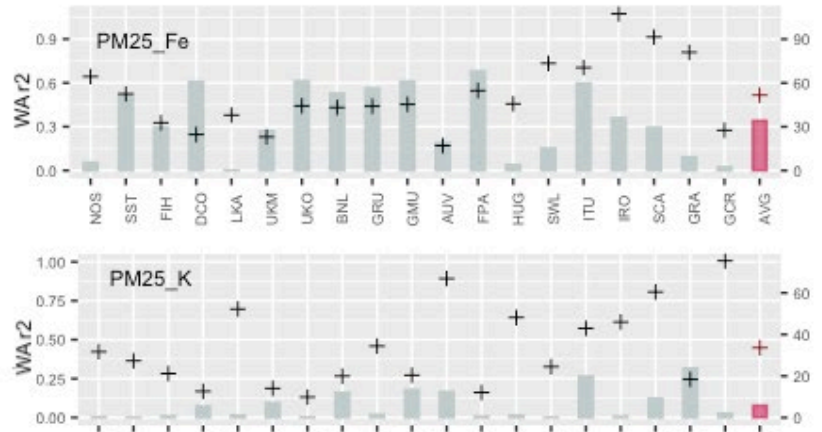

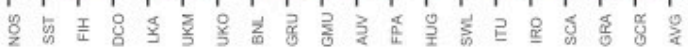

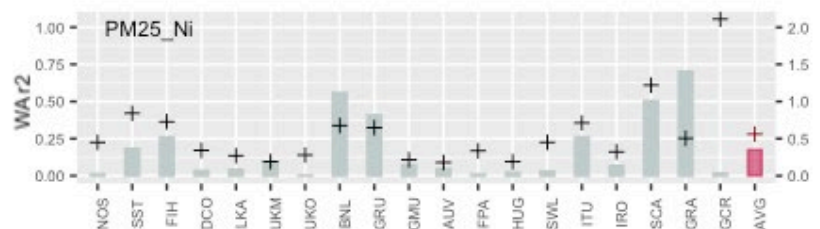
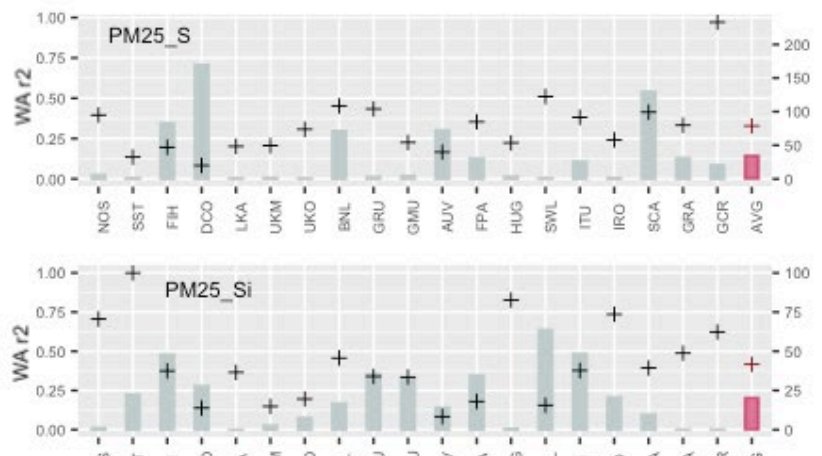

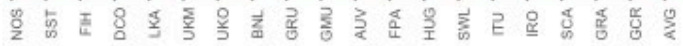

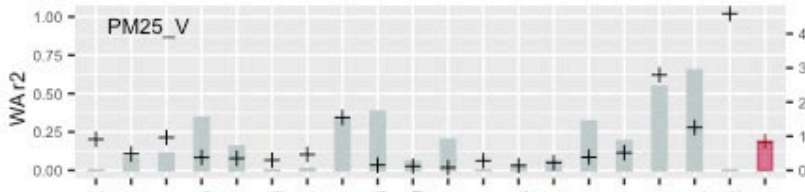

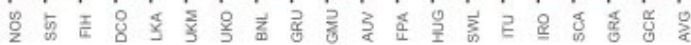

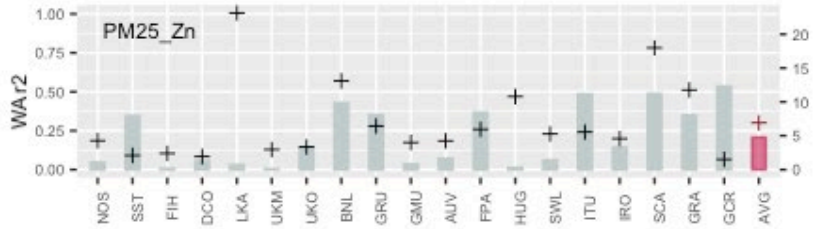

Two-step Random Forest, step1
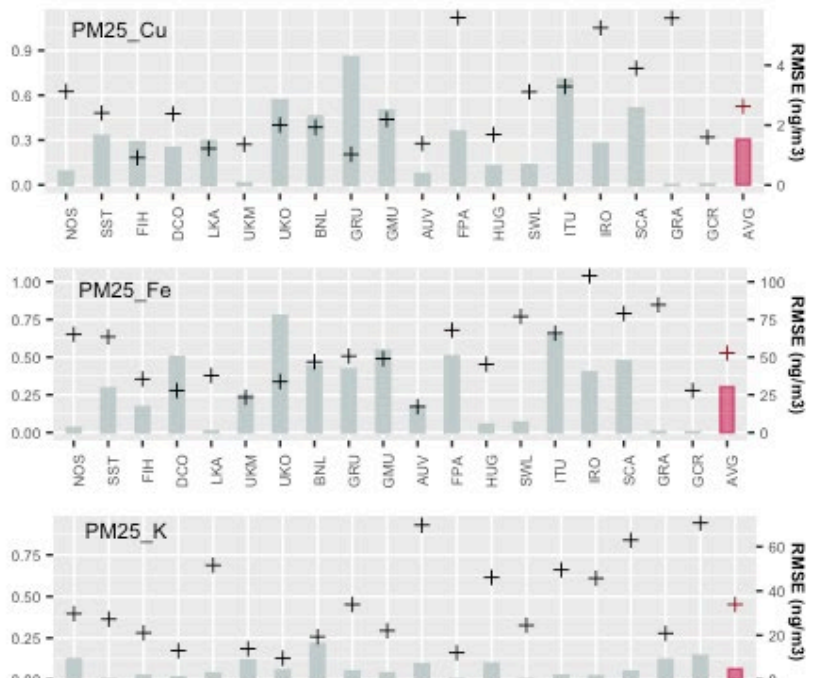

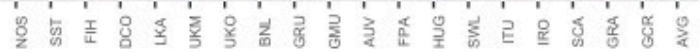

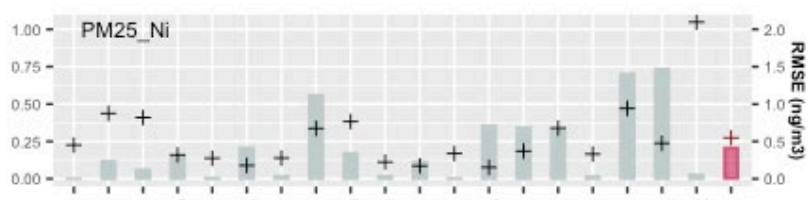

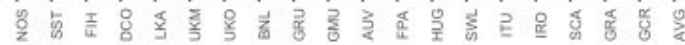
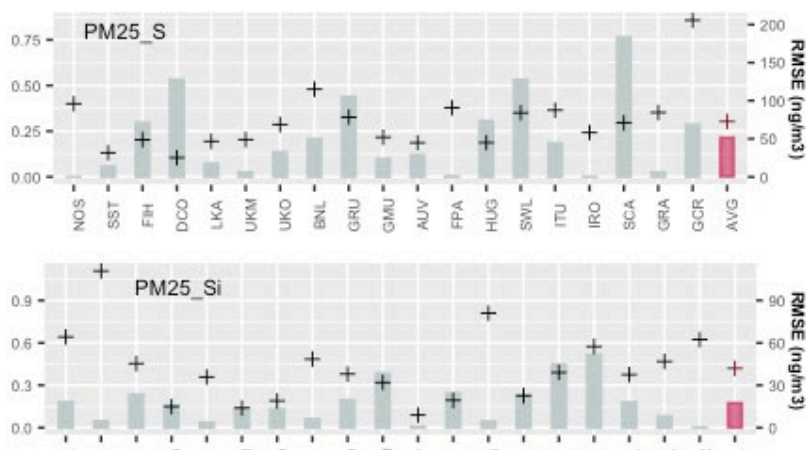

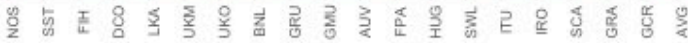

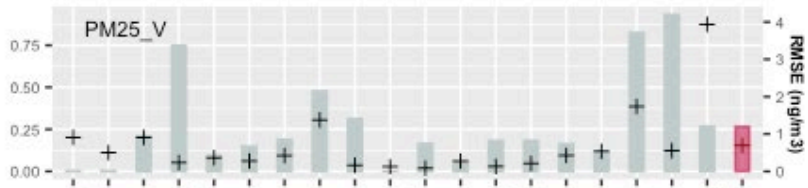

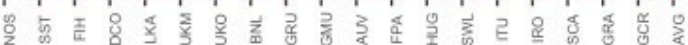

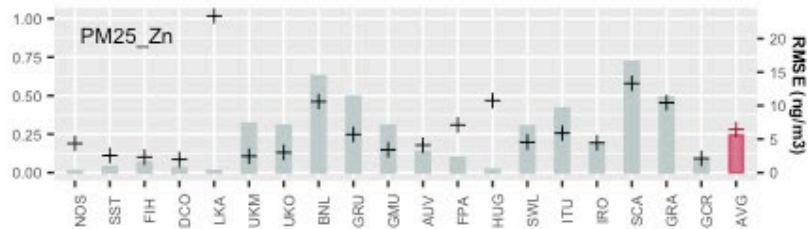



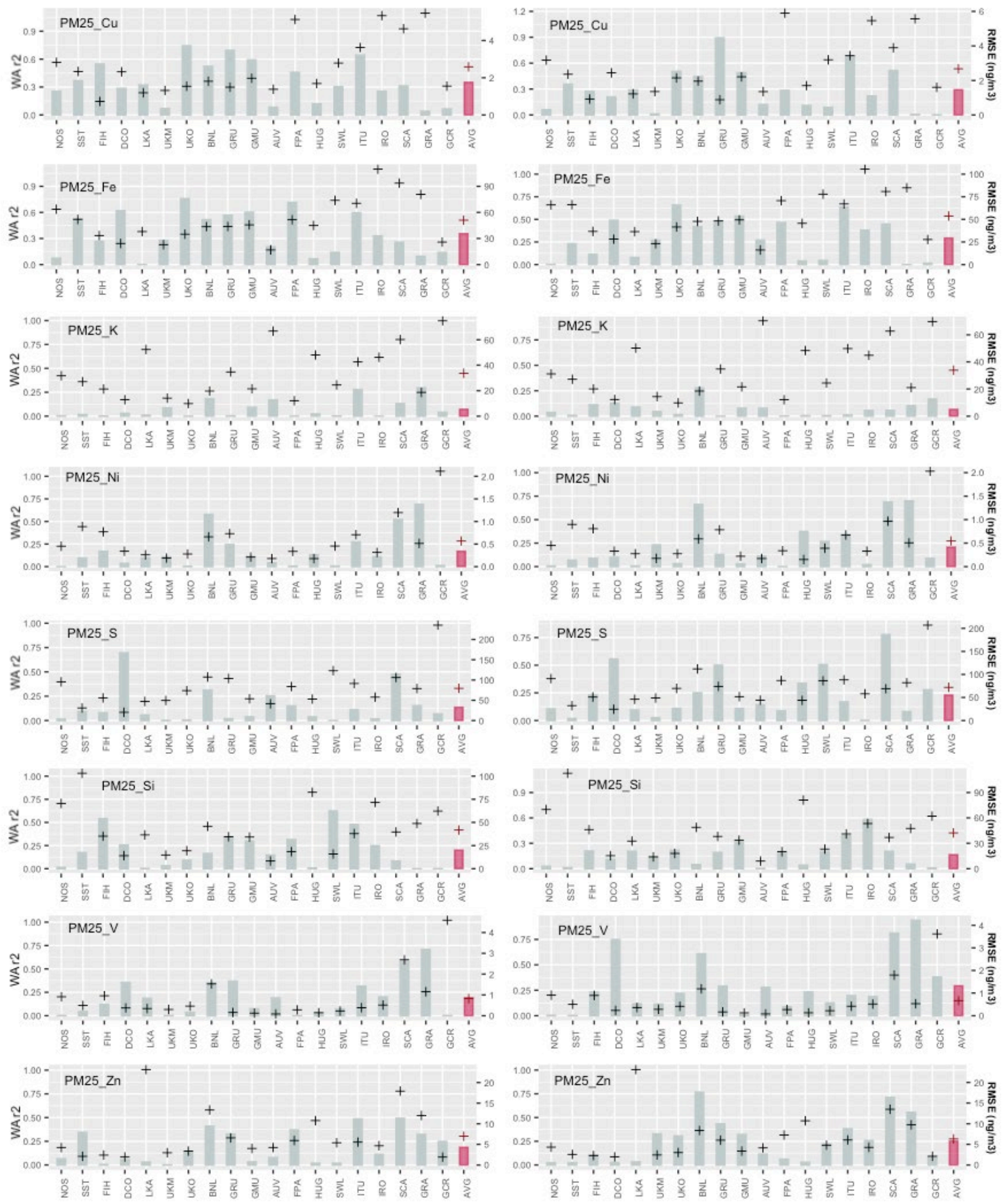

Study areas are shown from north to south. NOS: Oslo (Norway); SST: Stockholm County (Sweden); FIH: Helsinki/Turku (Finland); DCO: Copenhagen (Denmark); LKA: Kaunas (Lithuania); UKM: Manchester (United Kingdom); UKO: London/Oxford (United Kingdom); BNL: Netherlands/Belgium; GRU: Ruhr Area (Germany); GMU: Munich/Augsburg (Germany); AUV: Vorarlberg (Austria); FPA: Paris (France); HUG: Gyor (Hungary); SWL: Lugano (Switzerland); ITU: Turin (Italy); IRO: Rome (Italy); SCA: Catalunya (Spain); GRA: Athens (Greece); GCR: Heraklion (Greece); AVG = average. 
Figure S5. Maps of $\mathbf{P M}_{2.5}$ components

(a) Supervised Linear Regression models

(1) $\mathrm{PM}_{2.5} \mathrm{Cu}$

(2) $\mathrm{PM}_{2.5} \mathrm{Fe}$

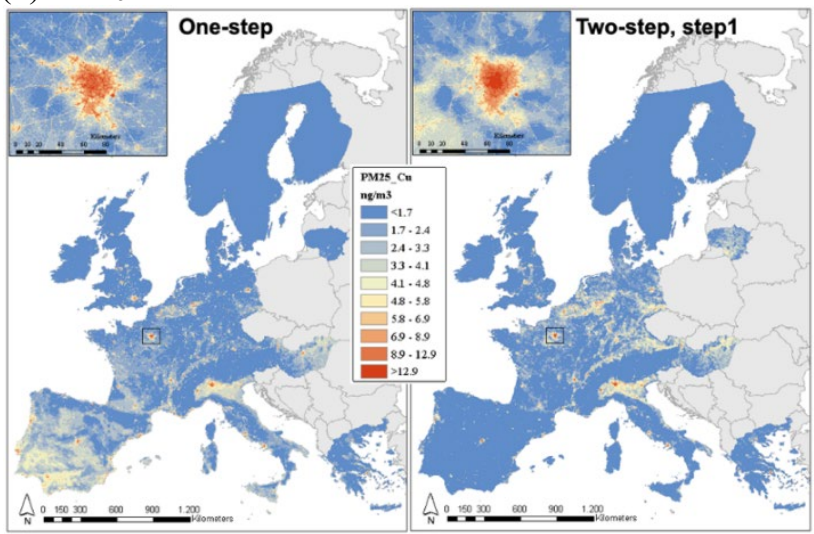

(3) $\mathrm{PM}_{2.5} \mathrm{~K}$

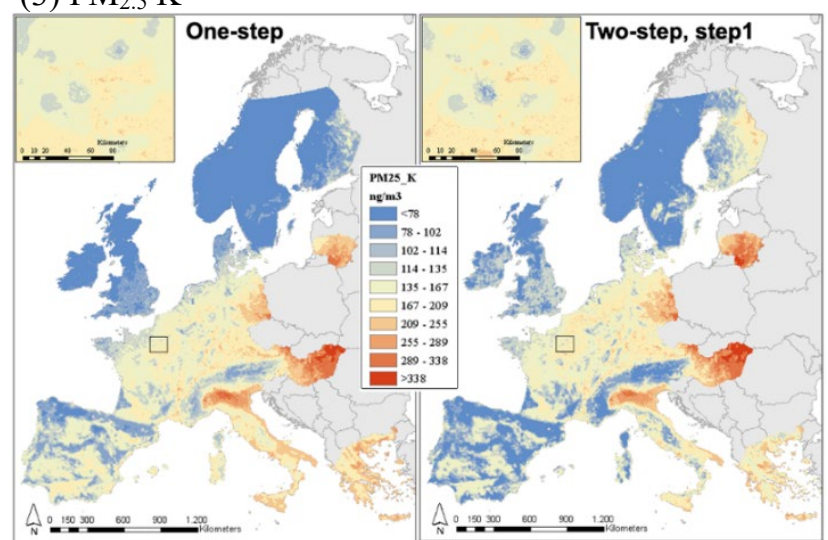

(5) $\mathrm{PM}_{2.5} \mathrm{~S}$

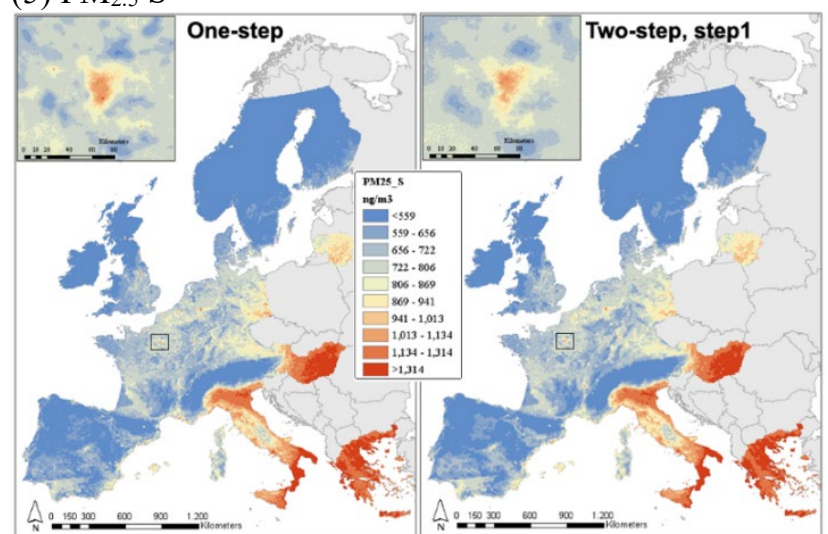

$\frac{\mathrm{PM}_{2.5} \mathrm{~V}}{\text { (7) }}$
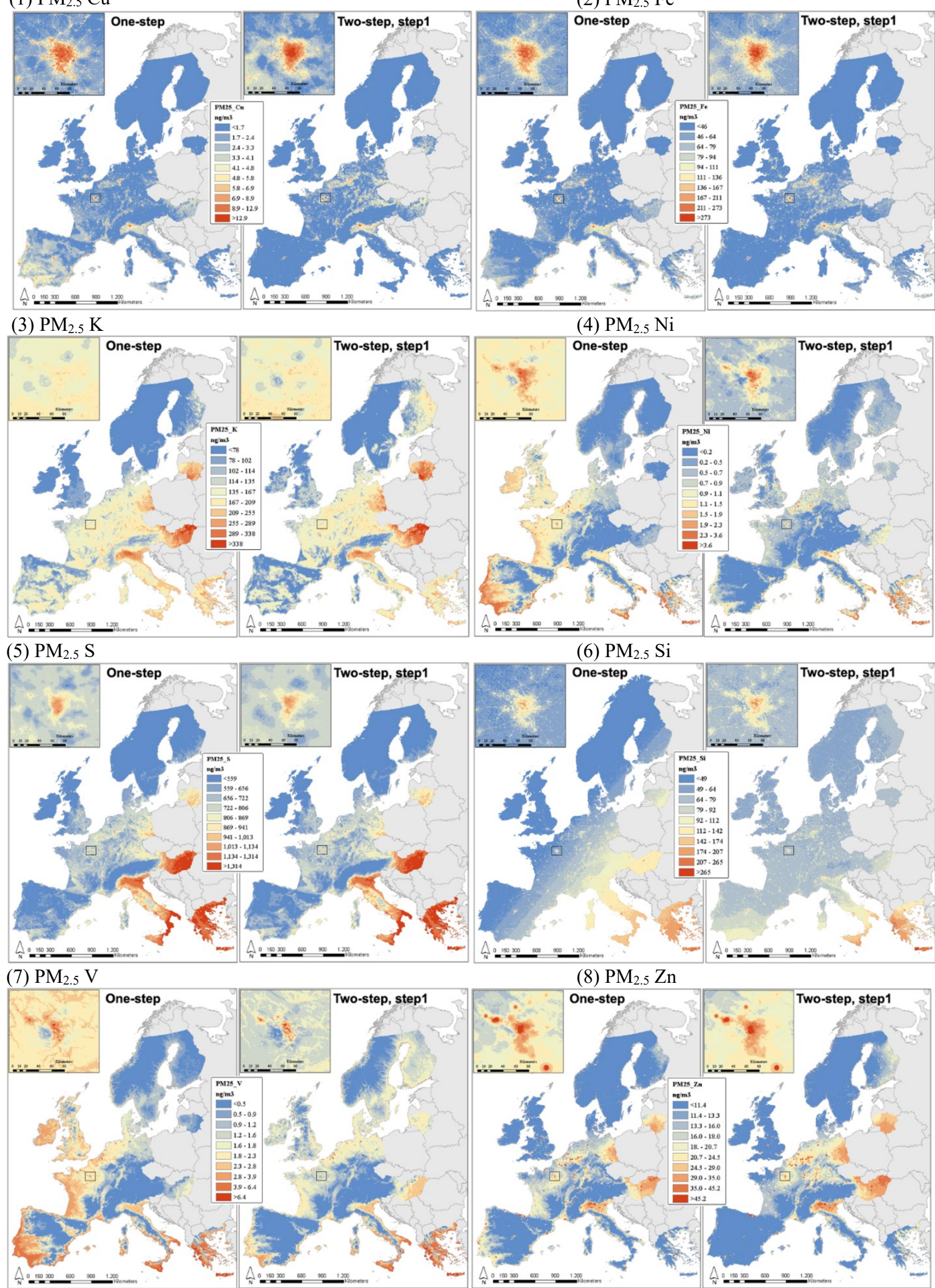

(4) $\mathrm{PM}_{2.5} \mathrm{Ni}$

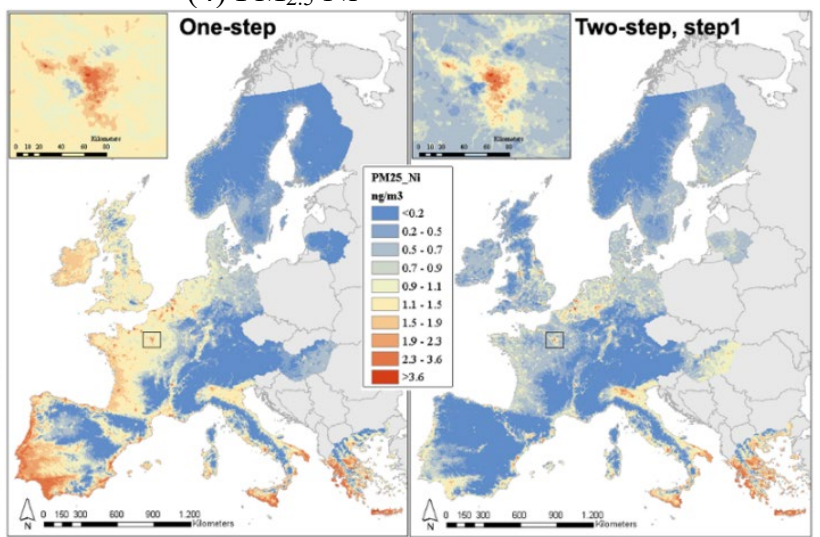

(6) $\mathrm{PM}_{2.5} \mathrm{Si}$

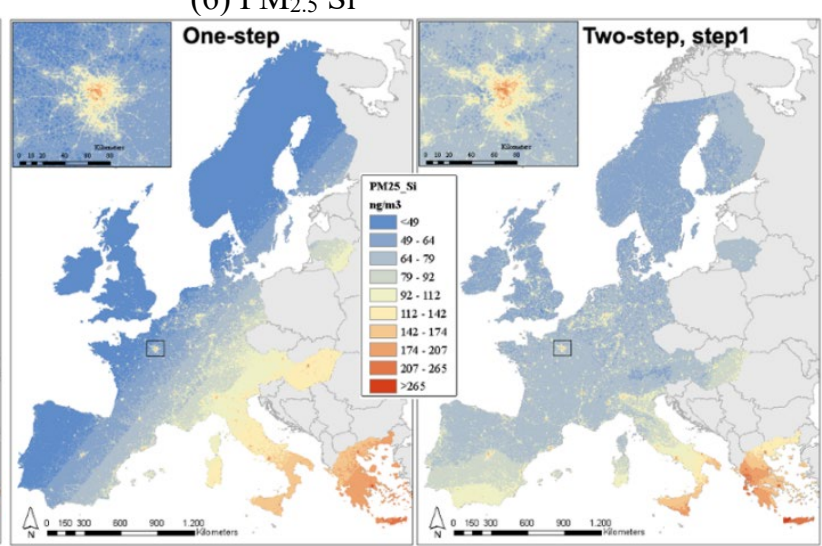

(8) $\mathrm{PM}_{2.5} \mathrm{Zn}$

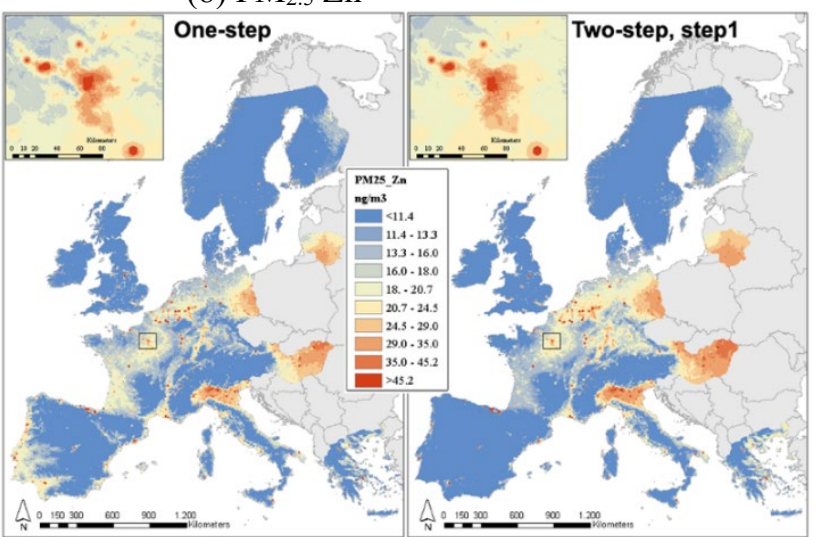


(b) Random Forest models

(1) $\mathrm{PM}_{2.5} \mathrm{Cu}$

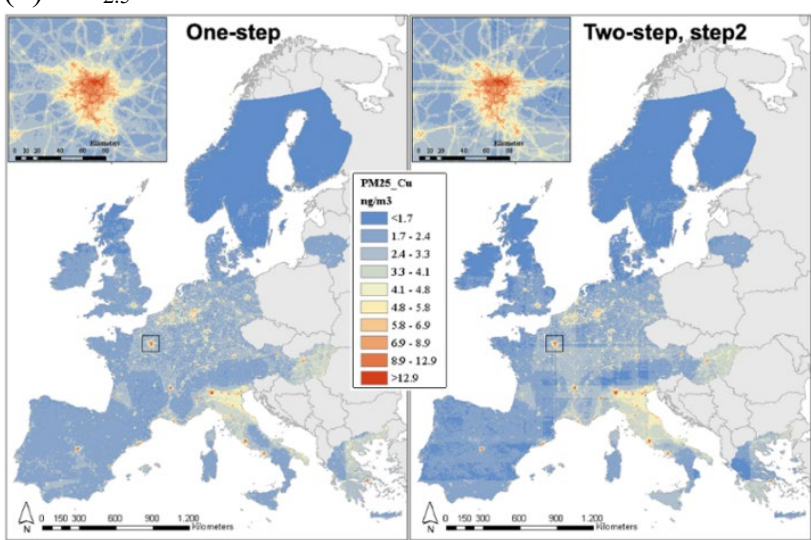

(3) $\mathrm{PM}_{2.5} \mathrm{~K}$

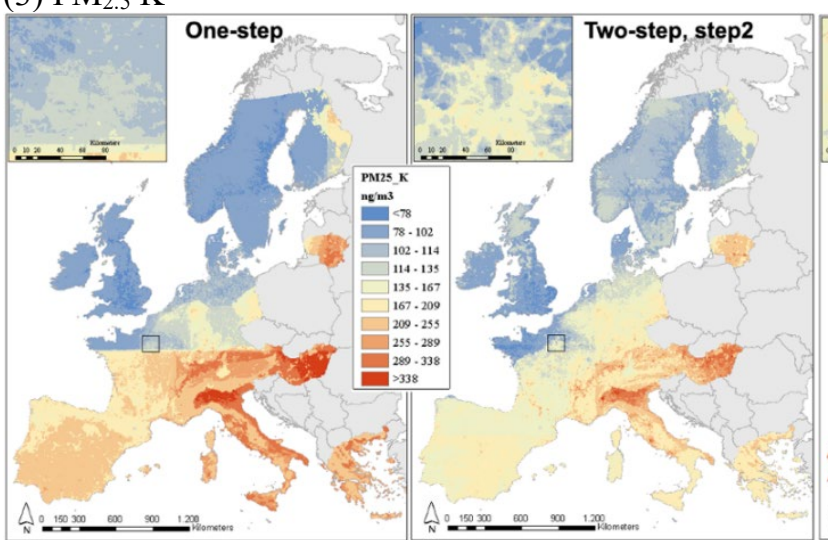

(5) $\mathrm{PM}_{2.5} \mathrm{~S}$

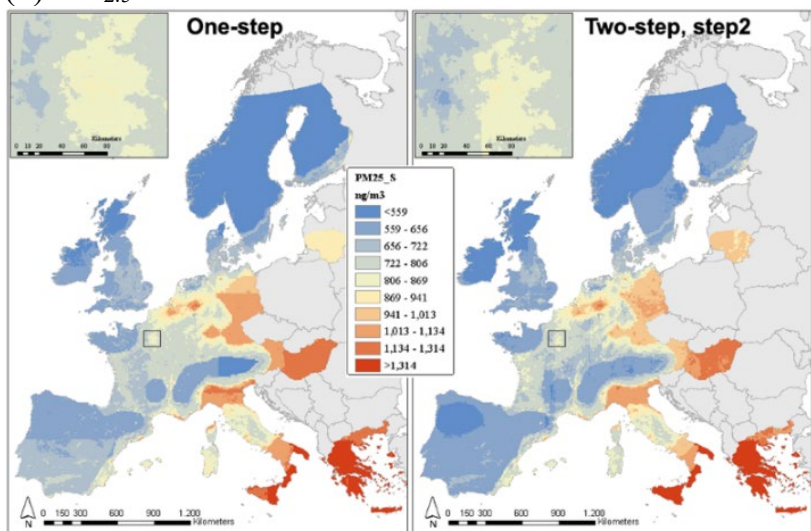

(7) $\mathrm{PM}_{2.5} \mathrm{~V}$
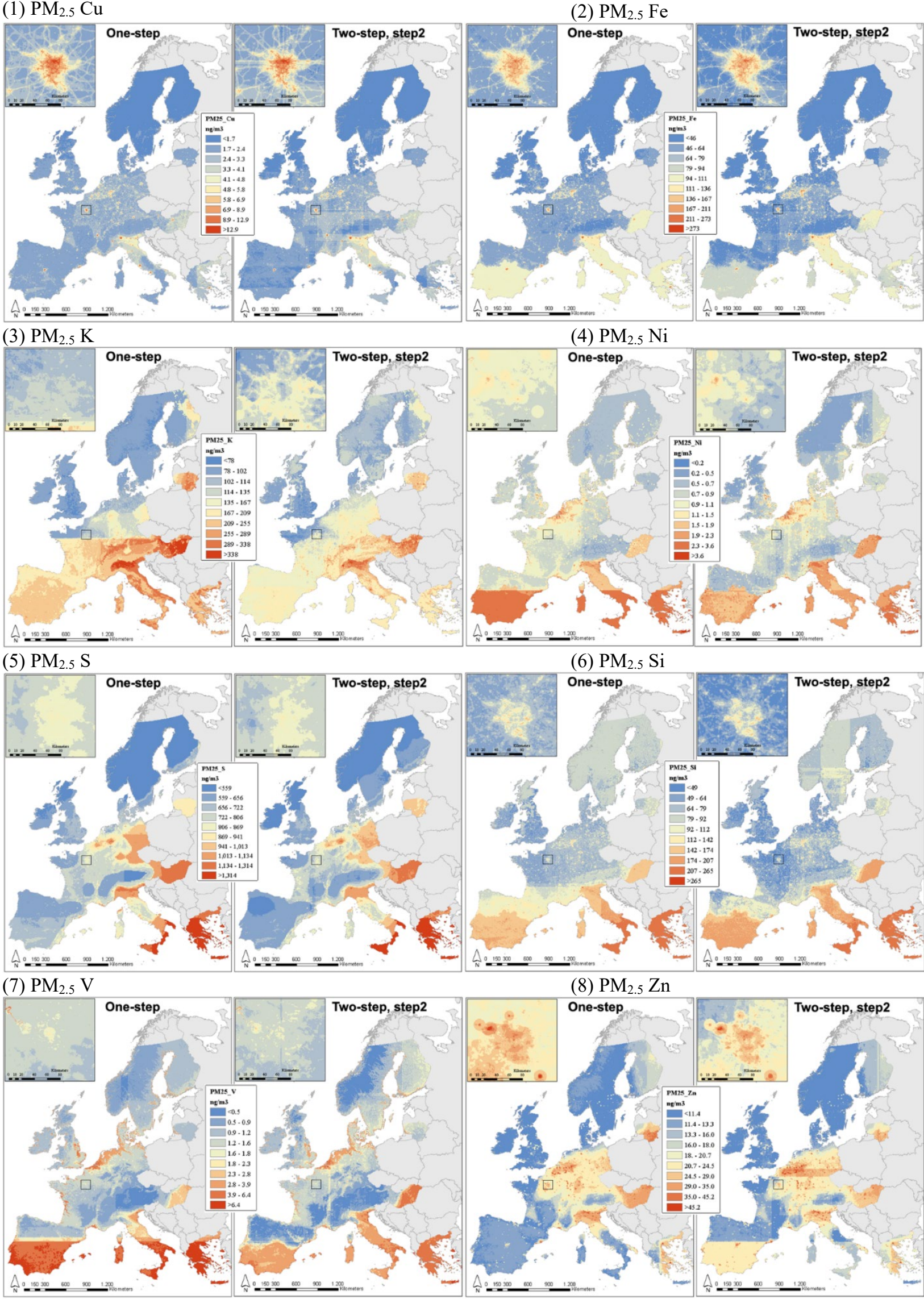

(4) $\mathrm{PM}_{2.5} \mathrm{Ni}$

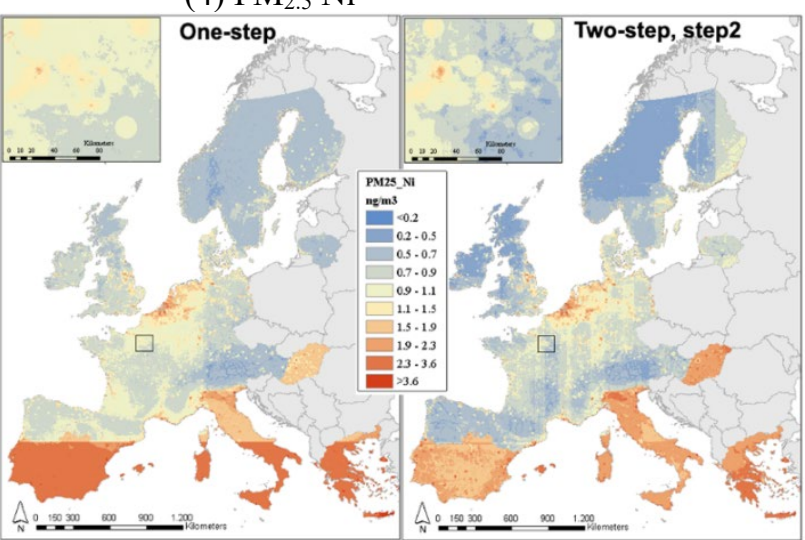

(6) $\mathrm{PM}_{2.5} \mathrm{Si}$

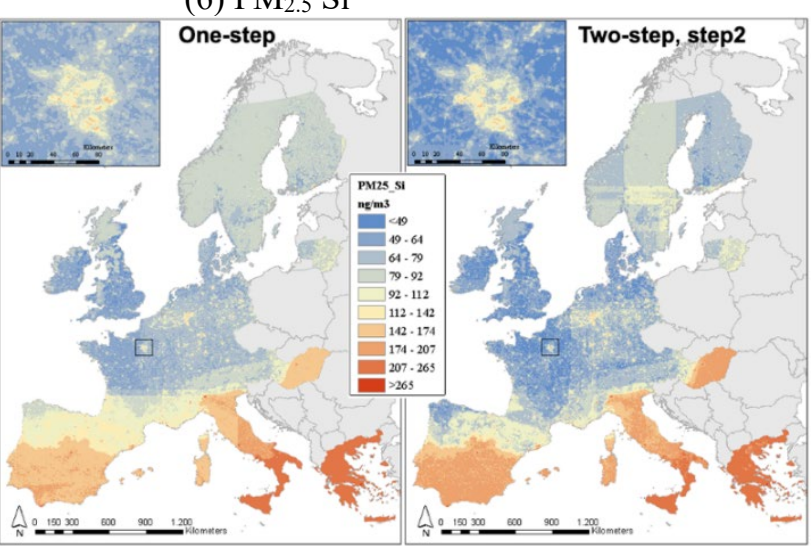

(8) $\mathrm{PM}_{2.5} \mathrm{Zn}$

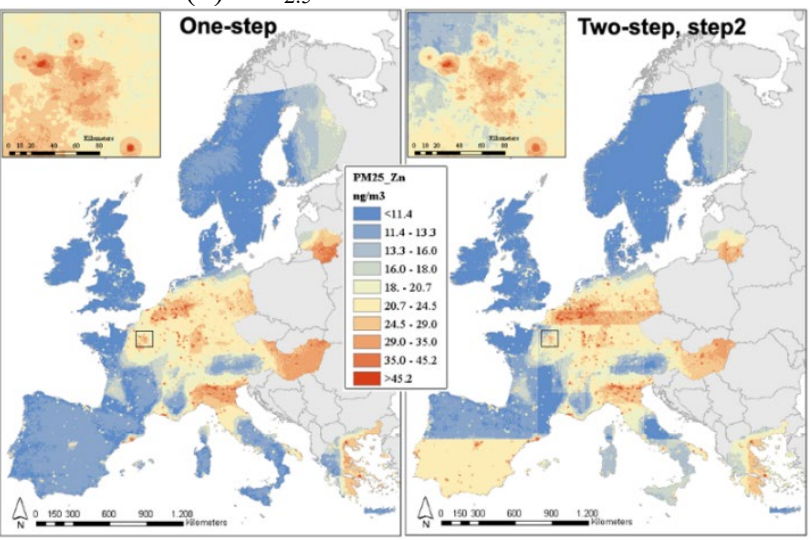




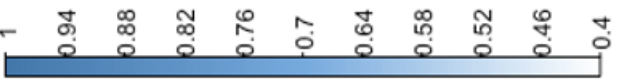
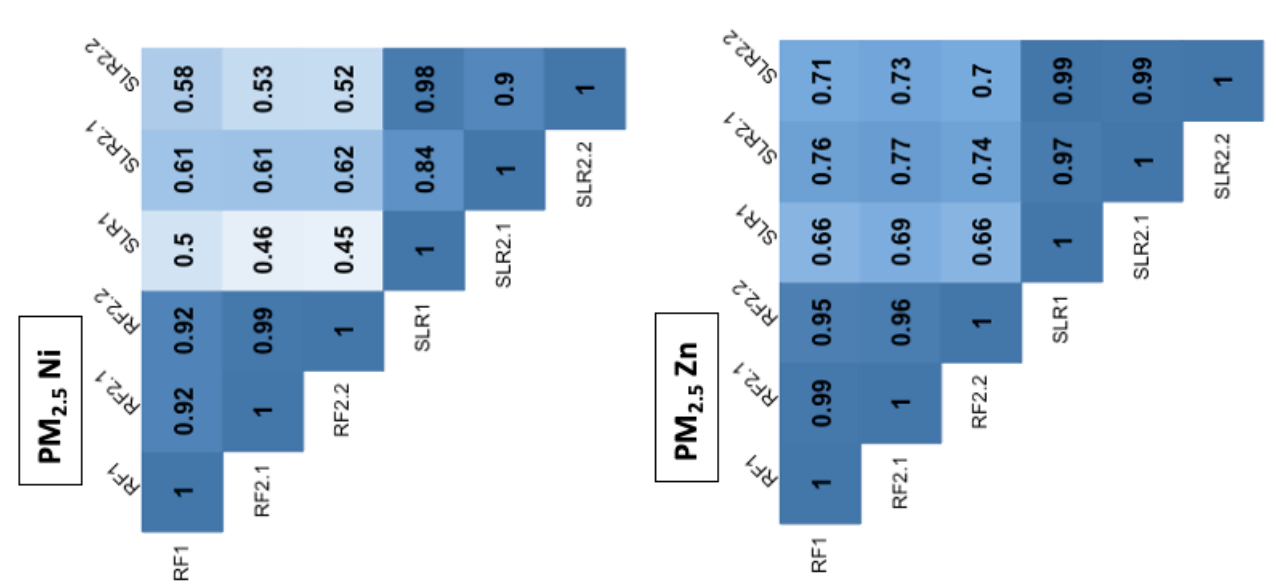

$\frac{\sqrt{2}}{\sqrt{2}}$
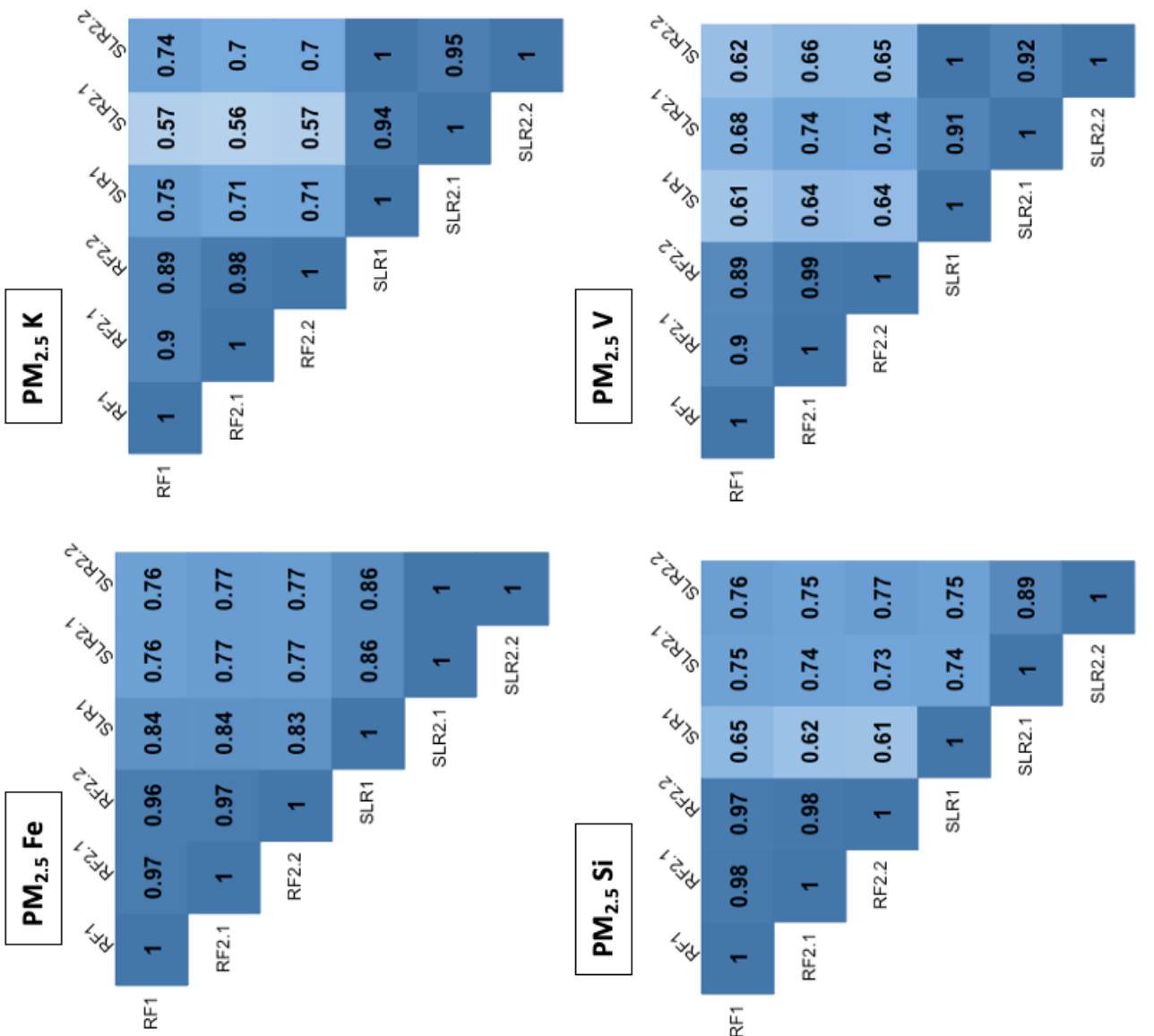

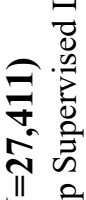

¿ 。ै

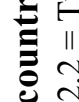
됭 党

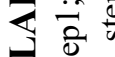
되 兽

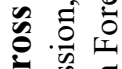
एँ 额 卷 晋寻 छ. 要 ज证

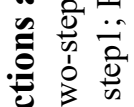
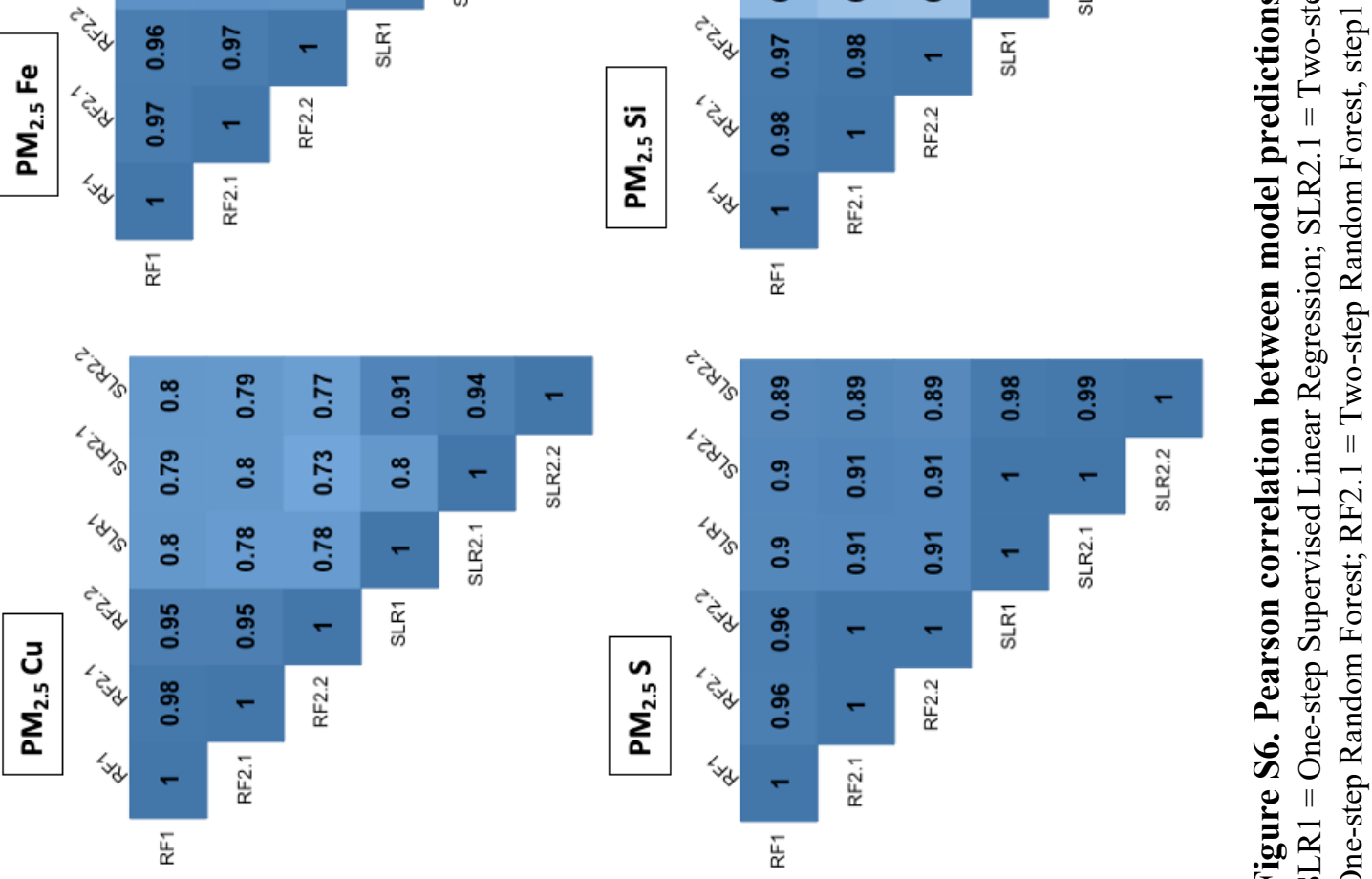
호 웜 छ छ

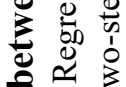
运

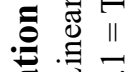

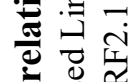
ठ․ 氞它 है के छ A के के 0 ฯ อ 11 के 홍목 


\section{Chapter}

\section{6}

\section{Long-Term Exposure to Fine Particle Elemental Components and Natural and Cause-Specific Mortality - A Pooled Analysis of Eight European Cohorts Within the ELAPSE Project}

Jie Chen, Sophia Rodopoulou, Kees de Hoogh, Maciej Strak, Zorana J. Andersen, Richard Atkinson, Mariska Bauwelinck, Tom Bellander, Jørgen Brandt, Giulia Cesaroni, Hans Concin, Daniela Fecht, Francesco Forastiere, John Gulliver, Ole Hertel, Barbara Hoffmann, Ulla Arthur Hvidtfeldt, Nicole A. H. Janssen, Karl-Heinz Jöckel, Jeanette Jørgensen, Klea Katsouyanni, Matthias Ketzel, Jochem O. Klompmaker, Anton Lager, Karin Leander, Shuo Liu, Petter Ljungman, Conor J. MacDonald, Patrik K.E. Magnusson, Amar Mehta, Gabriele Nagel, Bente Oftedal, Göran Pershagen, Annette Peters, Ole Raaschou-Nielsen, Matteo Renzi, Debora Rizzuto, Evangelia Samoli, Yvonne T. van der Schouw, Sara Schramm, Per Schwarze, Torben Sigsgaard, Mette Sørensen, Massimo Stafoggia, Anne Tjønneland, Danielle Vienneau, Gudrun Weinmayr, Kathrin Wolf, Bert Brunekreef* and Gerard Hoek*

*These authors contributed equally to this work

(Environmental Health Perspectives, 2021, 129(4): 047009) DOI: $10.1289 /$ EHP8368 


\begin{abstract}
Background Inconsistent associations between long-term exposure to particles with an aerodynamic diameter $\leq 2.5 \mu \mathrm{m}$ [fine particulate matter $\left(\mathrm{PM}_{2.5}\right)$ ] components and mortality have been reported, partly related to challenges in exposure assessment.
\end{abstract}

Objectives We investigated the associations between long-term exposure to $\mathrm{PM}_{2.5}$ elemental components and mortality in a large pooled European cohort; to compare health effects of $\mathrm{PM}_{2.5}$ components estimated with two exposure modeling approaches, namely supervised linear regression (SLR) and random forest (RF) algorithms.

Methods We pooled data from eight European cohorts with 323,782 participants, average age 49 y at baseline (1985-2005). Residential exposure to 2010 annual average concentration of eight $\mathrm{PM}_{2.5}$ components (copper, iron, potassium, nickel, sulfur, silicon, vanadium, and zinc) was estimated with Europe-wide SLR and RF models at a 100x100m scale. We applied Cox proportional hazards models to investigate the associations between components and natural and cause-specific mortality. In addition, two-pollutant analyses were conducted by adjusting each component for $\mathrm{PM}_{2.5}$ mass and nitrogen dioxide $\left(\mathrm{NO}_{2}\right)$ separately.

Results We observed 46,640 natural-cause deaths with 6,317,235 person-years and an average followup of $19.5 \mathrm{y}$. All SLR-modeled components were statistically significantly associated with naturalcause mortality in single-pollutant models with hazard ratios (HRs) from 1.05 to 1.27. Similar HRs were observed for RF-modeled copper, iron, potassium, sulfur, vanadium and zinc with wider confidence intervals (CIs). HRs for SLR-modeled nickel, sulfur, silicon, vanadium and zinc remained above unity and (almost) significant after adjustment for both $\mathrm{PM}_{2.5}$ and $\mathrm{NO}_{2}$. HRs only remained (almost) significant for RF-modeled potassium and vanadium in two-pollutant models. The HRs for vanadium were 1.03 (95\% CI: 1.02, 1.05) and 1.06 (95\% CI: 1.02, 1.10) for SLR- and RF-modeled exposures respectively per $2 \mathrm{ng} / \mathrm{m}^{3}$ adjusting for $\mathrm{PM}_{2.5}$ mass. Associations with cause-specific mortality were less consistent in two-pollutant models.

Conclusion Long-term exposure to vanadium in $\mathrm{PM}_{2.5}$ was most consistently associated with increased mortality. Associations for the other components were weaker for exposure modeled with RF than SLR in two-pollutant models. 


\section{Introduction}

The Global Burden of Disease (GBD 2015) study estimated that exposure to ambient particles with an aerodynamic diameter $\leq 2.5 \mu \mathrm{m}\left(\mathrm{PM}_{2.5}\right)$ was the fifth-ranking mortality risk factor, contributing to 4.2 million deaths per year (Cohen et al., 2017). $\mathrm{PM}_{2.5}$ is a mixture of a large number of components related to specific sources. Identifying which components of $\mathrm{PM}_{2.5}$ are main contributors to adverse health effects is important for targeted policy making. Although some studies have attempted to associate longterm exposure to specific $\mathrm{PM}_{2.5}$ components with mortality risks, the results are inconclusive. The California Teachers Study (Ostro et al., 2015) found an increased risk of ischemic heart disease (IHD) mortality in associations with exposure to nitrate, elemental carbon (EC), copper and secondary organics in $\mathrm{PM}_{2.5}$. The American Cancer Society (ACS) Cancer Prevention Study-II (CPS-II) suggested that long-term $\mathrm{PM}_{2.5}$ exposure from coal combustion and its key emission tracer elements (i.e., selenium and arsenic) were associated with increased IHD mortality risk, whereas exposure to silicon and potassium was not associated with mortality (Thurston et al., 2013; Thurston et al., 2016). In the Medicare population, the excess mortality risk associated with long-term $\mathrm{PM}_{2.5}$ exposure increased with relative concentration of EC, vanadium, copper, calcium and iron and decreased with nitrate, organic carbon and sulfate (Wang et al., 2017). The large European Study of Cohorts for Air Pollution Effects (ESCAPE) reported a robust relationship between natural-cause mortality and $\mathrm{PM}_{2.5}$ sulfur, and some evidence of associations with iron and copper in $\mathrm{PM}_{2.5}$ (Beelen et al., 2015). No statistically significant association with $\mathrm{PM}_{2.5}$ components was found for cardiovascular mortality in the ESCAPE study (Wang et al., 2014).

Long-term exposure assessment for particle components is more challenging than for $\mathrm{PM}_{2.5}$ mass because of limited regulatory routine monitoring (with the exception of nitrate, ammonium and sulfate) and less data on emission rates used as input to dispersion models (Holmes and Morawska, 2006). To date, the available epidemiological evidence used different exposure assessment methods, including direct monitoring (Ostro et al., 2011; Thurston et al., 2013; Thurston et al., 2016), chemical transport models at 4x4 km scale (Ostro et al., 2015) and fine spatial scale land use regression (LUR) models (Beelen et al., 2015; Wang et al., 2014). Different exposure assessment methods may lead to component-specific differences in exposure estimation error, potentially leading to bias (Adams et al., 2015). Studies have suggested that risk estimates of $\mathrm{PM}_{2.5}$ mass differed between exposure assessment methods (Jerrett et al., 2017; McGuinn et al., 2017). Studies comparing exposure assessment methods in their associations with health outcomes mainly focused on the comparison among direct monitoring, satellite products, dispersion/chemical transport models and LUR models. Recent developments in exposure assessment include combining different methods such as land-use or chemical transport modeling and monitoring data using a variety of approaches including linear regression and machine learning algorithms (Hoek, 2017). Comparisons have been made among exposure predictions developed with different algorithms in terms of prediction accuracy (Brokamp et al., 2017; Chen et al., 2019; Kerckhoffs et al., 2019). However, a simulation study suggested that improving the prediction accuracy of exposure models did not always improve the accuracy of health effect estimation, when bias is introduced into health effect estimation by the classical-like measurement error and the impact of Berkson-like measurement error on the health effect estimation error diminishes for large number of subjects (Szpiro et al., 2011). To our knowledge, no studies have compared exposure models developed with different algorithms regarding their relation with health outcomes.

The present study is part of the Effects of Low-level Air Pollution: a Study in Europe (ELAPSE). ELAPSE builds on the elemental composition, mortality and covariate data of the ESCAPE study (Beelen et al., 2014; Beelen et al., 2015; Wang et al., 2014). In ESCAPE each cohort was analyzed separately, whereas in ELAPSE respective ESCAPE cohorts were pooled to represent a contrast in lowlevel air pollution exposures. In addition, the follow-up data for mortality were extended from typically up to 2008 in ESCAPE to up to 2011-2017 in ELAPSE, which substantially increased the number of deaths and hence study power. Measurements for black carbon (BC) and elemental composition at 
individual ESCAPE study areas were pooled to develop Europe-wide exposure models covering combined study areas for application in the ELAPSE (Chen et al., 2020; De Hoogh et al., 2018). The combined ability to do pooled analyses, plus accounting for new insights in the robustness of LUR models related to the number of air pollution monitoring sites (Basagaña et al., 2012; Wang et al., 2012), strengthened the exposure assessment in ELAPSE. The Europe-wide models furthermore allowed better coverage of those ESCAPE cohorts in large study areas of which typically only a fraction was covered by dedicated monitoring campaigns (e.g., only Paris in the national French Etude Epidémiologique auprès de femmes de la Mutuelle Générale de l'Education Nationale (E3N) cohort) (de Hoogh et al., 2013; Tsai et al., 2015). The Europe-wide models for assessing 2010 annual average $\mathrm{PM}_{2.5}$ composition concentrations at a $100 \times 100 \mathrm{~m}$ scale were developed using two algorithms - the supervised linear regression (SLR) and the random forest (RF) algorithms, which is a machine learning algorithm (Chen et al., 2020). The RF models outperformed the SLR models at the Europe-wide level by $11-30 \%$ across components in hold-out-validation $R^{2}$, whereas the two models performed similarly in explaining variability within individual ESCAPE study areas. Despite the similar within-area performance, the exposure predictions at random sites derived from SLR and RF models correlated only moderately at the national level (the average of correlation coefficients at 11 ELAPSE countries range from 0.41 to 0.77 across components). We refer to correlations $<0.4$ as low, $0.4-0.7$ as moderate, and $>0.7$ as high. Although the focus of ELAPSE is on low-level air pollution defined as below the current air quality guidelines and standards, low-level is difficult to define for PM elemental composition because there are currently no guidelines/standards for PM elemental composition.

We previously observed significantly positive associations between $\mathrm{PM}_{2.5}$ and natural and causespecific mortality using the same pooled cohort in the framework of ELAPSE (Strak et al., submitted). Hazard ratio for natural cause mortality was $1.13(95 \%$ CI: $1.11,1.16)$ per $5 \mu \mathrm{g} / \mathrm{m}^{3}$ increase in $\mathrm{PM}_{2.5}$. The first aim of this study was to evaluate whether specific components of $\mathrm{PM}_{2.5}$ were associated with mortality. The second aim was to compare the health effects of $\mathrm{PM}_{2.5}$ components estimated with two different exposure modeling approaches, namely, the SLR and RF algorithms.

\section{Methods}

\subsection{Study populations}

The ELAPSE pooled cohort contains eight cohorts across six European countries able to participate in data pooling, areas with low-level air pollution exposure, and relatively recent recruitment dates (Table 1 and Figure S1). The cohorts are the following: the Cardiovascular Effects of Air Pollution and Noise in Stockholm (CEANS) cohort in Sweden, which was constructed from four sub-cohorts: the Stockholm Diabetes Prevention Program (SDPP)(Eriksson et al., 2008), the Stockholm Cohort of 60-Year-Olds (SIXTY) (Wändell et al., 2007), the Stockholm Screening Across the Lifespan Twin study (SALT) (Magnusson et al., 2013) and the Swedish National Study on Aging and Care in Kungsholmen (SNACK) (Lagergren et al., 2004); the Diet, Cancer and Health cohort (DCH) (Tjønneland et al., 2007) in Denmark; the Danish Nurse Cohort (DNC) (Hundrup et al., 2012) in Denmark, consisting at baseline of two surveys conducted in 1993 and 1999; the European Prospective Investigation into Cancer and Nutrition-Netherlands (EPIC-NL) cohort in the Netherlands, including the Monitoring Project on Risk Factors and Chronic Diseases in the Netherlands (MORGEN) and Prospect (Beulens et al., 2010); the Heinz Nixdorf Recall study (HNR) in Germany (Schmermund et al., 2002); the Etude Epidémiologique auprès de femmes de la Mutuelle Générale de l'Education Nationale (E3N) in France (Clavel-Chapelon and Group, 2015); the Cooperative Health Research in the Region of Augsburg (KORA) in Germany, consisting at baseline of two cross-sectional population-representative surveys conducted in 1994-1995 (S3) and 1999-2001 (S4); and the Vorarlberg Health Monitoring and Prevention Programme (VHM\&PP) in Austria (Ulmer et al., 2007). The study areas of most cohorts constituted a large city and its surrounding areas. Some cohorts, such as the French E3N cohort and the Danish DNC cohort, covered large regions of the country. All included cohort studies were approved by the medical ethics committees in their respective countries. Detailed information of each individual cohort is provided in 
Tables S1-S8. A lot of variable harmonization was done in the ESCAPE collaboration which formed the basis of the present study (Beelen et al., 2014; Cesaroni et al., 2014; Raaschou-Nielsen et al., 2013; Stafoggia et al., 2014). In ELAPSE, a joint codebook with exact definitions of variables were prepared, starting from the ESCAPE codebook. We asked each cohort to transfer the data to Utrecht University and checked the definition of variables according to the joint codebook. The variables in our confounder models did not require much harmonization. In the E3N cohort, smoking intensity was in classes. We assigned the midpoint as the actual value. Area-level socio-economic status (SES) was newly collected in ELAPSE. We specified the desired area-level SES variables with respect to spatial scale and variables before pooling the cohort data. The detailed harmonization process for area-level SES variables is described in the Supplemental Material in the section "Area-level socio-economic status (SES) variable harmonization".

\subsection{Air pollution exposure assessment}

Eight components were a priori selected in ESCAPE to represent major pollution sources: copper $(\mathrm{Cu})$, iron $(\mathrm{Fe})$ and zinc $(\mathrm{Zn})$ representing non-tailpipe traffic emissions; sulfur $(\mathrm{S})$ representing long-range transport of secondary inorganic aerosols; nickel $(\mathrm{Ni})$ and vanadium $(\mathrm{V})$ representing mixed oil burning/industry; silicon $(\mathrm{Si})$ representing crustal material; and potassium $(\mathrm{K})$ representing biomass burning (de Hoogh et al., 2013; Tsai et al., 2015). We assessed exposure to these eight elements in $\mathrm{PM}_{2.5}$ at the participants' baseline residential addresses using Europe-wide LUR models developed with two algorithms. The models have been described in detail elsewhere (Chen et al., 2020). Briefly, we estimated 2010 annual mean concentrations of $\mathrm{PM}_{2.5}$ elemental composition based on the standardized ESCAPE monitoring data. We offered large-scale satellite-model and chemical transport model estimates of components as predictors to represent background concentrations and land use, traffic, population and industrial point source data to model local spatial variability. We applied the supervised linear regression (SLR) (De Hoogh et al., 2018) and the random forest (RF) algorithms (Chen et al., 2019 ) to develop models for each component. The models explained a moderate to large fraction of the measured concentration variation at the European scale, ranging from $41 \%$ to $90 \%$ across components. Model performance evaluated by 5-fold hold-out validation reported in Chen et al. (2020) was extracted and is shown in Table S9. The RF models consistently outperformed the SLR models in explaining overall variability, including both between- and within- study area variability. The models explained within-area variability less well, with similar performance for SLR and RF models. The SLR and RF model predictions correlated moderately at the national level (the averaged correlation coefficients at six countries covered by the ELAPSE pooled cohort range from 0.43 to 0.78 across the components).

Exposure to 2010 annual mean concentration of $\mathrm{PM}_{2.5}$ mass and $\mathrm{NO}_{2}$ was assessed by Europe-wide LUR models developed previously (De Hoogh et al., 2018). The models were developed based on the European Environmental Agency AirBase routine monitoring data, with satellite-derived and chemical transport model air pollutants estimates, and land-use, traffic and population data as predictors. The $\mathrm{PM}_{2.5}$ model explained $72 \%$ of measured spatial variation in the annual average concentration across Europe, whereas the $\mathrm{NO}_{2}$ model explained $59 \%$.

We applied the exposure models to create $100 \times 100 \mathrm{~m}$ grids of the predicted concentrations of the pollutants covering the entire study area, and transferred the relevant parts to the participating centers for exposure assignment. Careful procedures were applied to ensure that correct exposure assignment occurred, including clarification of the correct coordinate system. Checking involved exposure assignment to a set of randomly selected coordinates by the participating centers and the coordinating center independently and by comparison of the exposure assignment. After assignment, anonymized data were returned to Utrecht University for checking and pooling.

We selected 2010 as the primary year of exposure modeling because 2009-2010 was the period of ESCAPE monitoring that we used to develop PM2.5 composition models (Chen et al., 2020). For PM2.5, this was the earliest year of a sufficiently wide coverage of PM2.5 monitoring across Europe (De Hoogh et al., 2018). For consistency, we used the year 2010 for NO2 as well. We assumed that the spatial 
variability of the relevant pollution concentrations remained reasonably stable to the baseline period (year 1985-2005). We also assumed that, for a mortality outcome, the exposure in the past few years was the most relevant exposure. We considered very high and negative predicted concentrations of elemental composition unrealistic. Truncations were performed to deal with unrealistic predicted concentrations (Chen et al., 2020). We defined a maximum predicted concentration for each component calculated by fitting the SLR model with the maximum predictor values at ESCAPE monitoring sites for positive slopes (or the minimum predictor values for negative slopes). We considered predicted concentrations larger than the maximum modeled values as unrealistic predictions and truncated them to the maximum predicted concentrations. The high unrealistic predictions were mostly related to a close distance to industrial sources. Negative predictions were set to zero. Truncation was performed in the main model population for SLR-modeled exposure: $11.3 \%$ for $\mathrm{Cu}, 0.5 \%$ for $\mathrm{Fe}, 11.6 \%$ for $\mathrm{Ni}, 14.3 \%$ for $\mathrm{V}$, and $2.6 \%$ for $\mathrm{Zn}$ (Table S10). The truncation was mostly performed for predictions below zero and mostly located in the North-European cohorts (i.e., CEANS, DNC) and KORA and VHM\&PP. The high truncation frequency in some cohorts indicates that these cohorts did not contribute much information to the analyses. Only 2,24 , and 240 observations ( $\leq 0.1 \%$ of all observations) were truncated because of high SLR predictions for $\mathrm{Cu}, \mathrm{Ni}$, and $\mathrm{Zn}$, respectively. No truncation was needed for RFmodeled exposure because the RF predictions were within the reasonable range, probably due to the flexible nature of the RF algorithm.

\subsection{Mortality outcome definition}

Identification of outcomes was based upon linkage to mortality registries. Natural mortality was defined based on the underlying cause of death recorded on death certificates according to the International Classification of Diseases, Ninth Revision (ICD-9) codes 001-779 and the International Statistical Classification of Diseases and Related Health Problems, Tenth Revision (ICD-10) codes A00-R99. We further defined mortality from cardiovascular disease (ICD-9 codes 400-440, ICD-10 codes I10-I70), respiratory disease (ICD-9 codes 460-519, ICD-10 codes J00-J99) and lung cancer (ICD-9 code 162, ICD-10 code C34).

\subsection{Statistical analyses}

\subsubsection{Main analyses}

To estimate hazard ratios (HRs) and 95\% confidence interval (CIs) for associations of the $\mathrm{PM}_{2.5}$ component exposure with natural and cause-specific mortality, we applied Cox proportional hazards models following the general ELAPSE analytical framework (Hvidtfeldt et al., 2021b; Liu et al., 2021a; Liu et al., 2021b; Samoli et al., 2021). We used strata for sub-cohorts contributing to the pooled cohort to account for differences in baseline hazard between the sub-cohorts unexplained by the available covariates. We used strata because the assumption of proportional hazards did not hold with respect to sub-cohort. Strata had a substantially better model performance compared to alternative specifications such as sub-cohort indicators. The decision to account for between cohort heterogeneity using strata implies that we mostly evaluated within-cohort exposure contrasts. Each $\mathrm{PM}_{2.5}$ component was included as a linear function in the Cox models as a reasonable summary of the association, allowing comparison with previous studies. HRs were calculated with a fixed increment for each $\mathrm{PM}_{2.5}$ component following the increments selected in previous publications from ESCAPE and ELAPSE (Beelen et al., 2015; Hvidtfeldt et al., 2021a): $\mathrm{PM}_{2.5} \mathrm{Cu}-5 \mathrm{ng} / \mathrm{m}^{3}, \mathrm{PM}_{2.5} \mathrm{Fe}-100 \mathrm{ng} / \mathrm{m}^{3}, \mathrm{PM}_{2.5} \mathrm{~K}-50 \mathrm{ng} / \mathrm{m}^{3}, \mathrm{PM}_{2.5} \mathrm{Ni}-1$ $\mathrm{ng} / \mathrm{m}^{3}, \mathrm{PM}_{2.5} \mathrm{~S}-200 \mathrm{ng} / \mathrm{m}^{3}, \mathrm{PM}_{2.5} \mathrm{Si}-100 \mathrm{ng} / \mathrm{m}^{3}, \mathrm{PM}_{2.5} \mathrm{~V}-2 \mathrm{ng} / \mathrm{m}^{3}, \mathrm{PM}_{2.5} \mathrm{Zn}-10 \mathrm{ng} / \mathrm{m}^{3}$. Censoring occurred at the time of the event of interest, death from other causes, emigration, loss to follow-up for other reasons, or at the end of follow-up, whichever came first. We a priori specified three confounder models with increasing control for individual and area-level covariates: Model 1 included only age (as the time scale), sub-cohort (as strata), sex (as strata), and year of enrollment; Model 2 added individuallevel covariates including marital status (married/cohabiting, divorced/separated, single, widowed), smoking status (never, former, current), smoking duration (years of smoking) for current smokers, smoking intensity (cigarettes/day) for current smokers, squared smoking intensity, body mass index 
(BMI) categories $\left(<18.5,18.5-24.9,25-29.9\right.$, and $\left.>30 \mathrm{~kg} / \mathrm{m}^{2}\right)$, and employment status (employed vs. unemployed); Model 3 further adjusted for neighborhood-level mean income in 2001. We determined the confounder models 2 and 3 by balancing the need to adjust for a comprehensive set of confounders and the availability in a large number of cohorts. BMI was included as a categorical variable because there is evidence of nonlinear relationships between continuous BMI and mortality (Di Angelantonio et al., 2016). We considered model 3 as the main model. Participants with missing exposure or incomplete information on model 3 covariates were excluded from all main analyses to ensure comparability between the model results.

Two-pollutant models were conducted with the main model 3 adjusting each component for $\mathrm{PM}_{2.5}$ mass and $\mathrm{NO}_{2}$ separately. We adjusted for $\mathrm{PM}_{2.5}$ mass to investigate whether the association with individual components reflecting specific sources remained after adjustment for generic $\mathrm{PM}_{2.5}$ mass for which we have strong evidence of associations (Beelen et al., 2014). We adjusted for $\mathrm{NO}_{2}$ in an attempt to disentangle the individual component effect from traffic exhaust emission for which $\mathrm{NO}_{2}$ is used as a marker. Adjustment for $\mathrm{NO}_{2}$ is especially important when assessing associations with the traffic nonexhaust components $\mathrm{Cu}, \mathrm{Fe}$ and $\mathrm{Zn}$. However, two-pollutant models can be difficult to interpret when the two pollutants reflect the same source or are strongly correlated. We did not model combinations of $\mathrm{PM}_{2.5}$ components in two-pollutant models because many were highly correlated (Figure S2) and we preferred to limit the complexity of analyses. The $\mathrm{PM}_{2.5}$ mass and $\mathrm{NO}_{2}$ estimates used in the twopollutant models were developed with the SLR algorithm (De Hoogh et al., 2018). We previously documented that, for $\mathrm{PM}_{2.5}$ mass and $\mathrm{NO}_{2}$ separately, SLR and RF models had similar performance, and that SLR- and RF-modeled exposure at external validation sites were highly correlated $\left(\mathrm{PM}_{2.5}\right.$ mass: Pearson $\mathrm{r}=0.89 ; \mathrm{NO}_{2}: \mathrm{r}=0.93$ ) (Chen et al., 2019). Consequently, only the SLR-modeled $\mathrm{PM}_{2.5}$ and $\mathrm{NO}_{2}$ exposures were linked to the individual cohorts.

We assessed the shape of the concentration-response functions (CRFs) for $\mathrm{PM}_{2.5}$ components and natural-cause mortality with natural cubic splines with 3 degrees of freedom. The CRFs can be difficult to interpret when there is limited variability in exposure contrasts.

In our interpretations, we attached more importance to two-pollutant models than single-pollutant models, acknowledging the difficulties in interpreting two-pollutant models. Given the similar performance of the SLR and RF model in explaining within-area variation (Table S9), and the fact that our analyses exploited primarily within-cohort exposure contrasts, we interpreted the two exposure methods equally. Therefore, we considered it more convincing when consistent associations between specific $\mathrm{PM}_{2.5}$ components and mortality were observed by applying two different exposure methods.

\subsubsection{Sensitivity analyses}

To evaluate the potential bias introduced by excluding participants with missing information on Model 3 covariates, we fitted Model 1 and Model 2 with participants with complete information on model 1 and model 2 covariates respectively.

To assess the sensitivity of our findings to using the 2010 exposures, we restricted analyses to followup periods starting from 2000, 2005 and 2008, with successively less temporal misalignment of the exposure model at the expense of shorter follow-up and fewer deaths.

To address potential residual confounding by socio-economic status (SES) factors, we further adjusted for individual level education, occupational status and additional neighborhood-level SES variables in cohorts that had such information.

All sensitivity analyses were performed for natural-cause mortality only.

All analyses were performed in R (version 3.4.0), using the packages survival, coxme, Matrix, foreach, glmnet, multcomp, survey, splines, Hmisc, mfp, VIM, ggplot2, frailtySurv, survsim, eha and stamod. Statistical significance was based on a $95 \%$ confidence interval of effect estimate, not including unity. 


\section{Results}

\subsection{Characteristics of the study population}

The total study population in the main model 3 (the most adjusted model) consisted of 323,782 subjects, contributing 6,317,235 person-years at risk. Most of the cohorts started in the mid-1990s with followup until 2011-2015. Fifteen percent of the total population was excluded from all main analyses owing to missing exposure $(0.5 \%)$, individual-level covariates $(12.7 \%)$ or neighborhood-level mean income $(1.8 \%)$. The excluded population was slightly younger (baseline age $46.4 \pm 14.7$ ) than the model 3 population (baseline age $48.7 \pm 13.4$ ). The proportion of females in the excluded population (64\%) was slightly lower than in the model 3 population (66\%). Table 1 and Tables S1 to S8 show the baseline characteristics of the participants in the individual sub-cohorts. The sub-cohorts differed in the number of participants, average years of follow-up, mean baseline age, percentage of female participants, lifestyle factors and neighborhood-level income, supporting the analysis accounting for difference in baseline hazards between sub-cohorts.

\subsection{Exposure distribution and correlations}

For $\mathrm{Cu}, \mathrm{Fe}, \mathrm{K}, \mathrm{S}$ and $\mathrm{Zn}$, concentrations were lower in the North European cohorts (i.e., CEANS, DCH and DNC; Figure S1) than in the other cohorts (Figure 1 and Table S11). The within-cohort contrast was substantial for $\mathrm{Cu}, \mathrm{Fe}$ and $\mathrm{Si}$ and limited for $\mathrm{K}, \mathrm{Ni}, \mathrm{S}, \mathrm{V}$ and $\mathrm{Zn}$ for both SLR- and RF-modeled exposures. Exposure distributions for the pooled cohort were similar for SLR- and RF-modeled estimates, although for most components the variability was smaller for RF. Our selected fixed increment reflected a larger exposure contrast than the interquartile ranges for most elements. For individual cohorts, large differences between the two algorithms were found, for example, $\mathrm{S}$ in the HNR study.

Correlations between exposure estimates derived from SLR and RF models were high for $\mathrm{Cu}$ and $\mathrm{Fe}$ (average within-cohort Spearman $\mathrm{r}=0.81$ for $\mathrm{Cu}, \mathrm{r}=0.84$ for $\mathrm{Fe}$ ) (Table 2). Correlations between SLRand RF-modeled exposure were moderate for $\mathrm{S}, \mathrm{Si}, \mathrm{Zn}$ and low for $\mathrm{K}, \mathrm{Ni}, \mathrm{V}$, with large variation between cohorts. We focused on within-cohort correlations because the epidemiological analysis exploited mostly within-cohort exposure contrast.

Correlations of composition with $\mathrm{PM}_{2.5}$ mass were mostly low to moderate (average of cohort-specific Spearman $r$ ranging from 0.13 to 0.49 ) for both SLR- and RF-modeled exposures (Table S12). Correlations with $\mathrm{NO}_{2}$ were mostly high for $\mathrm{Cu}$ and $\mathrm{Fe}$ (average of cohort-specific Spearman $r>0.7$ ) for both methods (Table S13). Correlations with $\mathrm{PM}_{2.5}$ mass and/or $\mathrm{NO}_{2}$ differed substantially in magnitude between cohorts, reflecting differences in study area size and the presence of major sources. Average of cohort-specific correlations between $\mathrm{Cu}$ and $\mathrm{Fe}$ were high, whereas both $\mathrm{Cu}$ and $\mathrm{Fe}$ were moderately correlated with Zn (Figure S2). Correlation between Ni and V modeled with the same algorithm was moderate, whereas the correlation was low when $\mathrm{Ni}$ and $\mathrm{V}$ were modeled with different algorithms. 


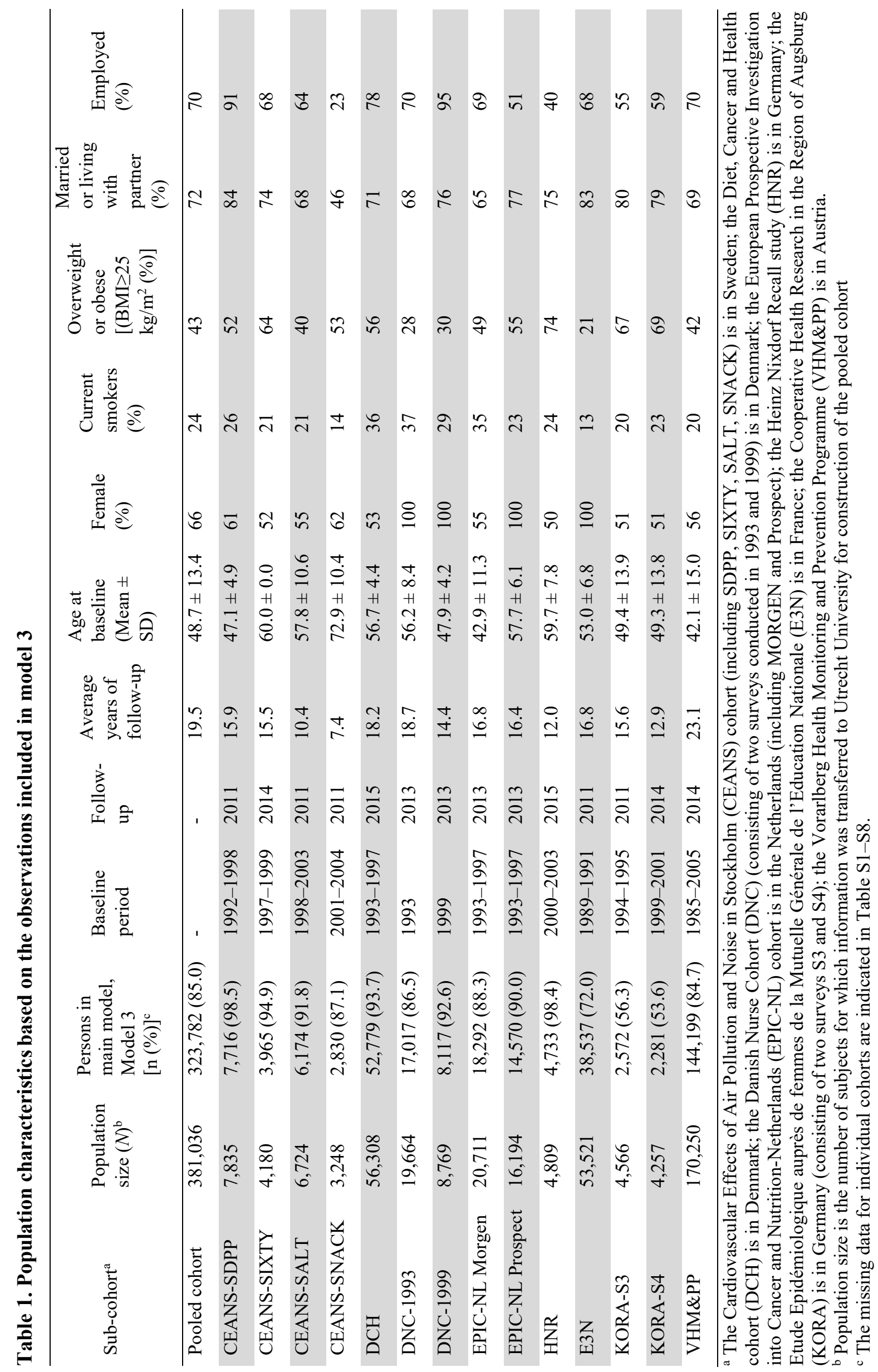


Chapter 6
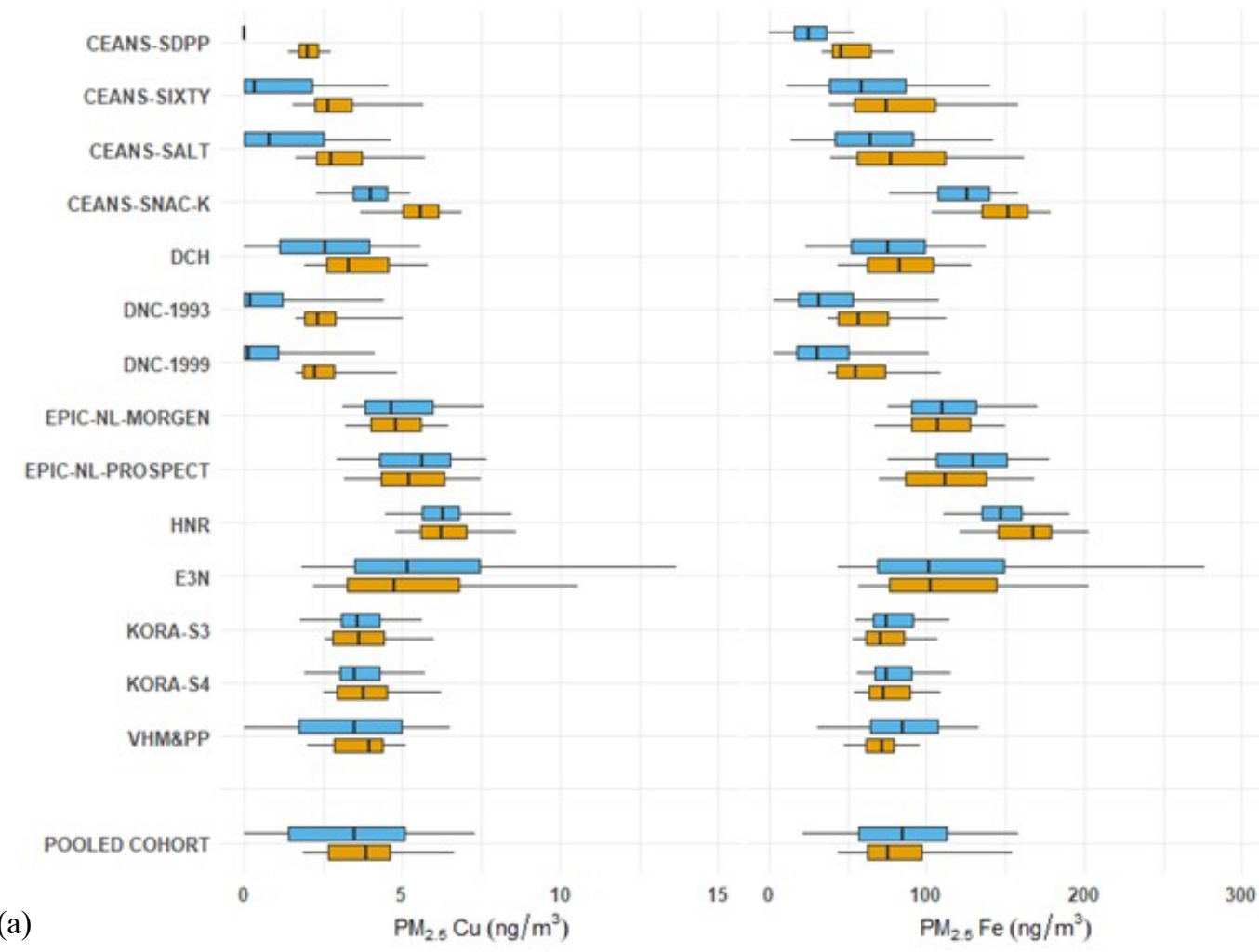

(a)

150

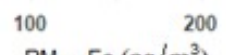

300
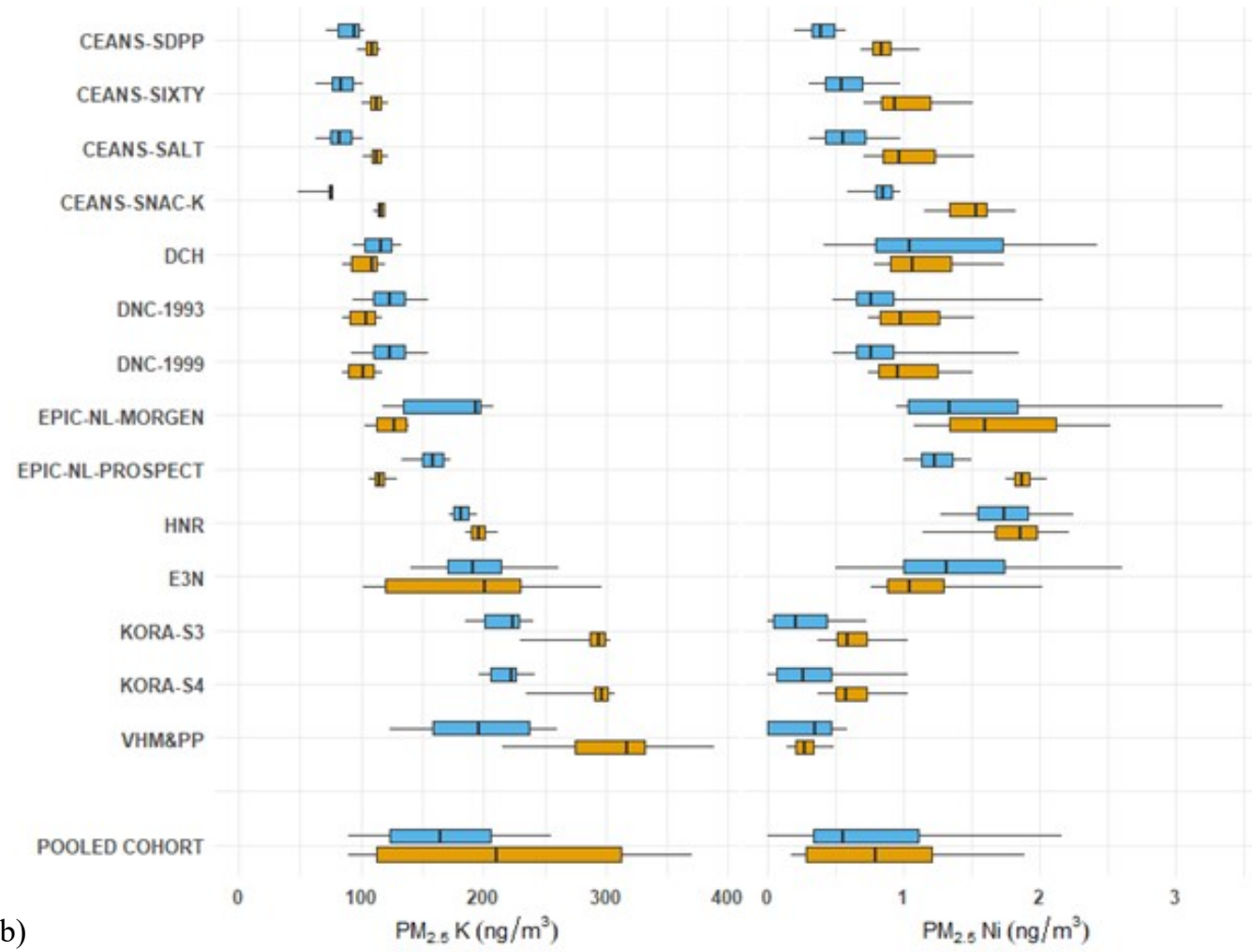

Exposure Model

宁 Random Forest

㝏 Supervised Linear Regression 


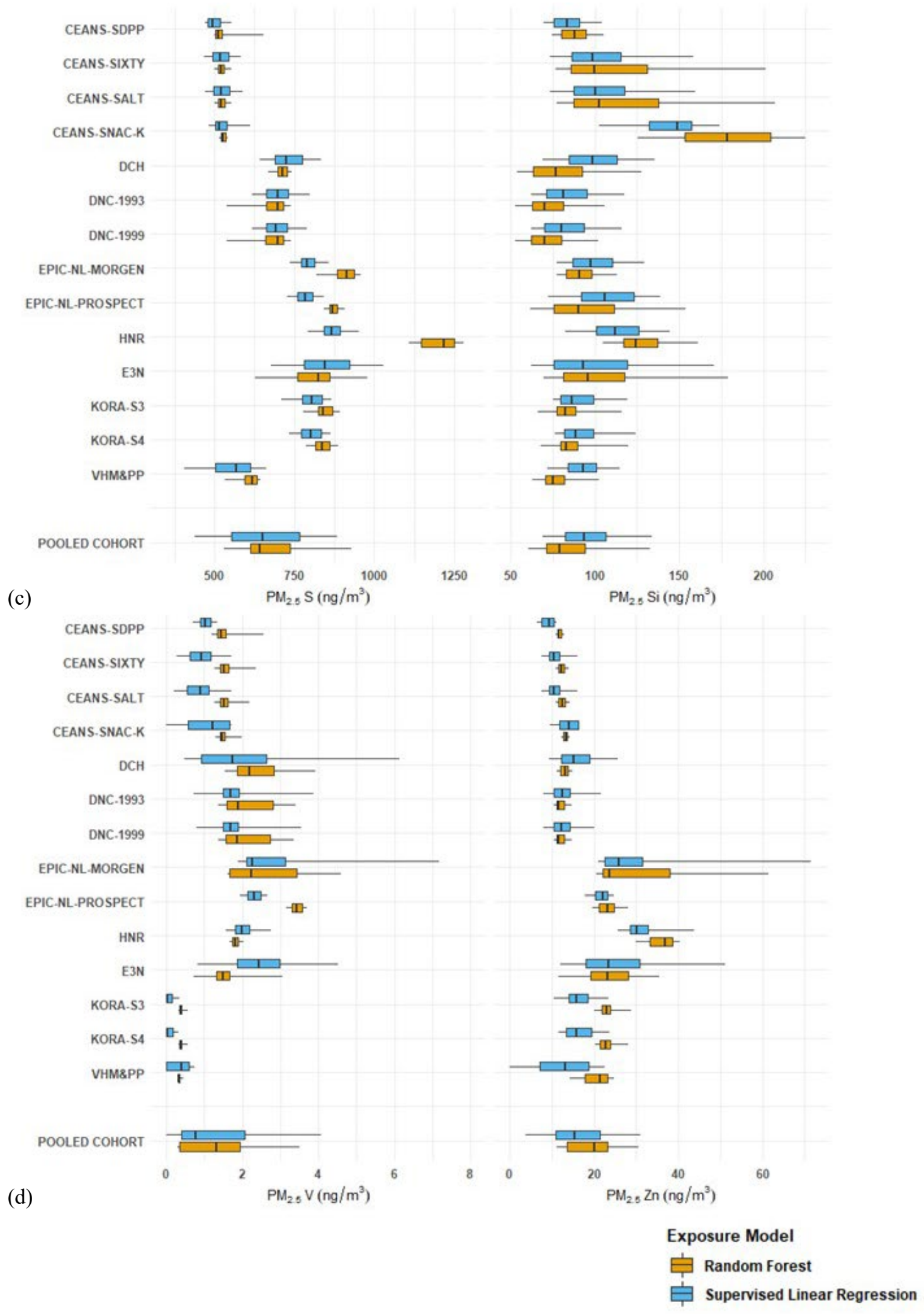

Figure 1. Distribution of component exposure at participant addresses estimated from Supervised Linear Regression and Random Forest models

The boundary of the box closest to zero indicates P25; furthest from zero - P75; bold vertical line inside the box - P50; whiskers indicate P5 and P95; see Table S11 for exposure distribution of components for the pooled cohort Sub-cohorts are shown from north to south 
Table 2. Spearman correlation coefficients between component exposure at participant addresses estimated from Supervised Linear Regression and Random Forest models $(\mathbf{N}=323,782)$

\begin{tabular}{lllllllll}
\hline Sub-cohort & $\mathrm{PM}_{2.5} \mathrm{Cu}$ & $\mathrm{PM}_{2.5} \mathrm{Fe}$ & $\mathrm{PM}_{2.5} \mathrm{~K}$ & $\mathrm{PM}_{2.5} \mathrm{Ni}$ & $\mathrm{PM}_{2.5} \mathrm{~S}$ & $\mathrm{PM}_{2.5} \mathrm{Si}$ & $\mathrm{PM}_{2.5} \mathrm{~V}$ & $\mathrm{PM}_{2.5} \mathrm{Zn}$ \\
\hline Average & 0.81 & 0.84 & 0.22 & 0.33 & 0.59 & 0.56 & 0.27 & 0.60 \\
CEANS-SDPP & 0.27 & 0.72 & 0.16 & 0.24 & 0.48 & -0.01 & 0.16 & 0.27 \\
CEANS-SIXTY & 0.86 & 0.89 & -0.09 & 0.44 & 0.39 & 0.76 & -0.07 & 0.45 \\
CEANS-SALT & 0.88 & 0.91 & -0.09 & 0.47 & 0.38 & 0.81 & -0.11 & 0.44 \\
CEANS-SNACK & 0.86 & 0.90 & 0.49 & 0.47 & 0.79 & 0.70 & 0.39 & 0.53 \\
DCH & 0.94 & 0.89 & -0.37 & 0.69 & 0.78 & 0.53 & 0.58 & 0.61 \\
DNC-1993 & 0.80 & 0.79 & 0.31 & 0.45 & 0.72 & 0.43 & 0.35 & 0.63 \\
DNC-1999 & 0.77 & 0.78 & 0.35 & 0.43 & 0.70 & 0.41 & 0.34 & 0.63 \\
EPIC-NL-Morgen & 0.92 & 0.93 & 0.82 & 0.89 & 0.20 & 0.59 & 0.7 & 0.52 \\
EPIC-NL-Prospect & 0.94 & 0.94 & 0.11 & 0.09 & 0.58 & 0.82 & -0.22 & 0.71 \\
HNR & 0.81 & 0.70 & -0.33 & 0.53 & 0.56 & 0.72 & 0.53 & 0.79 \\
E3N & 0.90 & 0.89 & 0.62 & 0.51 & 0.67 & 0.55 & 0.72 & 0.83 \\
KORA-S3 & 0.71 & 0.84 & 0.23 & -0.17 & 0.62 & 0.79 & -0.03 & 0.55 \\
KORA-S4 & 0.77 & 0.85 & -0.03 & 0.10 & 0.59 & 0.85 & 0.22 & 0.67 \\
VHM\&PP & 0.88 & 0.74 & 0.89 & -0.51 & 0.79 & -0.16 & 0.22 & 0.74
\end{tabular}

${ }^{a}$ Average of cohort-specific correlation coefficients. Cohort-specific correlations are shown because the analyses mostly exploit within-cohort exposure contrasts (i.e. stratified by sub-cohort id).

\subsection{Associations of $\mathrm{PM}_{2.5}$ composition with mortality}

Natural mortality

During the follow-up, we observed 46,640 (14.4\%) deaths from natural causes. Figure 2 and Table S14 show associations of $\mathrm{PM}_{2.5}$ composition with natural mortality. In the single-pollutant models, all components were significantly associated with natural mortality except for RF-modeled Ni and Si. For $\mathrm{Cu}, \mathrm{Fe}, \mathrm{K}, \mathrm{S}, \mathrm{V}$ and $\mathrm{Zn}$, the HR point estimates were similar for SLR- and RF-modeled exposures with generally wider confidence intervals (CIs) for RF. For Ni and Si, HRs were above unity for SLRmodeled and essentially unity for RF-modeled exposures.

In two-pollutant models, HRs strongly attenuated for most components, while HRs remained stable for $\mathrm{PM}_{2.5}$ mass and $\mathrm{NO}_{2}$ (Figure 2 and Table S14). For $\mathrm{Cu}$ and Fe, HR point estimates were similar for SLR- and RF-modeled exposures after adjustment for $\mathrm{PM}_{2.5}$ mass, with wider CIs observed for RFmodeled exposures. HRs for $\mathrm{Cu}$ and $\mathrm{Fe}$ decreased substantially and became mostly nonsignificant after adjustment for $\mathrm{NO}_{2}$ with HRs being above unity for SLR and below unity for RF. HRs for K were attenuated though still significantly above unity for SLR and RF after adjustment for $\mathrm{NO}_{2}$, whereas after adjustment for $\mathrm{PM}_{2.5}$ mass, the HRs reduced to unity for SLR but remained above unity for RF. For Ni, $\mathrm{S}, \mathrm{Si}$ and $\mathrm{Zn}$, HRs remained above unity and (almost) significant for SLR in two-pollutant models, whereas HRs reduced to essentially unity for RF. The HRs for V were reduced but remained above unity and (almost) significant in two-pollutant models, with similar estimates observed for SLR and RF.

We observed the strongest associations of natural mortality with all $\mathrm{PM}_{2.5}$ components in the minimally adjusted models (Model 1) (Table S15). HRs attenuated substantially after adjusting for individual level covariates (Model 2), except for K which remained stable. HRs increased slightly or remained stable 
after further adjustment for area-level covariates (Model 3). This pattern was observed both for SLRand RF-modeled exposures. For $\mathrm{Cu}, \mathrm{Fe}, \mathrm{K}, \mathrm{S}, \mathrm{V}$ and $\mathrm{Zn}$, the HR point estimates were similar between SLR- and RF-modeled exposures for all three models, with generally wider CIs for RF. For Ni and $\mathrm{Si}$, the effect estimates were larger for SLR- than for RF-modeled exposure in all models.

We generally observed linear or supra-linear concentration-response relationships for SLR-modeled elements and natural mortality (Figure S3). For some RF-modeled elements, there is no strong evidence of linear associations between exposure and mortality, mainly because of the limited variability in exposure concentrations.

\section{Cause-specific mortality}

We observed 15,492 (4.8\%) deaths from cardiovascular diseases during the follow-up. HRs were significantly above unity for all components in single pollutant models except for RF-modeled $\mathrm{Ni}$ and $\mathrm{Si}$, for which HRs were (nonsignificantly) below unity (Table S16). The magnitude of HR point estimates was similar to the HRs observed for natural mortality. In two-pollutant models, HRs for most components attenuated substantially, whereas HRs for $\mathrm{PM}_{2.5}$ and $\mathrm{NO}_{2}$ remained stable. HRs for $\mathrm{PM}_{2.5}$ and $\mathrm{NO}_{2}$ tended to be higher in models with RF-modeled than in SLR-modeled component exposure. With adjustment for $\mathrm{NO}_{2}$, HRs for $\mathrm{Cu}$ and $\mathrm{Fe}$ remained above unity for SLR but became unity or below unity for RF. HR point estimates for SLR-modeled Ni, S and Si were above unity in two-pollutant models adjusting for $\mathrm{PM}_{2.5}$ mass or $\mathrm{NO}_{2}$, whereas HRs were unity or below unity for RF. The HRs for $\mathrm{V}$ attenuated but remained above unity though nonsignificant after adjustment for $\mathrm{PM}_{2.5}$ mass or $\mathrm{NO}_{2}$, with similar estimates for SLR and RF.

We observed 2,846 (0.9\%) deaths from nonmalignant respiratory diseases during the follow-up. HRs above unity were observed for $\mathrm{Cu}, \mathrm{Fe}, \mathrm{Ni}$ and $\mathrm{V}$ with a similar magnitude for SLR- and RF-modeled exposures in single pollutant models (Table S17). For S, Si and Zn, HRs were above unity for SLRmodeled exposures, and were higher compared to RF-modeled exposures. HRs were close to unity for RF-modeled Si and Zn. In two-pollutant models, HRs remained stable after adjustment for $\mathrm{PM}_{2.5}$ mass. $\mathrm{HRs}$ were non-significantly below unity after adjustment for $\mathrm{NO}_{2}$ for components modeled with both algorithms except for $\mathrm{Ni}$ and $\mathrm{V}$, for which HRs attenuated but were still above unity for both SLR and RF. HRs for $\mathrm{NO}_{2}$ were stable in all models. HRs for $\mathrm{PM}_{2.5}$ varied from below to above unity in the different models, most of them were insignificant.

We observed 3,776 (1.2\%) deaths from lung cancer during the follow-up. HRs were above unity for all components in single pollutant models, though HRs for RF-modeled exposures were non-significant except for K, S and V (Table S18). In two-pollutant models with adjustment for $\mathrm{PM}_{2.5}$ mass or $\mathrm{NO}_{2}$, HRs stayed stable for SLR-modeled S, whereas HRs reduced substantially though remained nonsignificantly above unity for RF-modeled S. HRs for most other components reduced to unity or below unity and became non-significant in two-pollutant models. $\mathrm{HRs}$ for $\mathrm{PM}_{2.5}$ mass and $\mathrm{NO}_{2}$ remained stable in all models except for reduced HRs for SLR-modeled S.

Sensitivity analyses

Table S15 shows results derived from Model 1 using Model 1 and Model 3 populations, respectively, and results derived from Model 2 using Model 2 and Model 3 populations, respectively. The effect estimates were almost identical for the same model using different population, suggesting little selection bias was introduced by excluding subjects with missing covariates.

When restricting analyses to participants with follow-up time to after year 2000 (69\% of total personyears at risk, $84 \%$ of total death), after year 2005 ( $46 \%$ of total person-years at risk, $64 \%$ of total death), and after year 2008 (32\% of total person-years at risk, $47 \%$ of total death), we observed robust associations between $\mathrm{PM}_{2.5}$ composition and natural mortality (Table S19).

The effect estimates were not affected by additional adjustment for individual level education, occupational status (Table S20) and additional neighborhood-level SES variables (Table S21) in cohorts that had such information. 

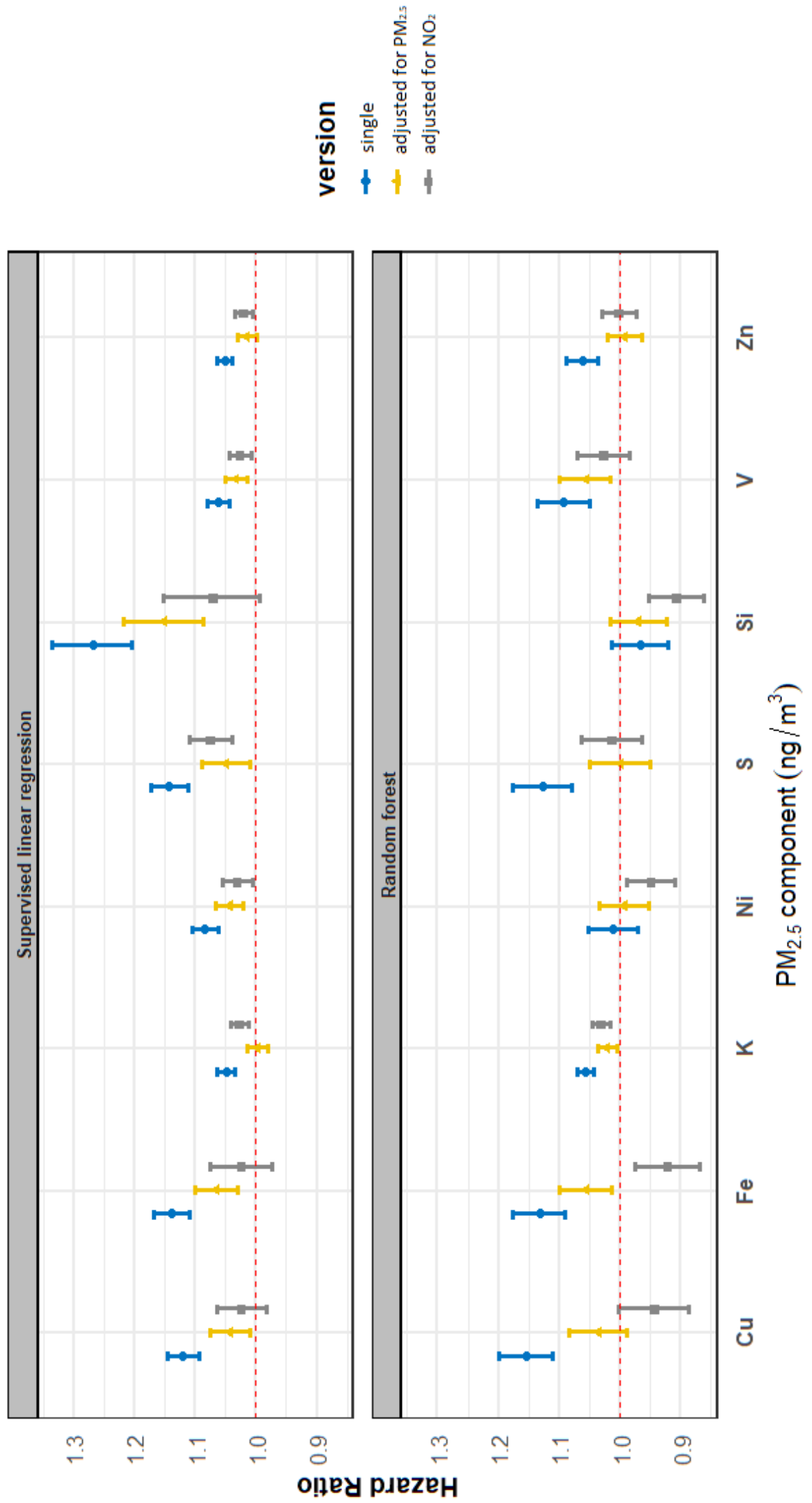

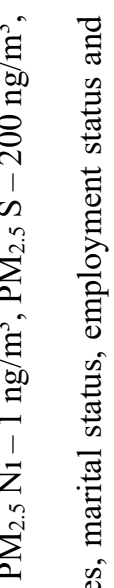

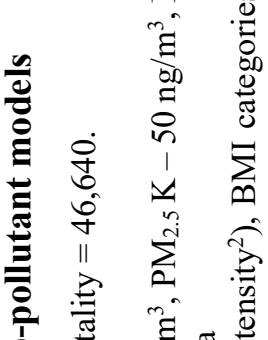

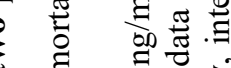

可 8.0.

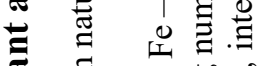

हี

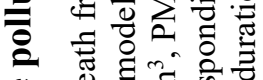

@ छ छ

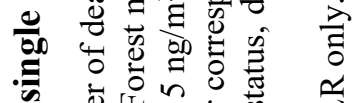

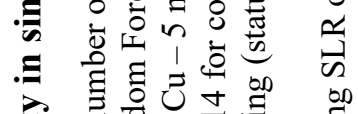

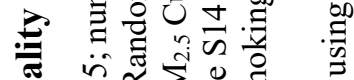

nิ $\sum_{0} 0$

तू 11 की की

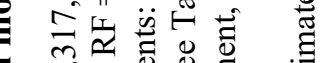

जु

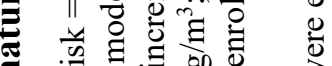

$\Rightarrow \quad \exists=000$

$=5.000$ की

क्षे

อ

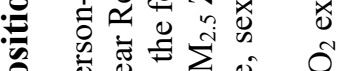

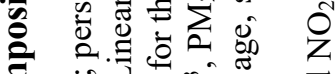

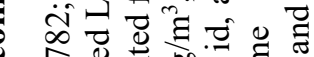

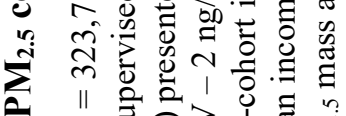

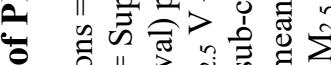

Q 일

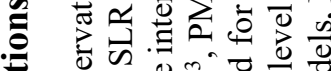

言

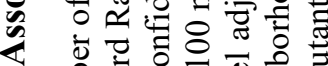

)

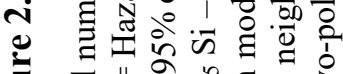

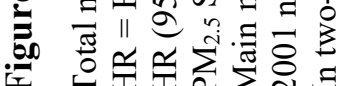




\section{Discussion}

We observed an elevated risk of mortality associated with long-term exposure to most $\mathrm{PM}_{2.5}$ elemental components in single pollutant models. In two-pollutant models with adjustment for $\mathrm{PM}_{2.5}$ mass or $\mathrm{NO}_{2}$, effect estimates were attenuated for almost all component-outcome pairs. Effect estimates for SLR- and RF-modeled exposures agreed well in single pollutant models, except for $\mathrm{Ni}$ and $\mathrm{Si}$, for which effect estimates for RF were lower. Effect estimates for RF modeled exposures were generally lower than for SLR in two-pollutant models.

\subsection{Comparison with previous studies}

Only a limited number of epidemiological studies have assessed associations between mortality and long-term exposure to $\mathrm{PM}_{2.5}$ elemental components. Among the components studied, sulfate has received the most attention. Sulfate is a secondary pollutant produced by atmospheric reactions of sulfur dioxide $\left(\mathrm{SO}_{2}\right)$ emitted by combustion of S-containing liquid and solid fuels. Because sulfate is primarily in the fine particle fraction, sulfate may travel for large distances, resulting in a relatively small within study area variability. Another important source is sea salt sulfate which is predominately in the coarse fraction but has a small fraction also in $\mathrm{PM}_{2.5}$ that is long-range transported (Belis et al., 2013). The California Teachers Study (Ostro et al., 2011) reported an increased hazard ratio (HR) of 1.06 (95\% CI: $0.97,1.16$ ) for natural cause mortality in association with a $2.2 \mu \mathrm{g} / \mathrm{m}^{3}$ increase in $\mathrm{PM}_{2.5}$ sulfate concentration, translating into a HR of 1.02 per $200 \mathrm{ng} / \mathrm{m}^{3}$ (the exposure contrast used in our analyses), assuming all $\mathrm{S}$ is present as sulfate (sulfate to $\mathrm{S}$ ratio of 3 ). Analyses of the ACS CPS-II data suggested that long-term $\mathrm{PM}_{2.5} \mathrm{~S}$ exposure was associated with all-cause mortality (HR ranged from 1.01 to 1.03 per $528.8 \mathrm{ng} / \mathrm{m}^{3}$ depending on the models) (Thurston et al., 2013). In ESCAPE, robust associations of $\mathrm{PM}_{2.5} \mathrm{~S}$ exposure with natural mortality were found (Beelen et al., 2015). The effect estimate observed in ESCAPE was similar to the estimate in the present study (HR 1.14, 95\% CI: $1.06,1.23 \mathrm{per} 200 \mathrm{ng} / \mathrm{m}^{3}$ in ESCAPE; HR 1.14, 95\% CI: $1.11,1.17$ and HR 1.13, 95\% CI: $1.08,1.18$ per $200 \mathrm{ng} / \mathrm{m}^{3}$ for SLRand RF-modeled exposures, respectively, in ELAPSE). In the present study we obtained a much narrower confidence interval, probably due to the longer follow-up and the pooling of cohort data. The effect estimate of $\mathrm{S}$ in our study was much larger than the estimates from the U.S. cohorts. One major difference is that the U.S. cohorts investigated between-area contrasts only whereas both ELAPSE and ESCAPE focused on within-area contrasts. Because the transported sulfate has relatively uniform spatial variation at the city scale, the exposure contrast was much smaller in our study than in the U.S. studies, thus a small effect in our study could be inflated when adopting it to the same increment of exposure as in the U.S. studies. Another explanation might be that we measured elemental composition between 2008 and 2011, when emission of $\mathrm{SO}_{2}$ had decreased compared to the baseline of all cohorts (EEA, 2015). The health effects in our study populations may be partly related to exposure levels and contrasts of $20 \mathrm{y}$ ago (most cohorts have baselines in the 1990s). Therefore, our S-related magnitude of health effect estimates may be overestimated.

In the present study, we also found robust associations between $\mathrm{S}$ and lung cancer mortality, which was observed in ACS CPS-II as well (Thurston et al., 2013). The effect estimates for lung cancer mortality were larger than for natural-cause mortality, with wider confidence intervals. In ESCAPE, robust associations were observed for S and lung cancer incidence (Raaschou-Nielsen et al., 2016). We observed elevated associations of S with cardiovascular mortality, which is consistent with previous findings in ESCAPE (Wang et al., 2014; Wolf et al., 2015) and in one of the ELAPSE sub-cohorts (i.e. the DCH cohort). The latter study reported an elevated risk of cardiovascular mortality associated with long-term exposure to secondary inorganic aerosols (Hvidtfeldt et al., 2019). The Women's Health Initiative-Observational Study (WHI-OS) found no association of sulfur with cardiovascular deaths (HR 1.01, 95\% CI: 0.92, 1.12 per $0.25 \mu \mathrm{g} / \mathrm{m}^{3}$ ), but a statistically significant association with cardiovascular events (HR 1.09, 95\% CI: 1.05, 1.14 per $0.25 \mu \mathrm{g} / \mathrm{m}^{3}$ ) (Vedal et al., 2013). In the California Teachers Study, IHD mortality was associated with long-term exposure to sulfate (Ostro et al., 2011) and high-S content fuel combustion (Ostro et al., 2015). 
Both $\mathrm{Ni}$ and $\mathrm{V}$ are suggested to be tracers of mixed industrial/ fuel-oil combustion and derived mainly from shipping emissions in Europe (Viana et al., 2008). Our study found a positive association of natural mortality with long-term exposure to Ni (HR 1.08, 95\% CI 1.06, 1.11 per $1 \mathrm{ng} / \mathrm{m}^{3}$ ) for SLR- and no association for RF-modeled exposures (HR 1.01, 95\% CI 0.97, 1.05 per $1 \mathrm{ng} / \mathrm{m}^{3}$ ). Our study found positive associations of natural mortality with long-term exposure to V (HR 1.06, 95\% CI 1.04, 1.08 and HR 1.09, 95\% CI 1.05, 1.14 per $2 \mathrm{ng} / \mathrm{m}^{3}$ for SLR- and RF-modeled exposures respectively). The effect estimates are similar to the estimates in ESCAPE for natural mortality (HR for Ni 1.05, 95\% CI 0.97, 1.13 per $1 \mathrm{ng} / \mathrm{m}^{3}$; HR for V 1.07, 95\% CI 0.93, 1.23 per $2 \mathrm{ng} / \mathrm{m}^{3}$ ) (Beelen et al., 2015), with much narrower CIs in ELAPSE. In ESCAPE, the accuracy of exposure estimates for $\mathrm{Ni}$ and $\mathrm{V}$ was limited because of the absence of specific sources of $\mathrm{Ni}$ and $\mathrm{V}$ in several study areas combined with limited measurement precision especially in areas with low pollution levels (de Hoogh et al., 2013). The Europe-wide models made use of both within and between area measurement contrasts and resulted in models with good performance for $\mathrm{Ni}$ and V (Chen et al., 2020). Compared to ESCAPE, the ELAPSE models further added industrial source data as potential predictors which improved the model performance. The improved exposure assessment may have allowed us to better detect the potential component-mortality associations. Our study also observed consistently positive associations between $\mathrm{V}$ and cause-specific mortality. Only a few studies have reported associations of mortality or morbidity with long-term exposure to $\mathrm{Ni}$ and $\mathrm{V}$. In ESCAPE, association was found between $\mathrm{PM}_{10} \mathrm{Ni}$ exposure and lung cancer incidence (Raaschou-Nielsen et al., 2016). In the Medicare population, stronger associations between long-term $\mathrm{PM}_{2.5}$ exposure and mortality were found for $\mathrm{PM}_{2.5}$ with higher $\mathrm{V}$ content (Wang et al., 2017). In the ACS CPS-II, associations between IHD mortality and Ni were reported (Thurston et al., 2013). The observed associations of $\mathrm{Ni}$ and $\mathrm{V}$ with mortality could be due to the components per se or to other components in emissions from oil combustion. Studies have suggested $\mathrm{V}$ in $\mathrm{PM}_{2.5}$ can induce oxidative stress, which is considered central to producing many of the negative health effects attributed to PM (Kelly and Fussell, 2020; Zhang et al., 2009). However, there is no stronger support from experimental studies for effects of $\mathrm{V}$ than for $\mathrm{Ni}, \mathrm{Fe}$ and $\mathrm{Cu}$, for which oxidative stress is also a major pathway.

In the present study, the effect estimates for the traffic-related components $\mathrm{Cu}$ and $\mathrm{Fe}$ remained after adjustment for $\mathrm{PM}_{2.5}$ mass but were reduced substantially after adjustment for $\mathrm{NO}_{2}$. The modestly wider confidence intervals for models with $\mathrm{PM}_{2.5}$ mass compared to the single pollutant models suggest these models provide interpretable results. Confidence intervals in two-pollutant models with $\mathrm{NO}_{2}$ widened somewhat more, due to the high correlations of $\mathrm{Cu}$ and $\mathrm{Fe}$ with $\mathrm{NO}_{2}$ in our study. Therefore, the substantial attenuation in effect estimates for $\mathrm{Cu}$ and $\mathrm{Fe}$ should be interpreted with caution, because effects of $\mathrm{NO}_{2}$ vs. those from $\mathrm{Cu}$ or $\mathrm{Fe}$ cannot be separated well. The high correlations of $\mathrm{Cu}$ and $\mathrm{Fe}$ with $\mathrm{NO}_{2}$ (average $\mathrm{R}$ of 0.75 ) in our study are consistent with correlations observed in the measured elemental components $(\mathrm{R}>0.75)$ that were used to develop the models (Tsai et al., 2015), suggesting the high correlations were not artificially introduced by the modeling methodology. Previous studies found mixed results regarding associations of mortality with $\mathrm{Cu}$ and $\mathrm{Fe}$. Using LUR models developed in ESCAPE, the Rome longitudinal study found associations of mortality with $\mathrm{Cu}$ and $\mathrm{Fe}$ in $\mathrm{PM}_{2.5}$ as well as tracers of tailpipe (i.e., $\mathrm{PM}_{2.5}$ absorbance) (Badaloni et al., 2017), but in the Badaloni study, no adjustment for $\mathrm{NO}_{2}$ was made. Positive associations were observed in the California Teachers Study between Fe and IHD mortality, but not with natural-cause, cardiopulmonary or pulmonary mortality (Ostro et al., 2011). Although the Ostro study did not adjust for $\mathrm{NO}_{2}$ or $\mathrm{PM}_{2.5}$ mass, adjustment for organic carbon did substantially reduced HRs. Analyses of ACS CPS-II data showed that traffic-related exposure was less strongly associated with excess mortality compared to coal combustion-related exposure (Thurston et al., 2013). However the ACS CPS-II study might have underestimated the effects of traffic-related air pollution because it investigated between-city variation, which represents only a small part of the expected overall variation in traffic-related air pollution.

Although $\mathrm{Zn}$ was a priori selected in ESCAPE to represent non-tailpipe traffic emissions, our Europewide models showed that a large fraction of the variation in the $\mathrm{Zn}$ measurements was explained by 
predictors representing industrial $\mathrm{Zn}$ emission (Chen et al., 2020), consistent with $\mathrm{Zn}$ being also a tracer for particles from industrial sources. This is consistent with source apportionment analyses in the ACS CPS-II, where $\mathrm{Zn}$ was considered as a source identifier for the metals industry (Thurston et al., 2016). The moderate correlations between $\mathrm{Zn}$ and $\mathrm{NO}_{2}$ (average $\mathrm{R}$ of 0.51 and 0.54 for SLR- and RF-modeled $\mathrm{Zn}$, respectively), suggest that $\mathrm{Zn}$ was not only related to traffic emission. The Rome longitudinal study found positive associations between $\mathrm{PM}_{2.5} \mathrm{Zn}$ and mortality from natural causes, cardiovascular diseases and IHD, using LUR models developed in ESCAPE (Badaloni et al., 2017). The ACS CPS-II also found some evidence of positive associations between $\mathrm{Zn}$ and mortality (Thurston et al., 2013). In the California Teachers Study, positive associations between Zn and IHD mortality were reported, but not with natural-cause, cardiopulmonary or pulmonary mortality (Ostro et al., 2011). Our study did not find clear evidence for associations of $\mathrm{Zn}$ with natural-cause or cause-specific mortality in two-pollutant models.

$\mathrm{K}$ was selected to represent biomass burning emission in ESCAPE (Tsai et al., 2015). While our new model included a plausible background predictor for biomass combustion (satellite-modeled organic matter), the model may have limited ability to capture within-area variability of biomass combustion emission because of the lack of reliable fine-scale predictor variables (Chen et al., 2020). Our study found elevated HRs for $\mathrm{K}$ exposure associated with mortality from natural-cause, cardiovascular diseases and lung cancer. HRs decreased after adjustment for $\mathrm{PM}_{2.5}$ mass to close to unity for SLRbased exposure, while they remained (significantly) elevated for RF exposures. K was reported to be associated with coronary events in ESCAPE (Wolf et al., 2015). $\mathrm{K}$ in ESCAPE was rather related to traffic (e.g., from resuspension of road dust) than to biomass burning. The California Teachers Study found positive associations between IHD mortality and K (Ostro et al., 2011), whereas the ACS CPSII consistently observed null association between $\mathrm{K}$ and mortality (Thurston et al., 2013).

$\mathrm{Si}$ was selected to represent crustal material, which is abundant in coarse particles. There was little evidence for an association between long-term coarse PM exposure and mortality (Adar et al., 2014; Hoek et al., 2013). The 2019 Integrated Science Assessment (ISA) rated the association between $\mathrm{PM}_{\text {coarse }}$ exposure and natural-cause mortality as "suggestive" (EPA, 2019). Our study found positive associations in single and two-pollutant models for $\mathrm{PM}_{2.5} \mathrm{Si}$ based upon SLR models, whereas associations were null or negative for RF models. The ACS CPS-II found that Si was consistently not associated with mortality across all models (Thurston et al., 2013). A negative and marginal association was observed for CVD events with Si in WHI-OS (Vedal et al., 2013). In contrast, analyses in the California Teachers Study showed positive associations of IHD mortality with Si (Ostro et al., 2011).

\subsection{Effect estimates using SLR- and RF-modeled exposures}

For most components, we observed generally consistent elevated mortality risks for SLR- and RFmodeled exposures in single pollutant models. However, less consistent associations for exposures by $\mathrm{RF}$ than SLR were found in two-pollutant models especially after adjusting for $\mathrm{NO}_{2}$. We do not have a clear explanation for these differences. There is no clear pattern of differences related to the spatial distribution of the components. We found differences both for components with a strong local contribution such as $\mathrm{Cu}$ and components with a predominantly large-scale variation such as $\mathrm{S}$. The less consistent association for RF-modeled exposure in two-pollutant models is not due to different correlation of components with $\mathrm{PM}_{2.5}$ mass or $\mathrm{NO}_{2}$, which were similar for RF- and SLR-modeled exposures. The two sets of models had similar performance in explaining within-area variability in internal cross-validations (Chen et al., 2020), which is the exposure contrast primarily exploited in the current analysis. The comparison of performance of the two algorithms is limited because we did not have external validation measurements. We therefore had no prior knowledge of which models had lower biases. We observed that the predicted variability of exposure was less for RF, explaining the wider confidence intervals in the epidemiological analyses using RF-modeled exposures. We note that RF models are more difficult to interpret in terms of how predictor variables act in the models, so a full analysis of the difference of specific predictors in the two algorithms is not possible. 


\subsection{Strengths and limitations}

One important strength is the highly standardized dataset used in this study, which was pooled from eight European cohorts with detailed individual and area-level covariate information, including smoking and BMI, which involved harmonizing variables between cohorts. The pooling of data allowed for more statistical power in our current analyses compared to the previous ESCAPE analyses. Another strength is the improvement in exposure assessment compared to ESCAPE. Analyses in ESCAPE may have had limited ability to detect component-specific mortality associations for $\mathrm{Ni}$ and $\mathrm{V}$ because of the lack of specific predictors in the exposure models for these components (de Hoogh et al., 2013). The Europe-wide $\mathrm{PM}_{2.5}$ composition models were able to make use of specific predictors representing pollution sources such as industrial sources, which explained a large proportion of the variation in measurements of specific components such as Zn (Chen et al., 2020). The Europe-wide models were developed based on a large number of measurement sites combined from individual ESCAPE study areas. A previous study has suggested that underestimation of the effect estimates was less serious when a large number of measurement sites was used for LUR modeling (Basagana et al., 2013).

One main limitation of our study is that the exposure models were developed based on measurements made in 2008-2011, whereas most included cohorts started in the mid-1990s. In the present study, we were not able to apply back-extrapolated exposure for $\mathrm{PM}_{2.5}$ components because we had insufficient information on the concentration of $\mathrm{PM}_{2.5}$ components in Europe over time. However, our results were robust when restricting the follow-up period to more recent start dates (year 2000, 2005 and 2008), indicating the impact of temporal misalignment by applying 2010 exposures was limited. Several studies in Europe have reported that the spatial contrast of $\mathrm{NO}_{2}$ remained stable for periods up to $10 \mathrm{y}$ (Cesaroni et al., 2012; Eeftens et al., 2011; Gulliver et al., 2013), suggesting that spatial contrast for traffic-related components such as $\mathrm{Cu}$ and $\mathrm{Fe}$ may be stable over time. For $\mathrm{Cu}$ and $\mathrm{Fe}$ contrasts may actually be more stable, given that non-tailpipe emissions have not been regulated, as opposed to tailpipe emissions. We cannot rule out the possibility that spatial contrast for components from other sources may have been less stable. For example the magnitude of our sulfur-related health estimates might be overestimated because of decreased $\mathrm{SO}_{2}$ emission over the years (EEA, 2015), which possibly resulted in a smaller contrast in sulfate exposure. The spatial pattern of major sources has likely not changed in a major way (Belis et al., 2013; Viana et al., 2008). Another limitation of the present study is that we did not consider residential mobility during follow-up. This may have resulted in measurement error, likely nondifferential and resulting in bias toward the null (Armstrong, 1998). The decision to focus on within-cohort exposure contrasts limited our ability to assess associations with components with relatively small within-area exposure contrasts such as S. However, we considered the potential confounding related to unmeasured differences between cohorts more critical. Last, the exposure maps for RF-modeled $\mathrm{K}$, Ni and V showed strong boundary effects that might affect the exposure estimates for some participants in the E3N cohort (Chen et al., 2020). However, we expected limited impact on the health effect estimation because few people live at the borders and the correlations between SLRand RF-modeled estimates did not stand out for these three elements, nor the E3N cohort.

\section{Conclusions}

Long-term exposures to especially vanadium in $\mathrm{PM}_{2.5}$ was associated with increased mortality risk, with associations observed for both SLR- and RF-modeled exposures. For the other components, associations were generally weaker when exposure was assessed with RF compared to SLR in twopollutant models. The consistency between SLR and RF could reflect the suitability of the models for estimating components rather than being evidence of a stronger effect on mortality. 


\section{References}

Adams K, Greenbaum DS, Shaikh R, et al. 2015. Particulate matter components, sources, and health: Systematic approaches to testing effects. J Air Waste Manag Assoc 65:544-558.

Adar SD, Filigrana PA, Clements N, et al. 2014. Ambient coarse particulate matter and human health: A systematic review and meta-analysis. Current environmental health reports 1:258-274.

Armstrong BG. 1998. Effect of measurement error on epidemiological studies of environmental and occupational exposures. Occup Environ Med 55:651-656.

Badaloni C, Cesaroni G, Cerza F, et al. 2017. Effects of long-term exposure to particulate matter and metal components on mortality in the rome longitudinal study. Environment international 109:146-154.

Basagana X, Aguilera I, Rivera M, et al. 2013. Measurement error in epidemiologic studies of air pollution based on land-use regression models. Am J Epidemiol 178:1342-1346.

Basagaña X, Rivera M, Aguilera I, et al. 2012. Effect of the number of measurement sites on land use regression models in estimating local air pollution. Atmospheric Environment 54:634-642.

Beelen R, Raaschou-Nielsen O, Stafoggia M, et al. 2014. Effects of long-term exposure to air pollution on natural-cause mortality: An analysis of 22 european cohorts within the multicentre escape project. The Lancet 383:785-795.

Beelen R, Hoek G, Raaschou-Nielsen O, et al. 2015. Natural-cause mortality and long-term exposure to particle components: An analysis of 19 european cohorts within the multi-center escape project. Environ Health Perspect 123:525-533.

Belis CA, Karagulian F, Larsen BR, et al. 2013. Critical review and meta-analysis of ambient particulate matter source apportionment using receptor models in europe. Atmospheric Environment 69:94-108.

Beulens JW, Monninkhof EM, Verschuren WM, et al. 2010. Cohort profile: The epic-nl study. International journal of epidemiology 39:1170-1178.

Brokamp C, Jandarov R, Rao MB, et al. 2017. Exposure assessment models for elemental components of particulate matter in an urban environment: A comparison of regression and random forest approaches. Atmos Environ (1994) 151:1-11.

Cesaroni G, Porta D, Badaloni C, et al. 2012. Nitrogen dioxide levels estimated from land use regression models several years apart and association with mortality in a large cohort study. Environmental Health 11:48.

Cesaroni G, Forastiere F, Stafoggia M, et al. 2014. Long term exposure to ambient air pollution and incidence of acute coronary events: Prospective cohort study and meta-analysis in 11 european cohorts from the escape project. BMJ 348:f7412.

Chen J, de Hoogh K, Gulliver J, et al. 2019. A comparison of linear regression, regularization, and machine learning algorithms to develop europe-wide spatial models of fine particles and nitrogen dioxide. Environment international 130:104934.

Chen J, de Hoogh K, Gulliver J, et al. 2020. Development of europe-wide models for particle elemental composition using supervised linear regression and random forest. Environ Sci Technol.

Clavel-Chapelon F, Group ENS. 2015. Cohort profile: The french e3n cohort study. International journal of epidemiology 44:801-809.

Cohen AJ, Brauer M, Burnett R, et al. 2017. Estimates and 25-year trends of the global burden of disease attributable to ambient air pollution: An analysis of data from the global burden of diseases study 2015. The Lancet 389:1907-1918.

de Hoogh K, Wang M, Adam M, et al. 2013. Development of land use regression models for particle composition in twenty study areas in europe. Environ Sci Technol 47:5778-5786.

De Hoogh K, Chen J, Gulliver J, et al. 2018. Spatial pm2. 5, no2, o3 and bc models for western europe-evaluation of spatiotemporal stability. Environment international 120:81-92.

Di Angelantonio E, Bhupathiraju SN, Wormser D, et al. 2016. Body-mass index and all-cause mortality: Individualparticipant-data meta-analysis of 239 prospective studies in four continents. The Lancet 388:776-786.

EEA. 2015. Https://www.Eea.Europa.Eu/data-and-maps/indicators/eea-32-sulphur-dioxide-so2-emissions-1/assessment-3.

Eeftens M, Beelen R, Fischer P, et al. 2011. Stability of measured and modelled spatial contrasts in no2 over time. Occupational and environmental medicine 68:765-770.

EPA US. 2019. Integrated science assessment (isa) for particulate matter (final report, 2019).

Eriksson AK, Ekbom A, Granath F, et al. 2008. Psychological distress and risk of pre-diabetes and type 2 diabetes in a prospective study of swedish middle-aged men and women. Diabetic Medicine 25:834-842.

Gulliver J, de Hoogh K, Hansell A, et al. 2013. Development and back-extrapolation of no2 land use regression models for historic exposure assessment in great britain. Environmental science \& technology 47:7804-7811.

Hoek G, Krishnan RM, Beelen R, et al. 2013. Long-term air pollution exposure and cardio-respiratory mortality: A review. Environmental health 12:43.

Hoek G. 2017. Methods for assessing long-term exposures to outdoor air pollutants. Curr Environ Health Rep 4:450-462.

Holmes NS, Morawska L. 2006. A review of dispersion modelling and its application to the dispersion of particles: An overview of different dispersion models available. Atmospheric environment 40:5902-5928.

Hundrup YA, Simonsen MK, Jørgensen T, et al. 2012. Cohort profile: The danish nurse cohort. International journal of epidemiology 41:1241-1247.

Hvidtfeldt UA, Geels C, Sorensen M, et al. 2019. Long-term residential exposure to pm2.5 constituents and mortality in a danish cohort. Environ Int 133:105268.

Hvidtfeldt UA, Chen J, Andersen ZJ, et al. 2021a. Long-term exposure to fine particle elemental components and lung cancer incidence in the elapse pooled cohort. Environ Res 193:110568.

Hvidtfeldt UA, Severi G, Andersen ZJ, et al. 2021b. Long-term low-level ambient air pollution exposure and risk of lung cancer - a pooled analysis of 7 european cohorts. Environ Int 146:106249.

Jerrett M, Turner MC, Beckerman BS, et al. 2017. Comparing the health effects of ambient particulate matter estimated using ground-based versus remote sensing exposure estimates. Environ Health Perspect 125:552-559.

Kelly FJ, Fussell JC. 2020. Toxicity of airborne particles-established evidence, knowledge gaps and emerging areas of importance. Philos Trans A Math Phys Eng Sci 378:20190322. 
Kerckhoffs J, Hoek G, Portengen Lt, et al. 2019. Performance of prediction algorithms for modeling outdoor air pollution spatial surfaces. Environmental science \& technology 53:1413-1421.

Lagergren M, Fratiglioni L, Hallberg IR, et al. 2004. A longitudinal study integrating population, care and social services data. The swedish national study on aging and care (snac). Aging clinical and experimental research 16:158-168.

Liu S, Jorgensen JT, Ljungman P, et al. 2021a. Long-term exposure to low-level air pollution and incidence of chronic obstructive pulmonary disease: The elapse project. Environ Int 146:106267.

Liu S, Jorgensen JT, Ljungman P, et al. 2021b. Long-term exposure to low-level air pollution and incidence of asthma: The elapse project. Eur Respir J.

Magnusson PK, Almqvist C, Rahman I, et al. 2013. The swedish twin registry: Establishment of a biobank and other recent developments. Twin Research and Human Genetics 16:317-329.

McGuinn LA, Ward-Caviness C, Neas LM, et al. 2017. Fine particulate matter and cardiovascular disease: Comparison of assessment methods for long-term exposure. Environ Res 159:16-23.

Ostro B, Lipsett M, Reynolds P, et al. 2011. Long-term exposure to constituents of fine particulate air pollution and mortality: Results from the california teachers study. Environ Health Perspect 118:363-369.

Ostro B, Hu J, Goldberg D, et al. 2015. Associations of mortality with long-term exposures to fine and ultrafine particles, species and sources: Results from the california teachers study cohort. Environ Health Perspect 123:549-556.

Raaschou-Nielsen O, Andersen ZJ, Beelen R, et al. 2013. Air pollution and lung cancer incidence in 17 european cohorts: Prospective analyses from the european study of cohorts for air pollution effects (escape). The Lancet Oncology 14:813-822. Raaschou-Nielsen O, Beelen R, Wang M, et al. 2016. Particulate matter air pollution components and risk for lung cancer. Environment international 87:66-73.

Samoli E, Rodopoulou S, Hvidtfeldt UA, et al. 2021. Modeling multi-level survival data in multi-center epidemiological cohort studies: Applications from the elapse project. Environment International 147:106371.

Schmermund A, Möhlenkamp S, Stang A, et al. 2002. Assessment of clinically silent atherosclerotic disease and established and novel risk factors for predicting myocardial infarction and cardiac death in healthy middle-aged subjects: Rationale and design of the heinz nixdorf recall study. American heart journal 144:212-218.

Stafoggia M, Cesaroni G, Peters A, et al. 2014. Long-term exposure to ambient air pollution and incidence of cerebrovascular events: Results from 11 european cohorts within the escape project. Environmental health perspectives 122:919-925.

Strak M, Weinmayr G, Rodopoulou S, et al. submitted. Long-term exposure to low-level air pollution and natural and causespecific mortality - a pooled analysis of eight european cohorts within the elapse project.

Szpiro AA, Paciorek CJ, Sheppard L. 2011. Does more accurate exposure prediction necessarily improve health effect estimates? Epidemiology 22:680-685.

Thurston G, Ito K, Lall R, et al. 2013. Npact study 4: Mortality and long-term exposure to pm2. 5 and its components in the american cancer society's cancer prevention study ii cohort. National Particle Component Toxicity (NPACT) Initiative: Integrated Epidemiologic and Toxicologic Studies of the Health Effects of Particulate Matter Components Health Effect Institute.

Thurston GD, Burnett RT, Turner MC, et al. 2016. Ischemic heart disease mortality and long-term exposure to source-related components of u.S. Fine particle air pollution. Environ Health Perspect 124:785-794.

Tjønneland A, Olsen A, Boll K, et al. 2007. Study design, exposure variables, and socioeconomic determinants of participation in diet, cancer and health: A population-based prospective cohort study of 57,053 men and women in denmark. Scandinavian journal of public health 35:432-441.

Tsai MY, Hoek G, Eeftens M, et al. 2015. Spatial variation of pm elemental composition between and within 20 european study areas--results of the escape project. Environ Int 84:181-192.

Ulmer H, Kelleher C, Fitz-Simon N, et al. 2007. Secular trends in cardiovascular risk factors: An age-period cohort analysis of 698954 health examinations in 181350 austrian men and women. Journal of internal medicine 261:566-576.

Vedal S, Campen M, McDonald J, et al. 2013. National particle component toxicity (npact) initiative report on cardiovascular effects. Research Report (Health Effects Institute):5-8.

Viana M, Kuhlbusch TAJ, Querol X, et al. 2008. Source apportionment of particulate matter in europe: A review of methods and results. Journal of Aerosol Science 39:827-849.

Wändell P-E, Wajngot A, De Faire U, et al. 2007. Increased prevalence of diabetes among immigrants from non-european countries in 60-year-old men and women in sweden. Diabetes \& metabolism 33:30-36.

Wang M, Beelen R, Eeftens M, et al. 2012. Systematic evaluation of land use regression models for no2. Environmental science \& technology 46:4481-4489.

Wang M, Beelen R, Stafoggia M, et al. 2014. Long-term exposure to elemental constituents of particulate matter and cardiovascular mortality in 19 european cohorts: Results from the escape and transphorm projects. Environ Int 66:97-106.

Wang Y, Shi L, Lee M, et al. 2017. Long-term exposure to pm2.5 and mortality among older adults in the southeastern us. Epidemiology 28:207-214.

Wolf K, Stafoggia M, Cesaroni G, et al. 2015. Long-term exposure to particulate matter constituents and the incidence of coronary events in 11 european cohorts. Epidemiology 26:565-574.

Zhang Z, Chau PY, Lai HK, et al. 2009. A review of effects of particulate matter-associated nickel and vanadium species on cardiovascular and respiratory systems. Int J Environ Health Res 19:175-185. 


\section{Supplementary Information}

\section{Area-level socio-economic status (SES) variable harmonization}

In the ELAPSE study manual (version 2, 31-10-2016) we identified that area-level SES variables were needed as potential confounders in the epidemiological analysis of the pooled cohort and the administrative cohorts. The Area-level SES Workgroup identified that harmonized SES data were not available from European databases for the neighborhood-scale. Therefore all local partners were asked to obtain the area-level SES data. We specified what data should be obtained and linked to the cohort data.

The area-level SES variables we aimed to obtain included composite score (combining different dimensions in one overall score), mean household income, low household income rate, income support rate, unemployment rate, low education rate, high education rate and ethnicity.

All variables were collected for a small area (neighborhood) and large area (region) to allow for confounding at commonly used spatial scales in previous cohort studies, realizing that not all data are necessary.

A neighborhood is a part of a city, with about 1,000 - 10,000 people. Ideally, we use a standard definition, referring to externally defined areas. If this is not available, postal codes were used, if they refer to the approximate number of people defined above. Examples include "buurt" and "wijk" in the Netherlands including on average 1,400 and 6,000 subjects; parish or census district ( 4,300 subjects) in Denmark. Quite a few of the ESCAPE cohorts have used municipality (or local administrative unit 2 (LAU2, former NUTS5)) in the ESCAPE project. We now aimed at a finer spatial scale, given that many of the included ESCAPE cohorts include a large metropolitan area with surrounding smaller towns. For smaller towns (e.g. below 10,000 subjects), the town (community) level was deemed to be fine. In some countries, data are available for multiple scales within the specified range. Availability of type of data (in multiple years) and comparability with other cohorts were criteria to select the scale. A very fine scale e.g. below 1,000 subjects is problematic for computational reasons (random effect models), particularly if the outcome is relatively rare.

The region is important for national cohorts. Each cohort defined this locally and judged whether a region scale is needed. When both a neighborhood and region scale is used, neighborhood should be nested within region.

SES has multiple dimensions, including income, education, occupation and employment. We use national composite scores that combine the different dimensions and in addition the main individual components as the association with air pollution and health may differ between dimensions. SES scores at regional scale were calculated by aggregating the raw variables to region level and then calculate the SES score. 


\section{Table S1. Characteristics of the Cardiovascular Effects of Air Pollution and Noise in Stockholm (CEANS) cohort}

All participants resided in Stockholm County, Sweden. The cohort is comprised of four sub-cohorts: The Stockholm Diabetes Preventive Program (SDPP) is a population-based prospective study of 7,949 subjects aged 35-54 years. The Stockholm Cohort of 60-year-olds (SIXTY) sub-cohort consists of a random population sample of one-third of all men and women living in Stockholm County turning 60 years between August 1997 and March 1999. The Screening Across the Lifespan Twin Study (SALT) sampled 7,043 individuals from the Swedish Twin Register born 1958 and earlier, who lived in Stockholm County. Lastly, The Swedish National Study of Aging and Care in Kungsholmen (SNAC-K) randomly sampled individuals 60+ years of age from a central area in Stockholm.

\begin{tabular}{|c|c|c|c|c|}
\hline \multirow{2}{*}{ Variable } & \multicolumn{4}{|c|}{ CEANS, sub-cohorts } \\
\hline & SDPP & SIXTY & SALT & SNAC-K \\
\hline Baseline year, range & 1992-1998 & 1997-1999 & $1998-2003$ & $2001-2004$ \\
\hline Enrolled, $\mathrm{N}^{\mathrm{a}}$ & 7,835 & 4,180 & 6,724 & 3,248 \\
\hline Included in mortality analyses & $7,716^{\mathrm{b}}$ & $3,965^{\mathrm{c}}$ & $6,174^{\mathrm{d}}$ & $2,830^{\mathrm{e}}$ \\
\hline \multicolumn{5}{|l|}{ Deaths, N (\%) } \\
\hline Natural cause & $337(4.4)$ & $593(15.0)$ & $891(14.4)$ & $960(33.9)$ \\
\hline Cardiovascular diseases & $74(1.0)$ & $163(4.1)$ & $326(5.3)$ & $416(14.7)$ \\
\hline Respiratory diseases & $12(0.2)$ & $38(1.0)$ & $57(0.9)$ & $61(2.2)$ \\
\hline Lung Cancer & $39(0.5)$ & $46(1.2)$ & $55(0.9)$ & $26(0.9)$ \\
\hline Age at baseline, yrs (mean \pm SD) & $47.1 \pm 4.9$ & $60.0 \pm 0.0$ & $57.8 \pm 10.6$ & $72.9 \pm 10.4$ \\
\hline Women, N (\%) & $4,721(61.2)$ & $2,065(52.1)$ & $3,416(55.3)$ & $1,767(62.4)$ \\
\hline Employed, N (\%) & $7,005(90.8)$ & $2,684(67.7)$ & $3,976(64.4)$ & $656(23.2)$ \\
\hline \multicolumn{5}{|l|}{ Marital status, N (\%) } \\
\hline Single & $1,271(16.5)$ & $182(4.6)$ & $863(14.0)$ & $459(16.2)$ \\
\hline Married & $6,445(83.5)$ & $2,930(73.9)$ & $4,179(67.7)$ & $1,300(45.9)$ \\
\hline Divorced & - & $649(16.4)$ & $693(11.2)$ & $388(13.7)$ \\
\hline Widowed & - & $204(5.1)$ & $439(7.1)$ & $683(24.1)$ \\
\hline \multicolumn{5}{|l|}{ Smoking status, N (\%) } \\
\hline Current & $2,035(26.4)$ & $839(21.2)$ & $1,311(21.2)$ & $404(14.3)$ \\
\hline Previous & $2,811(36.4)$ & $1,520(38.3)$ & $2,058(33.3)$ & $1,079(38.1)$ \\
\hline Never & $2,870(37.2)$ & $1,606(40.5)$ & $2,805(45.4)$ & $1,347(47.6)$ \\
\hline Smoking intensity ${ }^{\mathrm{af}}, \mathrm{g} / \mathrm{d}($ mean $\pm \mathrm{SD})$ & $13.5 \pm 7.4$ & $13.4 \pm 7.6$ & $12.7 \pm 8.0$ & $11.7 \pm 8.2$ \\
\hline Smoking duration ${ }^{\text {af }}$, yrs $($ mean \pm SD) & $27.9 \pm 8.6$ & $36.3 \pm 9.9$ & $37.9 \pm 9.3$ & $43.3 \pm 13.6$ \\
\hline \multicolumn{5}{|l|}{ BMI, $\mathrm{kg} / \mathrm{m}^{2}, \mathrm{~N}(\%)$} \\
\hline$<18.5$ & $54(0.7)$ & $26(0.7)$ & $94(1.5)$ & $78(2.8)$ \\
\hline $18.5-24.9$ & $3,688(47.8)$ & $1,397(35.2)$ & $3,622(58.7)$ & $1,251(44.2)$ \\
\hline $25.0-29.9$ & $3,007(39.0)$ & $1,767(44.6)$ & $2,054(33.3)$ & $1,134(40.1)$ \\
\hline $30.0+$ & $967(12.5)$ & $775(19.5)$ & $404(6.5)$ & $367(13.0)$ \\
\hline Neighborhood income ${ }^{g}($ mean \pm SD) & $24.3 \pm 4.2$ & $24.7 \pm 6.9$ & $25.3 \pm 6.6$ & $28.7 \pm 2.2$ \\
\hline
\end{tabular}

${ }^{a}$ The number of subjects for which information was transferred to Utrecht University for construction of the pooled cohort ${ }^{b}$ Subjects were excluded due to missing exposure (11), smoking status (6), smoking duration (23), smoking intensity (7), BMI (25), marital status (34), employment status (29), neighborhood income (4).

${ }^{\mathrm{c}}$ Subjects were excluded due to missing exposure (4), smoking status (124), smoking duration (171), smoking intensity (124), marital status (122), employment status (158).

${ }^{\mathrm{d}}$ Subjects were excluded due to missing exposure (2), smoking status (170), smoking duration (447), smoking intensity (170), BMI (253), marital status (103), employment status (29).

'Subjects were excluded due to fail in logical checks (1), missing smoking status (88), smoking duration (183), smoking intensity (139), BMI (290), marital status (7), employment status (73), neighborhood income (4).

${ }^{\mathrm{f}}$ For current smokers

${ }^{\mathrm{g}}$ EUR per 1,000, year 2001

References: (Eriksson et al., 2008; Lagergren et al., 2004; Lichtenstein et al., 2006; Wandell et al., 2007) 
Table S2. Characteristics of the Diet, Cancer and Health (DCH) cohort

Participants were recruited among persons aged 50-64 years from the areas of greater Copenhagen and Aarhus, Denmark, who were born in Denmark and free of cancer at baseline.

\begin{tabular}{|c|c|}
\hline \multirow{2}{*}{$\begin{array}{l}\text { Variable } \\
\text { Baseline year, range }\end{array}$} & DCH \\
\hline & 1993-1997 \\
\hline Enrolled, $\mathrm{N}^{\mathrm{a}}$ & 56,308 \\
\hline Included in mortality analyses ${ }^{b}$ & 52,779 \\
\hline \multicolumn{2}{|l|}{ Deaths, N (\%) } \\
\hline \begin{tabular}{l|l} 
& Natural cause \\
\end{tabular} & $10,490(19.9)$ \\
\hline Cardiovascular diseases & $2,143(4.1)$ \\
\hline Respiratory diseases & 861 (1.6) \\
\hline Lung Cancer & $1,282(2.4)$ \\
\hline Age at baseline, yrs (mean \pm SD) & $56.7 \pm 4.4$ \\
\hline Women, N (\%) & $27,709(52.5)$ \\
\hline Employed, N (\%) & $41,313(78.3)$ \\
\hline \multicolumn{2}{|l|}{ Marital status, N (\%) } \\
\hline Single & $3,220(6.1)$ \\
\hline \begin{tabular}{l|l} 
& Married \\
\end{tabular} & $37,665(71.4)$ \\
\hline \begin{tabular}{l|l} 
& Divorced \\
\end{tabular} & $8,980(17.0)$ \\
\hline \begin{tabular}{l|l} 
& Widowed \\
\end{tabular} & $2,914(5.5)$ \\
\hline \multicolumn{2}{|l|}{ Smoking status, N (\%) } \\
\hline \begin{tabular}{l|l} 
& Current \\
\end{tabular} & $19,175(36.3)$ \\
\hline \begin{tabular}{|l|l|} 
& Previous \\
\end{tabular} & $14,685(27.8)$ \\
\hline Never & $18,919(35.8)$ \\
\hline & $16.5 \pm 9.0$ \\
\hline $\begin{array}{l}\text { Smoking intensity }^{\mathrm{c}}, \mathrm{g} / \mathrm{d}(\text { mean } \pm \mathrm{SD}) \\
\left.\text { Smoking duration }^{\mathrm{c}} \text {, yrs (mean } \pm \mathrm{SD}\right)\end{array}$ & $36.3 \pm 7.7$ \\
\hline \multicolumn{2}{|l|}{$\mathrm{BMI}, \mathrm{kg} / \mathrm{m}^{2}, \mathrm{~N}(\%)$} \\
\hline \begin{tabular}{l|l} 
& $<18.5$ \\
\end{tabular} & $414(0.8)$ \\
\hline \begin{tabular}{l|l} 
& $18.5-24.9$ \\
\end{tabular} & $22,781(43.2)$ \\
\hline \begin{tabular}{l|l} 
& $25.0-29.9$ \\
\end{tabular} & $21,941(41.6)$ \\
\hline $30.0+$ & $7,643(14.5)$ \\
\hline Neighborhood income $^{\mathrm{d}}(\mathrm{mean} \pm \mathrm{SD})$ & $20.1 \pm 3.4$ \\
\hline
\end{tabular}

${ }^{\text {aT }}$ The number of subjects for which information was transferred to Utrecht University for construction of the pooled cohort

${ }^{b}$ Subjects were excluded due to missing exposure (907), smoking status (75), smoking duration (433), smoking intensity (1062), BMI (42), marital status (461), employment status (309), neighborhood income (930).

${ }^{\mathrm{c}}$ For current smokers

${ }^{\mathrm{d}}$ EUR per 1,000, year 2001

Reference: (Tjonneland et al., 2007) 


\section{Table S3. Characteristics of the Danish Nurse Cohort (DNC)}

The cohort was sampled among members of The Danish Nurse Organization (DNO) including both working and retired nurses. Questionnaires were mailed in 1993 to members aged 45+ years and again in 1999 with the inclusion of new members (45+ years).

\begin{tabular}{|c|c|c|}
\hline \multirow[b]{2}{*}{ Variable } & \multicolumn{2}{|c|}{ DNC, sub-cohorts } \\
\hline & DNC-1993 & DNC-1999 \\
\hline Baseline year & 1993 & 1999 \\
\hline Enrolled, $\mathrm{N}^{\mathrm{a}}$ & 19,664 & 8,769 \\
\hline Included in mortality analyses & $17,017^{\mathrm{b}}$ & $8,117^{\mathrm{c}}$ \\
\hline \multicolumn{3}{|l|}{ Deaths, N (\%) } \\
\hline Natural cause & $3,997(23.5)$ & $309(3.8)$ \\
\hline Cardiovascular diseases & $937(5.5)$ & $50(0.6)$ \\
\hline Respiratory diseases & $359(2.1)$ & $14(0.2)$ \\
\hline Lung Cancer & $351(2.1)$ & $30(0.4)$ \\
\hline Age at baseline, yrs (mean \pm SD) & $56.2 \pm 8.4$ & $47.9 \pm 4.2$ \\
\hline Women, N (\%) & $17,017(100.0)$ & $8,117(100.0)$ \\
\hline Employed, N (\%) & $11,907(70.0)$ & $7,693(94.8)$ \\
\hline \multicolumn{3}{|l|}{ Marital status, N (\%) } \\
\hline Single & $1,799(10.6)$ & $757(9.3)$ \\
\hline Married & $11,511(67.6)$ & $6,154(75.8)$ \\
\hline Divorced & $2,111(12.4)$ & $1,043(12.8)$ \\
\hline Widowed & $1,596(9.4)$ & $163(2.0)$ \\
\hline \multicolumn{3}{|l|}{ Smoking status, N (\%) } \\
\hline Current & $6,373(37.5)$ & $2,320(28.6)$ \\
\hline Previous & 4,864 (28.6) & $2,646(32.6)$ \\
\hline Never & $5,780(34.0)$ & $3,151(38.8)$ \\
\hline Smoking intensity ${ }^{\mathrm{d}}, \mathrm{g} / \mathrm{d}(\mathrm{mean} \pm \mathrm{SD})$ & $13.9 \pm 8.2$ & $13.3 \pm 7.3$ \\
\hline Smoking duration ${ }^{\mathrm{d}}$, yrs (mean \pm SD) & $31.6 \pm 9.9$ & $27.1 \pm 7.1$ \\
\hline \multicolumn{3}{|l|}{ BMI, kg/m², N (\%) } \\
\hline$<18.5$ & $499(2.9)$ & $142(1.7)$ \\
\hline $18.5-24.9$ & $11,742(69.0)$ & $5,539(68.2)$ \\
\hline $25.0-29.9$ & $3,893(22.9)$ & $1,897(23.4)$ \\
\hline $30.0+$ & $883(5.2)$ & $539(6.6)$ \\
\hline Neighborhood income $^{e}($ mean \pm SD) & $19.2 \pm 2.6$ & $19.0 \pm 2.4$ \\
\hline
\end{tabular}

${ }^{a}$ The number of subjects for which information was transferred to Utrecht University for construction of the pooled cohort

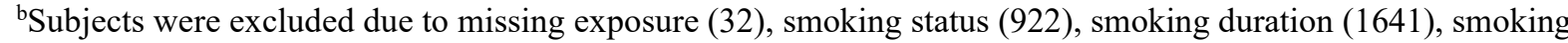
intensity (1314), BMI (156), marital status (201), employment status (590), neighborhood income (81).

${ }^{\mathrm{c}}$ Subjects were excluded due to missing exposure (11), smoking status (30), smoking duration (165), smoking intensity (190), BMI (40), marital status (42), employment status (310).

${ }^{\mathrm{d}}$ For current smokers

${ }^{\text {e}}$ EUR per 1,000, year 2001

Reference: (Hundrup et al., 2012) 
Table S4. Characteristics of the European Prospective Investigation into Cancer and Nutrition, the Netherlands (EPIC-NL)

The EPIC-NL combines two Dutch EPIC-cohorts: The Monitoring Project on Risk Factors and chronic diseases in the Netherlands (MORGEN) cohort which consists of a general population sample aged 2059 years from three Dutch towns (Amsterdam, Doetinchem and Maastricht). Prospect is a prospective cohort study among women aged 49-70, residing in the city of Utrecht or its vicinity, who participated in the nation-wide Dutch breast cancer screening programme between 1993 and 1997.

\begin{tabular}{|c|c|c|}
\hline \multirow{2}{*}{ Variable } & \multicolumn{2}{|c|}{ EPIC-NL, sub-cohorts } \\
\hline & MORGEN & PROSPECT \\
\hline Baseline year & 1993-1997 & 1993-1997 \\
\hline Enrolled, $\mathrm{N}^{\mathrm{a}}$ & 20,711 & 16,194 \\
\hline Included in mortality analyses & $18,292^{\mathrm{b}}$ & $14,570^{\mathrm{c}}$ \\
\hline \multicolumn{3}{|l|}{ Deaths, N (\%) } \\
\hline Natural cause & $1,180(6.5)$ & $1,997(13.7)$ \\
\hline Cardiovascular diseases & $261(1.4)$ & $459(3.2)$ \\
\hline Respiratory diseases & $63(0.3)$ & $126(0.9)$ \\
\hline Lung Cancer & $160(0.9)$ & $168(1.2)$ \\
\hline Age at baseline, yrs (mean \pm SD) & $42.9 \pm 11.3$ & $57.7 \pm 6.1$ \\
\hline Women, N $(\%)$ & $10,051(54.9)$ & $14,570(100.0)$ \\
\hline \multirow{2}{*}{\multicolumn{3}{|c|}{$\begin{array}{l}\text { Employed, N (\%) } \\
\text { Marital status, N (\%) }\end{array}$}} \\
\hline & & \\
\hline Single & $4629(25.3)$ & $836(5.7)$ \\
\hline Married & $11,916(65.1)$ & $11,179(76.7)$ \\
\hline Divorced & $1,380(7.5)$ & $1,172(8.0)$ \\
\hline Widowed & $367(2.0)$ & $1,383(9.5)$ \\
\hline \multicolumn{3}{|l|}{ Smoking status, N (\%) } \\
\hline Current & 6,357 (34.8) & $3,335(22.9)$ \\
\hline Previous & $5,153(28.2)$ & $4,795(32.9)$ \\
\hline Never & 6,782(37.1) & $6,440(44.2)$ \\
\hline Smoking intensity ${ }^{\mathrm{d}}, \mathrm{g} / \mathrm{d}($ mean $\pm \mathrm{SD})$ & $15.7 \pm 8.6$ & $13.7 \pm 8.7$ \\
\hline Smoking duration ${ }^{\mathrm{d}}$, yrs (mean \pm SD) & $24.8 \pm 10.6$ & $36.8 \pm 7.6$ \\
\hline \multicolumn{3}{|l|}{$\mathrm{BMI}, \mathrm{kg} / \mathrm{m}^{2}, \mathrm{~N}(\%)$} \\
\hline$<18.5$ & $188(1.0)$ & $87(0.6)$ \\
\hline $18.5-24.9$ & $9,122(49.9)$ & $6,505(44.6)$ \\
\hline $25.0-29.9$ & $6,869(37.6)$ & $5,790(39.7)$ \\
\hline $30.0+$ & $2,113(11.6)$ & $2,188(15.0)$ \\
\hline Neighborhood income $($ mean \pm SD) & $12.2 \pm 1.6$ & $13.1 \pm 1.4$ \\
\hline
\end{tabular}

${ }^{a}$ The number of subjects for which information was transferred to Utrecht University for construction of the pooled cohort

${ }^{b}$ Subjects were excluded due to fail in logical checks (28), missing exposure (11), smoking status (34), smoking duration (326), smoking intensity (1517), BMI (5), marital status (75), employment status (830), neighborhood income (2).

${ }^{c}$ Subjects were excluded due to fail in logical checks (1), missing smoking status (100), smoking duration (374), smoking intensity (716), BMI (20), marital status (101), employment status (104), neighborhood income (883).

${ }^{\mathrm{d}}$ For current smokers

${ }^{\mathrm{e}}$ EUR per 1,000, year 2001

Reference: (Beulens et al., 2010) 


\section{Table S5. Characteristics of the Heinz Nixdorf Recall study (HNR)}

The cohort consists of randomly sampled persons aged 45 to 75 years from the Ruhr area, Germany primarily in the three adjacent large cities Bochum, Essen, and Mülheim.

\begin{tabular}{|c|c|}
\hline Variable & HNR \\
\hline Baseline year, range & $2000-2003$ \\
\hline Enrolled, $\mathrm{N}^{\mathrm{a}}$ & 4,809 \\
\hline Included in mortality analyses ${ }^{\mathrm{b}}$ & 4,733 \\
\hline \multicolumn{2}{|l|}{ Deaths, N (\%) } \\
\hline Natural cause & $694(14.7)$ \\
\hline Cardiovascular diseases & $190(4.0)$ \\
\hline Respiratory diseases & $44(0.9)$ \\
\hline Lung Cancer & $63(1.3)$ \\
\hline Age at baseline, yrs (mean \pm SD) & $59.7 \pm 7.8$ \\
\hline Women, N (\%) & $2,382(50.3)$ \\
\hline Employed, N (\%) & $1,895(40.0)$ \\
\hline \multicolumn{2}{|l|}{ Marital status, N (\%) } \\
\hline Single & $274(5.8)$ \\
\hline Married & $3,538(74.8)$ \\
\hline Divorced & $472(10.0)$ \\
\hline Widowed & $449(9.5)$ \\
\hline \multicolumn{2}{|l|}{ Smoking status, N (\%) } \\
\hline Current & $1,113(23.5)$ \\
\hline Previous & $1,619(34.2)$ \\
\hline Never & $2,001(42.3)$ \\
\hline Smoking intensity ${ }^{\mathrm{c}}, \mathrm{g} / \mathrm{d}($ mean $\pm \mathrm{SD})$ & $18.6 \pm 12.0$ \\
\hline Smoking duration ${ }^{\mathrm{c}}$, yrs (mean \pm SD) & $34.5 \pm 9.4$ \\
\hline \multicolumn{2}{|l|}{$\mathrm{BMI}, \mathrm{kg} / \mathrm{m}^{2}, \mathrm{~N}(\%)$} \\
\hline$<18.5$ & $16(0.3)$ \\
\hline $18.5-24.9$ & $1,237(26.1)$ \\
\hline $25.0-29.9$ & $2,171(45.9)$ \\
\hline $30.0+$ & $1,309(27.7)$ \\
\hline Neighborhood income $^{\mathrm{d}}($ mean \pm SD) & $25.2 \pm 8.2$ \\
\hline
\end{tabular}

${ }^{a}$ The number of subjects for which information was transferred to Utrecht University for construction of the pooled cohort

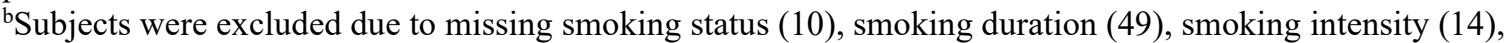
BMI (29), marital status (12), employment status (15).

${ }^{\mathrm{c}}$ For current smokers

${ }^{\mathrm{d} E U R}$ per 1,000, year 2001

Reference: (Schmermund et al., 2002) 
Table S6. Characteristics of the Etude Epidémiologique auprès de femmes de la Mutuelle Générale de l'Education Nationale (E3N)

The cohort was selected among French women aged 40 to 65 years who were insured through a national health system that primarily covered teachers. The cohort is nation-wide.

\begin{tabular}{|c|c|}
\hline Variable & E3N \\
\hline Baseline year, range & $1989-1991$ \\
\hline Enrolled, $\mathrm{N}^{\mathrm{a}}$ & 53,521 \\
\hline Included in mortality analyses ${ }^{b}$ & 38,537 \\
\hline \multicolumn{2}{|l|}{ Deaths, N (\%) } \\
\hline Natural cause & $1,941(5.0)$ \\
\hline Cardiovascular diseases & $266(0.7)$ \\
\hline Respiratory diseases & $59(0.2)$ \\
\hline Lung Cancer & $132(0.3)$ \\
\hline Age at baseline, yrs (mean \pm SD) & $53.0 \pm 6.8$ \\
\hline Women, N (\%) & $38,537(100.0)$ \\
\hline Employed, N (\%) & $26,158(67.9)$ \\
\hline \multicolumn{2}{|l|}{ Marital status, N (\%) } \\
\hline Single & $6,436(16.7)$ \\
\hline Married & $32,101(83.3)$ \\
\hline Divorced & - \\
\hline Widowed & - \\
\hline \multicolumn{2}{|l|}{ Smoking status, N (\%) } \\
\hline Current & $4,988(12.9)$ \\
\hline Previous & $7,411(19.2)$ \\
\hline Never & $26,138(67.8)$ \\
\hline Smoking intensity ${ }^{\mathrm{c}}, \mathrm{g} / \mathrm{d}($ mean $\pm \mathrm{SD})$ & $11.3(9.2)$ \\
\hline Smoking duration $^{\mathrm{c}}$, yrs $($ mean \pm SD) & $28.5(7.6)$ \\
\hline \multicolumn{2}{|l|}{ BMI, $\mathrm{kg} / \mathrm{m}^{2}, \mathrm{~N}(\%)$} \\
\hline$<18.5$ & $1,386(3.6)$ \\
\hline $18.5-24.9$ & $29,205(75.8)$ \\
\hline $25.0-29.9$ & $6,574(17.1)$ \\
\hline $30.0+$ & $1,372(3.6)$ \\
\hline Neighborhood income $^{\mathrm{d}}($ mean \pm SD $)$ & $11.2 \pm 3.0$ \\
\hline
\end{tabular}

aThe number of subjects for which information was transferred to Utrecht University for construction of the pooled cohort

${ }^{\mathrm{b}}$ Subjects were excluded due to fail in logical checks (14), missing exposure (629), smoking duration (8211), smoking intensity (9729), BMI (2644), marital status (1989), neighborhood income (222).

${ }^{\mathrm{c}}$ For current smokers

${ }^{\mathrm{d}}$ EUR per 1,000, year 2001

Reference: (Clavel-Chapelon and Group, 2015) 
Table S7. Characteristics of the Cooperative Health Research in the Region of Augsburg (KORA)

Two cross-sectional population-representative surveys were conducted in 1994-1995 (survey S3) and 1999-2001 (survey S4) in the city of Augsburg and two adjacent rural counties including inhabitants of German nationality aged 25 to 74 .

\begin{tabular}{|c|c|c|}
\hline \multirow{2}{*}{ Variable } & \multicolumn{2}{|c|}{ KORA, sub-cohorts } \\
\hline & $\mathbf{S 3}$ & S4 \\
\hline Baseline year, range & $1994-1995$ & 1999-2001 \\
\hline Enrolled, $\mathrm{N}^{\mathrm{a}}$ & 4,566 & 4,257 \\
\hline Included in mortality analyses & $2,572^{\mathrm{b}}$ & $2,281^{\mathrm{c}}$ \\
\hline \multicolumn{3}{|l|}{ Deaths, N (\%) } \\
\hline Natural cause & $391(15.2)$ & $215(9.4)$ \\
\hline Cardiovascular diseases & $159(6.2)$ & $72(3.2)$ \\
\hline Respiratory diseases & $33(1.3)$ & $20(0.9)$ \\
\hline Lung Cancer & $25(1.0)$ & $19(0.8)$ \\
\hline Age at baseline, yrs (mean \pm SD) & $49.4 \pm 13.9$ & $49.3 \pm 13.8$ \\
\hline Women, N (\%) & $1,308(50.9)$ & $1,173(51.4)$ \\
\hline Employed, N (\%) & $1,423(55.3)$ & $1,356(59.4)$ \\
\hline \multicolumn{3}{|l|}{ Marital status, N (\%) } \\
\hline Single & $227(8.8)$ & $184(8.1)$ \\
\hline Married & $2,060(80.1)$ & $1,807(79.2)$ \\
\hline Divorced & $108(4.2)$ & $151(6.6)$ \\
\hline Widowed & $177(6.9)$ & $139(6.1)$ \\
\hline \multicolumn{3}{|l|}{ Smoking status, N (\%) } \\
\hline Current & $519(20.2)$ & $523(22.9)$ \\
\hline Previous & $740(28.8)$ & $720(31.6)$ \\
\hline Never & $1,313(51.0)$ & $1,038(45.5)$ \\
\hline Smoking intensity ${ }^{\mathrm{d}}, \mathrm{g} / \mathrm{d}($ mean \pm SD $)$ & $16.5 \pm 9.5$ & $15.7 \pm 9.5$ \\
\hline Smoking duration $^{\mathrm{d}}$, yrs $($ mean \pm SD) & $25.2 \pm 12.1$ & $24.3 \pm 11.6$ \\
\hline \multicolumn{3}{|l|}{ BMI, $\mathrm{kg} / \mathrm{m}^{2}, \mathrm{~N}(\%)$} \\
\hline$<18.5$ & $13(0.5)$ & $8(0.4)$ \\
\hline $18.5-24.9$ & $837(32.5)$ & $710(31.1)$ \\
\hline $25.0-29.9$ & $1,116(43.4)$ & $996(43.7)$ \\
\hline $30.0+$ & $606(23.6)$ & $567(24.9)$ \\
\hline Neighborhood income $($ mean \pm SD $)$ & $36.7 \pm 4.4$ & $38.0 \pm 7.3$ \\
\hline
\end{tabular}

aThe number of subjects for which information was transferred to Utrecht University for construction of the pooled cohort

${ }^{\mathrm{b}}$ Subjects were excluded due to fail in logical checks (10), missing smoking duration (84), smoking intensity (129), BMI (52), neighborhood income (1825).

'Subjects were excluded due to missing smoking status (5), smoking duration (84), smoking intensity (18), BMI (37), marital status (5), employment status (6), neighborhood income (1892).

${ }^{\mathrm{d}}$ For current smokers

${ }^{\mathrm{e} E U R}$ per 1,000, year 2001

Reference: (Holle et al., 2005) 
Table S8. Characteristics of the Vorarlberg Health Monitoring and Prevention Programme (VHM\&PP)

The VHM\&PP is a population-based cohort recruited among all adults of the province of Vorarlberg, Austria. Vorarlberg is the western-most province of Austria consisting of towns and villages $(30,000$ inhabitants and smaller) and significant altitude differences.

\begin{tabular}{|c|c|}
\hline Variable & VHM\&PP \\
\hline Baseline year, range & $1985-2005$ \\
\hline Enrolled, $\mathrm{N}^{\mathrm{a}}$ & 170,250 \\
\hline Included in mortality analyses $^{\mathrm{b}}$ & 144,199 \\
\hline \multicolumn{2}{|l|}{ Deaths, N (\%) } \\
\hline Natural cause & $22,645(15.7)$ \\
\hline Cardiovascular diseases & $9,976(6.9)$ \\
\hline Respiratory diseases & $1,099(0.8)$ \\
\hline Lung Cancer & $1,380(1.0)$ \\
\hline Age at baseline, yrs (mean \pm SD) & $42.1 \pm 15.0$ \\
\hline Women, N (\%) & $81,017(56.2)$ \\
\hline Employed, N (\%) & $100,585(69.8)$ \\
\hline \multicolumn{2}{|l|}{ Marital status, N (\%) } \\
\hline Single & $24,832(17.2)$ \\
\hline Married & $99,400(68.9)$ \\
\hline Divorced & $9,762(6.8)$ \\
\hline Widowed & $10,205(7.1)$ \\
\hline \multicolumn{2}{|l|}{ Smoking status, N (\%) } \\
\hline Current & $28,871(20.0)$ \\
\hline Previous & $8,995(6.2)$ \\
\hline Never & $106,333(73.7)$ \\
\hline Smoking intensity ${ }^{\mathrm{c}}, \mathrm{g} / \mathrm{d}(\mathrm{mean} \pm \mathrm{SD})$ & $15.6 \pm 8.9$ \\
\hline Smoking duration ${ }^{\mathrm{c}}$, yrs (mean \pm SD) & $13.4 \pm 8.3$ \\
\hline \multicolumn{2}{|l|}{$\mathrm{BMI}, \mathrm{kg} / \mathrm{m}^{2}, \mathrm{~N}(\%)$} \\
\hline$<18.5$ & $4,450(3.1)$ \\
\hline $18.5-24.9$ & $78,575(54.5)$ \\
\hline $25.0-29.9$ & $45,533(31.6)$ \\
\hline $30.0+$ & $15,641(10.8)$ \\
\hline Neighborhood income ${ }^{\mathrm{d}}($ mean $\pm \mathrm{SD})$ & $22.9 \pm 1.7$ \\
\hline
\end{tabular}

${ }^{\text {aT }}$ The number of subjects for which information was transferred to Utrecht University for construction of the pooled cohort

bSubjects were excluded due to missing exposure (396), smoking duration (5789), smoking intensity (6376), BMI (11), marital status (9970), employment status (15056), neighborhood income (1895).

${ }^{\mathrm{c}}$ For current smokers

${ }^{\mathrm{d}}$ EUR per 1,000, year 2001

Reference: (Ulmer et al., 2007) 
Table S9. Performance of Europe-wide $\mathrm{PM}_{2.5}$ composition models in five-fold hold-out validation $^{\text {a }}$

\begin{tabular}{|c|c|c|c|c|c|c|c|c|}
\hline & $\mathrm{Cu}$ & $\mathrm{Fe}$ & $\mathrm{K}$ & $\mathrm{Ni}$ & $\mathrm{S}$ & $\mathrm{Si}$ & $\mathrm{V}$ & $\mathrm{Zn}$ \\
\hline \multicolumn{9}{|c|}{ Performance of $\mathrm{PM}_{2.5}$ composition models over Europe: $\mathrm{r}^{2}$} \\
\hline SLR & 0.48 & 0.48 & 0.59 & 0.56 & 0.79 & 0.46 & 0.63 & 0.41 \\
\hline $\mathrm{RF}$ & 0.59 & 0.61 & 0.80 & 0.76 & 0.90 & 0.62 & 0.86 & 0.71 \\
\hline \multicolumn{9}{|c|}{ Performance of $\mathrm{PM}_{2.5}$ composition models to assess within-area variation: average within-area $\mathrm{r}^{2}$} \\
\hline SLR & 0.35 & 0.36 & 0.07 & 0.17 & 0.14 & 0.20 & 0.19 & 0.19 \\
\hline $\mathrm{RF}$ & 0.29 & 0.29 & 0.07 & 0.21 & 0.23 & 0.17 & 0.29 & 0.25 \\
\hline
\end{tabular}

${ }^{a}$ Values extracted from Chen et al. (2020)

$\mathrm{r}^{2}=$ squared Pearson correlation, $\mathrm{SLR}=$ Supervised Linear Regression model, $\mathrm{RF}=$ Random Forest model

Table S10. Truncation frequency (Truncation performed for model 3 population, $\mathbf{N = 3 2 3 , 7 8 2 \text { ) }}$

\begin{tabular}{|c|c|c|c|}
\hline Pollutant & Exposure model & $\mathrm{N}$ below zero $(\%)$ & $\mathrm{N}$ above maximum $(\%)$ \\
\hline \multirow{2}{*}{$\mathrm{PM}_{2.5} \mathrm{Cu}$} & SLR & $36,683(11.3)^{\mathrm{a}}$ & $2(0)^{b}$ \\
\hline & $\mathrm{RF}$ & $0(0)$ & $0(0)$ \\
\hline \multirow{2}{*}{$\mathrm{PM}_{2.5} \mathrm{Fe}$} & SLR & $1,645(0.5)^{\mathrm{c}}$ & $0(0)$ \\
\hline & $\mathrm{RF}$ & $0(0)$ & $0(0)$ \\
\hline \multirow{2}{*}{$\mathrm{PM}_{2.5} \mathrm{~K}$} & SLR & $0(0)$ & $0(0)$ \\
\hline & $\mathrm{RF}$ & $0(0)$ & $0(0)$ \\
\hline \multirow{2}{*}{$\mathrm{PM}_{2.5} \mathrm{Ni}$} & SLR & $37,470(11.6)^{\mathrm{d}}$ & $24(0)^{\mathrm{e}}$ \\
\hline & $\mathrm{RF}$ & $0(0)$ & $0(0)$ \\
\hline \multirow{2}{*}{$\mathrm{PM}_{2.5} \mathrm{~S}$} & SLR & $0(0)$ & $0(0)$ \\
\hline & $\mathrm{RF}$ & $0(0)$ & $0(0)$ \\
\hline \multirow{2}{*}{$\mathrm{PM}_{2.5} \mathrm{Si}$} & SLR & $0(0)$ & $0(0)$ \\
\hline & $\mathrm{RF}$ & $0(0)$ & $0(0)$ \\
\hline \multirow{2}{*}{$\mathrm{PM}_{2.5} \mathrm{~V}$} & SLR & $46,243(14.3)^{f}$ & $0(0)$ \\
\hline & $\mathrm{RF}$ & $0(0)$ & $0(0)$ \\
\hline \multirow{2}{*}{$\mathrm{PM}_{2.5} \mathrm{Zn}$} & SLR & $8,154(2.5)^{\mathrm{g}}$ & $240(0.1)^{\mathrm{h}}$ \\
\hline & $\mathrm{RF}$ & $0(0)$ & $0(0)$ \\
\hline
\end{tabular}

$\mathrm{N}=$ Number of observations; SLR $=$ Supervised Linear Regression model, $\mathrm{RF}=$ Random Forest model

a $7464(97 \%)$ in CEANS-SDPP, $1754(44 \%)$ in CEANS-SIXTY, $2348(38 \%)$ in CEANS-SALT, $2(<1 \%)$ in CEANSSNACK, $3158(6 \%)$ in DCH, $7237(43 \%)$ in DNC-1993, $3564(44 \%)$ in DNC-1999, $1(<1 \%)$ in EPIC-NL-Morgen, $110(<1 \%)$ in E3N, $11045(8 \%)$ in VHM\&PP

b $2(<1 \%)$ in E3N

c $563(7 \%)$ in CEANS-SDPP, $30(1 \%)$ in CEANS-SIXTY, $53(1 \%)$ in CEANS-SALT, $58(<1 \%)$ in DCH, $640(4 \%)$ in DNC-1993, $301(4 \%)$ in DNC-1999,

d $51(1 \%)$ in CEANS-SDPP, $2(<1 \%)$ in CEANS-SIXTY, $3(<1 \%)$ in CEANS-SALT, $3(<1 \%)$ in DCH, $94(1 \%)$ in DNC-1993, $32(<1 \%)$ in DNC-1999, $438(1 \%)$ in E3N, $382(15 \%)$ in KORA-S3, 250 (11\%) in KORA-S4, 36215 $(25 \%)$ in VHM\&PP

e $4(<1 \%)$ in DCH, $3(<1 \%)$ in DNC-1993, $2(<1 \%)$ in EPIC-NL-Prospect, $10(<1 \%)$ in E3N, $5(<1 \%)$ in VHM\&PP f $37(1 \%)$ in CEANS-SIXTY, $68(1 \%)$ in CEANS-SALT, $183(6 \%)$ in CEANS- SNACK, $80(<1 \%)$ in DCH, $12(<1 \%)$ in DNC-1993, $5(<1 \%)$ in DNC-1999, $582(2 \%)$ in E3N, $1102(43 \%)$ in KORA-S3, 1078 (47\%) in KORA-S4, 43096 $(30 \%)$ in VHM\&PP

g $127(<1 \%)$ in E3N, $8027(6 \%)$ in VHM\&PP

h $1(<1 \%)$ in DNC-1993, $16(<1 \%)$ in EPIC-NL-Morgen, $3(<1 \%)$ in HNR, $220(1 \%)$ in E3N 


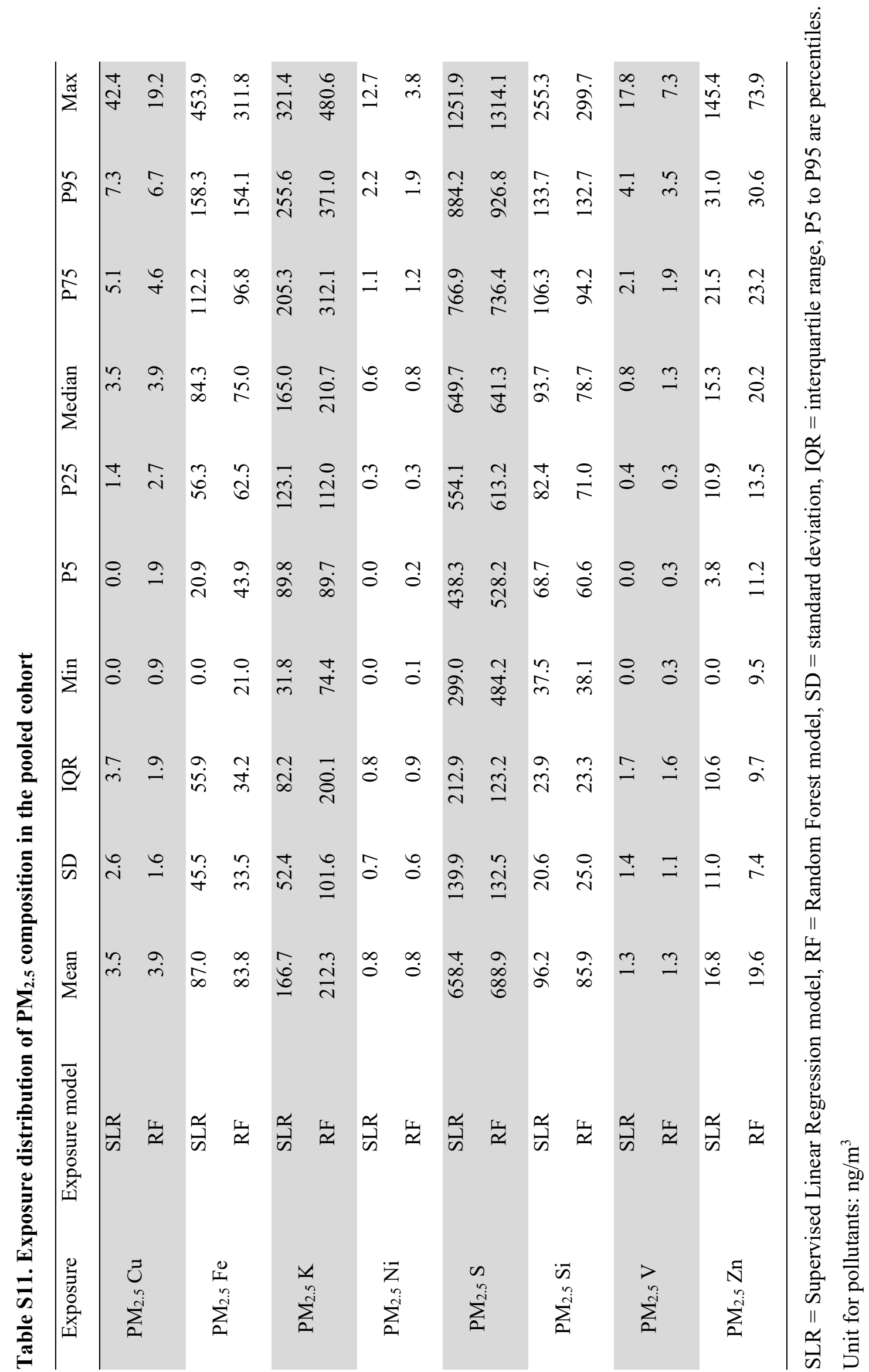




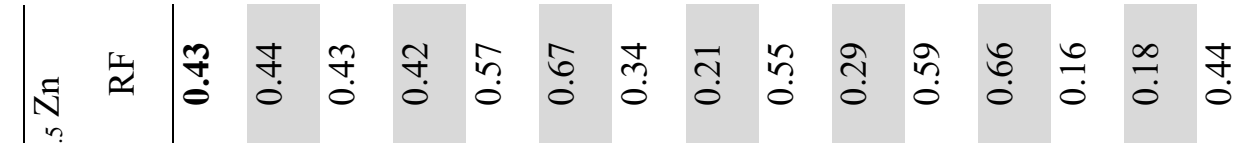

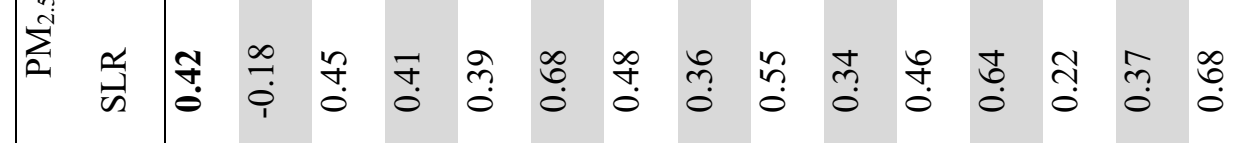

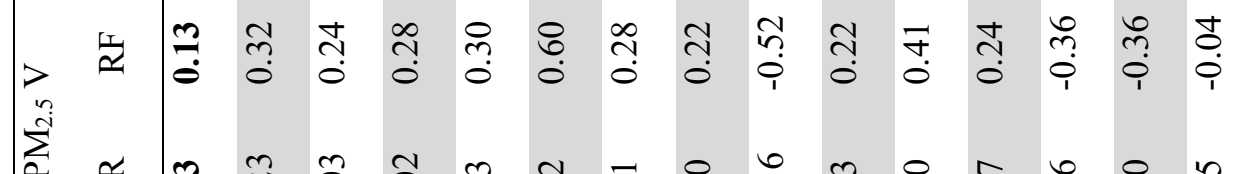

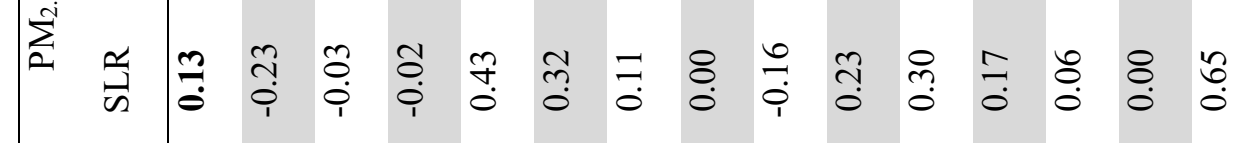

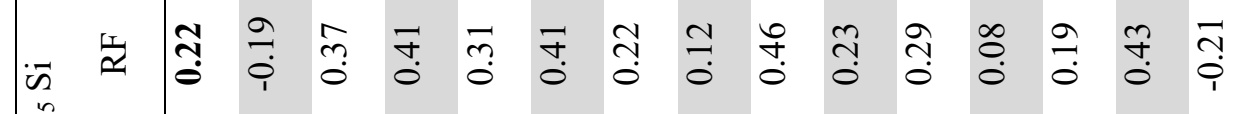

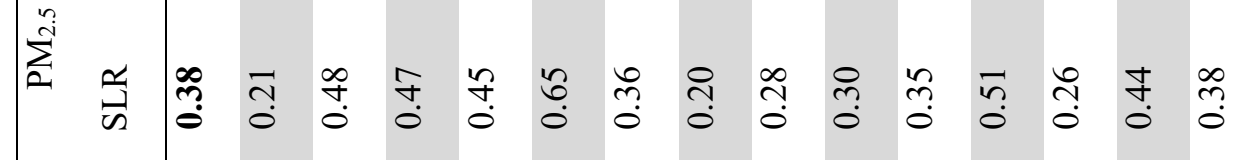

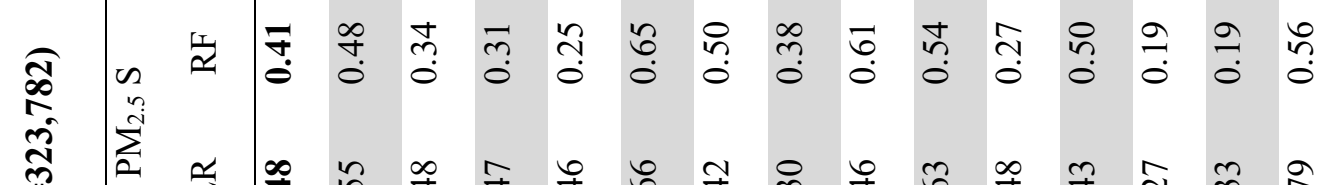

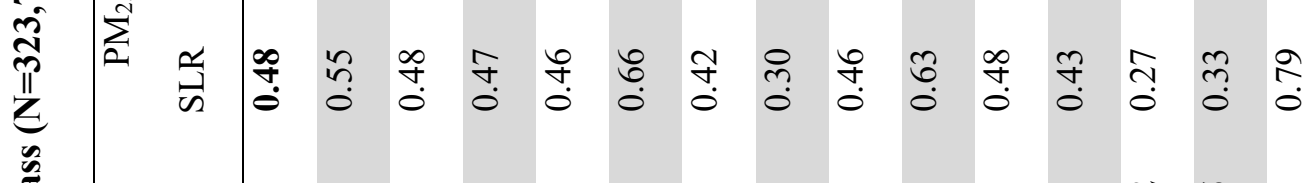

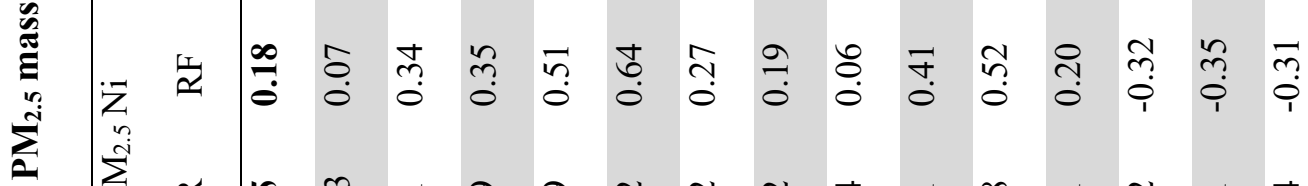

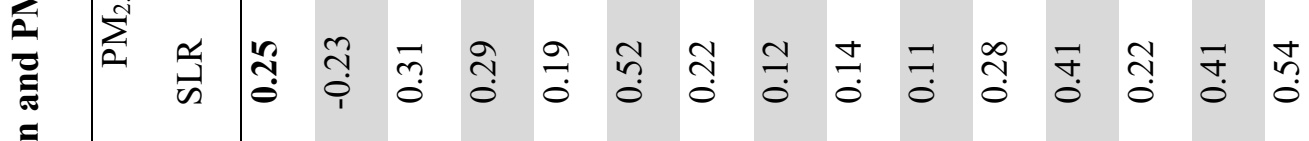

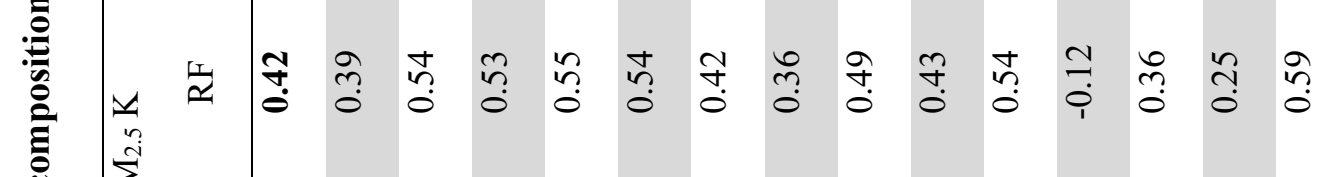

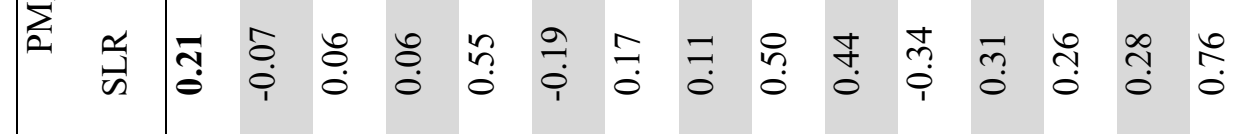

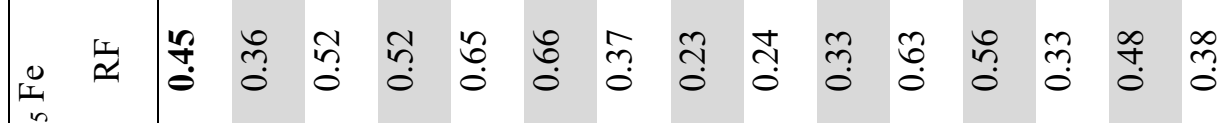

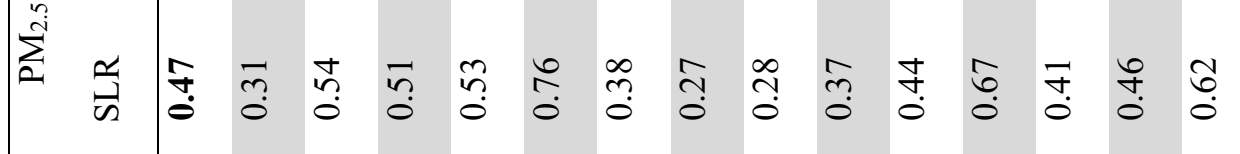

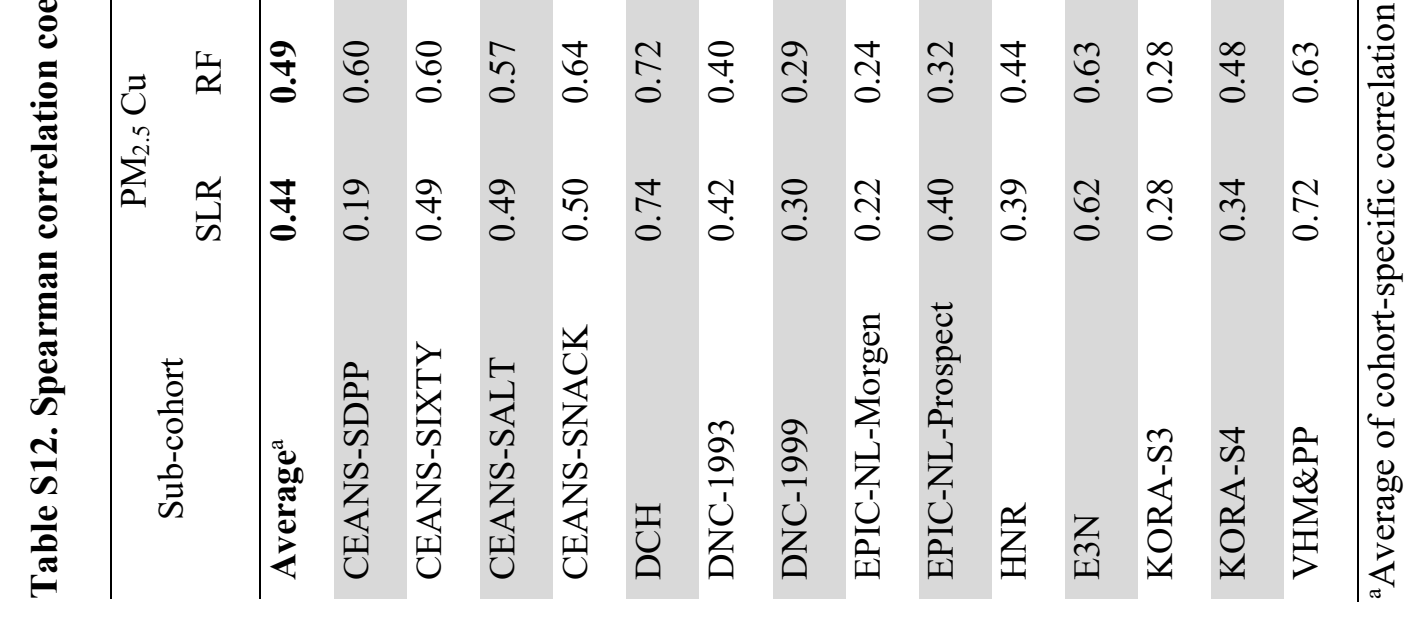

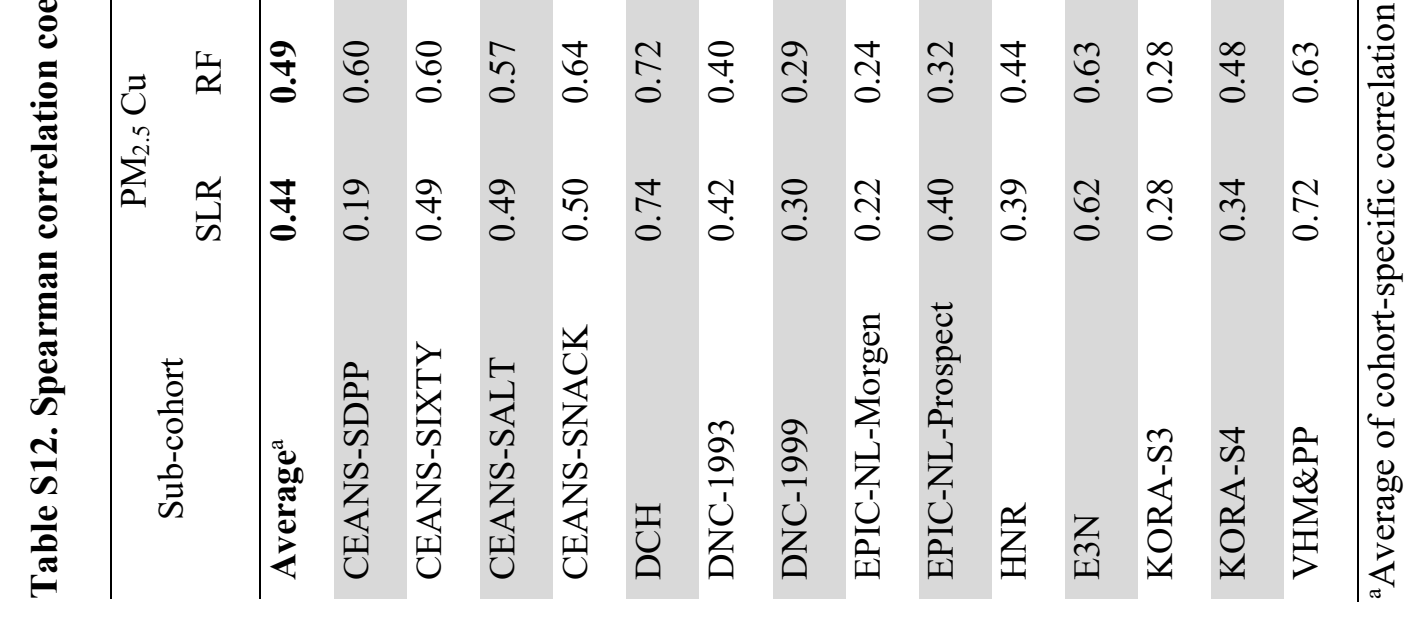

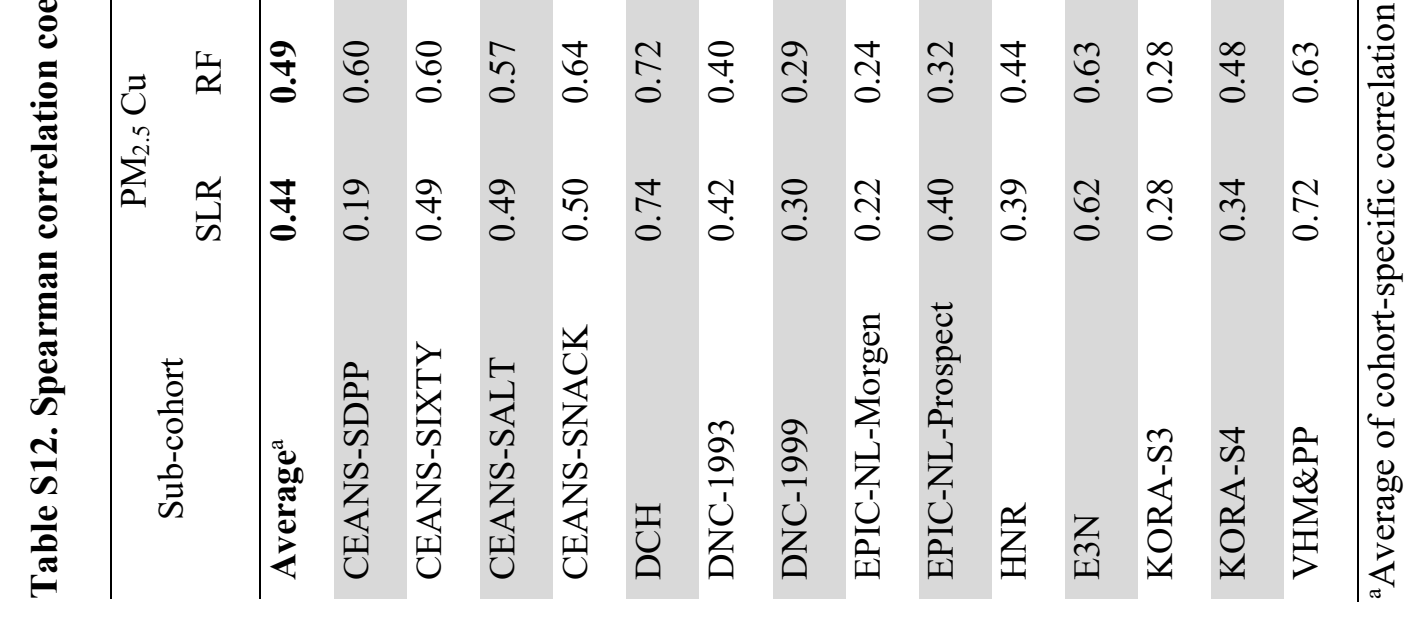

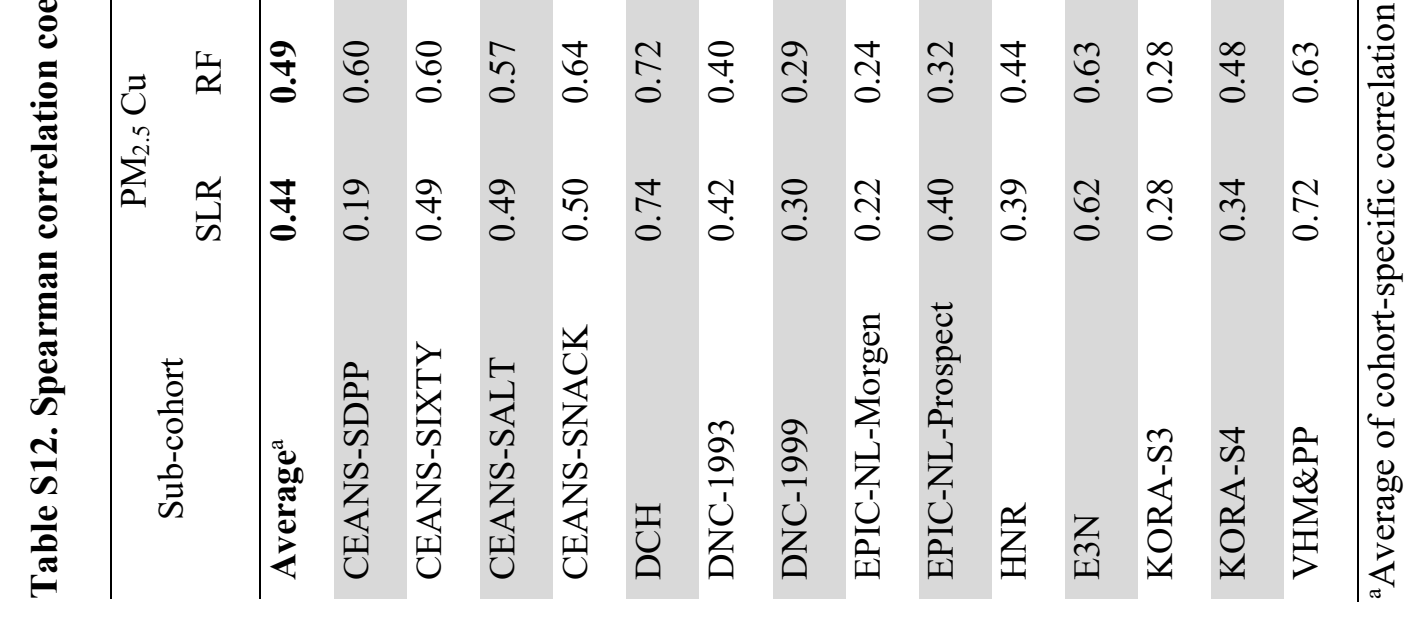




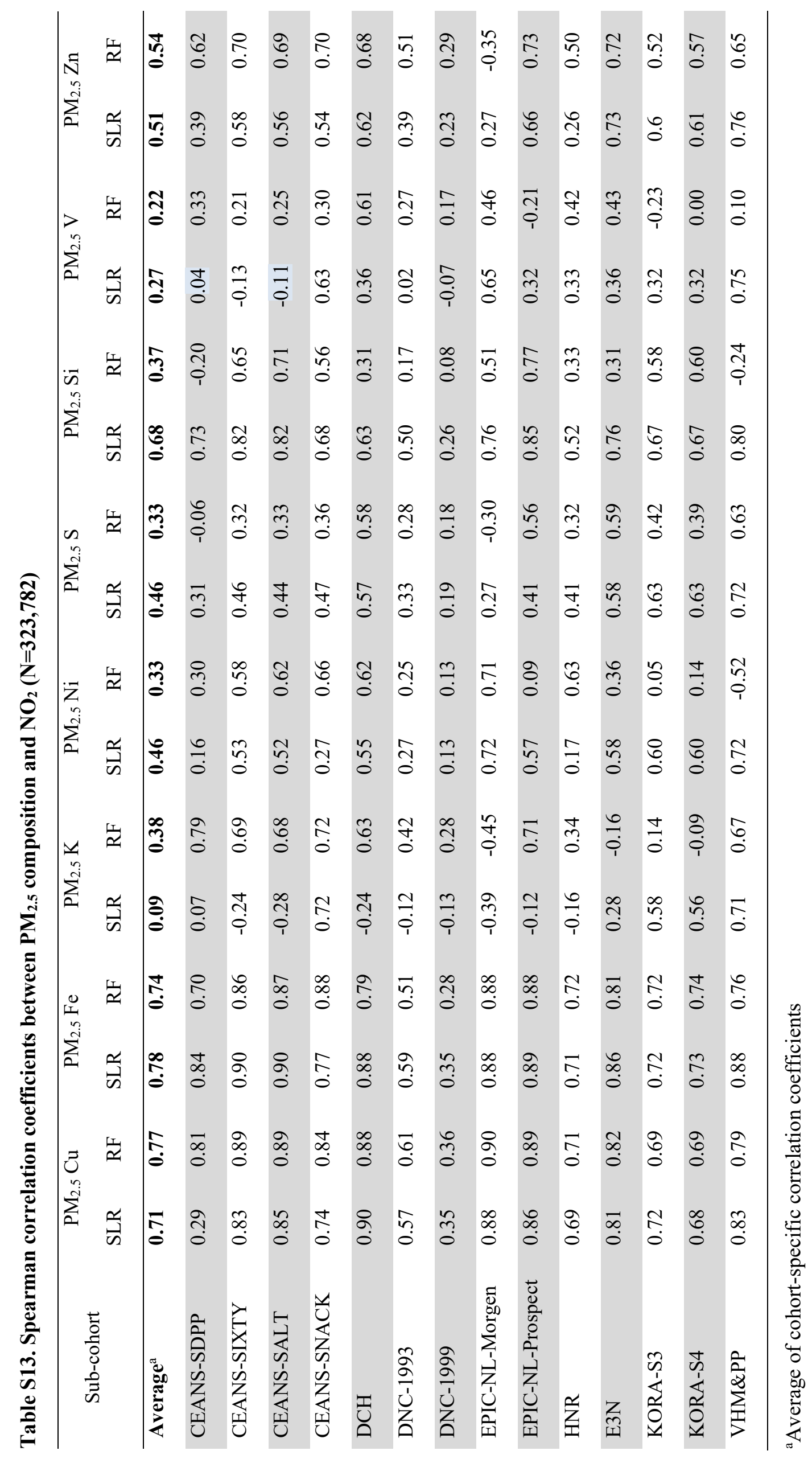




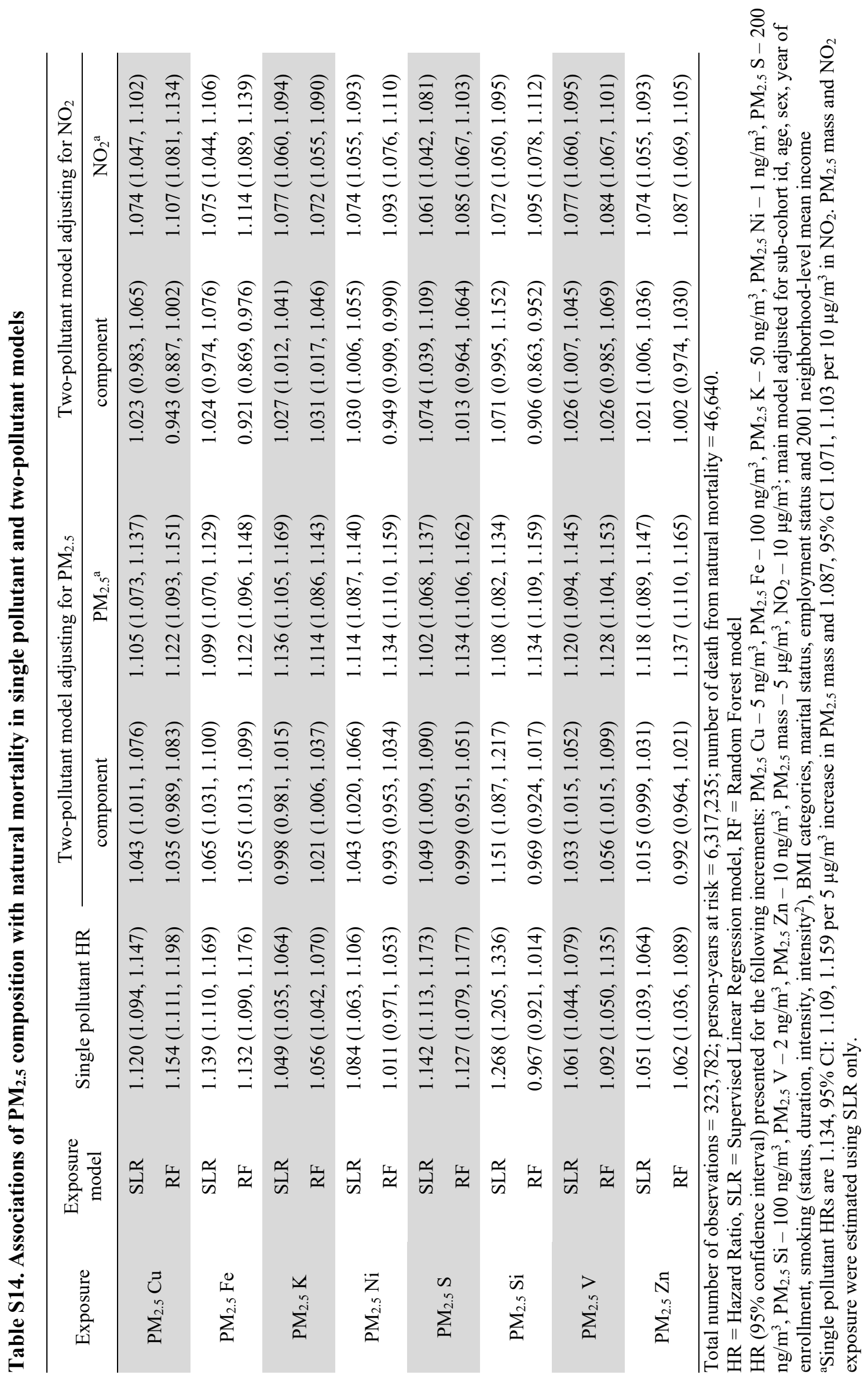




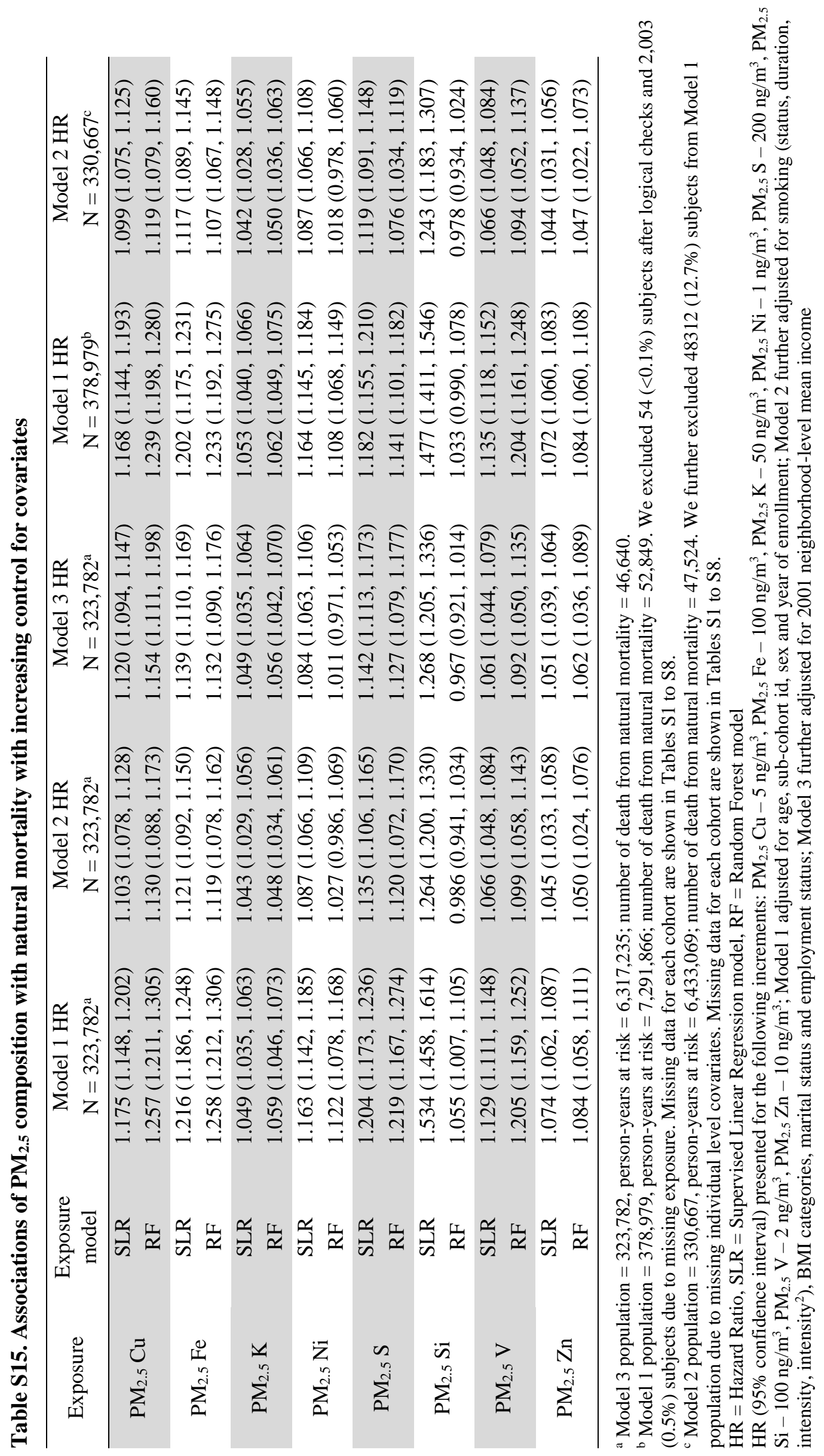




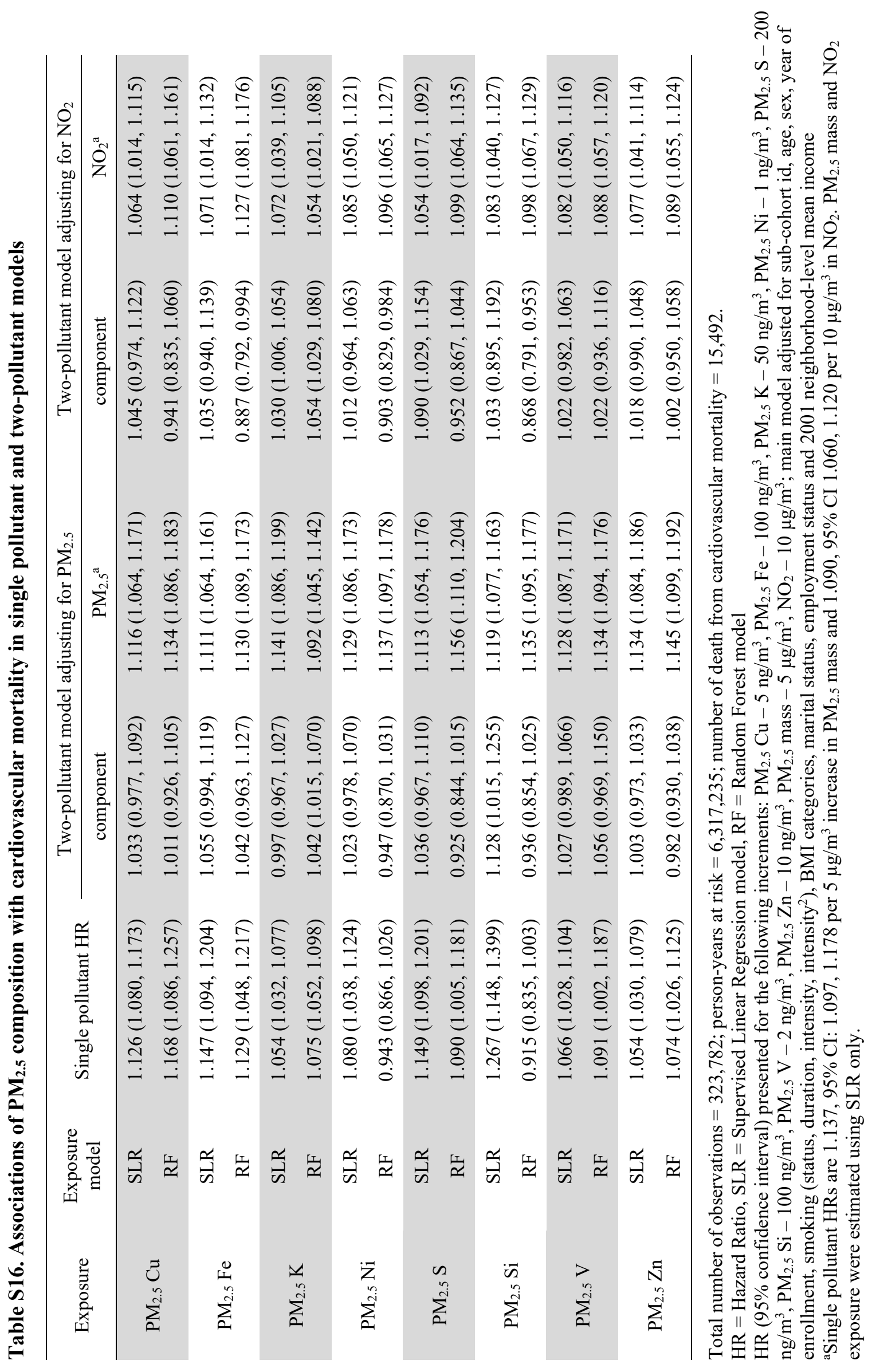




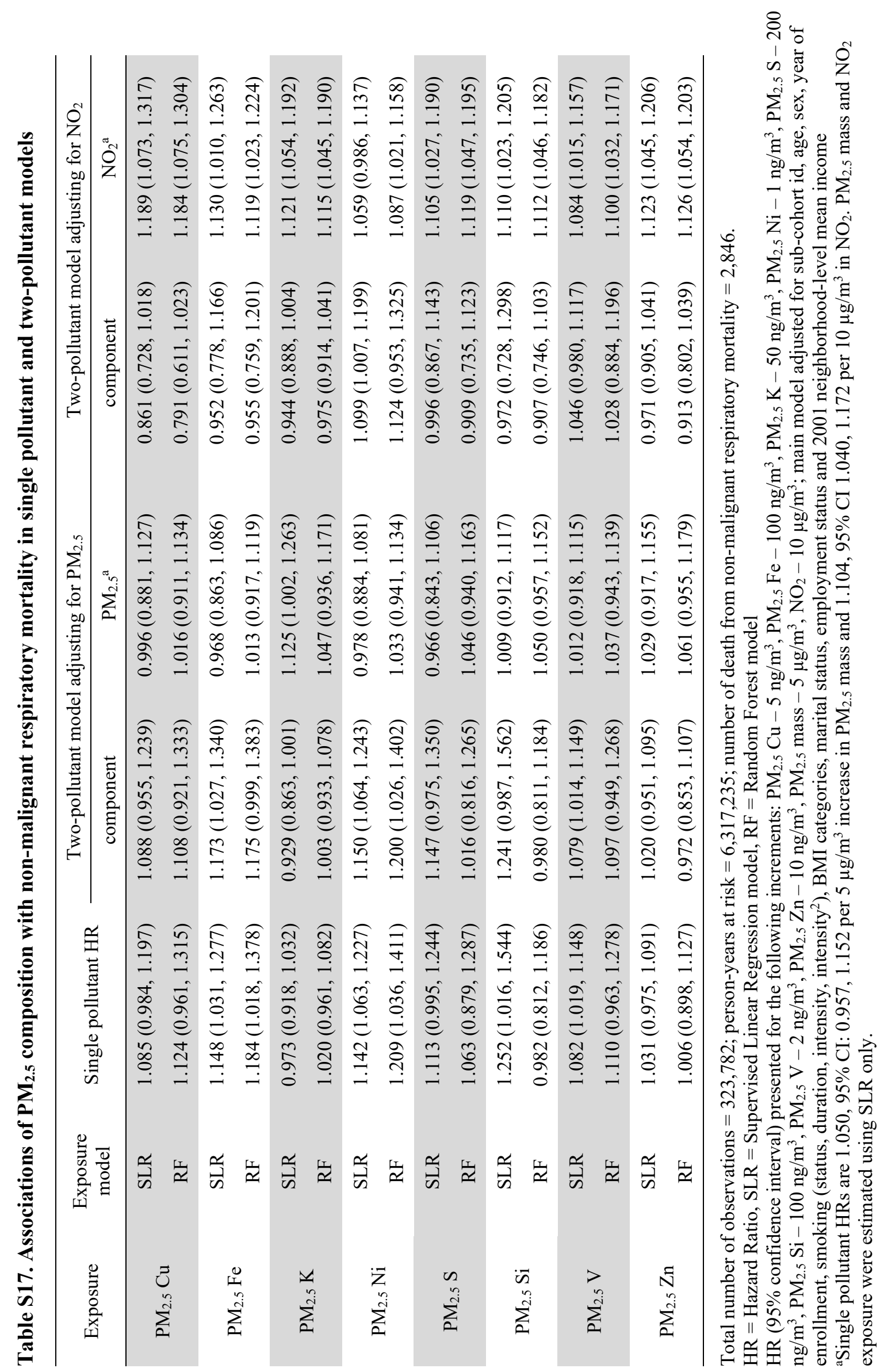




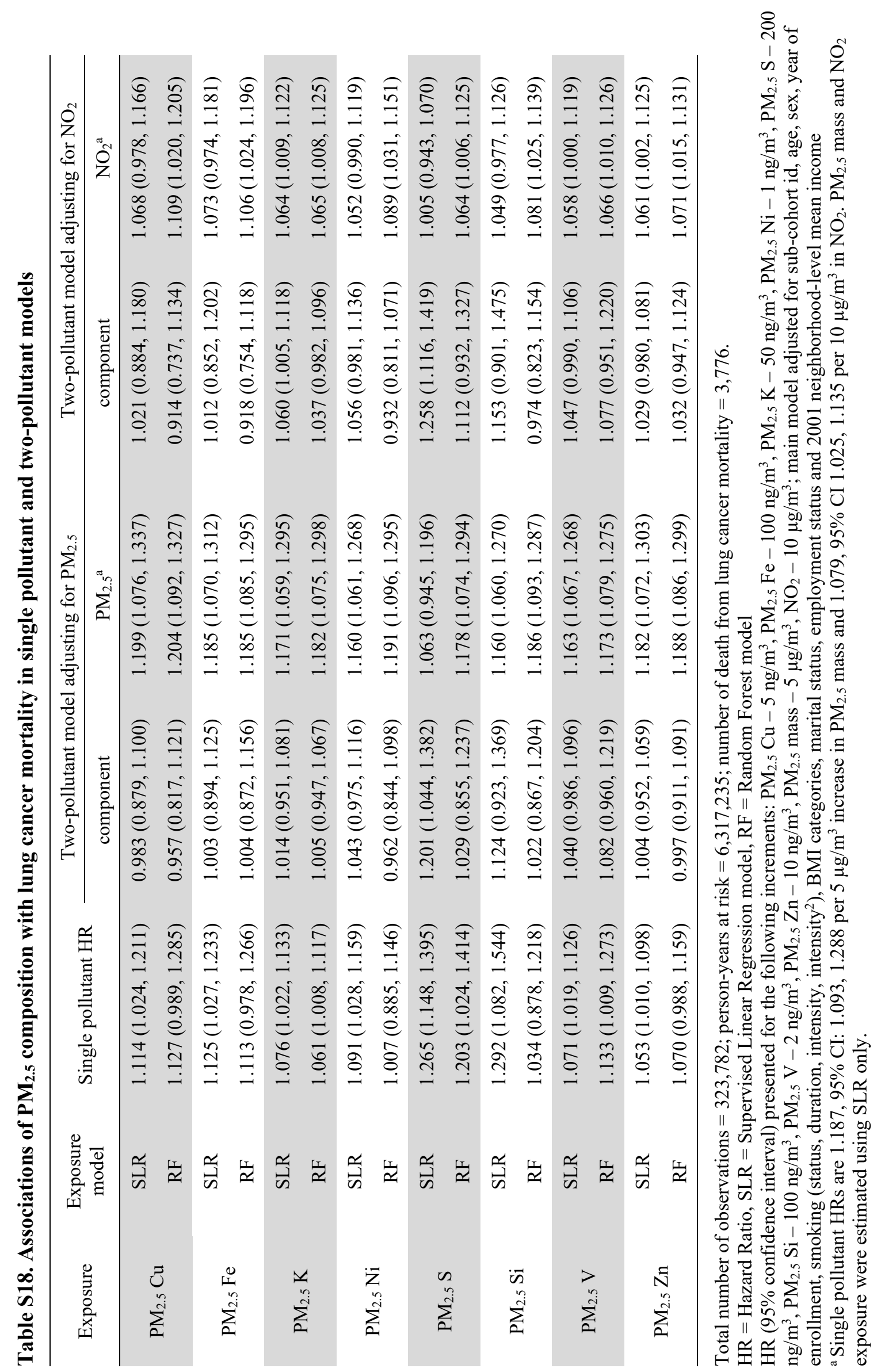




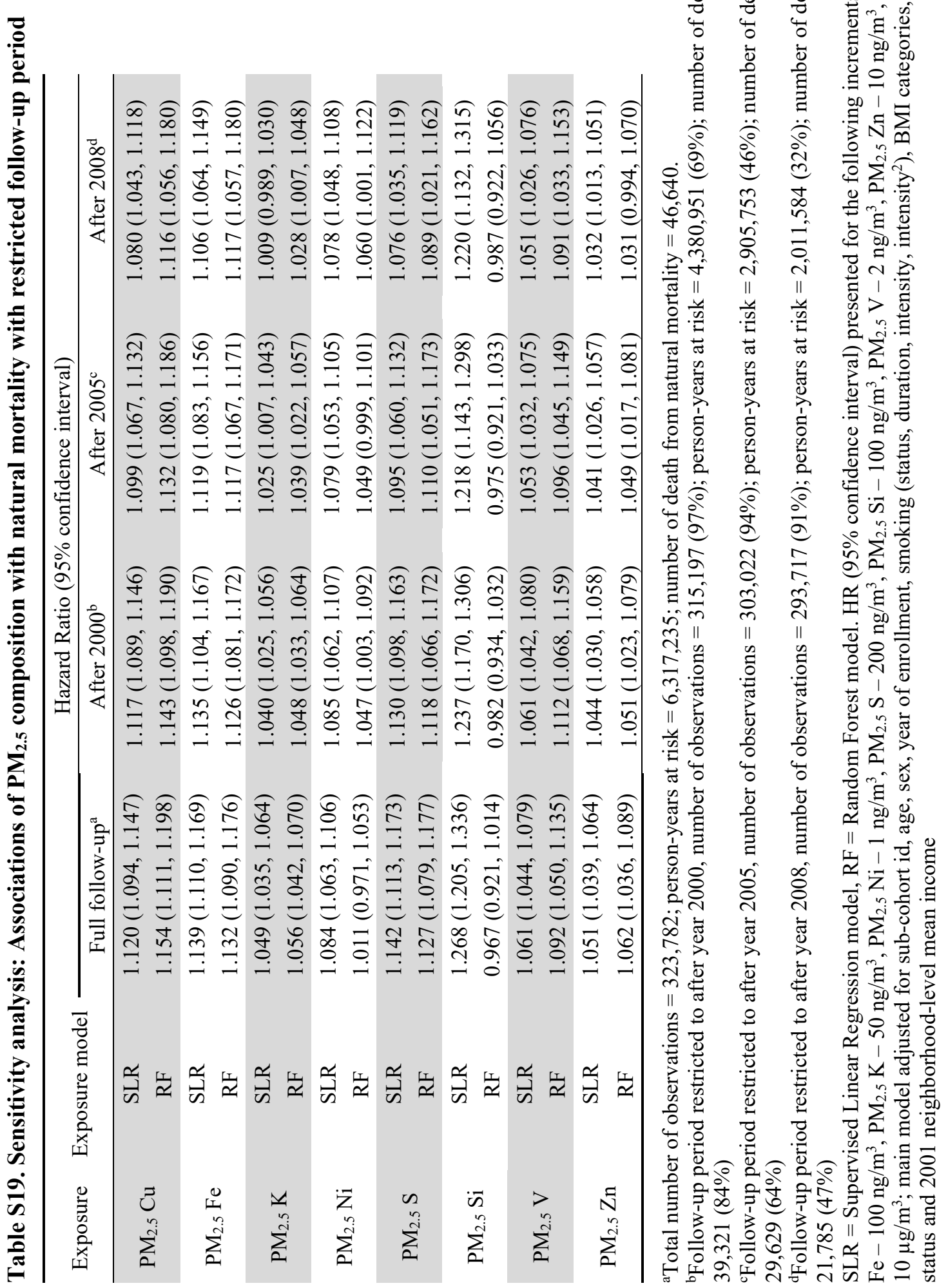




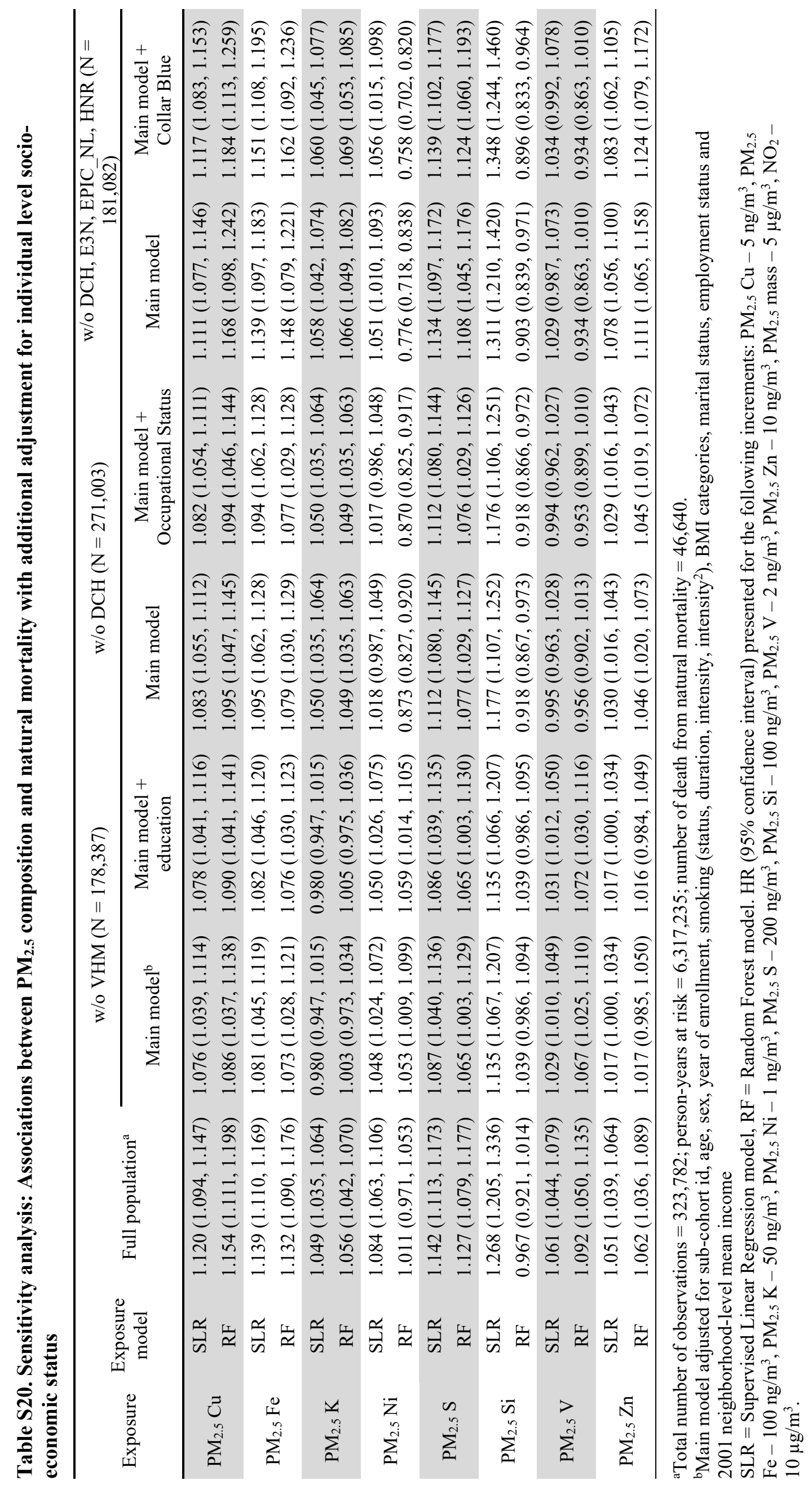




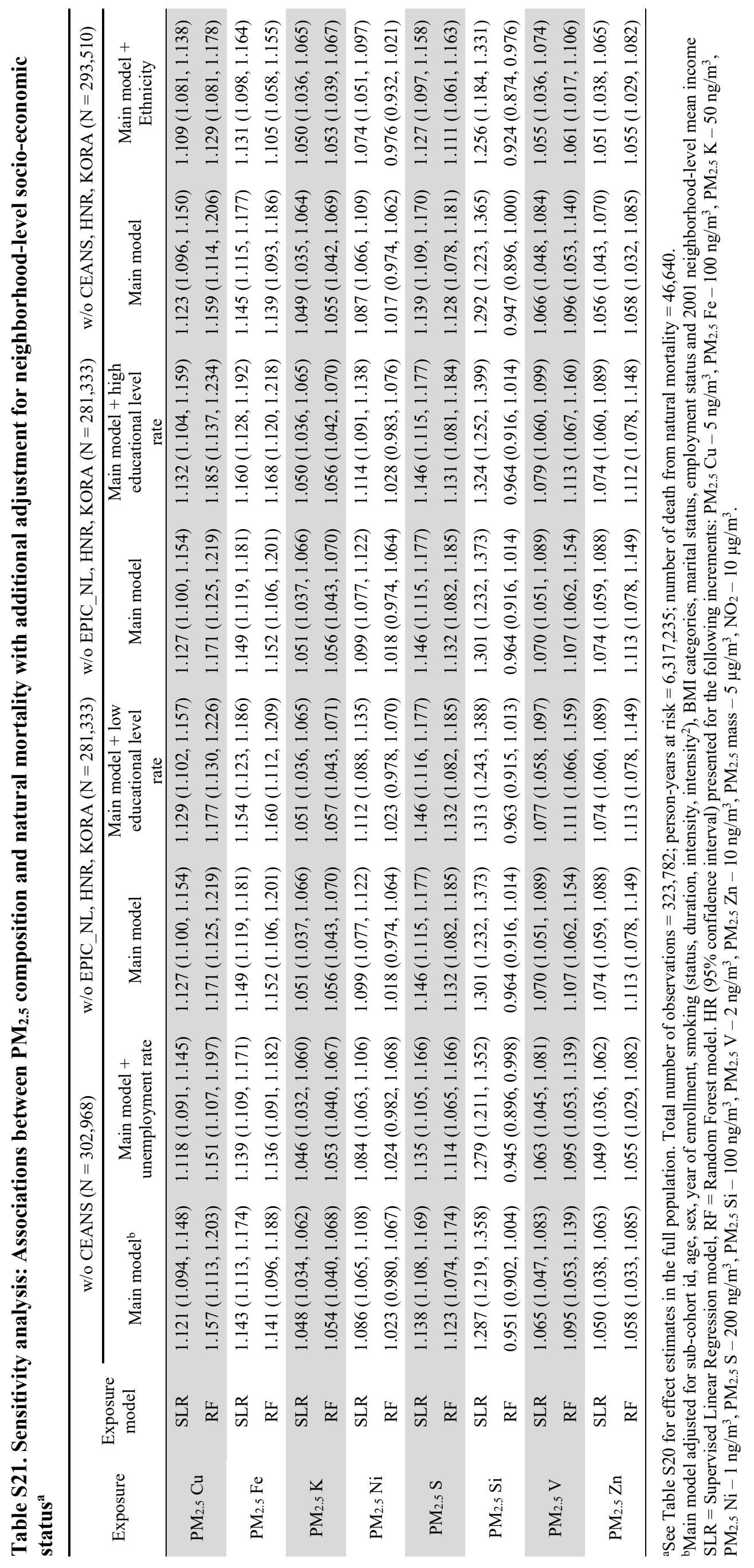




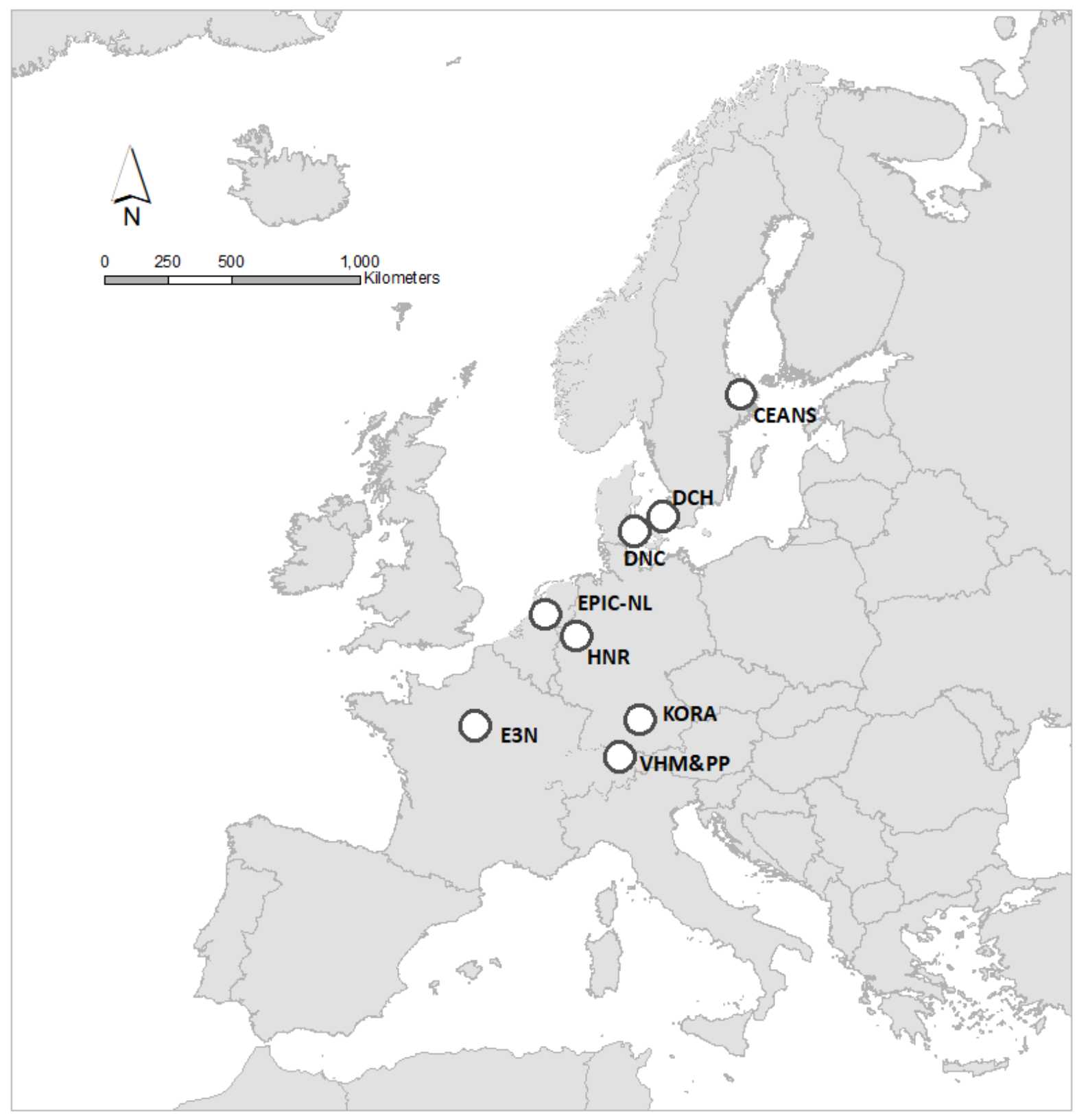

Figure S1. Map of study areas 


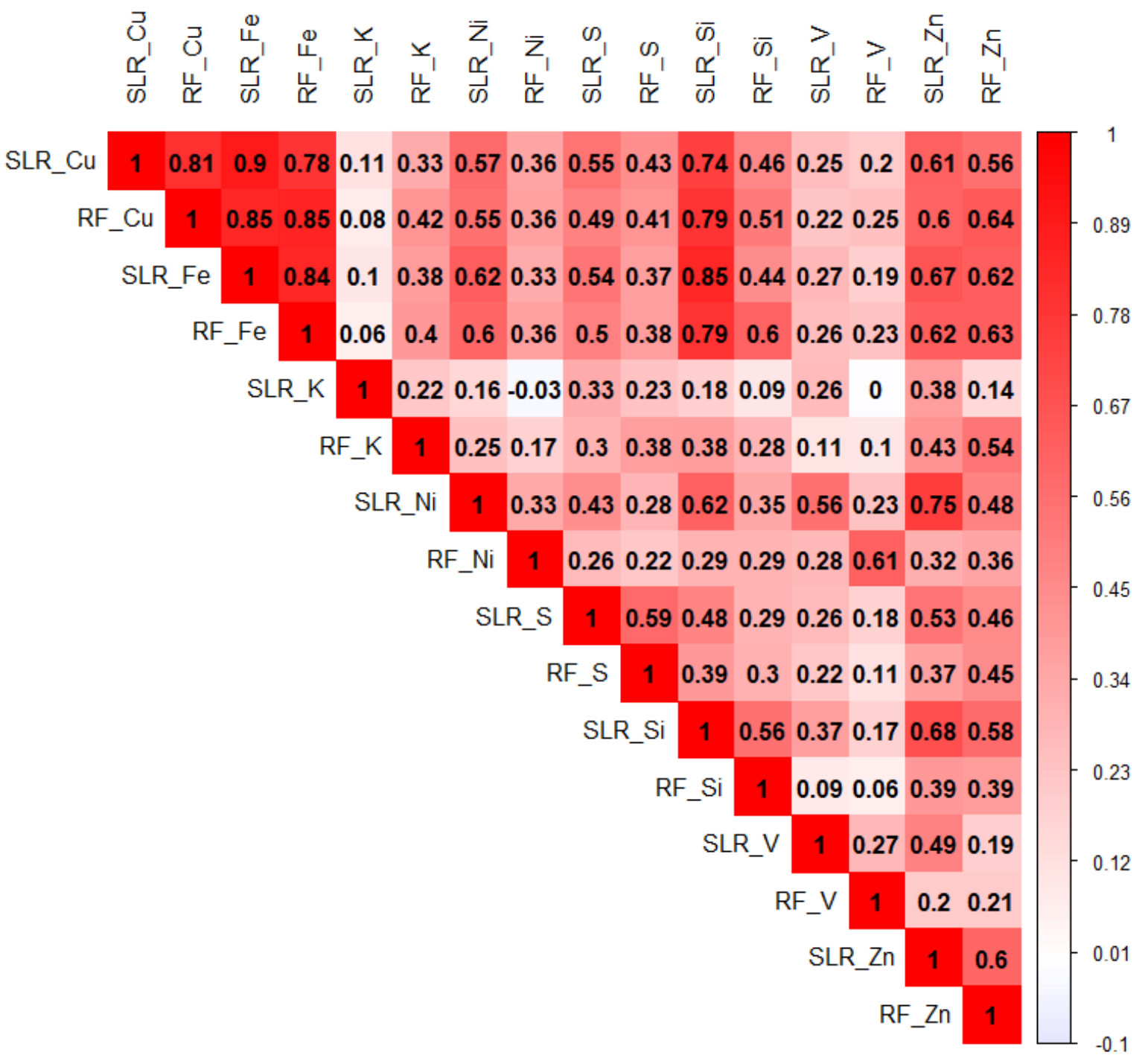

Figure S2. Average of cohort-specific Spearman correlation coefficients between $\mathbf{P M}_{2.5}$ composition 
Supervised Linear Regression
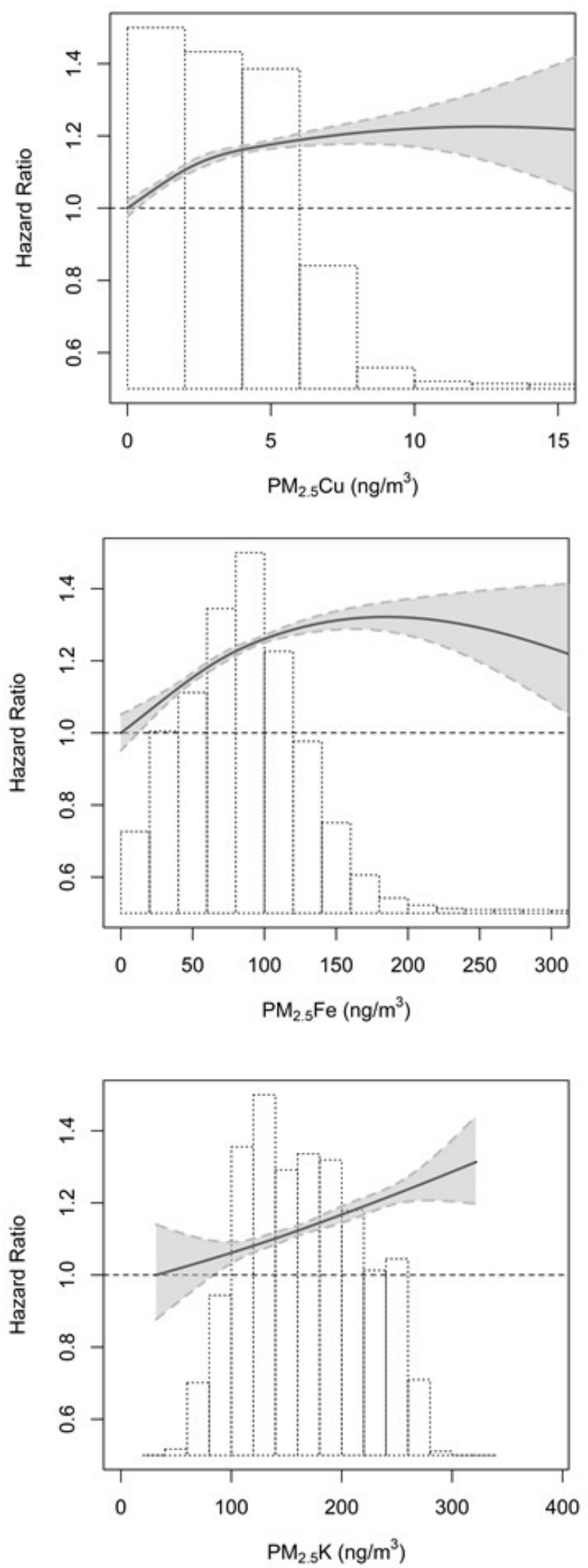

Random Forest
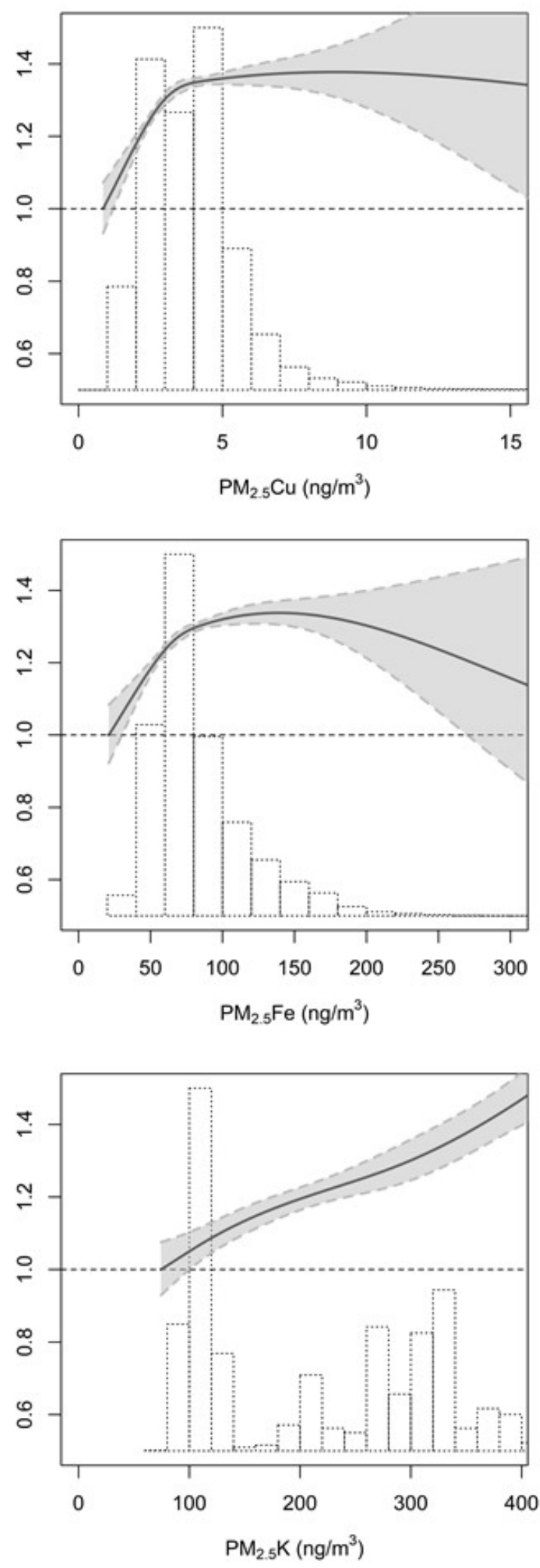

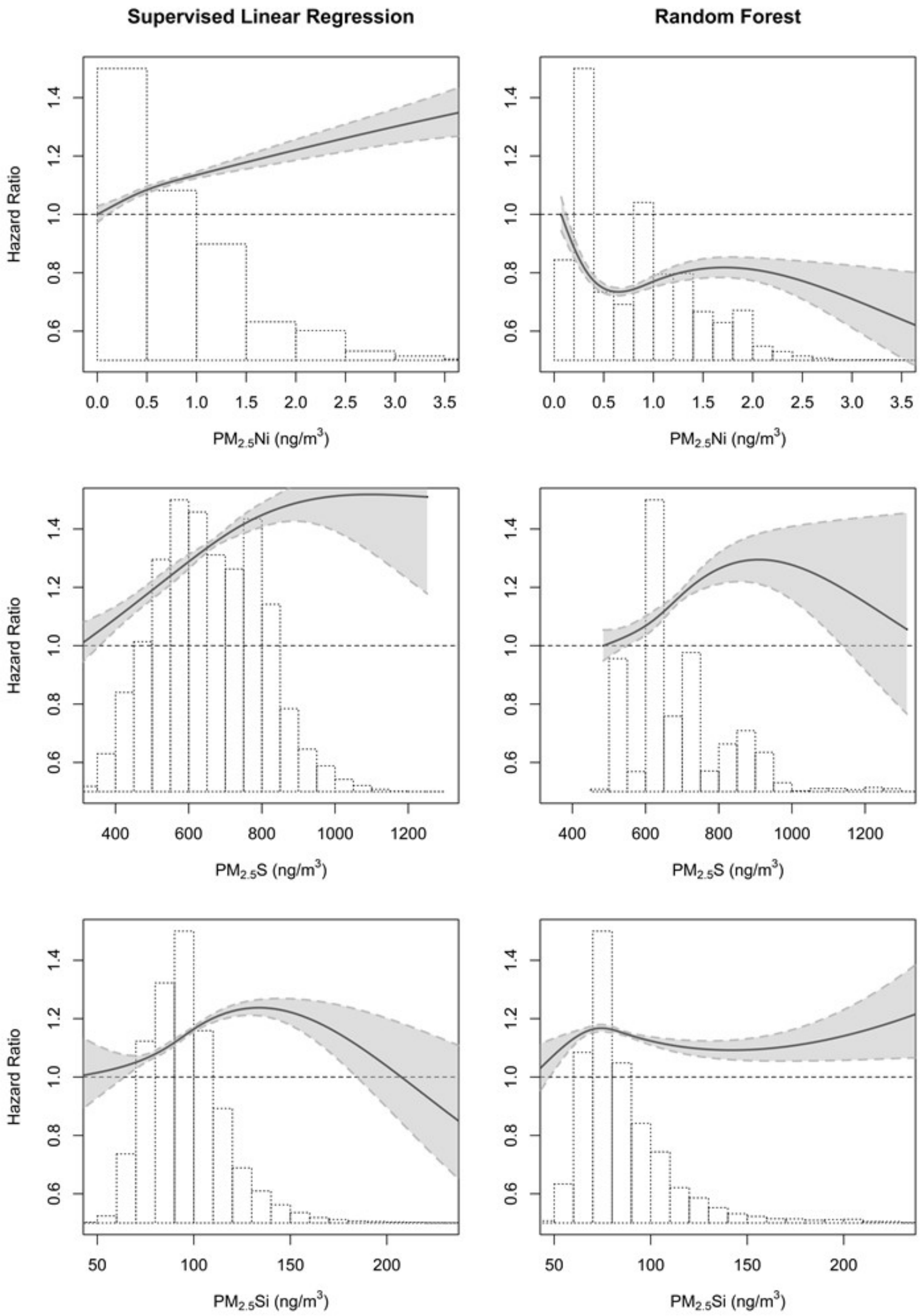

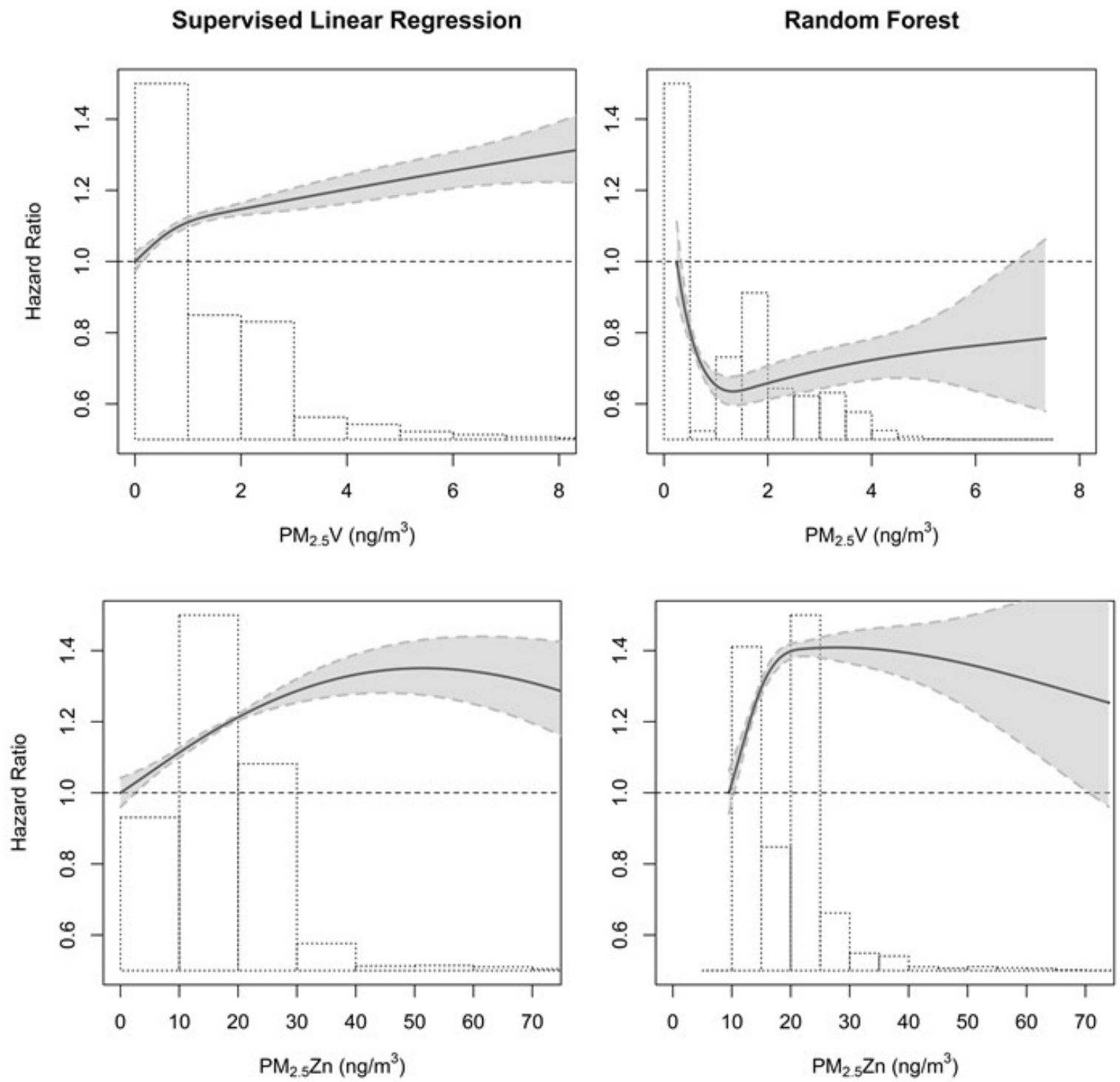

Figure S3. Natural spline (3 degree of freedom) for associations between $\mathbf{P M}_{2.5}$ composition and natural cause mortality

Histogram shows the exposure distribution (exposure distribution for each individual cohort is shown in Figure 1); Shaded: $95 \%$ confidence intervals; Y-axis truncated at 0.5 and 1.5 for all components; Hazard ratios are expressed relative to minimum exposure. 


\section{References}

Beelen R, Raaschou-Nielsen O, Stafoggia M, et al. 2014. Effects of long-term exposure to air pollution on natural-cause mortality: An analysis of 22 european cohorts within the multicentre escape project. The Lancet 383:785-795.

Beulens JW, Monninkhof EM, Verschuren WM, et al. 2010. Cohort profile: The epic-nl study. International journal of epidemiology 39:1170-1178.

Cesaroni G, Forastiere F, Stafoggia M, et al. 2014. Long term exposure to ambient air pollution and incidence of acute coronary events: Prospective cohort study and meta-analysis in 11 european cohorts from the escape project. BMJ 348:f7412.

Chen J, de Hoogh K, Gulliver J, et al. 2020. Development of europe-wide models for particle elemental composition using supervised linear regression and random forest. Environ Sci Technol.

Clavel-Chapelon F, Group ENS. 2015. Cohort profile: The french E3N cohort study. International journal of epidemiology 44:801-809.

Eriksson AK, Ekbom A, Granath F, et al. 2008. Psychological distress and risk of pre-diabetes and type 2 diabetes in a prospective study of swedish middle-aged men and women. Diabetic medicine : a journal of the British Diabetic Association 25:834-842.

Holle R, Happich M, Lowel H, et al. 2005. Kora--a research platform for population based health research. Gesundheitswesen 67 Suppl 1:S19-25.

Hundrup YA, Simonsen MK, Jorgensen T, et al. 2012. Cohort profile: The danish nurse cohort. International journal of epidemiology 41:1241-1247.

Lagergren M, Fratiglioni L, Hallberg IR, et al. 2004. A longitudinal study integrating population, care and social services data. The swedish national study on aging and care (snac). Aging clinical and experimental research 16:158-168.

Lichtenstein P, Sullivan PF, Cnattingius S, et al. 2006. The swedish twin registry in the third millennium: An update. Twin research and human genetics : the official journal of the International Society for Twin Studies 9:875-882.

Raaschou-Nielsen O, Andersen ZJ, Beelen R, et al. 2013. Air pollution and lung cancer incidence in 17 european cohorts: Prospective analyses from the european study of cohorts for air pollution effects (escape). The Lancet Oncology 14:813-822. Schmermund A, Mohlenkamp S, Stang A, et al. 2002. Assessment of clinically silent atherosclerotic disease and established and novel risk factors for predicting myocardial infarction and cardiac death in healthy middle-aged subjects: Rationale and design of the heinz nixdorf recall study. Risk factors, evaluation of coronary calcium and lifestyle. American heart journal 144:212-218

Stafoggia M, Cesaroni G, Peters A, et al. 2014. Long-term exposure to ambient air pollution and incidence of cerebrovascular events: Results from 11 european cohorts within the escape project. Environmental health perspectives 122:919-925.

Tjonneland A, Olsen A, Boll K, et al. 2007. Study design, exposure variables, and socioeconomic determinants of participation in diet, cancer and health: A population-based prospective cohort study of 57,053 men and women in denmark. Scandinavian journal of public health 35:432-441.

Ulmer H, Kelleher CC, Fitz-Simon N, et al. 2007. Secular trends in cardiovascular risk factors: An age-period cohort analysis of 698,954 health examinations in 181,350 austrian men and women. Journal of internal medicine 261:566-576.

Wandell PE, Wajngot A, de Faire U, et al. 2007. Increased prevalence of diabetes among immigrants from non-european countries in 60-year-old men and women in sweden. Diabetes \& metabolism 33:30-36. 



\section{Chapter

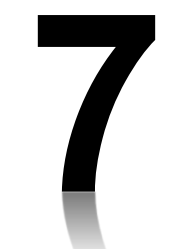

General Discussion 
The main objective of this thesis is to develop Europe-wide land use regression models for key pollutants $\mathrm{PM}_{2.5}, \mathrm{NO}_{2}, \mathrm{O}_{3}$, black carbon (BC) and $\mathrm{PM}_{2.5}$ elemental composition, as well as to evaluate the mortality effects of $\mathrm{PM}_{10}, \mathrm{PM}_{2.5}$ and $\mathrm{PM}_{2.5}$ composition. We compared exposure models developed with different statistical algorithms, and the health effects assessed by applying the different exposure models. This research has benefited from the unique air pollution dataset collected by a purposedesigned monitoring campaign conducted in 2008-2010 in the framework of the European Study of Cohorts for Air Pollution Effects (ESCAPE) ${ }^{1,2}$, and the large population in the Effects of Low-level Air Pollution: A Study in Europe (ELAPSE) pooled cohort with detailed individual-level covariates ${ }^{3}$. This research advances our knowledge on two of the current challenges in air pollution epidemiology: the health effects of air pollution at low concentrations and the most responsible components within PM for the adverse health effects. A systematic review of the literature of long-term exposure to PM air pollution and mortality was conducted to assess the current state of knowledge and assess research challenges.

This chapter begins with a summary of the main findings in this thesis. Then methodological issues related to confidence assessment of epidemiological evidence in systematic reviews, spatial scale and statistical algorithms for developing exposure models, existing evidence on health effects of long-term PM composition exposure, and the implications of this research are discussed.

\section{Main findings}

In Chapter 2, we systematically reviewed the existing evidence on associations of long-term exposure to $\mathrm{PM}_{10}$ and $\mathrm{PM}_{2.5}$ with natural-cause and cause-specific mortality. We identified 107 studies, of which 104 were cohort studies. The majority of studies were conducted in North America $(\mathrm{N}=62)$ and Europe $(\mathrm{N}=25)$. We performed meta-analysis to derive a combined risk ratio (RR) of 1.08 (95\% CI 1.06, 1.09) per $10 \mu \mathrm{g} / \mathrm{m}^{3}$ for $\mathrm{PM}_{2.5}$ and natural-cause mortality. Substantial heterogeneity was found across studies, probably related to differences in study populations, methodology, exposure level and composition of particles. Meta-analyses of studies conducted at low mean $\mathrm{PM}_{2.5}$ levels $\left(<25,20,15,12,10 \mu \mathrm{g} / \mathrm{m}^{3}\right.$ respectively) yielded RRs that were similar or higher compared to the overall RR, consistent with the finding of generally linear or supra-linear concentration-response functions (CRFs) in individual studies. There is evidence that associations between $\mathrm{PM}_{2.5}$ and increased mortality risk remained below the current EU limit exposure level $\left(25 \mu \mathrm{g} / \mathrm{m}^{3}\right)^{4}$, the US Environmental Protection Agency (EPA) National Ambient Air Quality Standards (NAAQS; $\left.12 \mu \mathrm{g} / \mathrm{m}^{3}\right)^{5}$, and the World Health Organization (WHO) air quality guideline value $\left(10 \mu \mathrm{g} / \mathrm{m}^{3}\right)^{6}$. Studies at low concentrations were mostly performed in North-America.

The ELAPSE project is conducted to provide new empirical evidence on associations between lowlevel air pollution and adverse health effects in Europe. In order to assess air pollution exposure for ELAPSE cohort participants, Europe-wide hybrid land use regression (LUR) models for 2010 annual mean concentrations of $\mathrm{PM}_{2.5}, \mathrm{NO}_{2}, \mathrm{BC}$ and $\mathrm{O}_{3}$ at $100 \times 100 \mathrm{~m}$ resolution were developed in Chapter 3 . The models were developed with a supervised linear regression (SLR) incorporating satellite observations, chemical transport model estimates, land use and traffic data as potential predictor variables. Kriging was performed to further explain spatial variations in residuals of the LUR models. Kriging significantly improved the $\mathrm{PM}_{2.5}$ and $\mathrm{O}_{3}$ but not the $\mathrm{NO}_{2}$ and $\mathrm{BC}$ models. The $\mathrm{PM}_{2.5}, \mathrm{NO}_{2}, \mathrm{O}_{3}$ warm season and $\mathrm{BC}$ models explained $66 \%, 58 \%, 60 \%$ and $51 \%$ of the variability in measured concentrations in five-fold cross-validation, respectively. The spatial structures of the models were stable in time at the West-European scale, allowing for application in long-term epidemiological studies with cohorts started one or two decades ago.

In view of the increasing number of air pollution exposure assessment studies exploring application of sophisticated and complex statistical algorithms beyond ordinary linear regression, we compared 16 different algorithms (linear regression, regularization and machine-learning algorithms) to develop 
Europe-wide $\mathrm{PM}_{2.5}$ and $\mathrm{NO}_{2}$ models in Chapter 4, using the same air pollution measurements and potential predictor variables used in Chapter 3 . We observed similar performance across algorithms evaluated by both five-fold cross-validation and external validation. The predicted concentrations from all algorithms at external validation sites were highly correlated $\left(\mathrm{R}^{2}>0.85\right)$.

In Chapter 5, we developed Europe-wide LUR models for eight a priori selected elemental components in $\mathrm{PM}_{2.5}$ - copper $(\mathrm{Cu})$, iron $(\mathrm{Fe})$, potassium $(\mathrm{K})$, nickel $(\mathrm{Ni})$, sulfur $(\mathrm{S})$, silicon $(\mathrm{Si})$, vanadium (V) and zinc (Zn), using supervised linear regression (SLR) and random forest (RF), a machine-learning algorithm. The models performed moderate to well across Europe, explaining $41 \%$ to $90 \%$ of the overall variability in measured concentrations in five-fold cross-validation. RF consistently outperformed SLR to explain overall concentration variability in the validation. Models explained within study area variation much less than the overall variation, with similar performance for RF and SLR for the within study variation. Maps proved a useful additional tool to evaluate the plausibility of model predictions beyond cross-validation statistics. Models differed substantially between elements in terms of the major predictor variables, broadly reflecting known sources. Agreement between the two algorithm predictions was high at the overall European level for $\mathrm{Cu}, \mathrm{K}, \mathrm{S}$ and $\mathrm{Zn}(\mathrm{r}>0.7)$, and varied substantially at the national level.

In Chapter 6, we assessed associations between $\mathrm{PM}_{2.5}$ elemental composition and mortality in the ELAPSE pooled cohort $(\mathrm{N}=323,782)$. We applied exposures derived from both supervised linear regression (SLR) and random forest (RF) models developed in Chapter 5 and compared their relation with health outcomes. All SLR-modeled components were statistically significantly associated with natural-cause mortality in single pollutant models with hazard ratios (HRs) from 1.05-1.27. Similar HRs were observed for RF-modeled $\mathrm{Cu}, \mathrm{Fe}, \mathrm{K}, \mathrm{S}, \mathrm{V}$ and $\mathrm{Zn}$ with wider confidence intervals (CIs). For both SLR- and RF-modeled V, HRs attenuated but remained above unity and (borderline) significant after adjustment for $\mathrm{PM}_{2.5}$ mass or $\mathrm{NO}_{2}$. The $\mathrm{HRs}$ for V were 1.03 (95\% CI: 1.02, 1.05) and $1.06(95 \%$ CI: 1.02, 1.10) for SLR- and RF-modeled exposures, respectively, per $2 \mathrm{ng} / \mathrm{m}^{3}$ adjusting for $\mathrm{PM}_{2.5}$ mass. Associations for the other components were weaker for exposure modeled with RF than SLR in twopollutant models.

\section{Risk of bias and certainty of evidence assessments in systematic reviews}

Risk of bias assessment tools are increasingly used in systematic reviews for appraisal of individual studies. The optimal method for assessing risk of bias within individual observational studies is currently an active area of research with no clear consensus ${ }^{7-9}$. Different risk of bias assessment tools include different bias domains and/or define the same domains differently. Risk of bias assessments of individual studies are part of evidence synthesis, and act as a domain for downgrading the overall quality of evidence in the GRADE (Grading of Recommendations Assessment, Development and Evaluation) system $^{10}$. The GRADE approach is a framework for rating the overall certainty of evidence in the collection of systematically reviewed studies (a 'body of evidence'). There are other frameworks currently being used by government and public health agencies for certainty assessment in evidence synthesis. For example, the International Agency for Research on Cancer (IARC) goes beyond direct evidence from epidemiologic studies to come to a final assessment regarding human carcinogenicity ${ }^{11}$. IARC synthesizes evidence from epidemiologic studies, animal studies, pharmacokinetic/mechanistic experiments, and surveys of human exposure. Likewise, the US EPA considers evidence across disciplines including atmospheric science, toxicology, epidemiology, and aquatic and terrestrial ecology, to develop an Integrated Science Assessment (ISA) for the criteria air pollutants ${ }^{12}$.

In the systematic review conducted in Chapter 2, we applied a domain-based risk of bias assessment too $^{13}$ and an adapted GRADE framework (see online Appendix 6a of Chapter 2: https://ars.elscdn.com/content/image/1-s2.0-S0160412020319292-mmc7.docx) that were specifically developed for the review purpose coordinated by the WHO. The intention of applying these assessment tools is to 
increase transparency and replicability, and to limit subjectivity in the evaluations. However, we struggled with applying these assessment tools when conducting the systematic review. Concerns have been raised regarding the implementation of these tools in the context of environmental epidemiological studies $^{14,15}$.

One major concern is the mechanistic application of these standardized algorithms without clear explanation of the reasoning behind the judgements ${ }^{14}$. Assessment of study validity is complex, and it requires substantive knowledge of the topic being examined. Mechanistically checking a list of study attributes without a thoughtful and informative guidance by subject-matter expertise is likely to yield erroneous conclusions and may dismiss important evidence. Algorithmic assessments of the same studies with different tools can result in opposite conclusions ${ }^{16}$. Therefore, in my opinion, the main value of risk of bias assessment derives from the clear reasoning behind the judgements, which inherently depends on subjective expert evaluation. To prevent purely mechanistic assessments, two suggestions are given below:

First, to identify the critical domains that have potential to introduce bias before conducting risk of bias assessments. The vulnerable aspects of a particular research topic are usually well-known to experts in that field and thus scrutiny should be focused on these domains. For example, misclassification in outcome measurement is unlikely to play a major role in mortality studies because mortality data are often derived from death registries and the causes of death are coded according to the international classification of diseases (ICD) typically using broad groups of causes (e.g., natural-cause, cardiovascular, respiratory). Whereas in morbidity studies, outcome measurement should be more carefully examined depending on the source of incidence data (e.g, self-reports, hospital discharge records), because the date of disease onset is not always well documented. The different data sources may introduce different biases and thus contribute to heterogeneity of effect estimates across studies.

Second, to characterize the direction, magnitude and relative importance of individual biases. Risk of bias tools typically only determine whether a risk for specific biases occurs without quantifying it. Furthermore, the tools assess risk of bias, not whether the bias actually occurs. Characterizing the biases helps to provide an informative summary and assessment when evaluating the evidence in an individual study or in a set of studies. For example, if an elevated risk is found and only classical nondifferential measurement errors in the exposure variables are present, we will still have confidence in the evidence of a positive association because these errors generally bias effect estimates towards null. If biases compensate for one another when one tends to produce an upward bias and the other produces a downward bias, confidence in the evidence for a positive association is still supported ${ }^{17}$. Identifying which of the potential biases are most influential also helps to give clear guidance for optimizing future studies on a topic.

Another major concern is that most risk of bias assessment tools and the GRADE framework consider hypothetical randomized controlled trials (RCTs) as ideal study ${ }^{15}$. This is because these tools are initially implemented for evaluating evidence to develop clinical guidelines ${ }^{18,19}$. As a result, observational studies are considered to produce evidence of a lower certainty compared to RCTs in the GRADE framework, and some of the domains in the risk of bias assessment tools are not applicable to observational studies.

Although RCTs are typically considered the strongest study design in epidemiology because of its ability to eliminate or strongly reduce confounding ${ }^{20}$, it is unethical and often impossible to randomize people to potentially harmful exposures with no perceived benefits in the context of environmental epidemiology. Besides, RCTs would have to involve impossibly long follow-up periods and large sample sizes to have sufficient statistical power for observing chronic disease onset or rare outcomes ${ }^{21}$. Moreover, as participants in RCTs often differ from the general population, the results of an RCT may not be generalizable. Observational studies, in contrast, are conducted in real life and thus - when based on random samples of the target population - reflect the nature of population-level exposures ${ }^{22}$. Outcomes with long latencies can be evaluated with long follow-up time, which is possible both 
prospectively in cohort studies and retrospectively in case-control studies. Furthermore, a series of sensitivity analyses is usually done in observational studies to ensure the robustness of findings and for critical appraisa ${ }^{23}$. Observational studies focus on characterizing concentration-response relationships, rather than simply comparing the outcome between exposed and unexposed groups as in RCTs. Given the challenges in conducting and interpreting RCTs in the context of environmental epidemiology, I argue, evidence from observational studies should not automatically be considered as weaker than evidence from RCTs.

One domain for downgrading the overall certainty of evidence in the GRADE framework is inconsistency/heterogeneity. However, heterogeneity is present in observational epidemiologic studies by nature because of factors such as different populations, exposure assessment methods, pollution mixtures, time periods, geographic locations or study designs. In fact, apparently conflicting results from epidemiological studies do not necessarily indicate that some are false positives or false negatives ${ }^{24}$. This might, for example, reflect differences in exposure levels or susceptibility of the populations. On the contrary, consistent findings from environmental epidemiological studies conducted in different populations under different study designs should be considered as a domain for upgrading the overall certainty of evidence, because it is unlikely that the same biases will occur in multiple studies with different underlying patterns of confounding. For example, it is more convincing that associations between respiratory symptoms and air pollution from truck traffic were observed in different locations across the world. The associations found in Western Europe and North America were not different from those found in other parts of the world ${ }^{25}$.

Statistical methodology is not included as a domain in the risk of bias assessment tools but is important for observational studies. Observational studies generally use models to assess the relationships between exposure and outcome and to control for potential biases. Misuse of models or violation of statistical assumptions may lead to biased or erroneous results. A methodological study conducted within the American Cancer Society cohort suggested failure to account for spatial autocorrelation can lead to an understatement of the uncertainty of the air pollution association with mortality ${ }^{26}$. Other concerns include over-adjustment for covariates, adjusting for causal intermediates, incorrect assignment of exposure ${ }^{27}$. Adding the statistical methodology domain may make it possible to infer the direction of resulting biases ${ }^{15}$.

To conclude, RCTs should not be considered as ideal study design when studying environmental exposures. The risk of bias and certainty of evidence assessment tools need to be adapted for better evaluation of observational studies. Mechanistic application of the tools is not informative for assessment of individual studies or the body of evidence. Clear reasoning behind the judgements is needed. The less mechanistic frameworks provided by IARC and the US EPA for evidence assessment provide a better starting point, particularly as these frameworks integrate assessments from more disciplines than epidemiology.

\section{Air pollution exposure model development}

\section{Spatial scale}

Air pollution exposure models with different spatial scales are developed to meet the requirements of the epidemiological studies. In chapter 3 and chapter 5, we developed Europe-wide LUR models for application in the ELAPSE project. ELAPSE is a multi-center project involving collaboration of cohorts from eleven countries across Europe ${ }^{3,28-31}$. One advantage of applying harmonized exposure models in such international studies is the consistency of exposure assessment methodology. Applying different exposure assessment approaches may affect conclusions drawn in epidemiological studies ${ }^{32}$, and may affect interpretations of multi-pollutant analyses if different exposure methods were applied for different pollutants in the same study ${ }^{33}$. Similarly, large-scale national or continental exposure models at a fine resolution have been developed previously to provide consistent exposure estimates for large 
study populations in North-American studies ${ }^{34,35}$. In the global studies such as the Global Burden of Diseases (GBD) assessment ${ }^{36}$ and the International Study of Asthma and Allergies in Childhood $(\text { ISAAC })^{37}$, global air pollution exposure models were used. Models applied in the global projects are usually derived from satellite estimates at a coarse resolution (e.g., $\left.0.1^{\circ} \times 0.1^{\circ}, \sim 10 \times 10 \mathrm{~km}\right)^{38-40}$. Although using models at a coarse resolution might be sufficiently accurate for pollutants that have relatively small spatial variability such as long-range transported sulfate ${ }^{41,42}$, it might introduce large uncertainties and potentially bias epidemiological associations when estimating exposure to pollutants that vary considerably at local scale (e.g., traffic-related pollutants) ${ }^{1,2,41}$. The Women's Health Initiative study showed larger cardiovascular mortality risks of $\mathrm{PM}_{2.5}$ related to within-city contrasts compared to between-city contrasts ${ }^{43}$. Effect estimates would be underestimated if only between-city contrasts were exploited. Therefore, it is necessary to develop models for a large domain with fine spatial resolution.

The Europe-wide models for BC and PM composition developed in this thesis are based on pooled measurements from individual ESCAPE study areas ${ }^{1,41}$. Area-specific models were developed previously for the same pollutants for application in the ESCAPE study ${ }^{44,45}$. One reason for developing Europe-wide models in this thesis is that the ESCAPE models do not meet the requirements for the ELAPSE project. Most ESCAPE study areas were a large city with surrounding smaller rural communities. The ELAPSE project covers a larger study area than the ESCAPE individual study areas. For example, ESCAPE models were developed for the city of Copenhagen and surrounding areas, while the ELAPSE project includes the population distributed across entire Denmark. Local LUR models are typically not transferable from one area to another ${ }^{46,47}$, though some studies suggested local calibration with data from a few monitoring sites may improve the transferability ${ }^{48,49}$. Large-area models can probably be applied within the domain the models were developed, in our case Western Europe.

One benefit of developing Europe-wide models by pooling measurements from the ESCAPE study areas is the increasing number of training sites in the modeling process. Methodological studies suggested more training sites in model development result in more robust LUR models with better performance $^{50,51}$, and therefore more accurate health effect estimates ${ }^{52}$. Another benefit is the ability to make use of large-scale satellite and chemical transport model (CTM) estimates for assessing the background concentrations of air pollutants at approximately $10 \times 10 \mathrm{~km}$ resolution. This benefit applies for pollutants that lack the main sources and have very low concentrations in individual areas. For these pollutants, no area-specific models could be developed in areas without the main source present, or poor models were developed mainly explained by predictors that are not representing the sources ${ }^{44}$. When developing Europe-wide models for these pollutants, the model was informed by areas where specific sources were present. Large-scale satellite or chemical transport model estimates contributed to assess the low background concentrations in areas where sources are lacking (Chapter 5). This is still helpful when applied in epidemiological studies exploiting between-area contrasts in pollution concentrations. On the other hand, the potential predictor variables offered for developing large-scale models are restricted to variables that are available for all study areas. For example, traffic intensity variables are only available for part of ESCAPE study areas ${ }^{53}$. Traffic intensity variables would have been a better predictor representing traffic emissions than road length used in our Europe-wide models ${ }^{54,55}$. The ESCAPE area-specific models for $\mathrm{NO}_{2}$ on average improved $10 \%$ by offering traffic intensity data compared to road length data $^{56}$. Therefore, in epidemiological studies involving only a single community, local models might be a better choice than larger scale models as these models will have been developed with the most extensive information available locally. The advantage of local models applies primarily when a sufficient number of sites is available for model training. Wang and coworkers suggested that for study areas in the ESCAPE study, fairly robust models could be developed with about 40 sites and more $^{50}$.

Depending on the requirements of the epidemiologic study, the most appropriate exposure model should be applied. To adapt to the development in quantifying health effects of air pollution at low 
concentrations using large populations, large-scale national or continental models at sufficiently fine spatial resolution are needed.

\section{Statistical algorithms}

LUR models are often developed with the ordinary least square linear regression algorithm ${ }^{57,58}$. There are four assumptions associated with a linear regression model: 1) the relationship between $X$ and the mean of $\mathrm{Y}$ is linear (linearity); 2) the variance of residuals is the same for any value of $\mathrm{X}$ (homoscedasticity); 3) observations are independent of each other (independence); 4) for any fixed value of $\mathrm{X}, \mathrm{Y}$ is normally distribute (normality) ${ }^{59}$. Violation of the assumptions would reduce the accuracy of LUR model estimates and/or affect regression coefficient estimation. Other concerns of applying a multiple linear regression algorithm in developing spatial models include overfitting, collinearity, spatial non-stationarity and spatial autocorrelation. In chapter 4, we compared 16 different algorithms to predict spatial variation in annual average of $\mathrm{PM}_{2.5}$ and $\mathrm{NO}_{2}$ across Europe. We observed similar performance for models developed with the different algorithms. Two of the concerns that were not fully addressed in chapter 4 are spatial non-stationarity and spatial autocorrelation. Spatial nonstationarity implies inconstant relationships among variables across space. This property leads to spatial instability of regression coefficients and can be associated with heteroskedastic regression residuals ${ }^{60}$. For example, one might expect that regression coefficient for road length might not be the same in Portugal and in the Netherlands. One approach to resolve spatial non-stationarity is the geographically weighted regression (GWR) algorithm ${ }^{61}$. In a GWR, spatially varying coefficients are estimated for predictors by developing local regression models at numerous local neighborhoods. GWR has been applied to model ground-level air pollution exposures based on satellite observations ${ }^{62-64}$, but not much in LUR modeling ${ }^{65}$. Spatial autocorrelation can be defined as similarity of observations over short distances ${ }^{66}$, and can result in autocorrelated residuals if the model does not fully explain the spatial patterns. Some studies suggested the residuals of LUR models were independent for models developed in metropolitan areas even though significant spatial autocorrelation was present in the measured concentrations ${ }^{67,68}$. One study resolved spatial autocorrelation in sulfur dioxide model residuals with a spatial autoregressive model ${ }^{69}$. In developing spatiotemporal models for $\mathrm{PM}_{2.5}$, convolutional layers aggregating neighboring information were used in a neural network algorithm to account for spatial and temporal autocorrelation ${ }^{70}$. In chapter 3, kriging was performed on the residuals from the supervised linear regression models to account for the spatial autocorrelation ${ }^{55}$. In chapter 4 , we observed kriging on the residual of the generalized boosted machine and neural network models, both are machinelearning algorithms, did not significantly improve the model performance. This indicated that the machine-learning algorithms have some ability to address spatial autocorrelation.

Machine-learning algorithms have increasingly been applied in air pollution exposure assessment ${ }^{34,70-}$ ${ }^{73}$. Although similar performance was observed for machine-learning algorithms and supervised linear regression algorithm in chapter 4 , when modeling spatial variation in annual average $\mathrm{PM}_{2.5}$ and $\mathrm{NO}_{2}$ concentrations, machine-learning models accounted for spatial trends across Europe better than the linear models for $\mathrm{PM}_{2.5}$ composition (chapter 5). The different findings could be explained by the different distribution of monitoring sites for model development. While the $\mathrm{PM}_{2.5}$ and $\mathrm{NO}_{2}$ models were developed on a large number of fairly evenly distributed routine monitoring sites, the $\mathrm{PM}_{2.5}$ composition models were based on clustered ESCAPE monitoring sites. Another possible explanation is that there might be more complex relationships between predictors and elemental composition than with the mass of $\mathrm{PM}_{2.5}$ and $\mathrm{NO}_{2}$. Studies generally reported a similar or better performance of machine-learning algorithms compared to linear regression algorithm. In a study developing local-scale $\mathrm{PM}_{2.5}$ composition exposure models based on 24 sites, random forest, a machine-learning model, outperformed linear models ${ }^{74}$. In another study modeling ultrafine particle (UFP) concentrations based on mobile monitoring, similar performance was observed for machine-learning and linear models ${ }^{75}$. More work is needed to understand the performance of different algorithms in different conditions. 
Machine-learning algorithms are attractive because they relax the assumptions of linear regression models and offer a flexible way to model the relationships including non-linearity and interactions ${ }^{59}$. They further require less theoretical knowledge (e.g., a priori transformation of predictor variables to fit the linearity assumption) than linear regression models. However, the opaque modeling process of the machine-learning models results in difficulty in interpretation. Although some machine-learning algorithms provide useful information such as relative importance of individual predictors, it is not as straightforward as the regression coefficients of predictors derived from linear regression models.

Understanding how the machine-learning algorithms work would help to interpret the derived models. For example, in Chapter 5 we observed strong boundary effects in exposure surfaces produced by random forest models. This can be explained by the classification nature of the random forest algorithm: depending on the value where decision trees are split, large difference in predictions will be produced below and above that value. Therefore, smoothing might be required before applying the exposure surfaces in epidemiological studies. Even without fully understanding the method, it is important to graphically document what the complex model predicts. In chapter 5 we illustrated the importance of mapping the predicted exposures. Providing scatterplots or three-dimensional surfaces of predicted concentrations versus important predictor variables is another tool to understand the predictions of the model ${ }^{76}$. In addition, models developed with machine-learning algorithms could be improved significantly with careful tuning and/or training ${ }^{59}$. For example in chapter 4 , the neural network performed the worst among the tested algorithms, whereas it achieved high predictive accuracy in modeling the spatiotemporal variation of $\mathrm{PM}_{2.5}$ over the continental $\mathrm{US}^{70}$. In the latter study, the authors trained the neural network with more sophisticated methodology including adding convolutional layers. We cannot rule out the possibility of better performance of our Europe-wide models with more sophisticated training.

Another suggestion for future application of statistical algorithms in air pollution exposure modeling is not assuming one algorithm a priori, but to find the best predictive model for the data. A study selected the optimal prediction model from 11 statistical algorithms to estimate population exposure to PM during wildfires ${ }^{77}$. Ensemble predictions can also be attractive because they leverage information from several individual models. In chapter 4 we developed two simple ensemble models by calculating the median value or a weighted average of the 16 individual model predictions. In a study modeling UFP concentrations, a 'Super Learner' algorithm was applied to combine results from a wide range of different modeling techniques ${ }^{75}$. Neither our simple ensemble models nor the 'Super Learner' algorithm improve upon the individual models. However, the idea to combine individual models and average out biases is attractive. Further research is needed.

Health effects related to air pollution exposure modelled with different algorithms have been even less compared than predicted exposure. In chapter 6 we illustrated that while supervised linear regression and random forest had similar performance in predicting particle component exposures, the estimated associations with linear regression were generally more consistent than with random forest. The estimated associations with health depend not only on the validity of the model, but also on the type of exposure measurement error (Berkson, classical), the predicted exposure contrast and the relationship with potential confounders. Szpiro et al. has provided a theoretical discussion of the discrepancy between models with the best exposure prediction and models with the least bias ${ }^{78}$.

Different statistical algorithms have their pros and cons. Understanding these would help to develop models with better prediction accuracy and help to interpret the models. With the development in computing capability, it is attractive to select the best model from a number of algorithms, or to develop an ensemble model from several models. 


\section{Long-term exposure to PM composition and health}

Only a few epidemiological studies have investigated health effects of long-term exposure to PM composition. The number of studies has been small because of the scarcity of measurements relative to regulated pollutants such as $\mathrm{PM}_{2.5}$ and $\mathrm{NO}_{2}$ and methodological challenges. So far, mixed findings have been reported for associations between health outcomes and a series of $\mathrm{PM}_{2.5}$ components including secondary inorganic aerosols (sulfate, nitrate, ammonium), elemental carbon (EC), metals and organic components. Some of the discrepancies can be explained by the different populations, exposure assessment methods and statistical modeling approaches applied in the studies.

Sulfate, sometimes quantified as elemental sulfur, is most consistently associated with a variety of health outcomes across studies. Two pivotal studies in the early 1990s - the Harvard Six Cities study ${ }^{79}$ and the American Cancer Society study ${ }^{80}$ - reported associations between long-term sulfate exposure and mortality from all causes, lung cancer and cardiopulmonary diseases. Later studies conducted in the North America and Europe continued to find elevated risk of mortality and morbidity in relation to long-term sulfate exposure ${ }^{27,81-86}$. Sulfate is long-range transported and thus has relatively uniform concentrations within cities. The relatively large-scale spatial variation of sulfate allows the U.S. studies, which typically relied on central site ambient air pollution monitoring networks, to assess the health effects related to sulfate exposure. However, it is difficult to disentangle effects of sulfate from that of $\mathrm{PM}_{2.5}$ mass because of the high proportion of sulfate in PM combined with its relatively low fine spatial scale variability ${ }^{87,88}$. In the ESCAPE meta-analysis of 19 European cohorts, association between $\mathrm{PM}_{2.5}$ sulfur and all-cause mortality was robust to adjustment for $\mathrm{PM}_{2.5}$ mass $^{81}$. In the ELAPSE pooled cohort, we observed the associations of $\mathrm{PM}_{2.5}$ sulfur with mortality attenuated substantially after adjustment for $\mathrm{PM}_{2.5}$ mass. The HR for SLR-modeled sulfur remained positive in two-pollutant model, whereas the HR for RF-modeled sulfur reduced to unity (Chapter 6).

Studies on other secondary inorganic aerosols are fewer compared to sulfate. In a cohort of more than 100,000 current and former female public school professionals in the California Teachers Study, nitrate was associated with mortality from cardiovascular and Ischemic Heart Disease (IHD), but not with pulmonary disease mortality ${ }^{89}$. Nitrate was also reported to be positively associated with mortality rate in studies within the U.S. Medicare beneficiaries and the U.S. veterans ${ }^{90-92}$. In the large Danish Diet, Cancer and Health cohort ${ }^{85}$, a positive association was observed between long-term nitrate exposure and mortality from all causes and respiratory diseases. The Danish study also reported negative associations between ammonium and mortality when taking into account overall secondary inorganic aerosols in multipollutant models. The negative associations can possibly be explained by the differences in exposure estimate precision combined with high correlation between pollutants instead of protective effect. We did not assess associations with nitrate or ammonium in the ELAPSE study.

There is mounting evidence on associations between adverse health outcomes and long-term exposure to black carbon particles, including black carbon (BC), black smoke (BS), absorption coefficient of PM $(\mathrm{Abs})$ and elemental carbon $(\mathrm{EC})^{93}$. These black carbon metrics are all indicators for carbon-rich combustion sources and are often used interchangeably in the literature. Important sources of BC include coal combustion, diesel-powered motorized traffic and off-road machinery and wood burning. $\mathrm{BC}$ is a primary pollutant emitted especially as fine and ultrafine particles. A meta-analysis conducted in 2013 reported statistically significantly positive pooled estimate for all-cause mortality and $\mathrm{EC}^{94}$. The EC exposure used in the meta-analysis includes BC or BS used as markers for soot in individual studies. Several later studies associated long-term EC exposure with risk of brain tumors and cardiovascular health outcomes ${ }^{27,85,89,95-98}$. In ELAPSE, we found significantly positive associations between $\mathrm{BC}$ and mortality and incidence of stroke, asthma and COPD hospital admissions in the pooled cohort $^{28,29,99}$. Findings for organic carbon (OC) are more mixed. While some studies suggested longterm OC exposure related to cardiovascular outcomes ${ }^{27,84}$, others did not ${ }^{83,90,98}$. OC is a mixture of primary organic carbon particles and secondary organic aerosol formed in the atmosphere. Because of the more secondary nature of OC compared to EC, OC has less fine spatial scale variation compared to 
$\mathrm{EC} / \mathrm{BC}^{100}$. OC consists of different components derived from very different sources (e.g., traffic, biomass burning). The varying relationships between $\mathrm{OC}$ and health endpoints can be partly explained by the different impacts of the specific components on health. One technical difficulty remains in reliably measuring the carbonaceous particles concentrations, which makes results across studies difficult to compare ${ }^{93}$. Furthermore, the carbonaceous particles are highly correlated with $\mathrm{NO}_{2}$ in concentrations, especially in the developed countries where an important source of both pollutants is diesel powered vehicles. The high correlations make it difficult to disentangle individual health effects. Another concern is that studies using limited number of monitors to represent exposure over large metropolitan areas do not sufficiently capture the large spatial gradients of the carbonaceous particles within a city ${ }^{83,98}$. In the Women's Health Initiative-Observational Study (WHI-OS) and the MultiEthnic Study of Atherosclerosis (MESA) ${ }^{84}$, the authors tried to address the sparseness of the EPA's Chemical Speciation monitoring Network (CSN) by adding additional monitoring data derived from a different measurement approach. However, the different measurement approach and sampling time used by the extra monitoring campaign might, on the contrary, increase the exposure measurement errors $^{101}$. In the earlier California Teacher's study, the spatial variability issues were accounted for by restricting the analyses to participants living within 8 - and 30-km buffers of a monitor ${ }^{27}$. While the 30$\mathrm{km}$ buffer might still be too large for capturing high spatial variation, the $8-\mathrm{km}$ buffer reduced the sample size significantly and thus the statistical power ${ }^{89}$. Recent studies typically applied fine spatial scale models to estimate exposure at the individual level (i.e., the outdoor concentrations at participants' residences) $)^{85,89,102}$. Validity of the fine-scale air pollution models needs to be carefully examined, because the estimated effect of pollutant can be transferred from a less precisely modelled constituent to a more adequately modelled constituent in multi-pollutant models ${ }^{103}$.

Mixed results have been reported for associations between health outcomes and long-term exposure to a variety of metals, including copper, iron, zinc, nickel, vanadium, potassium, calcium, lead, manganese, aluminum and sodium ${ }^{81,83,84,89,91}$. One challenge in studying health effects of metal composition is to reliably estimate their concentrations. Metals usually have low concentrations, sometimes with a large number of measurements below the limits of detection ${ }^{41,83}$. The low concentration of metals might hinder the assessment of their health impacts, as exposure estimates derived from models based on these low-concentration measurements are likely to be imprecise ${ }^{44}$. This issue applies especially to metals such as nickel, vanadium, lead, but much less for the traffic-related iron, zinc and copper. Some of the metals are difficult to model, because the GIS databases do not readily contain the relevant predictor variables such as shipping emissions or wood burning ${ }^{44}$. For these metals direct monitoring may be attractive. However, characterizing individual- or population-level metal exposures with one or a few monitors within a city may introduce large measurement error for metals with high spatial heterogeneity ${ }^{27,83}$. This may bias the epidemiological findings unless there is a large contrast in exposure between cities. Another challenge is to separate the effects of individual components within PM, which is not restricted to metal composition. Individual components or elements are typically linked to one or more specific sources while several elements often share the same source. Studies have linked source categories directly to health outcomes using source apportionment results as an alternative approach to studying specific components ${ }^{83,89,98}$. An analysis in the American Cancer Society Cancer Prevention Study II (ACS CPS-II) suggested exposure to coal combustion-related air pollution explained most associations between $\mathrm{PM}_{2.5}$ mass and increased risk of mortality from all causes, IHD and lung cancer ${ }^{83,98}$. In the California Teachers study, associations with IHD mortality were observed for sources including gas- and diesel-fueled vehicles, meat cooking and high-sulfur fuel combustion ${ }^{89}$. Other studies used source apportionment results of elements as tracers for specific sources to assist in the interpretation of the health effects ${ }^{81,84}$. For example, in the Rome longitudinal study, higher mortality risk was found to be associated with increased exposure to copper, iron and zinc, representing nontailpipe traffic emissions ${ }^{104}$. Applying the same set of exposure models, analyses conducted within the ESCAPE and TRANSPHORM (Transport related Air Pollution and Health impacts - Integrated Methodologies for Assessing Particulate Matter) concluded long-term exposure to transition metals 
originating from traffic and industry may be related to chronic systemic inflammation ${ }^{105}$. Linking source categories to health endpoints may represent the combined effects of multiple pollutants from the same source and is more readily translatable into air quality policy. However, it may introduce additional uncertainties and assumptions. Source apportionment results can be different depending on the different methodologies ${ }^{106}$. Furthermore, it is difficult to compare the quantitative effect estimates for pollution sources across studies that are varied in time and space. In ELAPSE, we did not directly assess which source categories are most responsible for health effects but used source apportionment results in Europe to assist in the interpretations as was done in ESCAPE ${ }^{81,107-109}$. Future studies are suggested to link both source categories and individual components to the health endpoints, with a focus on examining consistencies and differences between the two approaches, following the National Particle Component Toxicity (NPACT) study ${ }^{83,98}$. When source apportionment results are used, researchers should recognize and discuss the uncertainties introduced by the apportionment method.

The analyses within the ACS CPS-II found no association with mortality for soil and biomass combustion source categories and their elemental tracers (calcium, silicon and potassium) ${ }^{95}$. Consistently, a negative and marginal association was observed for CVD events with silicon in the WHI-OS ${ }^{84}$. Silicon is a specific tracer for crustal material that is a large component of soil and resuspended road dust. However, a distinction between soil and road dust is often not possible because of the overlapping source profiles ${ }^{42}$. Crustal materials are often abundant in coarse particles. There is little evidence for an association between long-term coarse PM exposure and adverse health effects ${ }^{94,110}$. The 2019 Integrated Science Assessment (ISA) rated the association between PM $_{\text {coarse }}$ exposure and natural-cause mortality as "suggestive"111. In contrast, a few studies have reported adverse cardiovascular effects of long-term exposure to silicon ${ }^{27,108}$. In the California Teachers Study, the authors interpreted the silicon exposure as a proxy either for toxic constituents found in road dust or for exposures to traffic-related pollutants ${ }^{27}$.

Recent studies have tried to associate adverse health effects with oxidative potential of particulate matter ${ }^{12-114}$, as it was proposed that oxidative potential may be a more health relevant metric than PM mass $^{115,116}$. However, it remains challenging to specifically model the concentrations for oxidative potential, because emission factors are not available and specific sources are not known ${ }^{117}$. We did not assess health effects of oxidative potential in ELAPSE, because Europe-wide data were not available.

Although multiple groups of PM components and sources have been linked to a variety of adverse health outcomes, the collective evidence has not yet isolated elements or sources that are most strongly and unequivocally related to specific health endpoints ${ }^{118}$. Similarly, the US EPA concluded in the Integrated Science assessment (ISA) for PM that there was evidence for associations of mortality with multiple elements, without a clear pattern of particularly important components ${ }^{111}$. Further research is needed to address the methodological challenges in this field and to provide more evidence about the relative harm of the specific components. Methodological challenges discussed in this section include: 1) more limited routine monitoring compared to regulated pollutants, which limits the possibilities to develop large-scale models and to assess historical exposures, as we experienced within ELAPSE; 2)Multiple comparison issues may apply if a large number of elements are included in an epidemiological study (risk of chance findings); 3) Multi-collinearity.

\section{Implications}

\section{Application of air pollution models in the ELAPSE project}

Europe-wide air pollution models developed in Chapter 2 and Chapter 5 for $\mathrm{PM}_{2.5}, \mathrm{NO}_{2}, \mathrm{BC}, \mathrm{O}_{3}$ and $\mathrm{PM}_{2.5}$ elemental composition were applied in the ELAPSE project to characterize individual exposures. Compared to the ESCAPE area-specific LUR models ${ }^{44,45,56}$, the Europe-wide models allow for the consistent exposure estimation for all participants in the ELAPSE project. The models further cover 
study areas that were not covered by the ESCAPE models. Moreover, methodological studies suggested more stable exposure models can be developed based on larger number of model training sites ${ }^{51,119}$.

The ELAPSE project aims to investigate health effects of long-term exposure to low concentrations of outdoor air pollution in Europe ${ }^{3}$. 'Low concentration' is defined as less than the current EU limit values, the US EPA NAAQS and/or the WHO air quality guideline values, respectively. The study assesses the shape of the CRFs with a focus at the shape at low exposures. The ELAPSE project consists of a pooled cohort of more than 300,000 participants and seven large administrative cohorts of more than 28 million adults. In the pooled cohort, data from eight existing cohorts in seven European countries with detailed lifestyle data were combined and analyzed centrally. Analyses of the administrative cohorts were conducted locally, with fewer possibilities to control for individual-level confounders. A summary of the main findings of the ELAPSE project is given below.

In the pooled cohort, 325,367 adults were followed for an average of 19.5 years. Almost all subjects had 2010 annual average exposures to $\mathrm{PM}_{2.5}$ below the EU Limit Value $\left(25 \mu \mathrm{g} / \mathrm{m}^{3}\right)$. More than 50,000 and 25,000 subjects had exposures below the US-EPA NAAQS $\left(12 \mu \mathrm{g} / \mathrm{m}^{3}\right)$ and WHO AQG $\left(10 \mu \mathrm{g} / \mathrm{m}^{3}\right)$, respectively. Nearly all subjects had 2010 annual average exposures to $\mathrm{NO}_{2}$ below the WHO AQG and EU Limit Values $\left(40 \mu \mathrm{g} / \mathrm{m}^{3}\right)$. Long-term exposure to $\mathrm{PM}_{2.5}, \mathrm{NO}_{2}, \mathrm{BC}$ were (borderline) significantly positively associated with mortality from natural-cause, cardiovascular and respiratory diseases ${ }^{120}$. Hazard ratios (HR, 95\% CI) for natural-cause mortality were $1.130(1.106,1.155)$ for a $5 \mu \mathrm{g} / \mathrm{m}^{3}$ increase in $\mathrm{PM}_{2.5}, 1.086(1.070,1.102)$ for a $10 \mu \mathrm{g} / \mathrm{m}^{3}$ increase in $\mathrm{NO}_{2}$ and $1.081(1.065,1.098)$ for a $0.5^{*} 10^{-5} / \mathrm{m}$ increase in BC. For these pollutants, a supra-linear CRF with steeper slopes at low exposures was observed, with no evidence of a threshold. Consistently, HRs were slightly larger in subsets of participants restricted to low exposures. The HR for natural-cause mortality was $1.296(1.140,1.474)$ for subjects with $\mathrm{PM}_{2.5}$ exposures below the US standard of $12 \mu \mathrm{g} / \mathrm{m}^{3}$. The HR for natural-cause mortality was $1.101(1.083,1.119)$ for subjects with $\mathrm{NO}_{2}$ exposures below the $\mathrm{WHO}$ and $\mathrm{EU}$ limit value of $40 \mu \mathrm{g} / \mathrm{m}^{3}$. Negative associations were found between mortality and ozone. This may be related to the narrow range of ozone exposure in the pooled cohort combined with the high negative correlations between ozone and especially $\mathrm{NO}_{2}$ and $\mathrm{BC}$. Associations between mortality and long-term exposure to elemental composition of $\mathrm{PM}_{2.5}$ were documented in Chapter 6 . We identified that exposure to vanadium in $\mathrm{PM}_{2.5}$ was most consistently associated with increased natural-cause mortality.

Significantly positive associations were found between long-term $\mathrm{PM}_{2.5}, \mathrm{NO}_{2}$ and $\mathrm{BC}$ exposure and incidence of cerebrovascular events and asthma and COPD hospital admissions ${ }^{28,29,99} . \mathrm{NO}_{2}$ was furthermore significantly associated with acute coronary heart disease and $\mathrm{PM}_{2.5}$ with lung cancer incidence $^{31,99}$. The CRFs generally displayed a linear to supra-linear association with no evidence of a threshold, except the sublinear association between $\mathrm{NO}_{2}$ and acute coronary heart disease. Subset analyses suggested that adverse health effects of $\mathrm{PM}_{2.5}$ remained below $20 \mu \mathrm{g} / \mathrm{m}^{3}$ and in some cases 12 $\mu \mathrm{g} / \mathrm{m}^{3}$. Associations with $\mathrm{NO}_{2}$ remained below 30 and possibly $20 \mu \mathrm{g} / \mathrm{m}^{3}$. In two-pollutant models, $\mathrm{NO}_{2}$ was most consistently associated with acute coronary heart disease, stroke, asthma and COPD hospital admissions. $\mathrm{PM}_{2.5}$ was not associated with these outcomes in two-pollutant models. $\mathrm{PM}_{2.5}$ was the only pollutant that was associated with lung cancer incidence in two-pollutant models. A further analysis showed that the associations between lung cancer incidence and $\mathrm{PM}_{2.5}$ composition were most robust for sulfur, potassium, nickel and vanadium ${ }^{30}$. Associations with ozone were negative, though not statistically significant in two-pollutant models.

In the administrative cohorts, analyses were conducted locally in Norway, Denmark, England, the Netherlands, Belgium, Switzerland and Rome (Italy ${ }^{121}$. Cohort-specific results were then pooled into a random-effects meta-analysis. All cohorts contributed to 28,146,444 subjects of observation with $3,593,741$ deaths from natural causes. Almost all participants had exposures below the EU limit values for both $\mathrm{PM}_{2.5}\left(25 \mu \mathrm{g} / \mathrm{m}^{3}\right)$ and $\mathrm{NO}_{2}\left(40 \mu \mathrm{g} / \mathrm{m}^{3}\right)$. More than 3.9 million (mostly from the Scandinavian cohorts) and 1.9 million (mostly from the Norwegian cohort) subjects had $2010 \mathrm{PM}_{2.5}$ exposure below the US-EPA NAAQS $\left(12 \mu \mathrm{g} / \mathrm{m}^{3}\right)$ and the WHO air quality guideline $\left(10 \mu \mathrm{g} / \mathrm{m}^{3}\right)$, respectively. 
Significantly positive associations were found between $\mathrm{PM}_{2.5}, \mathrm{NO}_{2}$ and $\mathrm{BC}$ and mortality from naturalcause, cardiovascular diseases, respiratory diseases and lung cancer. Pooled HRs of natural-cause mortality were 1.053 (95\% CI: 1.021, 1.085), 1.044 (95\% CI: 1.019, 1.069) and 1.039 (95\% CI: 1.018 , 1.059) per $5 \mu \mathrm{g} / \mathrm{m}^{3}$ increment in $\mathrm{PM}_{2.5}, 10 \mu \mathrm{g} / \mathrm{m}^{3}$ in $\mathrm{NO}_{2}$ and $0.5^{*} 10^{-5} / \mathrm{m}$ in $\mathrm{BC}$, respectively. Large heterogeneity was observed in the cohort-specific HRs. HRs were similar for cardiovascular and respiratory mortality, and highest for lung cancer mortality. Warm season ozone was negatively associated with both natural-cause and cause-specific mortality. Cohort-specific CRFs displayed differently between cohorts, though associations were generally linear for $\mathrm{PM}_{2.5}$ and supra-linear for $\mathrm{NO}_{2}$, with no indication of a threshold. The subset analyses supported the observations from the shape of the associations. For $\mathrm{PM}_{2.5}$ and $\mathrm{NO}_{2}$, HRs were modestly larger when the analysis was restricted to subjects exposed to low concentrations, i.e., HRs below $10 \mu \mathrm{g} / \mathrm{m}^{3}$ (the WHO guidelines) for $\mathrm{PM}_{2.5}$ and $20 \mu \mathrm{g} / \mathrm{m}^{3}$ for $\mathrm{NO}_{2}$ were $1.078(1.046,1.111)$ and $1.061(0.985,1.143)$, respectively. Copper and iron in $\mathrm{PM}_{2.5}$ were positively associated with mortality, especially for respiratory and lung cancer mortality.

The ELAPSE findings are generally consistent with findings from other recent studies on health effects of low-level air pollution ${ }^{122-125}$, including the other two studies funded by the Health Effects Institute ${ }^{126}$ : the Mortality-Air Pollution Associations in Low-Exposure Environments (MAPLE) study ${ }^{127}$ and the analyses in the Medicare enrollees ${ }^{128}$. The MAPLE study includes approximately 8.5 million participants from three Canadian Census Health and Environment Cohorts (CanCHEC), and about half million Canadian Community Health Survey (CCHS) participants with additional lifestyle and behavioral information ${ }^{127}$. The pooled HR across the three CanCHEC cohorts for nonaccidental mortality was $1.053(1.041,1.065)$ per $10 \mu \mathrm{g} / \mathrm{m}^{3}$ increment in long-term average $\mathrm{PM}_{2.5}$ exposure. In the CCHS cohort, an HR of $1.11(1.04,1.18)$ per $10 \mu \mathrm{g} / \mathrm{m}^{3}$ increase in $\mathrm{PM}_{2.5}$ for the nonaccidental mortality risk was reported. A supra-linear association was observed for long-term average $\mathrm{PM}_{2.5}$ and nonaccidental mortality, with a steep increase across lower concentrations (i.e., $<5 \mu \mathrm{g} / \mathrm{m}^{3}$ ), followed by a leveling off or a smaller increase after $\sim 10 \mu \mathrm{g} / \mathrm{m}^{3}$. No apparent threshold or sublinear association was suggested. In the Medicare cohort of around 61 million Americans aged 65 years and older, hazard ratios were derived from two-pollutant analyses ${ }^{128}$. A $7.3 \%$ (95\% CI: $\left.7.1 \%-7.5 \%\right)$ and a $1.1 \%(95 \%$ : $1.0 \%-1.2 \%$ ) higher risk of all-cause mortality were reported for a $10 \mu \mathrm{g} / \mathrm{m}^{3}$ and a $10 \mathrm{ppb}$ increase in annual average $\mathrm{PM}_{2.5}$ and average $\mathrm{O}_{3}$ concentrations in the warm season, respectively. When restricting the analyses to person-years with low exposures (concentrations less than $12 \mu \mathrm{g} / \mathrm{m}^{3} \mathrm{PM}_{2.5}$ and $50 \mathrm{ppb}$ $\mathrm{O}_{3}$ ), the mortality risk was $13.6 \%$ (95\% CI: $\left.13.1 \%-14.1 \%\right)$ and $1.0 \%$ (95\% CI: $\left.0.9 \%-1.1 \%\right)$ for the same increase in $\mathrm{PM}_{2.5}$ and $\mathrm{O}_{3}$ respectively. The CRFs from the two-pollutant models showed almost linear curves, with no indication of a threshold down to $5 \mu \mathrm{g} / \mathrm{m}^{3} \mathrm{PM}_{2.5}$ and $30 \mathrm{ppb} \mathrm{O}_{3}$. A recent study modeled an ensemble shape of the association between $\mathrm{PM}_{2.5}$ and natural-cause mortality using data from 41 cohorts from 16 countries $^{129}$. The curve displayed a supra-linear association over lower exposures and then a near-linear association at higher concentrations.

The findings from the ELAPSE project and other studies on health effects of low-level air pollution provide novel and potentially important insight into the shape of concentration-response functions at low concentrations. These studies might contribute to the future research on health effects of air pollution, global burden of disease assessments and revision of air quality guidelines and standards.

\section{Policy recommendations}

In 2015, an expert consultation was organized by the WHO calling for the need to update existing air quality guidelines ${ }^{130}$. The latest WHO air quality guidelines were released in 2006 based on studies mainly conducted in North America and Europe ${ }^{6}$. New evidence has emerged since then and showed associations between adverse health effects and air pollutants at levels below the existing WHO guideline values. A series of systematic reviews were conducted by different groups (including our group) coordinated by the WHO focusing on long-term and short-term health effects of $\mathrm{PM}, \mathrm{O}_{3}, \mathrm{NO}_{2}$, $\mathrm{SO}_{2}$ and $\mathrm{CO}^{131-134}$. The reviews documented clear associations between all pollutants and mortality and 
morbidity outcomes. These reviews have been applied in developing the updated WHO air quality guidelines. Among these pollutants, $\mathrm{PM}_{2.5}$ has received particular attention because it has been associated with increased risks in mortality and morbidity more than any other regulated air pollutant ${ }^{36,111}$. IARC has classified ambient PM as carcinogenic, with evidence of increased risk for cancer at levels below the current $\mathrm{PM}_{2.5}$ guideline value ${ }^{135}$. The systematic review documented in chapter 2 showed clear evidence for associations between mortality and long-term exposure to $\mathrm{PM}_{2.5}$ at levels below the current WHO guideline value of $10 \mu \mathrm{g} / \mathrm{m}^{3}$. This is further confirmed by findings of the ELAPSE project and other recent studies on health effects of low-level air pollution, as discussed above. One of the key findings of the ELAPSE project is the supra-linear concentration-response relationships between $\mathrm{PM}_{2.5}$ exposure and health effects, with no indication of a threshold below which there is no effects of $\mathrm{PM}_{2.5}$ on health. The supra-linear associations were not only observed for mortality outcomes, but also for a variety of incident endpoints including cerebrovascular events, acute coronary heart disease, lung cancer incidence, and asthma and COPD hospital admissions ${ }^{28,29,31,99}$. The evidence suggests the need to tighten the current guideline value.

Another ongoing discussion is whether PM mass is the most efficient metric for regulation purposes. Components of PM vary in space and time, and some components may be more responsible for the observed health effects than others. $\mathrm{PM}_{2.5}$ concentrations in parts of Africa would exceed the guideline value largely with probably little harm, because dust is a dominant component of ambient PM there ${ }^{136,137}$. Although regulatory control of PM might be improved with robust quantification of the toxicity of various constituents of the PM mixture and of their sources ${ }^{90}$, the existing evidence is not sufficient to support such component-specific regulations, as discussed in section 4. Consistently, the 2019 PM ISA concluded that there is no indication of any one source or component is consistently more closely related to specific health outcomes than $\mathrm{PM}_{2.5}$ mass ${ }^{111}$. More studies are needed to further contribute to the understanding of the associations between PM composition and health outcomes in order to recommend relevant regulatory controls.

\section{Conclusions}

A systematic review of long-term exposure to $\mathrm{PM}_{2.5}$ and $\mathrm{PM}_{10}$ documented significantly positive associations with mortality. Associations at low levels were predominantly found in North-American studies. Effect estimates were heterogenous, probably related to differences in particle composition, exposure level and methodological differences. We developed Europe-wide LUR models for assessing long-term exposure to $\mathrm{PM}_{2.5}, \mathrm{NO}_{2}, \mathrm{O}_{3}$, black carbon (BC) and $\mathrm{PM}_{2.5}$ elemental composition. We found only limited differences in model performance using supervised linear regression and a range of algorithms including machine-learning methods. Models were applied in the ELAPSE project to assign exposure at the individual level. Both $\mathrm{PM}_{2.5}$ and $\mathrm{PM}_{10}$ were associated with increased mortality from all causes, cardiovascular disease, respiratory disease and lung cancer. Associations remained below the current WHO air quality guideline level of $10 \mu \mathrm{g} / \mathrm{m}^{3}$ for $\mathrm{PM}_{2.5}$. Long-term exposures to especially vanadium in $\mathrm{PM}_{2.5}$ was associated with increased mortality risk, with associations observed for both random forest- and supervised linear regression-modeled exposures. For the other elemental components studied, associations were generally weaker when exposure was assessed with random forest compared to supervised linear regression in two-pollutant models. 


\section{References}

1. Eeftens M, Tsai M-Y, Ampe C, et al. Spatial variation of PM2.5, PM10, PM2.5 absorbance and PMcoarse concentrations between and within 20 European study areas and the relationship with NO2 - Results of the ESCAPE project. Atmospheric Environment 2012; 62: 303-17.

2. Cyrys J, Eeftens M, Heinrich J, et al. Variation of NO2 and NOx concentrations between and within 36 European study areas: results from the ESCAPE study. Atmospheric Environment 2012; 62: 374-90.

3. ELAPSE. Effects of Low-Level Air Pollution: A Study in Europe. http://wwwelapseprojecteu/ 2016.

4. EU. https://ec.europa.eu/environment/air/quality/standards.htm.

5. USEPA. https://www.epa.gov/criteria-air-pollutants/naaqs-table.

6. WHO. Air quality guidelines: global update 2005: particulate matter, ozone, nitrogen dioxide, and sulfur dioxide: World Health Organization; 2006.

7. Sterne JA, Hernán MA, Reeves BC, et al. ROBINS-I: a tool for assessing risk of bias in non-randomised studies of interventions. bmj 2016; 355.

8. NTP. Handbook for conducting a literature-based health assessment using OHAT approach for systematic review and evidence integration. Office of Health Assessment and Translation (OHAT), Division of the National Toxicology Program, National Institute of Environmental Health Sciences https://ntpniehsnihgov/ntp/ohat/pubs/handbookmarch2019 508pdf 2019.

9. Peterson J, Welch V, Losos M, Tugwell P. The Newcastle-Ottawa scale (NOS) for assessing the quality of nonrandomised studies in meta-analyses. Ottawa: Ottawa Hospital Research Institute 2011.

10. GRADE TGWG. GRADE handbook for grading quality of evidence and strength of recommendations. https://gdtgradeproorg/app/handbook/handbookhtm/ 2013.

11. IARC IAfRoC. IARC monographs on the identification of carcinogenic hazards to humans. World Health Organization 2019.

12. EPA U. Preamble to the Integrated Science Assessments (ISA). US Environmental Protection Agency Washington, DC; 2015.

13. WHO. Risk of bias assessment instrument for systematic reviews informing WHO global air quality guidelines. https://www.euro.who.int/en/health-topics/environment-and-health/air-quality/publications/2020/risk-of-bias-

assessment-instrument-for-systematic-reviews-informing-who-global-air-quality-guidelines-2020 (last access: 20201110). 2020.

14. Savitz DA, Wellenius GA, Trikalinos TA. The Problem With Mechanistic Risk of Bias Assessments in Evidence Synthesis of Observational Studies and a Practical Alternative: Assessing the Impact of Specific Sources of Potential Bias. Am J Epidemiol 2019; 188(9): 1581-5.

15. Steenland K, Schubauer-Berigan MK, Vermeulen R, et al. Risk of Bias Assessments and Evidence Syntheses for Observational Epidemiologic Studies of Environmental and Occupational Exposures: Strengths and Limitations. Environ Health Perspect 2020; 128(9): 95002.

16. Losilla J-M, Oliveras I, Marin-Garcia JA, Vives J. Three risk of bias tools lead to opposite conclusions in observational research synthesis. Journal of clinical epidemiology 2018; 101: 61-72.

17. Lawlor DA, Tilling K, Davey Smith G. Triangulation in aetiological epidemiology. Int J Epidemiol 2016; 45(6): 1866-

86.

18. Guyatt G, Oxman AD, Akl EA, et al. GRADE guidelines: 1. Introduction-GRADE evidence profiles and summary of findings tables. J Clin Epidemiol 2011; 64(4): 383-94.

19. Guyatt G, Oxman AD, Vist G, et al. GRADE guidelines: 4. Rating the quality of evidence--study limitations (risk of bias). J Clin Epidemiol 2011; 64(4): 407-15.

20. Allen RW, Barn PK, Lanphear BP. Randomized controlled trials in environmental health research: unethical or underutilized? PLoS Med 2015; 12(1): e1001775.

21. Peel JL, Baumgartner J, Wellenius GA, Clark ML, Smith KR. Are Randomized Trials Necessary to Advance Epidemiologic Research on Household Air Pollution? Current Epidemiology Reports 2015; 2(4): $263-70$.

22. Pearce N, Vandenbroucke JP, Lawlor DA. Causal inference in environmental epidemiology: old and new approaches. Epidemiology 2019; 30(3): 311-6.

23. Elwood M. Critical appraisal of epidemiological studies and clinical trials: Oxford University Press; 2017.

24. Pearce N, Blair A, Vineis P, et al. IARC monographs: 40 years of evaluating carcinogenic hazards to humans. Environ Health Perspect 2015; 123(6): 507-14.

25. Brunekreef B, Stewart AW, Anderson HR, et al. Self-reported truck traffic on the street of residence and symptoms of asthma and allergic disease: a global relationship in ISAAC phase 3. Environmental health perspectives 2009; 117(11): 1791-8.

26. Burnett RT, Ma R, Jerrett M, et al. The spatial association between community air pollution and mortality: A new method of analyzing correlated geographic cohort data. Environmental Health Perspectives 2001; 109(SUPPL. 3): $375-80$.

27. Ostro B, Lipsett M, Reynolds $\mathrm{P}$, et al. Long-term exposure to constituents of fine particulate air pollution and mortality: results from the California Teachers Study. Environ Health Perspect 2011; 118(3): 363-9.

28. Liu S, Jørgensen JT, Ljungman P, et al. Long-term exposure to low-level air pollution and incidence of chronic obstructive pulmonary disease: The ELAPSE project. Environment International 2021; 146. 
29. Liu S, Jørgensen JT, Ljungman P, et al. Long-term exposure to low-level air pollution and incidence of asthma: the ELAPSE project. European Respiratory Journal 2020.

30. Hvidtfeldt UA, Chen J, Andersen ZJ, et al. Long-term exposure to fine particle elemental components and lung cancer incidence in the ELAPSE pooled cohort. Environmental Research 2020.

31. Hvidtfeldt UA, Severi G, Andersen ZJ, et al. Long-term low-level ambient air pollution exposure and risk of lung cancer - A pooled analysis of 7 European cohorts. Environ Int 2021; 146: 106249.

32. Jerrett M, Turner MC, Beckerman BS, et al. Comparing the Health Effects of Ambient Particulate Matter Estimated Using Ground-Based versus Remote Sensing Exposure Estimates. Environ Health Perspect 2017; 125(4): 552-9.

33. Downward GS, van Nunen E, Kerckhoffs J, et al. Long-Term Exposure to Ultrafine Particles and Incidence of Cardiovascular and Cerebrovascular Disease in a Prospective Study of a Dutch Cohort. Environ Health Perspect 2018; 126(12): 127007.

34. Hu X, Belle JH, Meng X, et al. Estimating PM2.5 Concentrations in the Conterminous United States Using the Random Forest Approach. Environ Sci Technol 2017.

35. Hystad P, Setton E, Cervantes A, et al. Creating national air pollution models for population exposure assessment in Canada. Environmental health perspectives 2011; 119(8): 1123-9.

36. Cohen AJ, Brauer M, Burnett R, et al. Estimates and 25-year trends of the global burden of disease attributable to ambient air pollution: an analysis of data from the Global Burden of Diseases Study 2015. The Lancet 2017; 389(10082): 1907-18.

37. Anderson HR, Butland BK, van Donkelaar A, et al. Satellite-based estimates of ambient air pollution and global variations in childhood asthma prevalence. Environmental health perspectives 2012; 120(9): 1333-9.

38. Brauer M, Freedman G, Frostad J, et al. Ambient air pollution exposure estimation for the global burden of disease 2013. Environmental science \& technology 2016; 50(1): 79-88.

39. Shaddick G, Thomas ML, Green A, et al. Data integration model for air quality: a hierarchical approach to the global estimation of exposures to ambient air pollution. Journal of the Royal Statistical Society: Series C (Applied Statistics) 2018; 67(1): 231-53.

40. Van Donkelaar A, Martin RV, Brauer M, et al. Global estimates of fine particulate matter using a combined geophysical-statistical method with information from satellites, models, and monitors. Environmental science \& technology 2016; 50(7): 3762-72.

41. Tsai MY, Hoek G, Eeftens M, et al. Spatial variation of PM elemental composition between and within 20 European study areas--Results of the ESCAPE project. Environ Int 2015; 84: 181-92.

42. Belis CA, Karagulian F, Larsen BR, Hopke PK. Critical review and meta-analysis of ambient particulate matter source apportionment using receptor models in Europe. Atmospheric Environment 2013; 69: 94-108.

43. Miller KA, Siscovick DS, Sheppard L, et al. Long-term exposure to air pollution and incidence of cardiovascular events in women. New England Journal of Medicine 2007; 356(5): 447-58.

44. de Hoogh K, Wang M, Adam M, et al. Development of land use regression models for particle composition in twenty study areas in Europe. Environ Sci Technol 2013; 47(11): 5778-86.

45. Eeftens $M$, Beelen R, de Hoogh K, et al. Development of Land Use Regression models for PM(2.5), PM(2.5) absorbance, $\mathrm{PM}(10)$ and PM(coarse) in 20 European study areas; results of the ESCAPE project. Environ Sci Technol 2012; 46(20): 11195-205.

46. Johnson M, Isakov V, Touma JS, Mukerjee S, Özkaynak H. Evaluation of land-use regression models used to predict air quality concentrations in an urban area. Atmospheric Environment 2010; 44(30): 3660-8.

47. Allen RW, Amram O, Wheeler AJ, Brauer M. The transferability of NO and NO2 land use regression models between cities and pollutants. Atmospheric Environment 2011; 45(2): 369-78.

48. Briggs DJ, de Hoogh C, Gulliver J, et al. A regression-based method for mapping traffic-related air pollution: application and testing in four contrasting urban environments. Science of the Total Environment 2000; 253(1-3): 151-67.

49. Vienneau D, De Hoogh K, Beelen R, Fischer P, Hoek G, Briggs D. Comparison of land-use regression models between Great Britain and the Netherlands. Atmospheric Environment 2010; 44(5): 688-96.

50. Wang M, Beelen R, Eeftens M, Meliefste K, Hoek G, Brunekreef B. Systematic evaluation of land use regression models for NO2. Environmental science \& technology 2012; 46(8): 4481-9.

51. Basagaña X, Rivera M, Aguilera I, et al. Effect of the number of measurement sites on land use regression models in estimating local air pollution. Atmospheric Environment 2012; 54: 634-42.

52. Basagana X, Aguilera I, Rivera M, et al. Measurement error in epidemiologic studies of air pollution based on landuse regression models. Am J Epidemiol 2013; 178(8): 1342-6.

53. Wang $\mathrm{M}$, Beelen R, Bellander $\mathrm{T}$, et al. Performance of multi-city land use regression models for nitrogen dioxide and fine particles. Environ Health Perspect 2014; 122(8): 843-9.

54. Chen J, de Hoogh K, Gulliver J, et al. Development of Europe-Wide Models for Particle Elemental Composition Using Supervised Linear Regression and Random Forest. Environ Sci Technol 2020.

55. de Hoogh K, Chen J, Gulliver J, et al. Spatial PM2.5, NO2, O3 and BC models for Western Europe - Evaluation of spatiotemporal stability. Environ Int 2018; 120: 81-92.

56. Beelen R, Hoek G, Vienneau D, et al. Development of NO2 and NOx land use regression models for estimating air pollution exposure in 36 study areas in Europe-the ESCAPE project. Atmospheric Environment 2013; 72: 10-23.

57. Crouse DL, Goldberg MS, Ross NA. A prediction-based approach to modelling temporal and spatial variability of traffic-related air pollution in Montreal, Canada. Atmospheric environment 2009; 43(32): 5075-84. 
58. Hoek G, Beelen R, De Hoogh K, et al. A review of land-use regression models to assess spatial variation of outdoor air pollution. Atmospheric environment 2008; 42(33): 7561-78.

59. Friedman J, Hastie T, Tibshirani R. The elements of statistical learning: Springer series in statistics New York; 2001. 60. Bertazzon S, Johnson M, Eccles K, Kaplan GG. Accounting for spatial effects in land use regression for urban air pollution modeling. Spatial and spatio-temporal epidemiology 2015; 14-15: 9-21.

61. Fotheringham AS, Charlton ME, Brunsdon C. Geographically weighted regression: a natural evolution of the expansion method for spatial data analysis. Environment and planning A 1998; 30(11): 1905-27.

62. Van Donkelaar A, Martin RV, Spurr RJ, Burnett RT. High-resolution satellite-derived PM2. 5 from optimal estimation and geographically weighted regression over North America. Environmental science \& technology 2015; 49(17): $10482-91$.

63. Bai Y, Wu L, Qin K, Zhang Y, Shen Y, Zhou Y. A Geographically and Temporally Weighted Regression Model for Ground-Level PM2.5 Estimation from Satellite-Derived 500 m Resolution AOD. Remote Sensing 2016; 8(3): 262.

64. van Donkelaar A, Martin RV, Li C, Burnett RT. Regional Estimates of Chemical Composition of Fine Particulate Matter Using a Combined Geoscience-Statistical Method with Information from Satellites, Models, and Monitors. Environ Sci Technol 2019; 53(5): 2595-611.

65. Beloconi A, Chrysoulakis N, Lyapustin A, Utzinger J, Vounatsou P. Bayesian geostatistical modelling of PM10 and PM2.5 surface level concentrations in Europe using high-resolution satellite-derived products. Environ Int 2018; 121(Pt 1): 57-70.

66. Getis A. Reflections on spatial autocorrelation. Regional Science and Urban Economics 2007; 37(4): 491-6.

67. Jerrett $M$, Arain $M$, Kanaroglou $\mathrm{P}$, et al. Modeling the intraurban variability of ambient traffic pollution in Toronto, Canada. Journal of Toxicology and Environmental Health, Part A 2007; 70(3-4): 200-12.

68. Madsen C, Carlsen KCL, Hoek G, et al. Modeling the intra-urban variability of outdoor traffic pollution in Oslo, Norway-A GA2LEN project. Atmospheric Environment 2007; 41(35): 7500-11.

69. Kanaroglou PS, Adams MD, De Luca PF, Corr D, Sohel N. Estimation of sulfur dioxide air pollution concentrations with a spatial autoregressive model. Atmospheric Environment 2013; 79: 421-7.

70. Di Q, Kloog I, Koutrakis P, Lyapustin A, Wang Y, Schwartz J. Assessing PM2. 5 exposures with high spatiotemporal resolution across the continental United States. Environmental science \& technology 2016; 50(9): 4712-21.

71. de Hoogh K, Heritier H, Stafoggia M, Kunzli N, Kloog I. Modelling daily PM2.5 concentrations at high spatio-temporal resolution across Switzerland. Environmental pollution (Barking, Essex : 1987) 2018; 233: 1147-54.

72. Stafoggia M, Schwartz J, Badaloni C, et al. Estimation of daily PM10 concentrations in Italy (2006-2012) using finely resolved satellite data, land use variables and meteorology. Environ Int 2017; 99: 234-44.

73. Kim SY, Sheppard L, Bergen S, et al. Prediction of fine particulate matter chemical components with a spatiotemporal model for the Multi-Ethnic Study of Atherosclerosis cohort. J Expo Sci Environ Epidemiol 2016; 26(5): 520-8.

74. Brokamp C, Jandarov R, Rao MB, LeMasters G, Ryan P. Exposure assessment models for elemental components of particulate matter in an urban environment: A comparison of regression and random forest approaches. Atmos Environ (1994) 2017; 151: 1-11.

75. Kerckhoffs J, Hoek G, Portengen L, Brunekreef B, Vermeulen RC. Performance of Prediction Algorithms for Modeling Outdoor Air Pollution Spatial Surfaces. Environmental science \& technology 2019.

76. Apley DW, Zhu J. Visualizing the effects of predictor variables in black box supervised learning models. Journal of the Royal Statistical Society: Series B (Statistical Methodology) 2020; 82(4): 1059-86.

77. Reid CE, Jerrett M, Petersen ML, et al. Spatiotemporal prediction of fine particulate matter during the 2008 northern California wildfires using machine learning. Environ Sci Technol 2015; 49(6): 3887-96.

78. Szpiro AA, Paciorek CJ, Sheppard L. Does more accurate exposure prediction necessarily improve health effect estimates? Epidemiology 2011; 22(5): 680-5.

79. Dockery DW, Pope ICA, Xu X, et al. An association between air pollution and mortality in six U.S. cities. New England Journal of Medicine 1993; 329(24): 1753-9.

80. Pope CA, Thun MJ, Namboodiri MM, et al. Particulate air pollution as a predictor of mortality in a prospective study of U.S. Adults. American Journal of Respiratory and Critical Care Medicine 1995; 151(3 I): 669-74.

81. Beelen R, Hoek G, Raaschou-Nielsen O, et al. Natural-cause mortality and long-term exposure to particle components: an analysis of 19 European cohorts within the multi-center ESCAPE project. Environ Health Perspect 2015; 123(6): 525-33.

82. Pope CA, Burnett RT, Thun MJ, et al. Lung cancer, cardiopulmonary mortality, and long-term exposure to fine particulate air pollution. Jama 2002; 287(9): 1132-41.

83. Lippmann M, Chen LC, Gordon T, Ito K, Thurston GD. National Particle Component Toxicity (NPACT) Initiative: integrated epidemiologic and toxicologic studies of the health effects of particulate matter components. Research Report (Health Effects Institute) 2013; (177): 5-13.

84. Vedal S, Campen M, McDonald J, et al. National Particle Component Toxicity (NPACT) initiative report on cardiovascular effects. Research Report (Health Effects Institute) 2013; (178): 5-8.

85. Hvidtfeldt UA, Geels C, Sorensen M, et al. Long-term residential exposure to PM2.5 constituents and mortality in a Danish cohort. Environ Int 2019; 133(Pt B): 105268.

86. Crouse DL, Philip S, van Donkelaar A, et al. A New Method to Jointly Estimate the Mortality Risk of Long-Term Exposure to Fine Particulate Matter and its Components. Scientific reports 2016; 6: 18916.

87. McMurry PH, Shepherd MF, Vickery JS. Particulate matter science for policy makers: A NARSTO assessment: Cambridge University Press; 2004. 
88. Putaud J-P, Van Dingenen R, Alastuey A, et al. A European aerosol phenomenology-3: Physical and chemical characteristics of particulate matter from 60 rural, urban, and kerbside sites across Europe. Atmospheric Environment 2010; 44(10): 1308-20.

89. Ostro B, Hu J, Goldberg D, et al. Associations of mortality with long-term exposures to fine and ultrafine particles, species and sources: results from the California Teachers Study Cohort. Environ Health Perspect 2015; 123(6): 549-56.

90. Chung Y, Dominici F, Wang Y, Coull BA, Bell ML. Associations between long-term exposure to chemical constituents of fine particulate matter (PM2.5) and mortality in Medicare enrollees in the eastern United States. Environ Health Perspect 2015; 123(5): 467-74

91. Wang Y, Shi L, Lee M, et al. Long-term Exposure to PM2.5 and Mortality Among Older Adults in the Southeastern US. Epidemiology 2017; 28(2): 207-14.

92. Lipfert F, Baty J, Miller J, Wyzga R. PM2.5 constituents and related air quality variables as predictors of survival in a cohort of U.S. military veterans. Inhalation Toxicology 2006; 18(9): 645-57.

93. Janssen NA, Hoek G, Simic-Lawson M, et al. Black carbon as an additional indicator of the adverse health effects of airborne particles compared with PM10 and PM2.5. Environ Health Perspect 2011; 119(12): 1691-9.

94. Hoek G, Krishnan RM, Beelen R, et al. Long-term air pollution exposure and cardio- respiratory mortality: a review. Environmental health : a global access science source 2013; 12(1): 43.

95. Thurston G, Ito K, Lall R, et al. NPACT Study 4: Mortality and Long-Term Exposure to PM2. 5 and its Components in the American Cancer Society's Cancer Prevention Study II Cohort. National Particle Component Toxicity (NPACT) Initiative: Integrated Epidemiologic and Toxicologic Studies of the Health Effects of Particulate Matter Components Health Effect Institute 2013.

96. Harbo Poulsen A, Arthur Hvidtfeldt U, Sorensen M, et al. Components of particulate matter air-pollution and brain tumors. Environ Int 2020; 144: 106046.

97. Luben TJ, Nichols JL, Dutton SJ, et al. A systematic review of cardiovascular emergency department visits, hospital admissions and mortality associated with ambient black carbon. Environ Int 2017; 107: 154-62.

98. Thurston GD, Burnett RT, Turner MC, et al. Ischemic Heart Disease Mortality and Long-Term Exposure to SourceRelated Components of U.S. Fine Particle Air Pollution. Environ Health Perspect 2016; 124(6): 785-94.

99. Wolf K, Hoffmann B, Andersen ZJ, et al. Long-term exposure to low-level ambient air pollution and incidence of stroke and coronary heart disease - Results from six European cohorts within the ELAPSE Project. submitted.

100. Jedynska A, Hoek $G$, Eeftens $M$, et al. Spatial variations of $P A H$, hopanes/steranes and $E C / O C$ concentrations within and between European study areas. Atmospheric Environment 2014; 87: 239-48.

101. Adams K, Greenbaum DS, Shaikh R, van Erp AM, Russell AG. Particulate matter components, sources, and health: Systematic approaches to testing effects. J Air Waste Manag Assoc 2015; 65(5): 544-58.

102. Beelen R, Raaschou-Nielsen $\mathrm{O}$, Stafoggia $\mathrm{M}$, et al. Effects of long-term exposure to air pollution on natural-cause mortality: an analysis of 22 European cohorts within the multicentre ESCAPE project. The Lancet 2014; 383(9919): 785-95.

103. PHE PHE. Associations of long-term average concentrations of nitrogen dioxide with mortality. https://assetspublishingservicegovuk/government/uploads/system/uploads/attachment data/file/734799/COMEAP NO2 Reportpdf 2018.

104. Badaloni C, Cesaroni G, Cerza F, Davoli M, Brunekreef B, Forastiere F. Effects of long-term exposure to particulate matter and metal components on mortality in the Rome longitudinal study. Environment international 2017; 109: 146-54.

105. Hampel R, Peters A, Beelen R, et al. Long-term effects of elemental composition of particulate matter on inflammatory blood markers in European cohorts. Environ Int 2015; 82: 76-84.

106. Thurston GD, Ito K, Mar T, et al. Workgroup report: workshop on source apportionment of particulate matter health effects--intercomparison of results and implications. Environ Health Perspect 2005; 113(12): 1768-74.

107. Wang $M$, Beelen $\mathrm{R}$, Stafoggia $\mathrm{M}$, et al. Long-term exposure to elemental constituents of particulate matter and cardiovascular mortality in 19 European cohorts: results from the ESCAPE and TRANSPHORM projects. Environ Int 2014; 66: 97-106.

108. Wolf K, Stafoggia M, Cesaroni G, et al. Long-term exposure to particulate matter constituents and the incidence of coronary events in 11 European cohorts. Epidemiology 2015; 26(4): 565-74.

109. Raaschou-Nielsen $\mathrm{O}$, Beelen $\mathrm{R}$, Wang $\mathrm{M}$, et al. Particulate matter air pollution components and risk for lung cancer. Environment international 2016; 87: 66-73.

110. Adar SD, Filigrana PA, Clements N, Peel JL. Ambient coarse particulate matter and human health: a systematic review and meta-analysis. Current environmental health reports 2014; 1(3): 258-74.

111. USEPA. Integrated Science Assessment (ISA) for Particulate Matter (Final Report, 2019). US Environmental Protection Agency, Washington, DC, EPA/600/R-19/188, 2019 (https://cfpubepagov/ncea/isa/recordisplaycfm?deid=347534, last access: 20200128) 2019.

112. Strak M, Janssen N, Beelen R, et al. Long-term exposure to particulate matter, NO2 and the oxidative potential of particulates and diabetes prevalence in a large national health survey. Environ Int 2017; 108: 228-36.

113. Yang A, Janssen NA, Brunekreef B, Cassee FR, Hoek G, Gehring U. Children's respiratory health and oxidative potential of PM2.5: the PIAMA birth cohort study. Occup Environ Med 2016; 73(3): 154-60.

114. Weichenthal S, Crouse DL, Pinault L, et al. Oxidative burden of fine particulate air pollution and risk of cause-specific mortality in the Canadian Census Health and Environment Cohort (CanCHEC). Environ Res 2016; 146: 92-9.

115. Ayres JG, Borm P, Cassee FR, et al. Evaluating the toxicity of airborne particulate matter and nanoparticles by measuring oxidative stress potential--a workshop report and consensus statement. Inhal Toxicol 2008; 20(1): 75-99. 
116. Borm PJ, Kelly F, Kunzli N, Schins RP, Donaldson K. Oxidant generation by particulate matter: from biologically effective dose to a promising, novel metric. Occup Environ Med 2007; 64(2): 73-4.

117. Hoek G. Methods for Assessing Long-Term Exposures to Outdoor Air Pollutants. Curr Environ Health Rep 2017; 4(4): 450-62.

118. Stanek LW, Sacks JD, Dutton SJ, Dubois J-JB. Attributing health effects to apportioned components and sources of particulate matter: An evaluation of collective results. Atmospheric Environment 2011; 45(32): 5655-63.

119. Wang M, Beelen R, Basagana X, et al. Evaluation of land use regression models for NO2 and particulate matter in 20 European study areas: the ESCAPE project. Environmental science \& technology 2013; 47(9): 4357-64.

120. Strak M, Weinmayr G, Rodopoulou S, et al. Long-term exposure to low-level air pollution and natural and causespecific mortality - a pooled analysis of eight European cohorts within the ELAPSE project. submitted.

121. Stafoggia M, Oftedal B, Chen J, et al. Long-term Exposure to Low Ambient Air Pollution Concentrations and Causespecific Mortality among 28 Million Subjects - Results from seven European large cohorts within the ELAPSE Project. in preparation.

122. Hart JE, Liao X, Hong B, et al. The association of long-term exposure to PM2.5 on all-cause mortality in the Nurses' Health Study and the impact of measurement-error correction. Environmental health : a global access science source 2015; 14: 38.

123. Hvidtfeldt UA, Sørensen M, Geels C, et al. Long-term residential exposure to PM2. 5, PM10, black carbon, NO2, and ozone and mortality in a Danish cohort. Environment international 2019; 123: 265-72.

124. Hanigan IC, Rolfe MI, Knibbs LD, et al. All-cause mortality and long-term exposure to low level air pollution in the ' 45 and up study' cohort, Sydney, Australia, 2006-2015. Environment international 2019; 126: 762-70.

125. Di Q, Wang Y, Zanobetti A, et al. Air Pollution and Mortality in the Medicare Population. The New England journal of medicine 2017; 376(26): 2513-22.

126. HEI. RFA 14-3 Assessing Health Effects of Long-term Exposure to Low Levels of Ambient Air Pollution. https://wwwhealtheffectsorg/research/funding/rfa/14-3-assessing-health-effects-long-term-exposure-low-levels-ambientair-pollution 2014.

127. Brauer M, Brook JR, Christidis T, et al. Mortality-Air Pollution Associations in Low-Exposure Environments (MAPLE): Phase 1. Research Reports: Health Effects Institute 2019; 2019.

128. Dominici F, Schwartz J, Di Q, Braun D, Choirat C, Zanobetti A. Assessing adverse health effects of long-term exposure to low levels of ambient air pollution: phase 1. Research Reports: Health Effects Institute 2019; 2019.

129. Burnett $\mathrm{R}, \mathrm{Chen} \mathrm{H}$, Szyszkowicz $\mathrm{M}$, et al. Global estimates of mortality associated with long-term exposure to outdoor fine particulate matter. Proceedings of the National Academy of Sciences 2018; 115(38): 9592-7.

130. WHO. WHO expert consultation: available evidence for the future update of the WHO global air quality guidelines (AQGs). WHO: Geneva, Switzerland 2016.

131. Huangfu P, Atkinson R. Long-term exposure to NO2 and $\mathrm{O} 3$ and all-cause and respiratory mortality: A systematic review and meta-analysis. Environ Int 2020; 144: 105998.

132. Lee KK, Spath N, Miller MR, Mills NL, Shah ASV. Short-term exposure to carbon monoxide and myocardial infarction: A systematic review and meta-analysis. Environ Int 2020; 143: 105901.

133. Orellano P, Reynoso J, Quaranta N, Bardach A, Ciapponi A. Short-term exposure to particulate matter (PM10 and $\mathrm{PM}$ 2.5), nitrogen dioxide (NO2), and ozone (O3) and all-cause and cause-specific mortality: Systematic review and metaanalysis. Environ Int 2020; 142: 105876.

134. Chen J, Hoek G. Long-term exposure to PM and all-cause and cause-specific mortality: A systematic review and meta-analysis. Environ Int 2020: 105974.

135. Loomis D, Grosse Y, Lauby-Secretan B, et al. The carcinogenicity of outdoor air pollution. The Lancet Oncology 2013; 14(13): 1262-3.

136. Kotsyfakis M, Zarogiannis SG, Patelarou E. The health impact of Saharan dust exposure. International journal of occupational medicine and environmental health 2019; 32(6): 749-60.

137. Naidja L, Ali-Khodja H, Khardi S. Sources and levels of particulate matter in North African and Sub-Saharan cities: a literature review. Environmental Science and Pollution Research 2018; 25(13): 12303-28. 

Summary 
Air pollution concentrations in North America and Europe have decreased substantially over the past decades. Despite that, recent studies suggested that the well-documented adverse health effects of air pollution may persist at levels below the current air quality guidelines and standards. This has raised questions about the appropriateness of the existing standards and guideline values for regulated pollutants including particulate matter with an aerodynamic diameter $\leq 2.5$ and $10 \mu \mathrm{m}\left(\mathrm{PM}_{2.5}\right.$ and $\left.\mathrm{PM}_{10}\right)$, nitrogen dioxide $\left(\mathrm{NO}_{2}\right)$ and ozone $\left(\mathrm{O}_{3}\right)$. $\mathrm{PM}_{2.5}$ is considered to be particularly important among the pollutants. The composition of $\mathrm{PM}_{2.5}$ varies in time and space, which may result in differences in toxicity and risk to health of $\mathrm{PM}_{2.5}$. Mixed findings have been reported for associations between specific $\mathrm{PM}_{2.5}$ components and health endpoints.

Recent developments in air pollution epidemiology require air pollution exposure estimates covering large geographical areas at sufficiently fine spatial resolution. Land use regression (LUR) models have been widely used in air pollution exposure assessment and are often developed with linear regression algorithms. More flexible modeling algorithms, such as regularization algorithms and machine-learning algorithms, have been applied recently. Few studies have compared exposure models developed with different algorithms. No study has compared the health effects related to these different exposure models.

The Effects of Low-level Air Pollution: A Study in Europe (ELAPSE) is a Europe-wide collaboration which focuses on analyses of health effects of air pollutants at low concentrations, defined as less than the current European and United States' air quality standards and WHO air quality guideline values.

This thesis has the following specific aims:

1. To systematically evaluate the evidence of associations of long-term exposure to $\mathrm{PM}_{10}$ and $\mathrm{PM}_{2.5}$ with all-cause and cause-specific mortality with a focus on associations at low pollution levels

2. To develop Europe-wide LUR models for $\mathrm{PM}_{2.5}, \mathrm{NO}_{2}, \mathrm{O}_{3}$, black carbon (BC) and $\mathrm{PM}_{2.5}$ elemental composition

3. To compare LUR models developed with different algorithms in terms of prediction accuracy and interpretability

4. To investigate associations between long-term exposure to $\mathrm{PM}_{2.5}$ components and mortality, and to compare the health effects estimated using exposure models developed with different algorithms

In Chapter 2, we conducted a systematic review on associations of long-term exposure to $\mathrm{PM}_{2.5}$ and $\mathrm{PM}_{10}$ with natural-cause and cause-specific mortality. We identified 107 eligible articles, of which 104 were reporting results of cohort studies. The majority of studies were conducted in North America (N = 62) and Europe $(\mathrm{N}=25)$. By including the most recently published article for the same study population in the meta-analysis, we estimated a meta-analytical risk ratio (RR) of 1.08 (95\% CI 1.06, 1.09) per $10 \mu \mathrm{g} / \mathrm{m}^{3}$ for $\mathrm{PM}_{2.5}$ and natural-cause mortality $(\mathrm{N}=25)$. Substantial heterogeneity was found across studies, probably related to differences in study populations, methodology, exposure level and composition of particles. Meta-analytical RRs per $10 \mu \mathrm{g} / \mathrm{m}^{3}$ derived from studies conducted at low mean $\mathrm{PM}_{2.5}$ levels $\left(<25,20,15,12,10 \mu \mathrm{g} / \mathrm{m}^{3}\right.$ respectively) were higher compared to the overall $\mathrm{RR}$ and increased as $\mathrm{PM}_{2.5}$ concentration decreased (meta-analytical RRs at low concentrations ranged from 1.09 to 1.17). This is consistent with the finding of generally linear or supra-linear concentrationresponse functions (CRFs) in individual studies. Studies at low concentrations were predominantly conducted in North America where concentrations are lower than in most of Europe, in part because of lower population density. There is evidence that associations between $\mathrm{PM}_{2.5}$ and increased mortality risk remained below the current EU air quality limit exposure level $\left(25 \mu \mathrm{g} / \mathrm{m}^{3}\right)$, the USEPA National Ambient Air Quality Standards (NAAQS; $12 \mu \mathrm{g} / \mathrm{m}^{3}$ ), and even the WHO air quality guideline value $\left(10 \mu \mathrm{g} / \mathrm{m}^{3}\right)$. 
The ELAPSE project is conducted to provide new empirical evidence on associations between lowlevel air pollution and health outcomes in Europe, characterizing air quality with $\mathrm{PM}_{2.5}$ and additionally $\mathrm{NO}_{2}, \mathrm{BC}$ and $\mathrm{O}_{3}$. In Chapter 3, we developed Europe-wide hybrid LUR models for 2010 annual mean concentrations of $\mathrm{PM}_{2.5}, \mathrm{NO}_{2}, \mathrm{BC}$ and $\mathrm{O}_{3}$ at $100 \times 100 \mathrm{~m}$ resolution to assess air pollution exposure for ELAPSE cohort participants. The models were developed using a supervised linear regression algorithm with satellite observations, chemical transport model estimates, land use and traffic data as potential predictor variables. Kriging was performed to further explain spatial variations in residuals of the LUR models. Kriging significantly improved the $\mathrm{PM}_{2.5}$ and $\mathrm{O}_{3}$ models but not the $\mathrm{NO}_{2}$ and $\mathrm{BC}$ models. In five-fold cross-validation, the $\mathrm{PM}_{2.5}, \mathrm{NO}_{2}, \mathrm{O}_{3}$ warm season and $\mathrm{BC}$ models explained $66 \%$, $58 \%, 60 \%$ and $51 \%$ of the variability in measured concentrations, respectively. The spatial structures of the models were stable in time at the West-European scale, supporting application in long-term epidemiological studies with cohorts started one or two decades ago.

in Chapter 4, we compared 16 different algorithms (linear regression, regularization and machinelearning algorithms) to develop Europe-wide $\mathrm{PM}_{2.5}$ and $\mathrm{NO}_{2}$ models, using the same air pollution measurements and potential predictor variables used in Chapter 3. We observed similar performance across algorithms evaluated by five-fold cross-validation and an additional validation using an external dataset. The predicted concentrations from all algorithms at external validation sites were highly correlated $\left(\mathrm{R}^{2}>0.85\right)$.

In Chapter 5, we developed Europe-wide LUR models for elemental components in $\mathrm{PM}_{2.5}$. Eight elements were a priori selected to represent major sources: copper $(\mathrm{Cu})$, iron $(\mathrm{Fe})$ and zinc $(\mathrm{Zn})$ representing non-tailpipe traffic emissions; sulfur (S) representing long-range transport of secondary inorganic aerosols generated from combustion of sulfur containing solid and liquid fuels; nickel (Ni) and vanadium (V) representing mixed oil burning/industry; silicon (Si) representing crustal material; and potassium $(\mathrm{K})$ representing biomass burning. Models for each element were developed with supervised linear regression and random forest, a machine-learning algorithm. The models performed moderate to well across Europe, explaining $41 \%$ to $90 \%$ of the overall spatial variability in measured concentrations in five-fold cross-validation. Random forest models consistently outperformed supervised linear regression models in explaining the overall variability. Models explained within study area variation much less than the overall variation. Random forest and supervised linear regression models explained similar within study variation. Maps proved a useful additional tool to evaluate the plausibility of model predictions beyond cross-validation statistics. Models differed substantially between elements in terms of the major predictor variables. The major predictor variables included in the models broadly reflected the major sources of the corresponding elements. Agreement between the two algorithm predictions was high at the overall European level for $\mathrm{Cu}, \mathrm{K}, \mathrm{S}$ and $\mathrm{Zn}(\mathrm{r}>0.7)$, and varied substantially at the national level.

In Chapter 6, we assessed associations between exposure to $\mathrm{PM}_{2.5}$ elemental components and mortality in the ELAPSE pooled cohort $(\mathrm{N}=323,782)$. Exposure models developed in Chapter 5 with both supervised linear regression (SLR) and random forest (RF) algorithms were applied for comparison. All SLR-modeled components were statistically significantly associated with natural-cause mortality in single pollutant models with hazard ratios (HRs) from 1.05-1.27 over representative concentration ranges. Similar HRs were observed for RF-modeled $\mathrm{Cu}, \mathrm{Fe}, \mathrm{K}, \mathrm{S}, \mathrm{V}$ and $\mathrm{Zn}$ with wider confidence intervals (CIs). For both SLR- and RF-modeled V, HRs attenuated but remained above unity and (borderline) significant after adjustment for $\mathrm{PM}_{2.5}$ mass or $\mathrm{NO}_{2}$. The HRs for V were $1.03(95 \% \mathrm{CI}$ : $1.02,1.05)$ and $1.06(95 \%$ CI: 1.02, 1.10) for SLR- and RF-modeled exposures, respectively, per 2 $\mathrm{ng} / \mathrm{m}^{3}$ adjusting for $\mathrm{PM}_{2.5}$ mass. Associations for the other components were weaker for exposure modeled with RF than SLR in two-pollutant models. 
In Chapter 7, methodological issues related to confidence assessment of epidemiological evidence in systematic reviews, spatial scale and statistical algorithms for developing air pollution exposure models, existing evidence on health effects of long-term PM composition exposure are being discussed. The main ELAPSE findings by applying the air pollution models developed in this thesis were summarized. Finally, some policy recommendations were given based on the current state of knowledge. 
Samenvatting 
De concentraties luchtverontreiniging in Noord-Amerika en Europa zijn de afgelopen decennia aanzienlijk afgenomen. Desondanks suggereren recente studies dat de nadelige gezondheidseffecten van luchtverontreiniging kunnen aanhouden bij concentraties onder de huidige luchtkwaliteitsrichtlijnen en -normen. Dit roept vragen op over de geschiktheid van de bestaande normen en richtlijnen voor gereguleerde luchtvervuilende stoffen, waaronder fijnstof met een aerodynamische diameter van $\leq 2.5$ en $10 \mu \mathrm{m}\left(\mathrm{PM}_{2.5}\right.$ en $\left.\mathrm{PM}_{10}\right)$, stikstofdioxide $\left(\mathrm{NO}_{2}\right)$ en ozon $\left(\mathrm{O}_{3}\right)$. $\mathrm{PM}_{2.5}$ wordt beschouwd als een bijzonder belangrijke luchtvervuilende stof. De samenstelling van $\mathrm{PM}_{2.5}$ varieert in tijd en ruimte, wat kan leiden tot verschillen in de toxiciteit en gezondheidsrisico's van PM $_{2.5}$. Er zijn inconsistente bevindingen gerapporteerd voor associaties tussen specifieke componenten van $\mathrm{PM}_{2.5}$ en gezondheidsuitkomsten.

Recente ontwikkelingen in epidemiologisch onderzoek van luchtverontreiniging vereisen schattingen van de blootstelling aan luchtverontreiniging met een hoge ruimtelijke resolutie in grote geografische gebieden. Land use regressie (LUR) modellen worden veel gebruikt bij het schatten van de blootstelling aan luchtverontreiniging en worden vaak ontwikkeld met lineaire regressie algoritmen. Recentelijk zijn flexibeler, niet-lineaire modelleer algoritmen toegepast, zoals 'machine learning' algoritmen. Weinig studies hebben blootstellingsmodellen vergeleken die zijn ontwikkeld met verschillende algoritmen. Geen enkele studie heeft de gezondheidseffecten van luchtverontreiniging, samenhangend met blootstelling aan luchtverontreiniging zoals geschat met verschillende blootstellingsmodellen vergeleken.

ELAPSE (Effects of Low-level Air Pollution: A Study in Europe) is een Europa-brede samenwerking van onderzoeksinstituten die zich richt op het analyseren van gezondheidseffecten van luchtverontreiniging bij lage concentraties, gedefinieerd als concentraties lager dan de huidige Europese en Amerikaanse luchtkwaliteitsnormen en de advieswaarden van de WHO.

Dit proefschrift heeft de volgende specifieke doelstellingen:

1. Het systematisch evalueren van associaties tussen langdurige blootstelling aan $\mathrm{PM}_{10}$ en $\mathrm{PM}_{2.5}$ en totale sterfte en oorzaak specifieke sterfte, met een focus op associaties bij lage concentraties luchtverontreiniging

2. Het ontwikkelen van LUR modellen voor heel Europa voor $\mathrm{PM}_{2.5}, \mathrm{NO}_{2}, \mathrm{O}_{3}$, black carbon (BC) en een aantal elementaire componenten van $\mathrm{PM}_{2.5}$

3. Het vergelijken van de voorspellingsnauwkeurigheid en interpreteerbaarheid van LUR modellen die zijn ontwikkeld met verschillende algoritmen

4. Het bestuderen van associaties tussen langdurige blootstelling aan componenten van $\mathbf{P M}_{2.5}$ en sterfte, en het vergelijken van de gezondheidseffecten die zijn geschat met blootstellingsmodellen die zijn ontwikkeld met verschillende algoritmen

In Hoofdstuk 2 hebben we een systematisch review uitgevoerd van studies die associaties onderzochten tussen langdurige blootstelling aan $\mathrm{PM}_{2.5}$ en $\mathrm{PM}_{10}$ en natuurlijke en oorzaak specifieke sterfte. We identificeerden 107 artikelen die in aanmerking kwamen, waarvan 104 artikelen de resultaten van cohortonderzoeken rapporteerden. De meeste studies waren uitgevoerd in Noord-Amerika $(\mathrm{N}=62)$ en Europa $(\mathrm{N}=25)$. Wanneer er meer dan één artikel verscheen over dezelfde onderzoekspopulatie kozen we ervoor alleen het meest recente artikel op te nemen in de meta-analyse. Zodoende hebben we een meta-analytisch relatief risico (RR) van 1.08 (95\% CI 1.06, 1.09) per $10 \mu \mathrm{g} / \mathrm{m}^{3}$ geschat voor $\mathrm{PM}_{2.5}$ en natuurlijke sterfte ( $\mathrm{N}=25$ studies). We vonden aanzienlijke heterogeniteit tussen de studies, die waarschijnlijk is gerelateerd aan verschillen in onderzoekspopulaties, methodologie, blootstellingsniveaus en de samenstelling van de fijnstofdeeltjes. Meta-analytische RR's per $10 \mu \mathrm{g} / \mathrm{m}^{3}$, afgeleid van studies die zijn uitgevoerd bij lage gemiddelde $\mathrm{PM}_{2.5}$ concentraties (respectievelijk $<25$, $20,15,12,10 \mu \mathrm{g} / \mathrm{m}^{3}$ ), waren hoger dan het relatieve risico voor alle studies en namen toe naarmate de gemiddelde $\mathrm{PM}_{2.5}$ concentratie lager was (meta-analytische RR's bij lage concentraties varieerden van 1.09 tot 1.17). Dit komt overeen met de bevinding van in het algemeen lineaire of supra-lineaire concentratie-respons functies (CRF's) in individuele studies. Studies met lage concentraties 
luchtverontreiniging werden voornamelijk uitgevoerd in Noord-Amerika, waar de concentraties lager zijn dan in het grootste deel van Europa, deels vanwege de lagere bevolkingsdichtheid. Er zijn aanwijzingen voor associaties tussen $\mathrm{PM}_{2.5}$ en een verhoogd sterfterisico bij concentraties onder de huidige EU grenswaarde voor $\mathrm{PM}_{2.5}\left(25 \mu \mathrm{g} / \mathrm{m}^{3}\right)$, de US EPA National Ambient Air Quality Standards (NAAQS; $\left.12 \mu \mathrm{g} / \mathrm{m}^{3}\right)$, en zelfs de advieswaarde van de WHO $\left(10 \mu \mathrm{g} / \mathrm{m}^{3}\right)$.

Het ELAPSE project wordt uitgevoerd om associaties tussen lage concentraties aan luchtverontreiniging $\left(\mathrm{PM}_{2.5}, \mathrm{NO}_{2}, \mathrm{BC}\right.$ en $\left.\mathrm{O}_{3}\right)$ en gezondheidsuitkomsten in Europa te onderzoeken. In Hoofdstuk 3 hebben we Europa-brede hybride LUR modellen ontwikkeld voor de jaargemiddelde concentraties van $\mathrm{PM}_{2.5}, \mathrm{NO}_{2}, \mathrm{BC}$ en $\mathrm{O}_{3}$ in 2010 met een resolutie van $100 x 100 \mathrm{~m}$ om de blootstelling aan luchtverontreiniging voor deelnemers van het ELAPSE cohort te schatten. De modellen zijn ontwikkeld met behulp van een gesuperviseerd lineair regressie algoritme met satellietwaarnemingen, schattingen van chemische transportmodellen, landgebruik en verkeersgegevens als mogelijk voorspellende variabelen. Met behulp van 'kriging' hebben we de ruimtelijke variaties in de residuen van de LUR modellen verder verklaard. 'Kriging' heeft de $\mathrm{PM}_{2.5}$ en $\mathrm{O}_{3}$ modellen aanzienlijk verbeterd, maar niet de $\mathrm{NO}_{2}$ en $\mathrm{BC}$ modellen. In vijfvoudige kruisvalidatie verklaarden de $\mathrm{PM}_{2.5}, \mathrm{NO}_{2}, \mathrm{O}_{3}$ en $\mathrm{BC}$ modellen respectievelijk $66 \%, 58 \%, 60 \%$ en $51 \%$ van de variabiliteit in gemeten concentraties. De ruimtelijke structuren van de modellen waren stabiel in de tijd op West-Europese schaal. Dit ondersteunt de toepassing van deze modellen in langlopende epidemiologische studies met cohorten die één of twee decennia geleden zijn gestart.

In Hoofdstuk 4 vergeleken we 16 verschillende algoritmen (lineaire regressie, regularisatie en 'machine learning' algoritmen) om Europa-brede $\mathrm{PM}_{2.5}$ en $\mathrm{NO}_{2}$ modellen te ontwikkelen, met behulp van dezelfde luchtverontreinigingsmetingen en mogelijk voorspellende variabelen die in Hoofdstuk 3 werden gebruikt. We vonden dat de algoritmen gelijkwaardig presteerden, geëvalueerd door vijfvoudige kruisvalidatie en een aanvullende validatie met behulp van een externe dataset. De voorspelde concentraties van alle algoritmen op externe validatielocaties waren sterk gecorreleerd $\left(\mathrm{R}^{2}>\right.$ $0.85)$.

In Hoofdstuk 5 hebben we Europa-brede LUR modellen ontwikkeld voor een aantal elementaire componenten van $\mathrm{PM}_{2.5}$. Acht elementen werden a priori geselecteerd om de belangrijkste bronnen te vertegenwoordigen: koper $(\mathrm{Cu})$, ijzer $(\mathrm{Fe})$ en zink $(\mathrm{Zn})$, die niet-uitlaat verkeersemissies vertegenwoordigen; zwavel (S) staat voor transport over lange afstand van secundaire anorganische aerosolen afkomstig van verbranding van zwavelhoudende vaste en vloeibare brandstoffen; nikkel (Ni) en vanadium (V) vertegenwoordigen een mix van olieverbranding/industrie; silicium ( $\mathrm{Si}$ ), dat aardkorstmateriaal vertegenwoordigt; en kalium $(\mathrm{K})$, dat de verbranding van biomassa vertegenwoordigt. Voor elk element zijn modellen ontwikkeld met gesuperviseerde lineaire regressie en 'random forest', een 'machine learning' algoritme. De modellen presteerden matig tot goed in heel Europa en verklaarden $41 \%$ tot $90 \%$ van de totale ruimtelijke variabiliteit in gemeten concentraties in vijfvoudige kruisvalidatie. 'Random forest' modellen presteerden beter dan gesuperviseerde lineaire regressiemodellen bij het verklaren van de algehele variabiliteit. De LUR modellen verklaarden de variatie binnen een studiegebied veel minder goed dan de algehele variatie. 'Random forest' en gesuperviseerde lineaire regressie modellen waren vergelijkbaar in het verklaren van de binnen-studie variatie. Het gebruik van kaarten bleek een nuttig aanvullend hulpmiddel te zijn om de aannemelijkheid van de modelvoorspellingen te evalueren, naast kruisvalidatie statistieken. De modellen voor de verschillende elementen verschilden aanzienlijk wat betreft de belangrijkste voorspellende variabelen. De belangrijkste voorspellende variabelen die in de modellen waren opgenomen weerspiegelden in grote lijnen de belangrijkste bronnen van de overeenkomstige elementen. De overeenkomst tussen de twee algoritme voorspellingen was hoog op Europees niveau voor $\mathrm{Cu}, \mathrm{K}, \mathrm{S}$ en $\mathrm{Zn}(\mathrm{r}>0.7)$, en varieerde aanzienlijk op nationaal niveau.

In Hoofdstuk 6 hebben we associaties onderzocht tussen blootstelling aan elementaire componenten van $\mathrm{PM}_{2.5}$ en sterfte in het gepoolde ELAPSE cohort (met een totale populatie omvang van 323,782 
deelnemers). Ter vergelijking hebben we blootstellingsmodellen toegepast die zijn ontwikkeld in Hoofdstuk 5 met zowel gesuperviseerde lineaire regressie (SLR) als 'random forest' (RF) algoritmen. Alle SLR-gemodelleerde componenten waren statistisch significant geassocieerd met natuurlijke sterfte in modellen met éénelement, met hazard ratio's (HR's) van 1.05-1.27 over representatieve concentratie bereiken. Vergelijkbare HR's met bredere betrouwbaarheidsintervallen (CI's) werden gevonden voor RF-gemodelleerde $\mathrm{Cu}, \mathrm{Fe}, \mathrm{K}, \mathrm{S}, \mathrm{V}$ en $\mathrm{Zn}$. De HR's voor zowel SLR-als RF- gemodelleerde V verzwakten na correctie voor $\mathrm{PM}_{2.5}$-massa of $\mathrm{NO}_{2}$, maar bleven groter dan één en (borderline) significant. De HR's voor V waren 1.03 (95\% CI: 1.02, 1.05) en 1.06 (95\% CI: 1.02, 1.10) per $2 \mathrm{ng} / \mathrm{m}^{3}$ voor respectievelijk SLR- en RF-gemodelleerde blootstelling, gecorrigeerd voor $\mathrm{PM}_{2.5}$-massa. Associaties voor de andere componenten waren zwakker voor blootstelling gemodelleerd met RF dan SLR in modellen met twee elementen.

In Hoofdstuk 7 bespreken we methodologische kwesties met betrekking tot de betrouwbaarheidsbeoordeling van epidemiologische studies in systematische reviews, de ruimtelijke schaal en statistische algoritmen voor het ontwikkelen van modellen voor blootstelling aan luchtverontreiniging, en bestaande aanwijzingen voor gezondheidseffecten van langdurige blootstelling aan de componenten van fijnstof. De belangrijkste bevindingen van ELAPSE, door toepassing van de luchtverontreinigingsmodellen die in dit proefschrift zijn ontwikkeld, worden samengevat. Ten slotte worden enkele beleidsaanbevelingen gedaan gebaseerd op de huidige stand van kennis. 


\section{Affiliations of Coauthors}

Zorana J. Andersen

Department of Public Health, University of

Copenhagen, Copenhagen, Denmark

\section{Richard Atkinson}

Population Health Research, Institute St George's, University of London, London, UK

Mariska Bauwelinck

Interface Demography - Department of Sociology, Vrije Universiteit Brussel, Brussels, Belgium

\section{Tom Bellander}

Institute of Environmental Medicine, Karolinska

Institutet, Stockholm, Sweden

\section{Jørgen Brandt}

Department of Environmental Science, Aarhus University, Roskilde, Denmark

\section{Bert Brunekreef}

Institute for Risk Assessment Sciences, Utrecht

University, Utrecht, the Netherlands

\section{Giulia Cesaroni}

Department of Epidemiology, Lazio Region Health Service / ASL Roma 1, Rome, Italy

\section{Hans Concin}

Agency for Preventive and Social Medicine (aks), Bregenz, Austria

\section{Aaron van Donkelaar}

Department of Physics and Atmospheric Science, Dalhousie University, Halifax, Nova Scotia, Canada

\section{Daniela Fecht}

MRC Centre for Environment and Health, School of Public Health, Imperial College London, London, UK

\section{Francesco Forastiere}

Department of Epidemiology, Lazio Region Health Service / ASL Roma 1, Rome, Italy

\section{John Gulliver}

Centre for Environmental Health and Sustainability \& School of Geography, University of Leicester, Leicester, UK

\section{Ole Hertel}

Department of Environmental Science, Aarhus University, Roskilde, Denmark

\section{Gerard Hoek}

Institute for Risk Assessment Sciences, Utrecht

University, Utrecht, the Netherlands

\section{Barbara Hoffmann}

Institute for Occupational, Social and

Environmental Medicine, Heinrich Heine University

Düsseldorf, Germany

\section{Kees de Hoogh}

Swiss Tropical and Public Health Institute, Basel, Switzerland

\section{Ulla Arthur Hvidtfeldt}

Danish Cancer Society Research Center, Copenhagen, Denmark

Nicole A. H. Janssen

National Institute for Public Health and the Environment, Bilthoven, the Netherlands

\section{Karl-Heinz Jöckel}

Institute for Medical Informatics, Biometry and Epidemiology, University of Duisburg-Essen, Essen, Germany

\section{Jeanette Jørgensen}

Department of Public Health, University of Copenhagen, Copenhagen, Denmark

\section{Klea Katsouyanni}

Department of Hygiene, Epidemiology and Medical Statistics, National and Kapodistrian University of Athens, Athens, Greece

\section{Matthias Ketzel}

Department of Environmental Science, Aarhus University, Roskilde, Denmark

Jochem O. Klompmaker

Harvard T.H. Chan School of Public Health, Boston, MA, USA

\section{Anton Lager}

Department of Global Public Health, Karolinska Institutet, Stockholm, Sweden

\section{Karin Leander}

Institute of Environmental Medicine, Karolinska Institutet, Stockholm, Sweden

\section{Shuo Liu}

Department of Public Health, University of Copenhagen, Copenhagen, Denmark 


\section{Petter Ljungman}

Institute of Environmental Medicine, Karolinska Institutet, Stockholm, Sweden

\section{Conor J. MacDonald}

CESP, Faculté de Medicine, Université Paris-Saclay, Inserm U1018, Gustave Roussy, Villejuif, France

\section{Patrik K.E. Magnusson}

Department of Medical Epidemiology and Biostatistics, Karolinska Institutet, Stockholm, Sweden

\section{Randall V. Martin}

Department of Energy, Environmental \& Chemical Engineering, Washington University in St. Louis, St. Louis, Missouri, United States

\section{Amar Mehta}

Section of Epidemiology, Department of Public Health, University of Copenhagen, Copenhagen, Denmark

\section{Gabriele Nagel}

Institute of Epidemiology and Medical Biometry, Ulm University, Ulm, Germany

\section{Bente Oftedal}

Department of Environmental Health, Norwegian Institute of Public Health, Oslo, Norway

\section{Göran Pershagen}

Institute of Environmental Medicine, Karolinska Institutet, Stockholm, Sweden

\section{Annette Peters}

Institute of Epidemiology, Helmholtz Zentrum München, Neuherberg, Germany

\section{Ole Raaschou-Nielsen}

Danish Cancer Society Research Center, Copenhagen, Denmark

\section{Matteo Renzi}

Department of Epidemiology, Lazio Region Health Service / ASL Roma 1, Rome, Italy

\section{Debora Rizzuto}

Department of Neurobiology, Care Sciences, and Society, Karolinska Institutet, Stockholm, Sweden

\section{Sophia Rodopolou}

Department of Hygiene, Epidemiology and Medical Statistics, Medical School, National and Kapodistrian University of Athens, Athens, Greece

\section{Evangelia Samoli}

Department of Hygiene, Epidemiology and Medical Statistics, Medical School, National and Kapodistrian University of Athens, Athens, Greece

\section{Yvonne T. van der Schouw}

Julius Center for Health Sciences and Primary Care, University Medical Center Utrecht, Utrecht University, Utrecht, The Netherlands

\section{Sara Schramm}

Institute for Medical Informatics, Biometry and Epidemiology, University of Duisburg-Essen, University Hospital Essen, Germany

\section{Per Schwarze}

Norwegian Institute of Public Health, Oslo, Norway

\section{Torben Sigsgaard}

Department of Public Health, Environment Occupation and Health, Danish Ramazzini Centre, Aarhus University, Roskilde, Denmark

\section{Mette Sørensen}

Danish Cancer Society Research Center, Copenhagen, Denmark

\section{Massimo Stafoggia}

Department of Epidemiology, Lazio Region Health Service / ASL Roma 1, Rome, Italy

\section{Maciej Strak}

National Institute for Public Health and the Environment, Bilthoven, the Netherlands

\section{Anne Tjønneland}

Danish Cancer Society Research Center, Copenhagen, Denmark

\section{Roel Vermeulen}

Institute for Risk Assessment Sciences, Utrecht University, Utrecht, the Netherlands

\section{Danielle Vienneau}

Swiss Tropical and Public Health Institute, Basel, Switzerland

\section{Gudrun Weinmayr}

Institute of Epidemiology and Medical Biometry, Ulm University, Ulm, Germany

\section{Kathrin Wolf}

Institute of Epidemiology, Helmholtz Zentrum München, Neuherberg, Germany 


\section{Acknowledgements}

Completing a PhD is indeed a challenging task and a life-change experience. It would not have been possible without the support and guidance that I received from many people.

I would first like to thank my supervisors Prof. Dr. Bert Brunekreef, Dr. Gerard Hoek and Dr. Maciek Strak. I always feel honor and lucky to study and work under your excellent supervision. I benefitted a lot from our regular internal meetings, where you showed me how international cooperation works, and how to be an independent scientist. Bert, thank you for offering me the opportunity to work in this excellent ELAPSE project, where I received comprehensive training in exposure and epidemiology. Thank you for spending time regularly discussing articles with me at the start of my $\mathrm{PhD}$, regardless of your busy schedule. Thank you so much for all the knowledge and guidance that you have so generously shared with me. Besides the academic instructions, you supported me in applying the scholarship far before my arrival in the Netherlands and helped me settle down in the Netherlands. I only later realized that it is not common for a supervisor to pick up students at the airport ;) I'm so thankful that I have the privilege of being your student. Gerard, thank you for always being there, enlightening me when I got lost in the research. You guided my steps not always by giving instructions, but also by posing questions and leading me to think through what to do and why I would do it. I can always benefit from the inspiring discussions with you. Your passion for research has helped me find my own passion. I learnt everything about exposure science from you, and you encouraged me to learn more in GIS and molecular epidemiology. You also made sure that I loosen up a bit and enjoyed life outside the PhD. I feel so truly lucky to have a supervisor who shows all of the care, understanding and patience that you do. Maciek, you are more like an older brother than a supervisor to me. You helped to improve the performance of my office computer at the very beginning of my PhD, otherwise I might have wasted half of my PhD time on it. You coordinated all my needs at work and facilitated the smooth progression of my PhD. You are always happy to guide me whenever I need help, solving problems even before I realized them. You did an incredible job in coordinating the ELAPSE project. I was so worried that I cannot be as good as you when I first knew I would take over your work in project management. Your optimistic and humorous personality always motivates me. I wish you the absolute best going forward.

I would also like to thank my master supervisor Prof. Dr. Wei Huang at Peking University, who led me to the field of Environmental Epidemiology and encouraged me to pursue a PhD degree under the supervision of Prof. Dr. Bert Brunekreef. You are my role model in life. Many thanks to the China Scholarship Council for funding my PhD research in Utrecht.

I would like to thank the ELAPSE partners and coauthors for their contributions to my work and critical comments to improve my thesis. I would like to particularly acknowledge Dr. Kees de Hoogh, who collaborated and contributed to the exposure assessment work of this thesis. Dr. Danielle Vienneau, many thanks for your proofreading of my manuscripts before submission. The GIS course you organized was very helpful and I had a lot of fun with you that summer in Florence. Marjan, thanks for your amazing job in data management and detailed documentation. I can never forget that the auditors were unstinting in their praise when seeing your work. Aart, thanks for your always prompt responses to emails and for your efficiency in revolving problems. Shuo, we've been working together since our master's study. I hope you have a smooth PhD journey and we can work together in the future again. Prof. Dr. Roel Vermeulen, thanks for sharing your precious comments to improve my first publication.

I would like to thank my IRAS colleagues who collaborated and helped me during the last years. Ingrid, Christina and Petra, thanks for your hospitality and for coordinating logistics during my PhD. 
Gijs and Astrid, thanks for showing me almost everything at IRAS. Marije, thanks for helping me buy the first bike in the Netherlands. Jules, thanks for sharing the R script and your knowledge when I started my first paper. Jack, thanks for sharing the funny jokes and preparing the 'real Dutch pea soup'. Thanks to my roommates - Miaomiao, Adeylson, Bernice, Wouter, Eugenio, Mayu and Arnar - thanks so much for sharing experiences, great fun and lots of talking (in-person and online). Thanks to my fellow PhD students for the small talks, lunch walks and laugh-filled drinks - Myrna, Mariana, Daniel, Ilse, Calvin, Edith, Fleur, Warner, Hicham, Roosmarijn, Jeroen, Jochem, Joseph. Also thanks to other IRAS party committee members for all the fun - Anne, Emma and Sylvia. Big thanks to Lizan - thanks for your always valuable advices and your help with translating the summary of this thesis.

Many thanks to Dongsheng (杨东升) and Min (杨敏) for being my paranymphs of my defense. Dongsheng, thanks for being such a great roommate! Most of the time you just bring all the laughs, but sometimes you surprise me by being extremely considerate. Min, you are full of surprises! Thanks for sharing good recipes, delicious homemade food and crazy ideas. Thanks to Ruiwen (何睿 文), Dingyu (刘丁或), Yongliang (张永亮), Yujia (赵雨佳), Anran (蔡安然), Zhebin (虞哲涁), Cui (郭 萃)，刘明龙，孙丽风，陈度伸，刘燕燕，李泽实，朱林立，赵珊，郎一飞，付东龙，李婧，韦萱，符仲芳 for all the happy times, nice meals and common misery of PhD students we shared. The transition of moving to a new country would not be easy without your encouragements and help. I feel so lucky to meet you guys.

Dear Siqi, thank you for being my best friend and for inspiring me to be a better person. I get anxious easily and your words always calm me down. I know I can always count on you. I am forever indebted to you.

Xianke, thank you for sticking with me even after you've seen how moody and difficult I could get. Thank you for making time for me no matter how busy you are, and for always being supportive.

Finally and most importantly, I would like to express my infinite and endless gratitude to my family. Having a family like you makes me feel so deeply loved and gives me strength. Grandma, thanks for allowing me to grow and flourish in my way. I would not be the person I am today without your help. Mom and Dad, I know I am safe and loved with you, and that feeling is priceless. Thank you for always being there to support me. A special thank you to 子俊, my cousin, for making the cover of this thesis. I love it! 


\section{Curriculum Vitae}

Jie Chen was born on the $25^{\text {th }}$ of December, 1990 in Quanzhou, Fujian province, China. After graduating from high school in 2009, she studied Preventive Medicine at Peking University, China. She obtained her Bachelor of Science degree in 2014 and continued to study for a Master's degree in Environmental Health at Peking University, under the supervision of Prof. Wei Huang. During her master's study, she participated in several research projects focusing on acute health effects of air pollution and developed an interest in environmental epidemiology. In 2016, she started her PhD at the Institute for Risk Assessment Sciences, Utrecht University. She was supervised by Prof. Dr. Bert Brunekreef, Dr. Gerard Hoek and Dr. Maciek Strak. She has been working within the Health Effects Institute (HEI)-financed project 'Effects of Low-Level Air Pollution: A Study in Europe' (ELAPSE), which is coordinated by Utrecht University. Her research focused on air pollution exposure assessment and health effect evaluation. She has been working as the project manager of ELAPSE since 2019. She was also a member of the systematic review team, working for the update of the WHO Global Air Quality Guidelines (under the supervision of Dr. Gerard Hoek). Jie is currently doing her postdoctoral research within ELAPSE. 


\section{List of publications}

- Chen, J., Rodopoulou, S., de Hoogh, K., Strak, M., Andersen, Z. J., Atkinson, R., Bauwelinck, M., Bellander, T., Brandt, J., Cesaroni, G., Concin, H., Fecht, D., Forastiere, F., Gulliver, J., Hertel, O., Hoffmann, B., Hvidtfeldt, U. A., Janssen, N. A. H., Jöckel, K.-H., Jørgensen, J. T., Katsouyanni, K., Ketzel, M., Klompmaker, J. O., Lager, A., Leander, K., Liu, S., Ljungman, P. L. S., MacDonald, C. J., Magnusson, P. K. E., Mehta, A. J., Nagel, G., Oftedal, B., Pershagen, G., Peters, A., Raaschou-Nielsen, O., Renzi, M., Rizzuto, D., Samoli, E., Schouw, Y. T. v. d., Schramm, S., Schwarze, P., Sigsgaard, T., Sørensen, M., Stafoggia, M., Tjønneland, A., Vienneau, D., Weinmayr, G., Wolf, K., Brunekreef, B. \& Hoek, G. Long-term exposure to fine particle elemental components and natural and causespecific mortality - a pooled analysis of eight European cohorts within the ELAPSE project. Environmental Health Perspectives, 129(4): 047009, 2021.

- Hvidtfeldt, U. A., Chen, J., Andersen, Z. J., Atkinson, R., Bauwelinck, M., Bellander, T., Brandt, J., Brunekreef, B., Cesaroni, G., Concin, H., Fecht, D., Forastiere, F., van Gils, C. H., Gulliver, J., Hertel, O., Hoek, G., Hoffmann, B., de Hoogh, K., Janssen, N., Jørgensen, J. T., Katsouyanni, K., Jöckel, K.-H., Ketzel, M., Klompmaker, J. O., Lang, A., Leander, K., Liu, S., Ljungman, P. L. S., Magnusson, P. K. E., Mehta, A. J., Nagel, G., Oftedal, B., Pershagen, G., Peter, R. S., Peters, A., Renzi, M., Rizzuto, D., Rodopoulou, S., Samoli, E., Schwarze, P. E., Severi, G., Sigsgaard, T., Stafoggia, M., Strak, M., Vienneau, D., Weinmayr, G., Wolf, K. \& Raaschou-Nielsen, O. Long-term exposure to fine particle elemental components and lung cancer incidence in the ELAPSE pooled cohort. Environmental Research, 193:110568, 2021.

- Mortamais, M., Gutierrez, L.-A., de Hoogh, K., Chen, J., Vienneau, D., Carrière, I., Letellier, N., Helmer, C., Gabelle, A., Mura, T., Sunyer, J., Benmarhnia, T., Jacquemin, B. \& Berr, C. Long-term exposure to ambient air pollution and risk of dementia: Results of the prospective Three-City Study. Environment International 148, 106376, 2021.

- Samoli, E., Rodopoulou, S., Hvidtfeldt, U. A., Wolf, K., Stafoggia, M., Brunekreef, B., Strak, M., Chen, J., Andersen, Z. J., Atkinson, R., Bauwelinck, M., Bellander, T., Brandt, J., Cesaroni, G., Forastiere, F., Fecht, D., Gulliver, J., Hertel, O., Hoffmann, B., de Hoogh, K., Janssen, N. A. H., Ketzel, M., Klompmaker, J. O., Liu, S., Ljungman, P., Nagel, G., Oftedal, B., Pershagen, G., Peters, A., Raaschou-Nielsen, O., Renzi, M., Kristoffersen, D. T., Severi, G., Sigsgaard, T., Vienneau, D., Weinmayr, G., Hoek, G. \& Katsouyanni, K. Modeling multi-level survival data in multi-center epidemiological cohort studies: Applications from the ELAPSE project. Environment International 147, 106371, 2021.

- $\quad$ Liu, S., Jørgensen, J. T., Ljungman, P., Pershagen, G., Bellander, T., Leander, K., Magnusson, P. K. E., Rizzuto, D., Hvidtfeldt, U. A., Raaschou-Nielsen, O., Wolf, K., Hoffmann, B., Brunekreef, B., Strak, M., Chen, J., Mehta, A., Atkinson, R. W., Bauwelinck, M., Varraso, R., Boutron-Ruault, M.-C., Brandt, J., Cesaroni, G., Forastiere, F., Fecht, D., Gulliver, J., Hertel, O., de Hoogh, K., Janssen, N. A. H., Katsouyanni, K., Ketzel, M., Klompmaker, J. O., Nagel, G., Oftedal, B., Peters, A., Tjønneland, A., Rodopoulou, S. P., Samoli, E., Bekkevold, T., Sigsgaard, T., Stafoggia, M., Vienneau, D., Weinmayr, G., Hoek, G. \& Andersen, Z. J. Long-term exposure to low-level air pollution and incidence of chronic obstructive pulmonary disease: The ELAPSE project. Environment International 146, 106267, 2021.

- Klompmaker, J. O., Janssen, N., Andersen, Z. J., Atkinson, R., Bauwelinck, M., Chen, J., de Hoogh, K., Houthuijs, D., Katsouyanni, K., Marra, M., Oftedal, B., Rodopoulou, S., Samoli, E., Stafoggia, M., Strak, M., Swart, W., Wesseling, J., Vienneau, D., Brunekreef, B. \& Hoek, G. Comparison of associations between mortality and air pollution exposure estimated with a hybrid, a land-use regression and a dispersion model. Environment International 146, 106306, 2021.

- Hvidtfeldt, U. A., Severi, G., Andersen, Z. J., Atkinson, R., Bauwelinck, M., Bellander, T., Boutron-Ruault, M. C., Brandt, J., Brunekreef, B., Cesaroni, G., Chen, J., Concin, H., Forastiere, F., van Gils, C. H., Gulliver, J., Hertel, O., Hoek, G., Hoffmann, B., de Hoogh, K., Janssen, N., Jockel, K. H., Jorgensen, J. T., Katsouyanni, K., Ketzel, M., Klompmaker, J. O., Krog, N. H., Lang, A., Leander, K., Liu, S., Ljungman, P. L. S., Magnusson, P. K. E., Mehta, A. J., Nagel, G., Oftedal, B., Pershagen, G., Peter, R. S., Peters, A., Renzi, M., Rizzuto, D., Rodopoulou, S., Samoli, E., Schwarze, P. E., Sigsgaard, T., Simonsen, M. K., Stafoggia, M., Strak, M., Vienneau, D., Weinmayr, G., Wolf, K., Raaschou-Nielsen, O. \& Fecht, D. Long-term low-level ambient air pollution exposure and risk of lung cancer - A pooled analysis of 7 European cohorts. Environ Int 146: 106249, 2021.

- Liu, S., Jorgensen, J. T., Ljungman, P., Pershagen, G., Bellander, T., Leander, K., Magnusson, P. K. E., Rizzuto, D., Hvidtfeldt, U. A., Raaschou-Nielsen, O., Wolf, K., Hoffmann, B., Brunekreef, B., Strak, M., Chen, J., Mehta, A., Atkinson, R. W., Bauwelinck, M., Varraso, R., Boutron-Ruault, M. C., Brandt, J., Cesaroni, G., Forastiere, F., Fecht, D., Gulliver, J., Hertel, O., de Hoogh, K., Janssen, N. A. H., Katsouyanni, K., Ketzel, M., Klompmaker, J. O., Nagel, G., Oftedal, B., Peters, A., Tjonneland, A., Rodopoulou, S. P., Samoli, E., Kristoffersen, D. T., Sigsgaard, T., Stafoggia, M., Vienneau, D., Weinmayr, G., Hoek, G. \& Andersen, Z. J. Long-term exposure to low-level air pollution and incidence of asthma: the ELAPSE project. Eur Respir J, 2021. 
- Chen, J. \& Hoek, G. Long-term exposure to PM and all-cause and cause-specific mortality: A systematic review and meta-analysis. Environ Int, 105974, 2020.

- Chen, J., de Hoogh, K., Gulliver, J., Hoffmann, B., Hertel, O., Ketzel, M., Weinmayr, G., Bauwelinck, M., van Donkelaar, A., Hvidtfeldt, U. A., Atkinson, R., Janssen, N. A. H., Martin, R. V., Samoli, E., Andersen, Z. J., Oftedal, B. M., Stafoggia, M., Bellander, T., Strak, M., Wolf, K., Vienneau, D., Brunekreef, B. \& Hoek, G. Development of Europe-Wide Models for Particle Elemental Composition Using Supervised Linear Regression and Random Forest. Environ Sci Technol, 2020.

- Chen, J., de Hoogh, K., Gulliver, J., Hoffmann, B., Hertel, O., Ketzel, M., Bauwelinck, M., van Donkelaar, A., Hvidtfeldt, U. A., Katsouyanni, K., Janssen, N. A. H., Martin, R. V., Samoli, E., Schwartz, P. E., Stafoggia, M., Bellander, T., Strak, M., Wolf, K., Vienneau, D., Vermeulen, R., Brunekreef, B. \& Hoek, G. A comparison of linear regression, regularization, and machine learning algorithms to develop Europe-wide spatial models of fine particles and nitrogen dioxide. Environ Int 130, 104934, doi:10.1016/j.envint.2019.104934, 2019.

- $\quad$ Xu, H., Chen, J., Zhao, Q., Zhang, Y., Wang, T., Feng, B., Wang, Y., Liu, S., Yi, T., Liu, S., Wu, R., Zhang, Q., Fang, J., Song, X., Rajagopalan, S., Li, J., Brook, R. \& Huang, W. Ambient air pollution is associated with cardiac repolarization abnormalities in healthy adults. Environmental Research 171, 239-246, 2019.

- $\quad$ Xu, H., Wang, T., Liu, S., Brook, R., Feng, B., Zhao, Q., Song, X., Yi, T., Chen, J., Zhang, Y., Wang, Y., Zheng, L., Rajagopalan, S., Li, J. \& Huang, W. Extreme Levels of Air Pollution Associated With Changes in Biomarkers of Atherosclerotic Plaque Vulnerability and Thrombogenicity in Healthy Adults The Beijing AIRCHD Study. Circulation research 124, E30-E43, 2019.

- $\quad$ Xu, H., Brook, R. D., Wang, T., Song, X., Feng, B., Yi, T., Liu, S., Wu, R., Chen, J., Zhang, Y., Liu, S., Zhao, Q., Wang, Y., Zheng, L., Huo, Y., Rajagopalan, S., Li, J. \& Huang, W. Short-term effects of ambient air pollution and outdoor temperature on biomarkers of myocardial damage, inflammation and oxidative stress in healthy adults. Environmental Epidemiology 3, 2019.

- Wu, R., Song, X., Chen, D., Zhong, L., Huang, X., Bai, Y., Hu, W., Ye, S., Xu, H., Feng, B., Wang, T., Zhu, Y., Fang, J., Liu, S., Chen, J., Wang, X., Zhang, Y. \& Huang, W. Health benefit of air quality improvement in Guangzhou, China: Results from a long time-series analysis (2006-2016). Environment International 126, 552-559, 2019.

- $\quad$ Li, J., Zhou, C., Xu, H., Brook, R., Liu, S., Yi, T., Wang, Y., Feng, B., Zhao, M., Wang, X., Zhao, Q., Chen, J., Song, X., Wang, T., Liu, S., Zhang, Y., Wu, R., Gao, J., Pan, B., Pennathur, S., Rajagopalan, S., Huo, Y., Zheng, L. \& Huang, W. Ambient Air Pollution Is Associated With HDL (High-Density Lipoprotein) Dysfunction in Healthy Adults. Arterioscl Throm Vas 39, 513-522, 2019.

- $\quad$ Feng, B., Song, X., Dan, M., Yu, J., Wang, Q., Shu, M., Xu, H., Wang, T., Chen, J., Zhang, Y., Zhao, Q., Wu, R., Liu, S., Yu, J. Z., Wang, T. \& Huang, W. High level of source-specific particulate matter air pollution associated with cardiac arrhythmias. The Science of the total environment 657, 1285-1293, 2019.

- $\quad$ Feng, B., Li, L., Xu, H., Wang, T., Wu, R., Chen, J., Zhang, Y., Liu, S., Ho, S. S. H., Cao, J. \& Huang, W. PM2.5-bound polycyclic aromatic hydrocarbons (PAHs) in Beijing: Seasonal variations, sources, and risk assessment. Journal of Environmental Sciences 77, 11-19, 2019.

- de Hoogh, K., Chen, J., Gulliver, J., Hoffmann, B., Hertel, O., Ketzel, M., Bauwelinck, M., van Donkelaar, A., Hvidtfeldt, U. A., Katsouyanni, K., Klompmaker, J., Martin, R. V., Samoli, E., Schwartz, P. E., Stafoggia, M., Bellander, T., Strak, M., Wolf, K., Vienneau, D., Brunekreef, B. \& Hoek, G. Spatial $\mathrm{PM}_{2.5}, \mathrm{NO}_{2}, \mathrm{O}_{3}$ and $\mathrm{BC}$ models for Western Europe - Evaluation of spatiotemporal stability. Environ Int 120, 81-92, 2018.

- Wu, R., Song, X., Bai, Y., Chen, J., Zhao, Q., Liu, S., Xu, H., Wang, T., Feng, B., Zhang, Y., Zhong, L., Wang, X., Wu, F. \& Huang, W. Are current Chinese national ambient air quality standards on 24-hour averages for particulate matter sufficient to protect public health? J Environ Sci 71, 67-75, 2018.

- $\quad$ Liu, S., Chen, J., Zhao, Q., Song, X., Shao, D., Meliefste, K., Du, Y., Wang, J., Wang, M., Wang, T., Feng, B., Wu, R., $\mathrm{Xu}$, H., Bei, H., Brunekreef, B. \& Huang, W. Cardiovascular benefits of short-term indoor air filtration intervention in elderly living in Beijing: An extended analysis of BIAPSY study. Environ Res 167, 632-638, 2018.

- Huang, W., Wang, L., Li, J. P., Liu, M. C., Xu, H. B., Liu, S. C., Chen, J., Zhang, Y., Morishita, M., Bard, R. L., Harkema, J. R., Rajagopalan, S. \& Brook, R. D. Short-Term Blood Pressure Responses to Ambient Fine Particulate Matter Exposures at the Extremes of Global Air Pollution Concentrations. American Journal of Hypertension 31, 590-599, 2018.

- Shao, D., Du, Y., Liu, S., Brunekreef, B., Meliefste, K., Zhao, Q., Chen, J., Song, X., Wang, M., Wang, J., Xu, H., Wu, R., Wang, T., Feng, B., Lung, C. S., Wang, X., He, B. \& Huang, W. Cardiorespiratory responses of air filtration: A randomized crossover intervention trial in seniors living in Beijing: Beijing Indoor Air Purifier StudY, BIAPSY. The Science of the total environment 603-604, 541-549, 2017. 


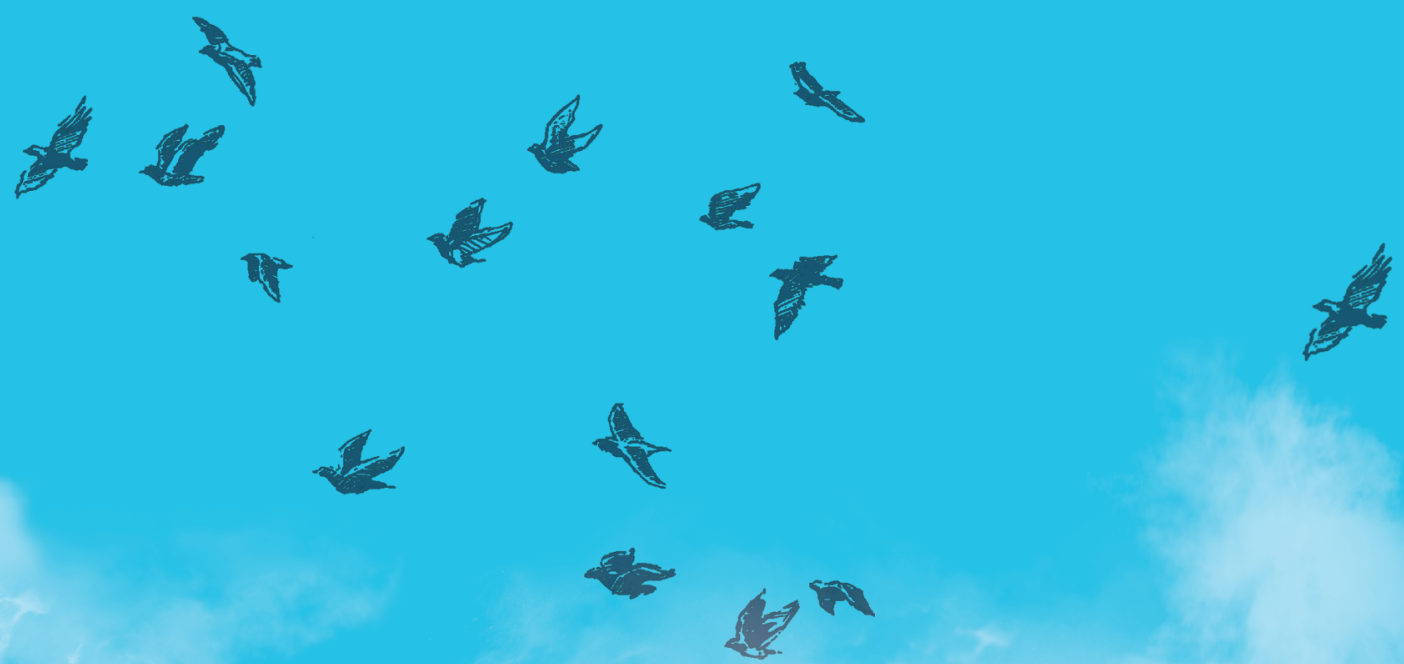